Supporting Information

\title{
Synthesis of Cyclobutanones by Gold(I)-Catalyzed [2+2] Cycloaddition of Ynol Ethers with Alkenes
}

\author{
Margherita Zanini, ${ }^{\dagger, \dagger}$ Andrea Cataffo,,$+\star \star \star A$ Antonio M. Echavarren $* \dagger,+$ \\ $\dagger$ Institute of Chemical Research of Catalonia (ICIQ), Barcelona Institute of Science and \\ Technology, Av. Països Catalans 16, 43007 Tarragona, Spain \\ * Departament de Química Analítica i Química Orgànica, Universitat Rovira i Virgili, \\ C/Marcel·li Domingo s/n, 43007 Tarragona, Spain.
}

Email:

aechavarren@iciq.es 


\section{Contents}

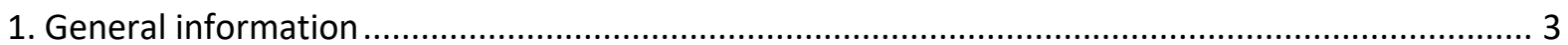

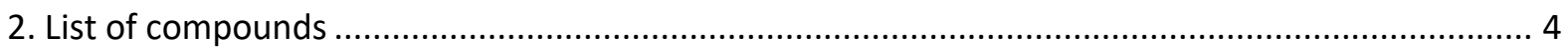

3. Optimization of the gold(I)-catalyzed (4+2) cycloaddition of internal ynol ethers with alkenes ...... 5

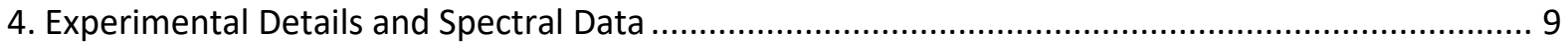

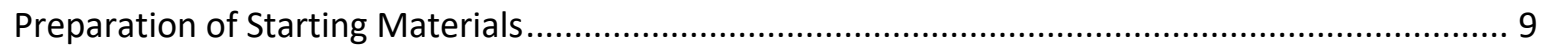

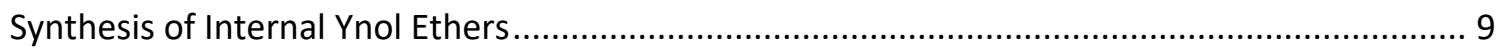

Scope of the gold(I)-catalyzed [2+2] cycloaddition reaction of ynol ethers with alkenes............... 21

General procedure B: Gold(I)-catalyzed reaction of terminal ynol ethers with alkenes ............. 21

General procedure C: Gold(I)-catalyzed reaction of internal ynol ethers with alkenes .............. 98

One-pot Synthesis of Cyclobutanones Starting from Ynol ethers ............................................... 134

General procedure D: one-pot synthesis of cyclobutanones 5 starting from ynol ethers 1 ..... 135

$1 \mathrm{mmol}$ scale one-pot synthesis of cyclobutanones 5 a starting from ynol ethers $1 \mathrm{a}$................ 136

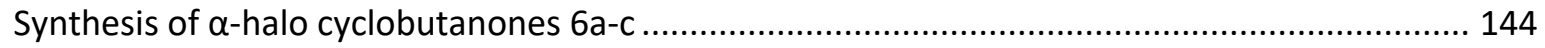

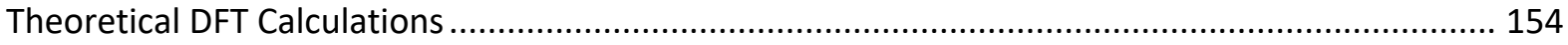

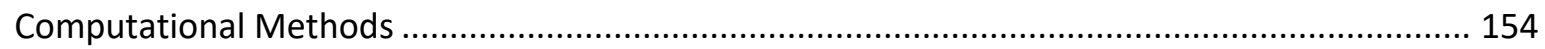

Mechanistic Studies on the Gold(I) Catalyzed Reactions of Ynol Ethers ..................................... 154

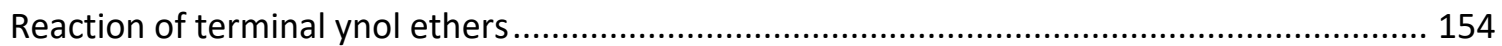

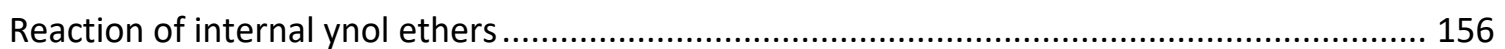

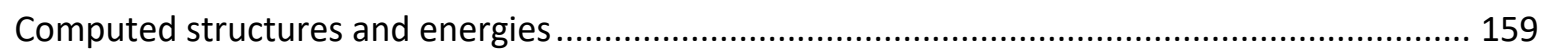

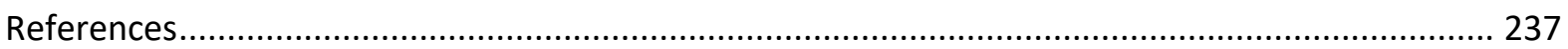




\section{General information}

Anhydrous reactions were performed under nitrogen or argon in solvents dried by passing through an activated alumina column on a PureSolv ${ }^{\mathrm{TM}}$ solvent purification system (Innovative Technologies, Inc., MA). Analytical thin layer chromatography was carried out using TLC-aluminium sheets with $0.2 \mathrm{~mm}$ of silica gel (Merck $\mathrm{GF}_{234}$ ) using UV light as the visualizing agent, and an acidic solution of vanillin in ethanol or a basic aqueous solution of $\mathrm{KMnO}_{4}$ as the developing agent. Purifications by chromatography were carried out using flash grade silica gel (SDS Chromatogel 60 ACC, 40-63 mm). Organic solutions were concentrated under reduced pressure on Büchi or IKA rotary evaporators. NMR spectra were recorded at $298 \mathrm{~K}$ on a Bruker Avance 300, Bruker Avance 400 Ultrashield and Bruker Avance 500 Ultrashield apparatuses. The signals are given as $\delta / \mathrm{ppm}$ (multiplicity, coupling constant (Hertz), number of protons) downfield from tetramethylsilane, with calibration on the residual protio-solvent used $\left(\delta_{\mathrm{H}}=7.26 \mathrm{ppm}\right.$ and $\delta_{\mathrm{C}}=77.16 \mathrm{ppm}$ for $\mathrm{CDCl}_{3}, \delta_{\mathrm{H}}=5.32 \mathrm{ppm}$ and $\delta_{\mathrm{C}}=54.00 \mathrm{ppm}$ for $\mathrm{CD}_{2} \mathrm{Cl}_{2}$,). Mass spectra were recorded on a Waters UPLC-QqTOF (Maxis Impact, Bruker Daltonics) with ESI and APCI, or a Waters Alliance HPLC-TOF (MicroTOF Focus, Bruker Daltonics) with ESI and APCI. Melting points were determined using a Büchi melting point apparatus. Infrared spectra were recorded on a Bruker ALPHA FTIR-ATR TR0 spectrometer with ATR module on a thin film (the sample was dissolved in a volatile solvent, then the solvent evaporated). The most intense absorption bands $(v)$ are listed in wavenumbers $\left(\mathrm{cm}^{-1}\right)$. Specific optical rotation measurements were carried out on a Jasco P-1030 model polarimeter equipped with a PMT detector using the sodium line at $589 \mathrm{~nm}$, and $2 \mathrm{~mL}$ (100 mm pathlength) cells.

All reagents were used as purchased, with no further purification. Complexes $\left[(t \mathrm{BuXPhos}) \operatorname{AuNCMe}_{\mathrm{BAAr}}{ }^{\mathrm{F}}(\mathbf{A}),{ }^{\mathrm{i}}\right.$ and [(IPr)AuNCMe]SbF6 (C) $)^{\text {ii }}$ were synthesized according to previously reported procedures. The NMR data are in agreement with the ones reported in the literature. 


\section{List of compounds}

Successful ynol ethers prepared (if known and prepared according to published procedure, the reference is reported below the structure) ${ }^{\mathrm{iii}}$<smiles>C#COc1ccccc1</smiles>

1a (Ref. 3)<smiles>C(#Cc1ccccc1)c1ccccc1</smiles>

$1 f$<smiles>C#COc1ccc(C(F)(F)F)cc1</smiles>

(Ref. 3)<smiles>C#COc1cc(OC)cc(OC)c1</smiles>

1c

(Ref. 3)<smiles>C#COc1cccc2ccccc12</smiles>

1d

(Ref. 3)<smiles>C#COc1ccc(Cl)cc1</smiles><smiles>COc1ccc(C#COc2ccccc2)cc1</smiles>

$1 \mathrm{~g}$<smiles>Cc1ccc(C#COc2ccccc2)cc1</smiles>

$1 \mathbf{i}$<smiles>COc1ccccc1OC#Cc1ccccc1</smiles>

1j

Successful alkenes prepared or purchased from commercial vendors (if prepared according to published procedure, the reference is reported below the structure $)^{\mathrm{iv}}$, v, vi , vii viii<smiles>C=C(C)c1ccccc1</smiles>

$\mathbf{2 a}$<smiles>CC(=Cc1ccc(C(C)(C)C)cc1)C(C)(C)C</smiles>

$2 b$ (Ref. 4)<smiles>C=C(C)c1ccc(F)cc1</smiles>

2c<smiles>C=C(C)c1ccc(-c2ccccc2)cc1</smiles>

2d

(Ref. 5)<smiles>C=C1CCCc2ccccc21</smiles>

$2 e$

(Ref. 6)<smiles>C=C(c1ccccc1)c1ccccc1</smiles>

2f

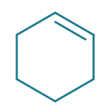

2I<smiles>C1=CC2CCCCCC2C1</smiles>

$2 m$<smiles>C1=CC2CCCCC(CCC1)C2</smiles>

2n<smiles>C1=CCCCCC=CC1</smiles>

20<smiles>CC1=CC[C@@H]2C(C1)C2(C)C</smiles>

$2 p$ 2j<smiles>C=C(C)CCc1ccccc1</smiles>

$2 q$

(Ref. 4)<smiles>CC=C(C)C</smiles><smiles>CC(C)=C1CCC1C</smiles>

2s<smiles>C=C1CC/C=C(/CC)CC[C@@H]2[C@@H]1CC2(C)C</smiles>

2u<smiles>C=C1CCCCC1</smiles>

2v<smiles>CCCCOC=C1CCCCC1</smiles>

2w (Ref. 7)<smiles></smiles>

2x

(Ref. 8) 


\section{Optimization of the gold(I)-catalyzed $(4+2)$ cycloaddition of internal ynol ethers with alkenes}

Table S.1: Catalyst screening for the intermolecular reaction of $\mathbf{1 f}$ with $\mathbf{2 a}{ }^{a}$

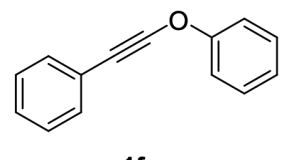

16

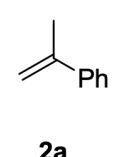

$2 a$

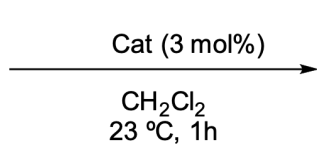

$23^{\circ} \mathrm{C}, 1 \mathrm{~h}$

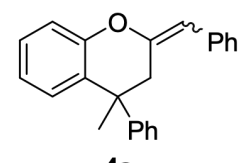

4a

\begin{tabular}{|c|c|c|c|}
\hline Entry & Catalyst & Yield $(\%)^{b}$ & $\mathrm{E}: Z^{b}$ \\
\hline 1 & B & 55 & $1: 3$ \\
\hline 2 & $\mathbf{A}$ & $79(76)$ & $1: 1$ \\
\hline 3 & $\mathbf{E}$ & 30 & $1: 2$ \\
\hline 4 & $\mathbf{F}$ & 44 & $1: 1.7$ \\
\hline 5 & G & 57 & $1: 4$ \\
\hline 6 & C & 49 & $1: 1.2$ \\
\hline 7 & D & 45 & $1: 3$ \\
\hline 8 & $\mathrm{InBr}_{3}$ & 40 & $>1: 20$ \\
\hline 9 & $\mathrm{InOTf}_{3}$ & $--^{c}$ & $--^{c}$ \\
\hline 10 & $\mathrm{InCl}_{3}$ & $--^{c}$ & $--^{c}$ \\
\hline 11 & TFA & $-d$ & $-d$ \\
\hline
\end{tabular}

${ }^{a}$ Substrates 1f:2a in a 1:2 ratio. ${ }^{b}$ Yields determined by ${ }^{1} \mathrm{H}$ NMR using trichloroethylene as internal standard. Isolated yields in parentheses. ${ }^{c}$ Decomposition of 1f. ${ }^{d}$ No reaction 


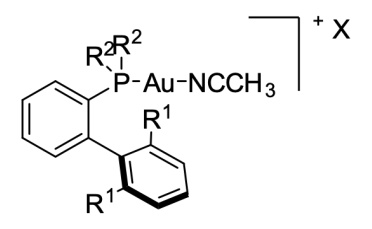

$A R^{1}=\mathbb{P r}, R^{2}=t B u, X=B A r_{4}{ }^{F-}$

B: $R^{1}=H, R^{2}=t B u, X=S b F_{6}$ -

$E: R^{1}=\operatorname{Pr}, R^{2}=t B u, X=S_{b F}$

$F: R^{1}=H, R^{2}=C y, X=S b F_{6}$

G: $R^{1}=H, R^{2}=t B u, X=B A r_{4}{ }^{F-}$

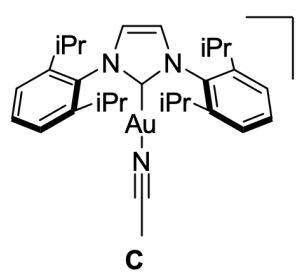

$\mathrm{SbF}_{6}^{-}$

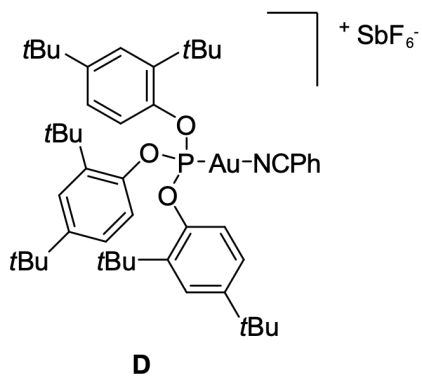

Table S.2. Solvent screening for the intermolecular reaction of $\mathbf{1 f}$ with $2 \mathbf{a}^{a}$<smiles>C(#Cc1ccccc1)c1ccccc1</smiles>

$1 f$

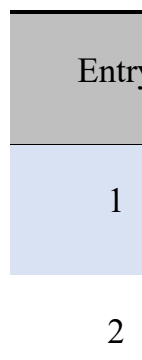

3

4

5

6

7

8

9

10

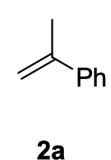

2a

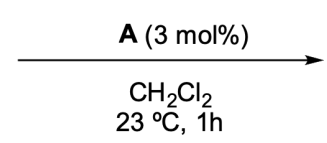

$23^{\circ} \mathrm{C}, 1 \mathrm{~h}$

Yield $(\%)^{b}$

$79(76)$

67

$1: 1$

77

$1.3: 1$

$\mathrm{PhCH}_{3}$

THF

47

$1: 2$

$\mathrm{PhCF}_{3}$

77

$1: 1$

$\mathrm{CH}_{3} \mathrm{NO}_{2}$

EtOAc

69

$1: 1$

Hexane

62

$1.5: 1$

1,4-Dioxane

79

$1.4: 1$

Neat

60

$1.3: 1$

${ }^{a}$ Substrates 1f:2a in a 1:2 ratio. ${ }^{b}$ Yields determined by ${ }^{1} \mathrm{H}$ NMR 
using trichloroethylene as internal standard. Isolated yields in parentheses.

Table S.3. Screening of conditions for the gold(I)-catalyzed (4+2) cycloaddition of $\mathbf{1 f}$ with $\mathbf{2 a}$.

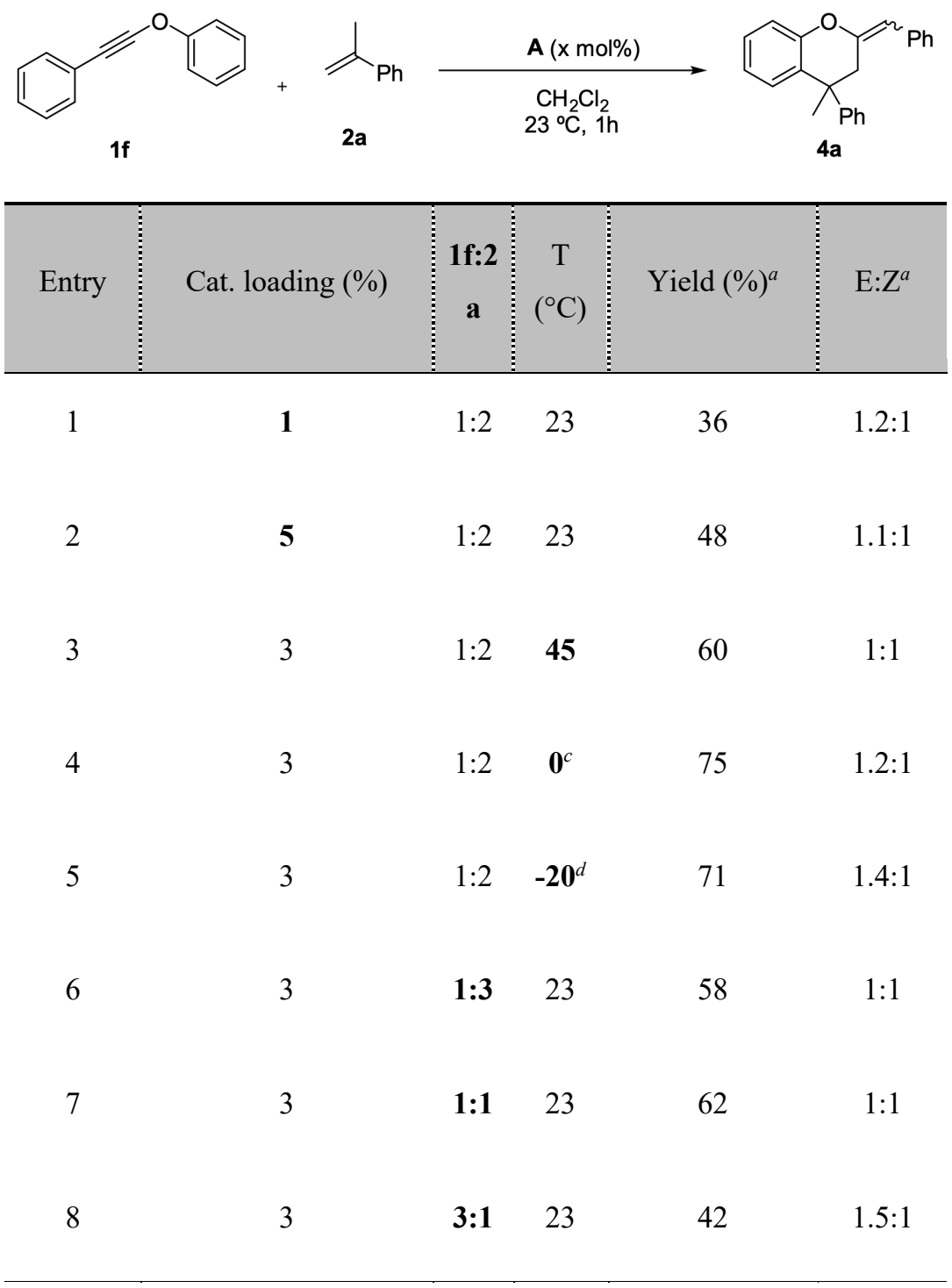

${ }^{a}$ Yields determined by ${ }^{1} \mathrm{H}$ NMR using trichloroethylene as internal standard.

${ }^{b}$ Reaction time 20 minutes. ${ }^{c}$ Reaction time 4 h. ${ }^{d}$ Reaction time $14 \mathrm{~h}$. 
During the purification of $\mathbf{4 a}$ by column chromatography on silica gel we observed the partial $\mathrm{E} / \mathrm{Z}$ isomerization of the double bond, leading also to the formation of a third product identified as endo-4a (Scheme S.1). According to DFT calculations $\mathbf{Z}-4 \mathbf{a}$ is slightly the most stable of the three isomers (by just $0.2 \mathrm{kcal} \mathrm{mol}^{-1}$ ).
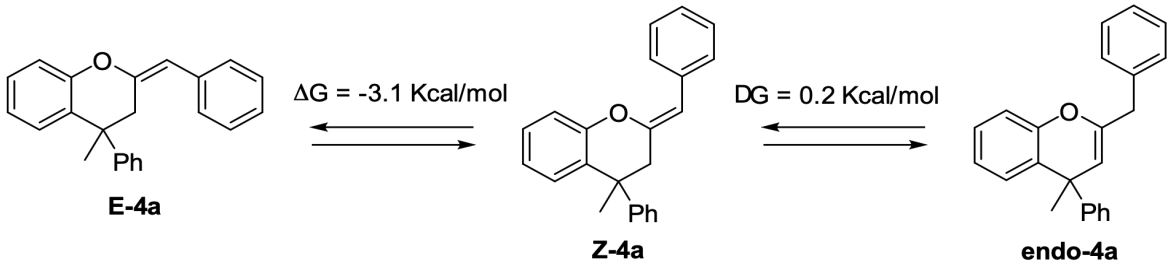

Scheme S.1. Equilibrium and energy barriers for the three stereoisomers of 4 a.

We attempted then to favor the isomerization of the double bond towards a single product, however without success (Table S.4).

Table S.4. Attempts towards the isomerization of the double bond in $\mathbf{4 a}$.

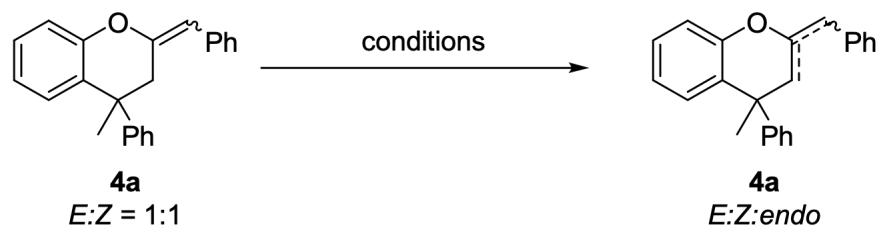

\begin{tabular}{|c|c|c|}
\hline Entry & Conditions $^{c}$ & $E: Z$ :endo $\left(\right.$ yield\%) ${ }^{a}$ \\
\hline 1 & Silica gel - 40 minutes & $-: 1: 2$ \\
\hline 2 & Silica gel - $4 \mathrm{~h}$ & $-: 1: 2$ \\
\hline 3 & $2 \mathrm{M} \mathrm{HCl}$ in water (1 equiv) $-15 \mathrm{~h}^{d}$ & $-: 1.3: 1$ \\
\hline 4 & Acetic acid (1 equiv) - $1 \mathrm{~h}$ & $-: 1: 2$ \\
\hline 5 & Citric acid (1 equiv) - $1 \mathrm{~h}$ & $-: 3: 1$ \\
\hline 6 & (1 equiv) $-1 \mathrm{~h}$ & $-:-: 1(40 \%)^{b}$ \\
\hline
\end{tabular}

${ }^{a}$ Ratio between the isomers determined by ${ }^{1} \mathrm{H}$ NMR using trichloroethylene as internal standard. ${ }^{b}$ Yield determined by ${ }^{1} \mathrm{H}$ NMR using trichloroethylene as internal standard. ${ }^{c}$ Reaction performed at 23 ${ }^{\circ} \mathrm{C} .{ }^{d}$ Reaction performed at $40{ }^{\circ} \mathrm{C}$. 


\section{Experimental Details and Spectral Data}

\section{Preparation of Starting Materials}

\section{Synthesis of Internal Ynol Ethers}

Internal ynol ethers 1f,h-j were synthesized following a modified reported procedure. ${ }^{\text {ix }}$

General procedure A: Synthesis of internal ynol ethers

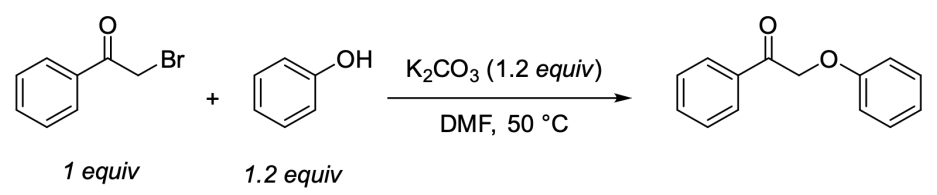

The phenol (6.0, mmol, 1.2 equiv) and $\mathrm{K}_{2} \mathrm{CO}_{3}(6.0 \mathrm{mmol}, 1.2$ equiv) were suspended in dry DMF $(0.3 \mathrm{M})$ and stirred for 30 minutes, then the needed 2-bromoketone ( $5.0 \mathrm{mmol}, 1$ equiv) was added and the mixture was stirred at $50{ }^{\circ} \mathrm{C}$ (silicon oil bath) until complete conversion was observed monitoring the reaction by GC-MS. The reaction was then quenched with water and the crude product was extracted three times with EtOAc. The collected organic phases were washed several times with $\mathrm{KOH}\left(2 \mathrm{M}\right.$ in $\left.\mathrm{H}_{2} \mathrm{O}\right)$ and Brine, dried over $\mathrm{Na}_{2} \mathrm{SO}_{4}$ and the solvent evaporated. The crude product was filtered through a plug of silica gel and used in the following step without further purifications.

Note: In some case larger excess of phenol was necessary and after the filtration through silica gel the product was obtained as a mixture with residual phenol. The presence of the phenol does not affect the following step as far as its amount remains less than the $20 \%$ of the total. In case of a larger excess a second washing with $\mathrm{KOH}$ $2 \mathrm{M}$ in $\mathrm{H}_{2} \mathrm{O}$ it will be necessary.

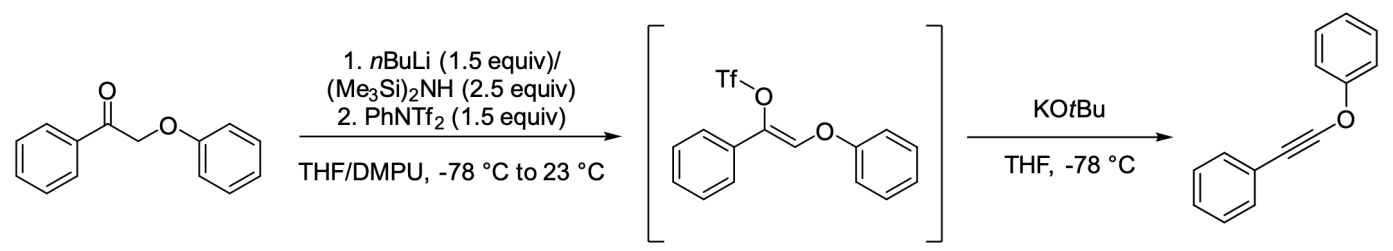

Bis(trimethylsilyl)amine (12.5 mmol, 2.5 equiv) dissolved in THF (5 mL, $1 \mathrm{M}$ with respect of the limiting reagent) under argon was cooled to $0^{\circ} \mathrm{C}$ in an ice bath and butyllithium $(7.5 \mathrm{mmol}, 2.5 \mathrm{M}$ in hexane, 1.5 equiv) was added dropwise. After 10 minutes, the mixture was cooled to $-78^{\circ} \mathrm{C}$ and a solution of the crude mixture from the previous step $(5.0 \mathrm{mmol}, 1$ equiv) in THF $(5 \mathrm{~mL}, 1 \mathrm{M})$ was added dropwise. The reaction mixture was stirred for $1 \mathrm{~h}$ at $78{ }^{\circ} \mathrm{C}$, and then a solution of 1,1,1-trifluoro-N-phenyl-N-((trifluoromethyl)sulfonyl)methanesulfonamide (7.5 mmol, 1.5 equiv) in 1:1 mixture DMPU:THF (total volume $5 \mathrm{~mL}: 1 \mathrm{M}$ with respect of the limiting reagent) was added rapidly (1s). The mixture was allowed to warm to room temperature and then was stirred for $1 \mathrm{~h}$. The reaction mixture was quenched with ice-cold saturated $\mathrm{NaHCO}_{3}$ solution and was then diluted with ether. The phases were separated, and the aqueous layer was back-extracted with ether. The combined organic phases were then dried over $\mathrm{Na}_{2} \mathrm{SO}_{4}$, filtered, and concentrated in vacuo. The crude triflate was dissolved in THF (5 mL, $\left.1 \mathrm{M}\right)$ under argon and cooled to $-78^{\circ} \mathrm{C}$. A solution of $\mathrm{KO} t \mathrm{Bu}$ in THF (12.5 mmol, 1 molar, 2.5 equiv) was added dropwise, and the reaction mixture darkened in color. After 20 minutes, a saturated solution $\mathrm{NaHCO}_{3}$ was added and the mixture was allowed to warm to room temperature with stirring. $\mathrm{Et}_{2} \mathrm{O}$ was added, and the phases were 
separated. The aqueous layer was back-extracted with $\mathrm{Et}_{2} \mathrm{O}$ and the combined organic phases were then dried over $\mathrm{Na}_{2} \mathrm{SO}_{4}$, filtered, and concentrated in vacuo. Purification of the residue by flash chromatography silica gel afforded the ynol ether.

Notes: 1) The crude triflate can be stored without solvent and under argon for up to three days in the freezer and then used in the second step without erosion of the final yield.

2) Highly electron-rich ynol ethers are generally unstable, and the final purification has to be carried on quickly and in some cases using neutral alumina instead of silica gel.

3) The reported procedure can be followed without modifications for amounts of starting material up to $1 \mathrm{~g}$. On larger scale the overall yield dropped drastically.

\section{(Phenoxyethynyl)benzene (1f)}<smiles>C(#Cc1ccccc1)c1ccccc1</smiles>

Ynol ether $\mathbf{1 f}$ was synthesized following general procedure A starting from 2-bromo-1-phenylethan-1-one (1.0 g, $5.00 \mathrm{mmol})$ and phenol (6.00 mmol, 1.2 equiv). The second step of the synthesis was carried out on $1 \mathrm{~g}$ of crude mixture (assumed $4.71 \mathrm{mmol}$ ) and after purification by flash chromatography on silica gel (Eluent: $100 \%$ Pentane) 1f was obtained as an orange oil (609 mg, 67\%).

${ }^{1} \mathbf{H}$ NMR $\left(300 \mathrm{MHz}, \mathrm{CDCl}_{3}\right) \delta 7.51-7.44(\mathrm{~m}, 2 \mathrm{H}), 7.44-7.28(\mathrm{~m}, 7 \mathrm{H}), 7.22-7.14(\mathrm{~m}, 1 \mathrm{H})$.

${ }^{13} \mathbf{C}$ NMR $\left(75 \mathrm{MHz}, \mathrm{CDCl}_{3}\right) \delta 156.1,131.8,129.8,128.4,127.5,124.6,122.9,115.1,92.5,46.4$.

Data in agreement with the one reported in literature. ${ }^{9}$ 
${ }^{1} \mathrm{H}$ NMR $\left(300 \mathrm{MHz}, \mathrm{CDCl}_{3}\right)$ of $\mathbf{1 g}$

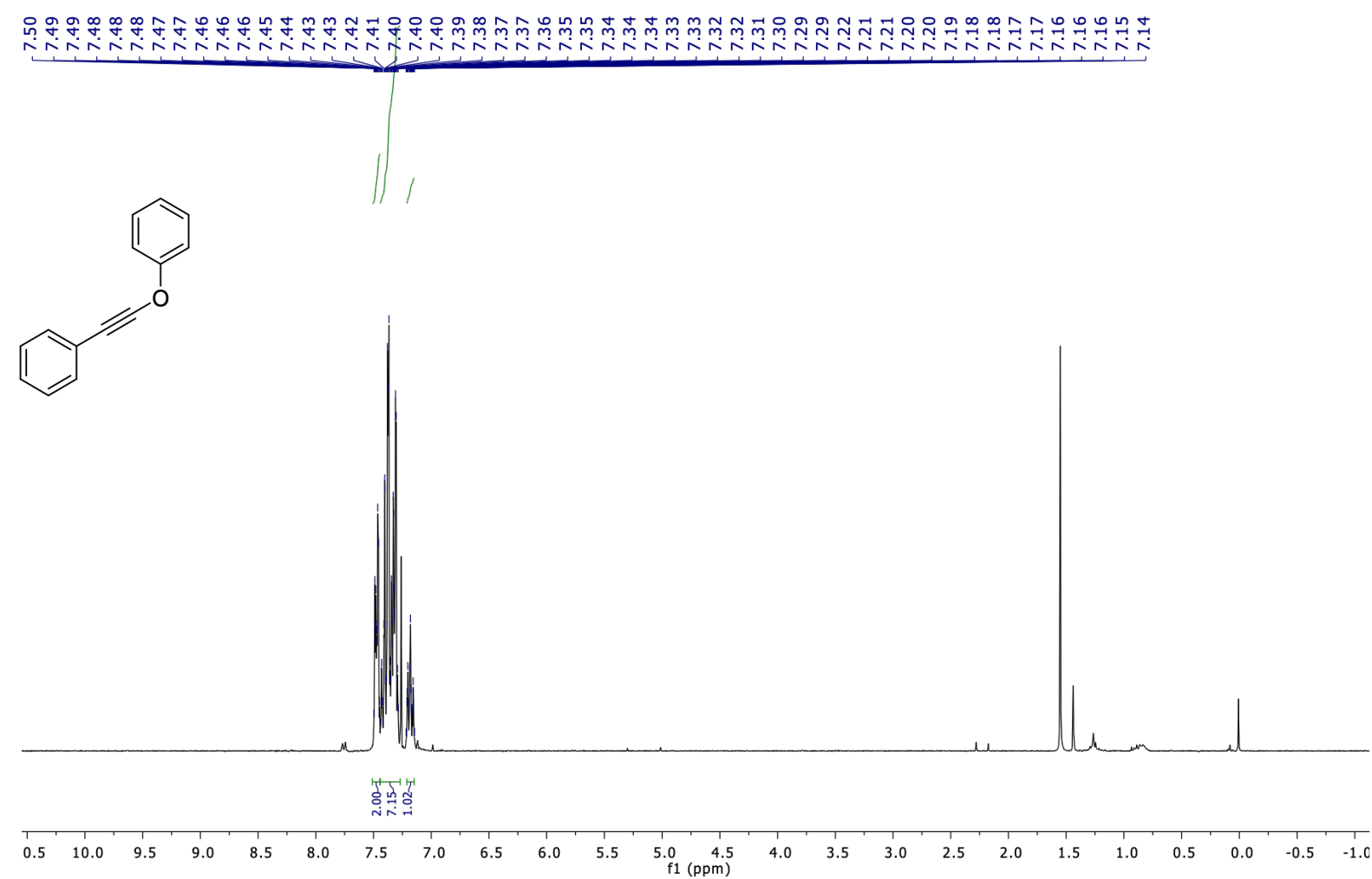

${ }^{13} \mathrm{C} \mathrm{NMR}\left(75 \mathrm{MHz}, \mathrm{CDCl}_{3}\right)$ of $\mathbf{1 f}$
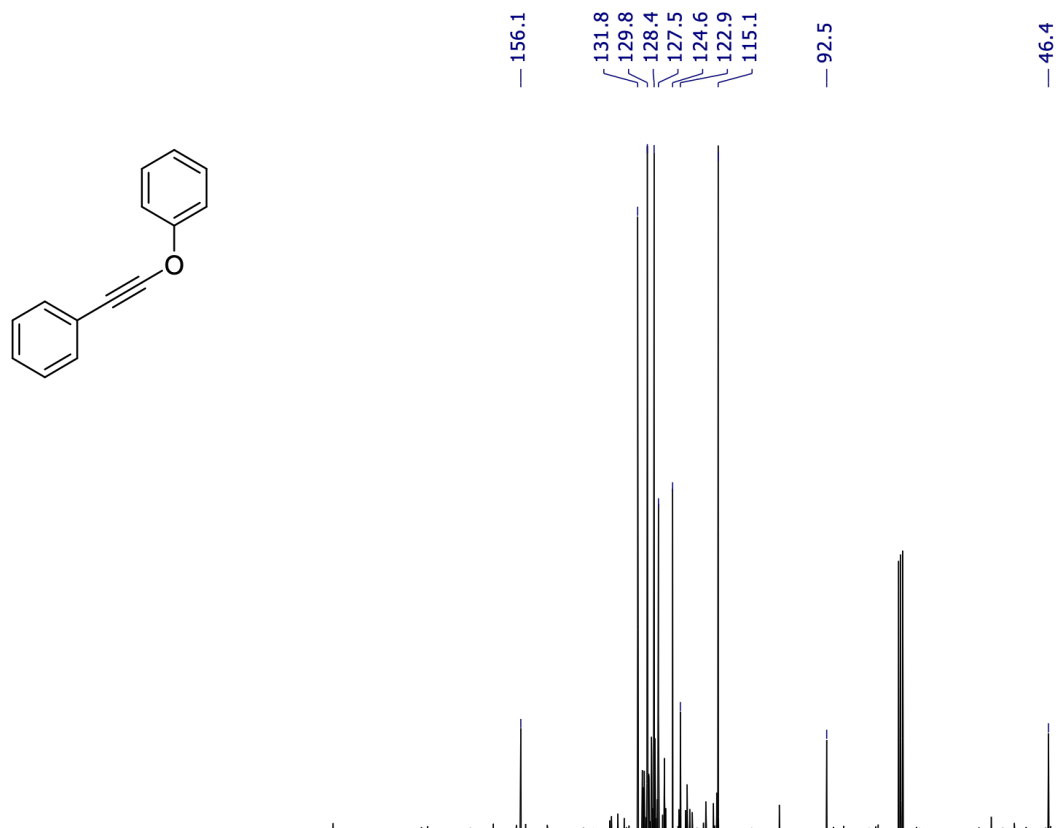

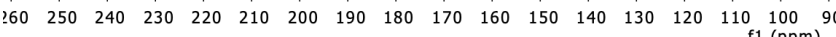


<smiles>FC(F)(F)c1ccccc1OC#Cc1ccccc1</smiles>

Ynol ether $\mathbf{1 h}$ was synthesized following general procedure A starting from 2-bromo-1-(2(trifluoromethyl)phenyl)ethan-1-one (1.35 g, $5.0 \mathrm{mmol})$ and phenol (6.00 mmol, 1.2 equiv). The second step of the synthesis was carried out on $390 \mathrm{mg}$ of crude mixture (assumed $1.4 \mathrm{mmol}$ ) and after purification by flash chromatography on silica gel (Eluent: 100\% Pentane) $1 \mathrm{~h}$ was obtained as an orange oil (179 mg, 49\%).

${ }^{1} \mathrm{H}$ NMR $\left(400 \mathrm{MHz}, \mathrm{CDCl}_{3}\right) \delta 7.67(\mathrm{ddd}, J=7.9,1.4,0.7 \mathrm{~Hz}, 1 \mathrm{H}), 7.61$ (ddt, $\left.J=7.7,1.4,0.7 \mathrm{~Hz}, 1 \mathrm{H}\right), 7.49$ (tdd, $J=7.6,1.4,0.7 \mathrm{~Hz}, 1 \mathrm{H}), 7.45-7.34(\mathrm{~m}, 5 \mathrm{H}), 7.21(\mathrm{tt}, J=6.6,1.5 \mathrm{~Hz}, 1 \mathrm{H})$.

${ }^{19}$ F NMR $\left(376 \mathrm{MHz}, \mathrm{CDCl}_{3}\right) \delta-62.5$.

${ }^{13}$ C NMR $\left(101 \mathrm{MHz}, \mathrm{CDCl}_{3}\right) \delta 156.1,134.1,131.5,130.0,127.1,126.0(\mathrm{q}, \mathrm{J}=5.1 \mathrm{~Hz}), 124.9,124.0(\mathrm{q}, \mathrm{J}=$ $273.2 \mathrm{~Hz}), 122.6,121.7$ (q, J = 2.3 Hz), 115.2, 97.4, 43.2.

HRMS (APCI) $m / z$ calculated for $\mathrm{C}_{15} \mathrm{H}_{10} \mathrm{~F}_{3} \mathrm{O}^{+}[\mathrm{M}+\mathrm{H}]^{+}: 263.0678$, found: 263.0670 .

${ }^{1} \mathrm{H}$ NMR $\left(400 \mathrm{MHz}, \mathrm{CDCl}_{3}\right)$ of $\mathbf{1 h}$
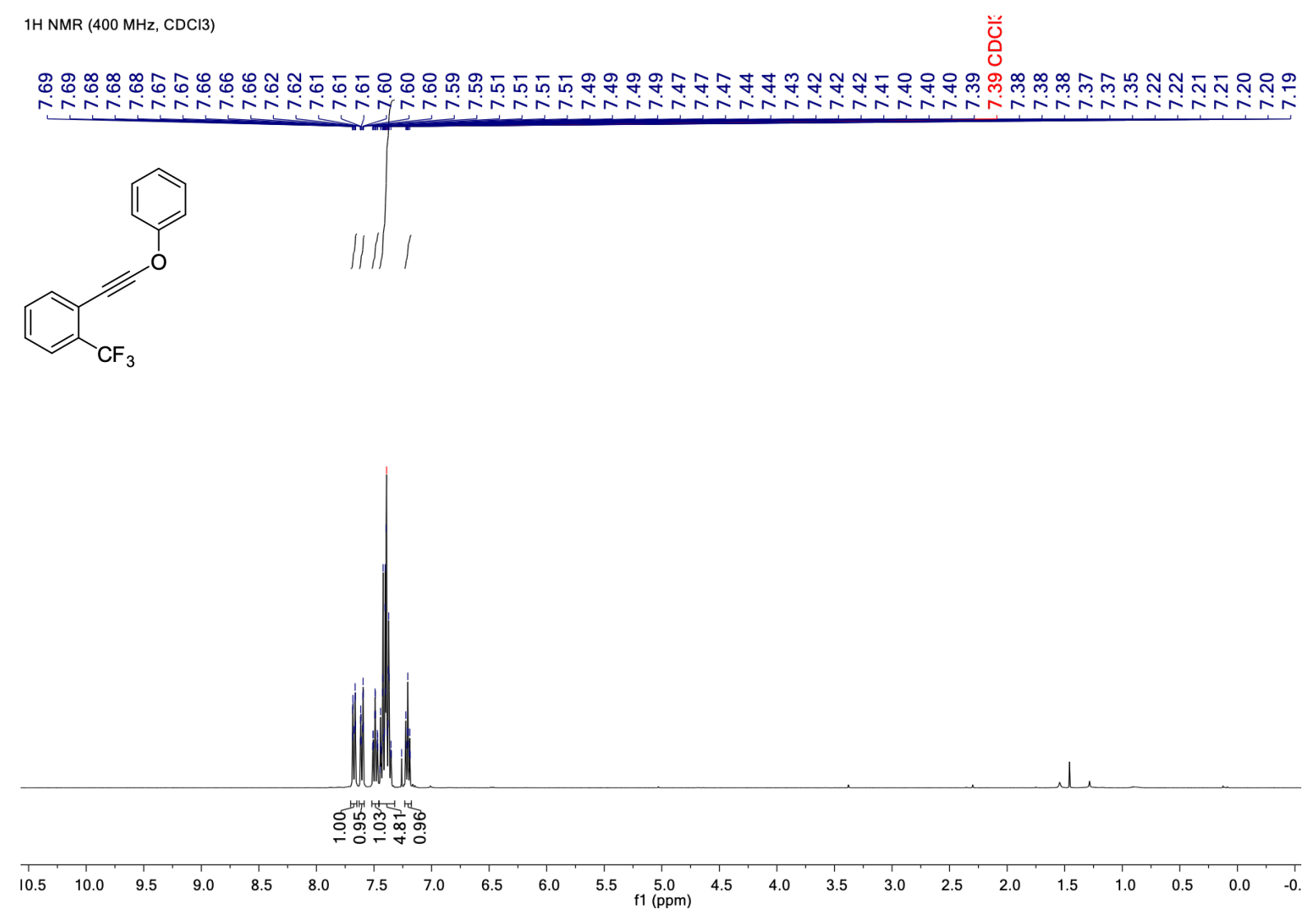
${ }^{19} \mathrm{~F}$ NMR $\left(376 \mathrm{MHz}, \mathrm{CDCl}_{3}\right)$ of $\mathbf{1 h}$

19F-NMR (376 MHz, CDCl3)

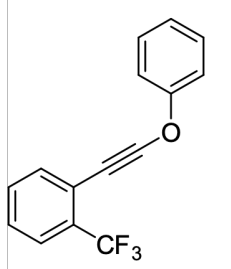

$\stackrel{\substack{n \\ \stackrel{n}{i}}}{i}$

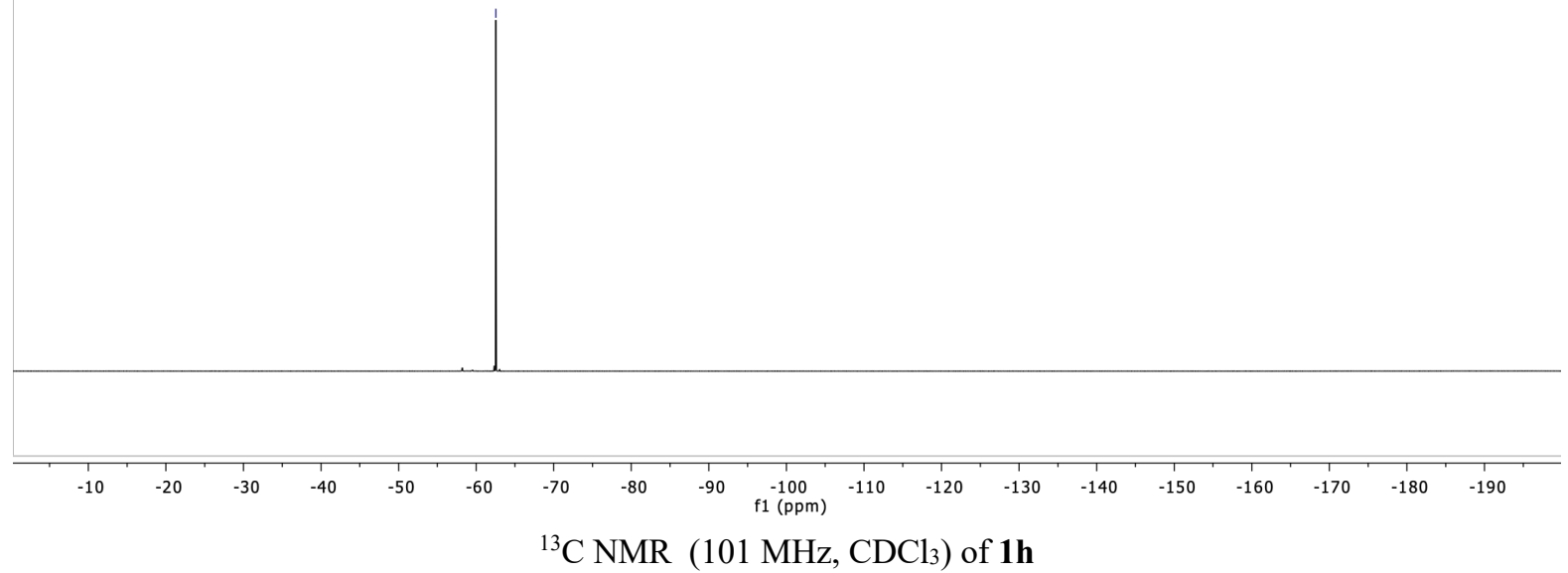

13C \{1H\} NMR (101 MHz, CDCl35; -

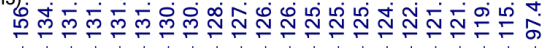<smiles>FC(F)(F)c1ccccc1C#COc1ccccc1</smiles> 


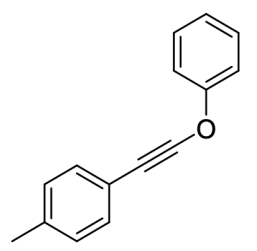

Ynol ether 1i was synthesized following general procedure A starting from 2-bromo-1-(p-tolyl)ethan-1-one (532 $\mathrm{mg}, 2.5 \mathrm{mmol}$ ) and phenol (3.0 mmol, 1.2 equiv). The second step of the synthesis was carried out on $340 \mathrm{mg}$ (assumed $1.5 \mathrm{mmol}$ ) of crude and after purification by flash chromatography on silica gel (Eluent: $100 \%$ Pentane) 1i was obtained as an orange oil ( $219 \mathrm{mg}, 70 \%)$.

${ }^{1} \mathbf{H}$ NMR $\left(400 \mathrm{MHz}, \mathrm{CDCl}_{3}\right) \delta 7.45-7.31(\mathrm{~m}, 7 \mathrm{H}), 7.22-7.11(\mathrm{~m}, 3 \mathrm{H}), 2.37(\mathrm{~s}, 3 \mathrm{H})$.

${ }^{13}$ C NMR $\left(101 \mathrm{MHz}, \mathrm{CDCl}_{3}\right) \delta 156.3,137.6,131.8,129.8,129.2,124.5,119.8,115.2,92.0,46.4,21.5$.

HRMS (APCI) $m / z$ calculated for $\mathrm{C}_{15} \mathrm{H}_{13} \mathrm{O}^{+}[\mathrm{M}+\mathrm{H}]^{+}:$209.0961, found: 209.0962 .

${ }^{1} \mathrm{H}$ NMR $\left(400 \mathrm{MHz}, \mathrm{CDCl}_{3}\right)$ of $\mathbf{1 i}$
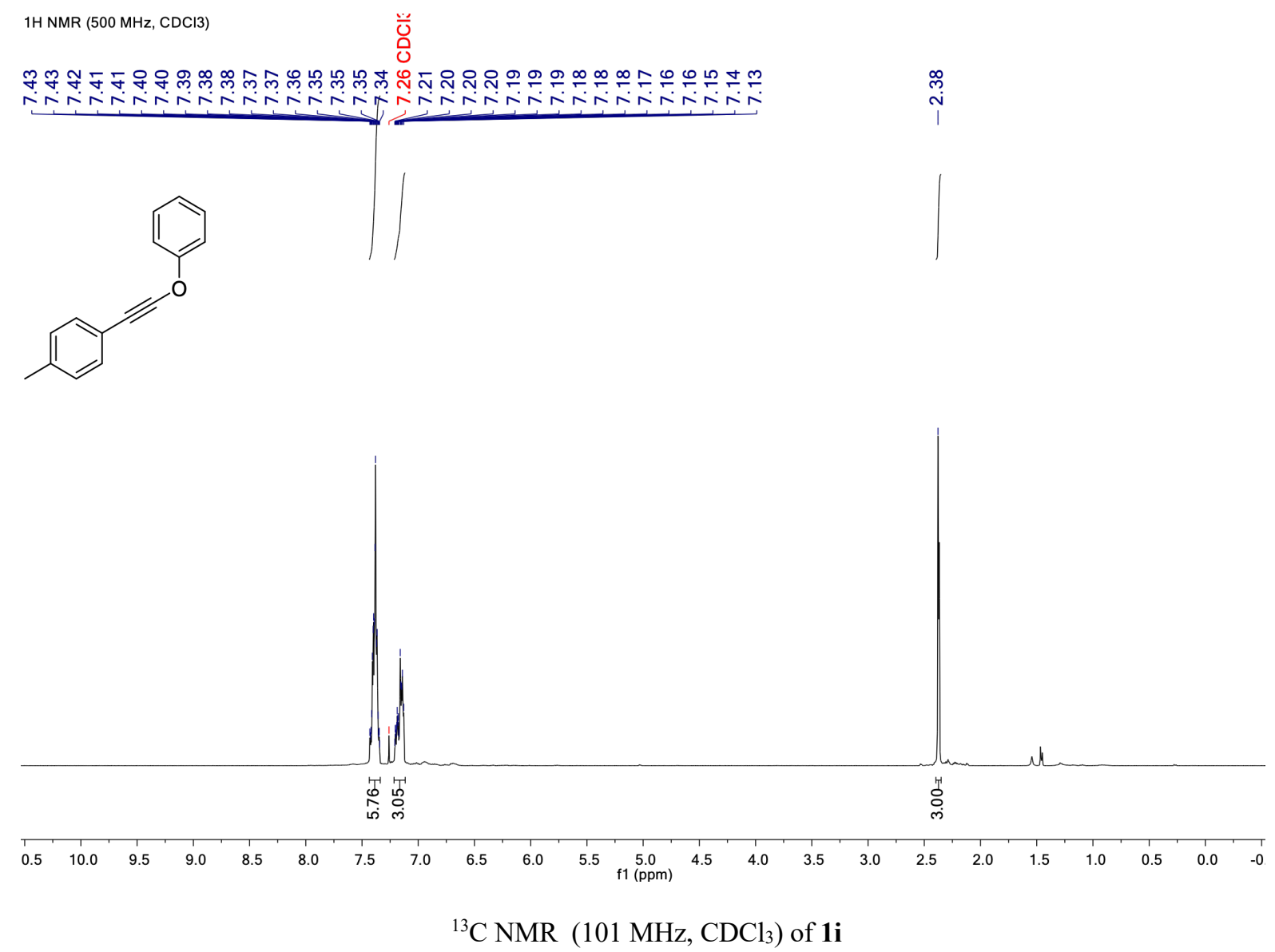


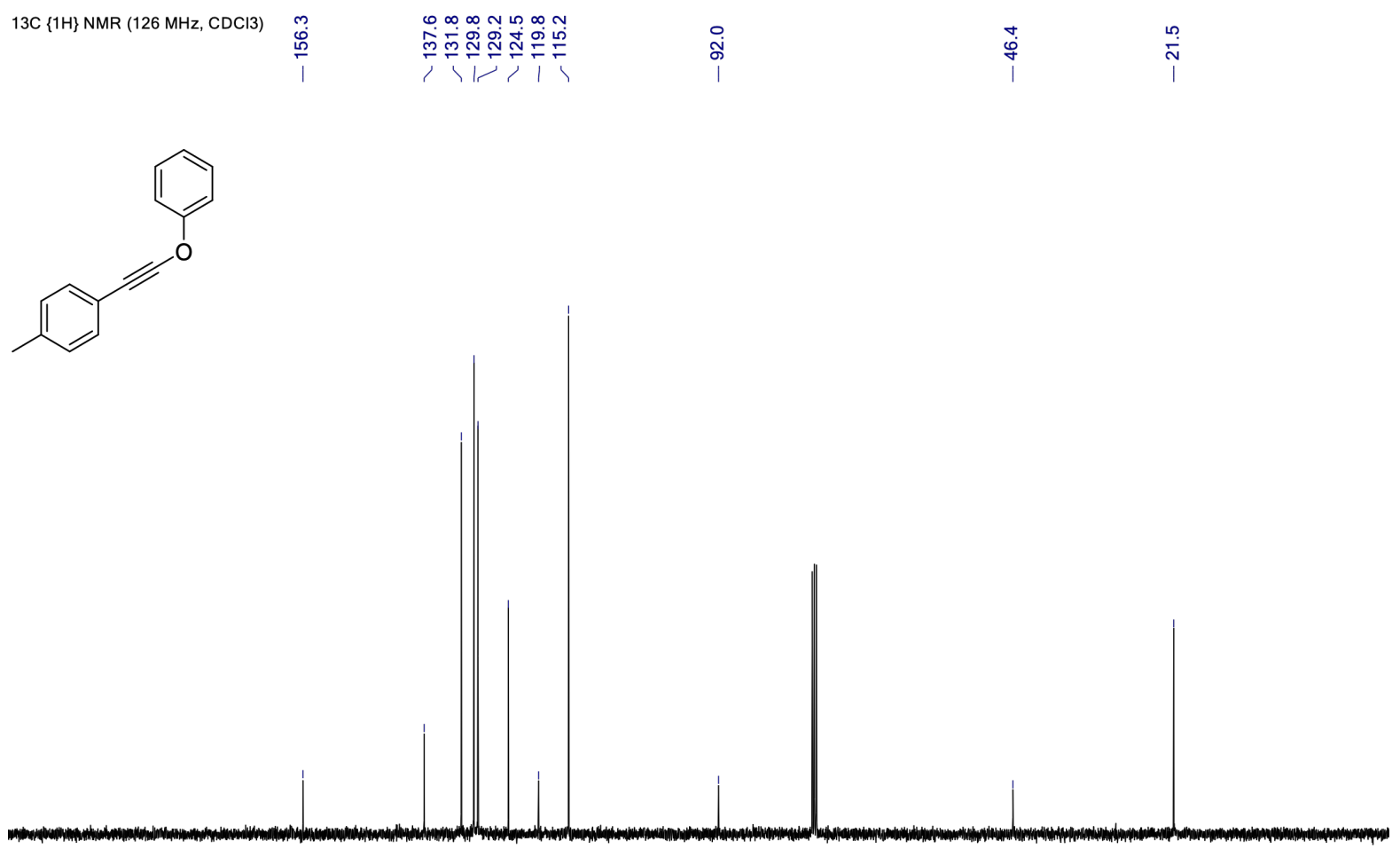

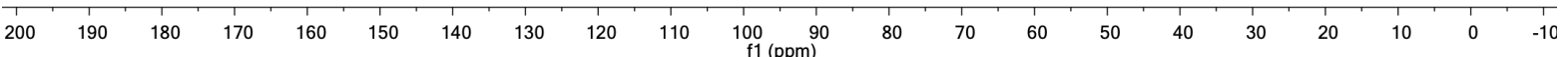


<smiles>COc1ccccc1OC#Cc1ccccc1</smiles>

Ynol ether 1j was synthesized following general procedure A starting from 2-bromo-1-phenylethan-1-one 2bromo-1-phenylethan-1-one $(1.0 \mathrm{~g}, 5.00 \mathrm{mmol})$ and 2-methoxyphenol $(1.4 \mathrm{~mL}, 12.5 \mathrm{mmol})$. The second step of the synthesis was carried out on $340 \mathrm{mg}$ of crude mixture (assumed $1.40 \mathrm{mmol}$ ) and after purification by flash chromatography on silica gel (Eluent: 20:1 Pentane/ $\mathrm{Et}_{2} \mathrm{O}$ ) $\mathbf{1 j}$ was obtained as an orange oil (239 $\mathrm{mg}, 76 \%$ ).

${ }^{1} \mathbf{H}$ NMR $\left(400 \mathrm{MHz}, \mathrm{CDCl}_{3}\right) \delta 7.56(\mathrm{dd}, J=8.1,1.6 \mathrm{~Hz}, 1 \mathrm{H}), 7.46-7.40(\mathrm{~m}, 2 \mathrm{H}), 7.31-7.23(\mathrm{~m}, 3 \mathrm{H}), 7.09$ (ddd, $J=8.0,7.6,1.6 \mathrm{~Hz}, 1 \mathrm{H}), 6.96(\mathrm{ddd}, J=8.1,7.6,1.5 \mathrm{~Hz}, 1 \mathrm{H}), 6.92(\mathrm{dd}, J=8.0,1.5 \mathrm{~Hz}, 1 \mathrm{H}), 3.87(\mathrm{~s}, 3 \mathrm{H})$.

${ }^{13}$ C NMR $\left(101 \mathrm{MHz}, \mathrm{CDCl}_{3}\right) \delta 148.3,145.5,131.9,128.4,127.5,125.1,123.1,120.9,115.1,112.7,92.7,56.2$, 46.3 .

HRMS (ESI) $m / z$ calculated for $\mathrm{C}_{15} \mathrm{H}_{12} \mathrm{NaO}_{2}{ }^{+}[\mathrm{M}+\mathrm{Na}]^{+}: 247.0730$, found: 247.0736

${ }^{1} \mathrm{H}$ NMR $\left(400 \mathrm{MHz}, \mathrm{CDCl}_{3}\right)$ of $\mathbf{1 j}$

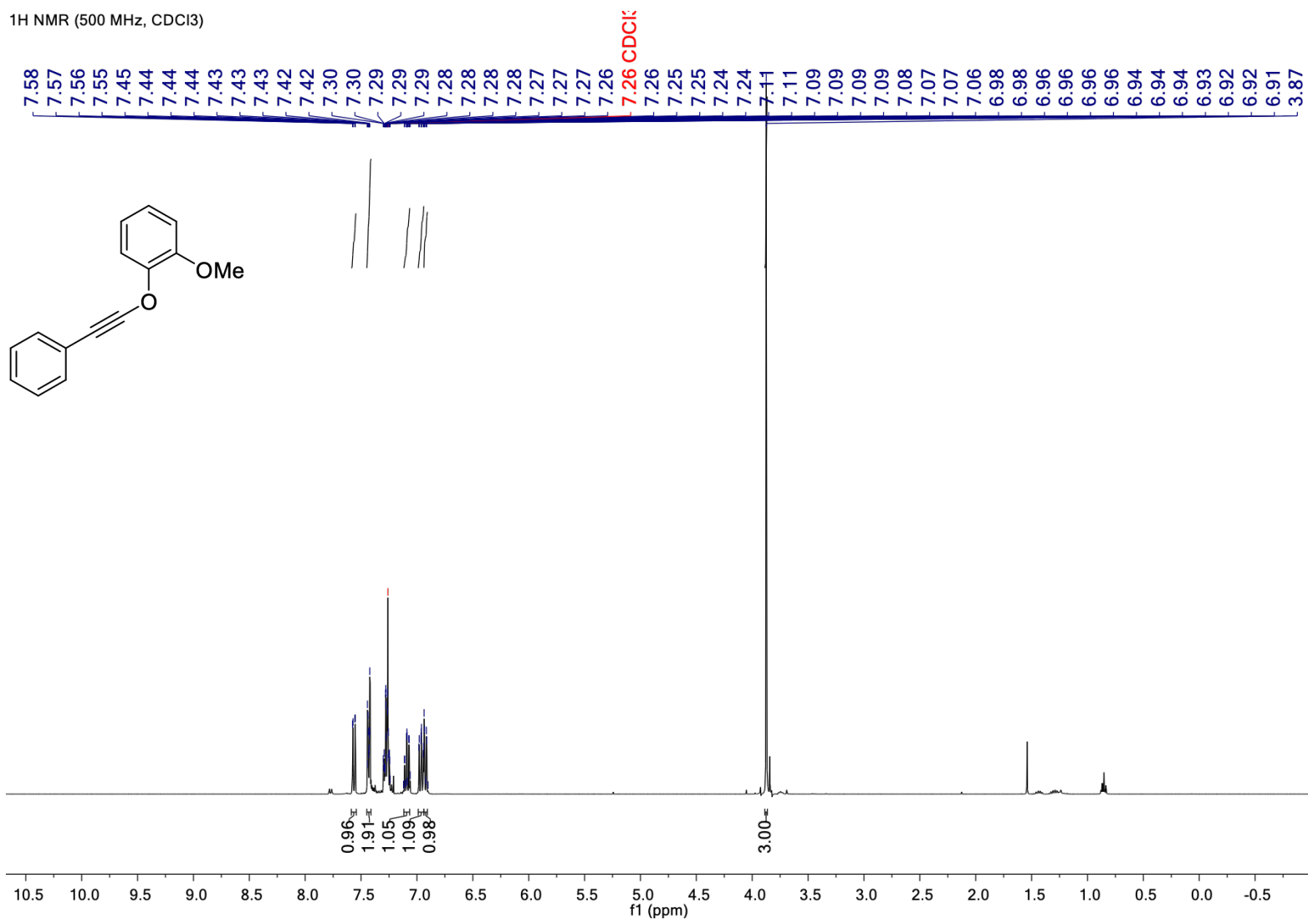


${ }^{13} \mathrm{C}$ NMR $\left(101 \mathrm{MHz}, \mathrm{CDCl}_{3}\right)$ of $\mathbf{1} \mathbf{j}$

$13 \mathrm{C}\{1 \mathrm{H}\} \mathrm{NMR}(126 \mathrm{MHz}, \mathrm{CDCl} 3)$

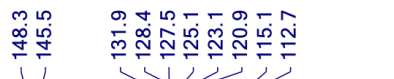

OMe

今ั่

离

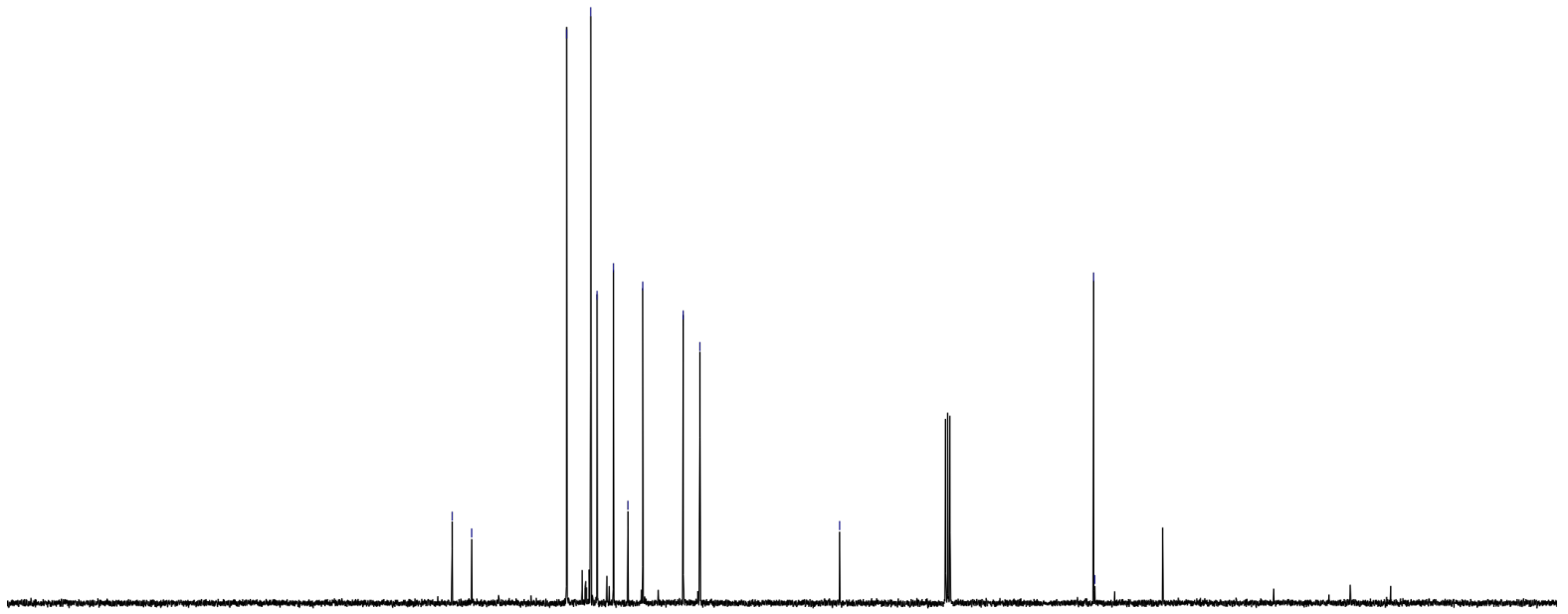

\begin{tabular}{lllllllllllllllllllllllllll}
\hline 210 & 200 & 190 & 180 & 170 & 160 & 150 & 140 & 130 & 120 & 110 & 100 & 90 & 80 & 70 & 60 & 50 & 40 & 30 & 20 & 10 & 0 & -1
\end{tabular} 


\section{1-Methoxy-4-(phenoxyethynyl)benzene (1g)}

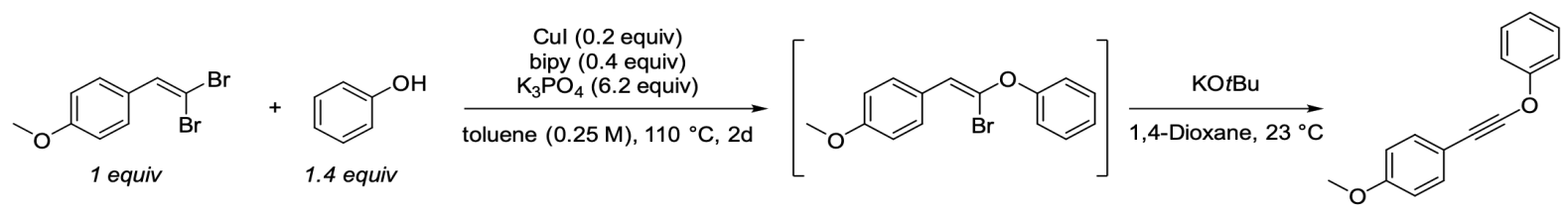

$15 \mathrm{~mL}$ pressure tube was charged with the phenol ( $3.2 \mathrm{mmol}, 1.4$ equiv), 2,2'-bipyridine ( $0.9 \mathrm{mmol}, 0.4$ equiv), $\mathrm{K}_{3} \mathrm{PO}_{4}$ (14.3 mmol, 6.2 equiv), and copper(I) iodide $(0.45 \mathrm{mmol}, 0.2$ equiv). The tube was fitted with a rubber septum, evacuated under high vacuum and backfilled with argon. Dry and degassed toluene (10 mL, $0.25 \mathrm{M})$ was next added followed by 1-(2,2-dibromovinyl)-4-methoxybenzene (672 $\mathrm{mg}, 2.3 \mathrm{mmol}, 1$ equiv.) The rubber septum was replaced by teflon-coated screw cap and the heterogeneous suspension heated at $110{ }^{\circ} \mathrm{C}$ for 2 days (silicon oil bath), cooled to room temperature, filtered through a plug of silica gel and concentrated under reduced pressure. The crude product was then dissolved in dry dioxane $(6 \mathrm{~mL}, 0.4 \mathrm{M})$ and treated with potassium tertbutoxide ( $4.6 \mathrm{mmol}, 2$ equiv). The resulting mixture was stirred overnight at room temperature, filtered through a plug of silica gel (washed with EtOAc), and concentrated in vacuo. The crude residue was purified by flash column chromatography eluting with hexane affording $1 \mathrm{~g}$ as an orange oil (162 $\mathrm{mg}, 31 \%)$.

${ }^{1} \mathbf{H}$ NMR $\left(500 \mathrm{MHz}, \mathrm{CDCl}_{3}\right) \delta 7.43-7.37(\mathrm{~m}, 2 \mathrm{H}), 7.38(\mathrm{~m}, 1 \mathrm{H}), 7.37-7.31(\mathrm{~m}, 2 \mathrm{H}), 7.19-7.12(\mathrm{~m}, 1 \mathrm{H}), 6.89$ $-6.82(\mathrm{~m}, 2 \mathrm{H}), 3.81(\mathrm{~s}, 3 \mathrm{H})$.

${ }^{13}$ C NMR $\left(126 \mathrm{MHz}, \mathrm{CDCl}_{3}\right) \delta 159.2,156.4,133.3,129.8,124.5,115.2,114.1,91.4,55.4,46.1,27.3$.

HRMS (APCI) $\mathrm{m} / z$ calculated for $\mathrm{C}_{15} \mathrm{H}_{13} \mathrm{O}_{2}{ }^{+}[\mathrm{M}+\mathrm{H}]^{+}: 225.0910$, found: $225.0909 . \mathrm{m} / z$ calculated for $\mathrm{C}_{15} \mathrm{H}_{13} \mathrm{O}_{2}{ }^{+}$ $\left[\mathrm{M}+\mathrm{CH}_{3} \mathrm{OH}+\mathrm{H}\right]^{+}:$257.1172, found: 257.1171 . 
${ }^{1} \mathrm{H}$ NMR $\left(500 \mathrm{MHz}, \mathrm{CDCl}_{3}\right)$ of $\mathbf{1 g}$

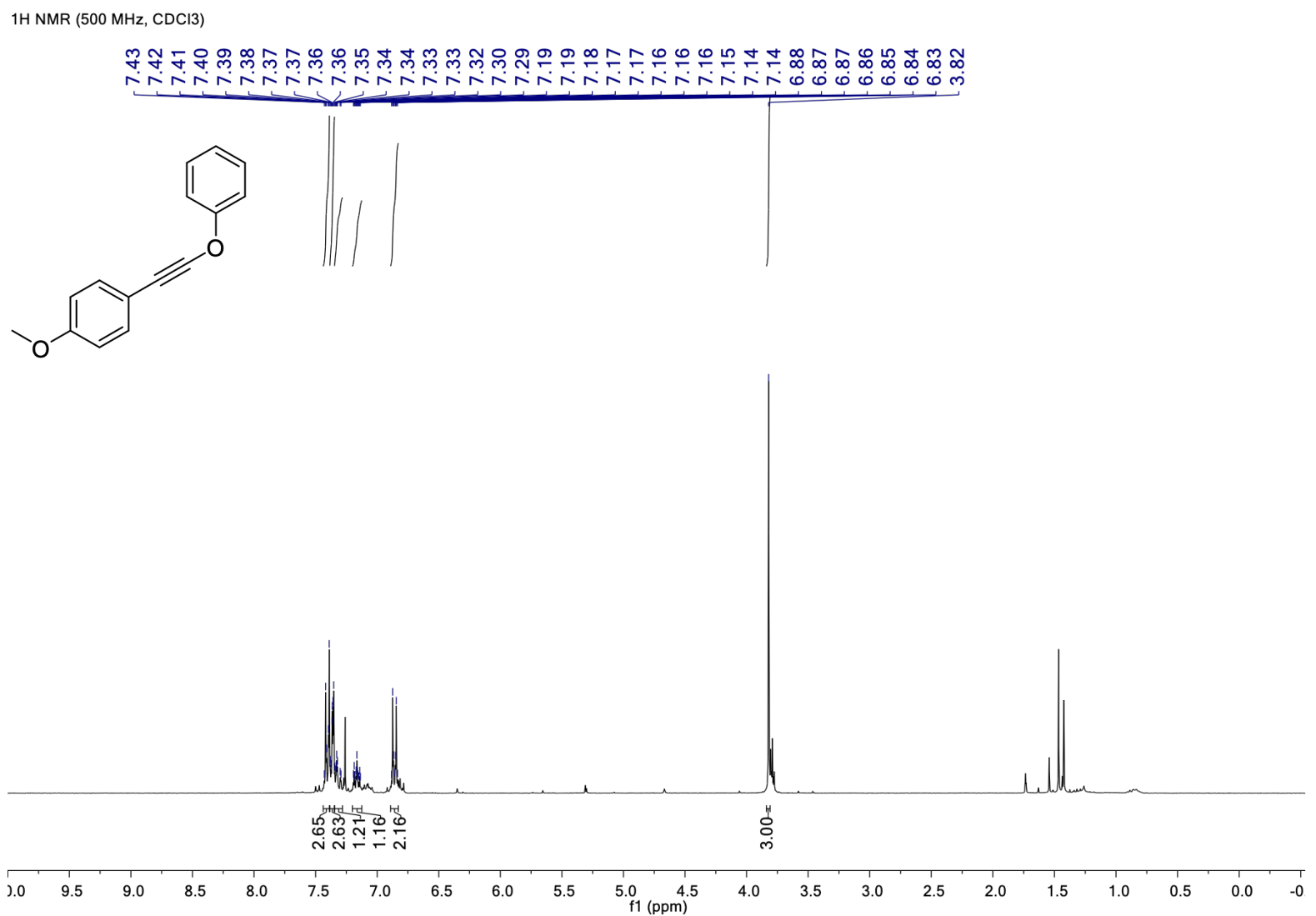

${ }^{13} \mathrm{C} \mathrm{NMR}\left(126 \mathrm{MHz}, \mathrm{CDCl}_{3}\right)$ of $\mathbf{1 g}$

$13 \mathrm{C}\{1 \mathrm{H}\}$ NMR $(126 \mathrm{MHz}, \mathrm{CDCl} 3)$

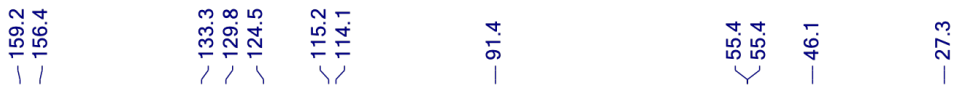<smiles>COc1ccc(C#COc2ccccc2)cc1</smiles>

$\begin{array}{lllllllllll}210 & 200 & 190 & 180 & 170 & 160 & 150 & 140 & 130 & 120 & 110 \begin{array}{l}100 \\ \mathrm{f} 1(\mathrm{ppm})\end{array}\end{array}$ 


\section{Scope of the gold(I)-catalyzed [2+2] cycloaddition reaction of ynol ethers with alkenes}

General procedure B: Gold(I)-catalyzed reaction of terminal ynol ethers with alkenes

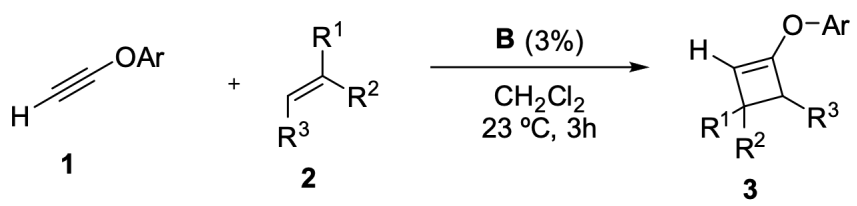

A GC-MS vial equipped with a magnetic stirring bar was charged with the ynol ether $(0.2 \mathrm{mmol}, 1$ equiv) and $\mathrm{CH}_{2} \mathrm{Cl}_{2}$ (HPLC grade, $0.2 \mathrm{~mL}, 1 \mathrm{M}$ ). The alkene $(0.6 \mathrm{mmol}, 3$ equiv) was then added followed by [(JohnPhos)AuNCMe] $\mathrm{SbF}_{6}(\mathbf{B}, 3 \mathrm{~mol} \%, 4.6 \mathrm{mg})$. The resulting mixture was stirred at $23{ }^{\circ} \mathrm{C}$ for $3 \mathrm{~h}$. The reaction was monitored by GC-MS or UHPLC-MSD. Once completed, the reaction was quenched with few drops of triethylamine and the solvent evaporated. The crude product was purified by flash chromatography on silica gel (eluent $=$ pentane:Et2O gradient from 100:0 to 50:1, otherwise stated) to obtain the pure oxy-cyclobutene.

\section{(1-Methyl-3-phenoxycyclobut-2-en-1-yl)benzene (3a)}

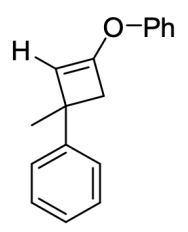

Cyclobutene 3a was synthesized following general procedure B starting from (ethynyloxy)benzene (23.6 mg, 0.2 mmol) and $\alpha$-methylstyrene $(78 \mu \mathrm{L}, 0.6 \mathrm{mmol})$. The crude product was purified by flash chromatography affording $3 \mathbf{a}$ as a colorless oil (40 $\mathrm{mg}, 85 \%)$.

${ }^{1}$ H NMR $\left(400 \mathrm{MHz}, \mathrm{CDCl}_{3}\right) \delta 7.42-7.30(\mathrm{~m}, 6 \mathrm{H}), 7.25-7.11(\mathrm{~m}, 4 \mathrm{H}), 5.19(\mathrm{~s}, 1 \mathrm{H}), 2.91(\mathrm{~d}, J=12.6 \mathrm{~Hz}, 1 \mathrm{H})$, $2.87(\mathrm{~d}, J=12.6 \mathrm{~Hz}, 1 \mathrm{H}), 1.61(\mathrm{~s}, 3 \mathrm{H})$.

${ }^{13}$ C NMR (101 MHz, $\left.\mathrm{CDCl}_{3}\right) \delta$ 155.0, 149.5, 148.0, 129.7, 128.2, 126.1, 125.9, 124.2, 119.5, 109.1, 47.3, 41.4, 28.4 .

HRMS (APCI) $m / z$ calculated for $\mathrm{C}_{17} \mathrm{H}_{17} \mathrm{O}^{+}[\mathrm{M}+\mathrm{H}]^{+}:$237.1274, found: 237.1273. 
${ }^{1} \mathrm{H}$ NMR $\left(400 \mathrm{MHz}, \mathrm{CDCl}_{3}\right)$ of $\mathbf{3 a}$

1H NMR (500 MHz, CDCl3)

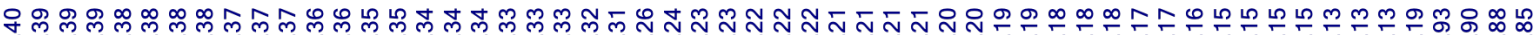

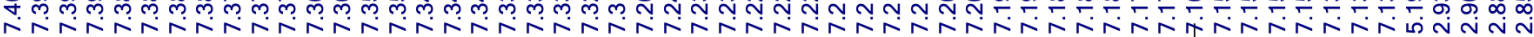<smiles></smiles>
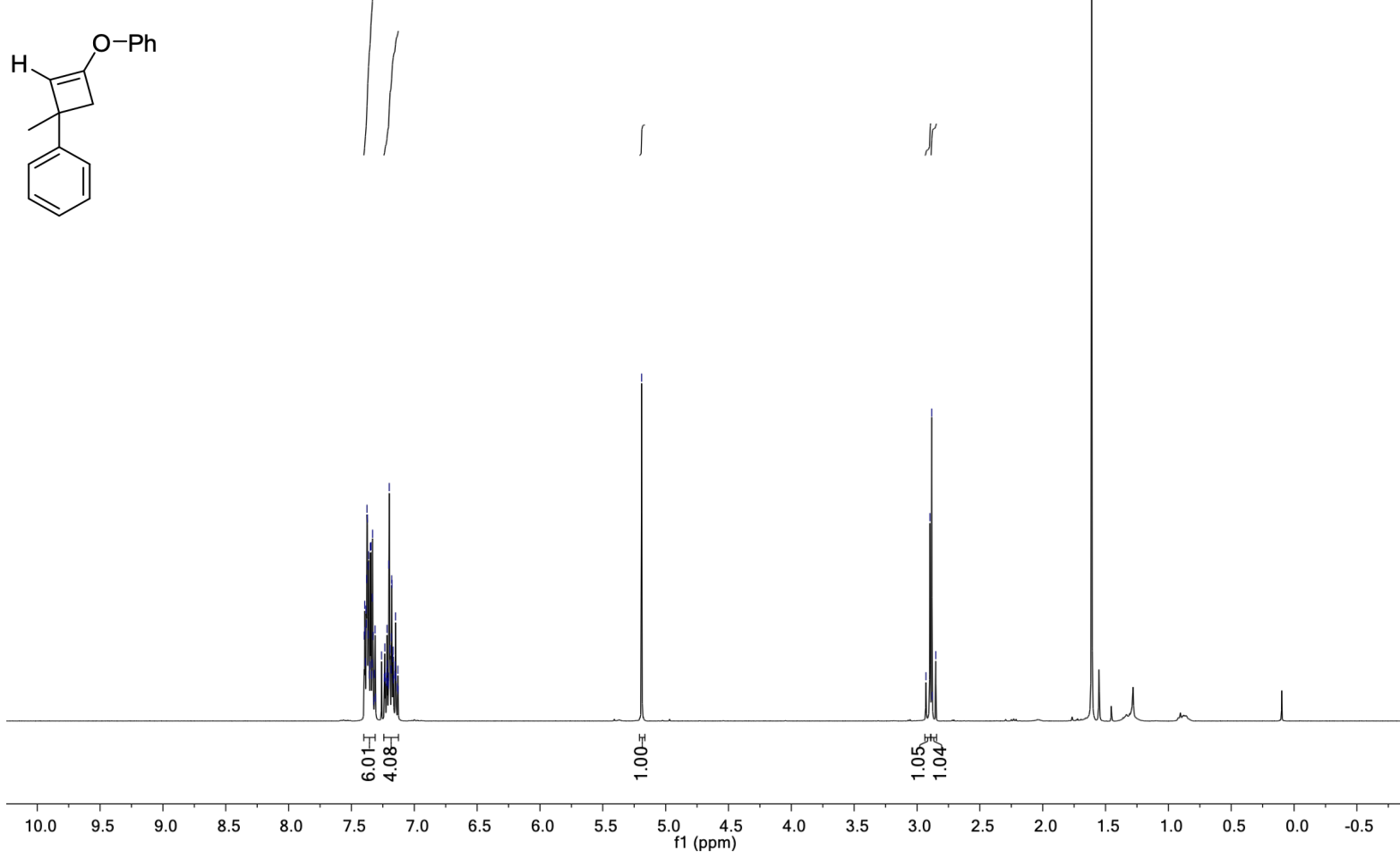

${ }^{13} \mathrm{C}$ NMR $\left(101 \mathrm{MHz}, \mathrm{CDCl}_{3}\right)$ of $\mathbf{3 a}$

$13 \mathrm{C}\{1 \mathrm{H}\} \mathrm{NMR}(126 \mathrm{MHz}, \mathrm{CDCl} 3)$<smiles>CC1(c2ccccc2)CC(Oc2ccccc2)C1</smiles>

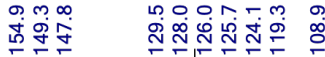

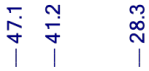

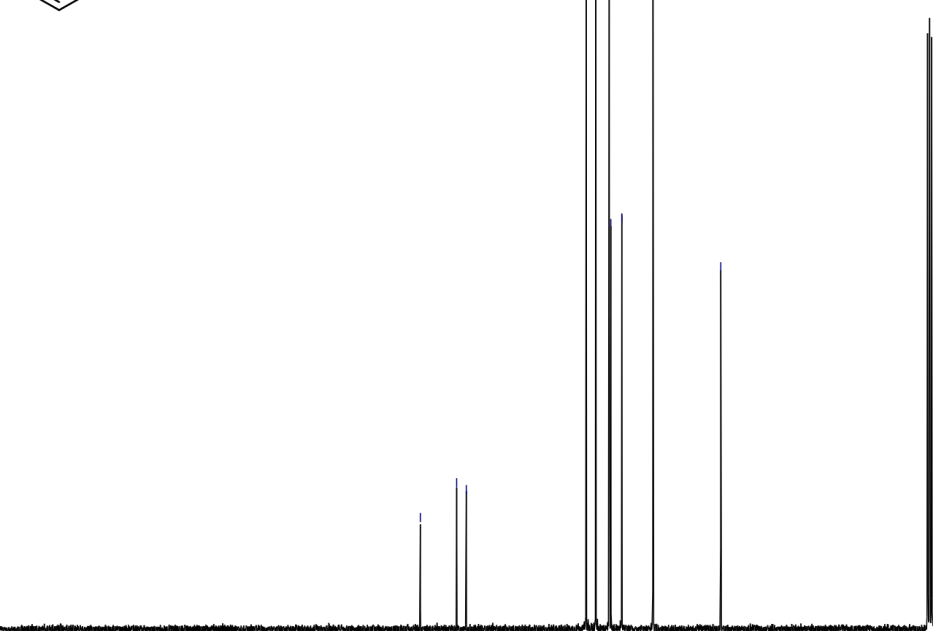

$\begin{array}{lllllllllll}210 & 200 & 190 & 180 & 170 & 160 & 150 & 140 & 130 & 120 & 110 \begin{array}{c}100 \\ \mathrm{f} 1(\mathrm{ppm})\end{array}\end{array}$ 


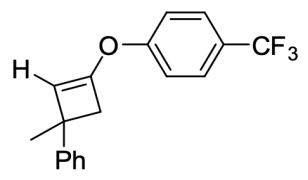

Cyclobutene 3b was synthesized following general procedure B starting from 1-(ethynyloxy)-4(trifluoromethyl)benzene $(37.2 \mathrm{mg}, 0.2 \mathrm{mmol})$ and $\alpha$-methylstyrene $(78 \mu \mathrm{L}, 0.6 \mathrm{mmol})$. The crude product was purified by flash chromatography affording $\mathbf{3 b}$ as a colorless oil (44 mg, $72 \%$ ).

${ }^{1}$ H NMR $\left(400 \mathrm{MHz}, \mathrm{CDCl}_{3}\right) \delta 7.72-7.54(\mathrm{~m}, 2 \mathrm{H}), 7.47-7.33(\mathrm{~m}, 4 \mathrm{H}), 7.31-7.14(\mathrm{~m}, 3 \mathrm{H}), 5.32(\mathrm{~s}, 1 \mathrm{H}), 2.94$ (d, $J=12.7 \mathrm{~Hz}, 1 \mathrm{H}), 2.89(\mathrm{~d}, J=12.7 \mathrm{~Hz}, 1 \mathrm{H}), 1.63(\mathrm{~s}, 3 \mathrm{H})$.

${ }^{19} \mathbf{F}$ NMR $\left(376 \mathrm{MHz}, \mathrm{CDCl}_{3}\right) \delta-62.0$.

${ }^{13}$ C NMR $\left(101 \mathrm{MHz}, \mathrm{CDCl}_{3}\right) \delta 157.7,148.4,147.5,128.3,127.1(\mathrm{q}, \mathrm{J}=3.7 \mathrm{~Hz}), 126.1,124.2(\mathrm{q}, \mathrm{J}=271.6 \mathrm{~Hz})$, $119.2,111.2,47.3,41.7,28.3$.

HRMS (APCI) $m / z$ calculated for $\mathrm{C}_{18} \mathrm{H}_{16} \mathrm{~F}_{3} \mathrm{O}^{+}[\mathrm{M}+\mathrm{H}]^{+}: 305.1148$, found: 305.1156 .

${ }^{1} \mathrm{H}$ NMR $\left(400 \mathrm{MHz}, \mathrm{CDCl}_{3}\right)$ of $\mathbf{3 b}$
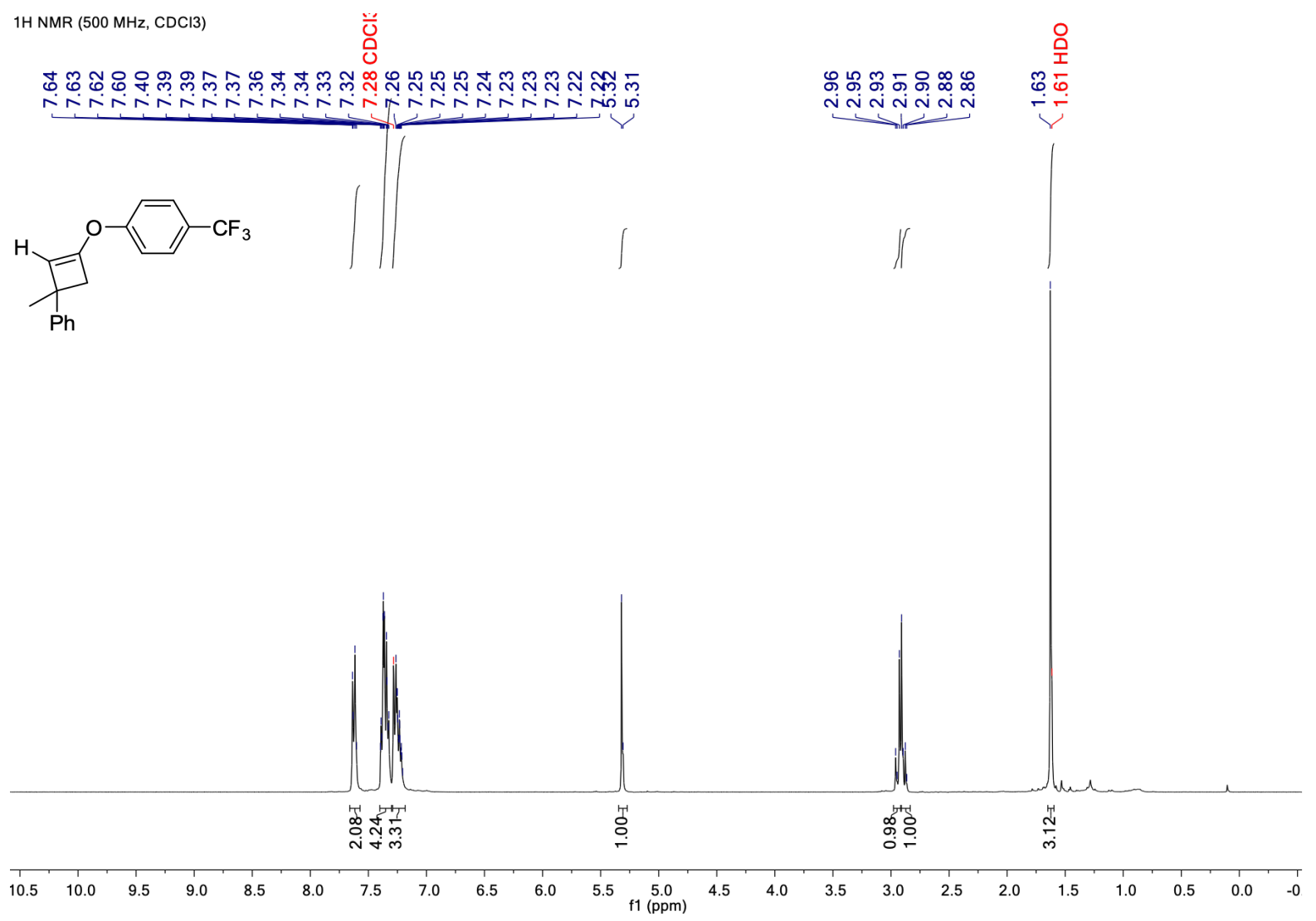
${ }^{19} \mathrm{~F}$ NMR $\left(376 \mathrm{MHz}, \mathrm{CDCl}_{3}\right)$ of $\mathbf{3 b}$

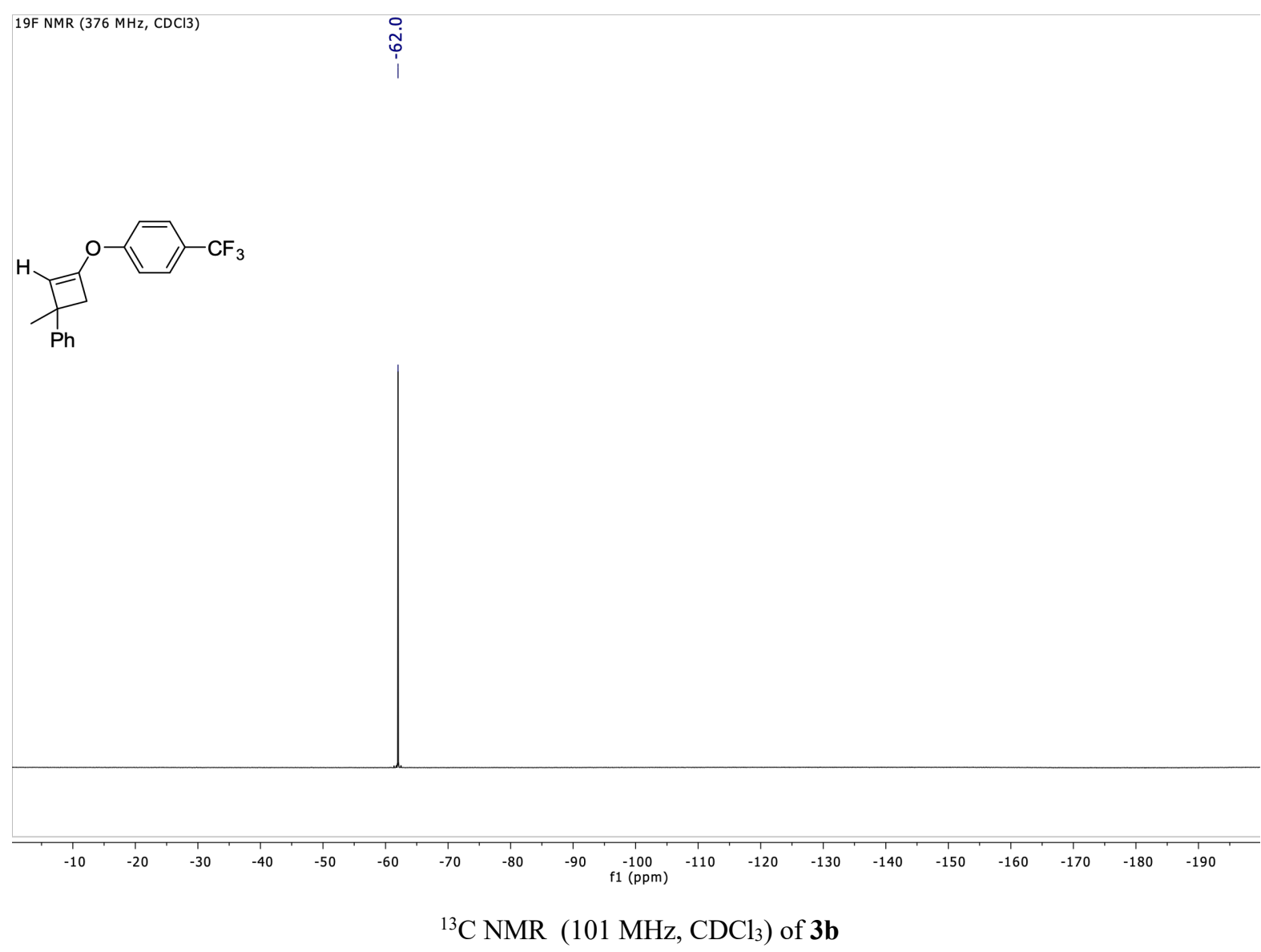

$13 \mathrm{C}\{1 \mathrm{H}\}$ NMR $(126 \mathrm{MHz}, \mathrm{CDCl} 3)$

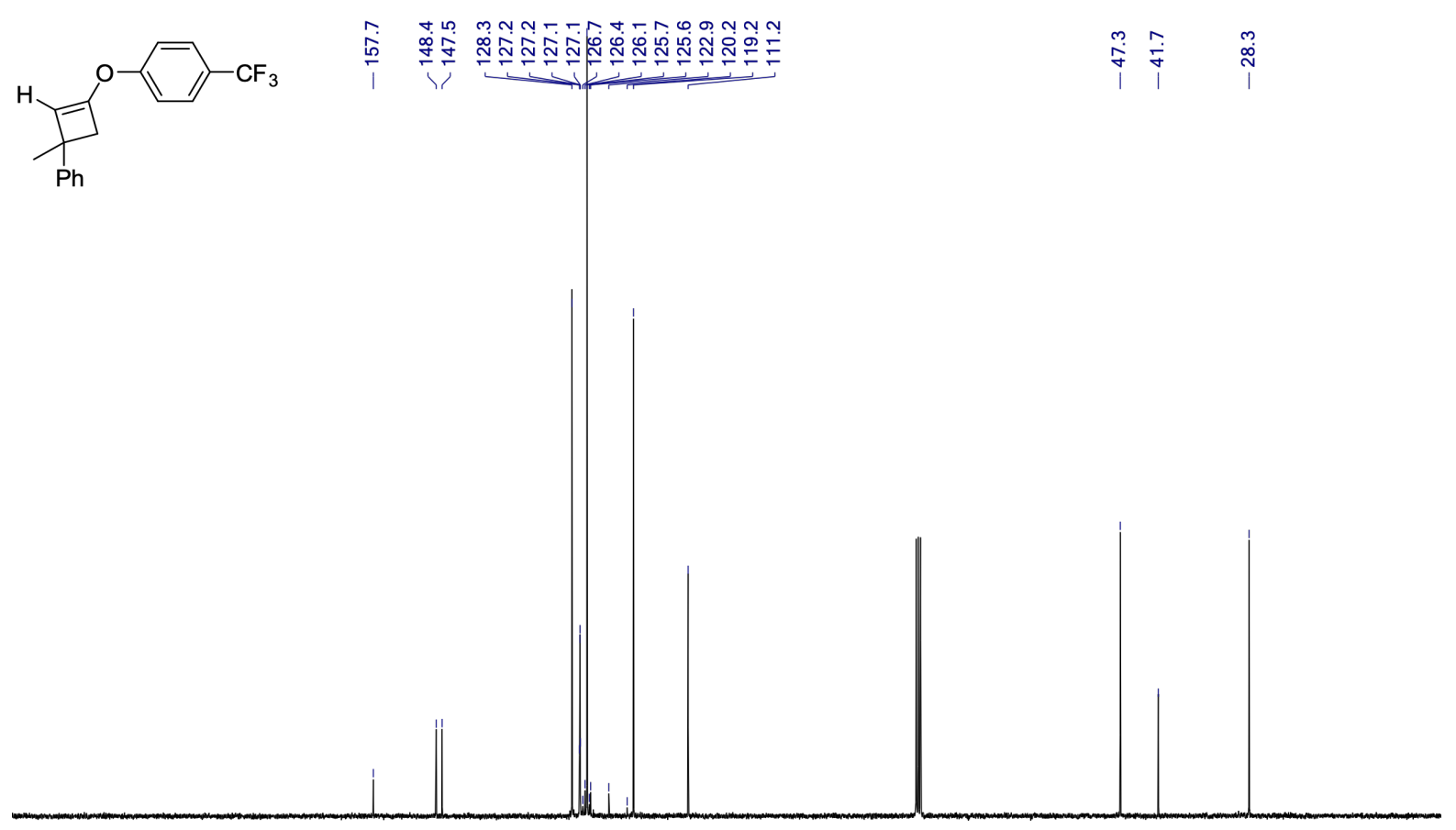

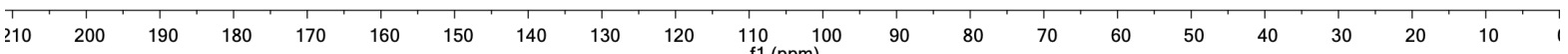




\section{1,3-Dimethoxy-5-((3-methyl-3-phenylcyclobut-1-en-1-yl)oxy)benzene (3c)}<smiles>COc1cc(OC)cc(OC2=CC(C)(c3ccccc3)C2)c1</smiles>

Cyclobutene 3c was synthesized following general procedure B starting from 1-(ethynyloxy)-3,5dimethoxybenzene (35.6 mg, $0.2 \mathrm{mmol})$ and $\alpha$-methylstyrene $(78 \mu \mathrm{L}, 0.6 \mathrm{mmol})$. The crude product was purified by flash chromatography affording $\mathbf{3 c}$ as a colorless oil (13 $\mathrm{mg}, 22 \%)$.

${ }^{1}$ H NMR $\left(300 \mathrm{MHz}, \mathrm{CDCl}_{3}\right) \delta 7.42-7.29(\mathrm{~m}, 4 \mathrm{H}), 7.21(\mathrm{ddt}, J=8.5,6.4,1.6 \mathrm{~Hz}, 1 \mathrm{H}), 6.36(\mathrm{~d}, J=2.2 \mathrm{~Hz}, 2 \mathrm{H})$, $6.26(\mathrm{t}, J=2.2 \mathrm{~Hz}, 1 \mathrm{H}), 5.27(\mathrm{~s}, 1 \mathrm{H}), 3.79(\mathrm{~s}, 6 \mathrm{H}), 2.88(\mathrm{~d}, J=12.8 \mathrm{~Hz}, 1 \mathrm{H}), 2.87(\mathrm{~d}, J=12.8 \mathrm{~Hz}, 1 \mathrm{H}), 1.60$ (s, $3 \mathrm{H})$.

${ }^{13}$ C NMR $\left(101 \mathrm{MHz} \mathrm{CDCl}_{3}\right) \delta 161.5,156.7,148.9,147.9,128.2,126.1,125.9,109.9,97.9,96.3,55.6,47.3$, $41.5,28.4$.

HRMS (APCI) $m / z$ calculated for $\mathrm{C}_{19} \mathrm{H}_{21} \mathrm{O}_{3}{ }^{+}[\mathrm{M}+\mathrm{H}]^{+}:$297.1485, found: 297.1484.

${ }^{1} \mathrm{H}$ NMR $\left(300 \mathrm{MHz}, \mathrm{CDCl}_{3}\right)$ of $\mathbf{3 c}$

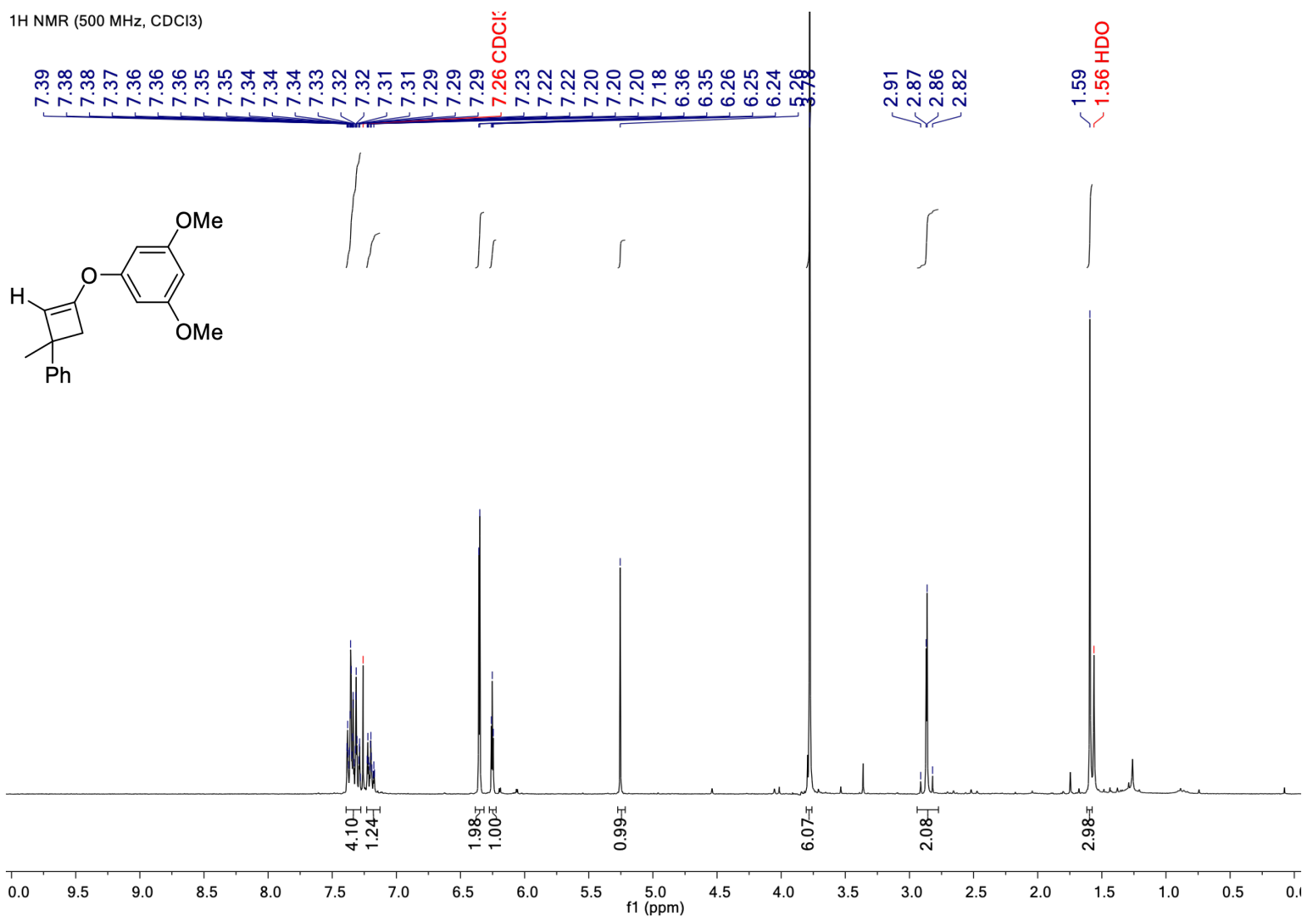


${ }^{13} \mathrm{C}$ NMR $\left(101 \mathrm{MHz}, \mathrm{CDCl}_{3}\right)$ of $\mathbf{3 c}$

$13 \mathrm{C}\{1 \mathrm{H}\} \mathrm{NMR}(126 \mathrm{MHz}, \mathrm{CDCl} 3)$

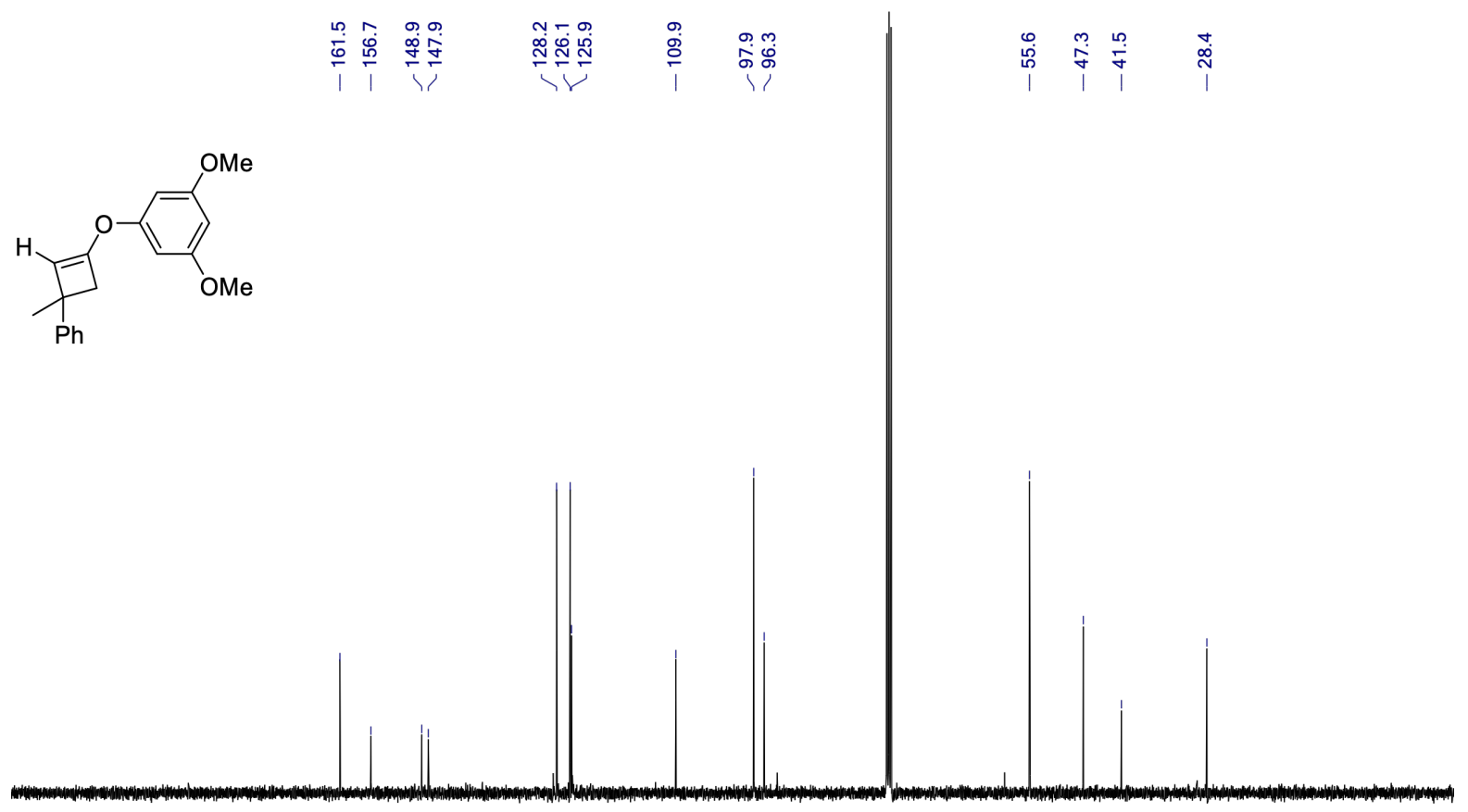

$\begin{array}{llllllllllllllllllllllll}210 & 200 & 190 & 180 & 170 & 160 & 150 & 140 & 130 & 120 & 110 & 100 & 90 & 80 & 70 & 60 & 50 & 40 & 30 & 20 & 10 & 0 & f(\mathrm{ppm}) & \end{array}$ 


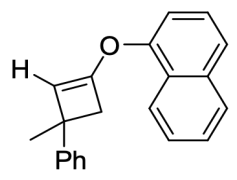

Cyclobutene 3d was synthesized following general procedure B starting from 1-(ethynyloxy)naphthalene (34 mg, $0.2 \mathrm{mmol}$ ) and $\alpha$-methylstyrene $(78 \mu \mathrm{L}, 0.6 \mathrm{mmol}$ ). The crude product was purified by flash chromatography affording $\mathbf{3 d}$ as a colorless oil z (34 $\mathrm{mg}, 58 \%)$.

${ }^{1} \mathbf{H}$ NMR $\left(400 \mathrm{MHz}, \mathrm{CDCl}_{3}\right) \delta 8.18(\mathrm{ddt}, J=6.4,3.6,0.8 \mathrm{~Hz}, 1 \mathrm{H}), 7.91-7.83(\mathrm{~m}, 1 \mathrm{H}), 7.65(\mathrm{dt}, J=8.2,1.0 \mathrm{~Hz}$, 1H), $7.57-7.49$ (m, 2H), 7.44 (dd, $J=8.2,7.6 \mathrm{~Hz}, 1 \mathrm{H}), 7.41-7.36$ (m, 2H), $7.36-7.29$ (m, 3H), $7.25-7.19$ (m, 1H), $5.20(\mathrm{~s}, 1 \mathrm{H}), 2.99(\mathrm{~d}, J=12.7 \mathrm{~Hz}, 1 \mathrm{H}), 2.95(\mathrm{~d}, J=12.7 \mathrm{~Hz}, 1 \mathrm{H}), 1.62(\mathrm{~s}, 3 \mathrm{H})$.

${ }^{13}$ C NMR $\left(101 \mathrm{MHz}, \mathrm{CDCl}_{3}\right) \delta 150.8,149.7,148.0,135.0,128.2,127.9,126.6,126.2,126.1,125.9,125.6,124.2$, 122.1, 114.4, 109.6, 47.3, 41.4, 28.4.

HRMS (APCI) $m / z$ calculated for $\mathrm{C}_{21} \mathrm{H}_{19} \mathrm{O}^{+}[\mathrm{M}+\mathrm{H}]^{+}: 287.1430$, found: 287.1431 .

${ }^{1} \mathrm{H}$ NMR $\left(400 \mathrm{MHz}, \mathrm{CDCl}_{3}\right)$ of $\mathbf{3 d}$

$1 \mathrm{H} \mathrm{NMR}(500 \mathrm{MHz}, \mathrm{CDCl} 3)$

苮

굳두

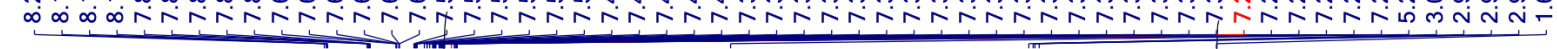<smiles>CC1(c2ccccc2)C=C(Oc2cccc3ccccc23)C1</smiles>
$1 /\|\|$

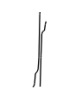

$\mathrm{Ph}$

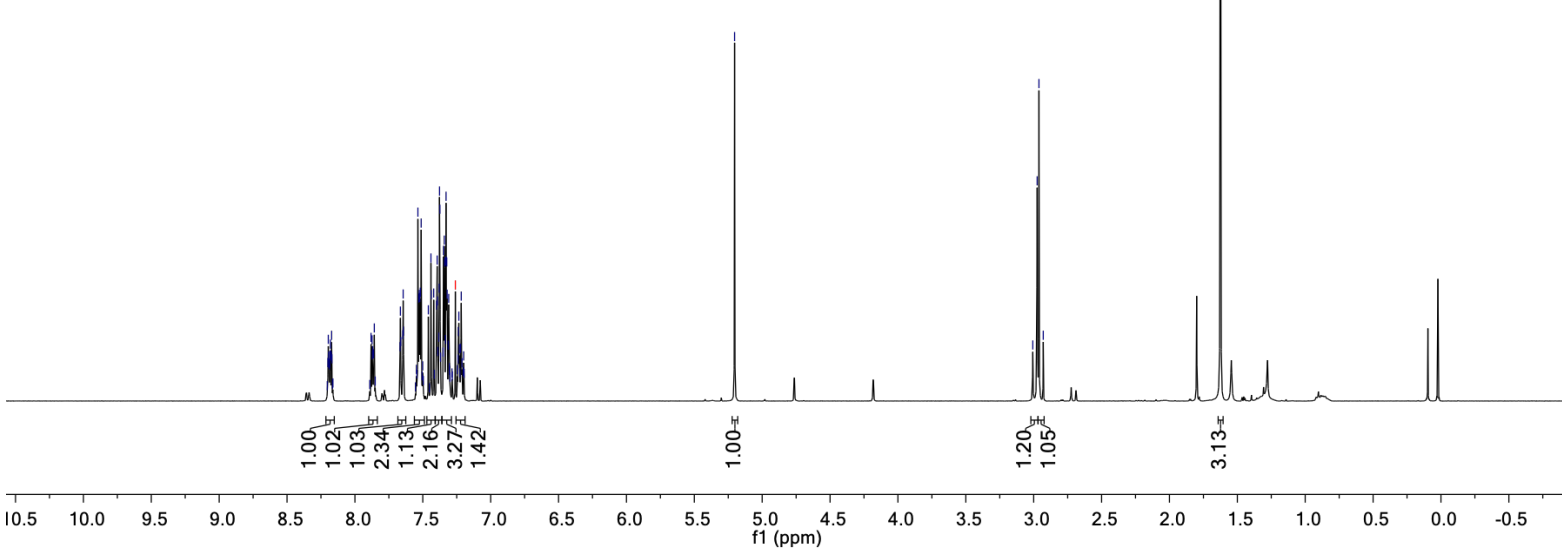

${ }^{13} \mathrm{C}$ NMR $\left(101 \mathrm{MHz}, \mathrm{CDCl}_{3}\right)$ of $\mathbf{3 d}$ 
<smiles>CC1(c2ccccc2)C=C(Oc2cccc3ccccc23)C1</smiles>
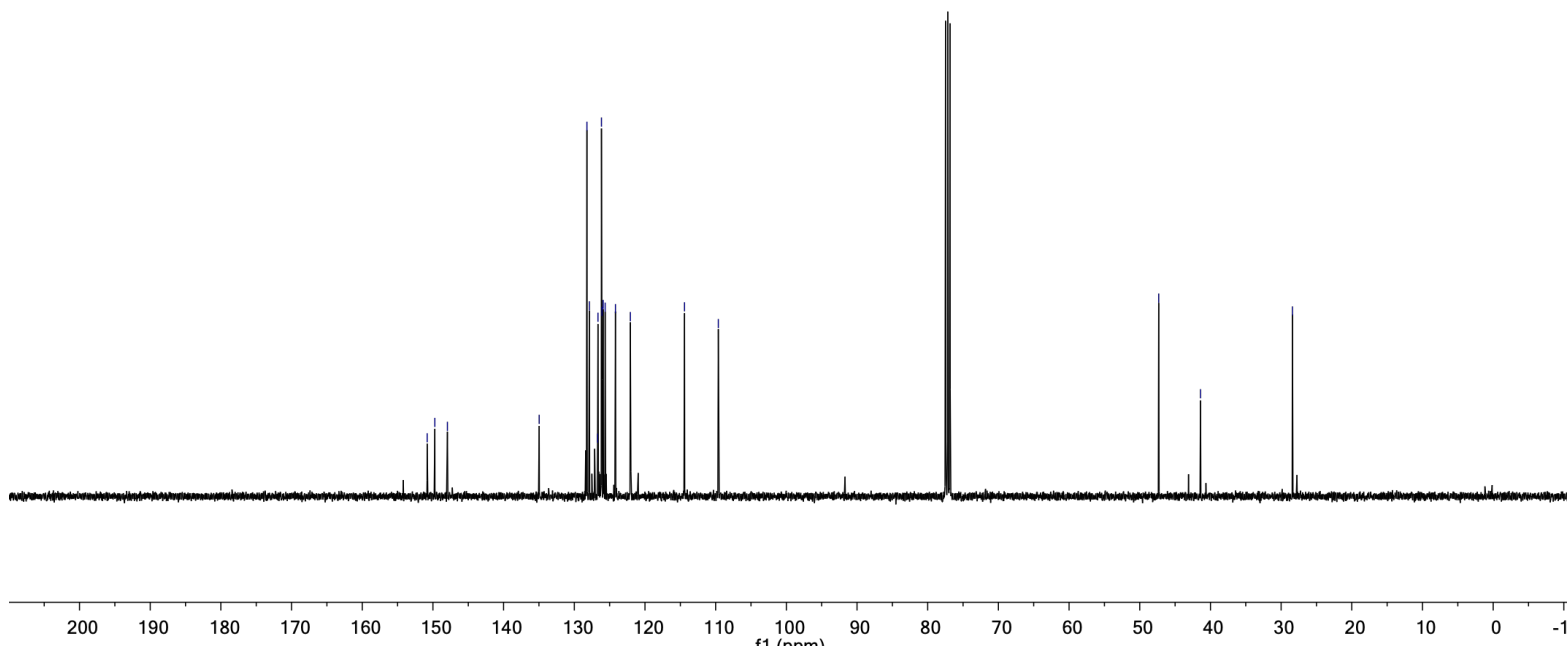

$110 \quad 100$
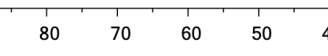

$\begin{array}{llllll}1 & 1 & 1 & 1 & \\ 30 & 20 & 10 & 0 & -1\end{array}$ 


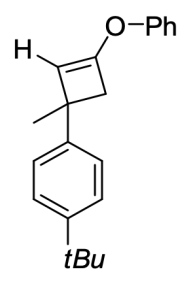

Cyclobutene 3e was synthesized following general procedure B starting from (ethynyloxy)benzene (23.6 mg, 0.2 $\mathrm{mmol}$ ) and 1-(tert-butyl)-4-(prop-1-en-2-yl)benzene (105 mg, $0.6 \mathrm{mmol})$. The crude product was purified by flash chromatography affording $3 \mathbf{e}$ as a colorless oil (52 $\mathrm{mg}, 89 \%)$.

${ }^{1}$ H NMR $\left(400 \mathrm{MHz}, \mathrm{CDCl}_{3}\right) \delta 7.40-7.30(\mathrm{~m}, 6 \mathrm{H}), 7.20(\mathrm{~m}, 2 \mathrm{H}), 7.18-7.12(\mathrm{~m}, 1 \mathrm{H}), 5.18(\mathrm{~s}, 1 \mathrm{H}), 2.91(\mathrm{~d}, J=$ $12.6 \mathrm{~Hz}, 1 \mathrm{H}), 2.85(\mathrm{~d}, J=12.6 \mathrm{~Hz}, 1 \mathrm{H}), 1.61(\mathrm{~s}, 3 \mathrm{H}), 1.35(\mathrm{~s}, 9 \mathrm{H})$.

${ }^{13}$ C NMR (101 MHz, $\left.\mathrm{CDCl}_{3}\right) \delta 155.1,149.4,148.6,144.9,129.7,125.8,125.1,124.2,119.5,109.3,47.3,41.0$, $34.5,31.6,28.3$.

HRMS (ESI) $m / z$ calculated for $\mathrm{C}_{21} \mathrm{H}_{25} \mathrm{O}^{+}[\mathrm{M}+\mathrm{H}]^{+}:$293.1900, found: 293.1898 .

${ }^{1} \mathrm{H}$ NMR $\left(400 \mathrm{MHz}, \mathrm{CDCl}_{3}\right)$ of $\mathbf{3 e}$

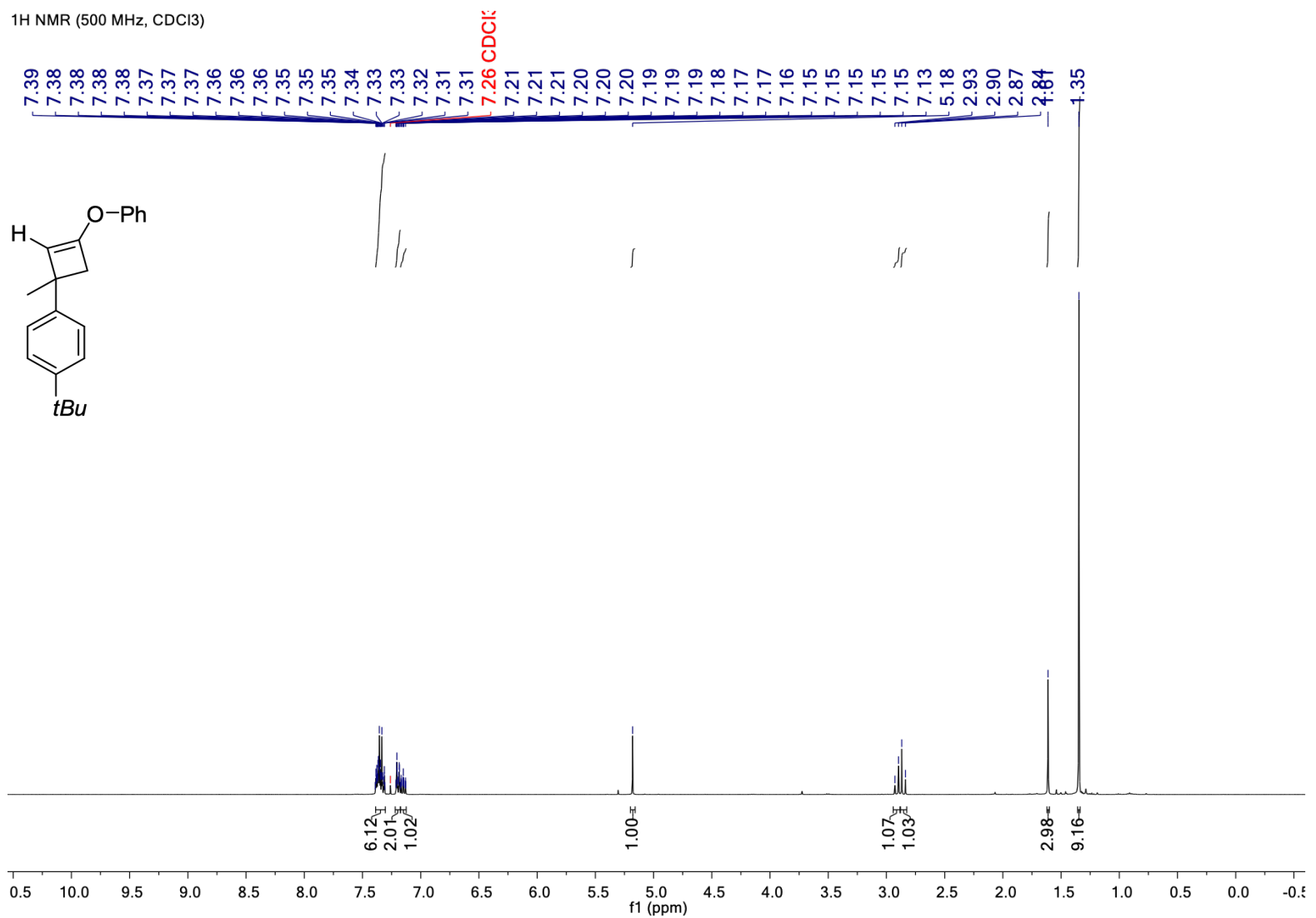


${ }^{13} \mathrm{C}$ NMR $\left(101 \mathrm{MHz}, \mathrm{CDCl}_{3}\right)$ of $\mathbf{3 e}$

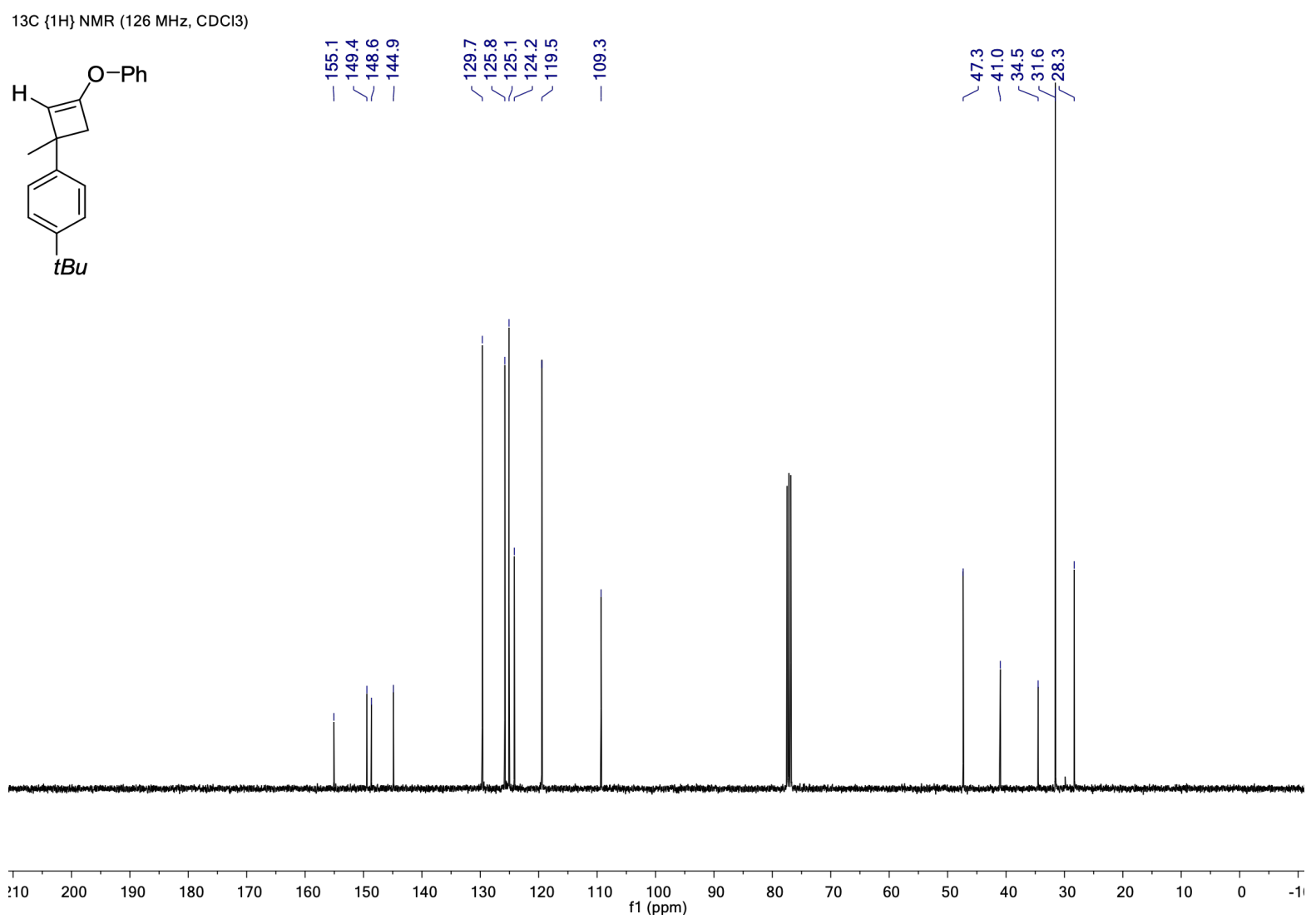




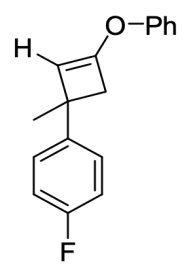

Cyclobutene 3f was synthesized following general procedure B starting from (ethynyloxy)benzene (23.6 mg, 0.2 $\mathrm{mmol}$ ) and 1-fluoro-4-(prop-1-en-2-yl)benzene $(82 \mathrm{mg}, 0.6 \mathrm{mmol})$. The crude product was purified by flash chromatography affording $\mathbf{3 f}$ as a colorless oil (34 mg, 67\%).

${ }^{1} \mathbf{H}$ NMR $\left(500 \mathrm{MHz}, \mathrm{CDCl}_{3}\right) \delta 7.35(\mathrm{tt}, J=6.8,1.0 \mathrm{~Hz}, 2 \mathrm{H}), 7.32-7.28(\mathrm{~m}, 2 \mathrm{H}), 7.19-7.14(\mathrm{~m}, 2 \mathrm{H}), 7.14-$ $7.11(\mathrm{~m}, 1 \mathrm{H}), 7.02-6.92(\mathrm{~m}, 2 \mathrm{H}), 5.13(\mathrm{~d}, J=0.7 \mathrm{~Hz}, 1 \mathrm{H}), 2.87-2.80(\mathrm{~m}, 2 \mathrm{H}), 1.57(\mathrm{~s}, 3 \mathrm{H})$.

${ }^{19}$ F NMR $\left(376 \mathrm{MHz}, \mathrm{CDCl}_{3}\right) \delta-117.87$.

${ }^{13}$ C NMR (126 MHz, $\left.\mathrm{CDCl}_{3}\right) \delta 162.3(\mathrm{~d}, J=243.7 \mathrm{~Hz}), 155.0,149.6,143.7,143.7,129.7,127.6(\mathrm{~d}, J=7.8 \mathrm{~Hz})$, $124.3,119.5,114.9(\mathrm{~d}, J=21.0 \mathrm{~Hz}), 108.9,47.4,40.9,28.4$.

HRMS (APCI) $m / z$ calculated for $\mathrm{C}_{17} \mathrm{H}_{16} \mathrm{FO}^{+}[\mathrm{M}+\mathrm{H}]^{+}: 255.1180$, found: 255.1170 .

\section{${ }^{1} \mathrm{H}$ NMR $\left(500 \mathrm{MHz}, \mathrm{CDCl}_{3}\right)$ of $\mathbf{3 f}$}

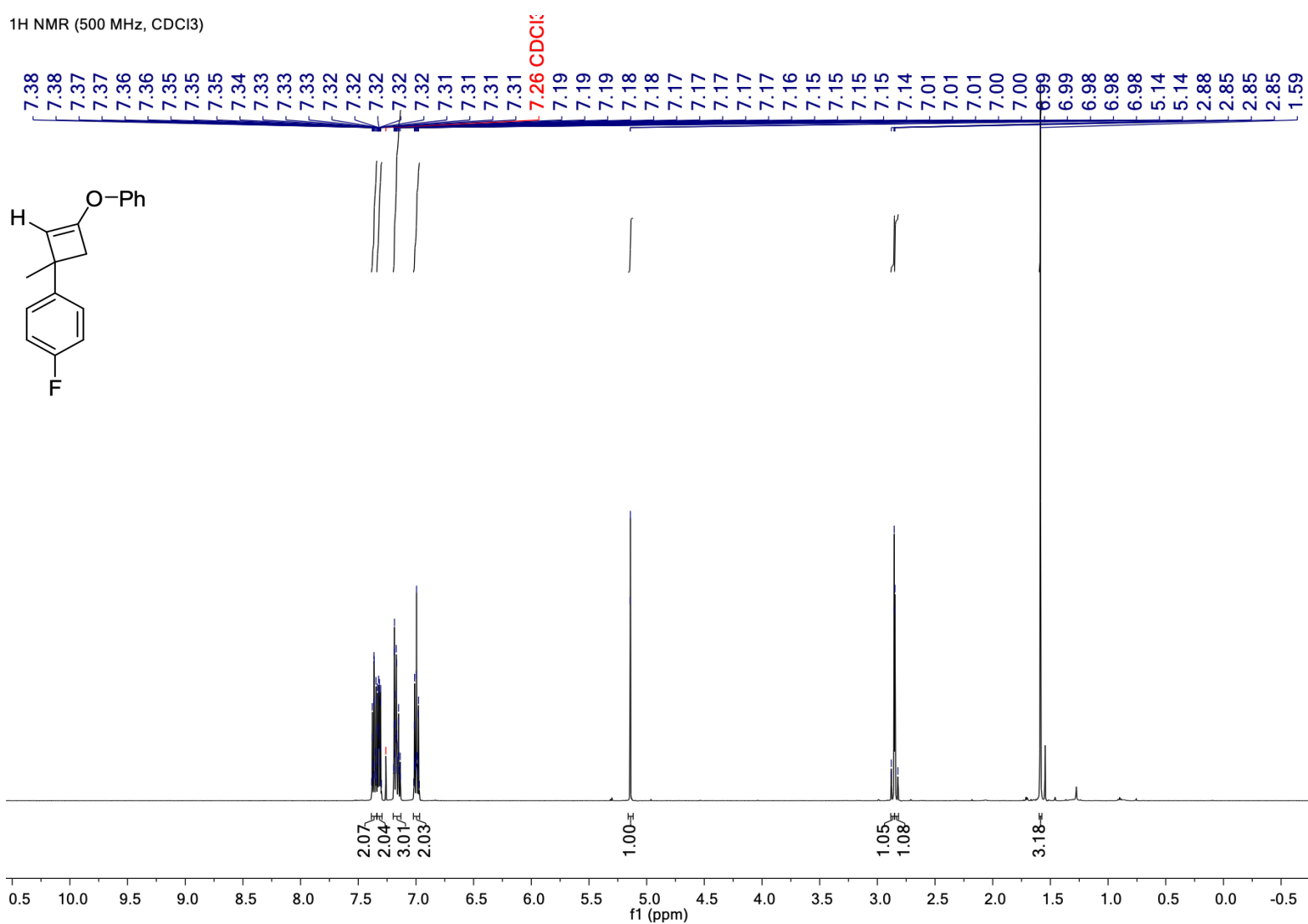


${ }^{19} \mathrm{~F}$ NMR $\left(376 \mathrm{MHz}, \mathrm{CDCl}_{3}\right)$ of $\mathbf{3 f}$

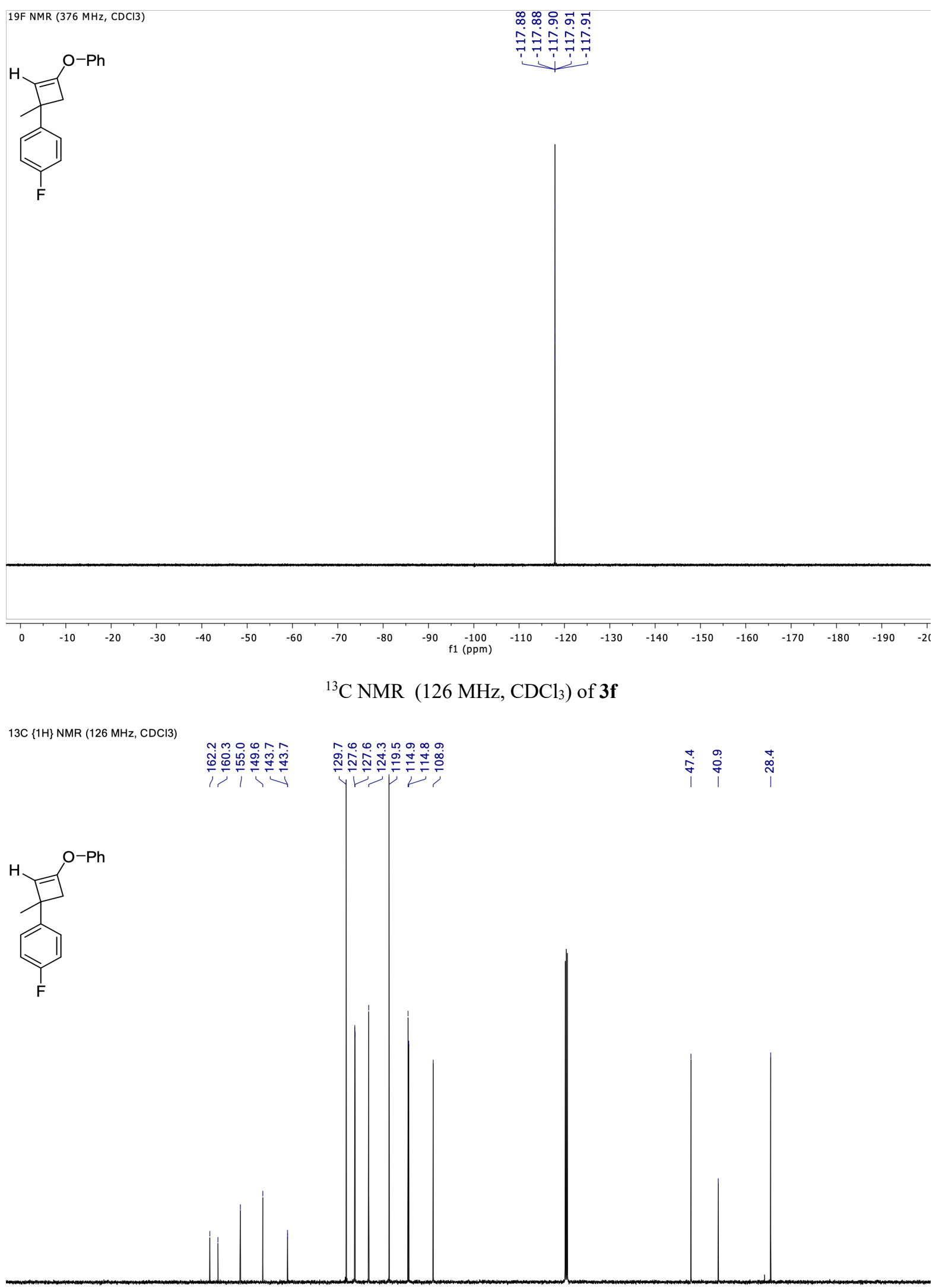

$\begin{array}{llllllllllllllllllllllll}110 & 200 & 190 & 180 & 170 & 160 & 150 & 140 & 130 & 120 & 110 & 100 & 90 & 80 & 70 & 60 & 50 & 40 & 30 & 20 & 10 & 0 & 0\end{array}$ 


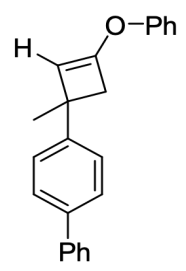

Cyclobutene 3g was synthesized following general procedure B starting from (ethynyloxy)benzene (23.6 mg, 0.2 mmol) and 4-(prop-1-en-2-yl)-1,1'-biphenyl (117 mg, $0.6 \mathrm{mmol})$. The crude product was purified by flash chromatography affording $\mathbf{3 g}$ as a white solid (56 $\mathrm{mg}, 90 \%)$.

${ }^{1} \mathbf{H}$ NMR $\left(500 \mathrm{MHz}, \mathrm{CDCl}_{3}\right) \delta 7.66-7.61(\mathrm{~m}, 2 \mathrm{H}), 7.61-7.55(\mathrm{~m}, 2 \mathrm{H}), 7.50-7.44(\mathrm{~m}, 4 \mathrm{H}), 7.42-7.33(\mathrm{~m}$, $3 \mathrm{H}), 7.24-7.21(\mathrm{~m}, 2 \mathrm{H}), 7.17(\mathrm{td}, J=7.3,1.2 \mathrm{~Hz}, 1 \mathrm{H}), 5.23(\mathrm{~s}, 1 \mathrm{H}), 2.97(\mathrm{~d}, J=12.7 \mathrm{~Hz}, 1 \mathrm{H}), 2.92(\mathrm{~d}, J=12.7$ $\mathrm{Hz}, 1 \mathrm{H}), 1.67$ (s, 3H).

${ }^{13}$ C NMR $\left(126 \mathrm{MHz}, \mathrm{CDCl}_{3}\right) \delta 155.0,149.6,147.1,141.2,138.9,129.7,128.9,127.2,127.2,127.0,126.6,124.3$, $119.5,109.1,47.4,41.2,28.3$.

HRMS (APCI) $m / z$ calculated for $\mathrm{C}_{23} \mathrm{H}_{21} \mathrm{O}^{+}[\mathrm{M}+\mathrm{H}]^{+}: 313.1587$, found: 313.1568 .

\section{${ }^{1} \mathrm{H} \mathrm{NMR}\left(500 \mathrm{MHz}, \mathrm{CDCl}_{3}\right)$ of $\mathbf{3 g}$}

1H NMR (500 MHz, CDCl3)

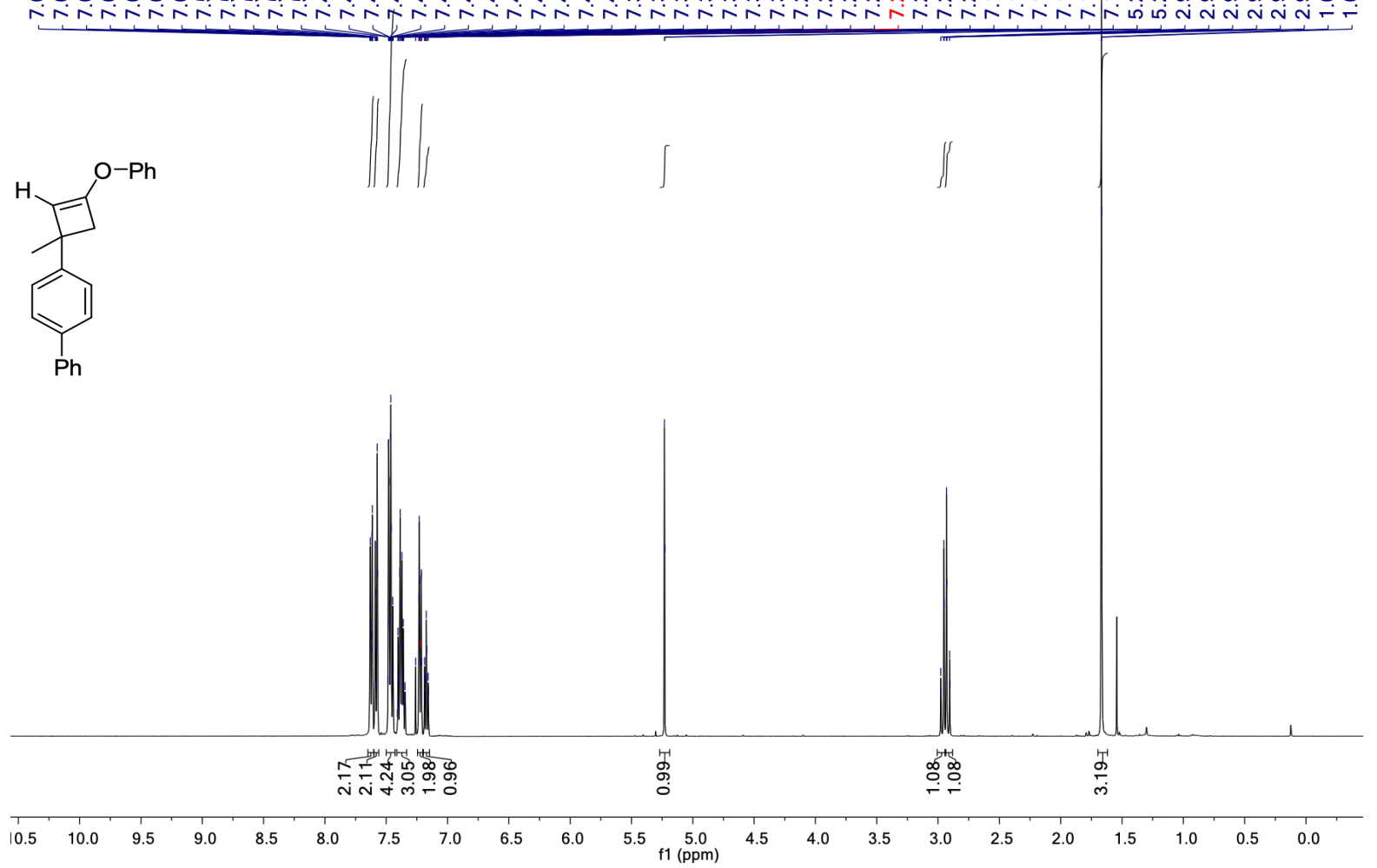


${ }^{13} \mathrm{C}$ NMR $\left(126 \mathrm{MHz}, \mathrm{CDCl}_{3}\right)$ of $\mathbf{3 g}$

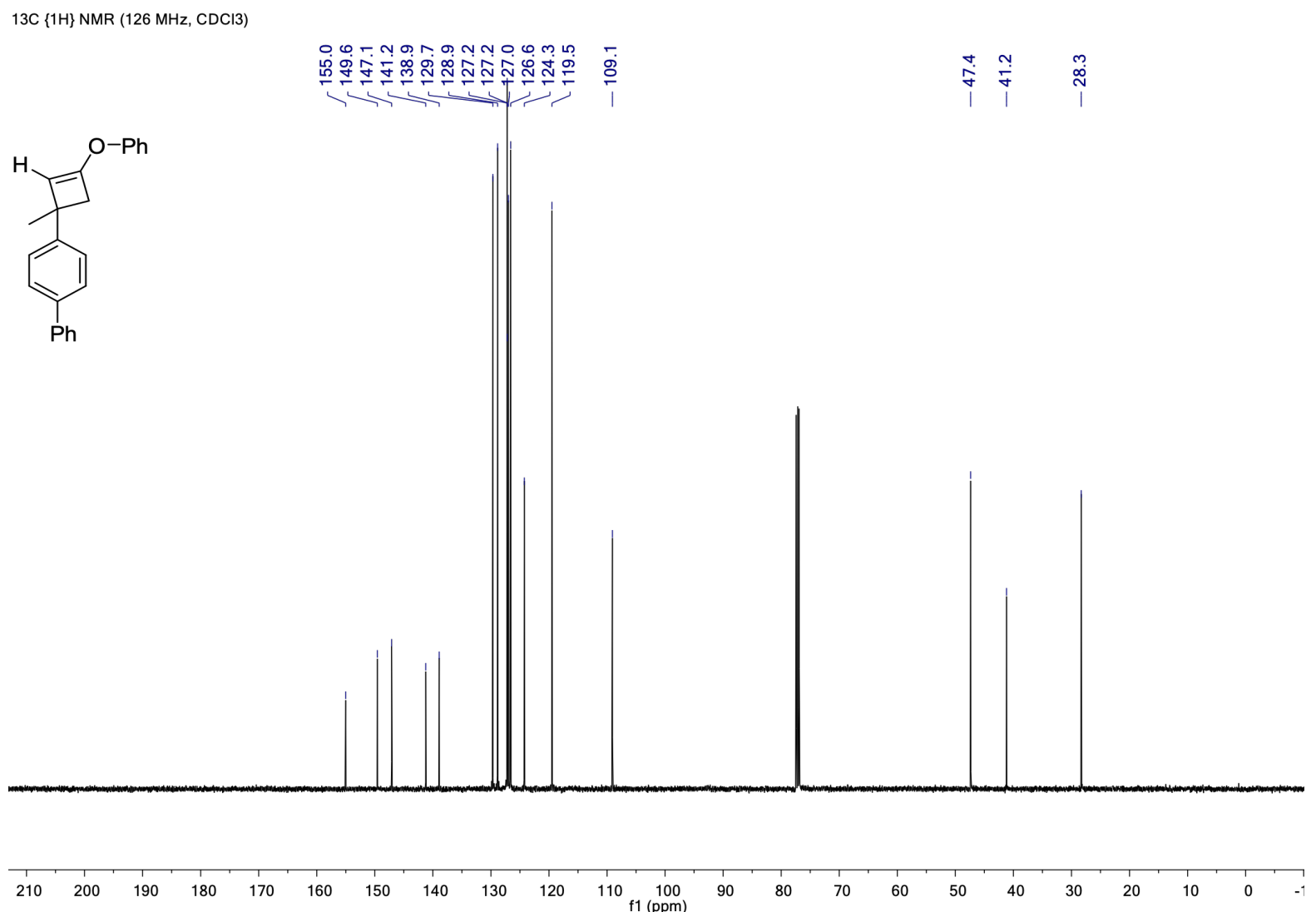




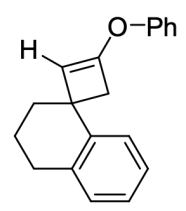

Cyclobutene 3h was synthesized following general procedure B starting from (ethynyloxy)benzene (23.6 mg, 0.2 mmol) and 1-methylene-1,2,3,4-tetrahydronaphthalene ( $87 \mathrm{mg}, 0.6 \mathrm{mmol})$. The crude product was purified by flash chromatography affording $\mathbf{3 h}$ as a brownish oil (21 $\mathrm{mg}, 40 \%)$.

${ }^{1} \mathbf{H}$ NMR $\left(400 \mathrm{MHz}, \mathrm{CDCl}_{3}\right) \delta 7.57(\mathrm{dd}, J=7.5,1.6 \mathrm{~Hz}, 1 \mathrm{H}), 7.42-7.34(\mathrm{~m}, 2 \mathrm{H}), 7.28-7.23(\mathrm{~m}, 2 \mathrm{H}), 7.21-$ $7.06(\mathrm{~m}, 4 \mathrm{H}), 5.00(\mathrm{~s}, 1 \mathrm{H}), 2.84(\mathrm{dd}, J=7.8,4.3 \mathrm{~Hz}, 2 \mathrm{H}), 2.73(\mathrm{~s}, 2 \mathrm{H}), 2.01-1.90(\mathrm{~m}, 3 \mathrm{H}), 1.82$ (dddd, $J=18.7$, 9.1, 7.5, $4.0 \mathrm{~Hz}, 1 \mathrm{H})$.

${ }^{13}$ C NMR $\left(126 \mathrm{MHz}, \mathrm{CDCl}_{3}\right) \delta 155.1,150.5,141.8,137.4,129.7,129.0,126.2,126.2,125.7,124.2,119.3,110.6$, $49.6,40.6,36.0,30.4,22.1$.

HRMS (APCI) $m / z$ calculated for $\mathrm{C}_{19} \mathrm{H}_{19} \mathrm{O}^{+}[\mathrm{M}+\mathrm{H}]^{+}: 263.1430$, found: 263.1429 .

${ }^{1} \mathrm{H}$ NMR (400 MHz, $\mathrm{CDCl}_{3}$ ) of $\mathbf{3 h}$

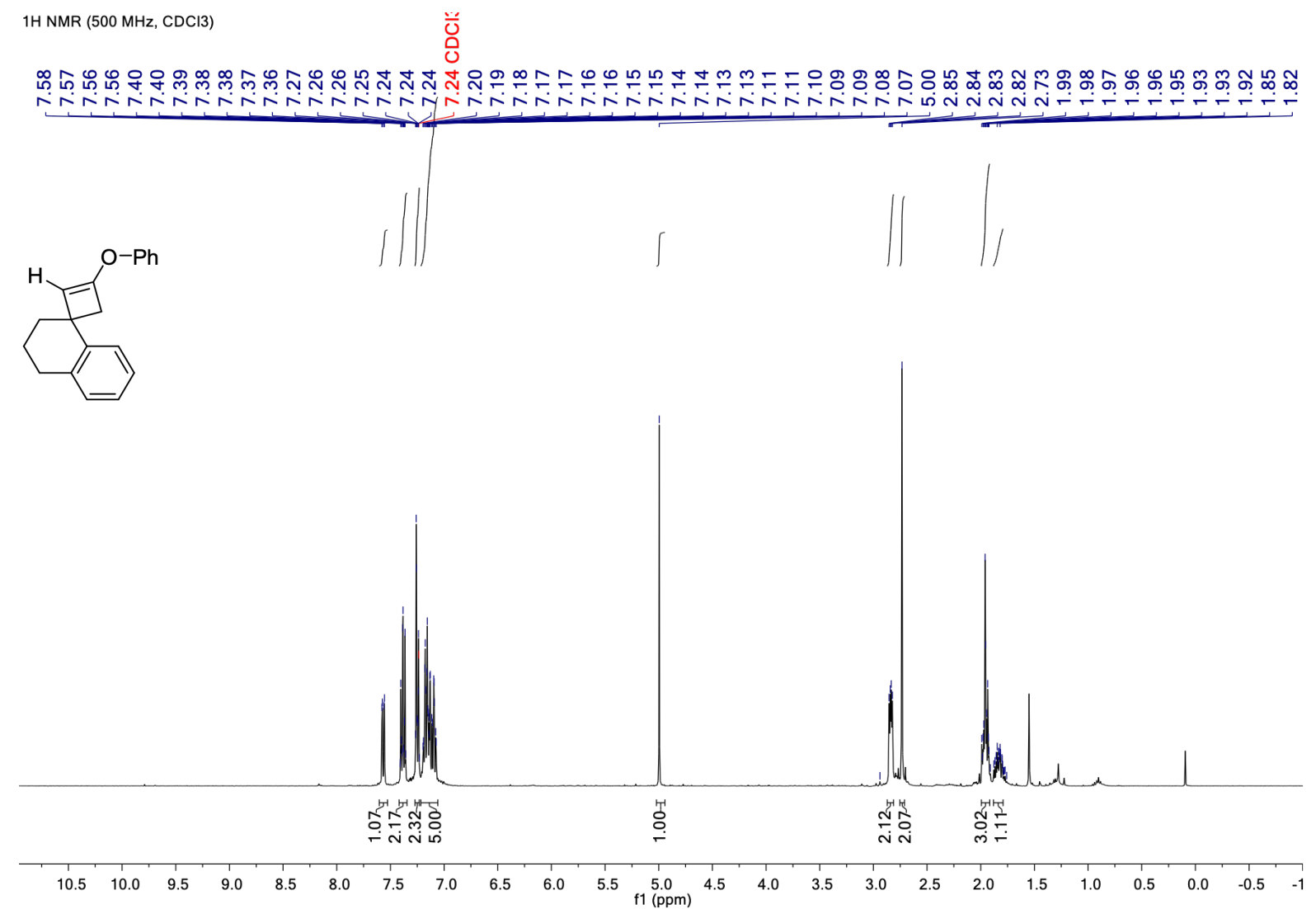


${ }^{13} \mathrm{C}$ NMR $\left(126 \mathrm{MHz}, \mathrm{CDCl}_{3}\right)$ of $\mathbf{3 h}$

$13 \mathrm{C}\{1 \mathrm{H}\} \mathrm{NMR}(126 \mathrm{MHz}, \mathrm{CDCl})$

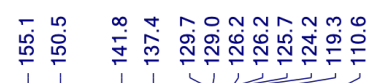

字
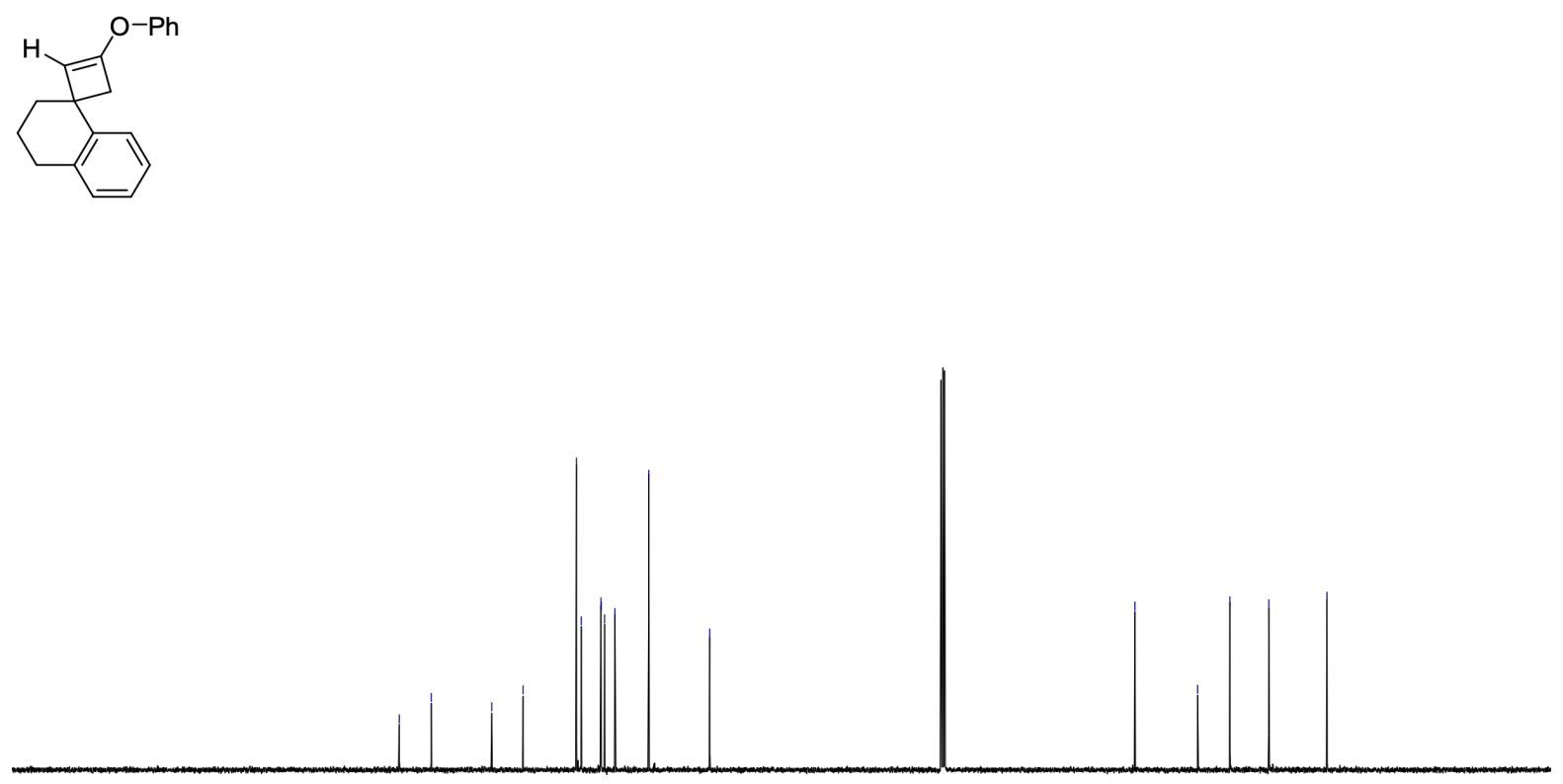

$\begin{array}{lllllllllllllllllllllllllllll}1 & 200 & 190 & 180 & 170 & 160 & 150 & 140 & 130 & 120 & 110 & 100 & 90 & 80 & 70 & 60 & 50 & 40 & 30 & 20 & 10 & 0 & -\end{array}$ 


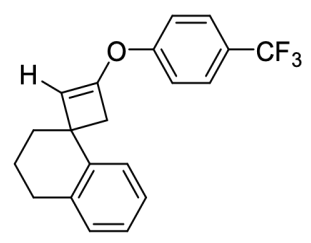

Cyclobutene 3i was synthesized following general procedure B starting from 1-(ethynyloxy)-4(trifluoromethyl)benzene (37.4 mg, $0.2 \mathrm{mmol}$ ) and 1-methylene-1,2,3,4-tetrahydronaphthalene (87 mg, 0.6 $\mathrm{mmol}$ ). The crude was purified by flash chromatography affording $\mathbf{3 i}$ as a yellow solid (59 $\mathrm{mg}, 89 \%)$.

M.p. $=61-69^{\circ} \mathrm{C}$.

${ }^{1} \mathbf{H}$ NMR $\left(500 \mathrm{MHz}, \mathrm{CDCl}_{3}\right) \delta 7.69-7.63(\mathrm{~m}, 2 \mathrm{H}), 7.55(\mathrm{dd}, J=7.6,1.7 \mathrm{~Hz}, 1 \mathrm{H}), 7.35(\mathrm{~d}, J=8.5 \mathrm{~Hz}, 2 \mathrm{H}), 7.23$ -7.09 (m, 3H), 5.14 (d, $J=0.7 \mathrm{~Hz}, 1 \mathrm{H}), 2.86$ (dd, $J=8.0,4.2 \mathrm{~Hz}, 2 \mathrm{H}), 2.78$ (p, $J=1.3 \mathrm{~Hz}, 2 \mathrm{H}), 2.08-1.93$ (m, $3 \mathrm{H}), 1.91-1.79(\mathrm{~m}, 1 \mathrm{H})$.

${ }^{19}$ F NMR $\left(376 \mathrm{MHz}, \mathrm{CDCl}_{3}\right) \delta-62.01$.

${ }^{13}$ C NMR (126 MHz, $\left.\mathrm{CDCl}_{3}\right) \delta 157.71,149.35,141.26,137.41,129.14,127.18$ (q, $\left.J=3.7 \mathrm{~Hz}\right), 126.39,126.24$, 126.18 (q, $J=32.8 \mathrm{~Hz}), 125.51,124.21$ (q, $J=271.5 \mathrm{~Hz}), 112.61,49.70,40.89,35.83,30.33,22.02$.

HRMS (ESI) $\mathrm{m} / \mathrm{z}$ calculated for $\mathrm{C}_{20} \mathrm{H}_{18} \mathrm{~F}_{3} \mathrm{O}^{+}[\mathrm{M}+\mathrm{H}]^{+}: 331.1304$, found: 331.1302 . 
${ }^{1} \mathrm{H}$ NMR $\left(400 \mathrm{MHz}, \mathrm{CDCl}_{3}\right)$ of $\mathbf{3 i}$

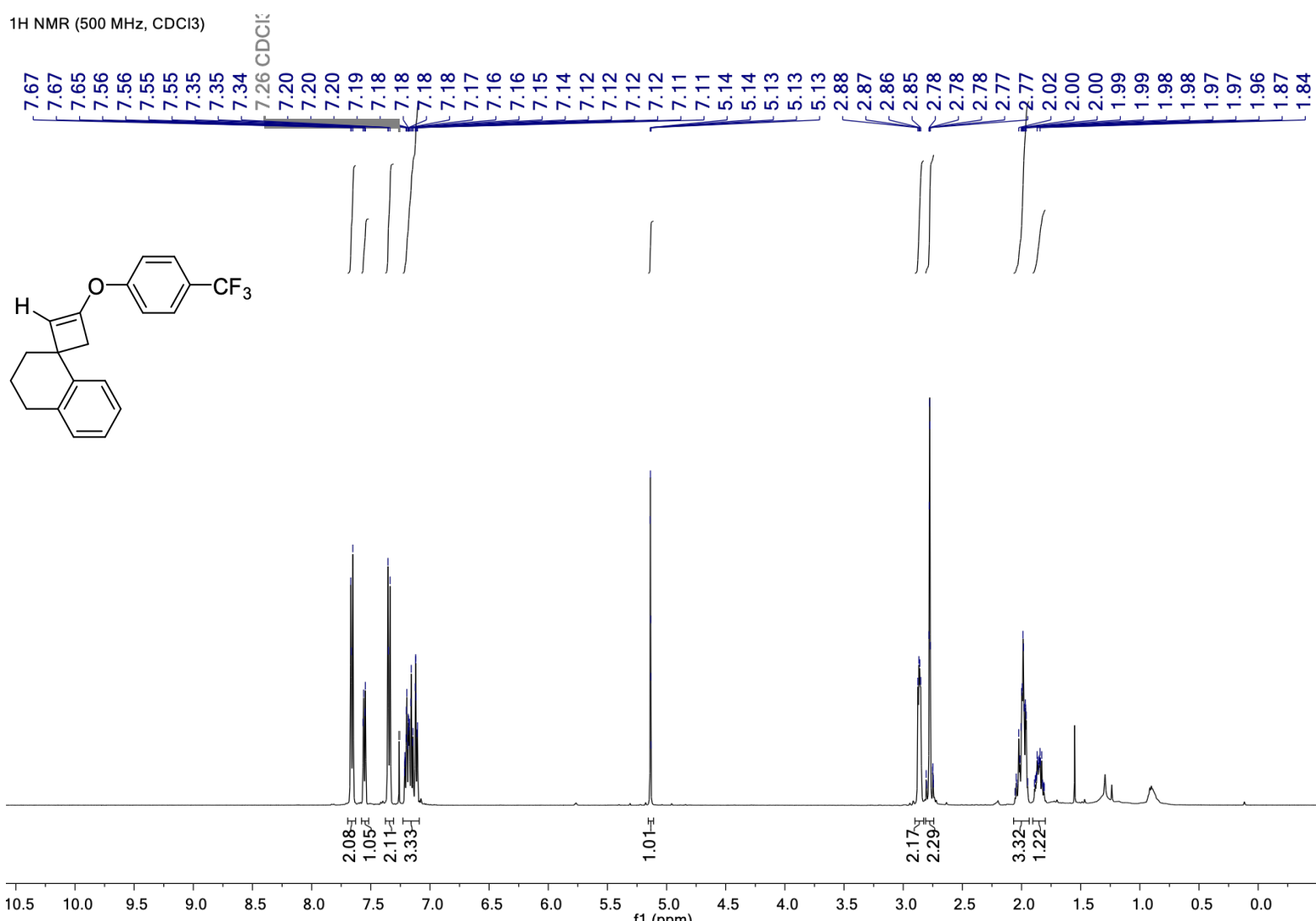

${ }^{19} \mathrm{~F}$ NMR $\left(376 \mathrm{MHz}, \mathrm{CDCl}_{3}\right)$ of $\mathbf{3 i}$

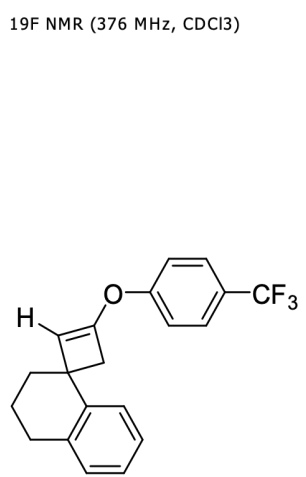

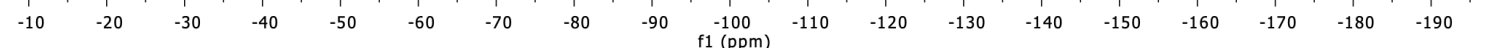


${ }^{13} \mathrm{C}$ NMR $\left(126 \mathrm{MHz}, \mathrm{CDCl}_{3}\right)$ of $\mathbf{3 i}$

$13 \mathrm{C}\{1 \mathrm{H}\} \mathrm{NMR}(126 \mathrm{MHz}, \mathrm{CDCl} 3)$
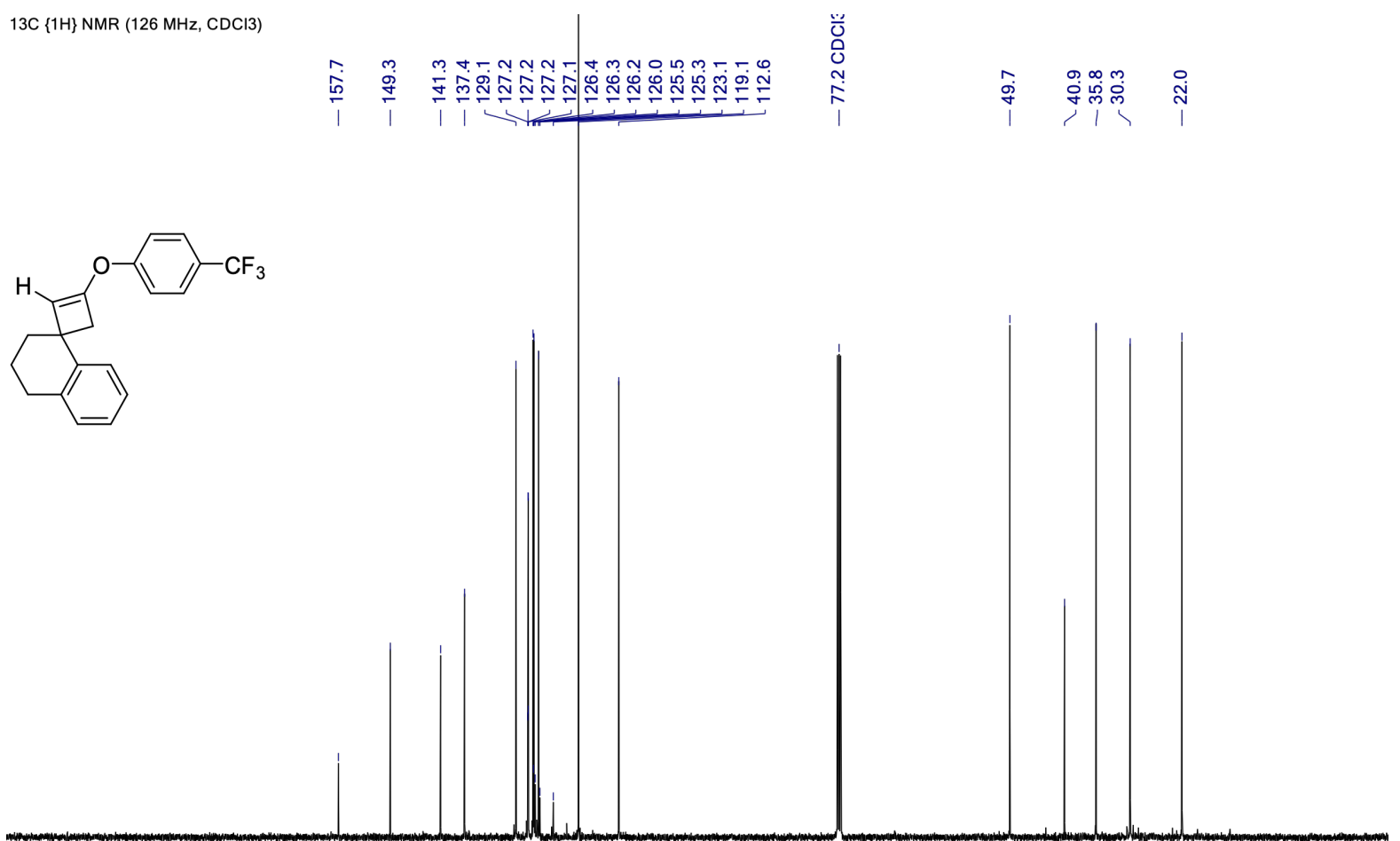

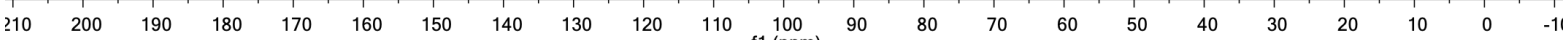




\section{(3-(4-(trifluoromethyl)phenoxy)cyclobut-2-ene-1,1-diyl)dibenzene (3j)}

Cyclobutene 3j was synthesized following general procedure B starting from 1-(ethynyloxy)-4(trifluoromethyl)benzene $(18.6 \mathrm{mg}, 0.1 \mathrm{mmol})$ and ethene-1,1-diyldibenzene $(54 \mathrm{mg}, 0.3 \mathrm{mmol})$. The crude was purified by flash chromatography affording $\mathbf{3} \mathbf{j}$ as a yellow solid (18 $\mathrm{mg}, 50 \%)$.

M.p. $=110-122^{\circ} \mathrm{C}$.

${ }^{1} \mathbf{H}$ NMR $\left(400 \mathrm{MHz}, \mathrm{CDCl}_{3}\right) \delta 7.67-7.59(\mathrm{~m}, 2 \mathrm{H}), 7.38-7.25(\mathrm{~m}, 10 \mathrm{H}), 7.22(\mathrm{ddt}, J=7.3,5.9,2.0 \mathrm{~Hz}, 2 \mathrm{H})$, $5.59(\mathrm{~s}, 1 \mathrm{H}), 3.39(\mathrm{~s}, 2 \mathrm{H})$.

${ }^{19}$ F NMR $\left(376 \mathrm{MHz}, \mathrm{CDCl}_{3}\right) \delta-61.66$.

${ }^{13}$ C NMR (101 MHz, $\left.\mathrm{CDCl}_{3}\right) \delta 157.4,149.8,146.1,128.4,127.3,127.2-127.1(\mathrm{~m}), 126.6(\mathrm{q}, J=32.7 \mathrm{~Hz})$, $126.3,124.2(\mathrm{q}, J=271.6 \mathrm{~Hz}), 119.5,109.4,49.0,47.9$.

HRMS (ESI) $\mathrm{m} / \mathrm{z}$ calculated for $\mathrm{C}_{23} \mathrm{H}_{18} \mathrm{~F}_{3} \mathrm{O}^{+}[\mathrm{M}+\mathrm{H}]^{+}: 367.1304$, found: 367.1304 .

${ }^{1} \mathrm{H}$ NMR $\left(400 \mathrm{MHz}, \mathrm{CDCl}_{3}\right)$ of $\mathbf{3 j}$

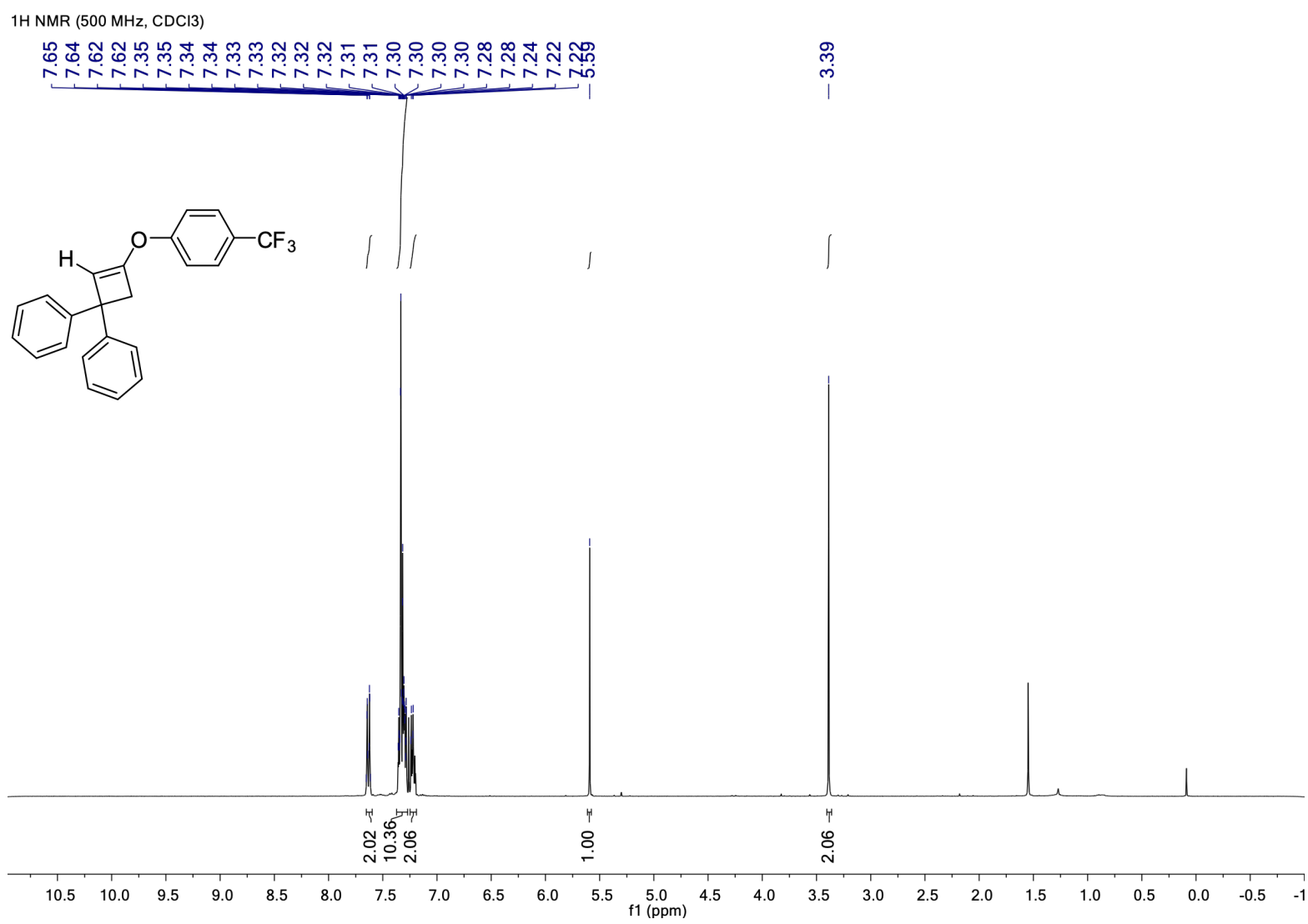


${ }^{19} \mathrm{~F}$ NMR (376 MHz, $\mathrm{CDCl}_{3}$ ) of $\mathbf{3 j}$

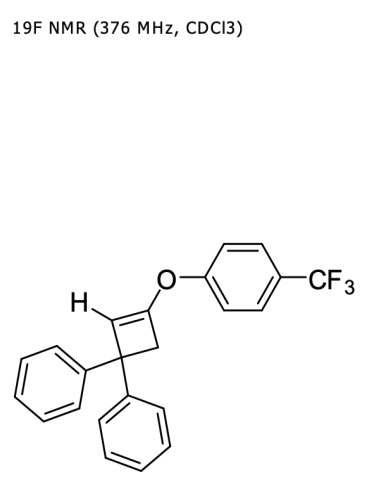

$\hat{i}$

\begin{tabular}{llllllllllllllllllllllllllllll}
\hline 30 & 20 & 10 & 0 & -10 & -20 & -30 & -40 & -50 & -60 & -70 & -80 & -90 & -100 & -110 & -120 & -130 & -140 & -150 & -160 & -170 & -180 & -190 & -200 & -210 & -220 & -23
\end{tabular}

${ }^{13} \mathrm{C}$ NMR $\left(101 \mathrm{MHz}, \mathrm{CDCl}_{3}\right)$ of $\mathbf{3 j}$

$13 \mathrm{C}\{1 \mathrm{H}\} \mathrm{NMR}(126 \mathrm{MHz}, \mathrm{CDCl} 3)$

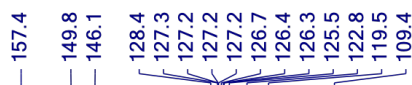

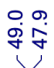
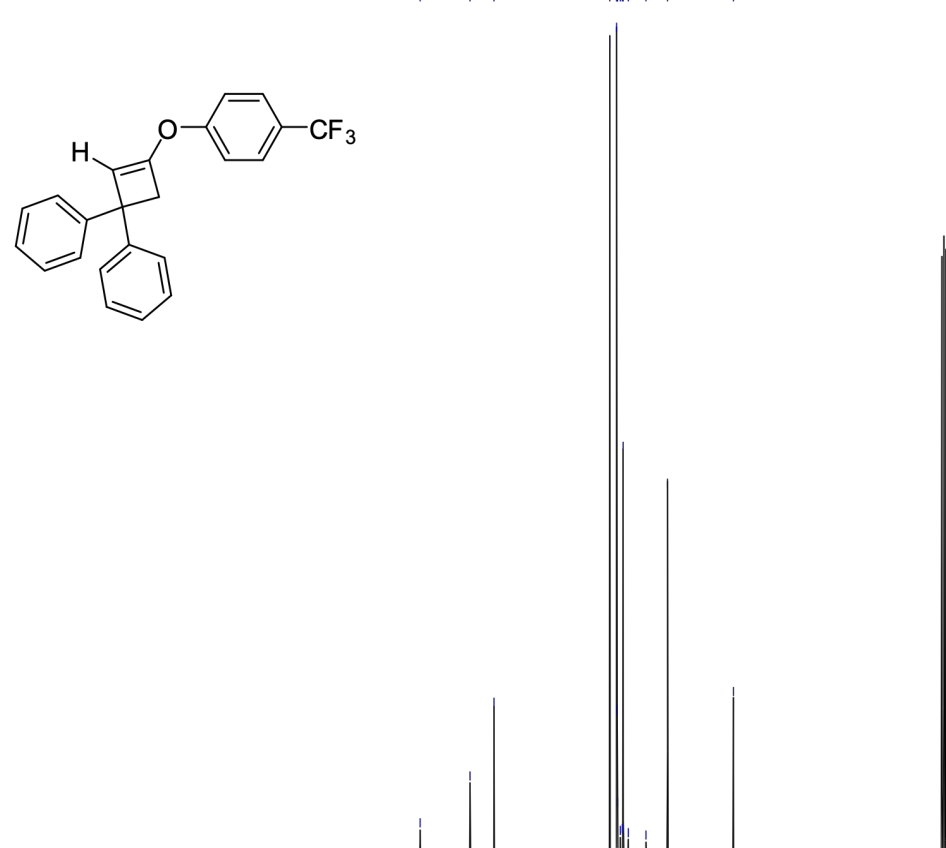

$\begin{array}{lllllllllllllllllllllll}210 & 200 & 190 & 180 & 170 & 160 & 150 & 140 & 130 & 120 & 110 & \begin{array}{c}100 \\ 100\end{array} & 90 & 80 & 70 & 60 & 50 & 40 & 30 & 20 & 10 & 0 & -10\end{array}$ 


\section{1-Methoxy-4-(3-phenoxycyclobut-2-en-1-yl)benzene (3k)}

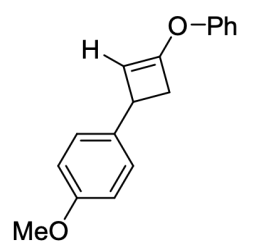

Cyclobutene 3k was synthesized following general procedure B starting from (ethynyloxy)benzene (23.6 mg, 0.2 mmol) and 1-methoxy-4-vinylbenzene $(81 \mathrm{mg}, 0.6 \mathrm{mmol})$. The crude product was purified by flash chromatography affording $\mathbf{3 k}$ as a yellow oil (19 $\mathrm{mg}, 37 \%)$.

${ }^{1} \mathbf{H}$ NMR $\left(400 \mathrm{MHz}, \mathrm{CDCl}_{3}\right) \delta 7.40-7.34(\mathrm{~m}, 2 \mathrm{H}), 7.28-7.19(\mathrm{~m}, 4 \mathrm{H}), 7.15(\mathrm{ddt}, J=8.5,7.0,1.2 \mathrm{~Hz}, 1 \mathrm{H}), 6.90$ - $6.84(\mathrm{~m}, 2 \mathrm{H}), 4.97(\mathrm{~d}, J=1.0 \mathrm{~Hz}, 1 \mathrm{H}), 3.81(\mathrm{~s}, 3 \mathrm{H}), 3.66(\mathrm{dt}, J=4.6,1.3 \mathrm{~Hz}, 1 \mathrm{H}), 3.24$ (dd, $J=13.0,4.6 \mathrm{~Hz}$, 1H), $2.52(\mathrm{dd}, J=13.0,1.6 \mathrm{~Hz}, 1 \mathrm{H})$.

${ }^{13}$ C NMR $\left(101 \mathrm{MHz}, \mathrm{CDCl}_{3}\right) \delta 158.4,155.0,150.1,136.2,129.7,127.7,124.2,119.4,113.9,105.3,55.5,41.9$, 36.8 .

HRMS (ESI) $m / z$ calculated for $\mathrm{C}_{17} \mathrm{H}_{17} \mathrm{O}_{2}^{+}[\mathrm{M}+\mathrm{H}]^{+}: 253.1223$, found: 253.1221 .

\section{${ }^{1} \mathrm{H} \mathrm{NMR}\left(400 \mathrm{MHz}, \mathrm{CDCl}_{3}\right)$ of $\mathbf{3 k}$}

1H NMR (500 MHz, CDCl3)

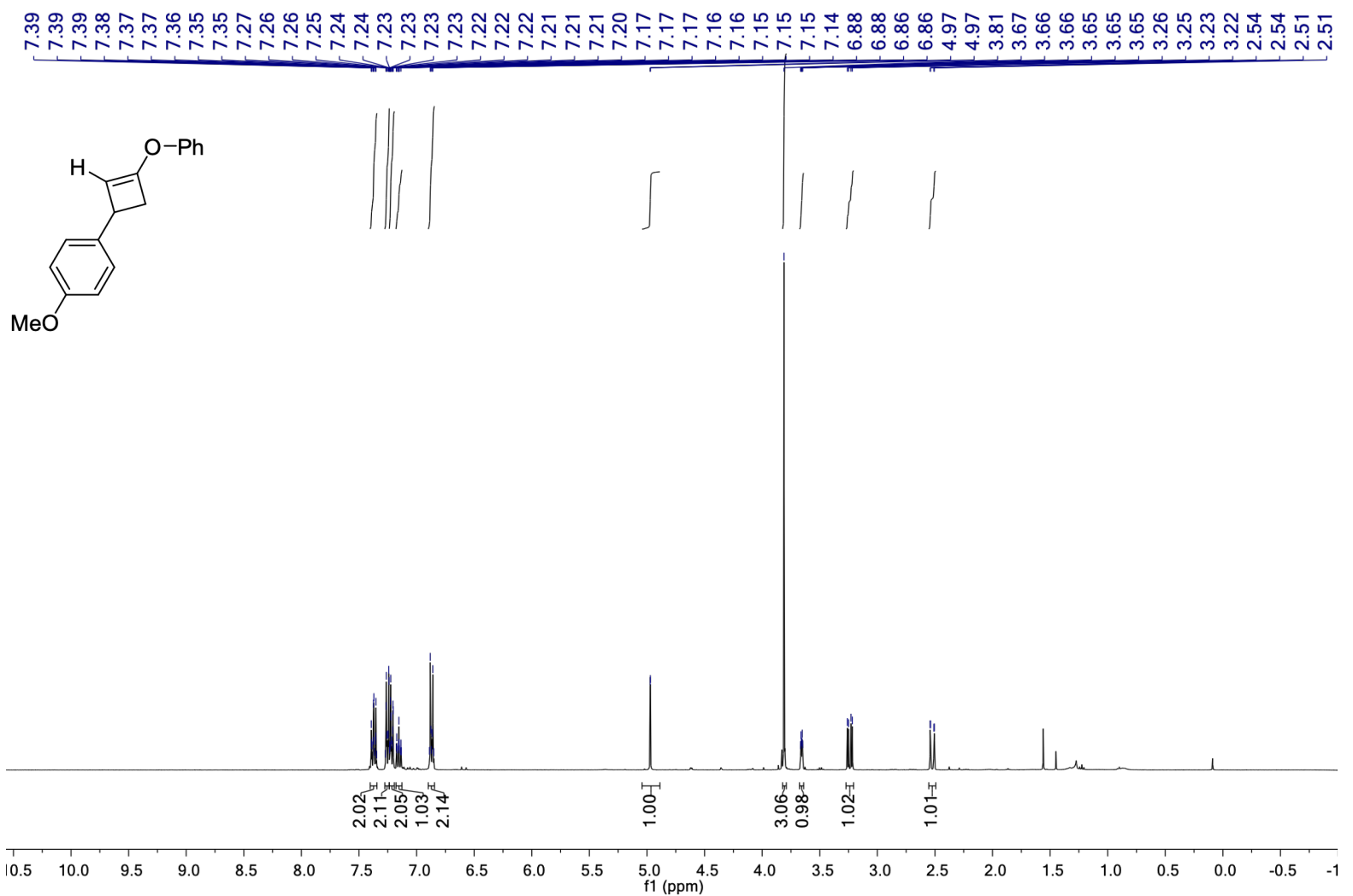


${ }^{13} \mathrm{C}$ NMR $\left(101 \mathrm{MHz}, \mathrm{CDCl}_{3}\right)$ of $\mathbf{3 k}$

$13 \mathrm{C}\{1 \mathrm{H}\}$ NMR $(126 \mathrm{MHz}, \mathrm{CDCl} 3)$

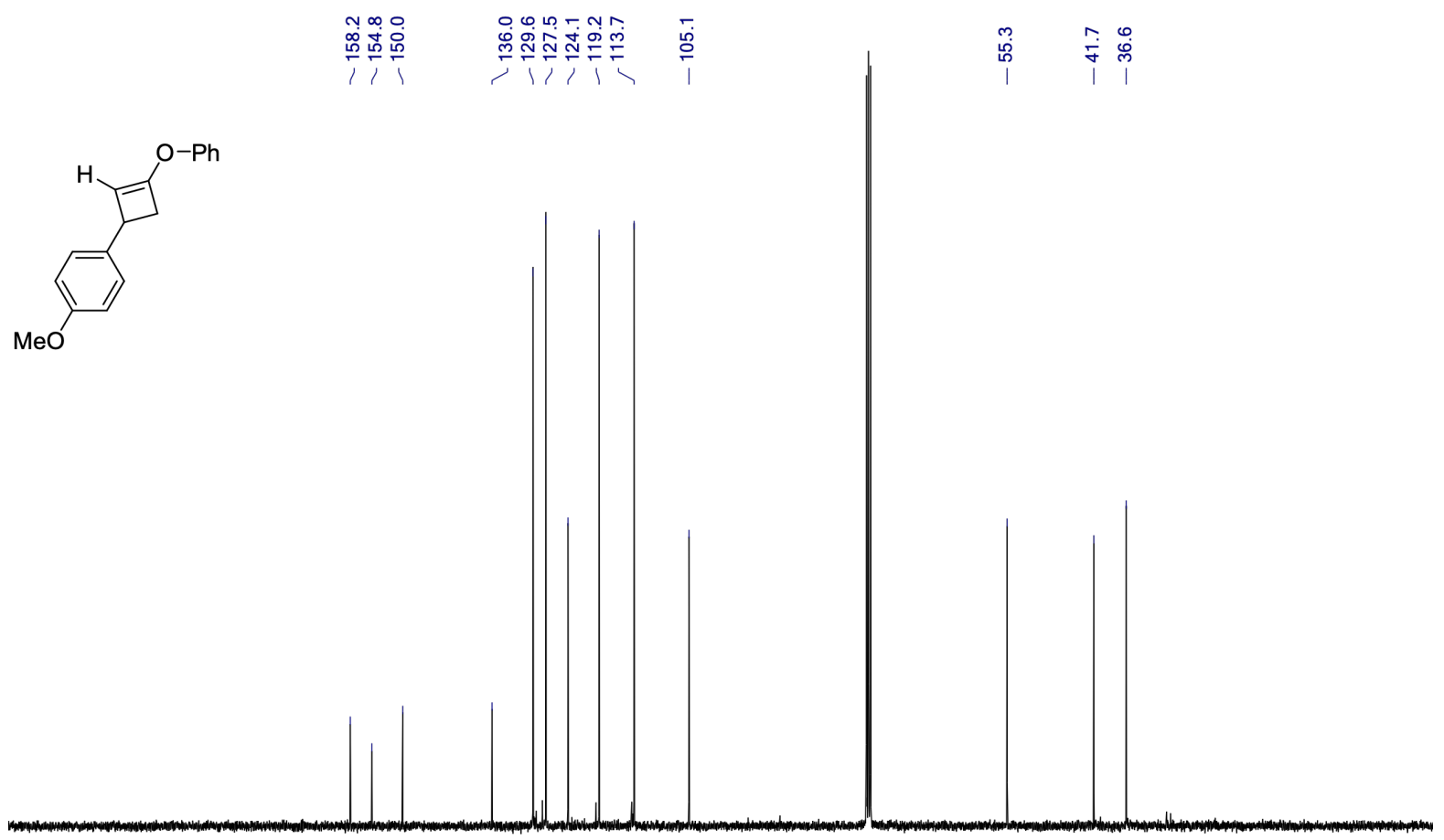

$\begin{array}{llllllllllllllllllllllll}210 & 200 & 190 & 180 & 170 & 160 & 150 & 140 & 130 & 120 & 110 & 100 & 90 & 80 & 70 & 60 & 50 & 40 & 30 & 20 & 10 & 0 & -11\end{array}$ 


\section{1-Chloro-4-((3-(4-methoxyphenyl)cyclobut-1-en-1-yl)oxy)benzene (3I)}

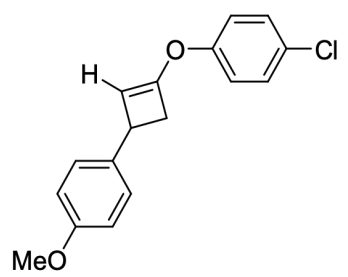

Cyclobutene 31 was synthesized following general procedure B starting from 1-chloro-4-(ethynyloxy)benzene (45.8 $\mathrm{mg}, 0.3 \mathrm{mmol}$ ) and 1-methoxy-4-vinylbenzene (121 mg, $0.9 \mathrm{mmol})$. The crude was purified by flash chromatography affording $\mathbf{3 l}$ as a transparent oil (33 $\mathrm{mg}, 38 \%)$.

${ }^{1}$ H NMR (400 MHz, $\left.\mathrm{CDCl}_{3}\right) \delta 7.36-7.27(\mathrm{~m}, 2 \mathrm{H}), 7.27-7.19(\mathrm{~m}, 2 \mathrm{H}), 7.19-7.10(\mathrm{~m}, 2 \mathrm{H}), 6.92-6.80(\mathrm{~m}$, 2H), $4.96(\mathrm{dd}, J=1.0,0.4 \mathrm{~Hz}, 1 \mathrm{H}), 3.80(\mathrm{~s}, 3 \mathrm{H}), 3.65(\mathrm{dt}, J=4.4,1.3 \mathrm{~Hz}, 1 \mathrm{H}), 3.22(\mathrm{dd}, J=13.0,4.5 \mathrm{~Hz}, 1 \mathrm{H})$, $2.51(\mathrm{ddd}, J=13.0,1.6,0.4 \mathrm{~Hz}, 1 \mathrm{H})$.

${ }^{13}$ C NMR $\left(101 \mathrm{MHz}, \mathrm{CDCl}_{3}\right) \delta 158.4,153.5,149.8,135.8,129.7,129.4,120.7,113.9,105.7,55.45,41.8,36.8$.

HRMS (ESI) m/z calculated for $\mathrm{C}_{17} \mathrm{H}_{16} \mathrm{ClO}_{2}{ }^{+}[\mathrm{M}+\mathrm{H}]^{+}: 287.0833$, found: 287.0832 .

${ }^{1} \mathrm{H}$ NMR (400 MHz, $\left.\mathrm{CDCl}_{3}\right)$ of $\mathbf{3 l}$

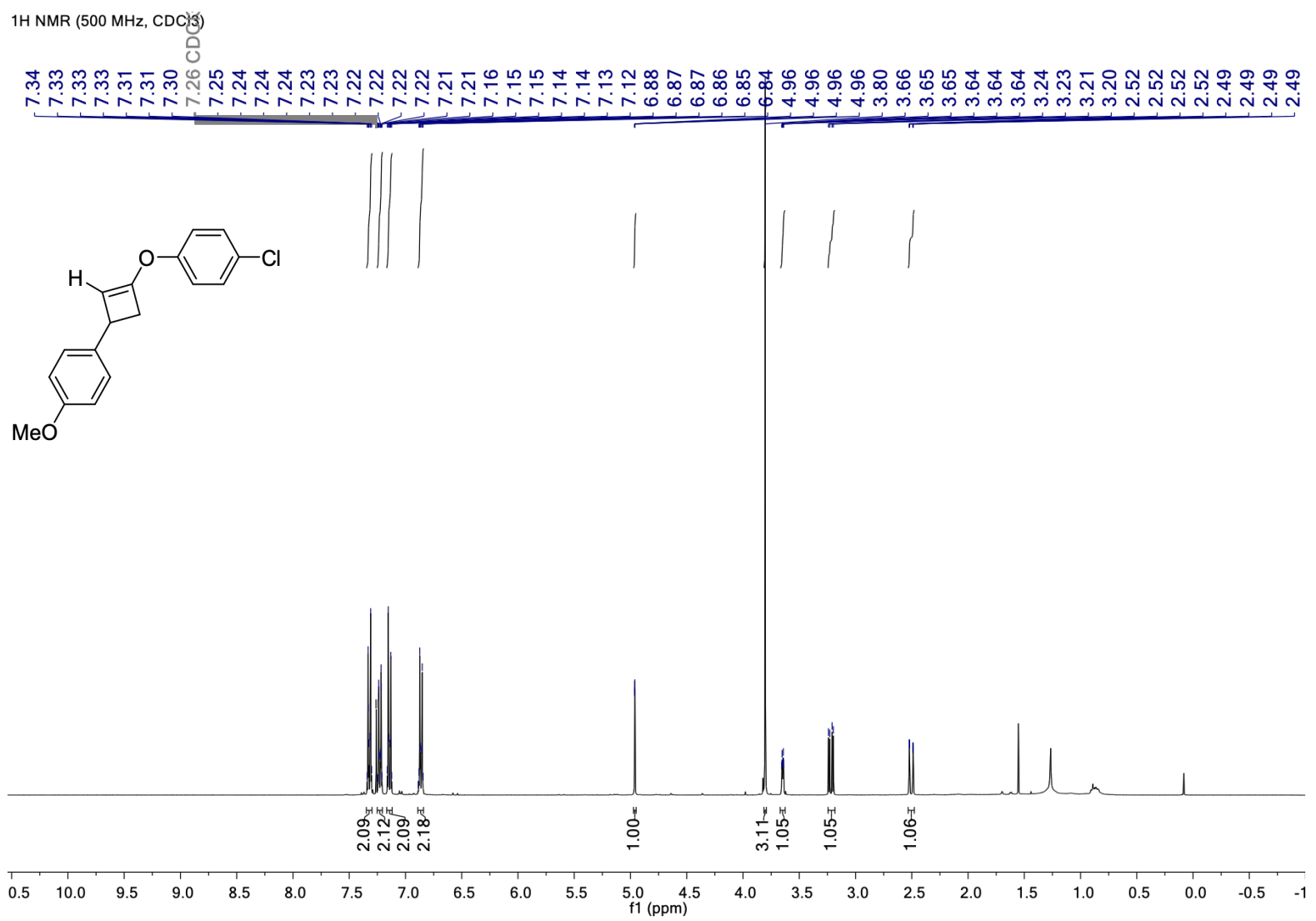


${ }^{13} \mathrm{C}$ NMR $\left(101 \mathrm{MHz}, \mathrm{CDCl}_{3}\right)$ of $3 \mathrm{I}$

$13 \mathrm{C}\{1 \mathrm{H}\} \mathrm{NMR}(126 \mathrm{MHz}, \mathrm{CDCl} 3)$

岤
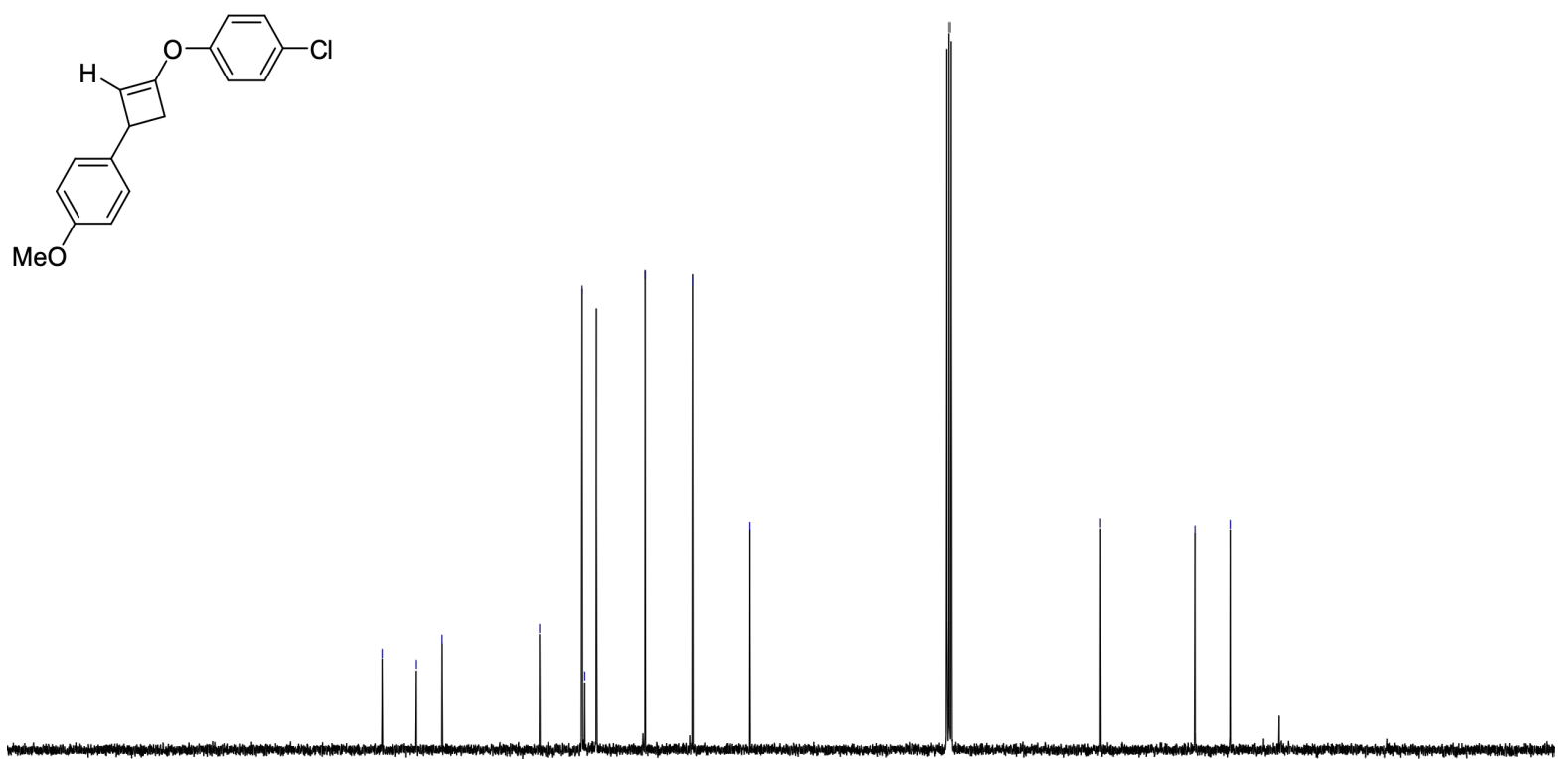

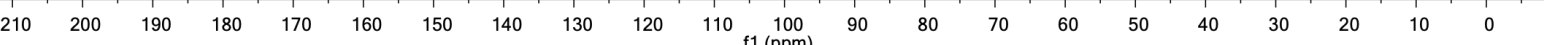




\section{1-Methoxy-4-(3-(4-(trifluoromethyl)phenoxy)cyclobut-2-en-1-yl)benzene (3m)}

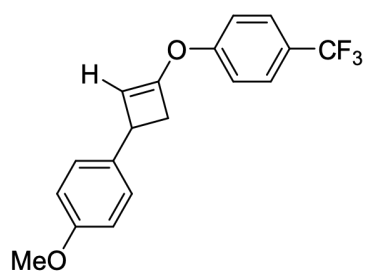

Cyclobutene 3m was synthesized following general procedure B starting from 1-(ethynyloxy)-4(trifluoromethyl)benzene $(55.8 \mathrm{mg}, 0.3 \mathrm{mmol})$ and 1-methoxy-4-vinylbenzene $(121 \mathrm{mg}, 0.9 \mathrm{mmol})$. The crude was purified by flash chromatography affording $3 \mathrm{~m}$ as a transparent oil (40 $\mathrm{mg}, 42 \%)$.

${ }^{1}$ H NMR (500 MHz, $\left.\mathrm{CDCl}_{3}\right) \delta 7.65-7.59(\mathrm{~m}, 2 \mathrm{H}), 7.32-7.27(\mathrm{~m}, 2 \mathrm{H}), 7.25-7.22(\mathrm{~m}, 2 \mathrm{H}), 6.89-6.85(\mathrm{~m}$, 2H), $5.11-5.07(\mathrm{~d}, 1 \mathrm{H}), 3.81(\mathrm{~s}, 3 \mathrm{H}), 3.69(\mathrm{dt}, J=4.6,1.3 \mathrm{~Hz}, 1 \mathrm{H}), 3.25$ (dd, $J=13.1,4.6 \mathrm{~Hz}, 1 \mathrm{H}), 2.55$ (ddd, $J$ $=13.1,1.6,0.4 \mathrm{~Hz}, 1 \mathrm{H})$.

${ }^{19}$ F NMR $\left(376 \mathrm{MHz}, \mathrm{CDCl}_{3}\right) \delta-62.03$

${ }^{13} \mathbf{C}$ NMR $\left(126 \mathrm{MHz}, \mathrm{CDCl}_{3}\right) \delta 158.5,157.6,148.9,135.5,127.7,127.2$ (q, $\left.J=3.7 \mathrm{~Hz}\right), 126.2(\mathrm{q}), 125.6(\mathrm{q}, J=$ $272.2 \mathrm{~Hz}), 119.1,114.0,107.4,55.5,42.0,37.0$.

HRMS (ESI) m/z calculated for $\mathrm{C}_{18} \mathrm{H}_{16} \mathrm{~F}_{3} \mathrm{O}_{2}{ }^{+}[\mathrm{M}+\mathrm{H}]^{+}: 321.1097$, found: 321.1101 .

${ }^{1} \mathrm{H}$ NMR $\left(500 \mathrm{MHz}, \mathrm{CDCl}_{3}\right)$ of $\mathbf{3 m}$

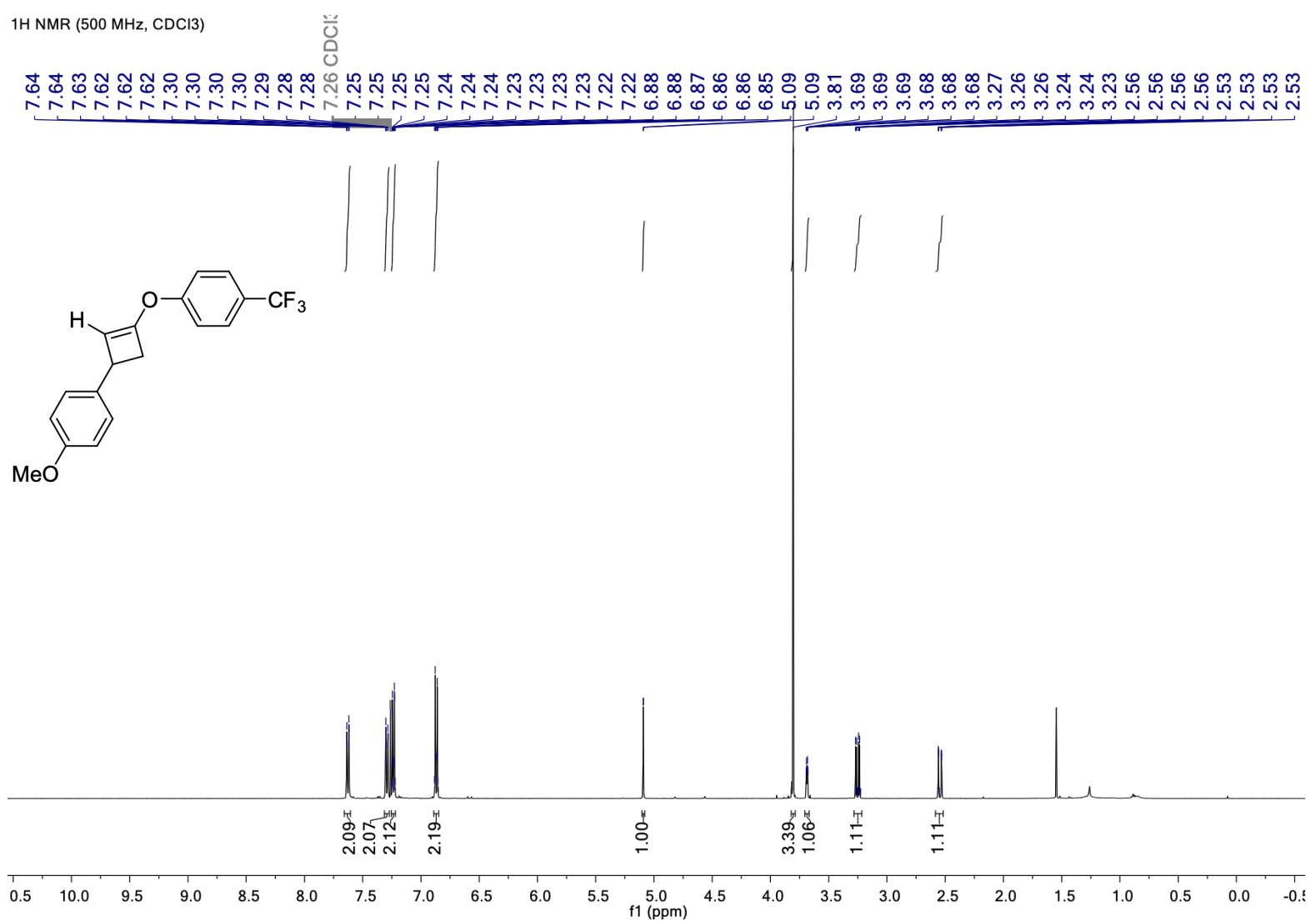


${ }^{19} \mathrm{~F} \mathrm{NMR}\left(376 \mathrm{MHz}, \mathrm{CDCl}_{3}\right)$ of $\mathbf{3 m}$
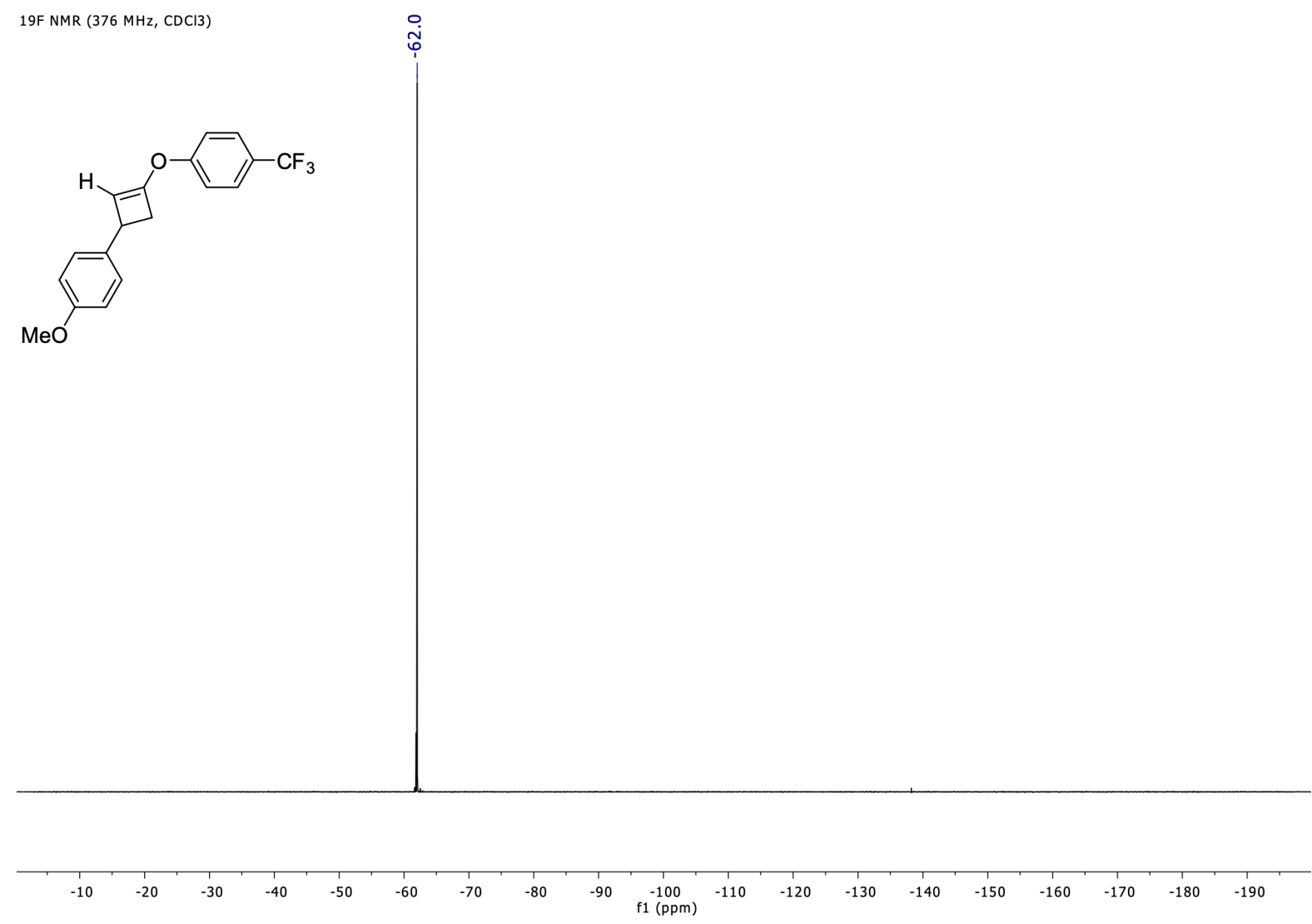

${ }^{13} \mathrm{C}$ NMR (126 MHz, $\left.\mathrm{CDCl}_{3}\right)$ of $\mathbf{3 m}$
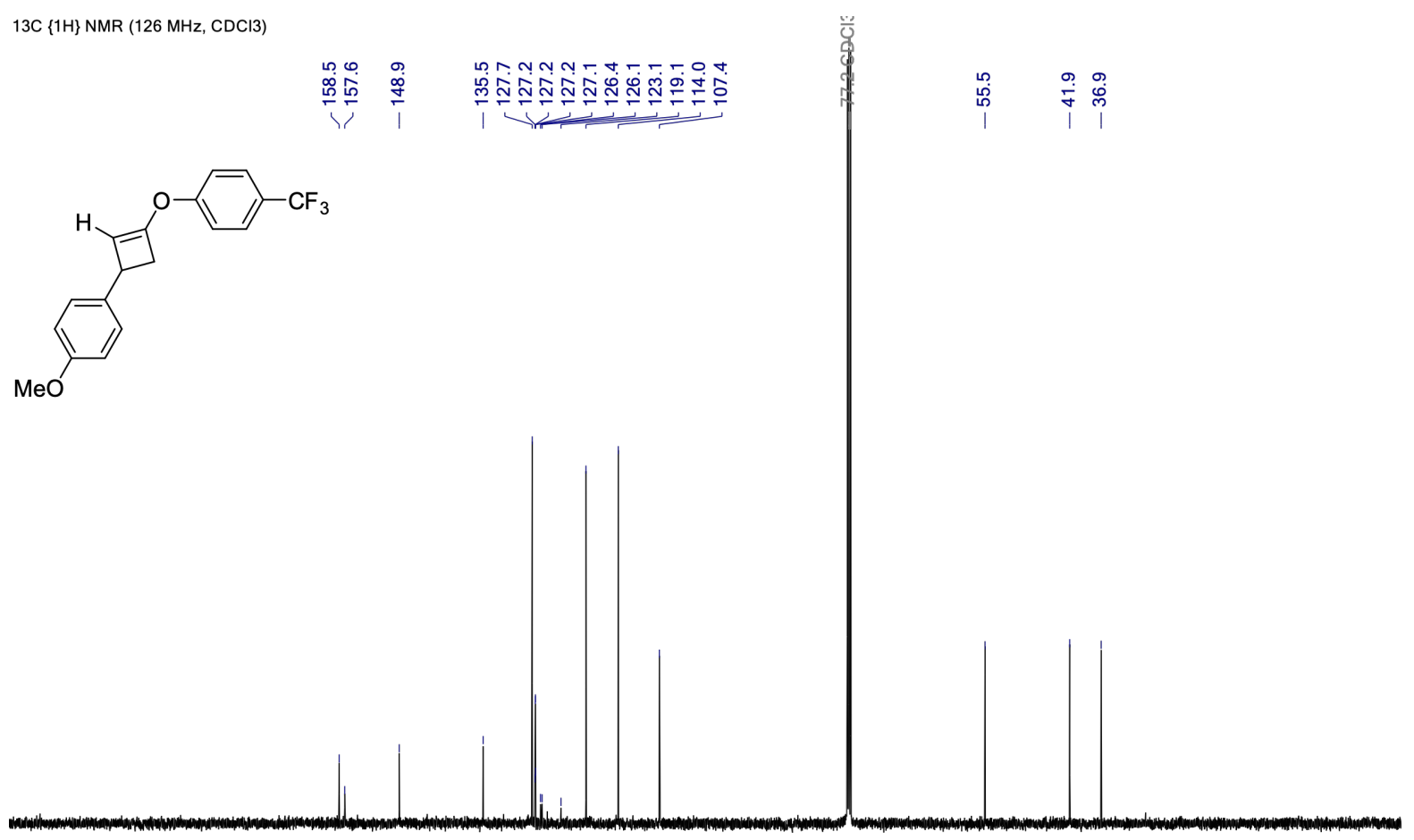

$\begin{array}{lllllllllllllllllllllll}210 & 200 & 190 & 180 & 170 & 160 & 150 & 140 & 130 & 120 & 110 & 100 & 90 & 80 & 70 & 60 & 50 & 40 & 30 & 20 & 10 & 0 & -1\end{array}$ 


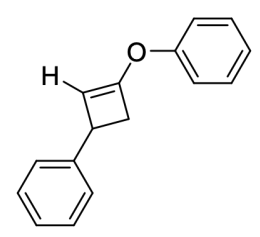

Cyclobutene 3n was synthesized following general procedure $\mathbf{B}$ starting from (ethynyloxy)benzene (23.6 mg, 0.2 $\mathrm{mmol}$ ) and styrene $(63 \mathrm{mg}, 0.3 \mathrm{mmol})$. The diagnostic signals of product were identified in the crude mixture and quantified by ${ }^{1} \mathrm{H}$ NMR using trichloroethylene as internal standard (16\%). However any attempt to isolate the product failed.

${ }^{1} \mathrm{H}$ NMR $\left(400 \mathrm{MHz}, \mathrm{CDCl}_{3}\right)$ of crude mixture of the reaction for the synthesis of $\mathbf{3 n}$

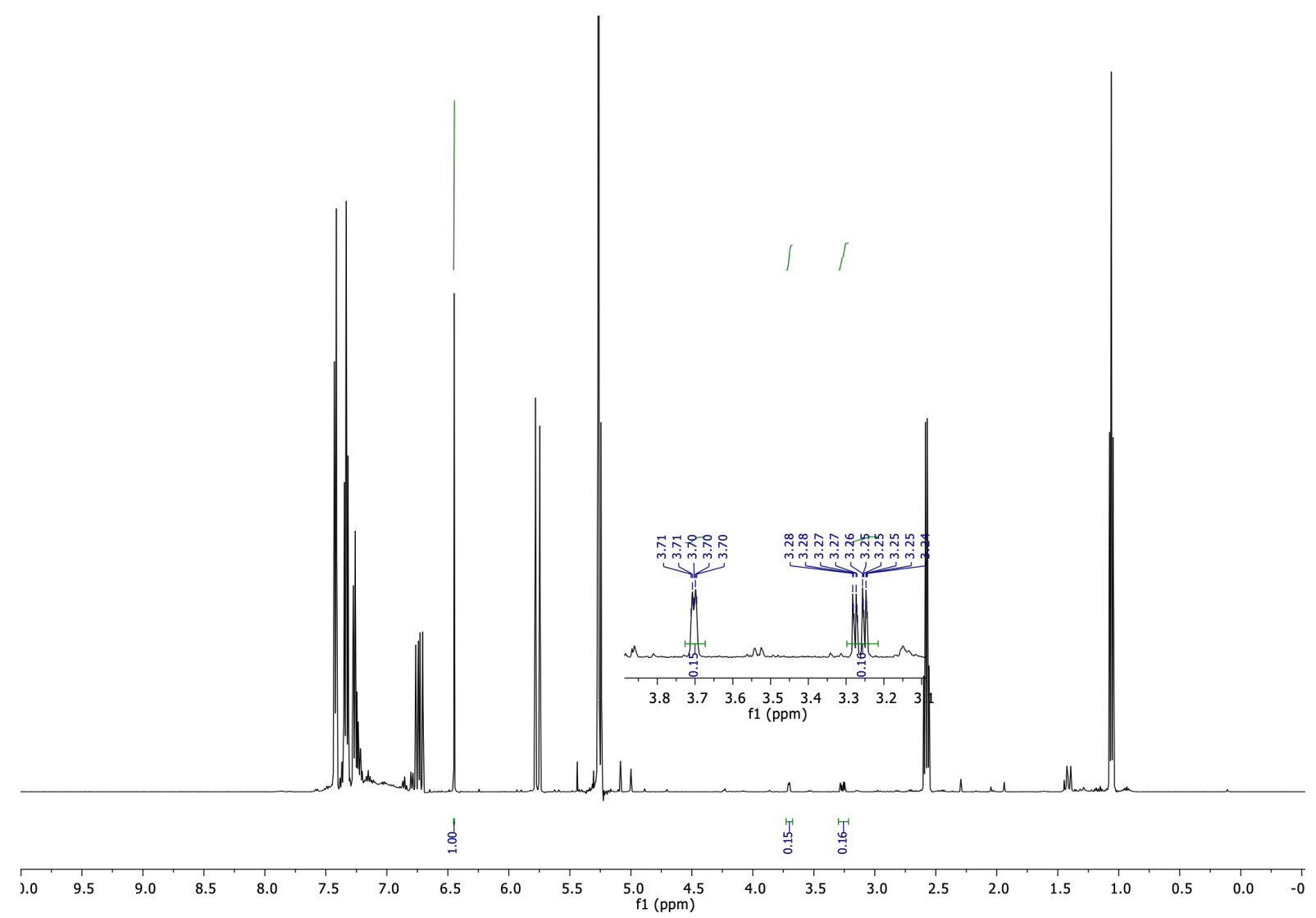

1-((3-phenylcyclobut-1-en-1-yl)oxy)-4-(trifluoromethyl)benzene (30)

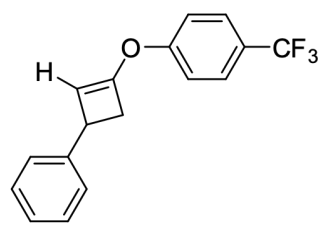

Cyclobutene 3o was synthesized following general procedure B starting from 1-(ethynyloxy)-4(trifluoromethyl)benzene (37.2 $\mathrm{mg}, 0.2 \mathrm{mmol})$ and styrene $(63 \mathrm{mg}, 0.3 \mathrm{mmol})$. The crude was purified by flash chromatography affording $3 \mathbf{0}$ as a transparent oil, 7:1 mixture of regioisomers (19 mg, 33\%). 
${ }^{1} \mathbf{H}$ NMR (400 MHz, $\left.\mathrm{CDCl}_{3}\right) \delta 7.68-7.57(\mathrm{~m}, 2.4 \mathrm{H}, \mathrm{Mr}+\mathrm{mr}), 7.37-7.27(\mathrm{~m}, 6.6 \mathrm{H}, \mathrm{Mr}+\mathrm{mr}), 7.29-7.19(\mathrm{~m}$, $1.1 \mathrm{H}, \mathrm{Mr}+\mathrm{mr}), 5.12(\mathrm{~m}, 1 \mathrm{H}, \mathrm{Mr}), 5.03(\mathrm{~s}, 0.1 \mathrm{H}, \mathrm{mr}), 4.26(\mathrm{dd}, J=4.6,1.5 \mathrm{~Hz}, 0.1 \mathrm{H}, \mathrm{mr}), 3.74(\mathrm{dt}, J=4.6,1.3$ $\mathrm{Hz}, 1 \mathrm{H}, \mathrm{Mr}), 3.29$ (dd, $J=13.07,4.57 \mathrm{~Hz}, 1 \mathrm{H}, \mathrm{Mr}), 2.71$ (ddd, $J=10.7,4.6,1.0 \mathrm{~Hz}, 0.1 \mathrm{H}, \mathrm{mr}), 2.61$ (dd, $J=13.1$, $1.6 \mathrm{~Hz}, 1 \mathrm{H}, \mathrm{Mr}), 2.09$ (ddd, $J=10.7,1.5,1.0 \mathrm{~Hz}, 0.1 \mathrm{H}, \mathrm{mr}$ ).

${ }^{19}$ F NMR $\left(376 \mathrm{MHz}, \mathrm{CDCl}_{3}\right) \delta-62.03$.

${ }^{13}$ C NMR (101 MHz, $\left.\mathrm{CDCl}_{3}\right) \delta 157.6(\mathrm{Mr}+\mathrm{mr}), 149.8(\mathrm{mr}), 149.0(\mathrm{Mr}), 143.5(\mathrm{Mr}), 140.2(\mathrm{mr}), 128.8(\mathrm{mr})$, $128.6(\mathrm{Mr}), 127.2$ (q, $J=3.8 \mathrm{~Hz}, \mathrm{Mr}+\mathrm{mr}), 127.0(\mathrm{mr}), 126.9(\mathrm{mr}), 126.8(\mathrm{Mr}), 126.7$ (Mr), 126.3 (q, $J=32.9$ Hz, Mr), 124.2 (q, J=271.5 Hz, Mr), 119.2 (Mr), 118.7 (mr), 107.2 (Mr), 103.8 (mr), 49.5 (mr), 41.8 (Mr), 37.6 (Mr), $31.6(\mathrm{mr})$. Two aromatic ${ }^{13} \mathrm{C}$ signals of the $\mathrm{mr}$ were not detected because of low intensity.

HRMS (ESI) $\mathrm{m} / \mathrm{z}$ calculated for $\mathrm{C}_{17} \mathrm{H}_{14} \mathrm{~F}_{3} \mathrm{O}^{+}[\mathrm{M}+\mathrm{H}]^{+}: 291.0991$, found: 291.0986 .

${ }^{1} \mathrm{H}$ NMR $\left(400 \mathrm{MHz}, \mathrm{CDCl}_{3}\right)$ of $\mathbf{3 o}$ 
1H NMR $(500 \mathrm{MHz}, \mathrm{CDCl} 3)$

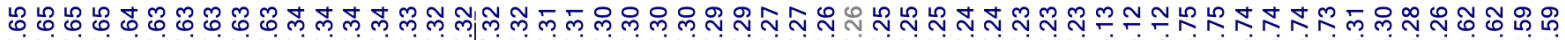

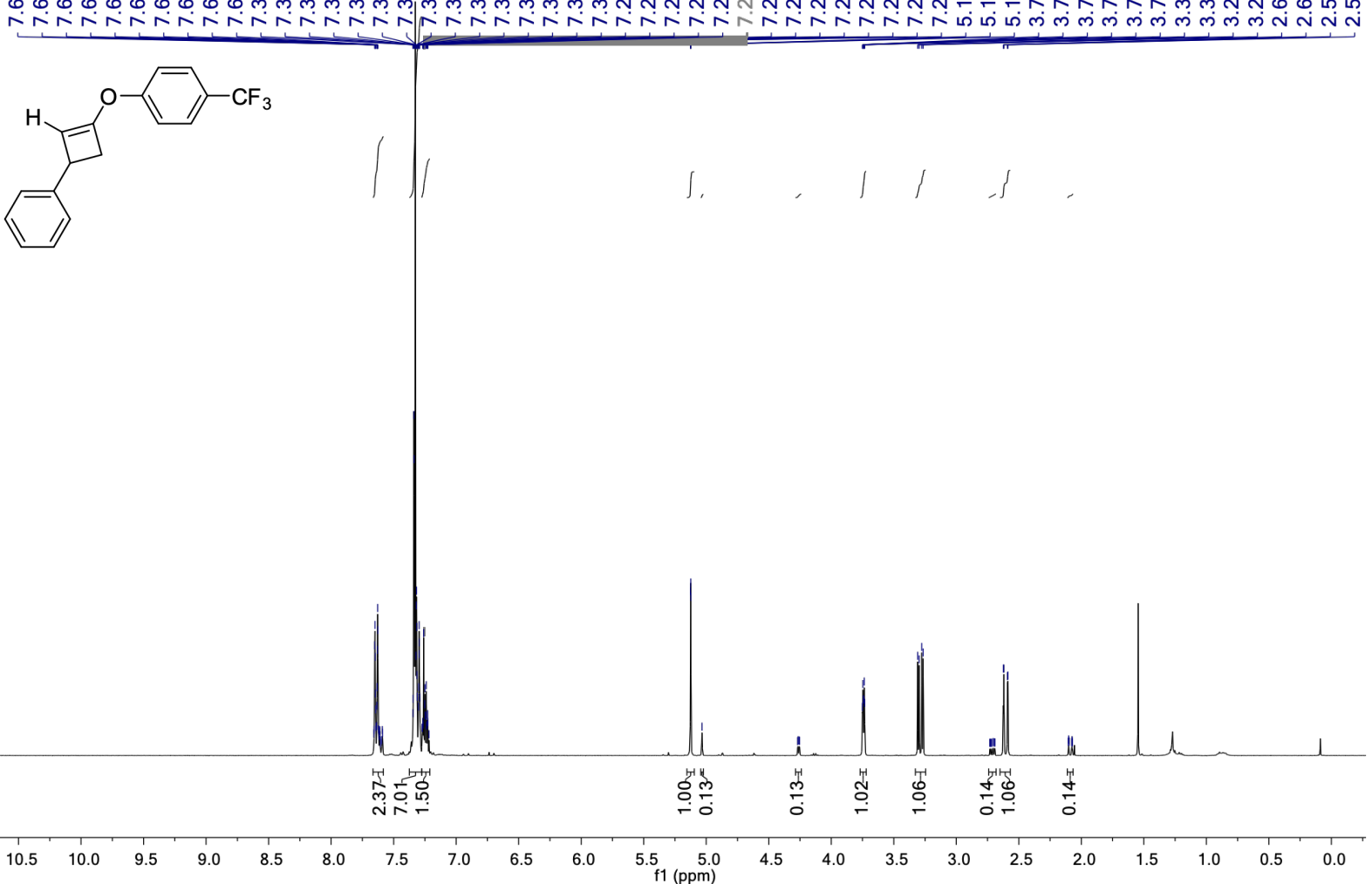

${ }^{19} \mathrm{~F}$ NMR (376 MHz, $\mathrm{CDCl}_{3}$ ) of $\mathbf{3 o}$

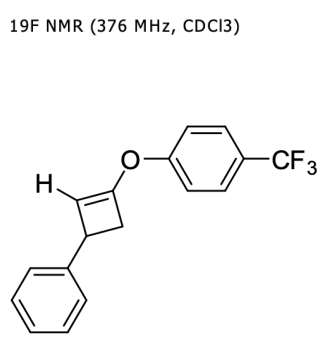

\begin{tabular}{|c|c|c|c|c|c|c|c|c|c|c|c|c|c|c|c|c|c|c|}
\hline-10 & -20 & $\begin{array}{l}1 \\
-30\end{array}$ & -40 & -50 & -60 & -70 & -80 & -90 & $\begin{array}{c}-100 \\
\text { f1 }(\mathrm{ppm})\end{array}$ & -110 & -120 & -130 & -140 & -150 & -160 & -170 & -180 & -190 \\
\hline
\end{tabular}

${ }^{13} \mathrm{C}$ NMR $\left(101 \mathrm{MHz}, \mathrm{CDCl}_{3}\right)$ of $\mathbf{3 o}$ 

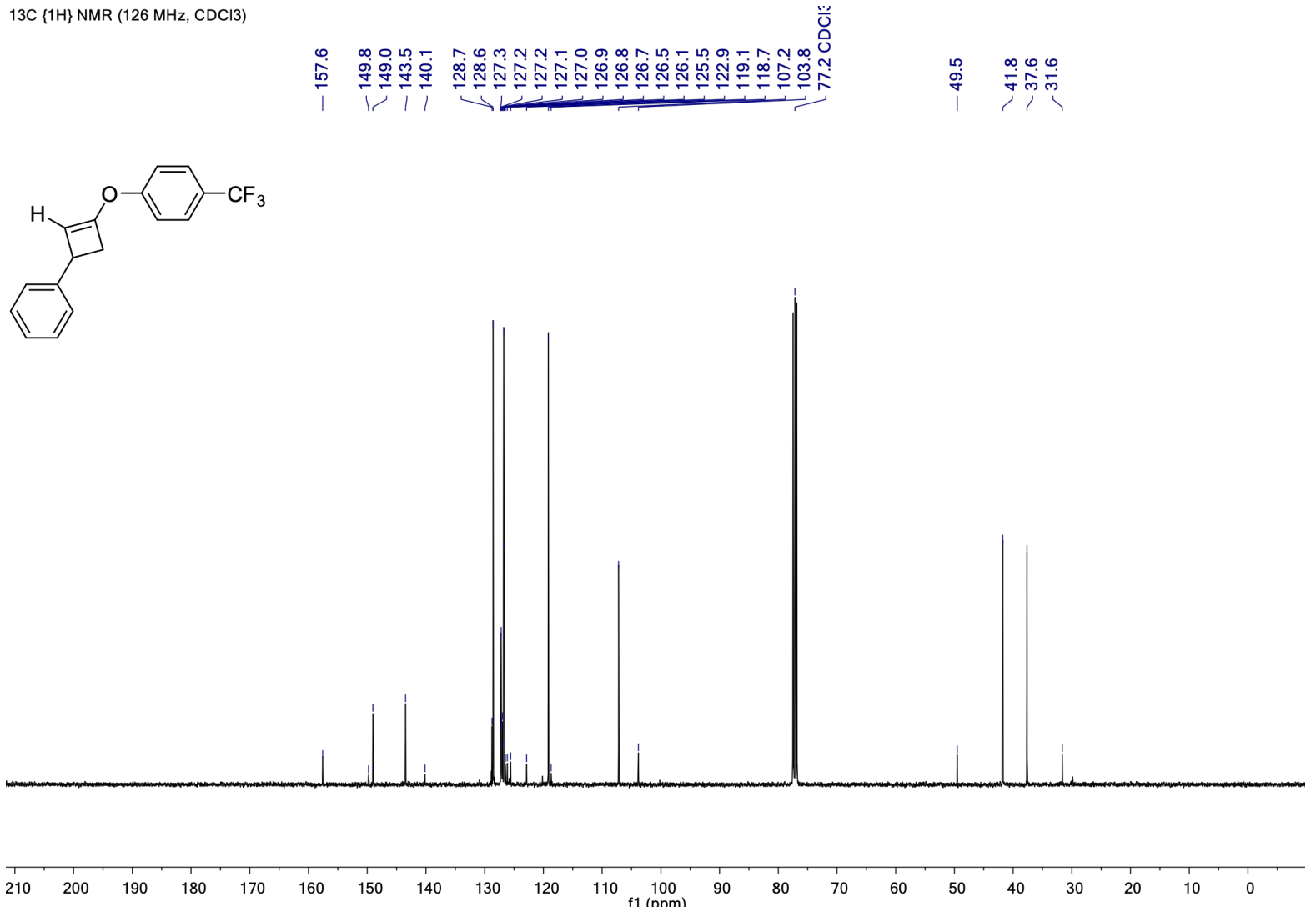


\section{Trimethyl((3-phenoxycyclobut-2-en-1-yl)methyl)silane (3p)}<smiles>CS(=O)(=O)CC1C=C(Oc2ccccc2)C1</smiles>

Cyclobutene 3p was synthesized following general procedure B starting from (ethynyloxy)benzene (23.6 mg, 0.2 $\mathrm{mmol}$ ) and allyltrimethylsilane $(95 \mathrm{ml}, 0.6 \mathrm{mmol})$. The crude product was purified by flash chromatography affording $\mathbf{3 p}$ as a colorless oil (11.6 $\mathrm{mg}, 24 \%)$.

${ }^{1}$ H NMR $\left(500 \mathrm{MHz}, \mathrm{CDCl}_{3}\right) \delta 7.40$ - $7.25(\mathrm{~m}, 2 \mathrm{H}), 7.15-7.11(\mathrm{~m}, 2 \mathrm{H}), 7.10(\mathrm{dt}, J=7.3,1.2 \mathrm{~Hz}, 1 \mathrm{H}), 4.80(\mathrm{~d}$, $J=0.9 \mathrm{~Hz}, 1 \mathrm{H}), 2.94(\mathrm{dd}, J=12.9,4.2 \mathrm{~Hz}, 1 \mathrm{H}), 2.57$ (tdt, $J=7.5,4.2,1.3 \mathrm{~Hz}, 1 \mathrm{H}), 2.22(\mathrm{dd}, J=12.8,1.5 \mathrm{~Hz}$, $1 \mathrm{H}), 0.79(\mathrm{~d}, J=7.6 \mathrm{~Hz}, 2 \mathrm{H}), 0.01(\mathrm{~s}, 9 \mathrm{H})$.

${ }^{13}$ C NMR $\left(126 \mathrm{MHz} \mathrm{CDCl}_{3}\right) \delta 155.2,148.3,129.6,123.9,119.3,108.7,40.9,29.8,23.3,-0.9$.

HRMS (APCI) $m / z$ calculated for $\mathrm{C}_{14} \mathrm{H}_{21} \mathrm{OSi}^{+}[\mathrm{M}+\mathrm{H}]^{+}: 233.1356$, found: 233.1357.

${ }^{1} \mathrm{H} \mathrm{NMR}\left(500 \mathrm{MHz}, \mathrm{CDCl}_{3}\right)$ of $\mathbf{3 p}$

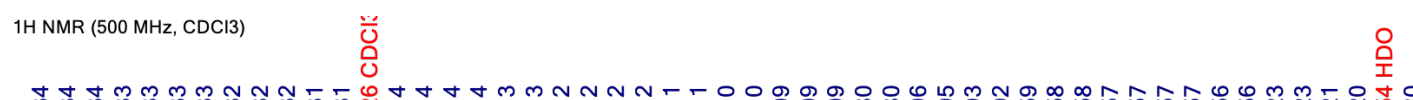

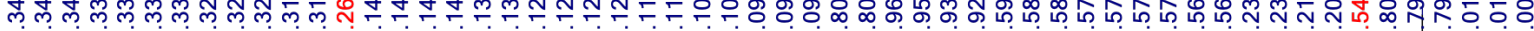

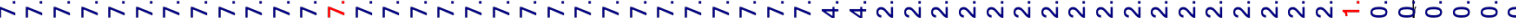
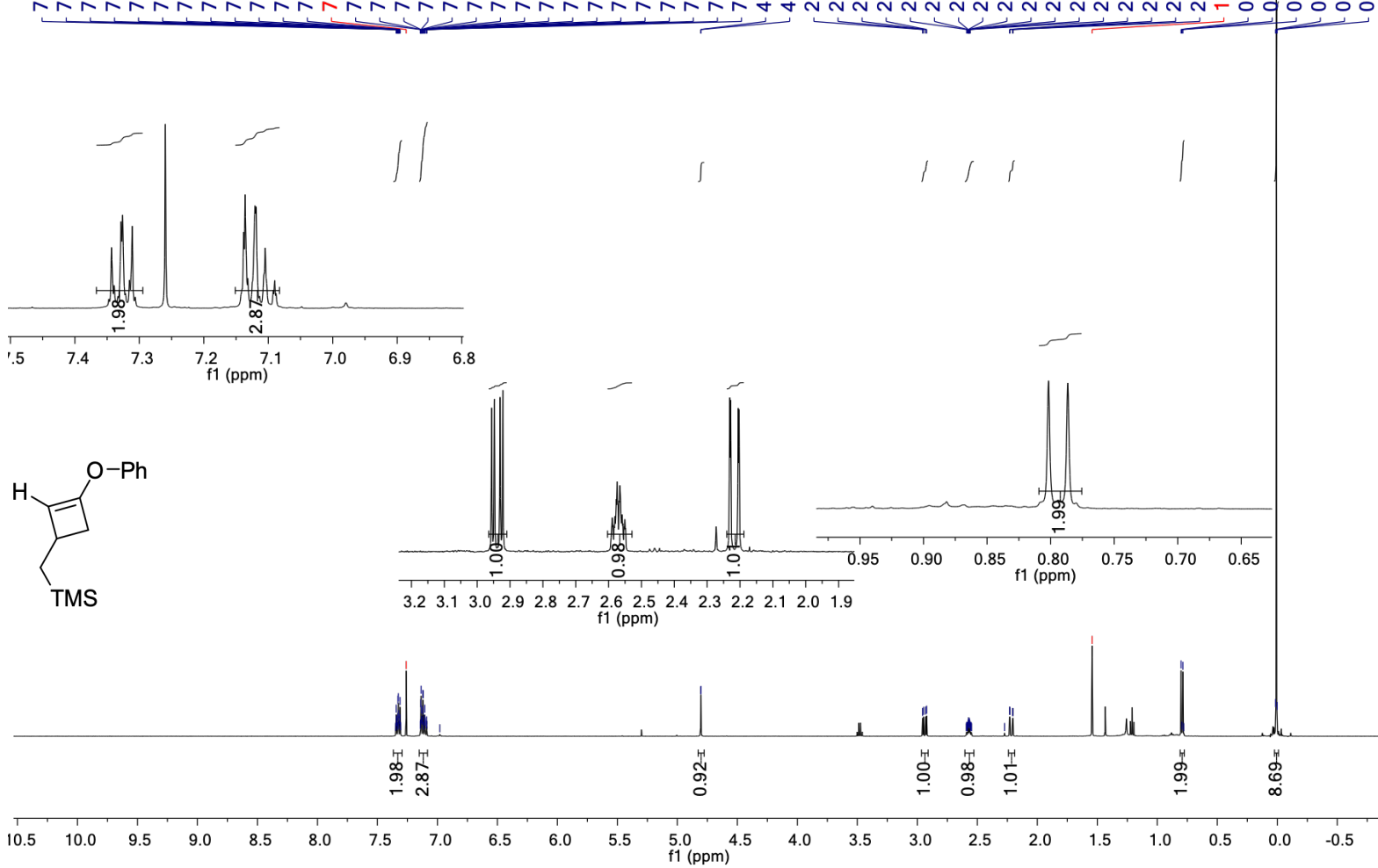
${ }^{13} \mathrm{C}$ NMR $\left(126 \mathrm{MHz}, \mathrm{CDCl}_{3}\right)$ of $\mathbf{3 p}$

$13 \mathrm{C}\{1 \mathrm{H}\} \mathrm{NMR}(126 \mathrm{MHz}, \mathrm{CDCl} 3)$

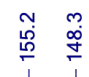

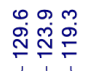

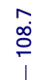

$\stackrel{\infty}{\circ} \quad \stackrel{\infty}{\sim} \stackrel{m}{i}$

$\stackrel{9}{i}$<smiles>CS(=O)(=O)CC1C=C(Oc2ccccc2)C1</smiles>

TMS

$\begin{array}{lllllllllllll}210 & 200 & 190 & 180 & 170 & 160 & 150 & 140 & 130 & 120 & 110 & 100 & 90\end{array}$ 


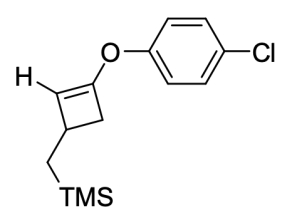

Cyclobutene 3q was synthesized following general procedure $\mathbf{B}$ starting from 1-chloro-4-(ethynyloxy)benzene $(45.8 \mathrm{mg}, 0.3 \mathrm{mmol})$ and allyltrimethylsilane $(103 \mathrm{mg}, 0.9 \mathrm{mmol})$. The crude was purified by flash chromatography affording $\mathbf{d}$ as a transparent oil ( $22 \mathrm{mg}, 28 \%)$.

${ }^{1} \mathbf{H}$ NMR $\left(500 \mathrm{MHz}, \mathrm{CDCl}_{3}\right) \delta 7.30-7.26(\mathrm{~m}, 2 \mathrm{H}), 7.08-7.03(\mathrm{~m}, 2 \mathrm{H}), 4.79(\mathrm{~d}, J=0.9 \mathrm{~Hz}, 1 \mathrm{H}), 2.92(\mathrm{dd}, J=$ $12.9,4.2 \mathrm{~Hz}, 1 \mathrm{H}), 2.56$ (tdt, $J=7.6,4.2,1.3 \mathrm{~Hz}, 1 \mathrm{H}), 2.20$ (dd, $J=12.9,1.4 \mathrm{~Hz}, 1 \mathrm{H}), 0.78(\mathrm{~d}, J=7.6 \mathrm{~Hz}, 2 \mathrm{H})$, $0.01(\mathrm{~s}, 9 \mathrm{H})$.

${ }^{13}$ C NMR $\left(101 \mathrm{MHz}, \mathrm{CDCl}_{3}\right) \delta 153.7,148.0,129.6,129.0,120.6,109.1,40.9,29.8,23.2,-0.92$.

HRMS (ESI) $\mathrm{m} / \mathrm{z}$ calculated for $\mathrm{C}_{14} \mathrm{H}_{20} \mathrm{ClOSi}^{+}[\mathrm{M}+\mathrm{H}]^{+}: 267.0966$, found: 267.0962 .

${ }^{1} \mathrm{H}$ NMR $\left(500 \mathrm{MHz}, \mathrm{CDCl}_{3}\right)$ of $\mathbf{3 q}$

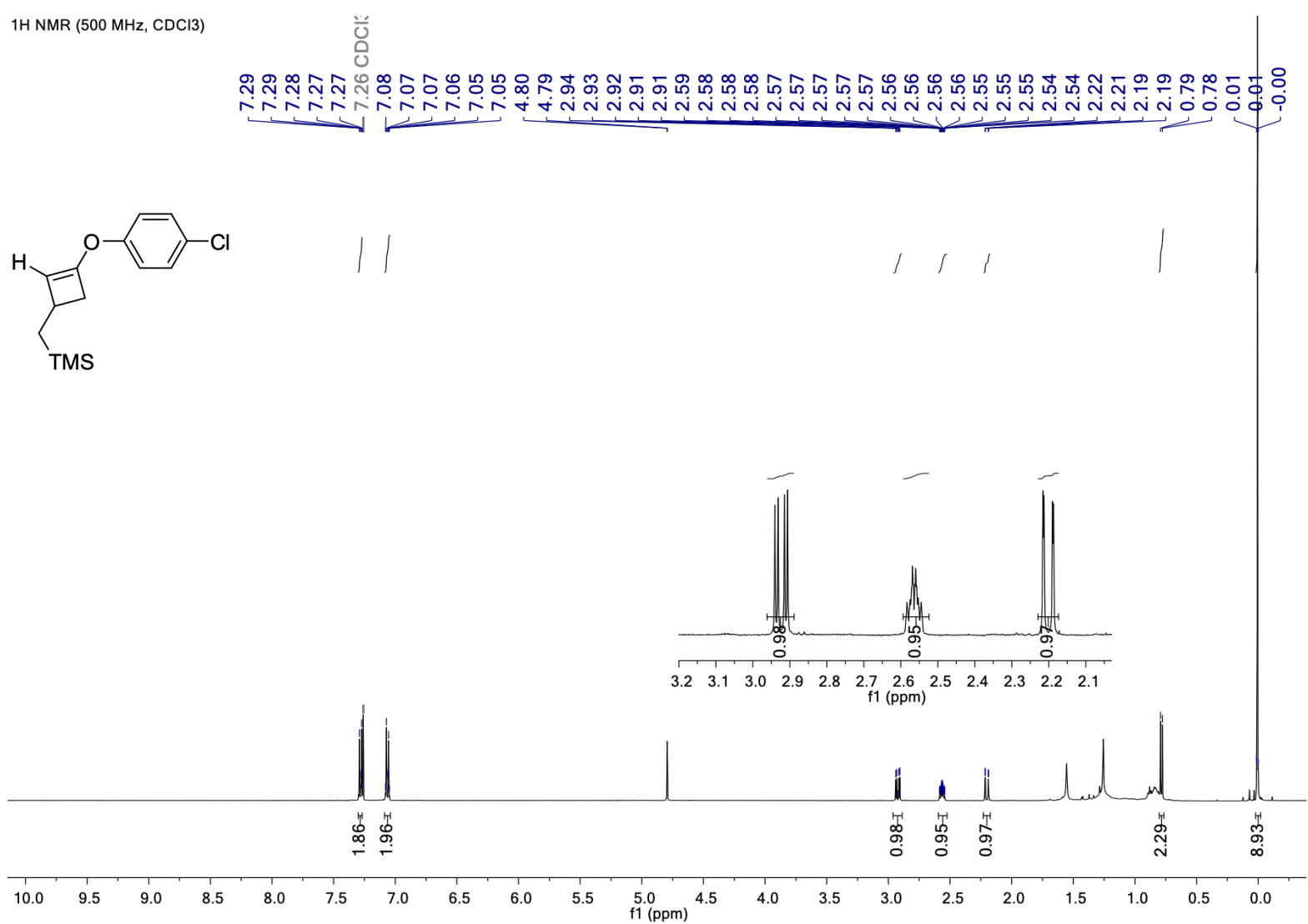


${ }^{13} \mathrm{C}$ NMR $\left(101 \mathrm{MHz}, \mathrm{CDCl}_{3}\right)$ of $\mathbf{3 q}$

$13 \mathrm{C}\{1 \mathrm{H}\} \mathrm{NMR}(126 \mathrm{MHz}, \mathrm{CDCl} 3)$

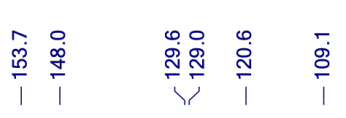

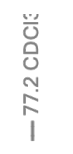

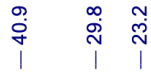

$$
\stackrel{?}{i}
$$
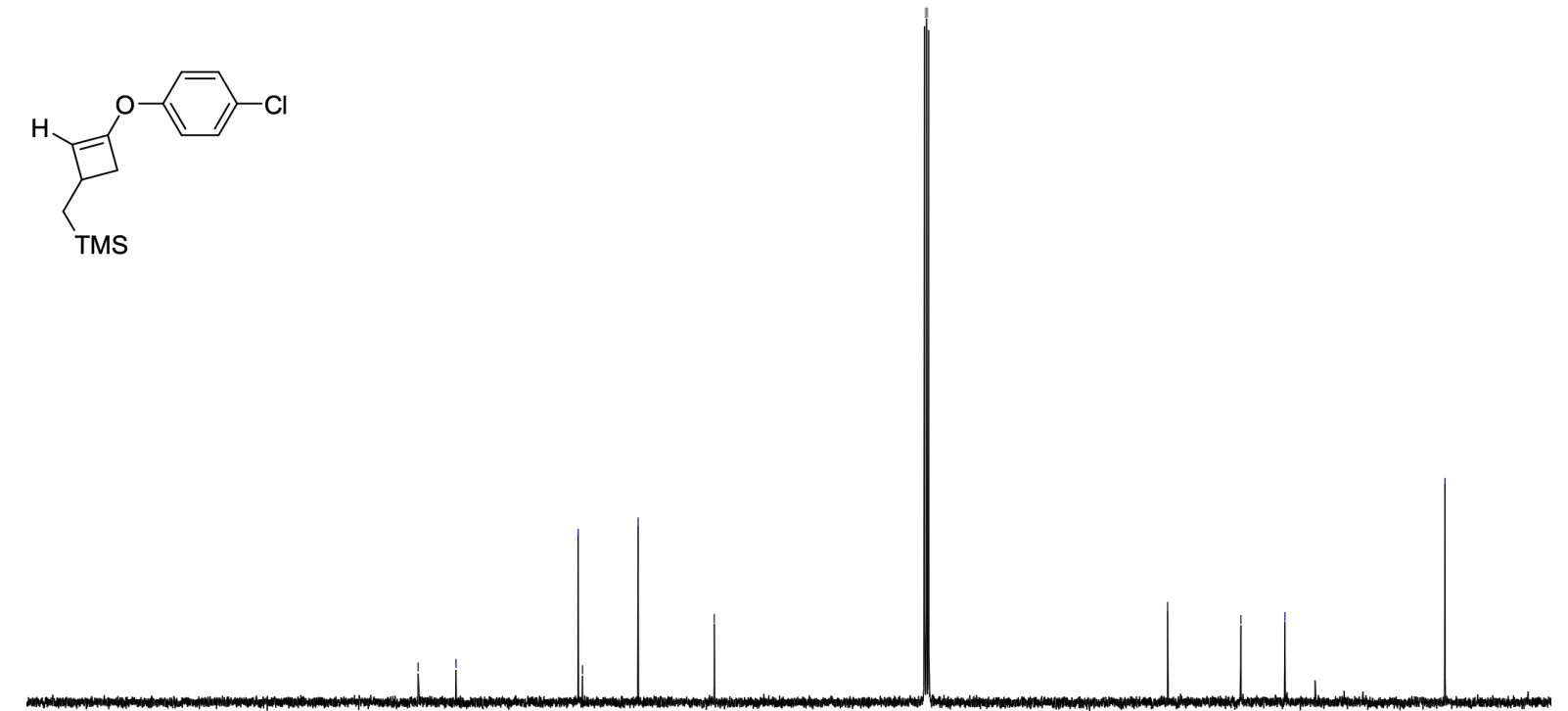

\begin{tabular}{lllllllllllllllllllllllllllll}
\hline 210 & 200 & 190 & 180 & 170 & 160 & 150 & 140 & 130 & 120 & 110 & 100 & 90 & 80 & 70 & 60 & 50 & 40 & 30 & 20 & 10 & 0 & -10
\end{tabular} 
Trimethyl((3-(4-(trifluoromethyl)phenoxy)cyclobut-2-en-1-yl)methyl)silane (3r)

Cyclobutene 3r was synthesized following general procedure B starting from 1-(ethynyloxy)-4(trifluoromethyl)benzene $(55.8 \mathrm{mg}, 0.3 \mathrm{mmol})$ and allyltrimethylsilane $(103 \mathrm{mg}, 0.9 \mathrm{mmol})$. The crude was purified by flash chromatography affording $3 \mathbf{r}$ as a transparent oil (27 $\mathrm{mg}, 30 \%)$.

${ }^{1}$ H NMR $\left(500 \mathrm{MHz} \mathrm{CDCl}_{3}\right) \delta 7.62-7.56(\mathrm{~m}, 2 \mathrm{H}), 7.24-7.18(\mathrm{~m}, 2 \mathrm{H}), 4.94(\mathrm{~d}, J=1.0 \mathrm{~Hz}, 1 \mathrm{H}), 2.97(\mathrm{dd}, J=$ 12.9, $4.2 \mathrm{~Hz}, 1 \mathrm{H}), 2.65-2.57(\mathrm{~m}, 1 \mathrm{H}), 2.25(\mathrm{dd}, J=12.9,1.4 \mathrm{~Hz}, 1 \mathrm{H}), 0.81(\mathrm{~d}, J=7.6 \mathrm{~Hz}, 2 \mathrm{H}), 0.02(\mathrm{~s}, 9 \mathrm{H})$.

${ }^{19}$ F NMR $\left(376 \mathrm{MHz}, \mathrm{CDCl}_{3}\right) \delta-61.99$.

${ }^{13} \mathbf{C}$ NMR $\left(126 \mathrm{MHz}, \mathrm{CDCl}_{3}\right) \delta 157.9,147.1,127.1(\mathrm{q}, J=3.8 \mathrm{~Hz}), 125.8(\mathrm{q}), 125.3(\mathrm{q}, J=272.3 \mathrm{~Hz}), 118.9$, $111.0,41.1,30.1,23.1,-0.9$.

HRMS (ESI) $\mathrm{m} / \mathrm{z}$ calculated for $\mathrm{C}_{15} \mathrm{H}_{20} \mathrm{~F}_{3} \mathrm{OSi}^{+}[\mathrm{M}+\mathrm{H}]^{+}: 301.1230$, found: 301.1225 .

${ }^{1} \mathrm{H}$ NMR $\left(500 \mathrm{MHz}, \mathrm{CDCl}_{3}\right)$ of $\mathbf{3 r}$
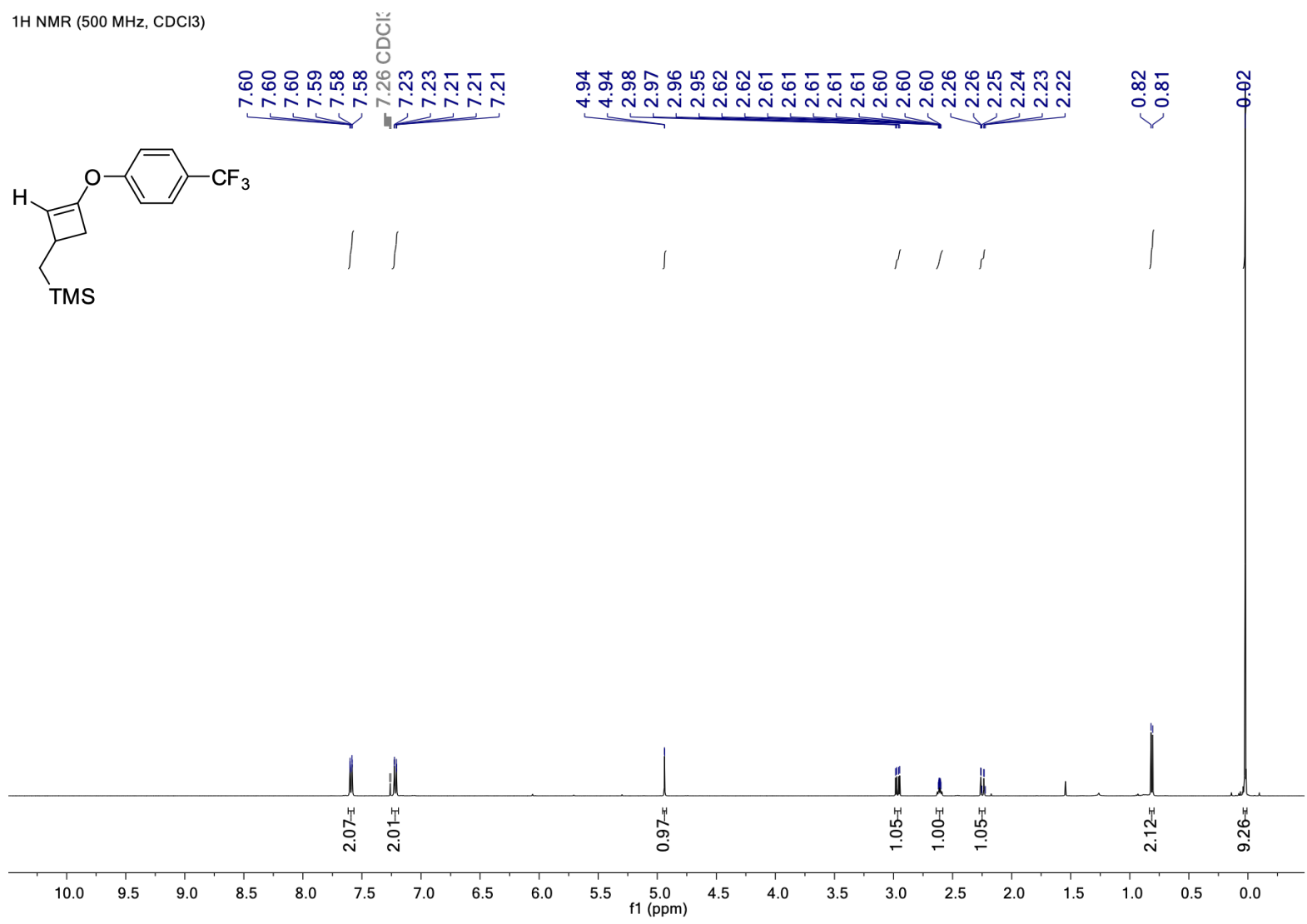
$\left.{ }^{19} \mathrm{~F} \mathrm{NMR} \mathrm{(376} \mathrm{MHz,} \mathrm{CDCl}_{3}\right)$ of $\mathbf{3 r}$
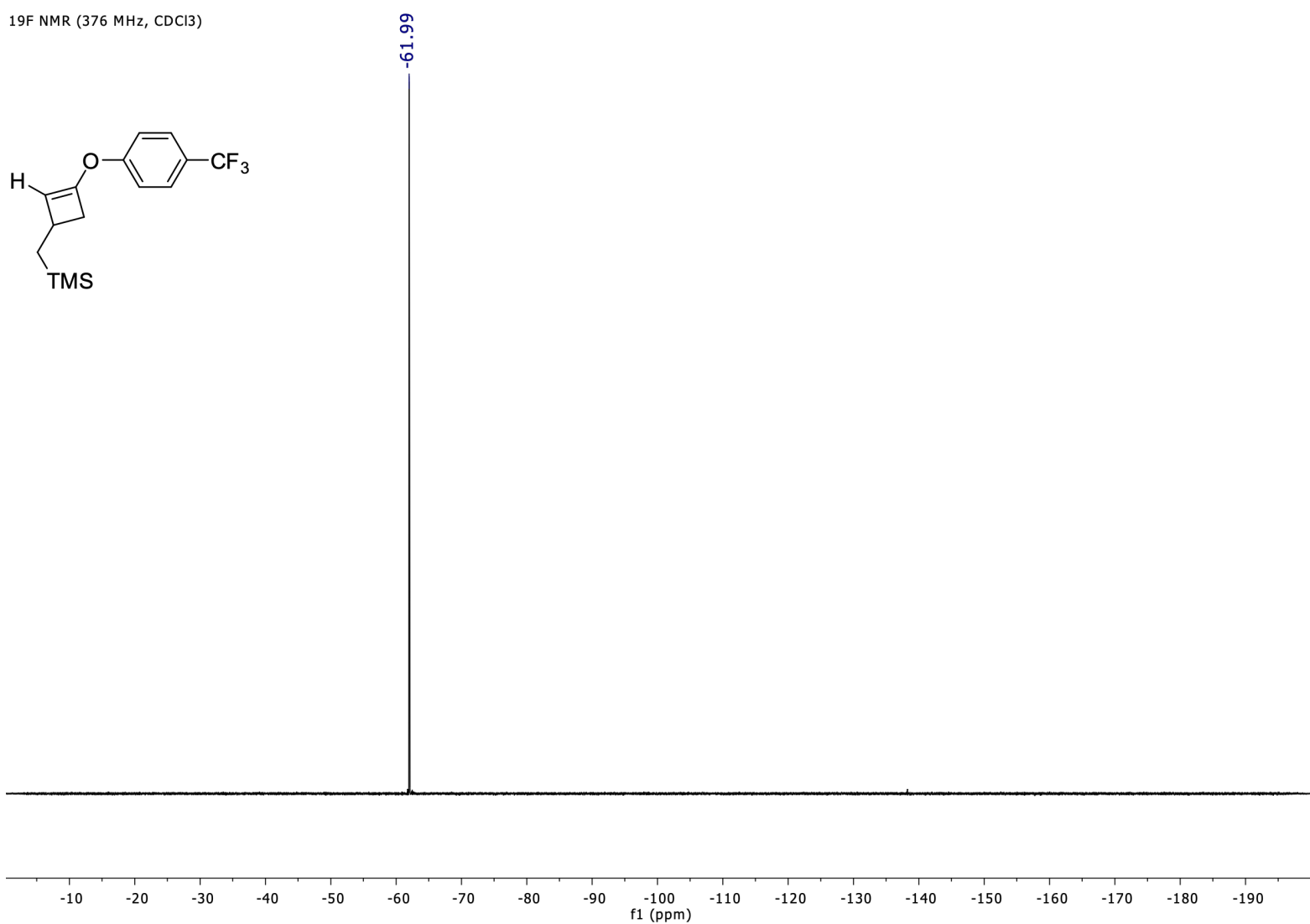

${ }^{13} \mathrm{C}$ NMR $\left(126 \mathrm{MHz}, \mathrm{CDCl}_{3}\right)$ of $\mathbf{3 r}$

$13 \mathrm{C}\{1 \mathrm{H}\} \mathrm{NMR}(126 \mathrm{MHz}, \mathrm{CDCl} 3)$

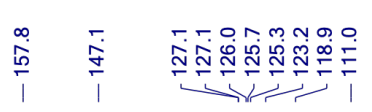<smiles>CS(=O)(=O)CC1C=C(Oc2ccc(C(F)(F)F)cc2)C1</smiles>
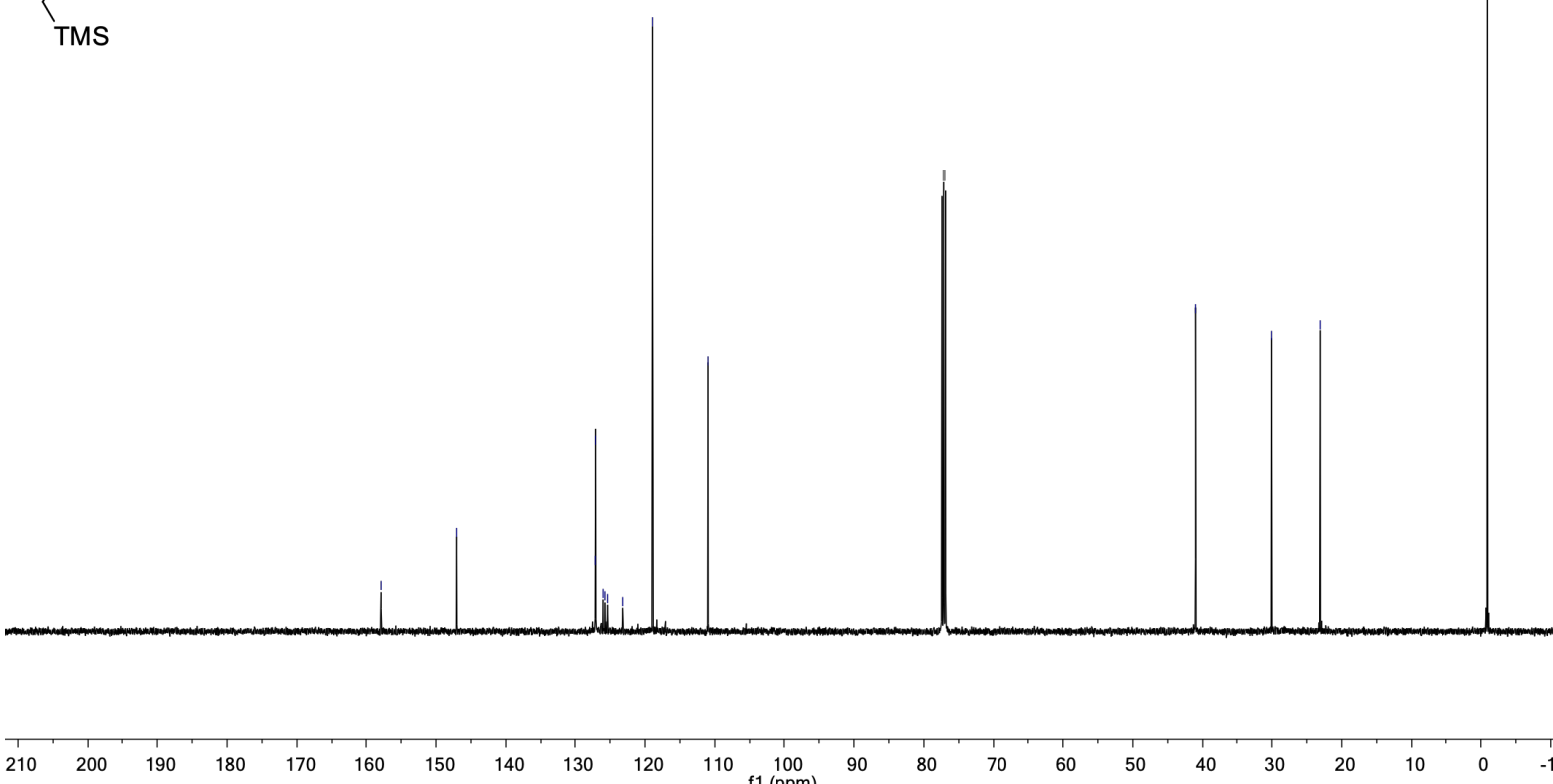


\section{8-phenoxybicyclo[5.2.0]non-8-ene (3s)}

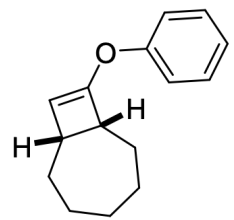

Cyclobutene 3s was synthesized following general procedure B starting from (ethynyloxy)- benzene (35.4 mg, $0.3 \mathrm{mmol}$ ) and cycloheptene $(87 \mathrm{mg}, 0.9 \mathrm{mmol})$. The crude was purified by flash chromatography affording $3 \mathrm{~s}$ as a transparent oil $(7 \mathrm{mg}, 11 \%)$.

${ }^{1} \mathbf{H}$ NMR $\left(500 \mathrm{MHz}, \mathrm{CD}_{2} \mathrm{Cl}_{2}\right) \delta 7.37-7.29(\mathrm{~m}, 2 \mathrm{H}), 7.17-7.07(\mathrm{~m}, 3 \mathrm{H}), 4.70(\mathrm{~d}, J=1.0 \mathrm{~Hz}, 1 \mathrm{H}), 3.12(\mathrm{dt}, J=$ $11.3,4.3 \mathrm{~Hz}, 1 \mathrm{H}), 2.60(\mathrm{dt}, J=10.4,4.2 \mathrm{~Hz}, 1 \mathrm{H}), 1.87-1.67(\mathrm{~m}, 5 \mathrm{H}), 1.52-1.21(\mathrm{~m}, 5 \mathrm{H})$.

${ }^{13}$ C NMR $\left(126 \mathrm{MHz}, \mathrm{CD}_{2} \mathrm{Cl}_{2}\right) \delta 155.52,129.84,124.14,119.51,104.80,49.51,39.75,32.37,31.91,28.3,28.3$, 28.2

HRMS (ESI) m/z calculated for $\mathrm{C}_{15} \mathrm{H}_{19} \mathrm{O}^{+}[\mathrm{M}+\mathrm{H}]^{+}: 215.1430$, found: 215.1428 .

${ }^{1} \mathrm{H}$ NMR $\left(500 \mathrm{MHz}, \mathrm{CDCl}_{3}\right)$ of $\mathbf{3 s}$

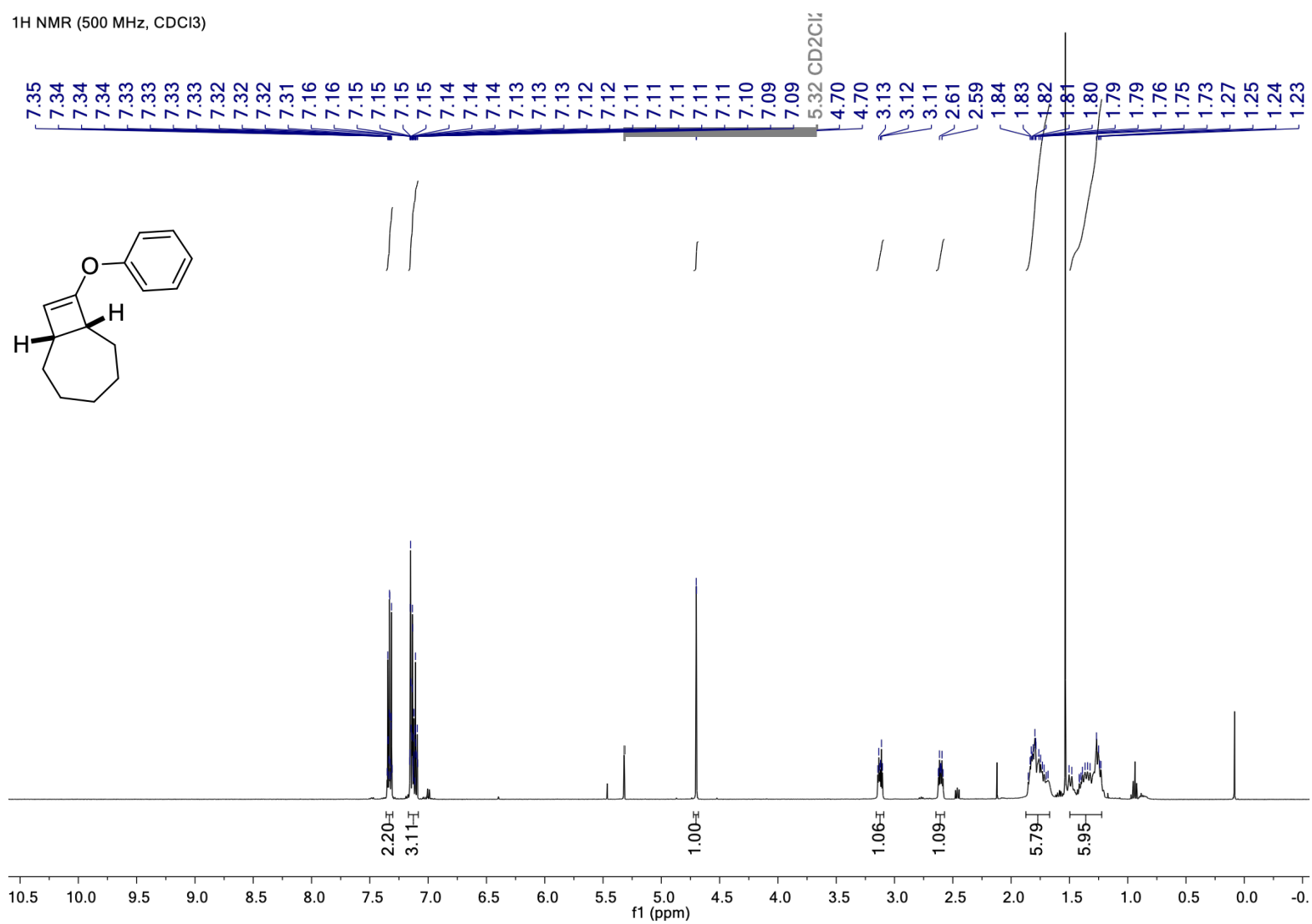


${ }^{13} \mathrm{C}$ NMR $\left(126 \mathrm{MHz}, \mathrm{CDCl}_{3}\right)$ of $\mathbf{3 s}$

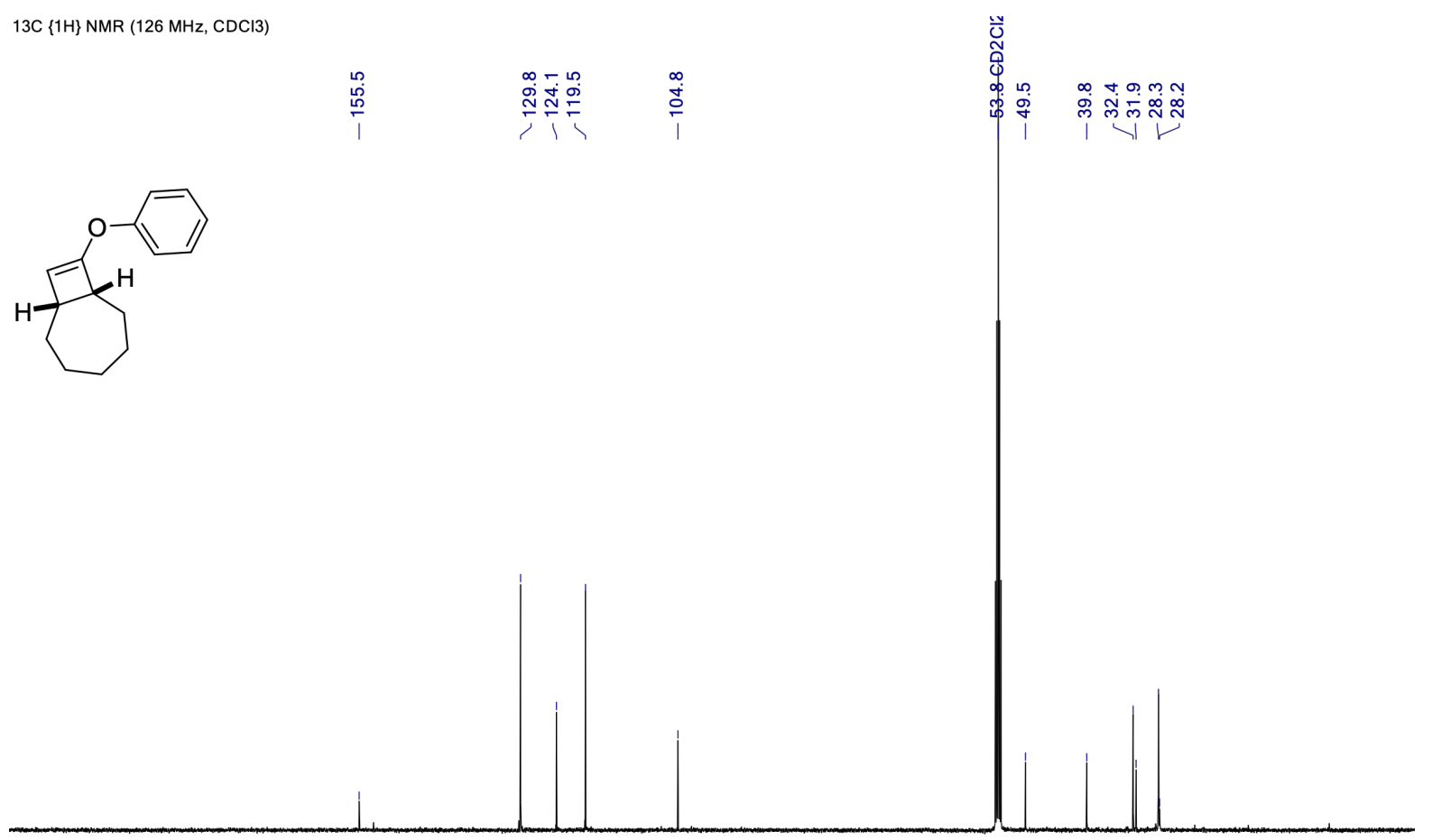

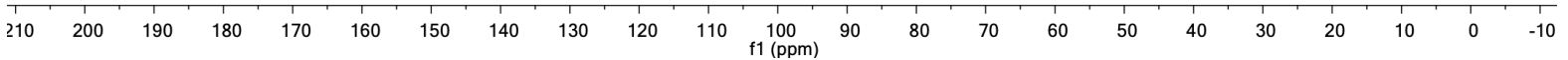




\section{8-(4-(trifluoromethyl)phenoxy)bicyclo[5.2.0]non-8-ene (3t)}

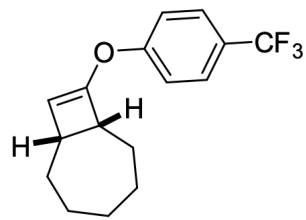

Cyclobutene 3t was synthesized following general procedure B starting from 1-(ethynyloxy)-4(trifluoromethyl)benzene $(37.2 \mathrm{mg}, 0.2 \mathrm{mmol})$ and cycloheptene $(58 \mathrm{mg}, 0.6 \mathrm{mmol})$. The crude was purified by flash chromatography affording $\mathbf{3 t}$ as a transparent oil (21 mg, 37\%).

${ }^{1} \mathbf{H}$ NMR $\left(500 \mathrm{MHz}, \mathrm{CDCl}_{3}\right) \delta 7.62-7.55(\mathrm{~m}, 2 \mathrm{H}), 7.27-7.21(\mathrm{~m}, 2 \mathrm{H}), 4.84(\mathrm{~d}, J=1.0 \mathrm{~Hz}, 1 \mathrm{H}), 3.15(\mathrm{dt}, J=$ 11.4, 4.4 Hz, 1H), 2.64 (dt, $J=10.4,4.2 \mathrm{~Hz}, 1 \mathrm{H}), 1.92-1.64(\mathrm{~m}, 5 \mathrm{H}), 1.54-1.18(\mathrm{~m}, 5 \mathrm{H})$.

${ }^{19}$ F NMR $\left(376 \mathrm{MHz}, \mathrm{CDCl}_{3}\right) \delta-61.58$.

${ }^{13}$ C NMR $\left(126 \mathrm{MHz} \mathrm{CDCl}_{3}\right) \delta 158.0,151.7,127.0(\mathrm{q}, J=3.8 \mathrm{~Hz}), 125.8(\mathrm{q}, J=32.6 \mathrm{~Hz}), 124.3(\mathrm{q}, J=271.7$ Hz), 119.0, 106.8, 49.4, 39.6, 32.0, 31.4, 28.0.

HRMS (ESI) $\mathrm{m} / \mathrm{z}$ calculated for $\mathrm{C}_{16} \mathrm{H}_{16} \mathrm{~F}_{3} \mathrm{O}^{+}[\mathrm{M}+\mathrm{H}]^{+}: 283.1304$, found: 283.1304 .

${ }^{1} \mathrm{H} \mathrm{NMR}\left(500 \mathrm{MHz}, \mathrm{CDCl}_{3}\right)$ of $\mathbf{3 t}$

$1 \mathrm{H}$ NMR $(500 \mathrm{MHz}, \mathrm{CDCl} 3)$

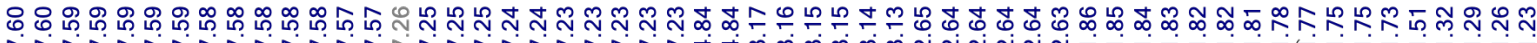
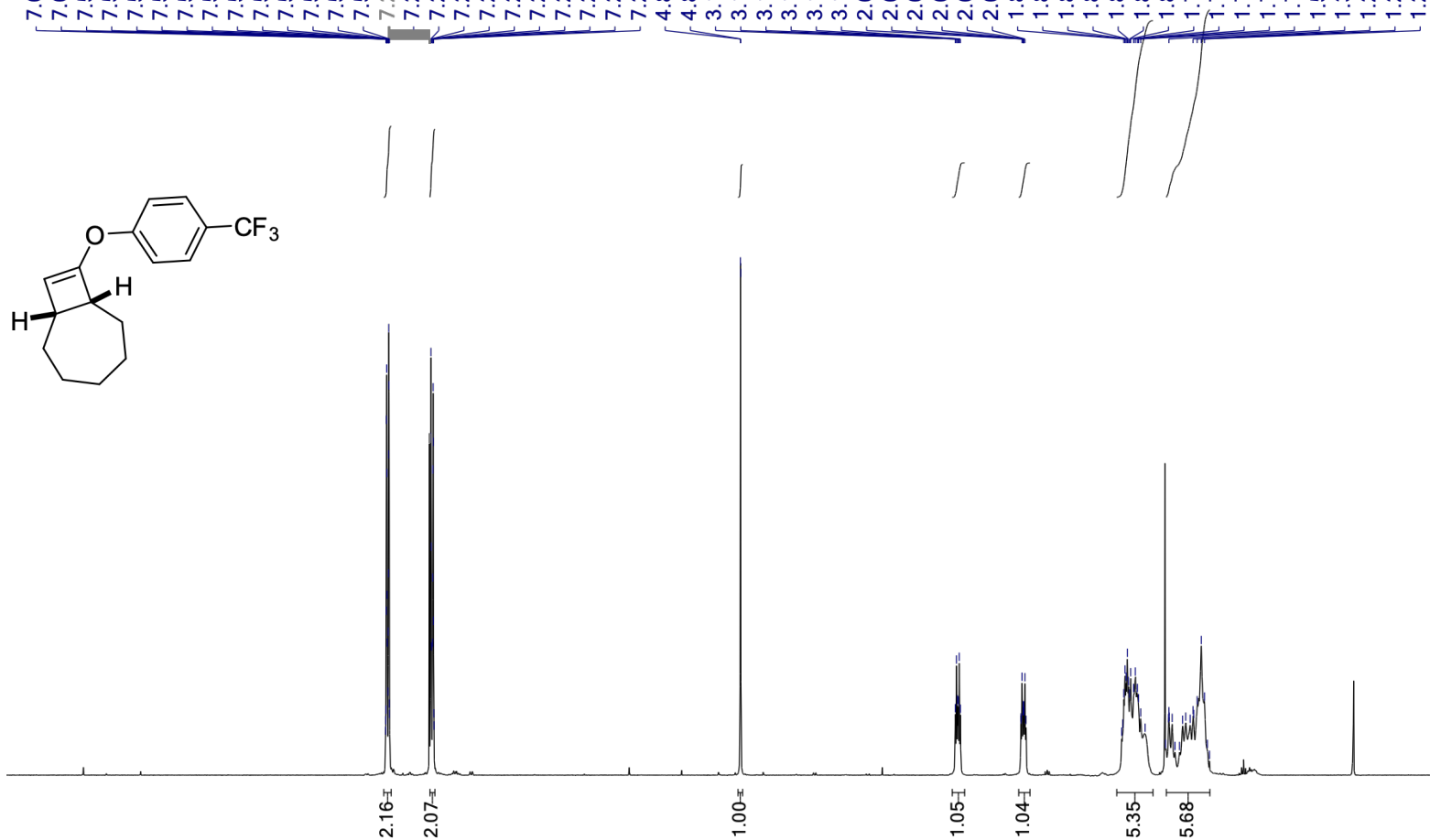

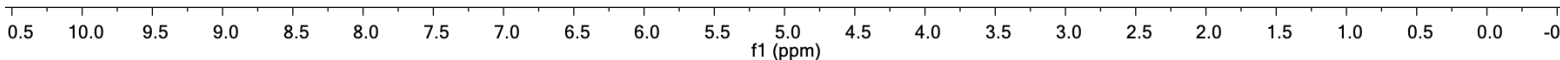


${ }^{19} \mathrm{~F}$ NMR (376 MHz, $\left.\mathrm{CDCl}_{3}\right)$ of $\mathbf{3 t}$

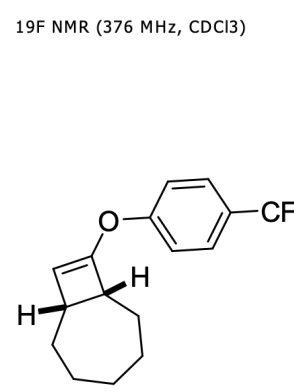

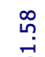

\begin{tabular}{llllllllllllllllllllllllllllllllll}
\hline 30 & 20 & 10 & 0 & -10 & -20 & -30 & -40 & -50 & -60 & -70 & -80 & -90 & -100 & -110 & -120 & -130 & -140 & -150 & -160 & -170 & -180 & -190 & -200 & -210 & -220 & -23
\end{tabular}

${ }^{13} \mathrm{C}$ NMR $\left(126 \mathrm{MHz}, \mathrm{CDCl}_{3}\right)$ of $\mathbf{3 t}$

$13 \mathrm{C}\{1 \mathrm{H}\} \mathrm{NMR}(126 \mathrm{MHz}, \mathrm{CDCl} 3)$

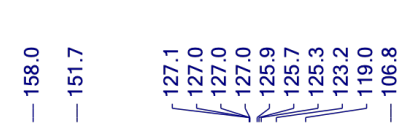

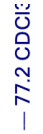

广.j.
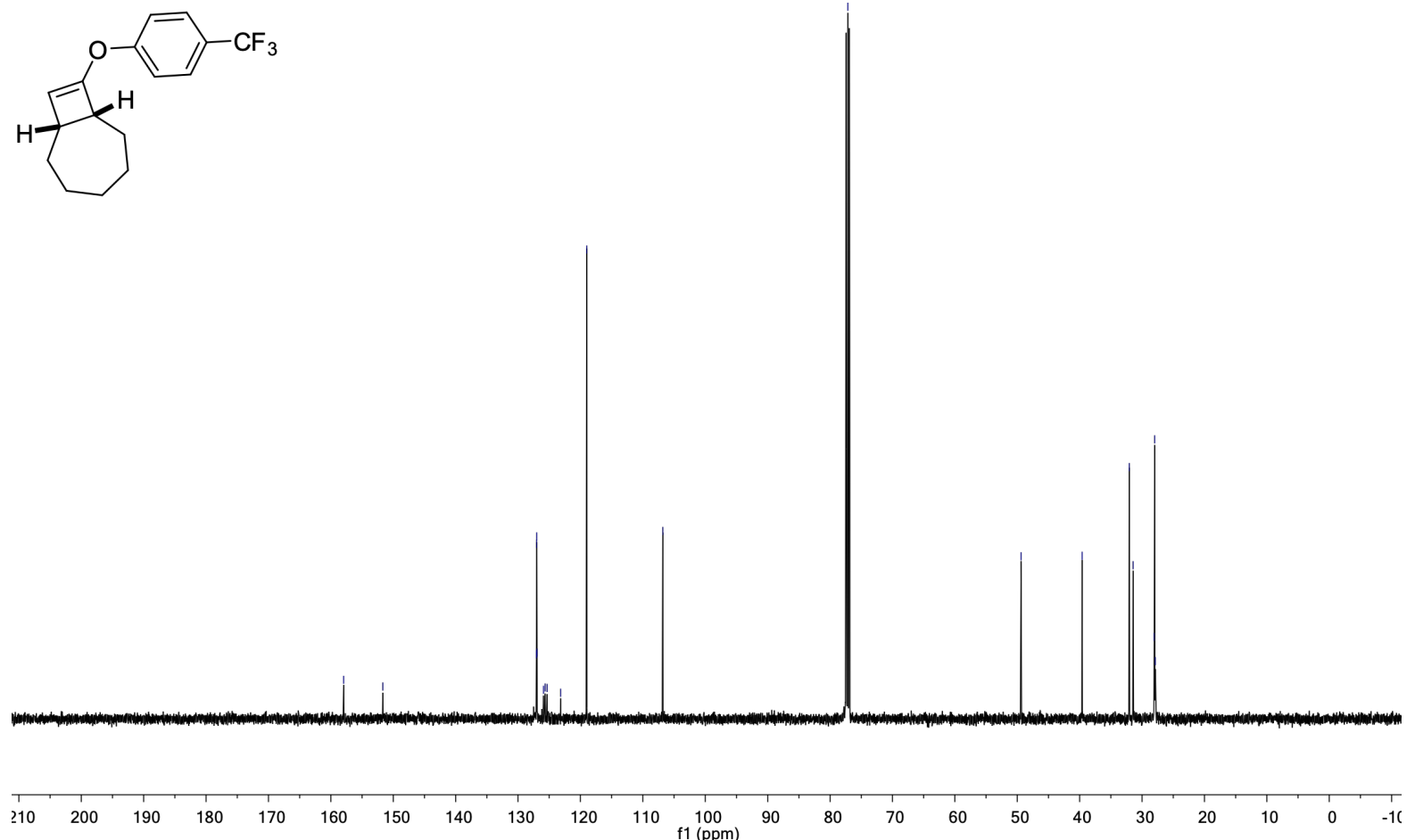


\section{9-Phenoxybicyclo[6.2.0]dec-9-ene (3u)}<smiles>C1=C(Oc2ccccc2)C2CCCCCCC12</smiles>

Cyclobutene 3u was synthesized following general procedure B starting from (ethynyloxy)benzene (23.6 mg, 0.2 $\mathrm{mmol}$ ) and (Z)-cyclooctene $(78 \mathrm{~mL}, 0.6 \mathrm{mmol})$. The crude product was purified by flash chromatography affording $3 \mathbf{u}$ as a yellow oil $(21 \mathrm{mg}, 45 \%)$.

${ }^{1}$ H NMR $\left(400 \mathrm{MHz}, \mathrm{CDCl}_{3}\right) \delta 7.32(\mathrm{tt}, J=7.5,2.2 \mathrm{~Hz}, 2 \mathrm{H}), 7.18-7.12(\mathrm{~m}, 2 \mathrm{H}), 7.12-7.06(\mathrm{~m}, 1 \mathrm{H}), 4.60(\mathrm{~d}, J$ $=1.0 \mathrm{~Hz}, 1 \mathrm{H}), 2.96(\mathrm{ddd}, J=12.0,4.2,2.0 \mathrm{~Hz}, 1 \mathrm{H}), 2.48-2.37(\mathrm{~m}, 1 \mathrm{H}), 1.91(\mathrm{dtd}, J=14.4,3.9,1.9 \mathrm{~Hz}, 1 \mathrm{H})$, $1.76-1.68(\mathrm{~m}, 1 \mathrm{H}), 1.68-1.57(\mathrm{~m}, 2 \mathrm{H}), 1.53-1.23(\mathrm{~m}, 8 \mathrm{H})$.

${ }^{13} \mathbf{C}$ NMR $\left(101 \mathrm{MHz}, \mathrm{CDCl}_{3}\right) \delta 155.1,151.8,129.4,123.6,119.0,104.3,48.4,39.0,30.4,29.9,28.1,26.2,26.1$, 24.3.

HRMS (ESI) $m / z$ calculated for $\mathrm{C}_{16} \mathrm{H}_{21} \mathrm{O}^{+}[\mathrm{M}+\mathrm{H}]^{+}:$229.1587, found: 229.1586 .

${ }^{1} \mathrm{H} \mathrm{NMR}\left(400 \mathrm{MHz}, \mathrm{CDCl}_{3}\right)$ of $\mathbf{3 u}$

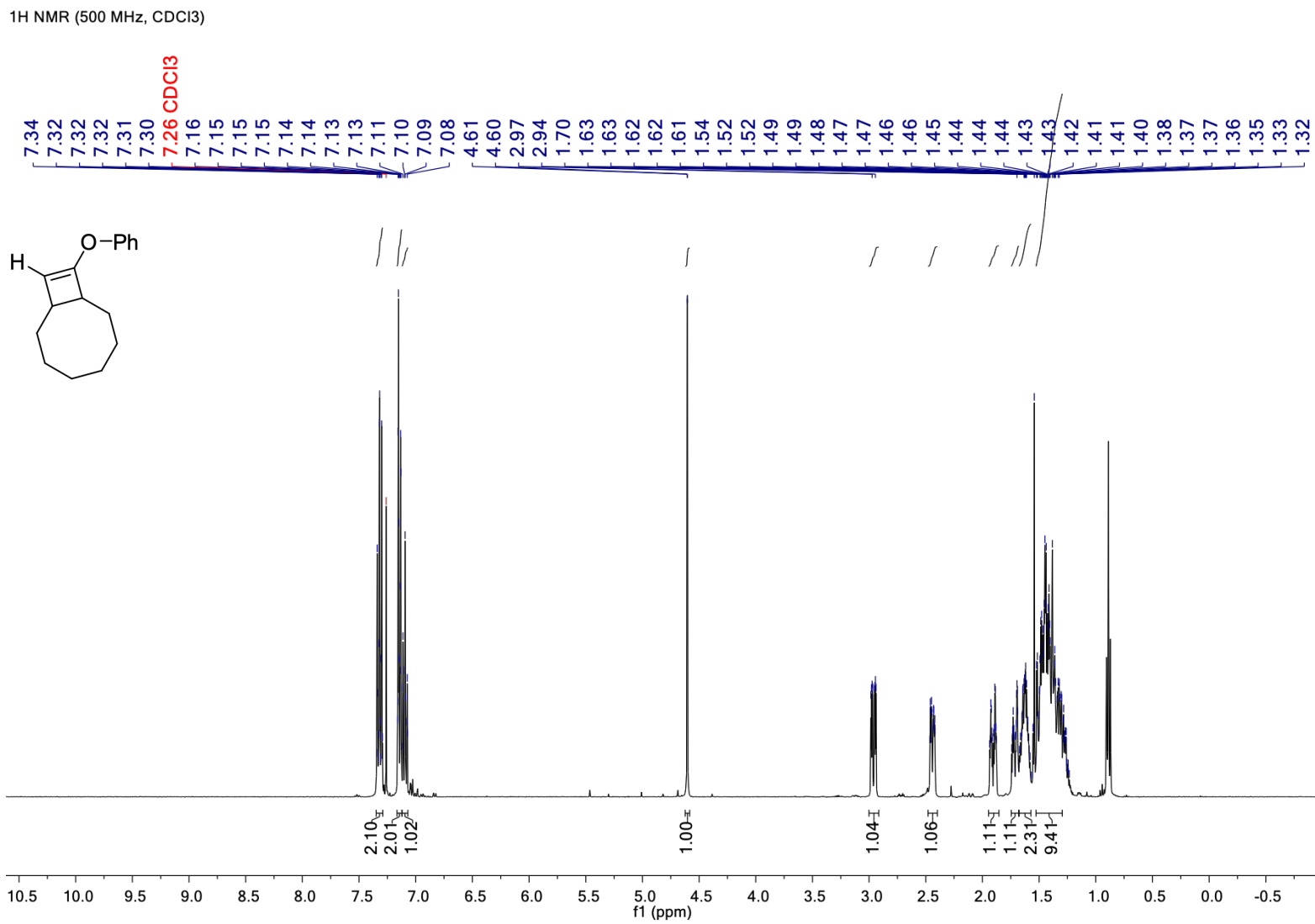


${ }^{13} \mathrm{C}$ NMR $\left(101 \mathrm{MHz}, \mathrm{CDCl}_{3}\right)$ of $\mathbf{3 u}$

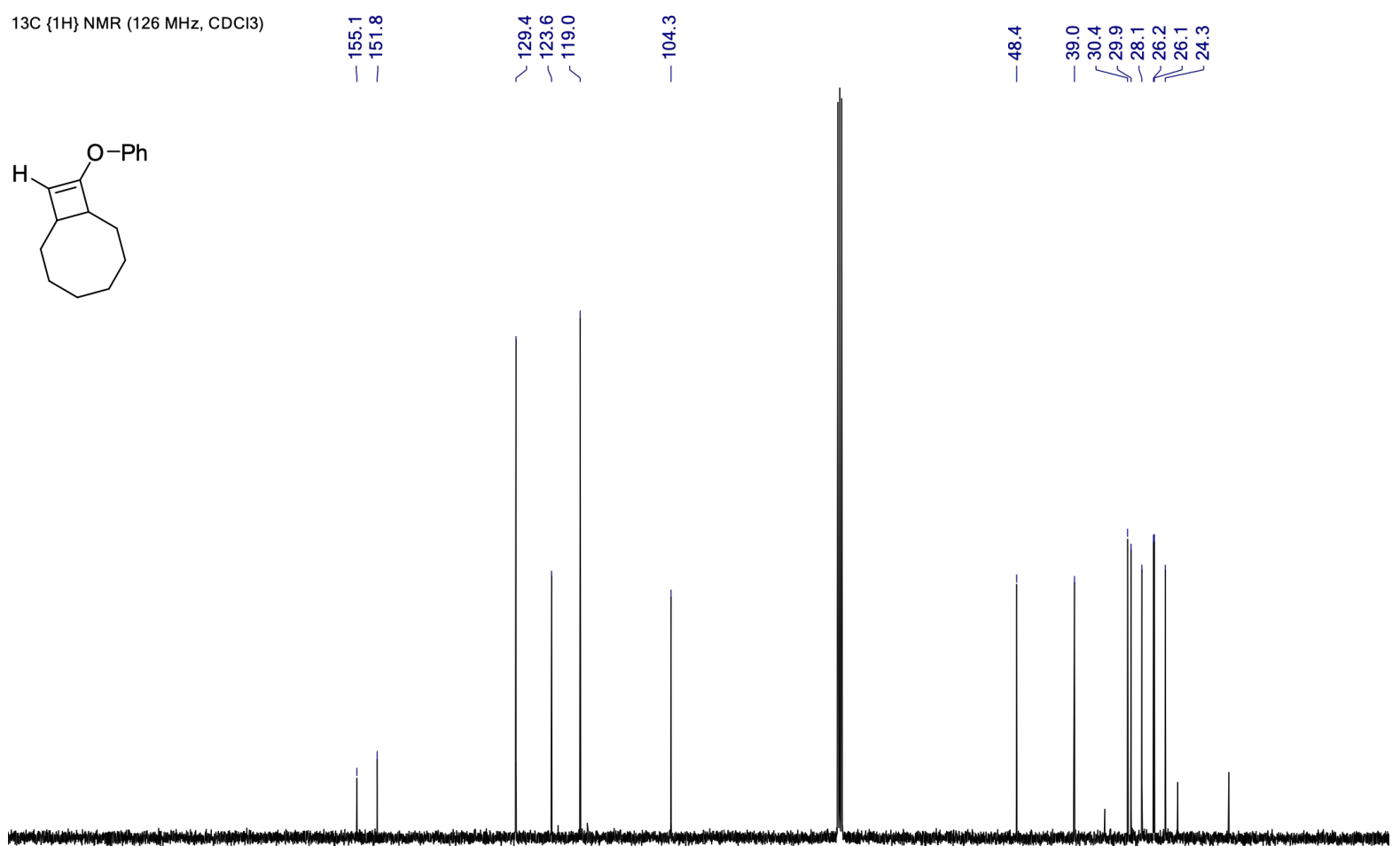

\begin{tabular}{lllllllllllllllllllllllll}
\hline 210 & 200 & 190 & 180 & 170 & 160 & 150 & 140 & 130 & 120 & 110 & 100 & 90 & 80 & 70 & 60 & 50 & 40 & 30 & 20 & 10 & 0 & $-1 \mathrm{C}$
\end{tabular} 


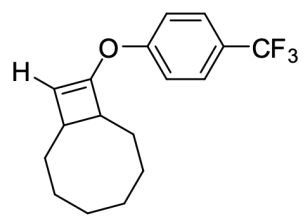

Cyclobutene $3 \mathbf{v}$ was synthesized following general procedure $\mathbf{B}$ starting from 1-(ethynyloxy)-4(trifluoromethyl)benzene (55.8 $\mathrm{mg}, 0.3 \mathrm{mmol}$ ) and (Z)-cyclooctene $(99 \mathrm{mg}, 0.9 \mathrm{mmol})$. The crude was purified by flash chromatography affording $3 \mathbf{v}$ as a transparent oil (64 $\mathrm{mg}, 72 \%$ ).

${ }^{1}$ H NMR $\left(500 \mathrm{MHz}, \mathrm{CDCl}_{3}\right) \delta 7.62-7.55(\mathrm{~m}, 2 \mathrm{H}), 7.26-7.20(\mathrm{~m}, 2 \mathrm{H}), 4.74(\mathrm{~d}, J=1.0 \mathrm{~Hz}, 1 \mathrm{H}), 2.98(\mathrm{ddd}, J=$ 12.0, 4.2, $2.0 \mathrm{~Hz}, 1 \mathrm{H}), 2.48$ (dddd, $J=11.5,4.1,1.8,1.0 \mathrm{~Hz}, 1 \mathrm{H}), 1.88$ (dtd, $J=14.5,3.9,2.0 \mathrm{~Hz}, 1 \mathrm{H}), 1.74$ (dtd, $J=12.5,3.1,1.9 \mathrm{~Hz}, 1 \mathrm{H}), 1.64$ (dddd, $J=14.2,13.0,6.5,3.9 \mathrm{~Hz}, 2 \mathrm{H}), 1.54-1.27$ (m, 8H).

${ }^{19}$ F NMR $\left(376 \mathrm{MHz}, \mathrm{CDCl}_{3}\right) \delta-61.98$.

${ }^{13}$ C NMR (126 MHz, $\left.\mathrm{CDCl}_{3}\right) \delta 158.0,150.7,127.0$ (q, $\left.J=3.8 \mathrm{~Hz}\right), 125.7$ (q, $\left.J=32.9 \mathrm{~Hz}\right), 124.3$ (q, $J=271.4$ $\mathrm{Hz}), 118.8,106.7,48.7,39.4,30.5,30.0,28.1,26.4,26.2,24.4$.

HRMS (ESI) $\mathrm{m} / \mathrm{z}$ calculated for $\mathrm{C}_{17} \mathrm{H}_{20} \mathrm{~F}_{3} \mathrm{O}^{+}[\mathrm{M}+\mathrm{H}]^{+}:$297.1461, found: 297.1455 .

${ }^{1} \mathrm{H}$ NMR $\left(500 \mathrm{MHz}, \mathrm{CDCl}_{3}\right)$ of $\mathbf{3 v}$
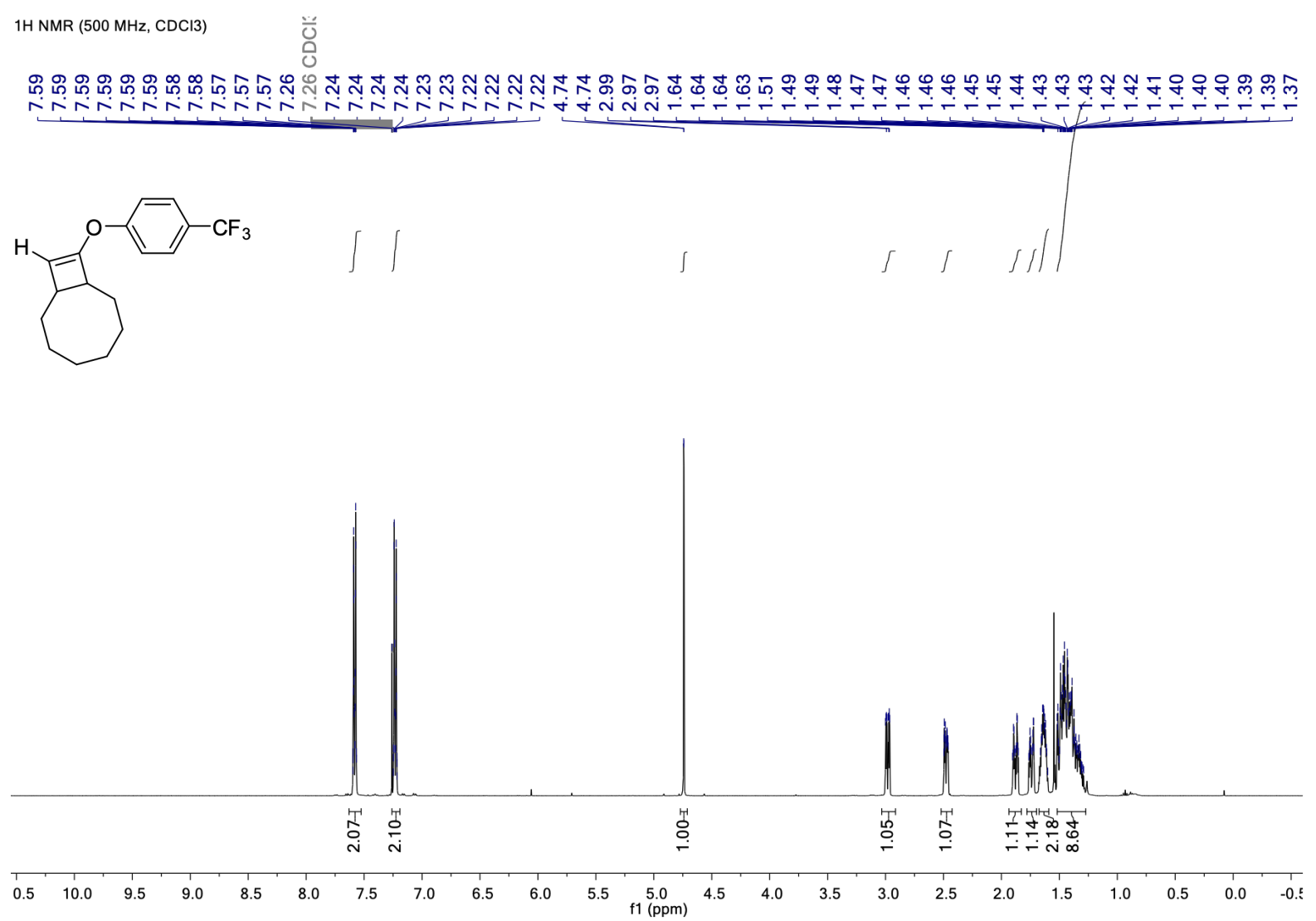
${ }^{19} \mathrm{~F}$ NMR (376 MHz, $\left.\mathrm{CDCl}_{3}\right)$ of $\mathbf{3 v}$
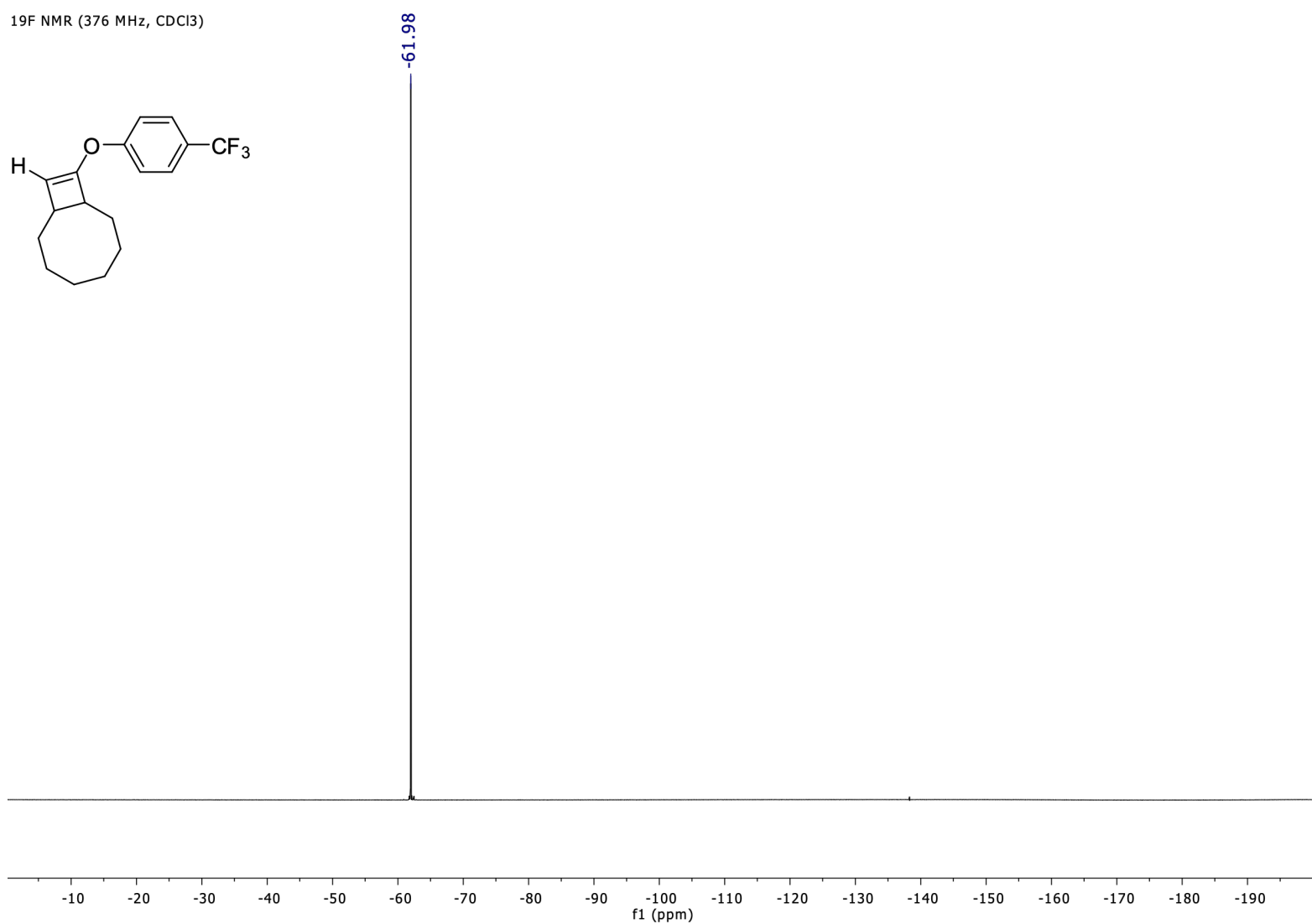

${ }^{13} \mathrm{C}$ NMR $\left(126 \mathrm{MHz}, \mathrm{CDCl}_{3}\right)$ of $\mathbf{3 v}$

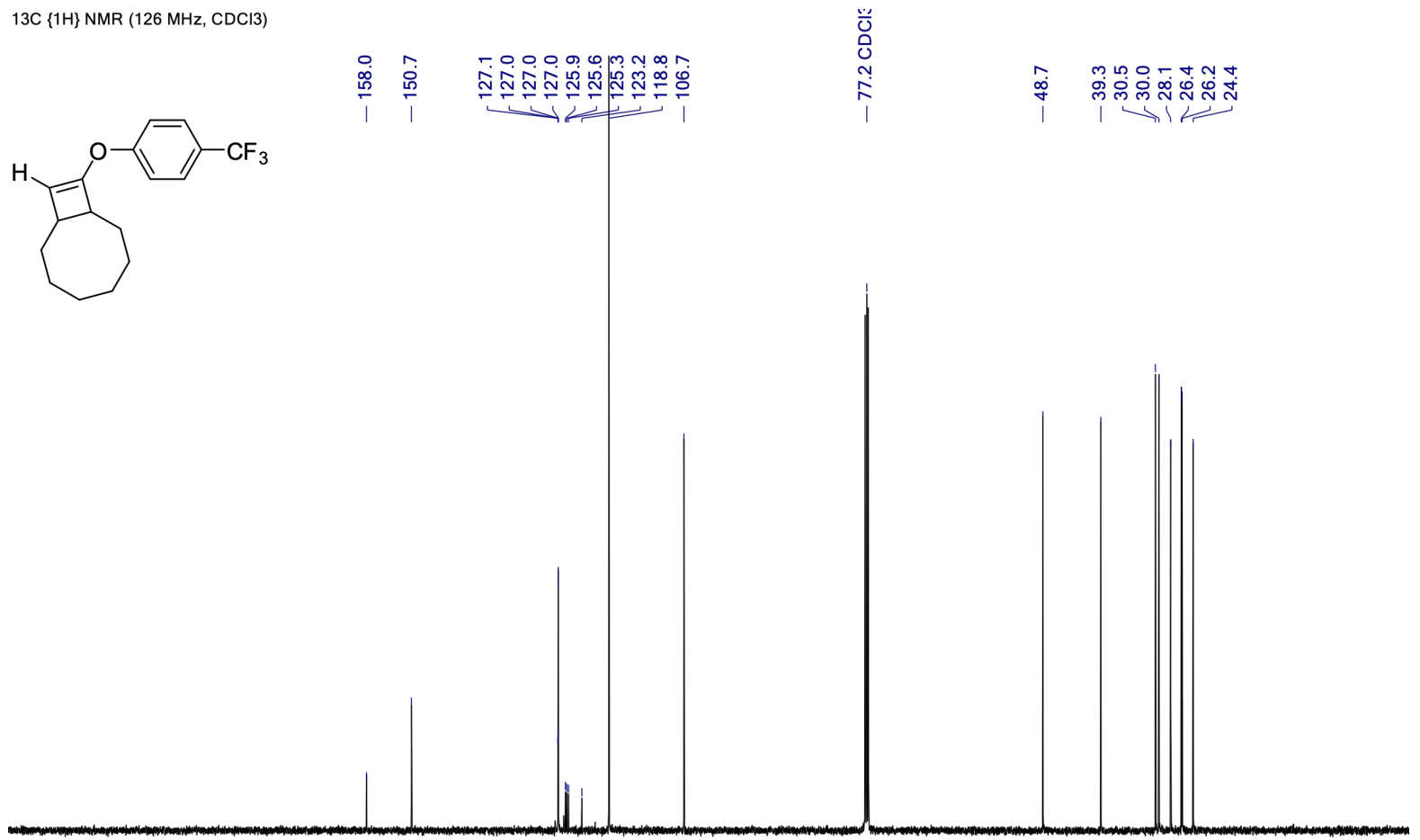

$\begin{array}{llllllllllllllllllllllllllll}210 & 200 & 190 & 180 & 170 & 160 & 150 & 140 & 130 & 120 & 110 & 100 & 90 & 80 & 70 & 60 & 50 & 40 & 30 & 20 & 10 & 0 & -1\end{array}$ 


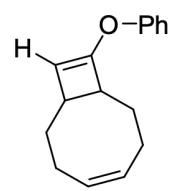

Cyclobutene 3w was synthesized following general procedure B starting from (ethynyloxy)- benzene (35.4 mg, $0.3 \mathrm{mmol}$ ) and 1,5-cyclooctadiene $(97 \mathrm{mg}, 0.9 \mathrm{mmol})$. The crude was purified by flash chromatography affording 3w as a transparent oil (12 $\mathrm{mg}, 18 \%)$.

${ }^{1}$ H NMR $\left(500 \mathrm{MHz}, \mathrm{CD}_{2} \mathrm{Cl}_{2}\right) \delta 7.36-7.28(\mathrm{~m}, 2 \mathrm{H}), 7.15-7.07(\mathrm{~m}, 3 \mathrm{H}), 5.76-5.65(\mathrm{~m}, 2 \mathrm{H}), 4.62(\mathrm{~d}, J=1.0$ $\mathrm{Hz}, 1 \mathrm{H}), 3.27$ (dt, $J=12.3,4.2 \mathrm{~Hz}, 1 \mathrm{H}), 2.75$ (dddd, $J=12.1,4.6,3.9,1.0 \mathrm{~Hz}, 1 \mathrm{H}), 2.41-2.26$ (m, $2 \mathrm{H}), 2.14-$ $2.01(\mathrm{~m}, 2 \mathrm{H}), 2.03-1.86(\mathrm{~m}, 2 \mathrm{H}), 1.72(\mathrm{dddd}, J=14.4,12.3,7.2,5.1 \mathrm{~Hz}, 1 \mathrm{H}), 1.62$ (dddd, $J=14.4,12.3,7.3$, $5.2 \mathrm{~Hz}, 1 \mathrm{H})$.

${ }^{13}$ C NMR $\left(126 \mathrm{MHz}, \mathrm{CD}_{2} \mathrm{Cl}_{2}\right) \delta 155.5,152.8,132.0,131.5,129.8,124.2,119.4,104.50,49.6,40.4,31.0,27.0$, $25.2,24.7$.

HRMS (ESI) m/z calculated for $\mathrm{C}_{16} \mathrm{H}_{19} \mathrm{O}^{+}[\mathrm{M}+\mathrm{H}]^{+}:$: 227.1430, found: 227.1429.

\section{${ }^{1} \mathrm{H}$ NMR $\left(500 \mathrm{MHz}, \mathrm{CD}_{2} \mathrm{Cl}_{2}\right)$ of $\mathbf{3 w}$}

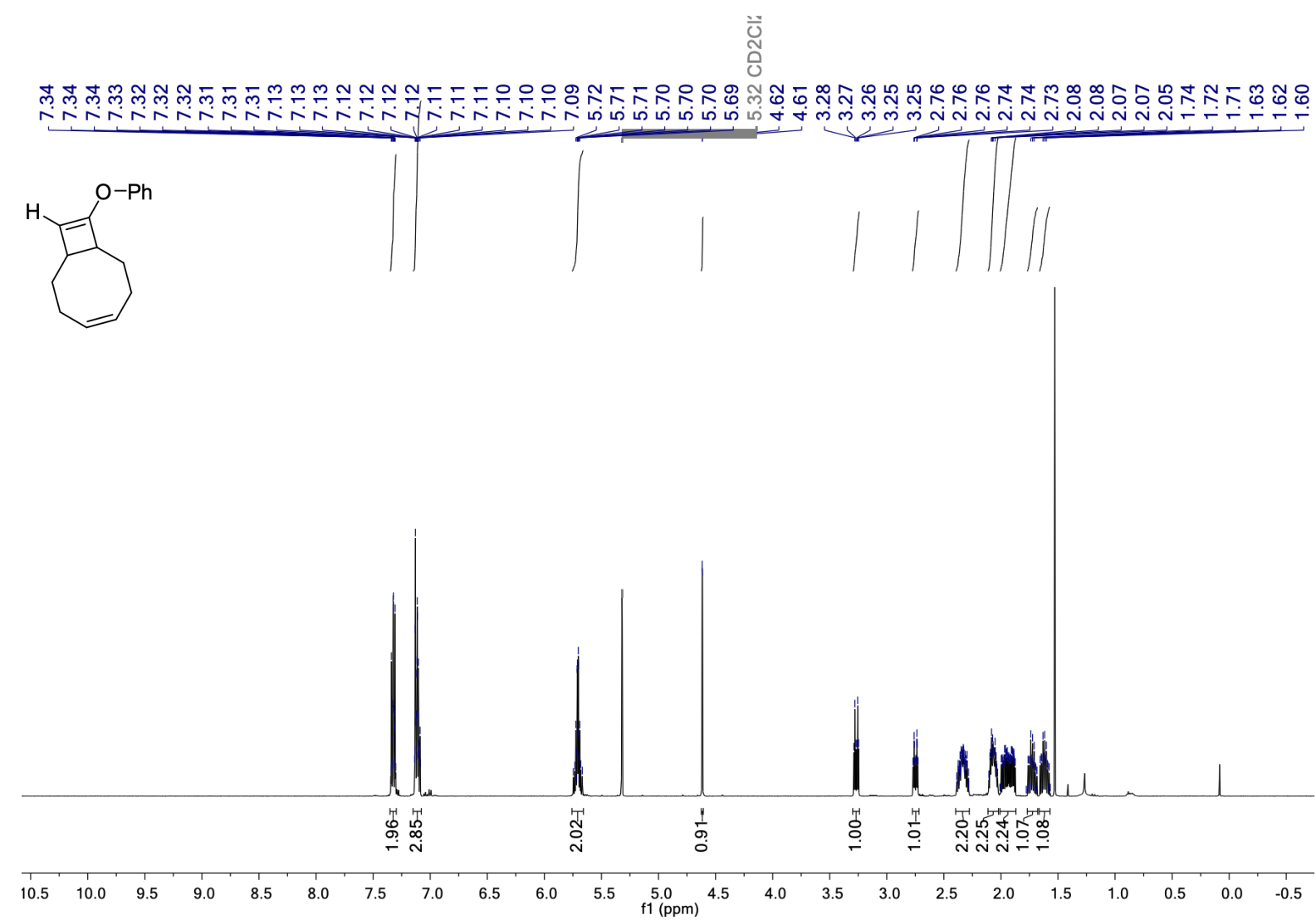


${ }^{13} \mathrm{C}$ NMR $\left(126 \mathrm{MHz}, \mathrm{CD}_{2} \mathrm{Cl}_{2}\right)$ of $\mathbf{3 w}$

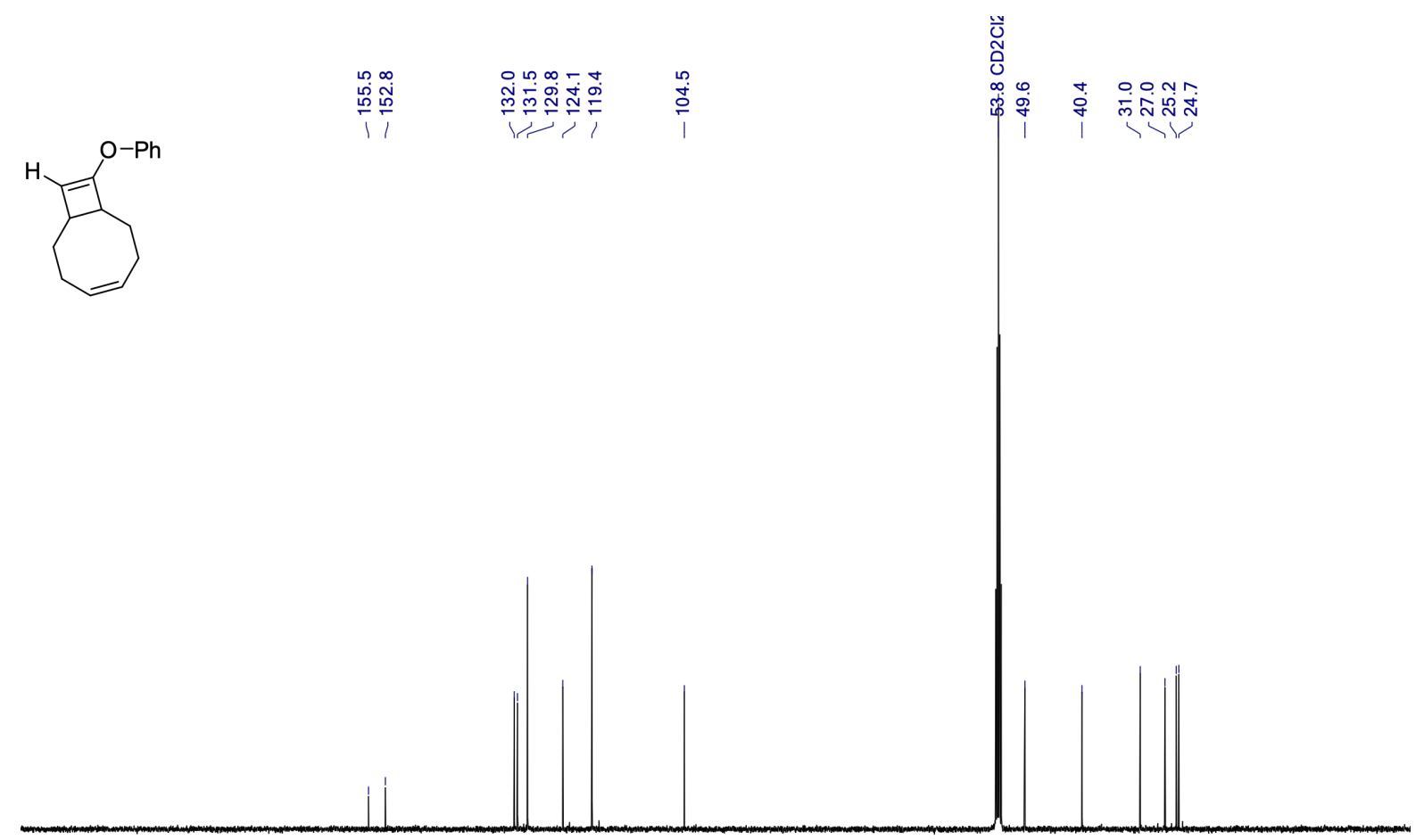

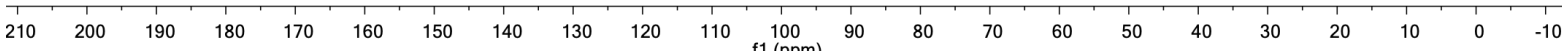




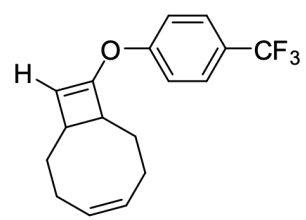

Cyclobutene 3x was synthesized following general procedure B starting from 1-(ethynyloxy)-4(trifluoromethyl)benzene $(37.2 \mathrm{mg}, 0.2 \mathrm{mmol})$ and 1,5-cyclooctadiene ( $65 \mathrm{mg}, 0.6 \mathrm{mmol})$. The crude was purified by flash chromatography affording $\mathbf{3 x}$ as a transparent oil (28 $\mathrm{mg}, 48 \%)$.

${ }^{1} \mathbf{H}$ NMR $\left(400 \mathrm{MHz}, \mathrm{CDCl}_{3}\right) \delta 7.63-7.54(\mathrm{~m}, 2 \mathrm{H}), 7.22(\mathrm{dt}, J=7.8,0.9 \mathrm{~Hz}, 2 \mathrm{H}), 5.78-5.64(\mathrm{~m}, 2 \mathrm{H}), 4.76(\mathrm{~d}$, $J=1.0 \mathrm{~Hz}, 1 \mathrm{H}), 3.29(\mathrm{dt}, J=12.3,4.2 \mathrm{~Hz}, 1 \mathrm{H}), 2.83-2.73(\mathrm{~m}, 1 \mathrm{H}), 2.43-2.26(\mathrm{~m}, 2 \mathrm{H}), 2.17-2.00(\mathrm{~m}, 2 \mathrm{H})$, $2.04-1.87(\mathrm{~m}, 2 \mathrm{H}), 1.82-1.57(\mathrm{~m}, 2 \mathrm{H})$.

${ }^{19}$ F NMR $\left(376 \mathrm{MHz}, \mathrm{CDCl}_{3}\right) \delta-61.99$.

${ }^{13}$ C NMR $\left(101 \mathrm{MHz}, \mathrm{CDCl}_{3}\right) \delta 157.9,151.2,131.7,131.2,127.0(\mathrm{q}, J=3.7 \mathrm{~Hz}), 125.8(\mathrm{q}, J=32.7 \mathrm{~Hz}), 124.3$ (q, $J=271.7 \mathrm{~Hz}), 118.9,106.5,49.4,40.1,30.4,26.6,24.9,24.5$.

HRMS (ESI) $\mathrm{m} / \mathrm{z}$ calculated for $\mathrm{C}_{17} \mathrm{H}_{18} \mathrm{~F}_{3} \mathrm{O}^{+}[\mathrm{M}+\mathrm{H}]^{+}:$295.1304, found: 295.1304 .

${ }^{1} \mathrm{H}$ NMR (400 MHz, $\left.\mathrm{CDCl}_{3}\right)$ of $\mathbf{3 x}$

1H NMR $(500 \mathrm{MHz}, \mathrm{CDCl} 3)$

㒸

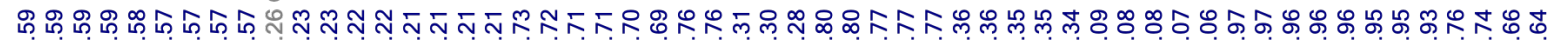

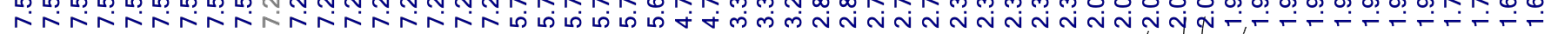<smiles></smiles>
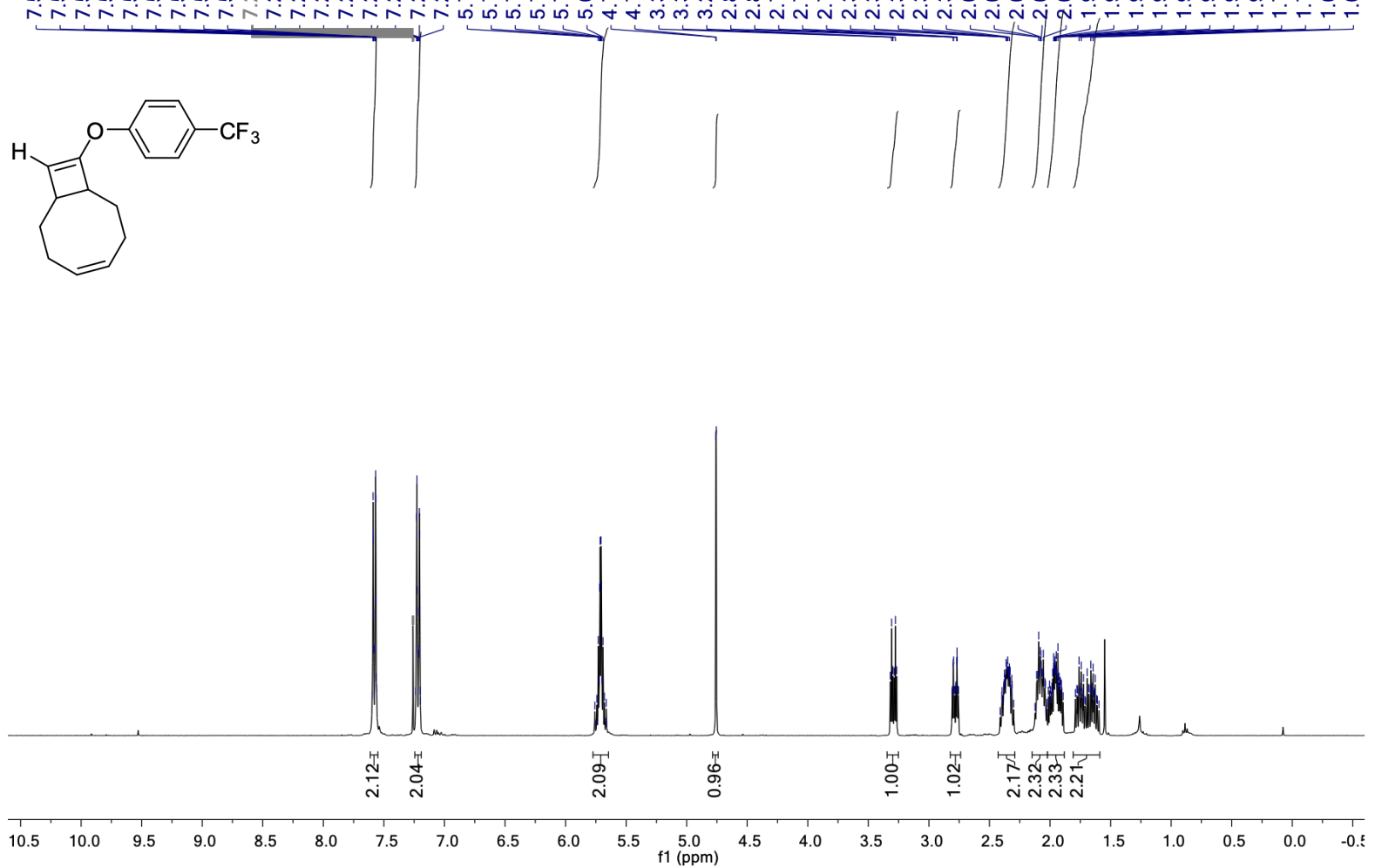
${ }^{19} \mathrm{~F}$ NMR (376 MHz, $\left.\mathrm{CDCl}_{3}\right)$ of $\mathbf{3 x}$
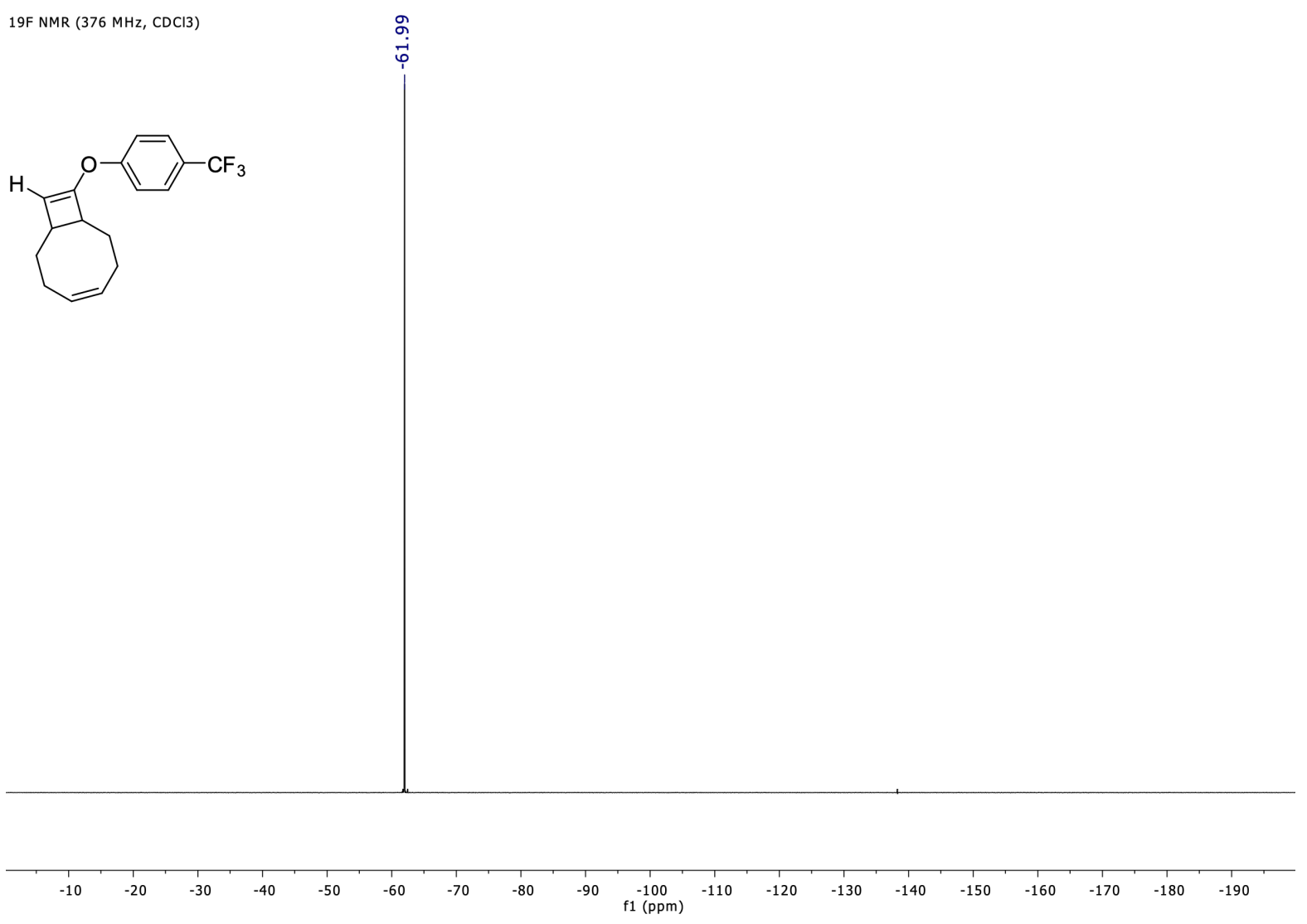

${ }^{13} \mathrm{C}$ NMR (101 MHz, $\left.\mathrm{CDCl}_{3}\right)$ of $\mathbf{3 x}$

$13 \mathrm{C}\{1 \mathrm{H}\} \mathrm{NMR}(126 \mathrm{MHz}, \mathrm{CDCl} 3)$

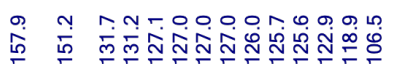
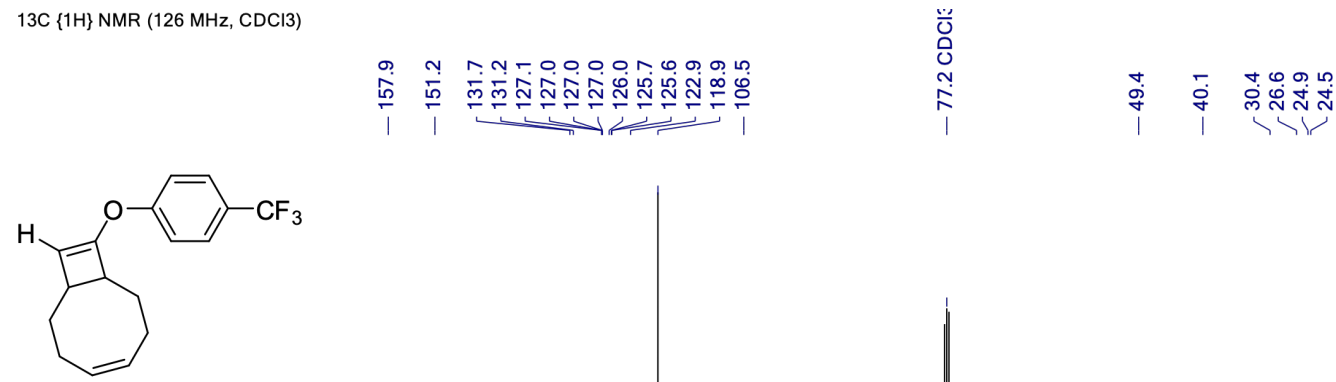

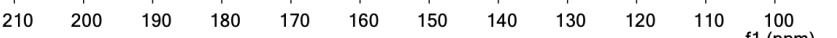

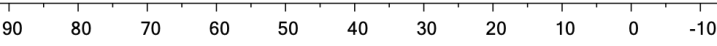




\section{1,4,4-trimethyl-8-(4-(trifluoromethyl)phenoxy)tricyclo[5.2.0.0 ${ }^{3,5}$ ]non-8-ene (3y)}<smiles>CC1(C)CCC2(C)CCC1CC2Oc1ccccc1</smiles>

Cyclobutene 3y was synthesized following general procedure B starting from (ethynyloxy)- benzene (35.4 mg, $0.3 \mathrm{mmol}$ ) and (+)-3-carene $(136 \mathrm{mg}, 0.9 \mathrm{mmol})$. The crude was purified by flash chromatography affording the single diastereoisomer $\mathbf{3 y}$ as a transparent oil $\left(24 \mathrm{mg}, 31 \%, d r\right.$ determined by ${ }^{1} \mathrm{H}$ NMR of the crude mixture $=$ $6: 1)$.

${ }^{1} \mathbf{H}$ NMR $\left(500 \mathrm{MHz}, \mathrm{CDCl}_{3}\right) \delta 7.36-7.29(\mathrm{~m}, 2 \mathrm{H}), 7.21-7.14(\mathrm{~m}, 2 \mathrm{H}), 7.10(\mathrm{ddt}, J=8.5,7.1,1.1 \mathrm{~Hz}, 1 \mathrm{H}), 4.68$ (s, 1H), 2.51 (dd, $J=4.0,2.3 \mathrm{~Hz}, 1 \mathrm{H}), 1.93$ (ddd, $J=15.1,7.9,2.4 \mathrm{~Hz}, 1 \mathrm{H}), 1.76$ (dd, $J=14.7,7.8 \mathrm{~Hz}, 1 \mathrm{H}), 1.13$ (s, 3H), $1.11-1.04(\mathrm{~m}, 1 \mathrm{H}), 1.04$ (s, 3H), $0.98(\mathrm{~s}, 3 \mathrm{H}), 0.96-0.87$ (m, 1H), $0.67-0.53(\mathrm{~m}, 2 \mathrm{H})$.

${ }^{13} \mathbf{C}$ NMR $\left(126 \mathrm{MHz}, \mathrm{CDCl}_{3}\right) \delta 155.45,150.65,129.56,123.75,119.25,108.66,48.41,35.90,28.92,28.50,25.84$, $19.72,18.81,17.57,16.84,15.85$.

HRMS (ESI) $\mathrm{m} / \mathrm{z}$ calculated for $\mathrm{C}_{18} \mathrm{H}_{23} \mathrm{O}^{+}[\mathrm{M}+\mathrm{H}]^{+}:$255.1743, found: 255.1741 .

$[\alpha]^{25.9^{\circ}}=-3.8\left(\mathrm{c}=0.55, \mathrm{CHCl}_{3}\right)$. 
${ }^{1} \mathrm{H}$ NMR (500 MHz, $\left.\mathrm{CDCl}_{3}\right)$ of $\mathbf{3 y}$

$1 \mathrm{H} \mathrm{NMR}(500 \mathrm{MHz}, \mathrm{CDCl} 3)$

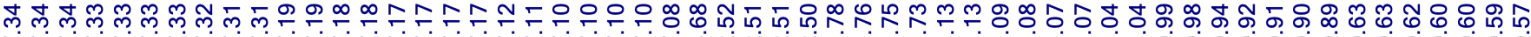<smiles>CC1[C@@H]2C[C@H]3C(Oc4ccccc4)=C[C@@H]3C[C@@H]2C1(C)C</smiles><smiles>C#CC</smiles>
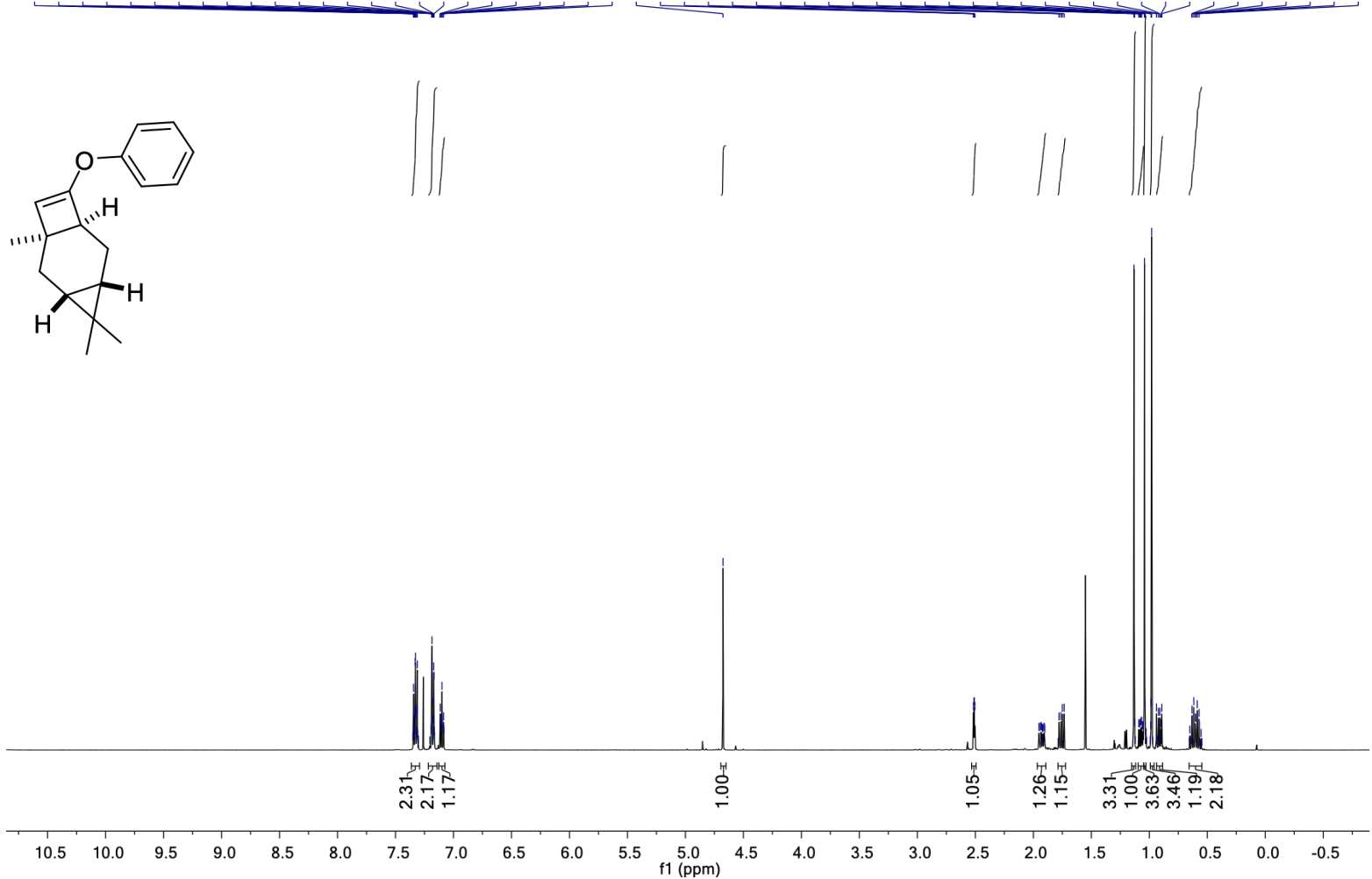

${ }^{1} \mathrm{H}-{ }^{-1} \mathrm{H}$ NOESY NMR $\left(\mathrm{CDCl}_{3}\right)$ of $\mathbf{3 y}$

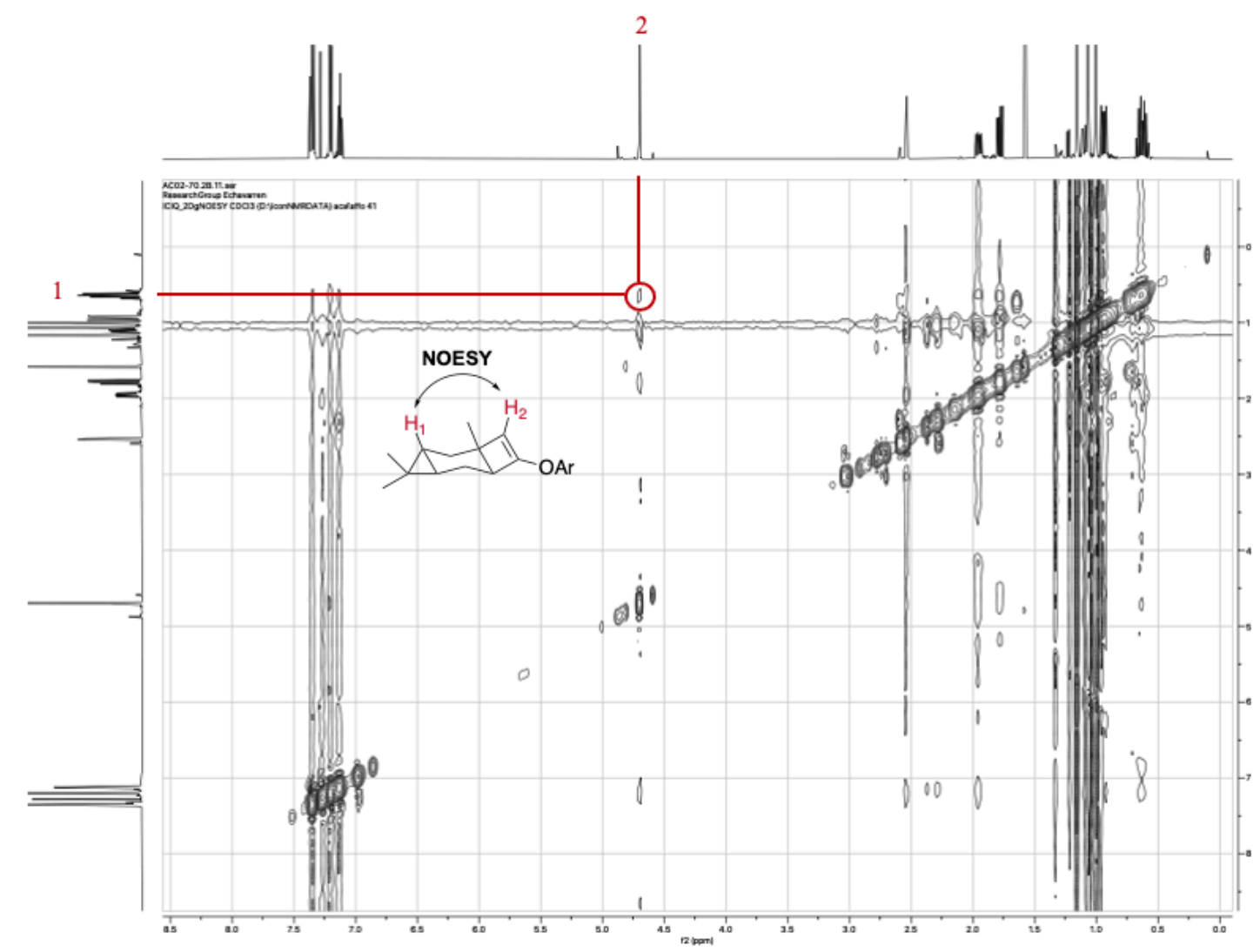


$\left.{ }^{13} \mathrm{C} \mathrm{NMR} \mathrm{(126} \mathrm{MHz,} \mathrm{CDCl}_{3}\right)$ of $\mathbf{3 y}$

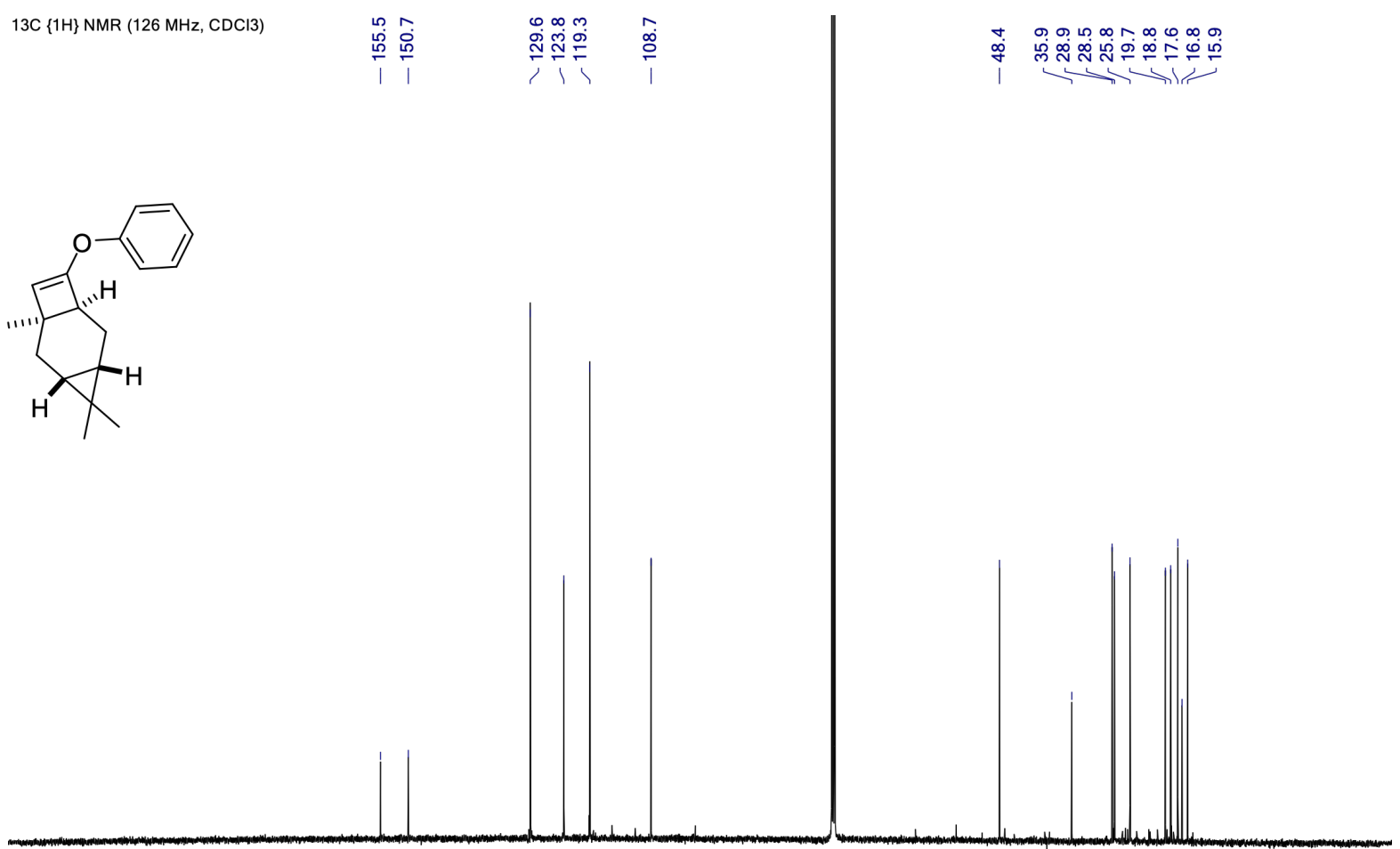

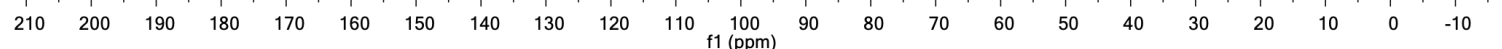




\section{1,4,4-trimethyl-8-(4-(trifluoromethyl)phenoxy)tricyclo[5.2.0.0 ${ }^{3,5}$ ]non-8-ene (3z)}<smiles>CC1(C)CC2(C)CC(Oc3ccc(C(F)(F)F)cc3)CC1C2(C)C</smiles>

Cyclobutene $\mathbf{3 z}$ was synthesized following general procedure $\mathbf{B}$ starting from 1-(ethynyloxy)-4(trifluoromethyl)benzene (18.6 mg, $0.1 \mathrm{mmol})$ and (+)-3-carene $(41 \mathrm{mg}, 0.3 \mathrm{mmol})$. The crude was purified by flash chromatography affording $3 \mathbf{z}$ as a transparent oil, 6:1 mixture of diastereomers (21 $\mathrm{mg}, 65 \%, d r$ determined by ${ }^{1} \mathrm{H}$ NMR of the crude mixture $=4: 1$ ).

${ }^{1} \mathbf{H}$ NMR $\left(400 \mathrm{MHz}, \mathrm{CDCl}_{3}\right) \delta 7.59-7.51(\mathrm{~m}, 2.4 \mathrm{H}, \mathrm{Md}+\mathrm{md}), 7.22(\mathrm{dt}, J=9.0,0.9 \mathrm{~Hz}, 2.4 \mathrm{H}, \mathrm{Md}+\mathrm{md}), 4.76$ (s, 1H, Md), 4.66 (d, $J=0.9 \mathrm{~Hz}, 0.2 \mathrm{H}, \mathrm{md}), 2.49$ (dd, $J=4.0,2.3 \mathrm{~Hz}, 1 \mathrm{H}, \mathrm{Md}), 2.07$ (t, $J=3.0 \mathrm{~Hz}, 0.2 \mathrm{H}, \mathrm{md}$ ), $1.93-1.77(\mathrm{~m}, 1.2 \mathrm{H}, \mathrm{Md}+\mathrm{md}), 1.74(\mathrm{dd}, J=14.7,7.9 \mathrm{~Hz}, 1.2 \mathrm{H}, \mathrm{Md}+\mathrm{md}), 1.11(\mathrm{~s}, 3.6 \mathrm{H}, \mathrm{Md}+\mathrm{md}), 1.10-$ $1.00(\mathrm{~m}, 1.2 \mathrm{H}, \mathrm{Md}+\mathrm{md}), 0.99(\mathrm{~s}, 3.6 \mathrm{H}, \mathrm{Md}+\mathrm{md}), 0.94$ (s, 3.6H, Md + md), $0.94-0.78(\mathrm{~m}, 1.2 \mathrm{H}, \mathrm{Md}+\mathrm{md})$, $0.63-0.44(\mathrm{~m}, 2.4 \mathrm{H}, \mathrm{Md}+\mathrm{md})$.

${ }^{19} \mathbf{F}$ NMR $\left(376 \mathrm{MHz}, \mathrm{CDCl}_{3}\right) \delta-61.96$.

${ }^{13}$ C NMR (101 MHz, CDCl $) \delta 158.2(\mathrm{Md}+\mathrm{md}), 153.7$ (md), $149.5(\mathrm{Md}), 127.0$ (q, $\left.J=3.7 \mathrm{~Hz} \mathrm{Md}+\mathrm{md}\right), 125.7$ (q, $J=33.0 \mathrm{~Hz}, \mathrm{Md}), 124.3$ (q, $J=271.5 \mathrm{~Hz}, \mathrm{Md}), 119.1$ (md), 118.9 (Md), 110.8 (Md), $103.2(\mathrm{md}), 48.7$ (Md), $46.2(\mathrm{md}), 40.0(\mathrm{md}), 36.3(\mathrm{Md}), 28.9(\mathrm{Md}+\mathrm{md}), 28.4(\mathrm{Md}), 26.1(\mathrm{md}), 25.7(\mathrm{Md}), 22.5(\mathrm{md}), 21.0(\mathrm{md}), 19.67$ (Md), $19.4(\mathrm{md}), 18.8(\mathrm{Md}), 17.5(\mathrm{Md}+\mathrm{md}), 16.9(\mathrm{Md}), 15.8(\mathrm{Md}+\mathrm{md})$. Two aromatic ${ }^{13} \mathrm{C}$ signals of the $\mathrm{mr}$ were not detected because of low intensity.

HRMS (ESI) m/z calculated for $\mathrm{C}_{19} \mathrm{H}_{22} \mathrm{~F}_{3} \mathrm{O}^{+}[\mathrm{M}+\mathrm{H}]^{+}: 323.1617$, found: 323.1614 . 
${ }^{1} \mathrm{H}$ NMR $\left(400 \mathrm{MHz}, \mathrm{CDCl}_{3}\right)$ of $\mathbf{3 z}$

1H NMR (500 MHz, CDCl3)

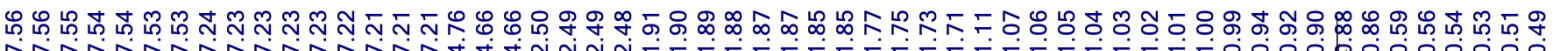
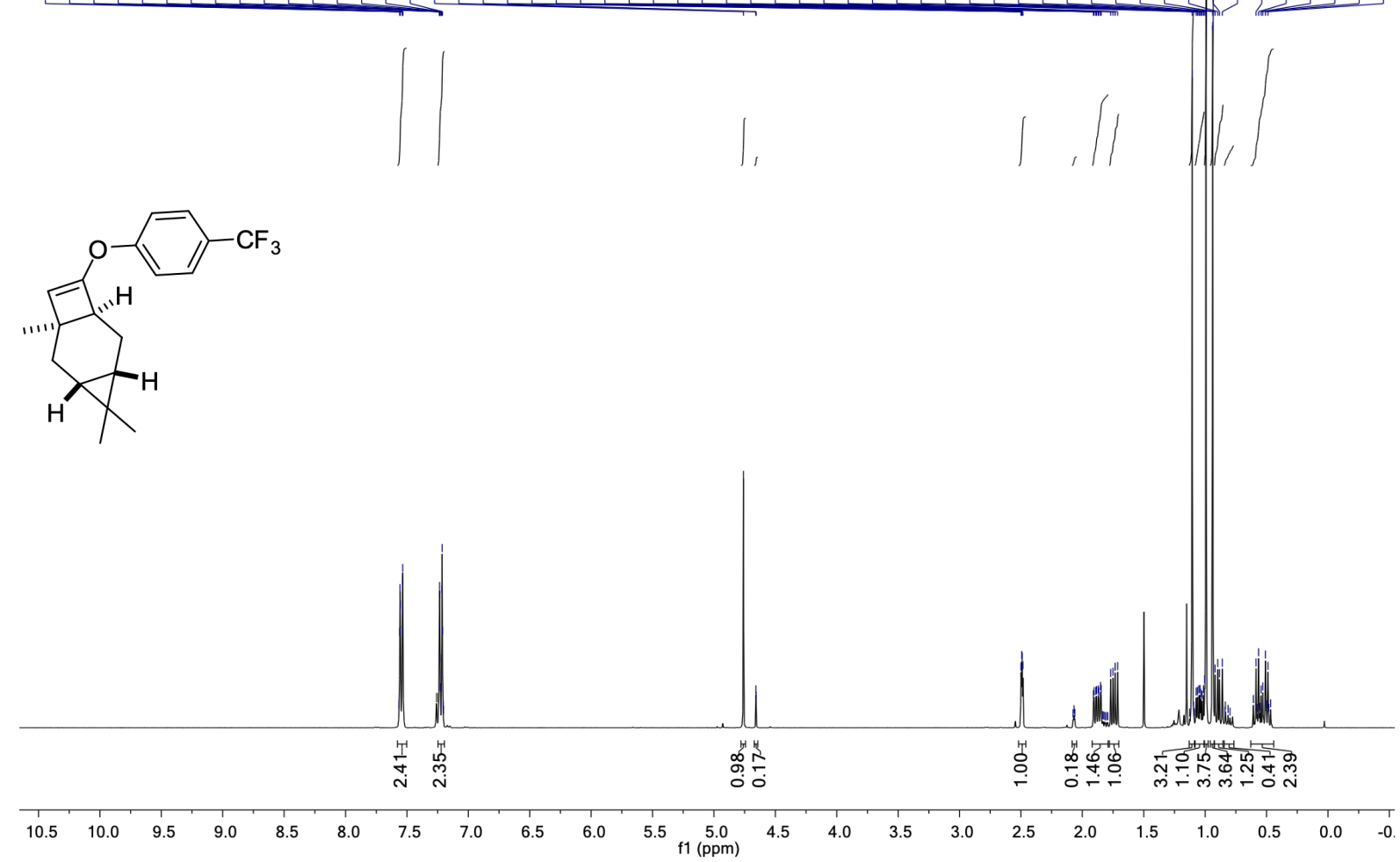

${ }^{1} \mathrm{H}-{ }^{1} \mathrm{H}$ NOESY NMR $\left(\mathrm{CDCl}_{3}\right)$ of $\mathbf{3 z}$

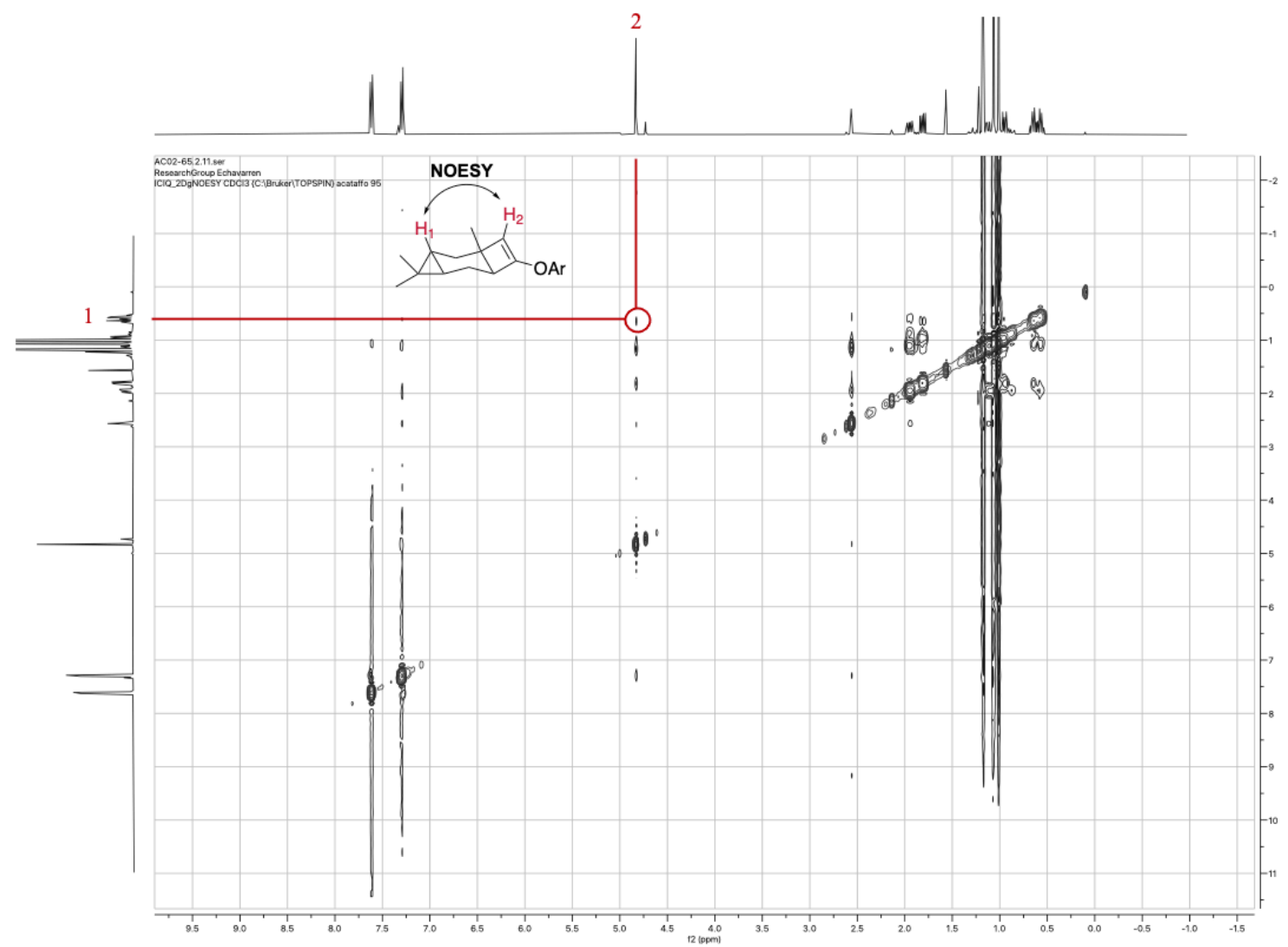


${ }^{19} \mathrm{~F}$ NMR $\left(376 \mathrm{MHz}, \mathrm{CDCl}_{3}\right)$ of $\mathbf{3 z}$

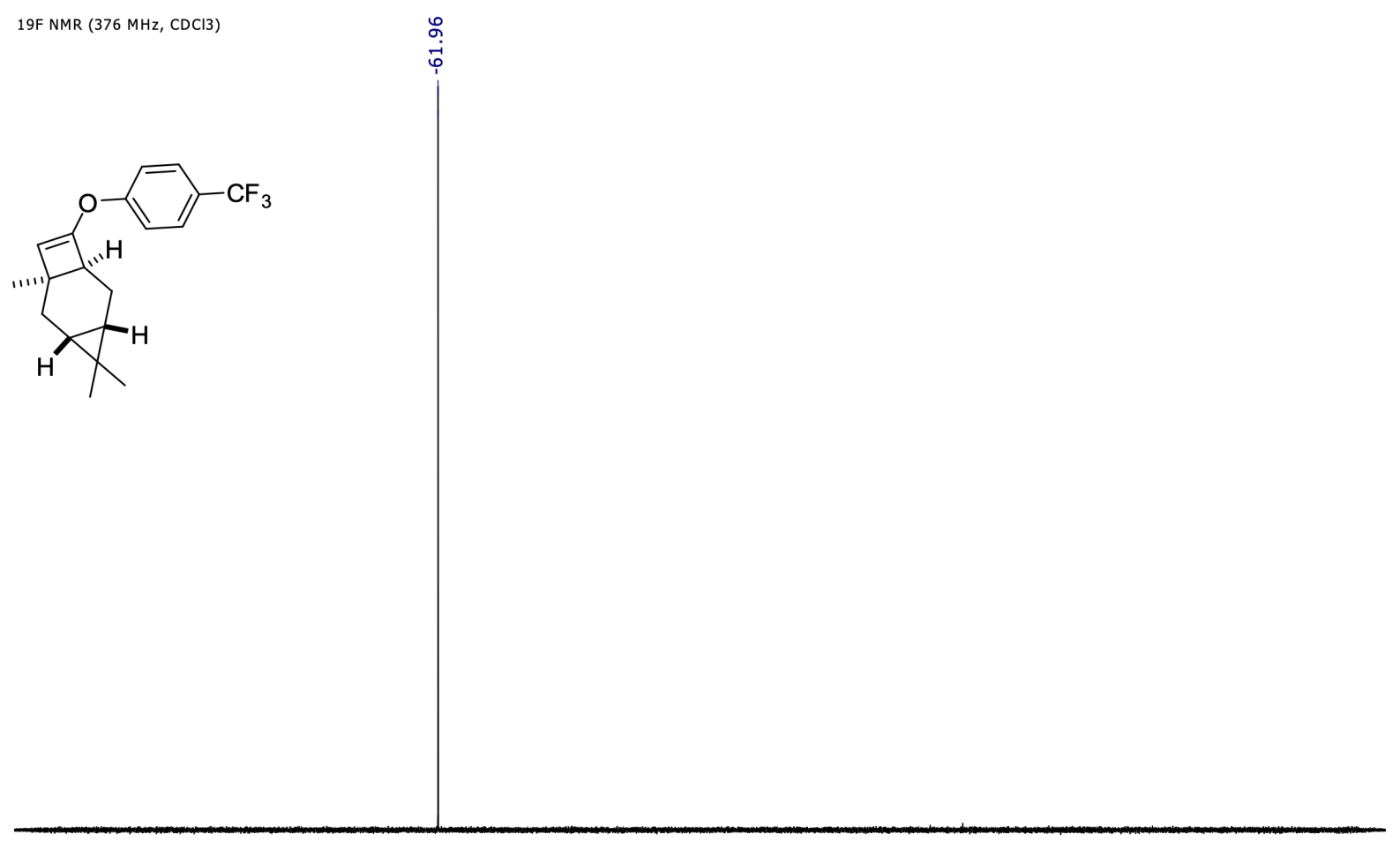

\begin{tabular}{|c|c|c|c|c|c|c|c|c|c|c|c|c|c|c|c|c|c|c|}
\hline-10 & -20 & -30 & -40 & -50 & -60 & -70 & -80 & -90 & $\begin{array}{c}-100 \\
\mathrm{f} 1(\mathrm{ppm})\end{array}$ & -110 & -120 & -130 & -140 & -150 & -160 & -170 & -180 & -190 \\
\hline
\end{tabular}

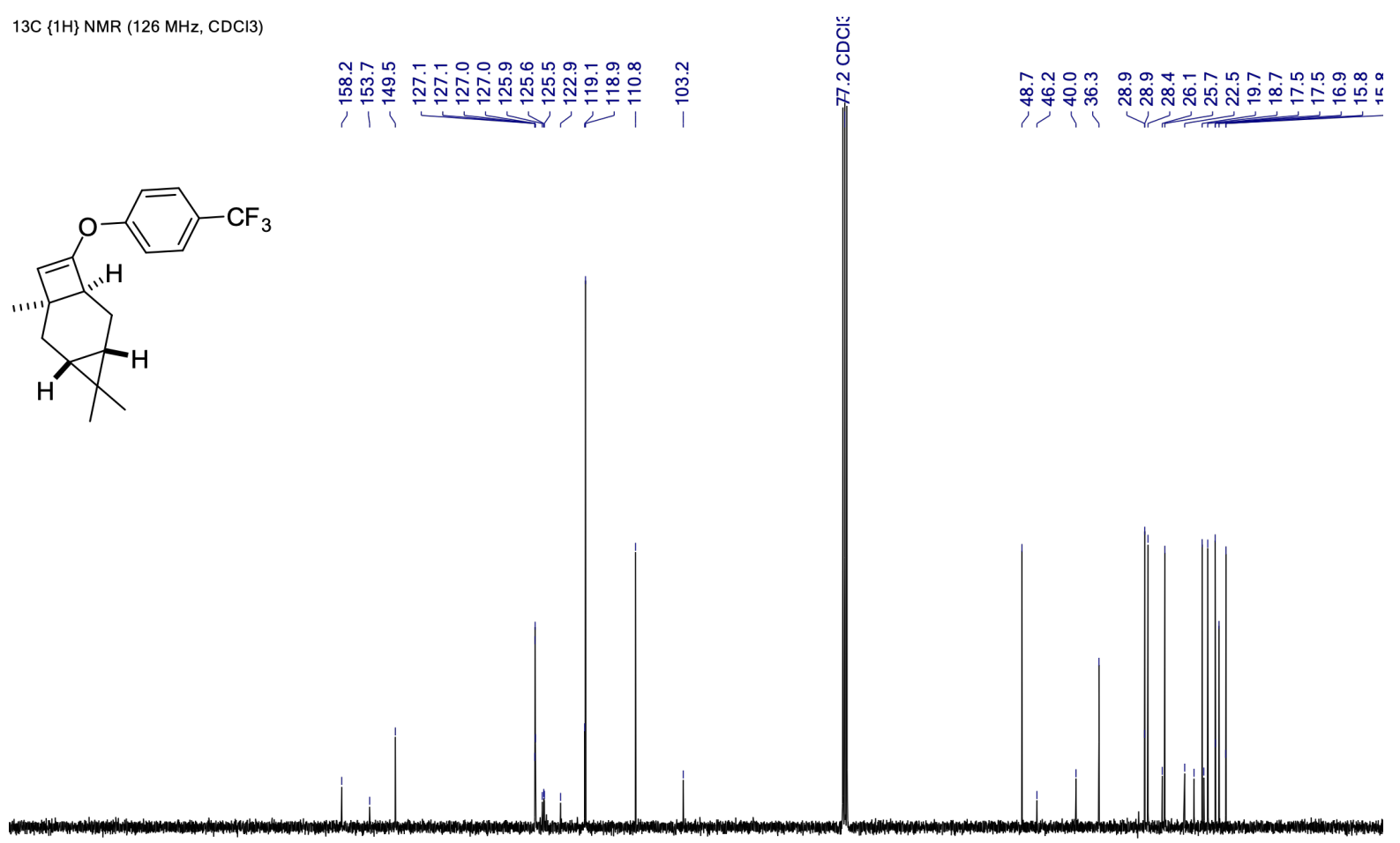

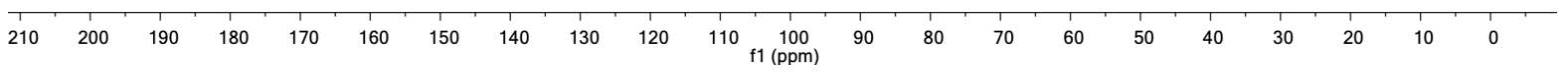




\section{2-Phenoxyspiro[3.5]non-1-ene (3aa)}<smiles>c1ccc(OC2CC3(CCCC3)C2)cc1</smiles>

Cyclobutene 3aa was synthesized following general procedure B starting from (ethynyloxy)benzene (23.6 mg, $0.2 \mathrm{mmol}$ ) and methylenecyclohexane $(58 \mathrm{mg}, 0.6 \mathrm{mmol})$. The crude product was purified by flash chromatography affording $3 \mathbf{a a}$ as a colorless oil (32 $\mathrm{mg}, 74 \%)$.

${ }^{1} \mathbf{H}$ NMR $\left(500 \mathrm{MHz}, \mathrm{CDCl}_{3}\right) \delta 7.36-7.29(\mathrm{~m}, 2 \mathrm{H}), 7.16-7.13(\mathrm{~m}, 2 \mathrm{H}), 7.11(\mathrm{tt}, J=7.3,1.2 \mathrm{~Hz}, 1 \mathrm{H}), 4.98(\mathrm{~s}$, $1 \mathrm{H}), 2.37(\mathrm{~s}, 2 \mathrm{H}), 1.62-1.38(\mathrm{~m}, 10 \mathrm{H})$.

${ }^{13} \mathbf{C}$ NMR $\left(126 \mathrm{MHz}, \mathrm{CDCl}_{3}\right) \delta 155.3,149.9,129.6,123.9,119.3,110.8,43.4,39.5,37.3,26.0,25.1$.

HRMS (APCI) $m / z$ calculated for $\mathrm{C}_{15} \mathrm{H}_{19} \mathrm{O}^{+}[\mathrm{M}+\mathrm{H}]^{+}: 215.1430$, found: 215.1428 .

${ }^{1} \mathrm{H} \mathrm{NMR}\left(500 \mathrm{MHz}, \mathrm{CDCl}_{3}\right)$ of $\mathbf{3 a a}$

$1 \mathrm{H} \mathrm{NMR}(500 \mathrm{MHz}, \mathrm{CDCl} 3)$

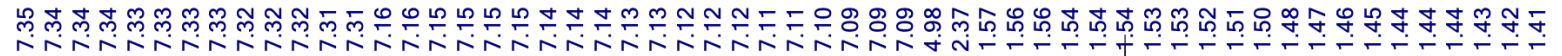
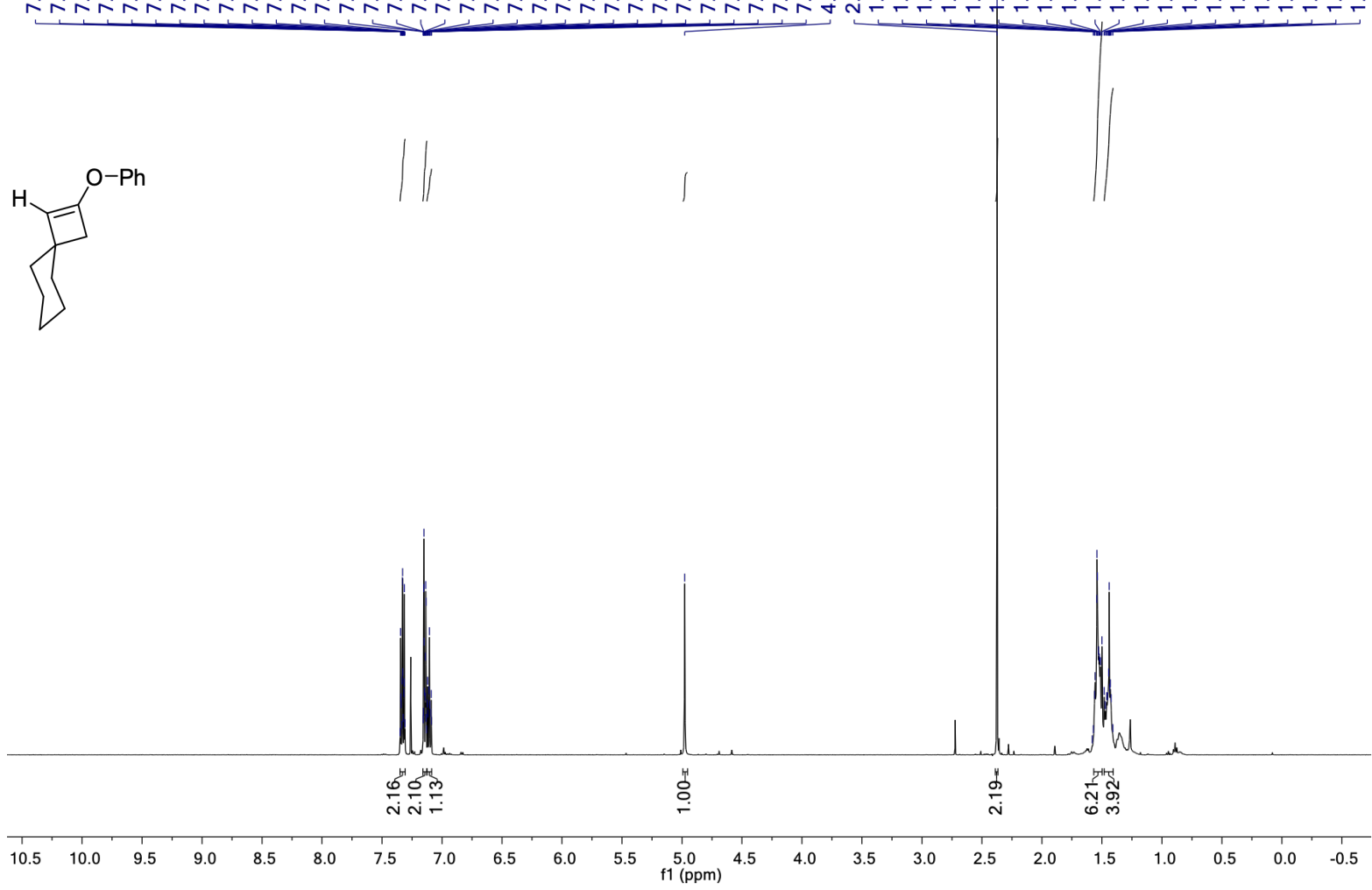
${ }^{13} \mathrm{C}$ NMR $\left(126 \mathrm{MHz}, \mathrm{CDCl}_{3}\right)$ of $\mathbf{3 a a}$

$13 \mathrm{C}\{1 \mathrm{H}\} \mathrm{NMR}(126 \mathrm{MHz}, \mathrm{CDCl} 3)$

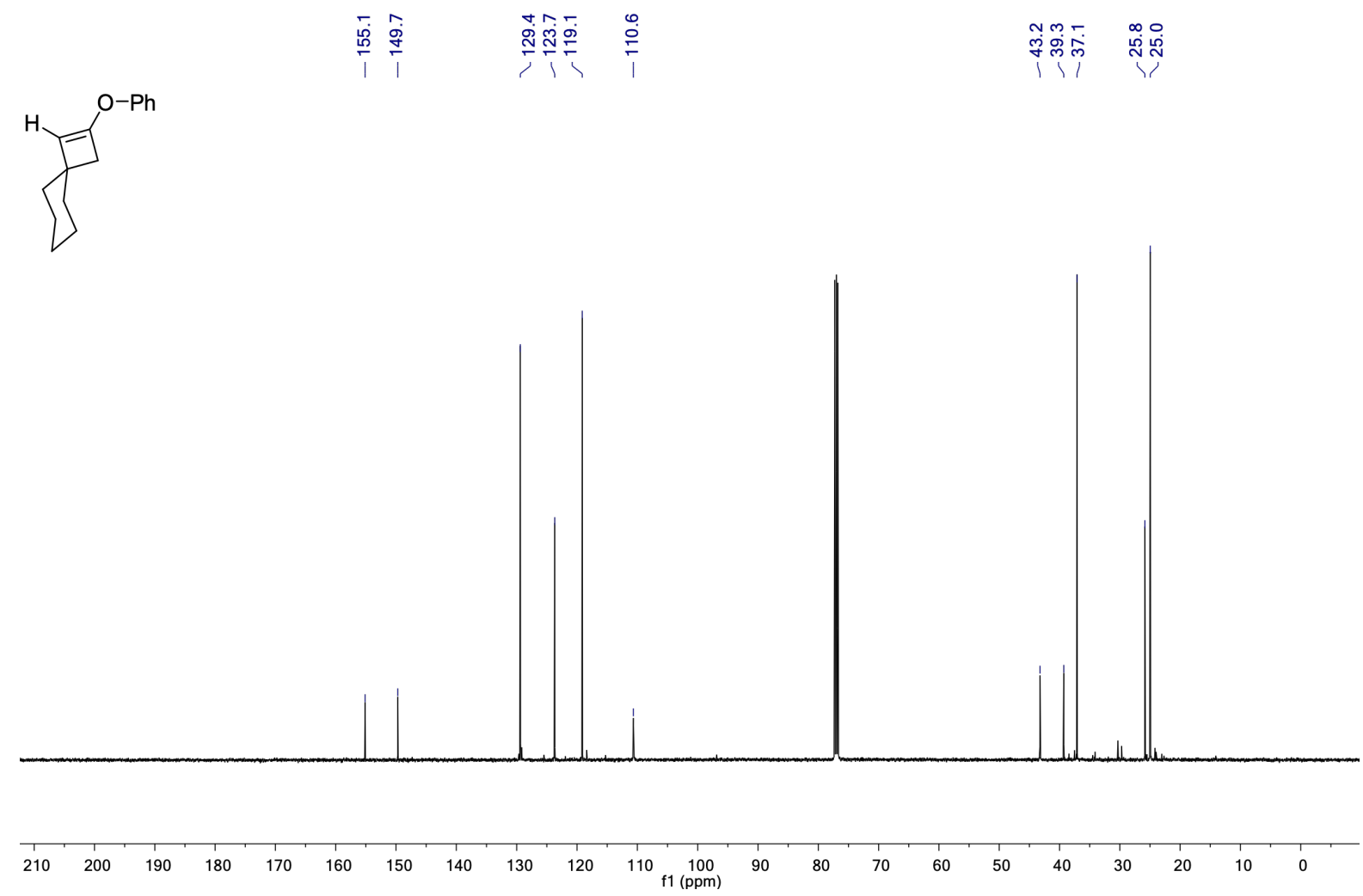




\section{(3-Methyl-3-phenethylcyclobut-1-en-1-yl)oxy)benzene (3bb)}<smiles>CC1(CCc2ccccc2)C=C(Oc2ccccc2)C1</smiles>

Cyclobutene 3bb was synthesized following general procedure B starting from (ethynyloxy)benzene (23.6 mg, $0.2 \mathrm{mmol}$ ) and (3-methylbut-3-en-1-yl)benzene $(87.6 \mathrm{mg}, 0.6 \mathrm{mmol})$. The crude product was purified by flash chromatography affording $3 \mathbf{b b}$ as a colorless oil (29 mg, 54\%).

${ }^{1} \mathbf{H}$ NMR $\left(500 \mathrm{MHz}, \mathrm{CDCl}_{3}\right) \delta 7.39-7.32(\mathrm{~m}, 2 \mathrm{H}), 7.29(\mathrm{t}, J=7.5 \mathrm{~Hz}, 2 \mathrm{H}), 7.24-7.18(\mathrm{~m}, 3 \mathrm{H}), 7.18-7.10(\mathrm{~m}$, $3 \mathrm{H}), 4.88(\mathrm{~s}, 1 \mathrm{H}), 2.73-2.61(\mathrm{~m}, 2 \mathrm{H}), 2.54(\mathrm{~d}, J=12.7 \mathrm{~Hz}, 1 \mathrm{H}), 2.44(\mathrm{~d}, J=12.7 \mathrm{~Hz}, 1 \mathrm{H}), 1.90-1.78(\mathrm{~m}, 2 \mathrm{H})$, $1.31(\mathrm{~s}, 3 \mathrm{H})$.

${ }^{13}$ C NMR $\left(126 \mathrm{MHz}_{\mathrm{CDCl}}\right) \delta 155.2,148.7,143.1,129.6,128.4,125.8,123.9,119.3,110.7,44.3,42.7,37.9$, $32.8,24.8$.

HRMS (APCI) $m / z$ calculated for $\mathrm{C}_{19} \mathrm{H}_{21} \mathrm{O}^{+}[\mathrm{M}+\mathrm{H}]^{+}: 265.1587$, found: 265.1585.

${ }^{1} \mathrm{H} \mathrm{NMR}\left(500 \mathrm{MHz}, \mathrm{CDCl}_{3}\right)$ of $\mathbf{3 b b}$

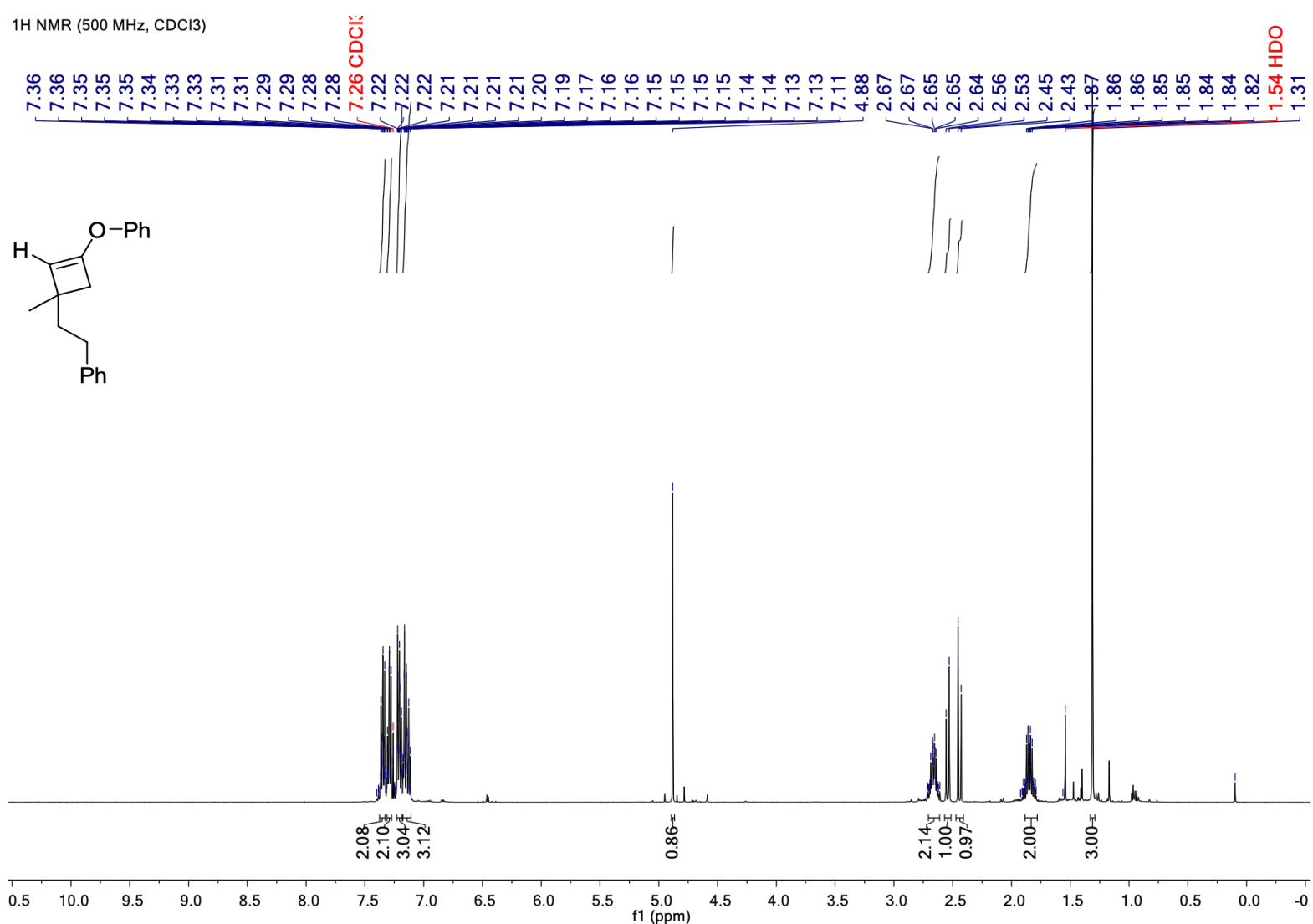


${ }^{13} \mathrm{C}$ NMR $\left(126 \mathrm{MHz}, \mathrm{CDCl}_{3}\right)$ of $\mathbf{3 b b}$

$13 \mathrm{C}\{1 \mathrm{H}\}$ NMR $(126 \mathrm{MHz}, \mathrm{CDCl})$

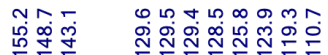

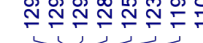

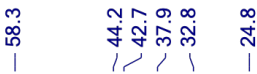
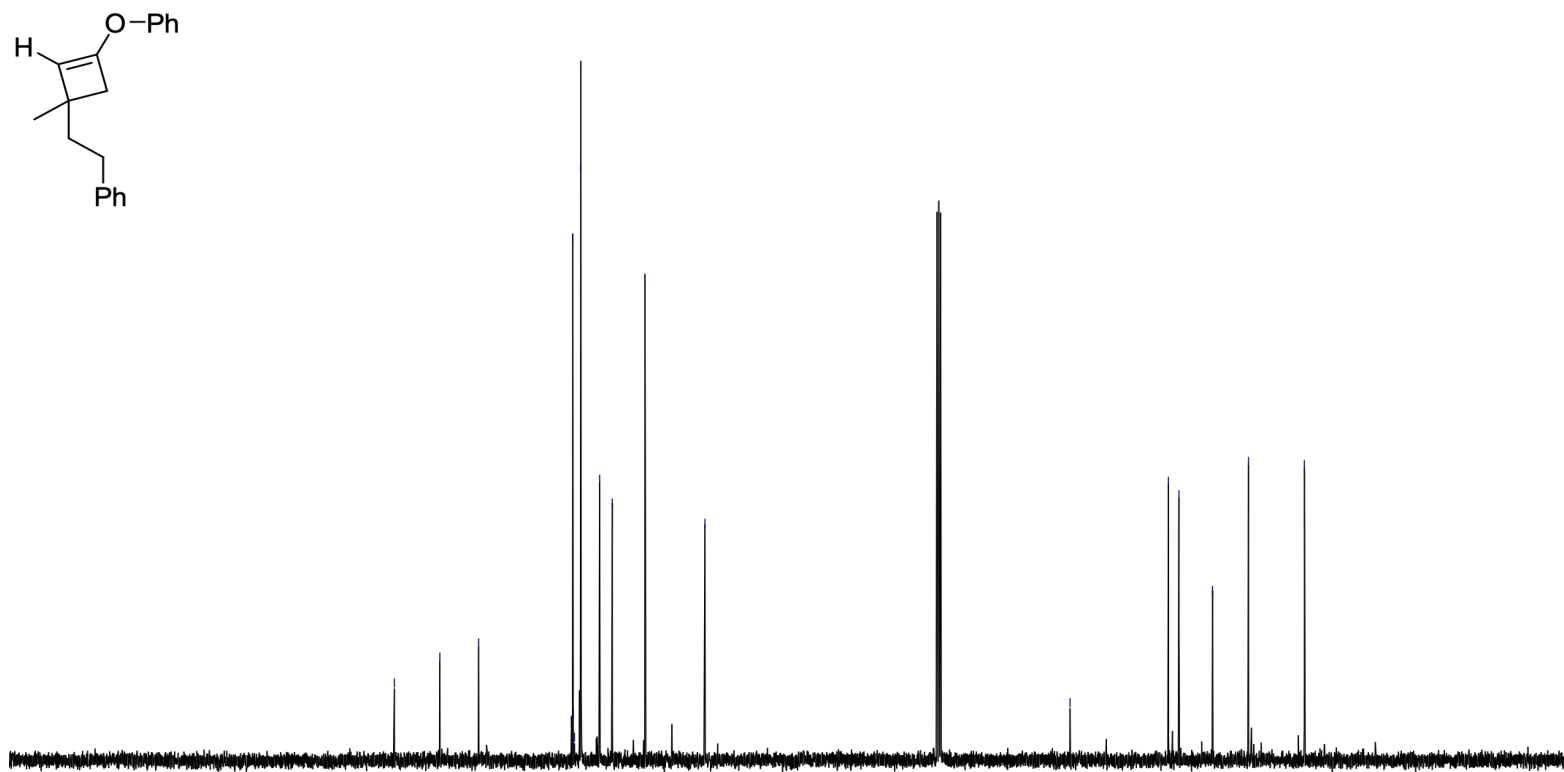

$10 \quad 200$ $\begin{array}{lllll}190 & 180 & 170 & 160 & 150\end{array}$ $140 \quad 130$ $\begin{array}{lll}120 & 110 & 100\end{array} 90$ $\begin{array}{lllllllll}70 & 60 & 50 & 40 & 30 & 20 & 10 & 0 & -10\end{array}$ 


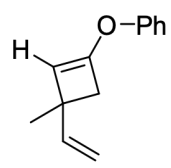

Cyclobutene 3cc was synthesized following general procedure B starting from (ethynyloxy)benzene (23.6 mg, $0.2 \mathrm{mmol})$ and isoprene $(60 \mathrm{ml}, 0.6 \mathrm{mmol})$. The crude product was purified by flash chromatography affording 3cc as a colorless oil (13 mg, 34\%).

${ }^{1} \mathbf{H}$ NMR $\left(500 \mathrm{MHz}, \mathrm{CDCl}_{3}\right) \delta 7.38-7.29(\mathrm{~m}, 2 \mathrm{H}), 7.17-7.14(\mathrm{~m}, 2 \mathrm{H}), 7.14-7.10(\mathrm{~m}, 1 \mathrm{H}), 6.06(\mathrm{dd}, J=17.2$, $10.4 \mathrm{~Hz}, 1 \mathrm{H}), 5.06$ (dd, $J=17.2,1.6 \mathrm{~Hz}, 1 \mathrm{H}), 4.94$ (dd, $J=10.4,1.6 \mathrm{~Hz}, 1 \mathrm{H}), 4.84(\mathrm{~s}, 1 \mathrm{H}), 2.61(\mathrm{~d}, J=12.7 \mathrm{~Hz}$, $1 \mathrm{H}), 2.55(\mathrm{~d}, J=12.7 \mathrm{~Hz}, 1 \mathrm{H}), 1.34(\mathrm{~s}, 3 \mathrm{H})$.

${ }^{13}$ C NMR $\left(126 \mathrm{MHz}, \mathrm{CDCl}_{3}\right) \delta 155.1,148.9,145.8,129.7,129.6,124.1,119.3,111.2,109.4,45.5,39.8,24.1$.

HRMS (ESI) $m / z$ calculated for $\mathrm{C}_{13} \mathrm{H}_{15} \mathrm{O}^{+}[\mathrm{M}+\mathrm{H}]^{+}: 187.1117$, found: 187.1122 .

${ }^{1} \mathrm{H}$ NMR $\left(500 \mathrm{MHz}, \mathrm{CDCl}_{3}\right)$ of $\mathbf{3 c c}$

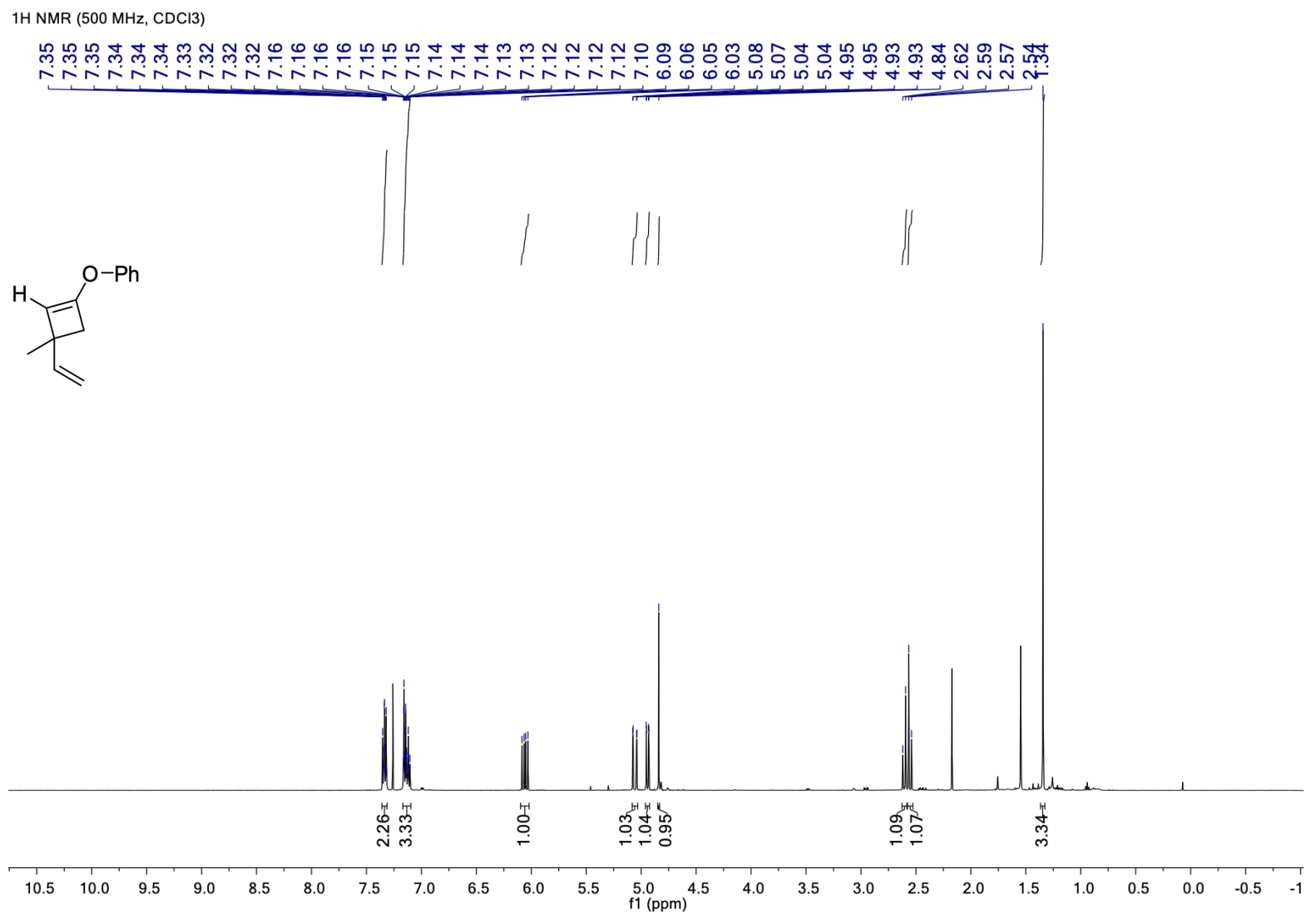


${ }^{13} \mathrm{C}$ NMR $\left(126 \mathrm{MHz}, \mathrm{CDCl}_{3}\right)$ of $\mathbf{3 c c}$

$13 \mathrm{C}\{1 \mathrm{H}\}$ NMR $(126 \mathrm{MHz}, \mathrm{CDCl} 3)$

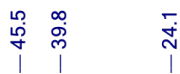
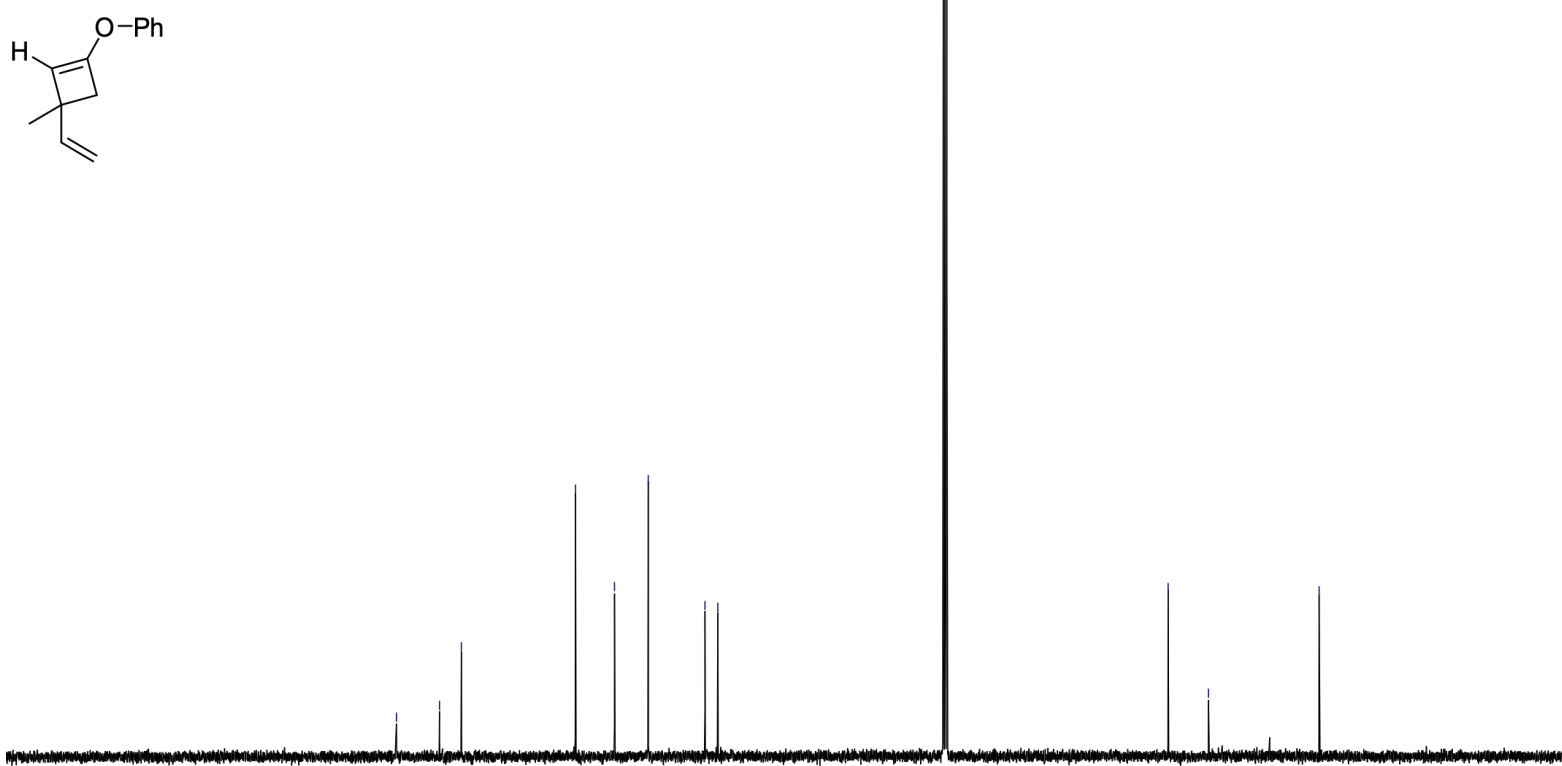

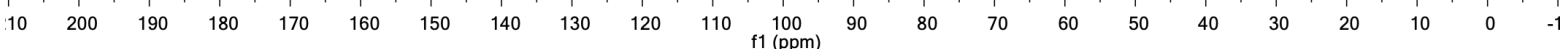




\section{((3-Methyl-3-vinylcyclobut-1-en-1-yl)oxy)-4-(trifluoromethyl)benzene (3dd)}

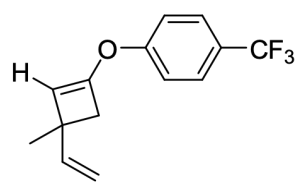

Cyclobutene 3dd was synthesized following general procedure B starting from 1-(ethynyloxy)-4(trifluoromethyl)benzene $(37.7 \mathrm{mg}, 0.2 \mathrm{mmol}$ ) and isoprene $(41 \mathrm{mg}, 0.6 \mathrm{mmol})$. The crude was purified by flash chromatography affording $3 \mathbf{d d}$ as a transparent oil (24 mg, 47\%).

${ }^{1} \mathbf{H}$ NMR $\left(500 \mathrm{MHz}, \mathrm{CDCl}_{3}\right) \delta 7.63-7.57(\mathrm{~m}, 2 \mathrm{H}), 7.27-7.21(\mathrm{~m}, 2 \mathrm{H}), 6.06(\mathrm{dd}, J=17.3,10.4 \mathrm{~Hz}, 1 \mathrm{H}), 5.08$ (dd, $J=17.2,1.5 \mathrm{~Hz}, 1 \mathrm{H}), 4.97(\mathrm{~s}, 1 \mathrm{H}), 4.97(\mathrm{dd}, J=10.4,1.5 \mathrm{~Hz}, 1 \mathrm{H}), 2.63(\mathrm{~d}, J=12.8 \mathrm{~Hz}, 1 \mathrm{H}), 2.58(\mathrm{~d}, J=$ $12.8 \mathrm{~Hz}, 1 \mathrm{H}), 1.36(\mathrm{~s}, 3 \mathrm{H})$.

${ }^{19}$ F NMR $\left(376 \mathrm{MHz}, \mathrm{CDCl}_{3}\right) \delta-61.64$.

${ }^{13}$ C NMR (126 MHz, $\left.\mathrm{CDCl}_{3}\right) \delta 157.7,147.8,145.3,127.1$ (q, $\left.J=3.7 \mathrm{~Hz}\right), 126.1(\mathrm{q}, J=32.7 \mathrm{~Hz}), 124.2(\mathrm{q}, J=$ $271.4 \mathrm{~Hz}), 119.0,111.6,111.5,45.6,40.1,24.0$.

HRMS (ESI) $\mathrm{m} / \mathrm{z}$ calculated for $\mathrm{C}_{14} \mathrm{H}_{14} \mathrm{~F}_{3} \mathrm{O}^{+}[\mathrm{M}+\mathrm{H}]^{+}:$255.0991, found: 255.0986 .

\section{${ }^{1} \mathrm{H}$ NMR $\left(500 \mathrm{MHz}, \mathrm{CDCl}_{3}\right)$ of $\mathbf{3 d d}$}

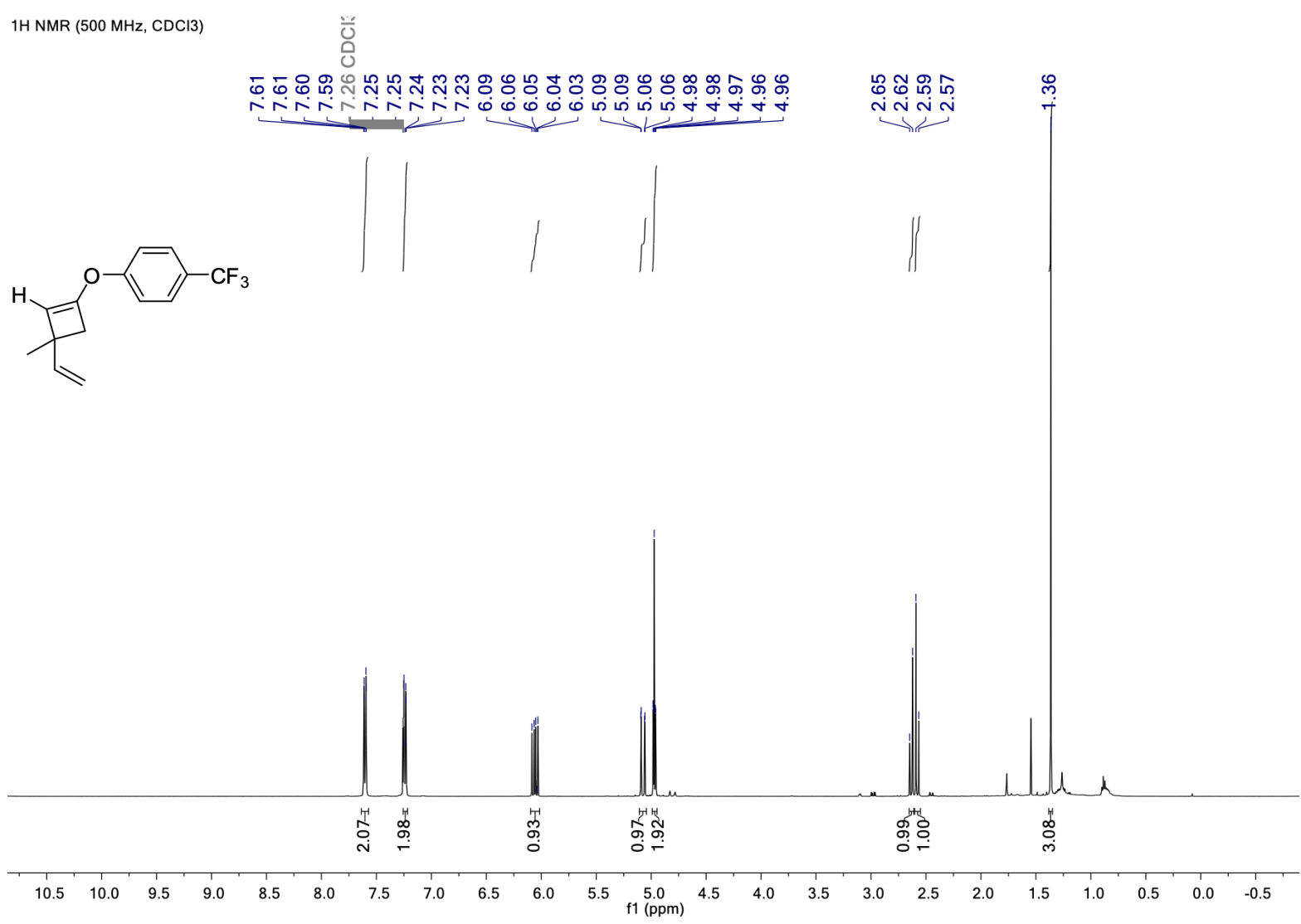


$\left.{ }^{19} \mathrm{~F} \mathrm{NMR} \mathrm{(376} \mathrm{MHz,} \mathrm{CDCl}_{3}\right)$ of $\mathbf{3 d d}$

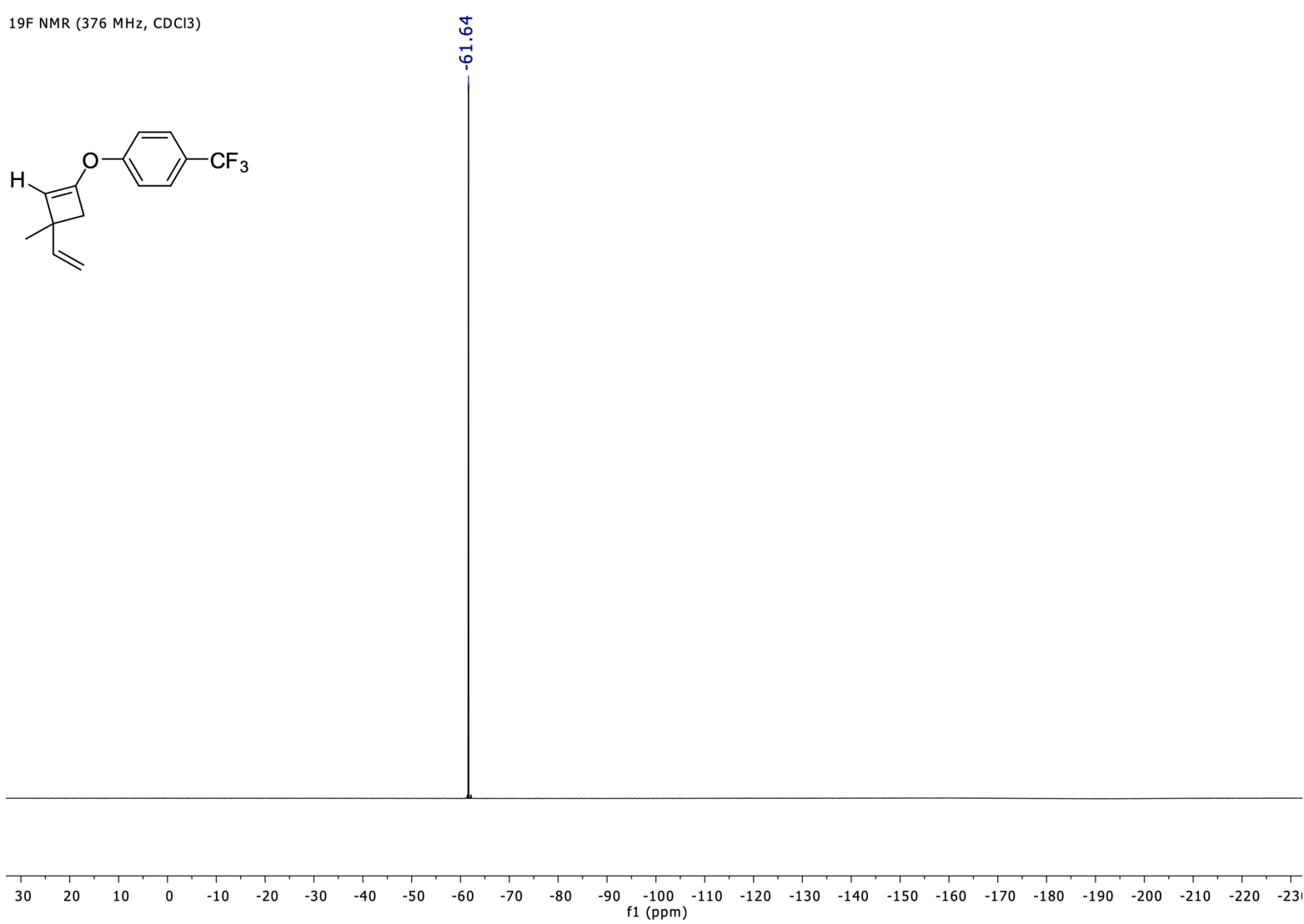

${ }^{13} \mathrm{C}$ NMR $\left(126 \mathrm{MHz}, \mathrm{CDCl}_{3}\right)$ of $\mathbf{3 d d}$

$13 \mathrm{C}\{1 \mathrm{H}\} \mathrm{NMR}(126 \mathrm{MHz}, \mathrm{CDCl} 3)$
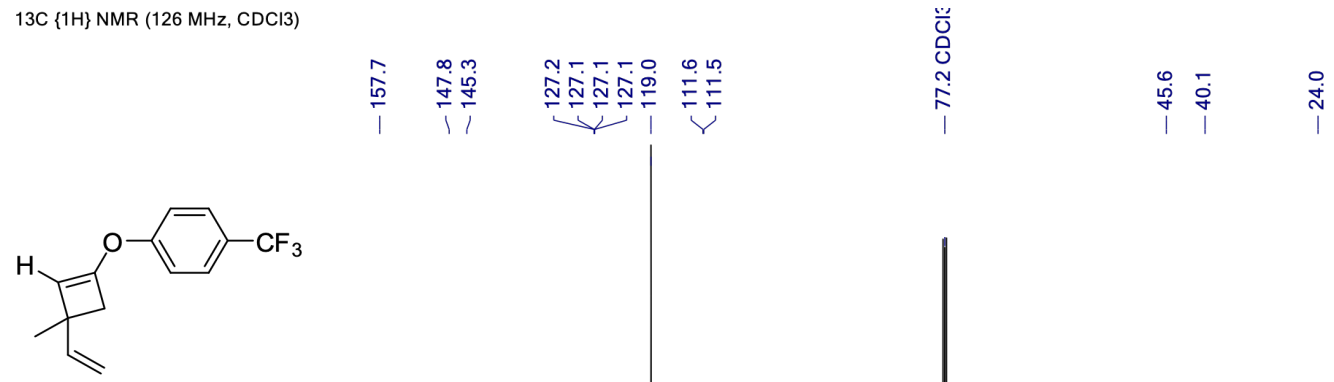

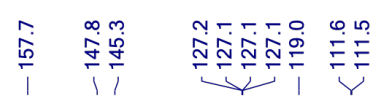

$\stackrel{1}{i} \underset{8}{1}$

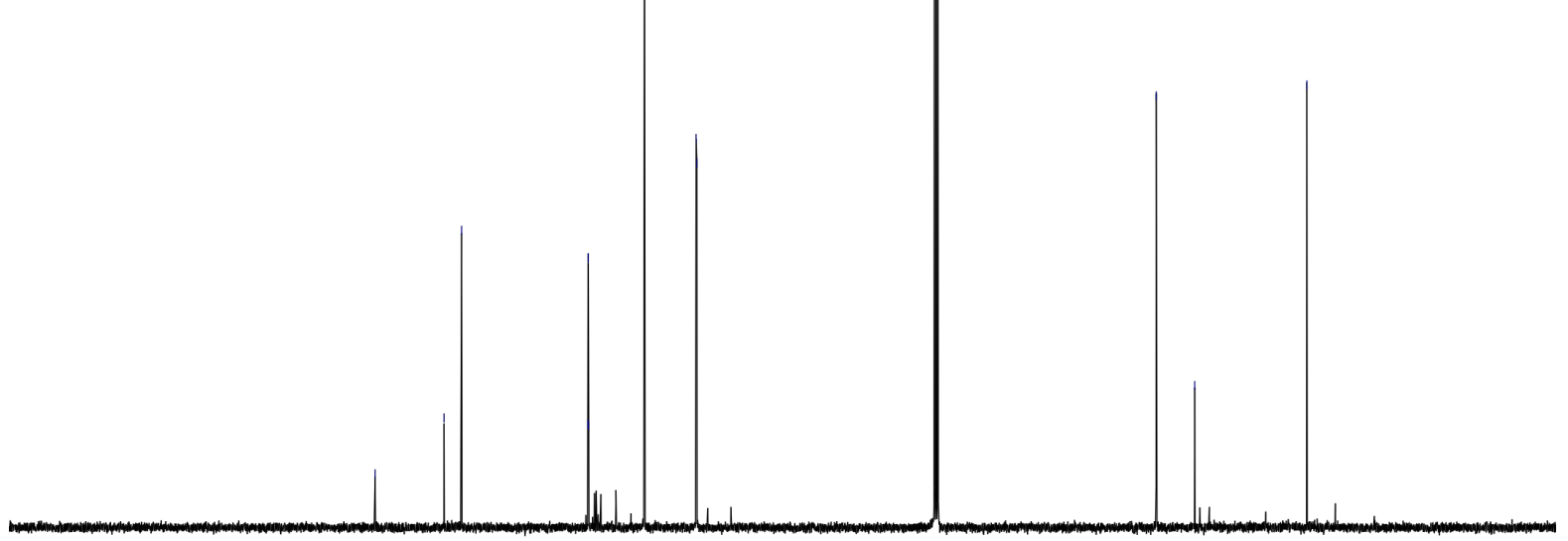

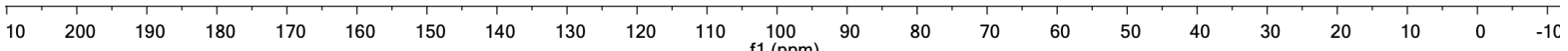




\section{((3,3,4-Trimethylcyclobut-1-en-1-yl)oxy)benzene (3ee)}<smiles>CC1C=C(Oc2ccccc2)C1C</smiles>

Cyclobutene 3ee was synthesized following general procedure B starting from (ethynyloxy)benzene (23.6 mg, $0.2 \mathrm{mmol}$ ) and 2-methylbut-2-ene $(64 \mathrm{~mL}, 0.6 \mathrm{mmol})$. The crude product was purified by flash chromatography affording 3ee as a yellow oil 7:1 mixture of regioisomers (29 mg, 77\%).

Major regiosomer (Mr), minor regioisomer (mr).

${ }^{1}$ H NMR (400 MHz, $\left.\mathrm{CDCl}_{3}\right) \delta 7.36-7.29$ (m, 3H, Mr + mr), $7.19-7.12(\mathrm{~m}, 2 \mathrm{H}, \mathrm{Mr}+\mathrm{mr}), 7.09$ (td, $J=7.3,1.2$ $\mathrm{Hz}, 1 \mathrm{H}, \mathrm{Mr}), 7.06-6.96$ (m, 0.15H, mr), 4.73 (s, 1H, Mr), 4.56 (d, J=1.0 Hz, 0.15H, mr), 2.69 (q, $J=7.1 \mathrm{~Hz}$, 1H, Mr), $2.21-2.14$ (m, 0.15H, mr), 1.19 (s, 3H, Mr), 1.18 (s, 0.7H, mr), 1.12 (s, 0.7H, mr), 1.09 (d, J=7.2 Hz, 3H, Mr), 1.07 (s, 3H, Mr), 1.03 (d, $J=6.8 \mathrm{~Hz}, 1 \mathrm{H}, \mathrm{mr})$.

${ }^{13}$ C NMR (101 MHz, CDCl $) \delta 155.4(\mathrm{mr}), 152.6(\mathrm{Mr}), 129.5$ (Mr), 129.5 (Mr), 123.8 (Mr), 122.7 (mr), 119.4 (mr), 119.2 (Mr), 117.9 (mr), 110.0 (Mr), 102.2 (mr), 48.8 (Mr), 45.1 (mr), 40.4 (mr), 37.3 (mr), 28.1 (Mr), 24.8 (Mr), $22.7(\mathrm{Mr}), 19.6(\mathrm{mr}), 15.6(\mathrm{mr}), 12.6(\mathrm{Mr})$.

HRMS (ESI) $m / z$ calculated for $\mathrm{C}_{13} \mathrm{H}_{17} \mathrm{O}^{+}[\mathrm{M}+\mathrm{H}]^{+}$: 189.1274 , found: 189.1273 .

\section{${ }^{1} \mathrm{H}$ NMR $\left(400 \mathrm{MHz}, \mathrm{CDCl}_{3}\right)$ of 3ee}

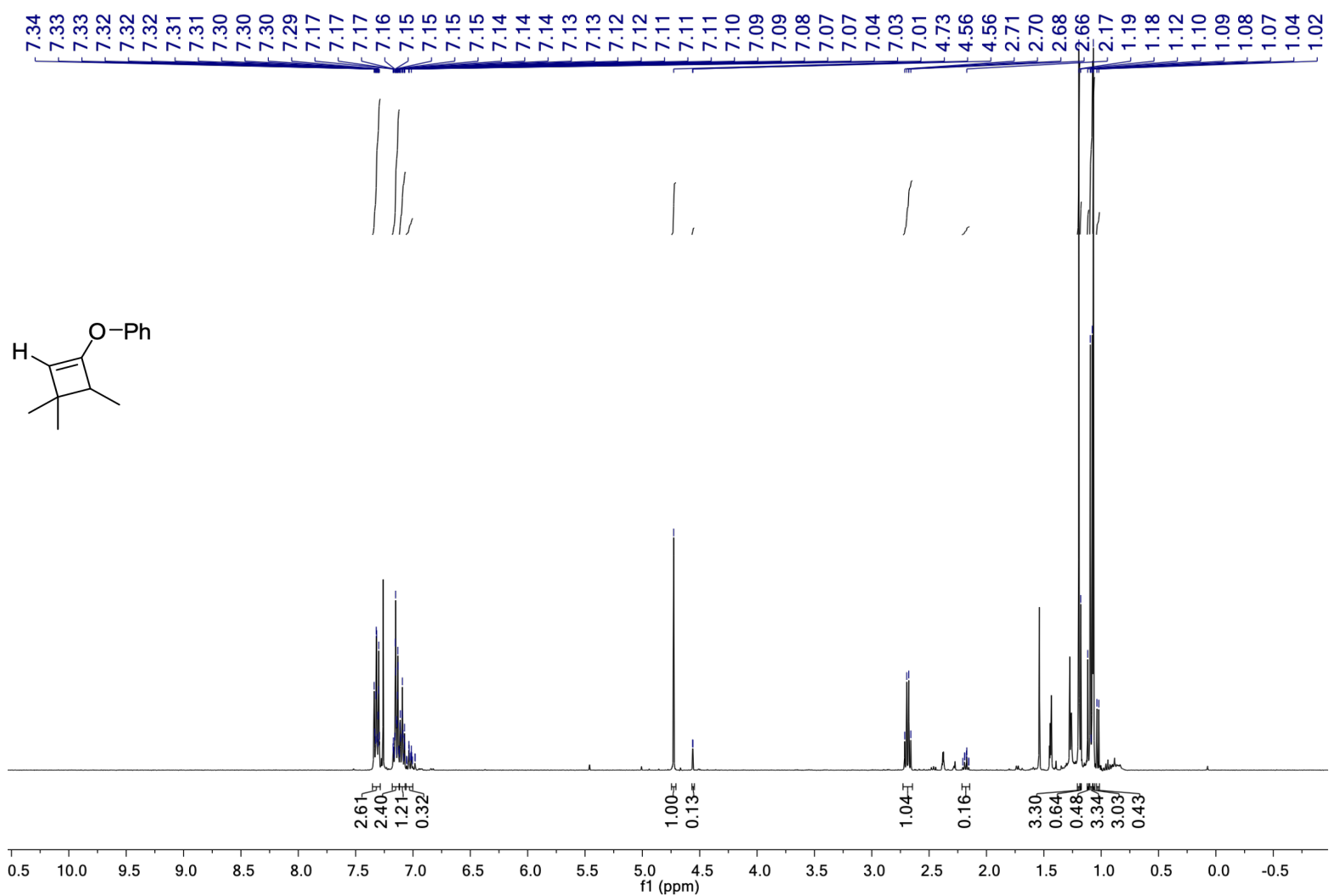


${ }^{13} \mathrm{C}$ NMR $\left(101 \mathrm{MHz}, \mathrm{CDCl}_{3}\right)$ of 3ee

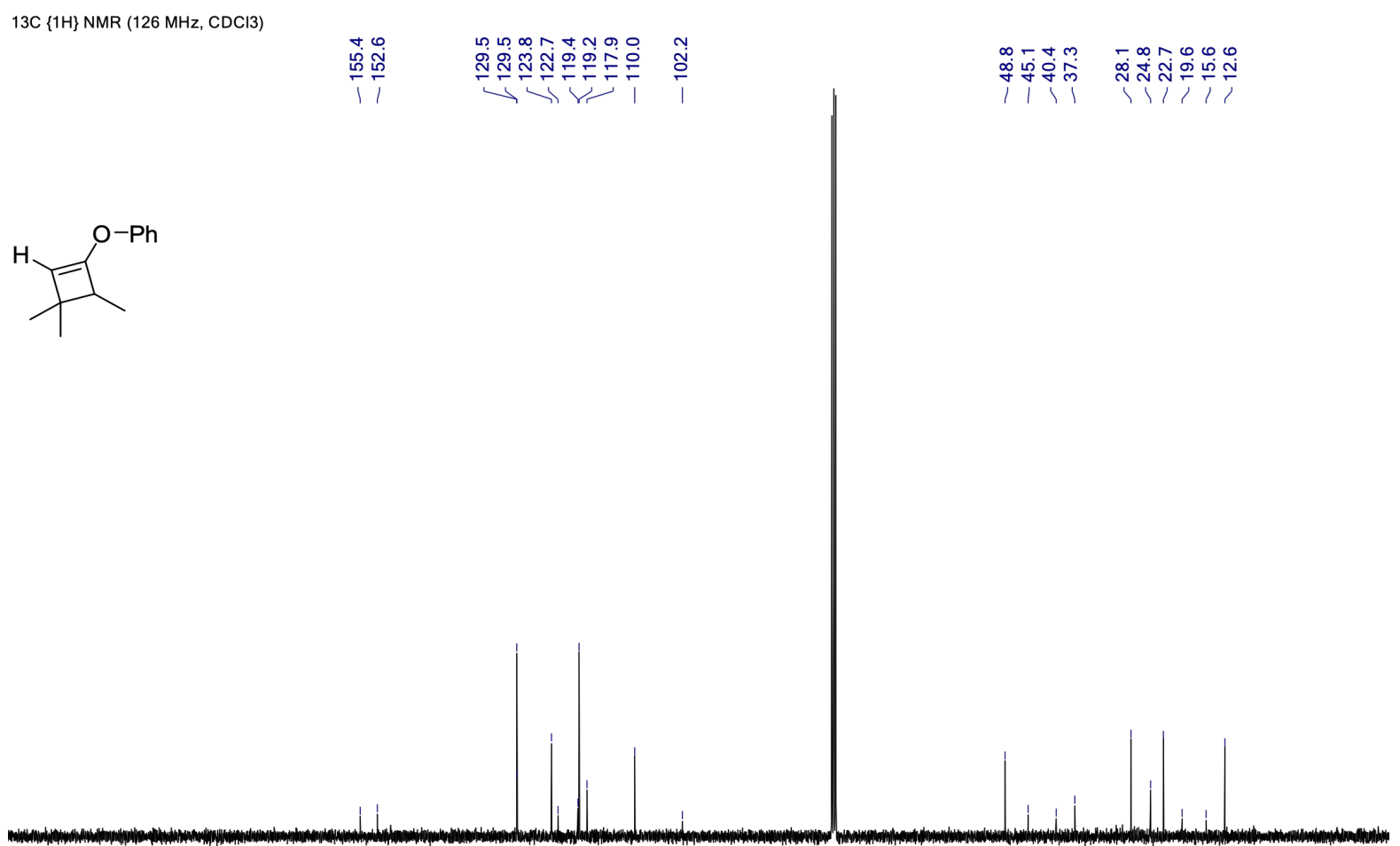

\begin{tabular}{llllllllllllllllllllllllll}
\hline 210 & 200 & 190 & 180 & 170 & 160 & 150 & 140 & 130 & 120 & 110 & 100 & 90 & 80 & 70 & 60 & 50 & 40 & 30 & 20 & 10 & 0 & -10
\end{tabular} 


\section{(3,3,4,4-Tetramethylcyclobut-1-en-1-yl)oxy)benzene (3ff)}

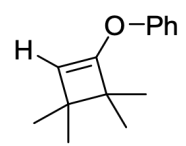

Cyclobutene 3ff was synthesized following general procedure B starting from (ethynyloxy)benzene (23.6 mg, 0.2 $\mathrm{mmol})$ and 2,3-dimethylbut-2-ene $(72 \mathrm{~mL}, 0.6 \mathrm{mmol})$. The crude product was purified by flash chromatography affording $\mathbf{3 f f}$ as a colorless oil (24 $\mathrm{mg}, 59 \%$ ).

${ }^{1} \mathbf{H}$ NMR $\left(400 \mathrm{MHz}, \mathrm{CDCl}_{3}\right) \delta 7.36-7.28(\mathrm{~m}, 2 \mathrm{H}), 7.19-7.14(\mathrm{~m}, 2 \mathrm{H}), 7.09(\mathrm{ddt}, J=7.7,7.0,1.2 \mathrm{~Hz}, 1 \mathrm{H}), 4.60$ (s, 1H), 1.19 (s, 6H), $1.10(\mathrm{~s}, 6 \mathrm{H})$.

${ }^{13}$ C NMR $\left(101 \mathrm{MHz}, \mathrm{CDCl}_{3}\right) \delta 156.5,155.5,129.5,123.8,119.4,107.4,48.8,40.5,24.3,21.5$.

HRMS (ESI) $m / z$ calculated for $\mathrm{C}_{14} \mathrm{H}_{19} \mathrm{O}^{+}[\mathrm{M}+\mathrm{H}]^{+}: 203.1430$, found: 203.1432 .

${ }^{1} \mathrm{H}$ NMR $\left(400 \mathrm{MHz}, \mathrm{CDCl}_{3}\right)$ of $\mathbf{3 f f}$

1H NMR (500 MHz, CDCl3)

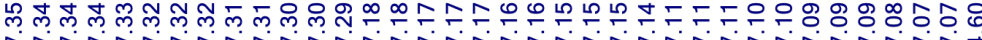

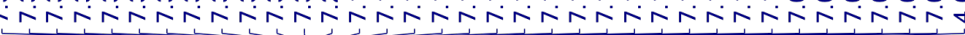

in
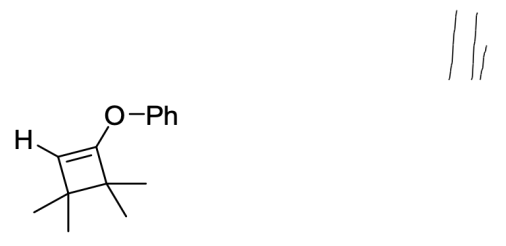

11

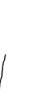

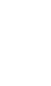

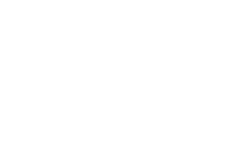


${ }^{13} \mathrm{C}$ NMR $\left(101 \mathrm{MHz}, \mathrm{CDCl}_{3}\right)$ of $\mathbf{3 f f}$

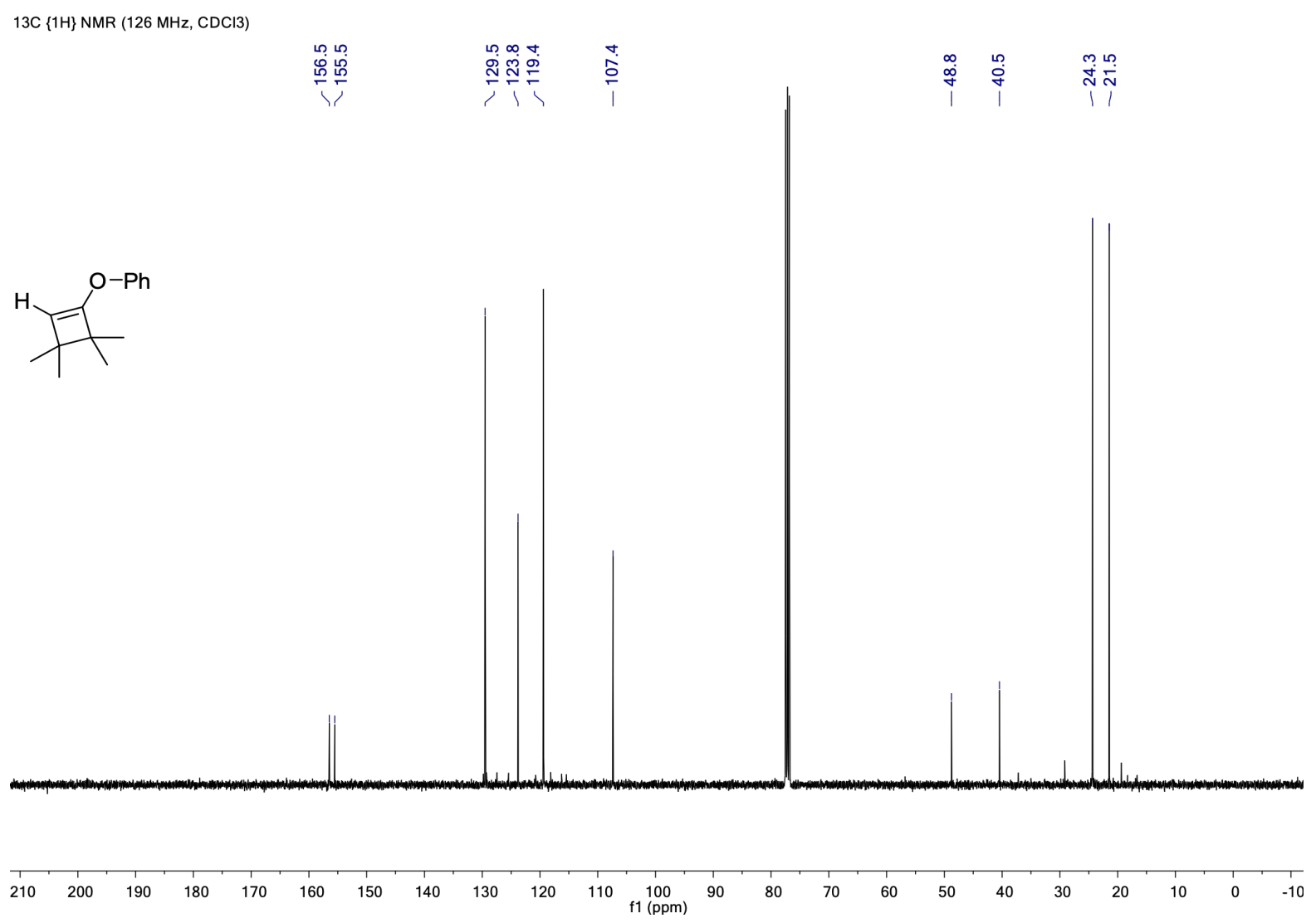




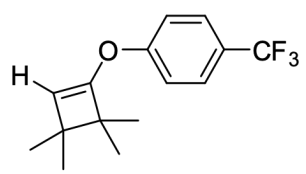

Cyclobutene 3gg was synthesized following general procedure B starting from 1-(ethynyloxy)-4(trifluoromethyl)benzene $(55.8 \mathrm{mg}, 0.3 \mathrm{mmol}$ ) and 2,3-dimethylbut-2-ene (76 $\mathrm{mg}, 0.9 \mathrm{mmol})$. The crude was purified by flash chromatography affording $\mathbf{3 g g}$ as a transparent oil (50 $\mathrm{mg}, 62 \%)$.

${ }^{1}$ H NMR $\left(500 \mathrm{MHz}, \mathrm{CDCl}_{3}\right) \delta 7.58(\mathrm{dt}, J=8.4,0.7 \mathrm{~Hz}, 2 \mathrm{H}), 7.28-7.23(\mathrm{~m}, 2 \mathrm{H}), 4.73(\mathrm{~s}, 1 \mathrm{H}), 1.19(\mathrm{~s}, 6 \mathrm{H}), 1.12$ $(\mathrm{s}, 6 \mathrm{H})$.

${ }^{19}$ F NMR $\left(376 \mathrm{MHz}, \mathrm{CDCl}_{3}\right) \delta-61.98$.

${ }^{13} \mathbf{C ~ N M R}\left(126 \mathrm{MHz}, \mathrm{CDCl}_{3}\right) \delta 158.3,155.2,127.0(\mathrm{q}, J=3.8 \mathrm{~Hz}), 125.8(\mathrm{q}, J=33.5 \mathrm{~Hz}), 124.3(\mathrm{q}, J=271.6$ Hz), 119.1, 109.3, 49.1, 40.8, 24.2, 21.4.

HRMS (ESI) $\mathrm{m} / \mathrm{z}$ calculated for $\mathrm{C}_{15} \mathrm{H}_{18} \mathrm{~F}_{3} \mathrm{O}^{+}[\mathrm{M}+\mathrm{H}]^{+}: 271.1304$, found: 271.1307 .

${ }^{1} \mathrm{H}$ NMR $\left(500 \mathrm{MHz}, \mathrm{CDCl}_{3}\right)$ of $\mathbf{3 g g}$

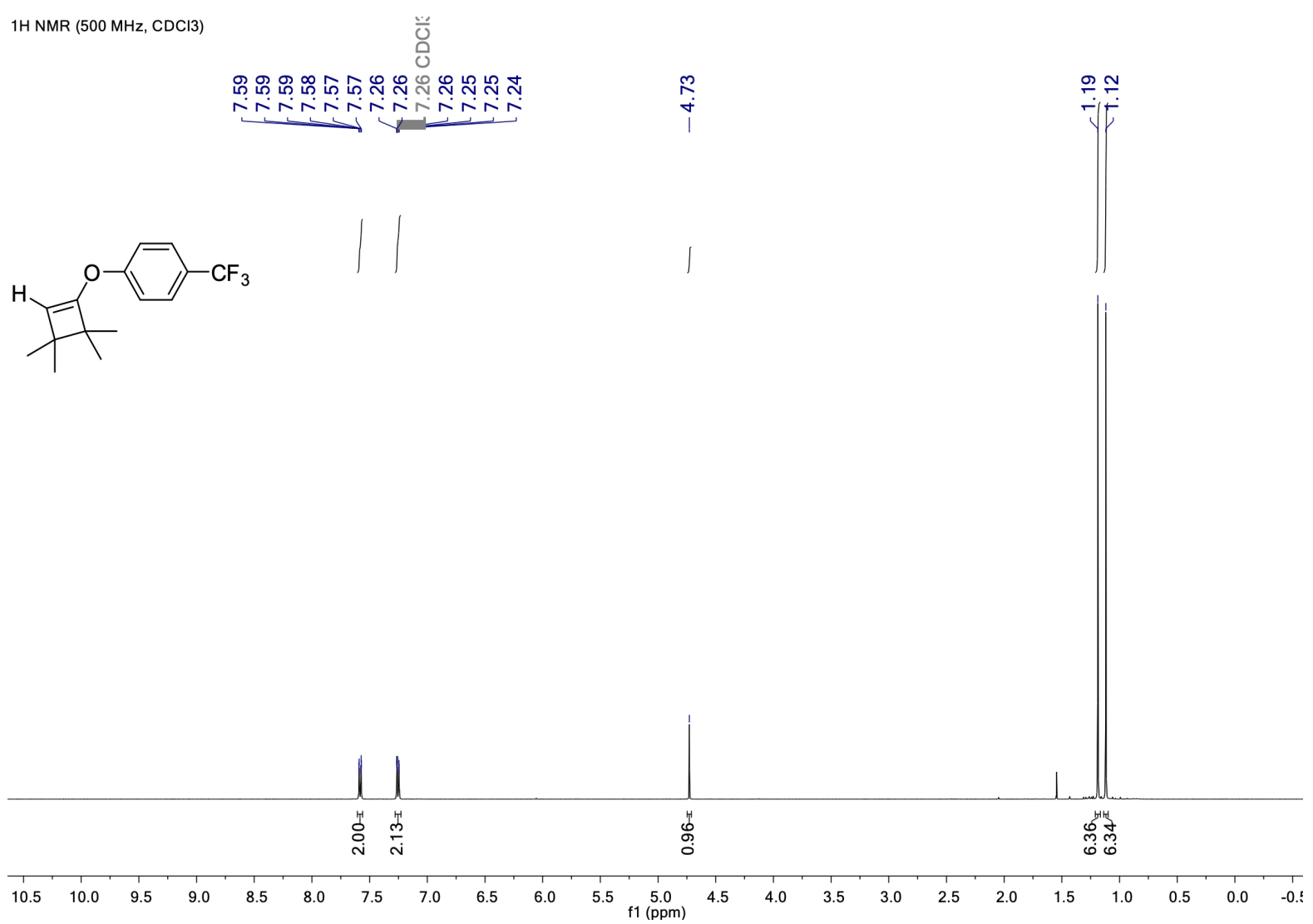


${ }^{19} \mathrm{~F}$ NMR (376 MHz, $\left.\mathrm{CDCl}_{3}\right)$ of $\mathbf{3 g g}$
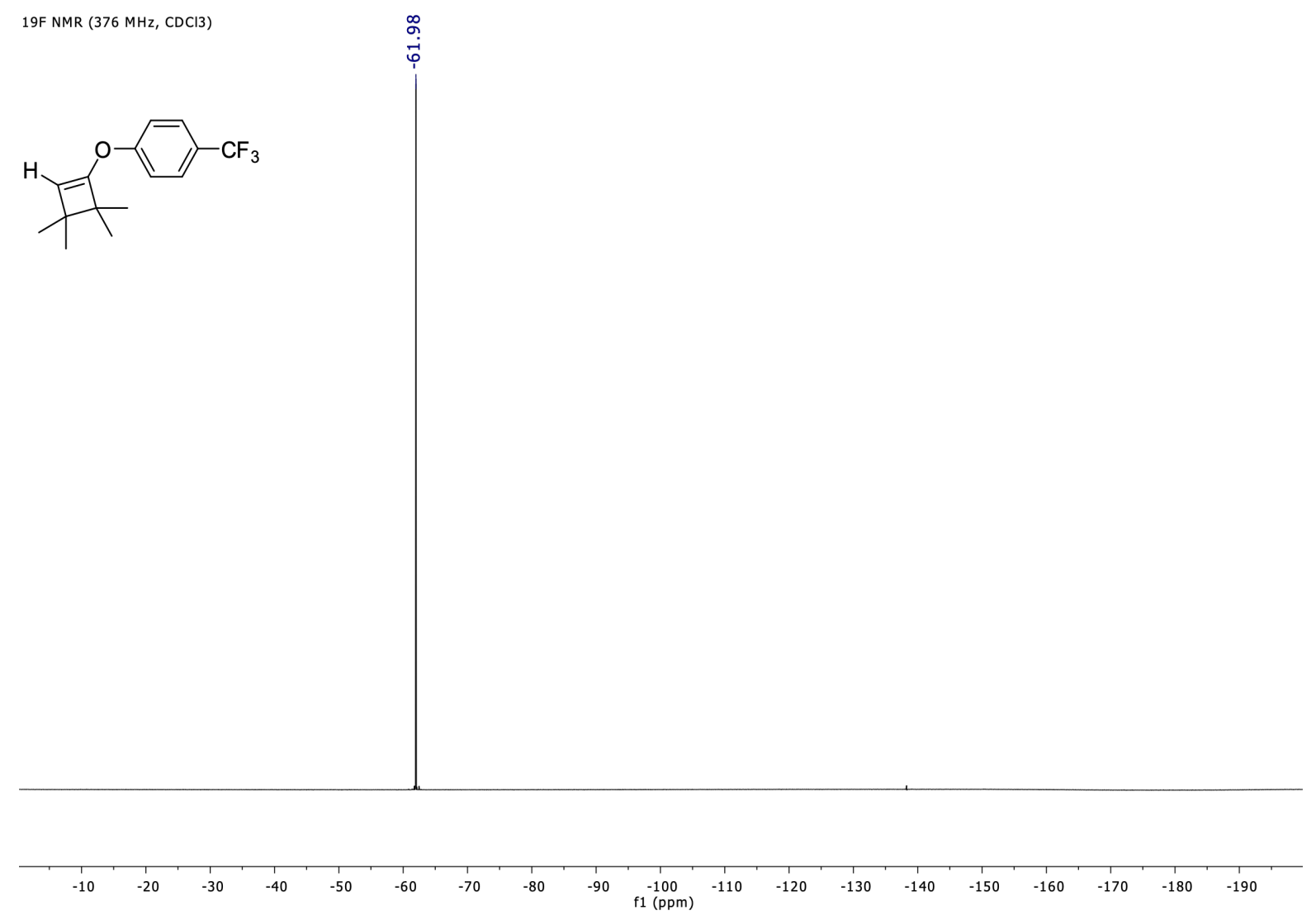

${ }^{13} \mathrm{C} \mathrm{NMR}\left(126 \mathrm{MHz}, \mathrm{CDCl}_{3}\right)$ of $\mathbf{3 g g}$

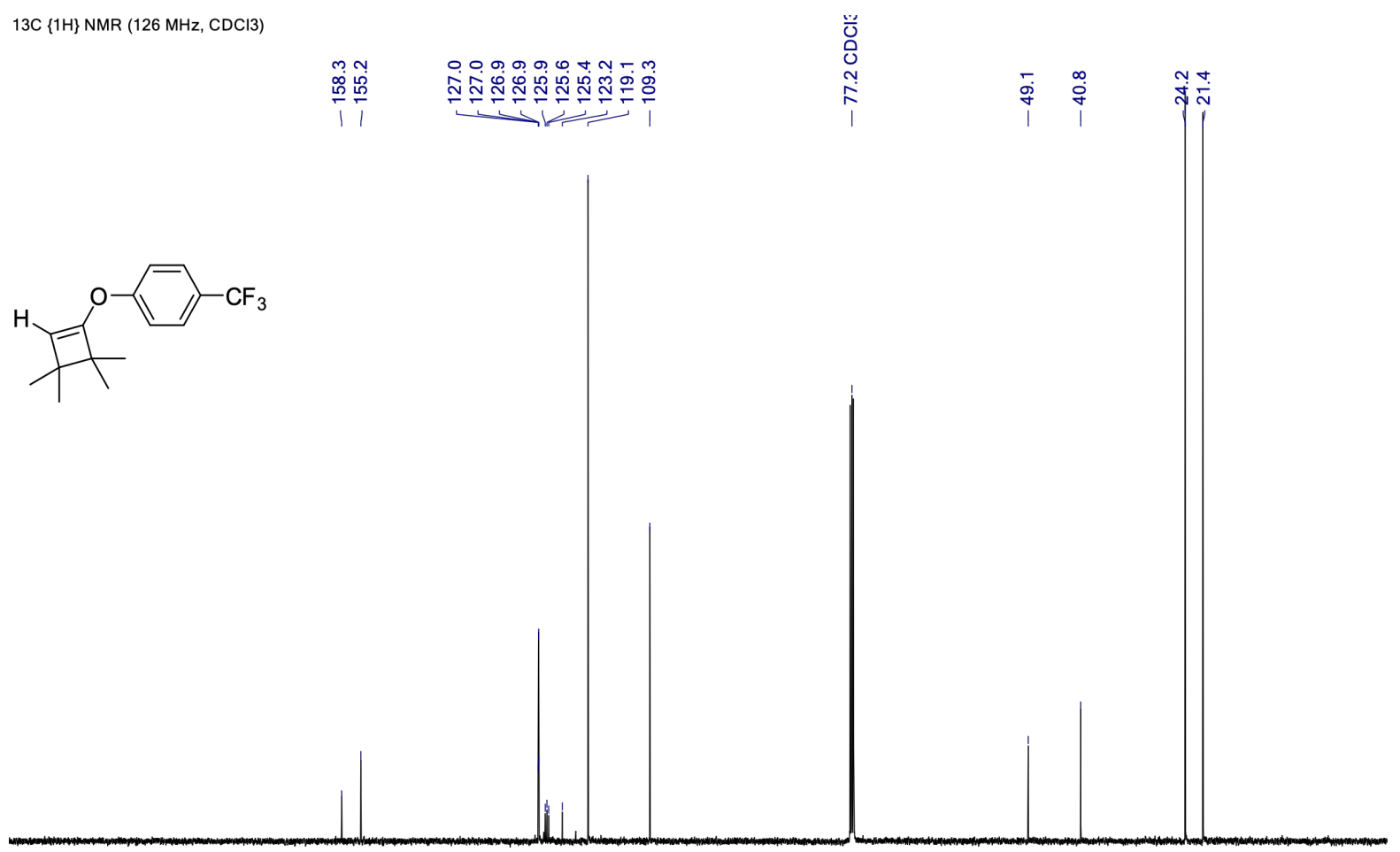

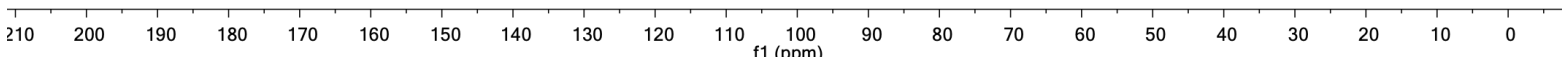


$(1 R, 11 S)-4,13,13-T r i m e t h y l-10-m e t h y l e n e-6-p h e n o x y t r i c y c l o[9.2 .0 .04,7]$ tridec-5-ene (3hh) and $(1 R, 11 S)-$ 4,13,13-trimethyl-10-methylenetricyclo[9.2.0.04,7]tridecan-6-one (5c)
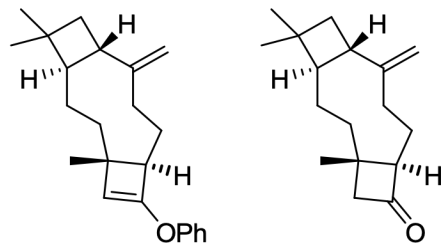

The cyclobutene $\mathbf{3 h h}$ and the cyclobutanone 5c were synthesized following general produced $\mathbf{B}$ starting from (ethynyloxy)benzene (23.6 mg, $0.2 \mathrm{mmol})$ and $\beta$-Caryophyllene $(170 \mathrm{ml}, 0.6 \mathrm{mmol})$. The yield (57\%, $3: 1 \mathrm{r} . r$.$) of$ 3hh was determined by ${ }^{1} \mathrm{H}$ NMR analysis of the crude reaction mixture using trichloroethylene as the internal standard. The crude product was purified by flash chromatography affording a mixture of $\mathbf{3 h h}$ and $\mathbf{5 c}$ in a ratio changing during time. Few fractions of pure $3 \mathbf{h h}$ as 1:1 mixture of regioisomers were separated (8 $\mathrm{mg}, 10 \%)$ and used to assign the structure by ${ }^{1} \mathrm{H}$ NMR and ${ }^{13} \mathrm{C}$ NMR, however $3 \mathrm{hh}$ was fully converted into $\mathbf{5 c}$ overnight, and no HRMS was measured. A second purification by flash chromatography afforded $\mathbf{5 c}$ as a yellow oil, 3:1 mixture of regioisomers $(21 \mathrm{mg}, 43 \%)$.

$(1 R, 11 S)-4,13,13-T r i m e t h y l-10-m e t h y l e n e-6-p h e n o x y t r i c y c l o[9.2 .0 .04,7]$ tridec-5-ene $(3 \mathrm{hh})(\mathrm{Mr}=$ major regioisomer, $\mathrm{mr}=$ minor regioisomer)

${ }^{1} \mathbf{H}$ NMR $\left(400 \mathrm{MHz}, \mathrm{CDCl}_{3}\right) \delta 7.36-7.30(\mathrm{~m}, 4 \mathrm{H}, \mathrm{Mr}+\mathrm{mr}), 7.16(\mathrm{dq}, J=7.9,1.3 \mathrm{~Hz}, 4 \mathrm{H}, \mathrm{Mr}+\mathrm{mr}), 7.13-$ $7.08(\mathrm{~m}, 2 \mathrm{H}, \mathrm{Mr}+\mathrm{mr}), 4.95(\mathrm{t}, J=1.3 \mathrm{~Hz}, 1 \mathrm{H}, \mathrm{mr}), 4.93(\mathrm{~s}, 1 \mathrm{H}, \mathrm{Mr}), 4.86(\mathrm{~d}, J=1.5 \mathrm{~Hz}, 1 \mathrm{H}, \mathrm{mr}), 4.85(\mathrm{~s}, 1 \mathrm{H}$, Mr), $4.66(\mathrm{~s}, 1 \mathrm{H}, \mathrm{Mr}), 4.48(\mathrm{~d}, J=1.1 \mathrm{~Hz}, 1 \mathrm{H}, \mathrm{mr}), 2.86(\mathrm{dd}, J=12.9,2.7 \mathrm{~Hz}, 1 \mathrm{H}, \mathrm{Mr}), 2.54-2.38(\mathrm{~m}, 6 \mathrm{H}, \mathrm{Mr}$ $+\mathrm{mr}), 2.09-2.00(\mathrm{~m}, 1 \mathrm{H}, \mathrm{mr}), 1.95-1.86(\mathrm{~m}, 3 \mathrm{H}, \mathrm{Mr}+\mathrm{mr}), 1.86-1.69(\mathrm{~m}, 5 \mathrm{H}, \mathrm{Mr}+\mathrm{mr}), 1.63(\mathrm{~m}, 6 \mathrm{H}, \mathrm{Mr}+$ $\mathrm{mr}), 1.56-1.40(\mathrm{~m}, 3 \mathrm{H}, \mathrm{Mr}+\mathrm{mr}), 1.41-1.25(\mathrm{~m}, 2 \mathrm{H}, \mathrm{Mr}+\mathrm{mr}), 1.23(\mathrm{~s}, 3 \mathrm{H}, \mathrm{mr}), 1.14(\mathrm{~s}, 3 \mathrm{H}, \mathrm{Mr}), 1.04(\mathrm{~s}, 3 \mathrm{H}$, $\mathrm{mr}), 1.02(\mathrm{~s}, 3 \mathrm{H}, \mathrm{Mr}), 1.00(\mathrm{~s}, 6 \mathrm{H}, \mathrm{Mr}+\mathrm{mr})$.

${ }^{13}$ C NMR $\left(101 \mathrm{MHz}, \mathrm{CDCl}_{3}\right) \delta 155.3,153.2,152.6,151.7,129.5,123.8,119.3,119.2,110.4,109.5,101.0,54.8$, 50.2, 49.3, 49.2, 43.1, 41.9, 40.6, 39.7, 36.9, 34.4, 34.0, 34.0, 33.9, 32.9, 30.1, 30.1, 29.2, 26.9, 26.3, 25.7, 24.8, $24.5,21.8,19.3$. 


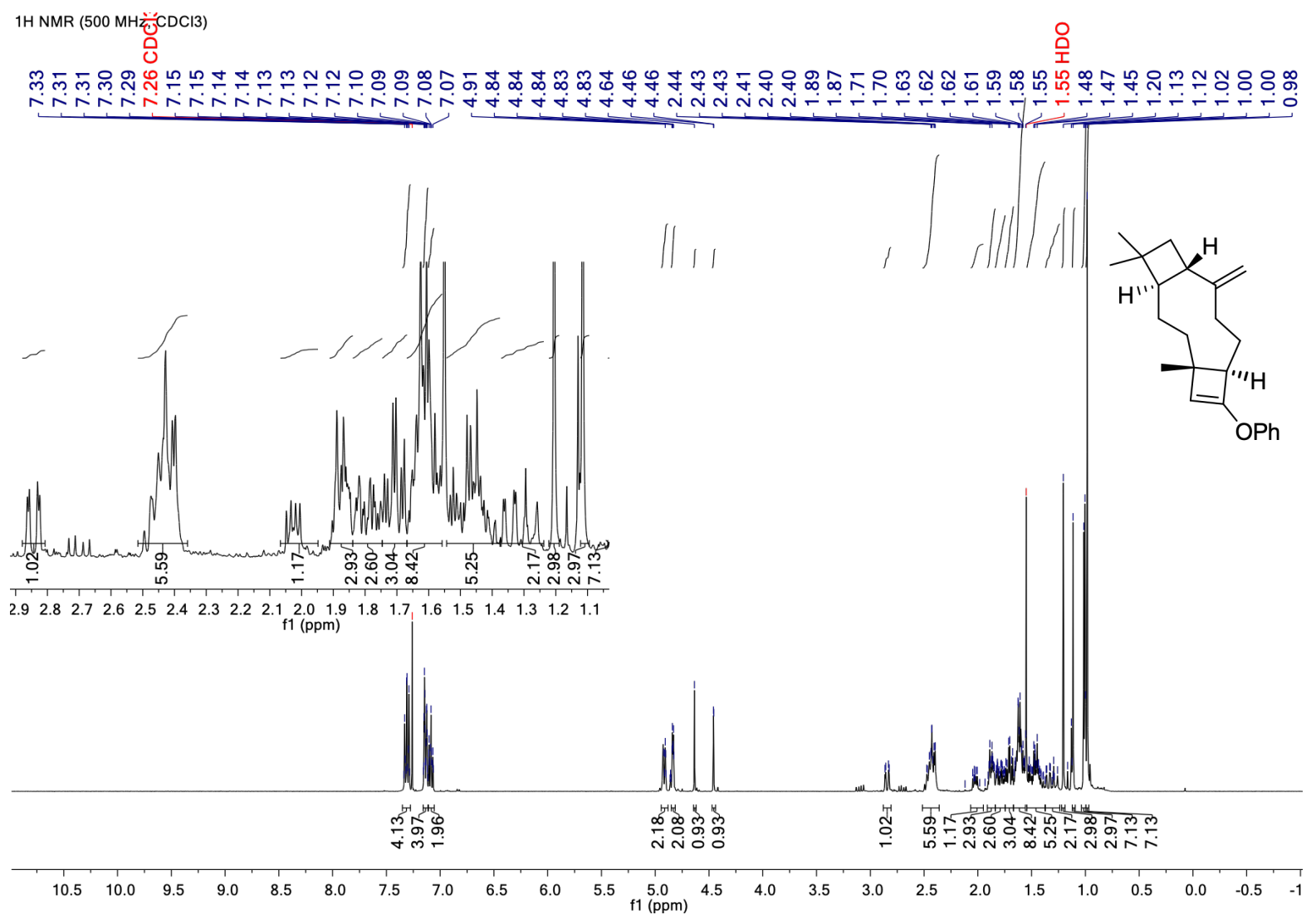

${ }^{13} \mathrm{C}$ NMR $\left(101 \mathrm{MHz}, \mathrm{CDCl}_{3}\right)$ of $\mathbf{3 h h}$

$13 \mathrm{C}\{1 \mathrm{H}\} \mathrm{NMR}(126 \mathrm{MHz}, \mathrm{CDCl} 3)$

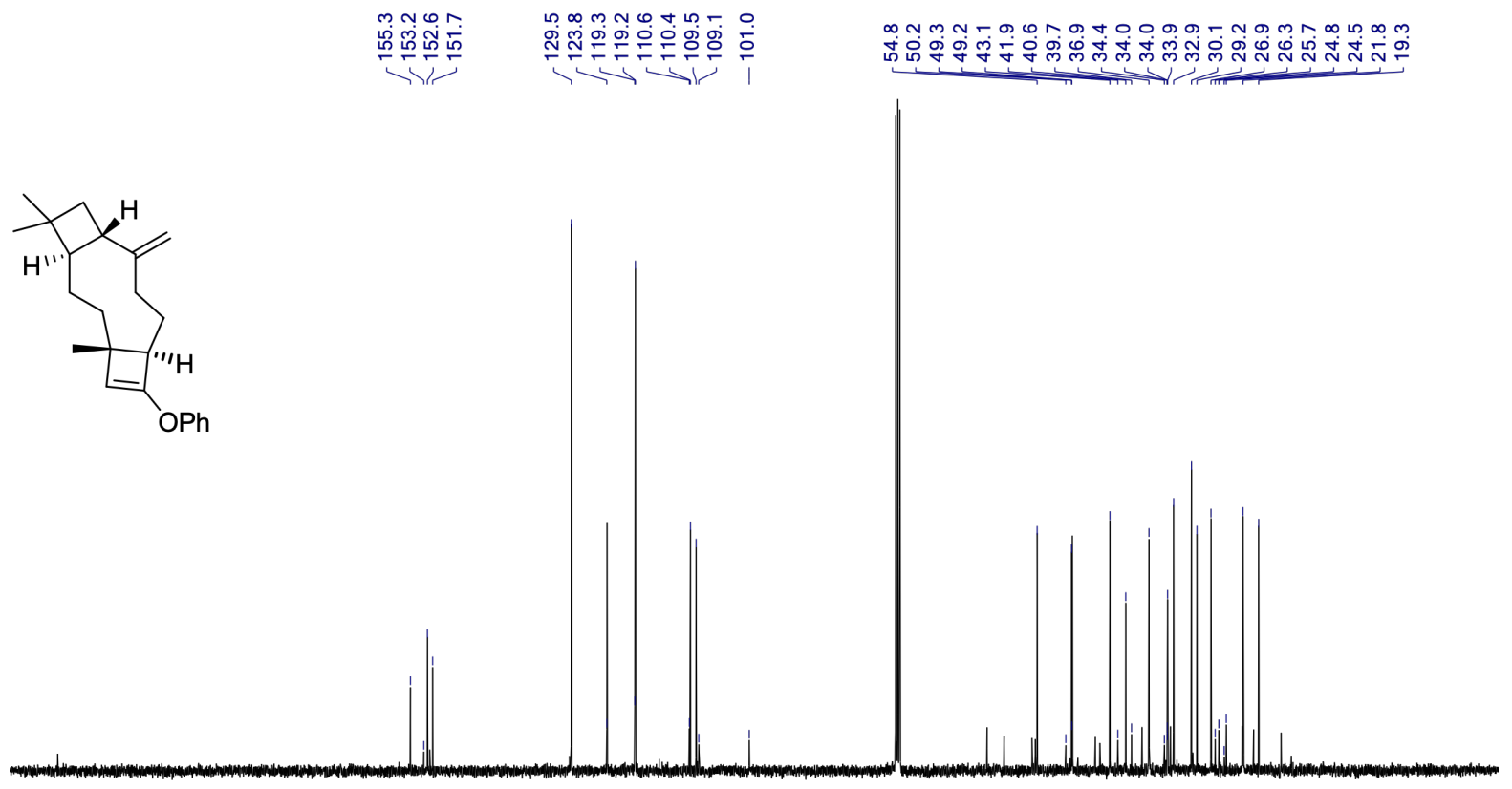

$\begin{array}{llllllllllllllllllllllll}210 & 200 & 190 & 180 & 170 & 160 & 150 & 140 & 130 & 120 & 110 & 100 & 90 & 80 & 70 & 60 & 50 & 40 & 30 & 20 & 10 & 0 & -10\end{array}$ 
$(1 \mathrm{R}, 11 \mathrm{~S})-4,13,13-$ Trimethyl-10-methylenetricyclo[9.2.0.04,7]tridecan-6-one $(5 \mathrm{c})(\mathrm{Mr}=$ major regioisomer, $\mathrm{mr}=$ minor regioisomer)

${ }^{1} \mathbf{H}$ NMR $\left(400 \mathrm{MHz}, \mathrm{CDCl}_{3}\right) \delta 4.95(\mathrm{~d}, J=1.2 \mathrm{~Hz}, 1 \mathrm{H}, \mathrm{Mr}), 4.92(\mathrm{t}, J=1.3 \mathrm{~Hz}, 0.3 \mathrm{H}, \mathrm{mr}), 4.85(\mathrm{~d}, J=1.6 \mathrm{~Hz}$, $0.3 \mathrm{H}, \mathrm{mr}), 4.84$ (q, $J=1.1 \mathrm{~Hz}, 1 \mathrm{H}, \mathrm{Mr}), 3.15-3.07$ (m, 0.3H, mr), $3.06-2.99$ (m, 1H, Mr ), 2.78 (dd, $J=16.8$, $2.9 \mathrm{~Hz}, 1 \mathrm{H}, \mathrm{Mr}), 2.69$ (dd, $J=18.0,8.1 \mathrm{~Hz}, 0.3 \mathrm{H}, \mathrm{mr}), 2.55$ (dd, $J=16.8,2.1 \mathrm{~Hz}, 1 \mathrm{H}, \mathrm{Mr}), 2.49-2.33$ (m, $2 \mathrm{H}$, $\mathrm{Mr}+\mathrm{mr}), 2.33-2.22(\mathrm{~m}, 1 \mathrm{H}, \mathrm{Mr}), 2.05-1.97(\mathrm{~m}, 1 \mathrm{H}, \mathrm{Mr}), 1.96-1.80(\mathrm{~m}, 3 \mathrm{H}, \mathrm{Mr}+\mathrm{mr}), 1.69-1.51(\mathrm{~m}, 8 \mathrm{H}$, $\mathrm{Mr}+\mathrm{mr}), 1.51-1.33(\mathrm{~m}, 2 \mathrm{H}, \mathrm{Mr}+\mathrm{mr}), 1.12(\mathrm{~s}, 1 \mathrm{H}, \mathrm{mr}), 1.12(\mathrm{~s}, 3 \mathrm{H}, \mathrm{Mr}), 1.00$ (s, 6H, Mr), 0.99 (s, 1H, mr), $0.98(\mathrm{~s}, 1 \mathrm{H}, \mathrm{mr})$.

${ }^{13}$ C NMR (126 MHz, $\left.\mathrm{CDCl}_{3}\right) \delta$ 216.3(mr), 211.6 (Mr), $152.6(\mathrm{mr}), 152.2(\mathrm{Mr}), 110.6(\mathrm{Mr}), 109.3(\mathrm{mr}), 63.9(\mathrm{mr})$, $62.9(\mathrm{Mr}), 60.1$ (Mr), $55.6(\mathrm{Mr}), 54.9$ (mr), 49.6, 48.2, 45.5, 44.8, 38.1, 37.0, 36.9, 34.4, 34.3 (mr), 34.2, 33.9, 33.5, 32.9, 32.4, 30.1 (mr), 30.1 (Mr), 25.7, 24.8 (mr), 24.5 (Mr), 21.9 (Mr), 21.7 (mr), 20.1 (Mr), 14.1 (mr).

HRMS (ESI) $m / z$ calculated for $\mathrm{C}_{17} \mathrm{H}_{26} \mathrm{NaO}^{+}[\mathrm{M}+\mathrm{Na}]^{+}: 269.1876$, found: 269.1867 .

${ }^{1} \mathrm{H}$ NMR $\left(400 \mathrm{MHz}, \mathrm{CDCl}_{3}\right)$ of $\mathbf{5 c}$

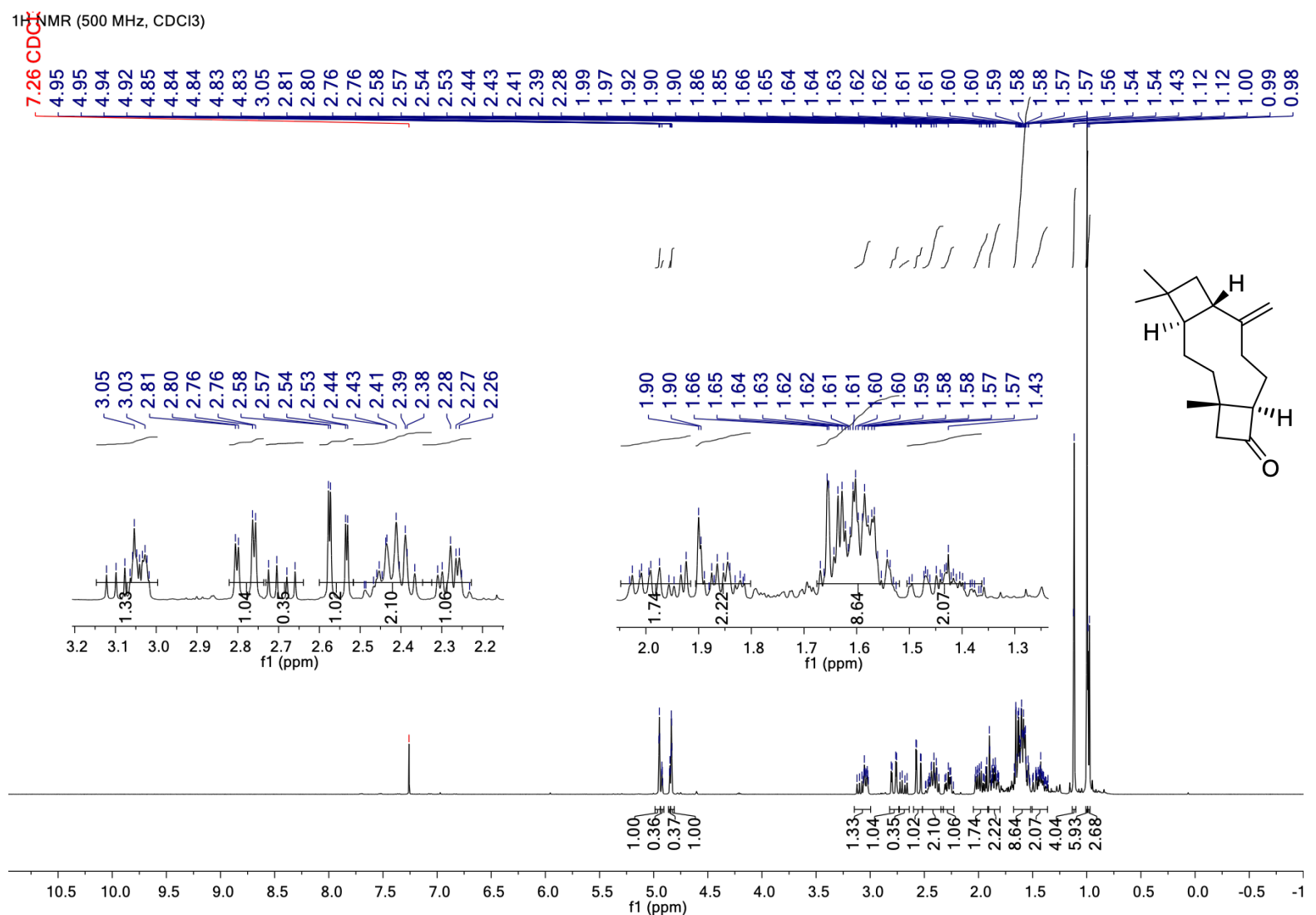

${ }^{13} \mathrm{C}$ NMR $\left(126 \mathrm{MHz}, \mathrm{CDCl}_{3}\right)$ of $\mathbf{5 c}$ 
$13 \mathrm{C}\{1 \mathrm{H}\}$ NMR $(126 \mathrm{MHz}, \mathrm{CDCl} 3)$

\begin{tabular}{|c|c|c|c|}
\hline 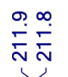 & 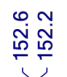 & $\begin{array}{l}00 \% \\
\stackrel{0}{0} \stackrel{\circ}{\circ}\end{array}$ & Oூ \\
\hline
\end{tabular}
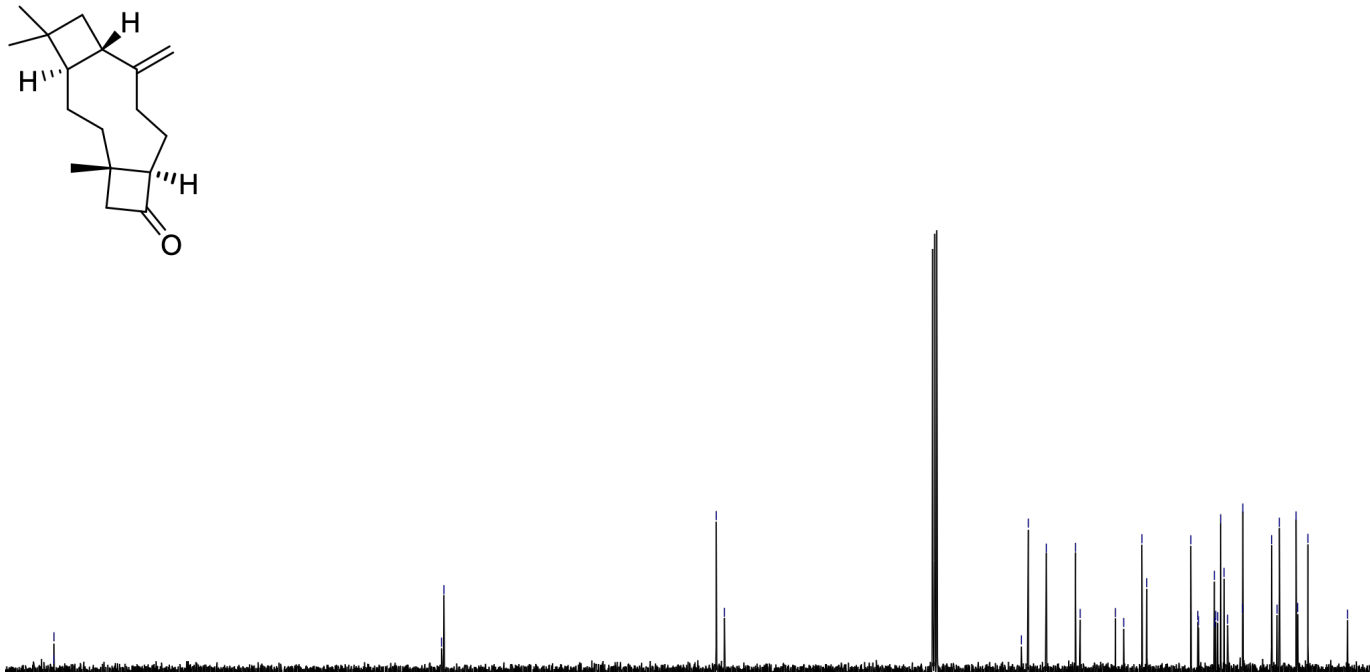


\section{tert-Butyldimethyl((3-phenoxyspiro[3.5]non-2-en-1-yl)oxy)silane (3ii)}

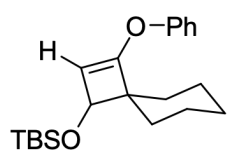

Cyclobutene 3ii was synthesized following general procedure B starting from (ethynyloxy)benzene ( $23.6 \mathrm{mg}, 0.2$ mmol) and tert-butyl(cyclohexylidenemethoxy)dimethylsilane $(136 \mathrm{mg}, 0.6 \mathrm{mmol})$. The crude product was purified by flash chromatography affording 3ii as a yellow oil (54 mg, 79\%).

${ }^{1} \mathbf{H}$ NMR $\left(400 \mathrm{MHz}, \mathrm{CDCl}_{3}\right) \delta 7.37-7.28(\mathrm{~m}, 2 \mathrm{H}), 7.18-7.09(\mathrm{~m}, 3 \mathrm{H}), 4.58(\mathrm{~d}, J=0.8 \mathrm{~Hz}, 1 \mathrm{H}), 4.12(\mathrm{~d}, J=$ $0.8 \mathrm{~Hz}, 1 \mathrm{H}), 1.82-1.70(\mathrm{~m}, 2 \mathrm{H}), 1.70-1.61(\mathrm{~m}, 2 \mathrm{H}), 1.61-1.45(\mathrm{~m}, 2 \mathrm{H}), 1.44-1.32(\mathrm{~m}, 2 \mathrm{H}), 1.32-1.12(\mathrm{~m}$, $2 \mathrm{H}), 0.90(\mathrm{~s}, 9 \mathrm{H}), 0.07$ (s, 3H), $0.06(\mathrm{~s}, 3 \mathrm{H})$.

${ }^{13}$ C NMR $\left(101 \mathrm{MHz}, \mathrm{CDCl}_{3}\right) \delta 162.0,155.2,129.6,124.4,119.8,100.8,72.8,55.5,33.8,30.4,26.1,26.1,24.4$, $23.5,18.4,-4.2,-4.5$

HRMS (ESI) $m / z$ calculated for $\mathrm{C}_{21} \mathrm{H}_{33} \mathrm{O}_{2} \mathrm{Si}^{+}[\mathrm{M}+\mathrm{H}]^{+}: 345.2244$, found: 345.2249 .

\section{${ }^{1} \mathrm{H}$ NMR $\left(400 \mathrm{MHz}, \mathrm{CDCl}_{3}\right)$ of 3ii}

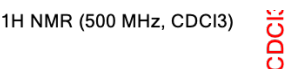

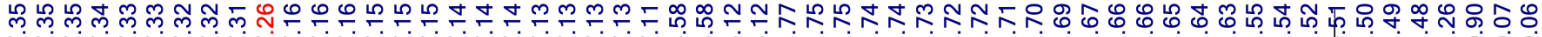
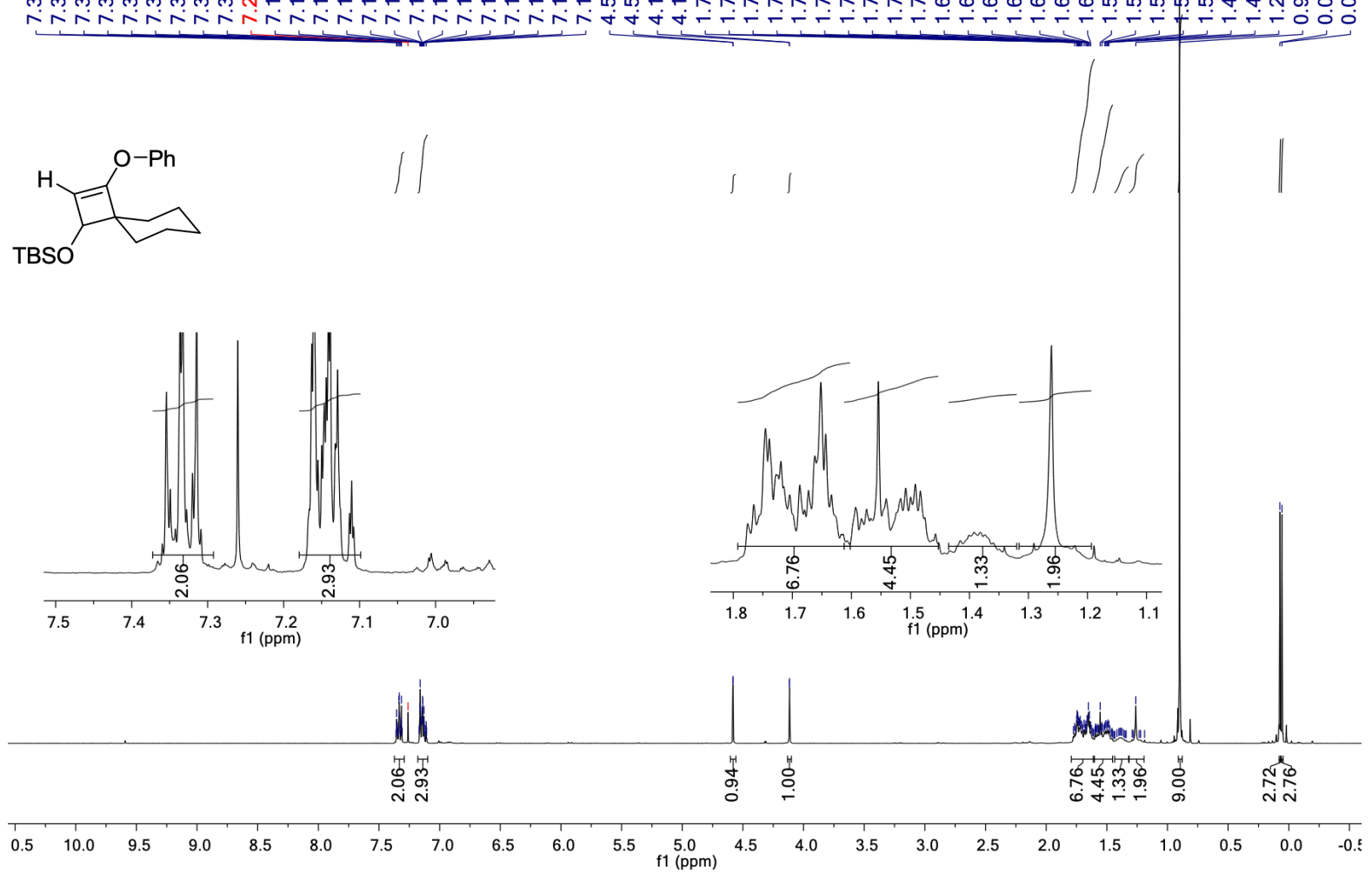
${ }^{13} \mathrm{C}$ NMR $\left(101 \mathrm{MHz}, \mathrm{CDCl}_{3}\right)$ of $3 \mathbf{i i}$

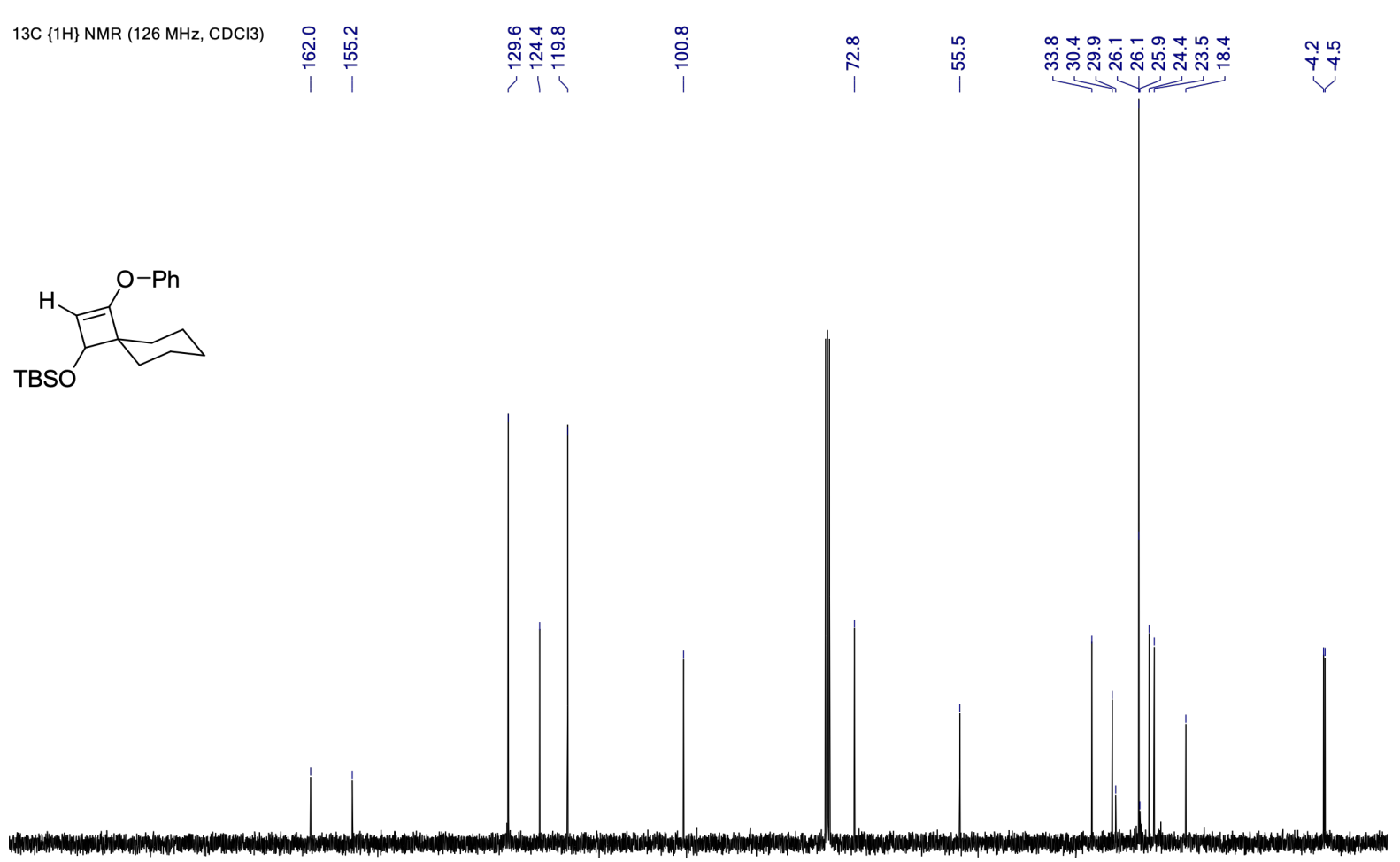

\begin{tabular}{llllllllllllllllllllllllll}
\hline 210 & 200 & 190 & 180 & 170 & 160 & 150 & 140 & 130 & 120 & 110 & 100 & 90 & 80 & 70 & 60 & 50 & 40 & 30 & 20 & 10 & 0 & -10
\end{tabular} 
tert-Butyl 3-methoxy-1-phenoxy-7-azaspiro[3.5]non-1-ene-7-carboxylate (3jj)

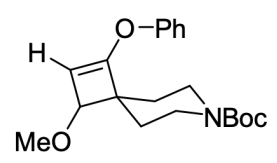

Cyclobutene 3jj was synthesized following general procedure B starting from (ethynyloxy)benzene ( $23.6 \mathrm{mg}, 0.2$ $\mathrm{mmol}$ ) and tert-butyl 4-(methoxymethylene)piperidine-1-carboxylate $(136 \mathrm{mg}, 0.6 \mathrm{mmol})$. The crude product was purified by flash chromatography affording $\mathbf{3 j j}$ as a colorless oil (64 $\mathrm{mg}, 92 \%)$.

${ }^{1} \mathbf{H}$ NMR $\left(400 \mathrm{MHz}, \mathrm{CDCl}_{3}\right) \delta 7.39-7.30(\mathrm{~m}, 2 \mathrm{H}), 7.20-7.11(\mathrm{~m}, 3 \mathrm{H}), 4.78(\mathrm{~d}, J=0.8 \mathrm{~Hz}, 1 \mathrm{H}), 3.77(\mathrm{~d}, J=$ $0.8 \mathrm{~Hz}, 1 \mathrm{H}), 3.70-3.47$ (m, 4H), 3.33 (s, 3H), $1.93-1.79$ (m, 3H), 1.72 (ddd, $J=12.7,7.8,3.9 \mathrm{~Hz}, 1 \mathrm{H}), 1.47$ (s, $9 \mathrm{H})$.

${ }^{13}$ C NMR $\left(101 \mathrm{MHz}, \mathrm{CDCl}_{3}\right) \delta 161.8,155.2,154.7,129.7,124.8,119.7,98.7,80.1,79.4,56.9,53.2,28.6,28.6$.

HRMS (ESI) $m / z$ calculated for $\mathrm{C}_{20} \mathrm{H}_{27} \mathrm{NNaO}_{4}^{+}[\mathrm{M}+\mathrm{H}]^{+}: 368.1832$, found: 368.1831 .

${ }^{1} \mathrm{H}$ NMR $\left(400 \mathrm{MHz}, \mathrm{CDCl}_{3}\right)$ of $\mathbf{3} \mathbf{j j}$

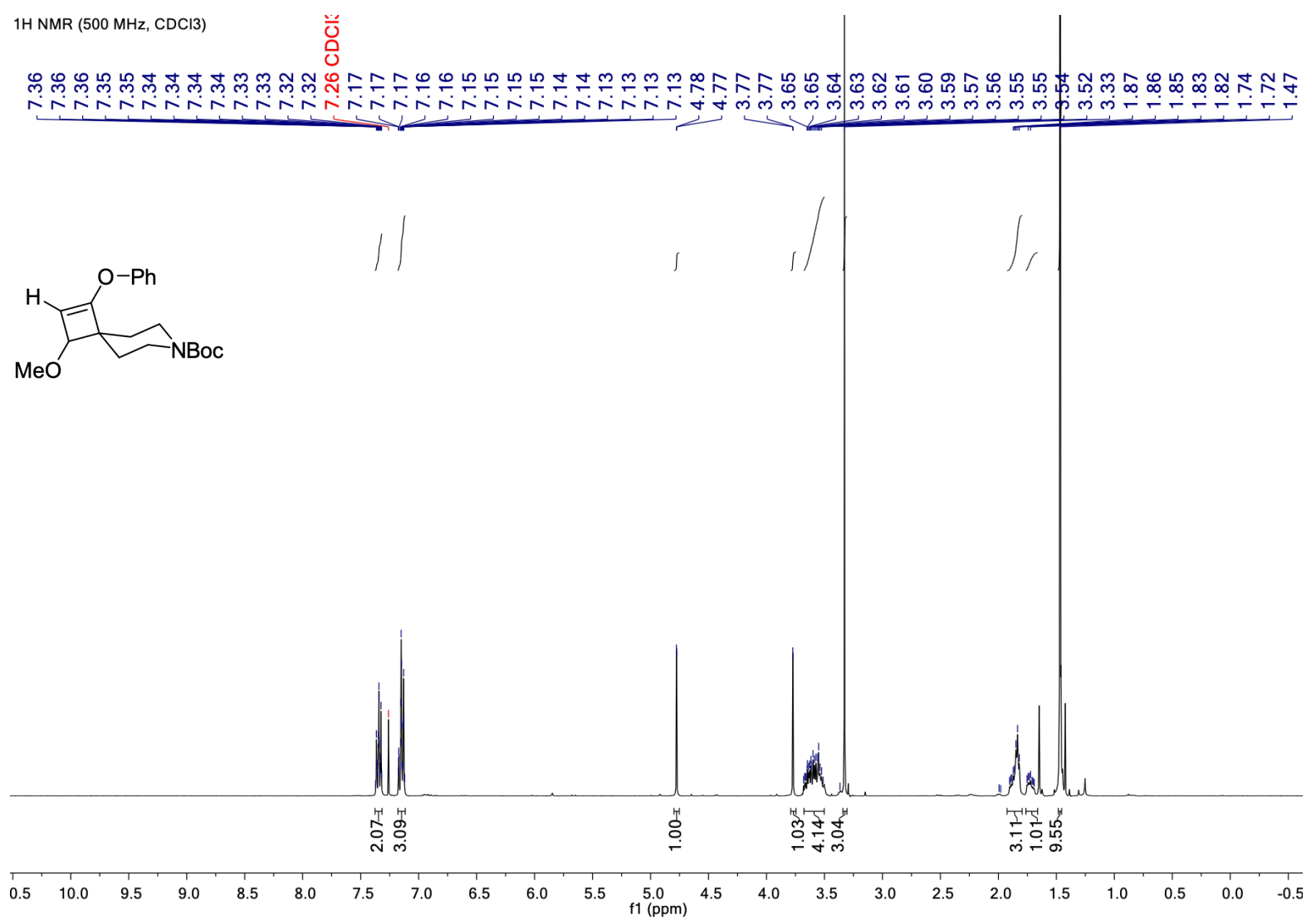


${ }^{13} \mathrm{C}$ NMR $\left(101 \mathrm{MHz}, \mathrm{CDCl}_{3}\right)$ of $\mathbf{3} \mathbf{j} \mathbf{j}$

$13 \mathrm{C}\{1 \mathrm{H}\} \mathrm{NMR}(126 \mathrm{MHz}, \mathrm{CDCl} 3)$

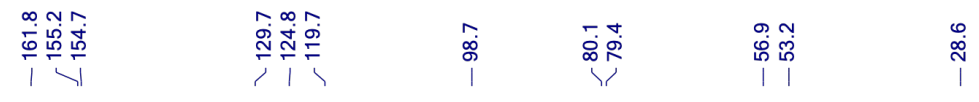
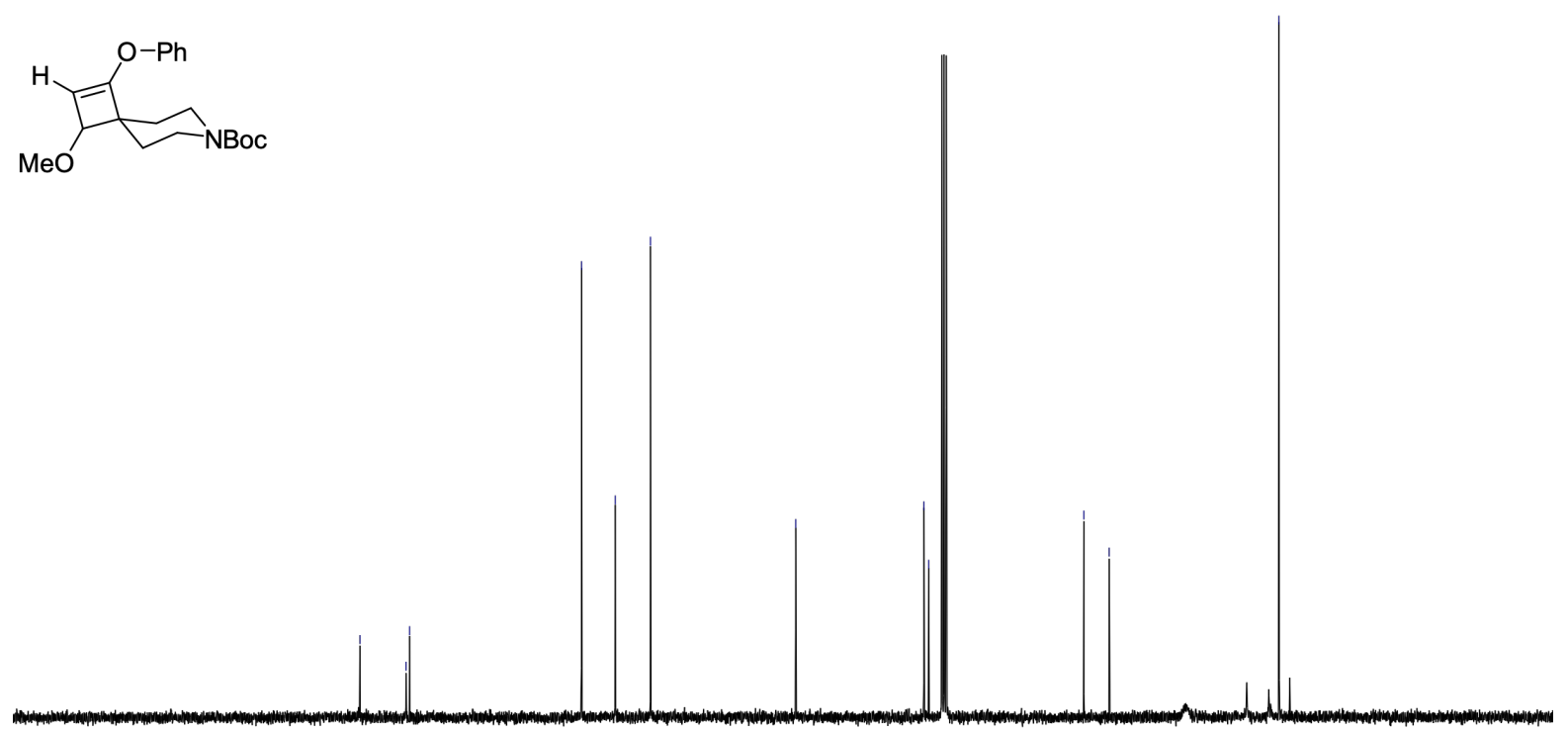

\begin{tabular}{lllllllllllllllllllllllllllll}
\hline & 110 & 200 & 190 & 180 & 170 & 160 & 150 & 140 & 130 & 120 & 110 & 100 & 90 & 80 & 70 & 60 & 50 & 40 & 30 & 20 & 10 & 0 & -1
\end{tabular} 
General procedure C: Gold(I)-catalyzed reaction of internal ynol ethers with alkenes<smiles>[R]C#COc1ccc([R])cc1</smiles>

1<smiles>[R]C=C([R])[R]</smiles>

2

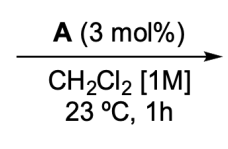

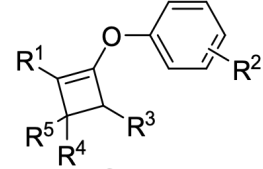

3<smiles>[R][R]1ccc2c(c1)C([R])([R])C(=Cc1c[R]:[R1]cc1)O2</smiles>

4

A GC-MS vial equipped with a magnetic stirring bar was charged with the ynol ether ( $0.2 \mathrm{mmol}, 1$ equiv) and $\mathrm{CH}_{2} \mathrm{Cl}_{2}$ (HPLC grade, $0.2 \mathrm{~mL}, 1 \mathrm{M}$ ). The alkene $(0.4 \mathrm{mmol}, 2$ equiv) was then added followed by $[(t \mathrm{BuXPhos}) \operatorname{AuNCMe}] \mathrm{BAr}_{4}{ }^{\mathrm{F}}(\mathbf{A}, 3 \mathrm{~mol} \%, 9.2 \mathrm{mg})$. The resulting mixture was stirred at $23{ }^{\circ} \mathrm{C}$ for $1 \mathrm{~h}$. The reaction was monitored by GC-MS or UHPLC-MSD. Once completed, the reaction was quenched with few drops of triethylamine and the solvent evaporated. The crude product was purified by flash chromatography on silica gel (eluent $=$ pentane:Et2O gradient from 100:0 to 50:1, otherwise stated) to obtain the pure product.

\section{6-Phenoxy-7-phenylbicyclo[3.2.0]hept-6-ene (3kk)}<smiles>c1ccccc1</smiles>

Cyclobutene 3kk was synthesized following general procedure $\mathbf{C}$ starting from (phenoxyethynyl)benzene (38 $\mathrm{mg}$, $0.2 \mathrm{mmol}$ ) and cyclopentene $(37 \mu \mathrm{L}, 0.4 \mathrm{mmol})$. The crude product was purified by flash chromatography affording 3kk as a colorless oil ( $24 \mathrm{mg}, 46 \%$ ).

${ }^{1}$ H NMR $\left(400 \mathrm{MHz}, \mathrm{CDCl}_{3}\right) \delta 7.51-7.45(\mathrm{~m}, 2 \mathrm{H}), 7.38-7.28(\mathrm{~m}, 4 \mathrm{H}), 7.21-7.15(\mathrm{~m}, 1 \mathrm{H}), 7.15-7.08(\mathrm{~m}$, $3 \mathrm{H}), 3.52(\mathrm{dd}, J=7.3,3.6 \mathrm{~Hz}, 1 \mathrm{H}), 3.21(\mathrm{dd}, J=6.7,3.6 \mathrm{~Hz}, 1 \mathrm{H}), 1.87(\mathrm{dd}, J=12.6,5.8 \mathrm{~Hz}, 1 \mathrm{H}), 1.80-1.63(\mathrm{~m}$, $2 \mathrm{H}), 1.57-1.48(\mathrm{~m}, 1 \mathrm{H}), 1.32(\mathrm{tt}, J=12.3,6.8 \mathrm{~Hz}, 1 \mathrm{H}), 1.11(\mathrm{ddt}, J=13.0,12.2,7.2 \mathrm{~Hz}, 1 \mathrm{H})$.

${ }^{13}$ C NMR $\left(101 \mathrm{MHz}, \mathrm{CDCl}_{3}\right) \delta 155.1,143.0,133.1,129.6,128.5,126.4,126.3,123.6,119.4,119.0,47.9,38.6$, $26.1,24.6,23.3$.

HRMS (APCI) $m / z$ calculated for $\mathrm{C}_{19} \mathrm{H}_{19} \mathrm{O}^{+}[\mathrm{M}+\mathrm{H}]^{+}: 263.1430$, found: 263.1432 . 
${ }^{1} \mathrm{H}$ NMR $\left(400 \mathrm{MHz}, \mathrm{CDCl}_{3}\right)$ of $\mathbf{3 k k}$

$1 \mathrm{H}$ NMR $(500 \mathrm{MHz}, \mathrm{CDCl} 3)$

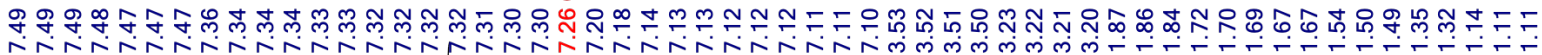

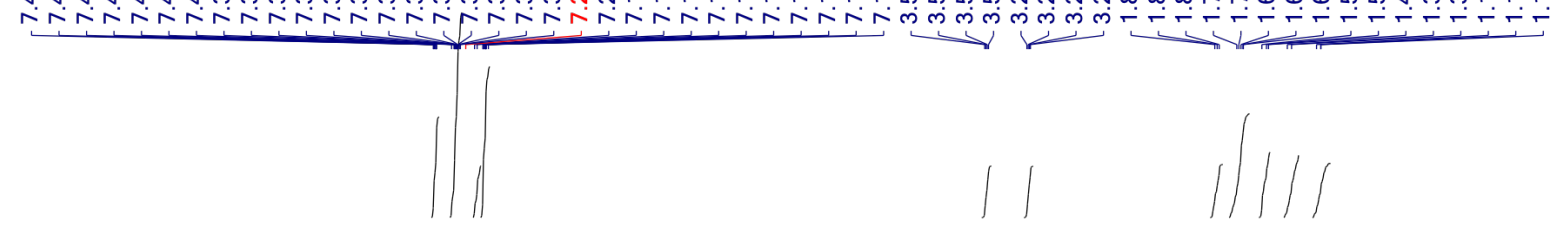

${ }^{\mathrm{Ph}}$ 


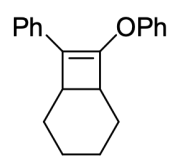

Cyclobutene 3ll was synthesized following general procedure $\mathbf{C}$ starting from (phenoxyethynyl)benzene (38 $\mathrm{mg}$, $0.2 \mathrm{mmol}$ ) and cyclohexene ( $42 \mu \mathrm{L}, 0.4 \mathrm{mmol})$. The crude product was purified by flash chromatography affording 3 II as a colorless oil ( $24 \mathrm{mg}, 43 \%)$.

${ }^{1}$ H NMR $\left(400 \mathrm{MHz}, \mathrm{CDCl}_{3}\right) \delta 7.50-7.45(\mathrm{~m}, 2 \mathrm{H}), 7.32(\mathrm{qd}, J=6.7,1.8 \mathrm{~Hz}, 4 \mathrm{H}), 7.20-7.15(\mathrm{~m}, 1 \mathrm{H}), 7.14-$ 7.07 (m, 3H), 3.23 (q, $J=5.1 \mathrm{~Hz}, 1 \mathrm{H}), 2.94(\mathrm{q}, J=5.1 \mathrm{~Hz}, 1 \mathrm{H}), 1.89$ (ddt, $J=14.1,9.6,4.4 \mathrm{~Hz}, 1 \mathrm{H}), 1.83-1.72$ $(\mathrm{m}, 1 \mathrm{H}), 1.67-1.55(\mathrm{~m}, 2 \mathrm{H}), 1.52-1.24(\mathrm{~m}, 4 \mathrm{H})$.

${ }^{13} \mathrm{C}$ NMR $\left(101 \mathrm{MHz}, \mathrm{CDCl}_{3}\right) \delta 155.2,146.3,134.0,129.6,128.5,126.3,126.2,123.7,121.4,119.1,41.5,32.4$, $23.7,22.4,18.6,18.2$.

HRMS (APCI) $m / z$ calculated for $\mathrm{C}_{20} \mathrm{H}_{21} \mathrm{O}^{+}[\mathrm{M}+\mathrm{H}]^{+}: 277.1587$, found: 277.1585 .

${ }^{1} \mathrm{H}$ NMR $\left(400 \mathrm{MHz}, \mathrm{CDCl}_{3}\right)$ of $\mathbf{3 I I}$

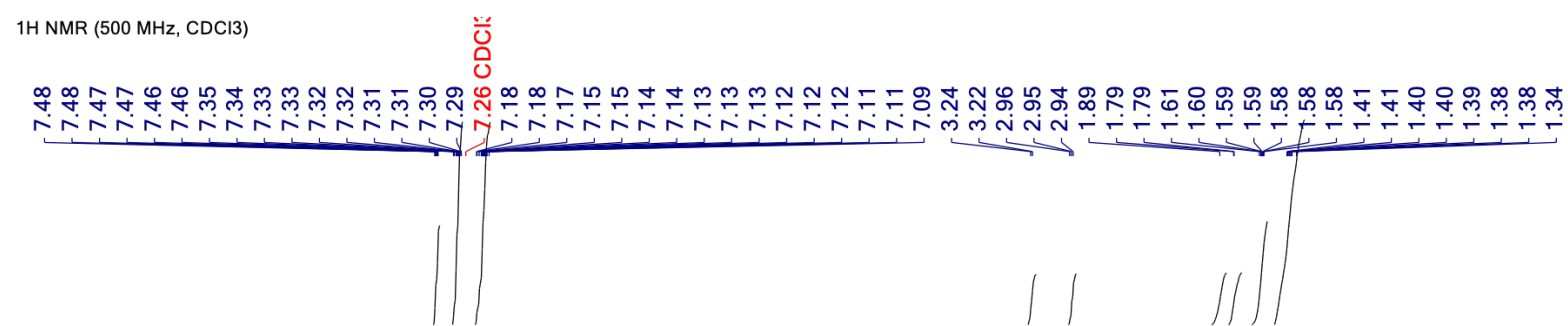<smiles>C(=C1CCCCC1Oc1ccccc1)c1ccccc1</smiles>

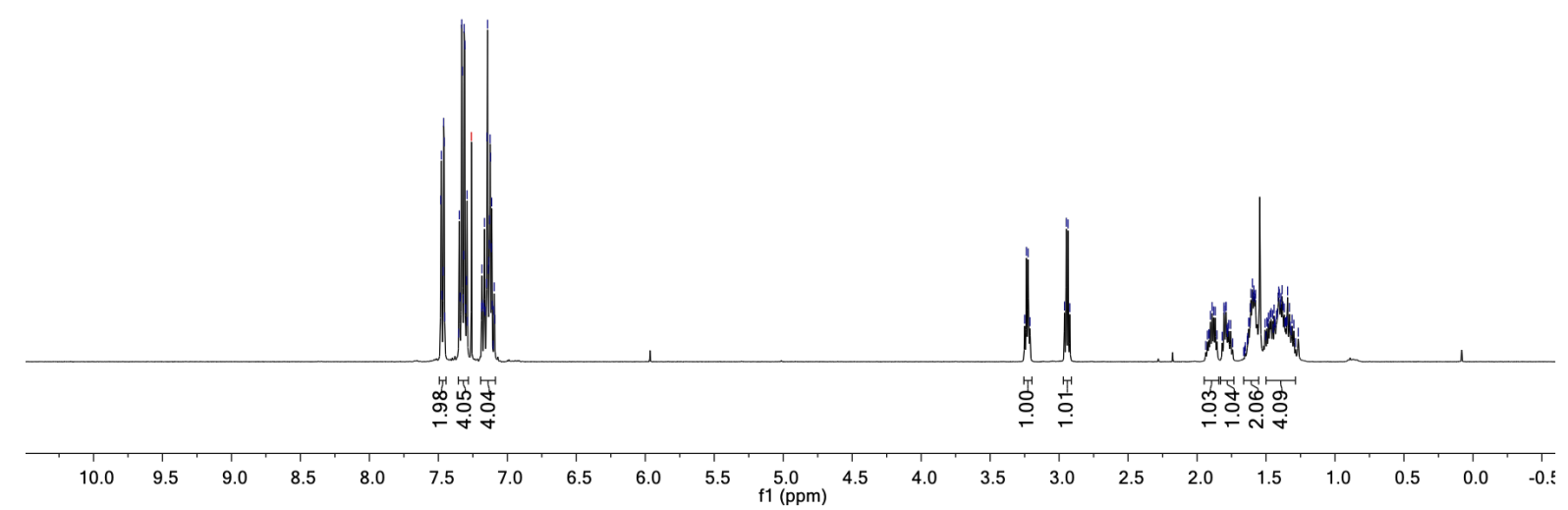


${ }^{13} \mathrm{C}$ NMR $\left(101 \mathrm{MHz}, \mathrm{CDCl}_{3}\right)$ of 3 II

$13 \mathrm{C}\{1 \mathrm{H}\}$ NMR $(126 \mathrm{MHz}, \mathrm{CDCl} 3)$

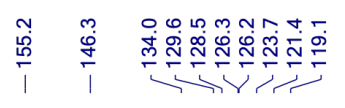

吕<smiles>c1ccc(OC2=C(c3ccccc3)C3CCCCC23)cc1</smiles> 


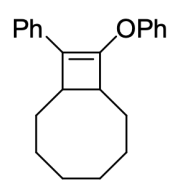

Cyclobutene $\mathbf{3 m m}$ was synthesized following general procedure $\mathbf{C}$ starting from (phenoxyethynyl)benzene (38 $\mathrm{mg}, 0.2 \mathrm{mmol})$ and $(\mathrm{Z})$-cyclooctene $(52 \mu \mathrm{L}, 0.4 \mathrm{mmol})$. The crude product was purified by flash chromatography affording $\mathbf{3 m m}$ as a colorless oil (52 $\mathrm{mg}, 85 \%)$.

${ }^{1} \mathbf{H}$ NMR $\left(400 \mathrm{MHz} \mathrm{CDCl}_{3}\right) \delta 7.41(\mathrm{dt}, J=8.3,1.3 \mathrm{~Hz}, 2 \mathrm{H}), 7.30(\mathrm{qd}, J=6.8,1.7 \mathrm{~Hz}, 4 \mathrm{H}), 7.21-7.12(\mathrm{~m}, 1 \mathrm{H})$, $7.12-7.05(\mathrm{~m}, 3 \mathrm{H}), 3.16(\mathrm{ddd}, J=11.4,4.6,2.4 \mathrm{~Hz}, 1 \mathrm{H}), 2.88(\mathrm{dd}, J=10.5,4.3 \mathrm{~Hz}, 1 \mathrm{H}), 2.23-2.04(\mathrm{~m}, 1 \mathrm{H})$, $1.75-1.63(\mathrm{~m}, 1 \mathrm{H}), 1.61-1.43(\mathrm{~m}, 8 \mathrm{H}), 1.42-1.30(\mathrm{~m}, 1 \mathrm{H}), 1.25-1.13(\mathrm{~m}, 1 \mathrm{H})$.

${ }^{13}$ C NMR $\left(101 \mathrm{MHz}, \mathrm{CDCl}_{3}\right) \delta 155.3,145.2,132.9,129.6,128.4,126.6,126.3,123.2,123.0,118.0,48.0,39.4$, $30.4,30.1,26.3,26.1,25.4,24.4$.

HRMS (APCI) $m / z$ calculated for $\mathrm{C}_{22} \mathrm{H}_{25} \mathrm{O}^{+}[\mathrm{M}+\mathrm{H}]^{+}: 305.1900$, found: 305.1906 .

\section{${ }^{1} \mathrm{H} \mathrm{NMR}\left(400 \mathrm{MHz}, \mathrm{CDCl}_{3}\right)$ of $\mathbf{3 m m}$}

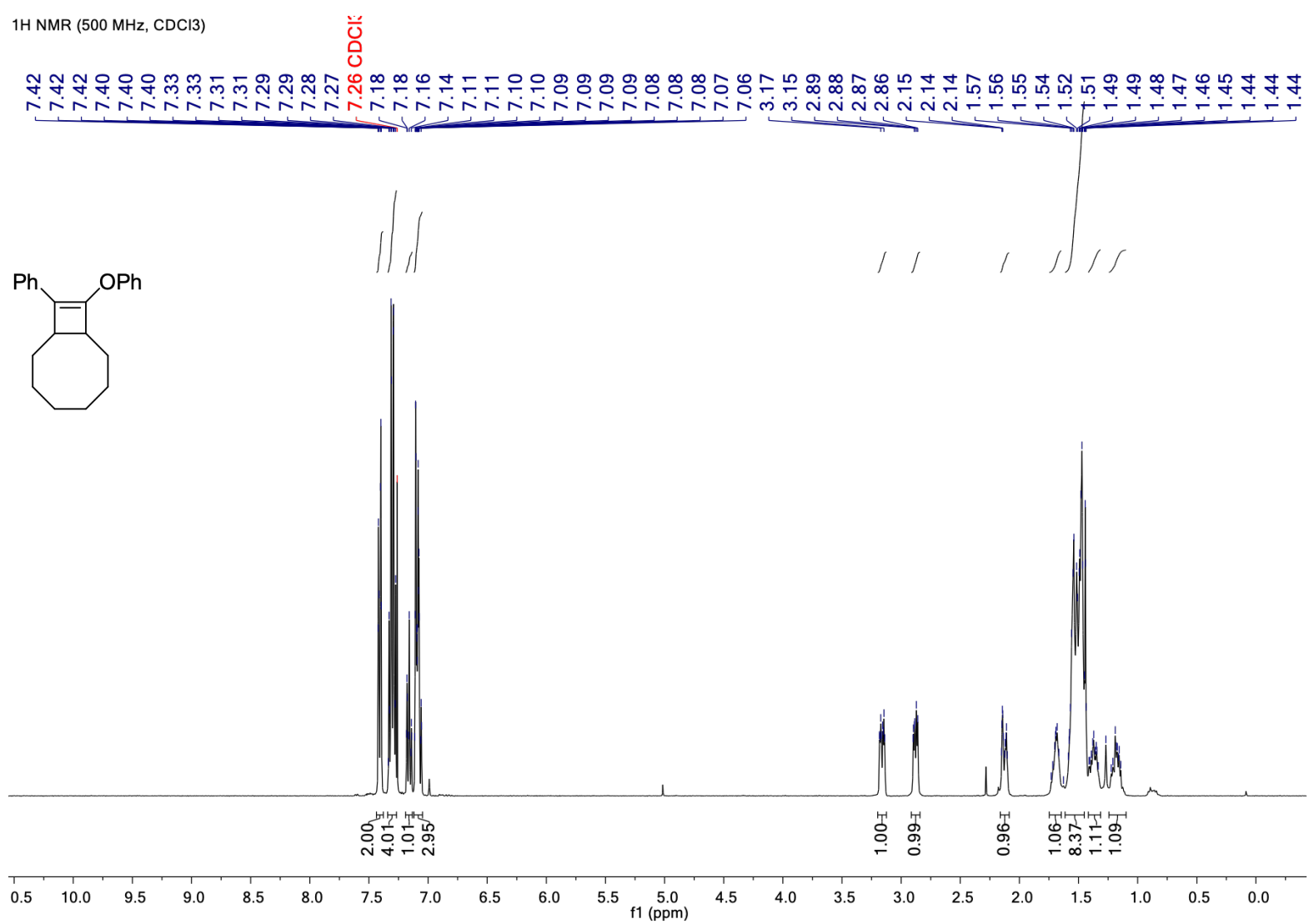


${ }^{13} \mathrm{C}$ NMR $\left(101 \mathrm{MHz}, \mathrm{CDCl}_{3}\right)$ of $\mathbf{3} \mathbf{m m}$

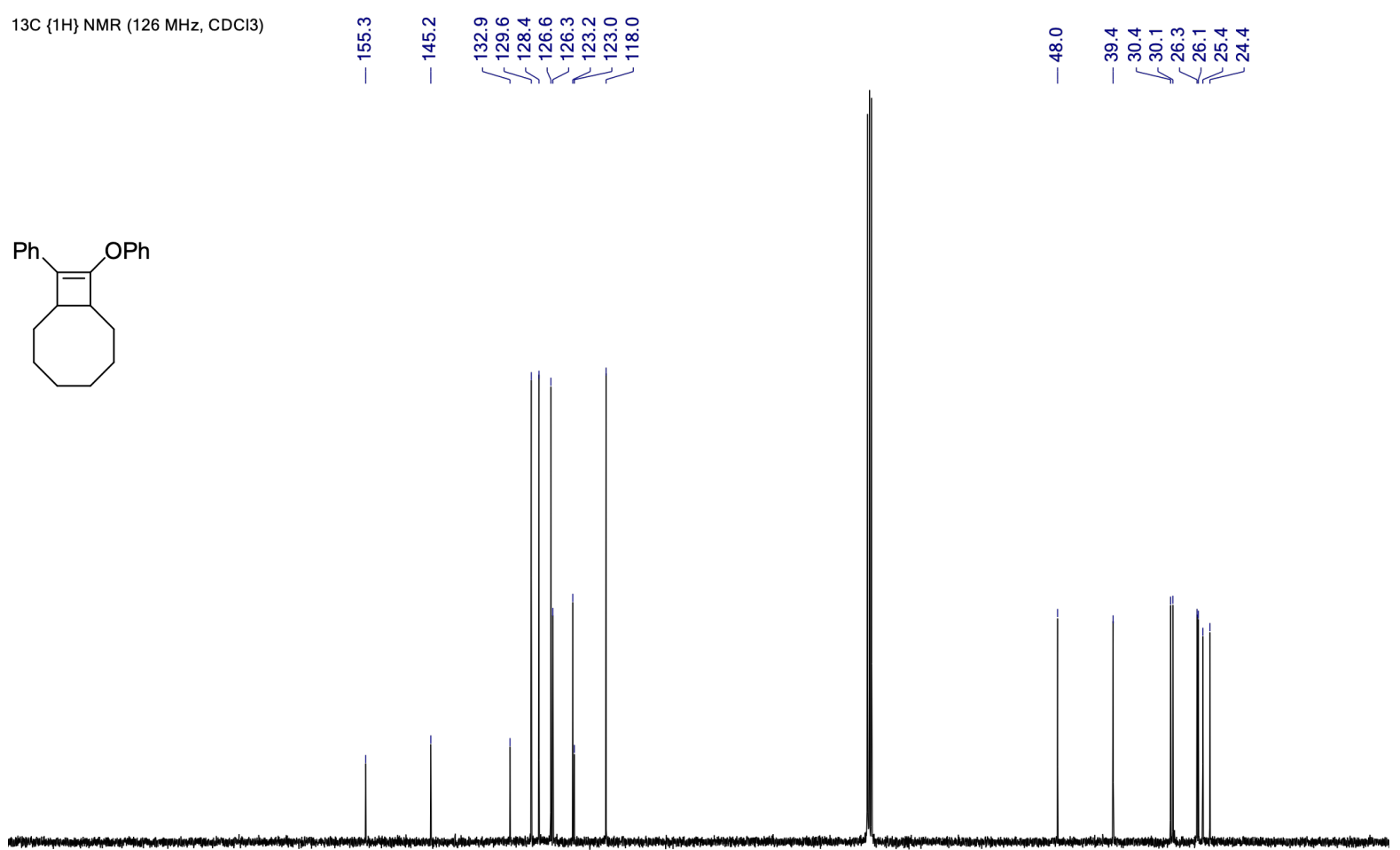

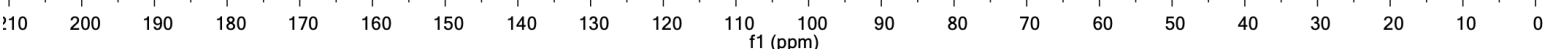



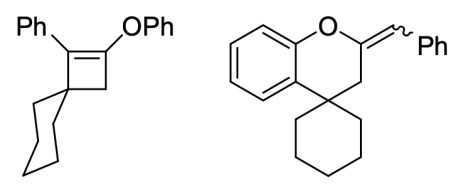

S1

Cyclobutene 3nn was synthesized following general procedure $\mathbf{C}$ starting from (phenoxyethynyl)benzene (39 mg, $0.2 \mathrm{mmol}$ ) and methylenecyclohexane $(48 \mathrm{ml}, 0.4 \mathrm{mmol})$. The crude product was purified by flash chromatography affording $\mathbf{3 n n}$ as yellow solid, (25 mg, 43\%). In the crude product also chromene $\mathbf{S 1}$ was identified and quantified in the crude mixture by ${ }^{1} \mathrm{H}$ NMR using trichloroethylene as internal standard $(27 \%, E: Z$ $=4.4: 1)$.

\section{(3-Phenoxycyclobut-2-ene-1,2-diyl)dibenzene (3nn)}

${ }^{1}$ H NMR (500 MHz, CDCl3) $\delta 7.60-7.52(\mathrm{~m}, 2 \mathrm{H}), 7.38-7.28(\mathrm{~m}, 4 \mathrm{H}), 7.18-7.14(\mathrm{~m}, 1 \mathrm{H}), 7.13-7.08(\mathrm{~m}$, $3 \mathrm{H}), 2.46$ (s, 2H), 2.06 (td, $J=13.1,3.7 \mathrm{~Hz}, 2 \mathrm{H}), 1.77$ (dt, $J=12.8,3.2 \mathrm{~Hz}, 2 \mathrm{H}), 1.72(\mathrm{dtt}, J=12.4,3.2,1.5 \mathrm{~Hz}$, $1 \mathrm{H}), 1.65(\mathrm{dq}, J=13.7,2.0 \mathrm{~Hz}, 2 \mathrm{H}), 1.36(\mathrm{qt}, J=12.7,3.3 \mathrm{~Hz}, 2 \mathrm{H}), 1.29-1.21(\mathrm{~m}, 1 \mathrm{H})$.

${ }^{13}$ C NMR $\left(126 \mathrm{MHz}, \mathrm{CDCl}_{3}\right) \delta 155.1,143.9,133.4,129.6,128.5,126.8,126.6,125.9,123.7,119.1,42.9,41.6$, 35.4, 25.9, 24.9.

HRMS (APCI) $m / z$ calculated for $\mathrm{C}_{21} \mathrm{H}_{23} \mathrm{O}^{+}[\mathrm{M}+\mathrm{H}]^{+}:$291.1743, found: 291.1748 . 
${ }^{1} \mathrm{H}$ NMR $(500 \mathrm{MHz}, \mathrm{CDCl} 3)$ of $\mathbf{3 m m}$

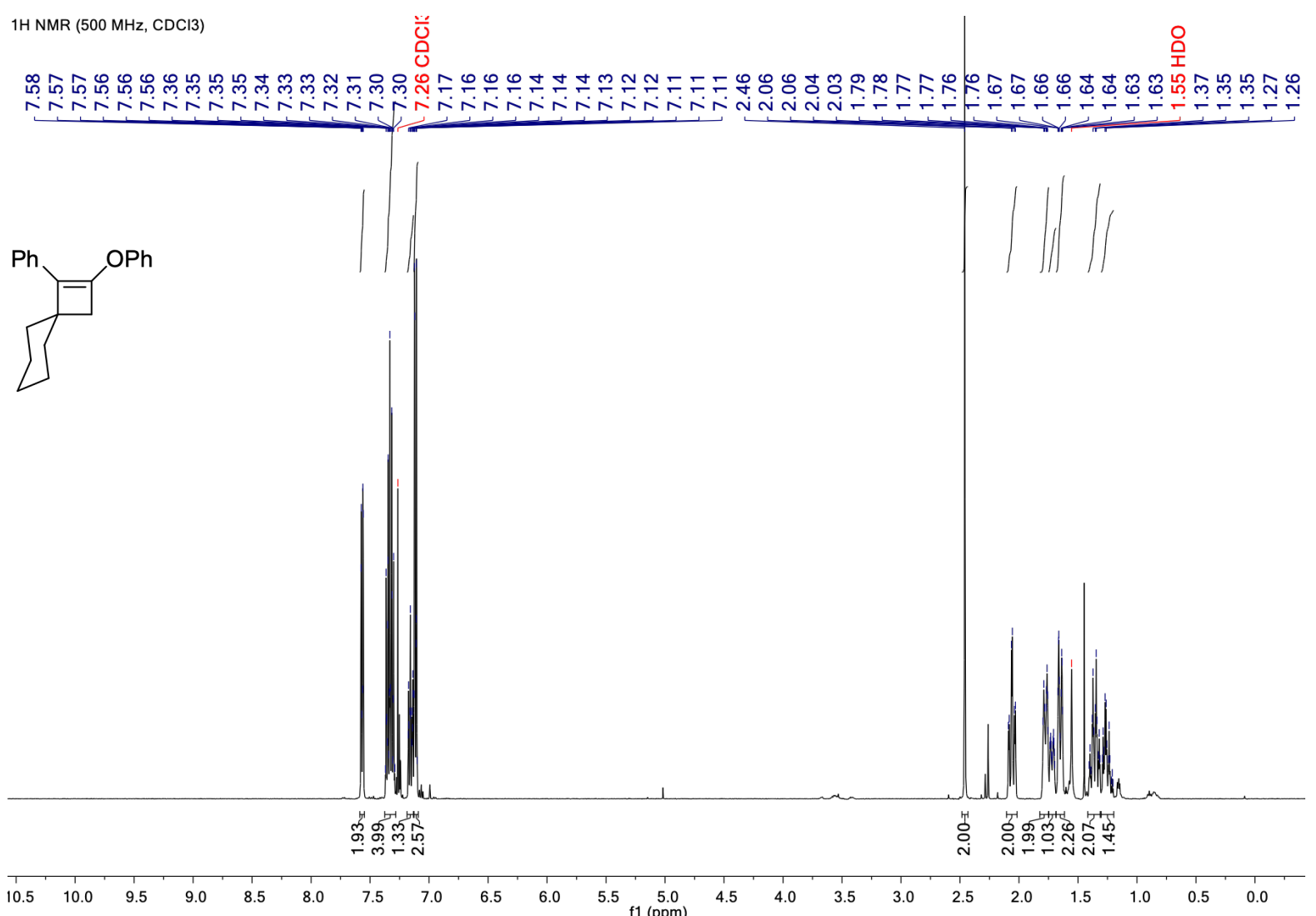

13C NMR (126 MHz, $\mathrm{CDCl}_{3}$ ) of $\mathbf{3 n n}$

$13 \mathrm{C}\{1 \mathrm{H}\} \mathrm{NMR}(126 \mathrm{MHz}, \mathrm{CDCl} 3)$

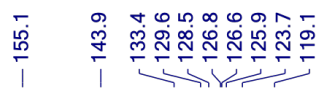

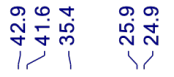
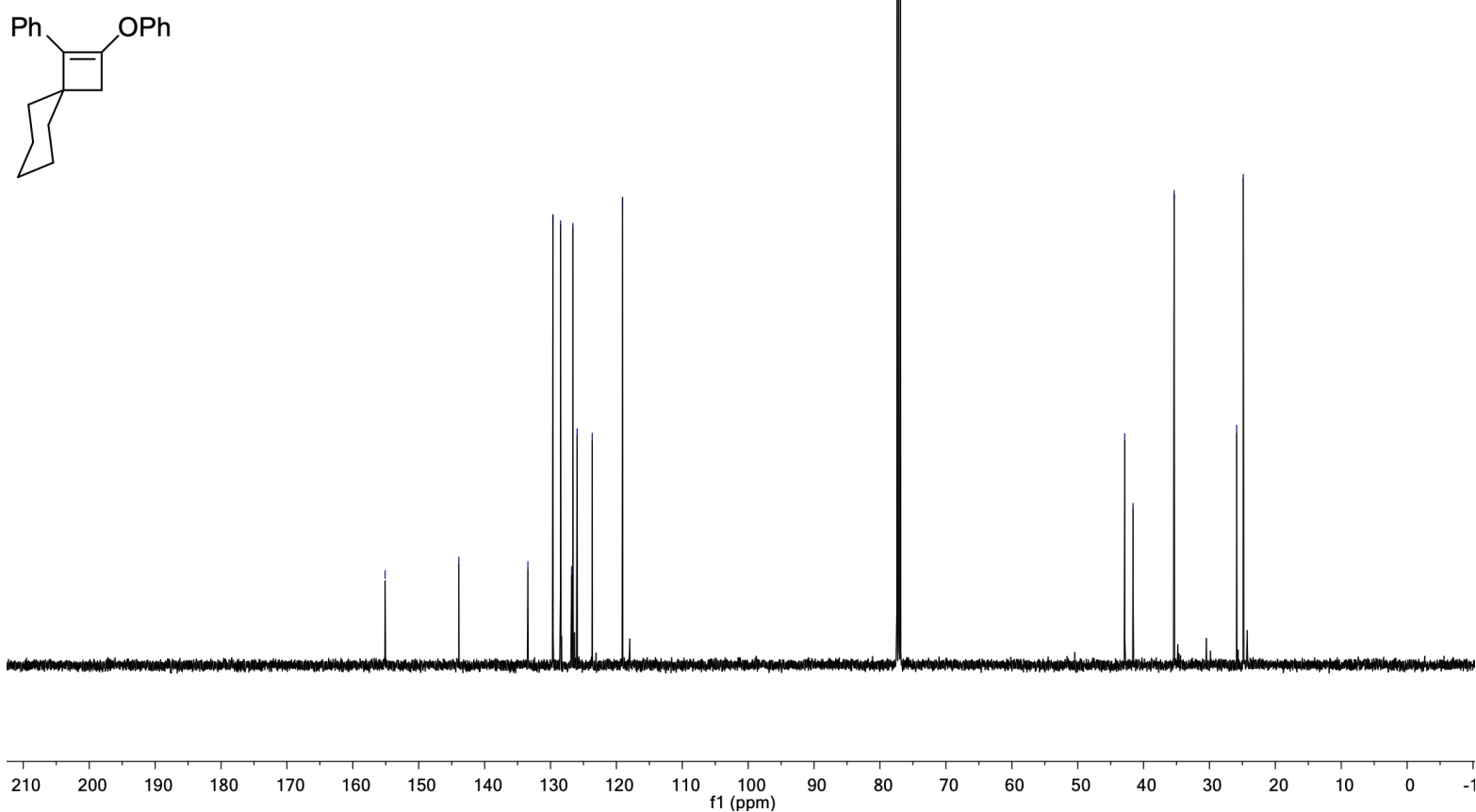
<smiles>C(=C1CC(c2ccccc2)c2ccccc2O1)c1ccccc1</smiles>

S2

The two products were synthesized following general procedure $\mathbf{C}$ starting from (phenoxyethynyl)benzene (39 $\mathrm{mg}, 0.2 \mathrm{mmol}$ ) and styrene $(42 \mathrm{mg}, 0.4 \mathrm{mmol})$. The two products were partially separated by flash chromatography affording $\mathbf{S 2}$ as a yellow oil, $E: Z=2.6: 1$ (32\% assigned by ${ }^{1} \mathrm{H}$ NMR using trichloroethylene as internal standard) and 300 as yellow oil, 4:1 mixture of regioisomers (36\% assigned by ${ }^{1} \mathrm{H}$ NMR using trichloroethylene as internal standard)

\section{(3-Phenoxycyclobut-2-ene-1,2-diyl)dibenzene (30o)}

${ }^{1}$ H NMR (400 MHz, $\left.\mathrm{CD}_{2} \mathrm{Cl}_{2}\right) \delta 7.42-7.27$ (m, 10H, Mr+mr), $7.28-7.09$ (m, 10H, Mr+mr), $7.07-6.96(\mathrm{~m}, 1 \mathrm{H}$, Mr), $4.33(\mathrm{dd}, J=4.9,1.7 \mathrm{~Hz}, 0.3 \mathrm{H}, \mathrm{mr}), 3.97$ (dd, $J=4.9,1.7 \mathrm{~Hz}, 1 \mathrm{H}, \mathrm{Mr}), 3.23$ (dd, $J=13.7,4.8 \mathrm{~Hz}, 1 \mathrm{H}, \mathrm{Mr})$, $3.00(\mathrm{dd}, J=10.5,4.9 \mathrm{~Hz}, 0.3 \mathrm{H}, \mathrm{mr}), 2.51$ (dd, $J=13.7,1.7 \mathrm{~Hz}, 1 \mathrm{H}, \mathrm{Mr}), 2.32$ (dd, $J=10.5,1.7 \mathrm{~Hz}, 0.3 \mathrm{H}, \mathrm{mr}$ ), 1.53 (s, 3H, Mr), 1.27 (s, 0.9H, mr).

${ }^{13}$ C NMR $\left(126 \mathrm{MHz}, \mathrm{CDCl}_{3}\right) \delta 154.8,143.6,142.8,133.0,129.8,129.5,128.7,128.5,128.4,127.1,126.7,126.4$, 126.4, 124.0, 121.1, 119.0, 118.9, 47.1, 40.9, 38.5, 31.6. The signals of ${ }^{13} \mathrm{C}$ NMR were not assigned to each regioisomer.

HRMS (APCI) $m / z$ calculated for $\mathrm{C}_{21} \mathrm{H}_{23} \mathrm{O}^{+}[\mathrm{M}+\mathrm{H}]^{+}: 298.1358$, found: 298.1360 

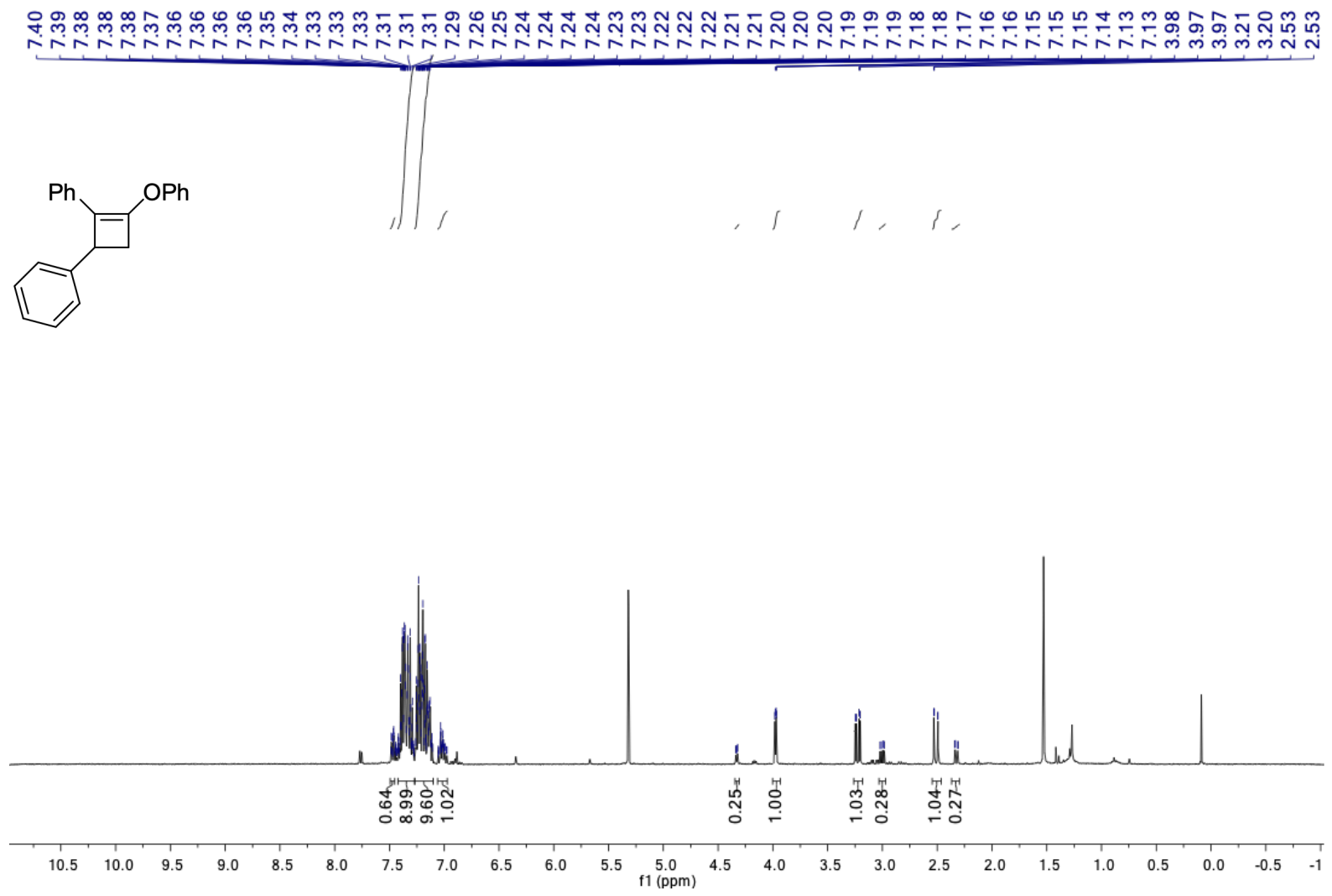

${ }^{13} \mathrm{C}$ NMR $\left(126 \mathrm{MHz}, \mathrm{CDCl}_{3}\right)$ of $\mathbf{3 o o}$

$13 \mathrm{C}\{1 \mathrm{H}\} \mathrm{NMR}(126 \mathrm{MHz}, \mathrm{CDCl} 3)$
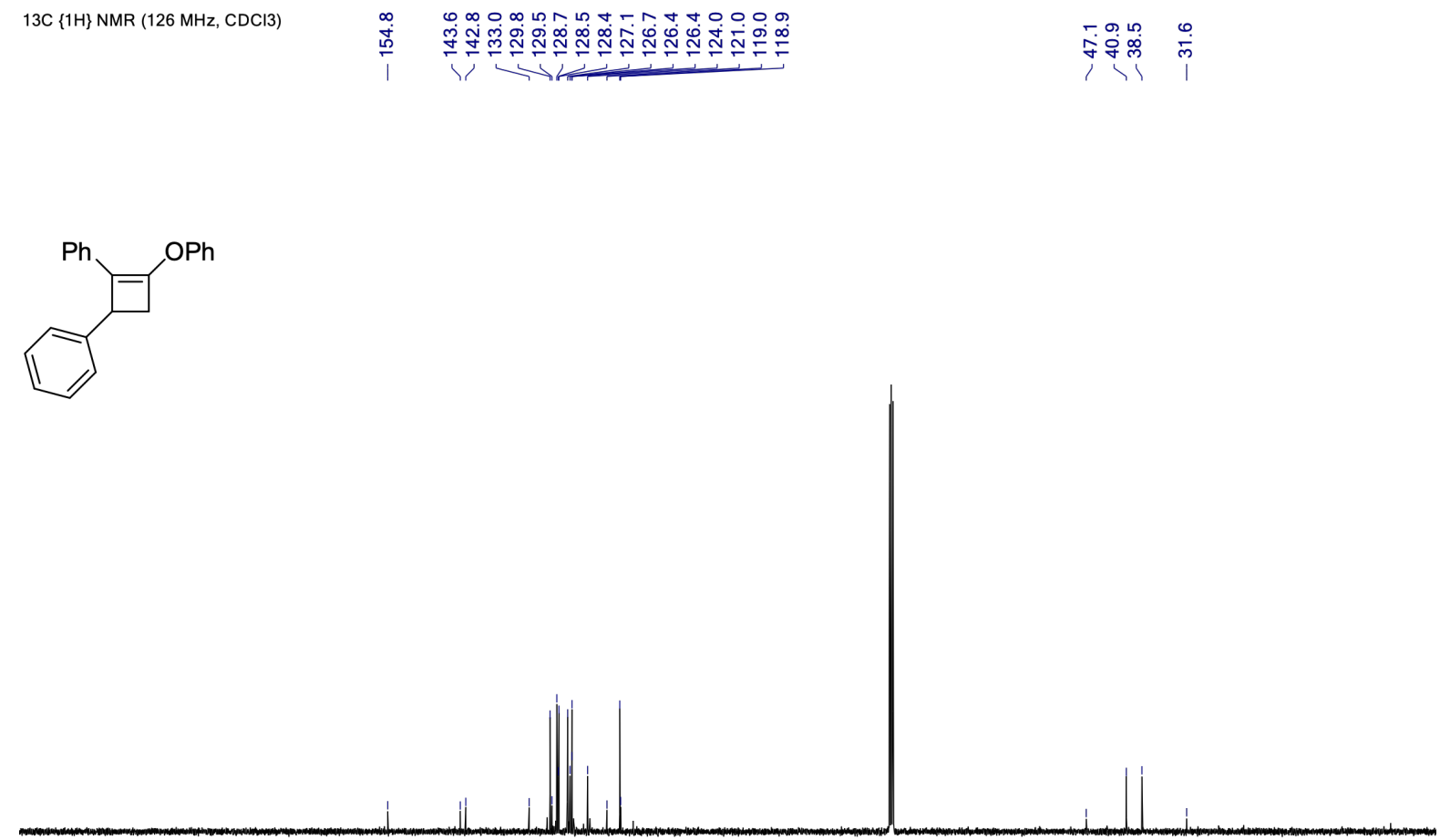

$\begin{array}{llllllllllllllllllllllll}10 & 200 & 190 & 180 & 170 & 160 & 150 & 140 & 130 & 120 & 110 & 100 & 90 & 80 & 70 & 60 & 50 & 40 & 30 & 20 & 10 & 0\end{array}$

\section{2-Benzylidene-4-phenylchromane (S2)}


${ }^{1} \mathbf{H}$ NMR (400 MHz, $\left.\mathrm{CD}_{2} \mathrm{Cl}_{2}\right) \delta 7.38-7.27(\mathrm{~m}, 4 \mathrm{H}, \mathrm{E}+\mathrm{Z}), 7.27-7.19(\mathrm{~m}, 5 \mathrm{H}, \mathrm{E}+\mathrm{Z}), 7.18-7.08(\mathrm{~m}, 4 \mathrm{H}, \mathrm{E}+\mathrm{Z})$, $7.06-6.96(\mathrm{~m}, 5 \mathrm{H}, \mathrm{E}+\mathrm{Z}), 6.96-6.80(\mathrm{~m}, 4 \mathrm{H}, \mathrm{E}+\mathrm{Z}), 6.34(\mathrm{~s}, 1 \mathrm{H}, \mathrm{E}), 5.67(\mathrm{~s}, 0.4 \mathrm{H}, \mathrm{Z}), 4.32(\mathrm{t}, J=7.9 \mathrm{~Hz}, 0.4 \mathrm{H}$, Z), $4.21-4.14$ (t, J = 6.6 Hz, 1H, E), 3.11 (ddd, $J=14.4,5.4,1.1 \mathrm{~Hz}, 1 \mathrm{H}, \mathrm{E}), 3.03$ (ddd, $J=14.4,6.9,0.9 \mathrm{~Hz}$, 1H, E), 2.95 (ddd, $J=16.6,7.4,1.1 \mathrm{~Hz}, 0.4 \mathrm{H}, \mathrm{Z}), 2.82$ (dd, $J=16.6,8.5 \mathrm{~Hz}, 0.4 \mathrm{H}, \mathrm{Z}), 1.53$ (s, 3H, E), $1.40-1.13$ $(\mathrm{m}, 1.2 \mathrm{H}, \mathrm{Z})$.

${ }^{13}$ C NMR (126 MHz, $\left.\mathrm{CDCl}_{3}\right) \delta 153.1,150.1,143.0,135.6,129.8,129.2,128.92$ 128.7, 128.7, 128.5, 128.4, 128.3, $127.8,127.1,127.1,126.8,126.1,125.9,125.8,125.7,124.3,121.6,120.6,116.4,108.8,104.9,45.3,41.2,34.8$, 31.0, 29.9. The signals of ${ }^{13} \mathrm{C} \mathrm{NMR}$ were not assigned to each diastereoisomer.

${ }^{1} \mathrm{H} \mathrm{NMR}\left(400 \mathrm{MHz}, \mathrm{CD}_{2} \mathrm{Cl}_{2}\right)$ of $\mathbf{S 2}$

$1 \mathrm{H}$ NMR $(500 \mathrm{MHz}, \mathrm{CD} 2 \mathrm{Cl} 2)$

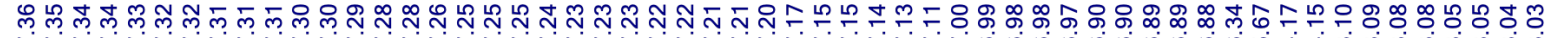

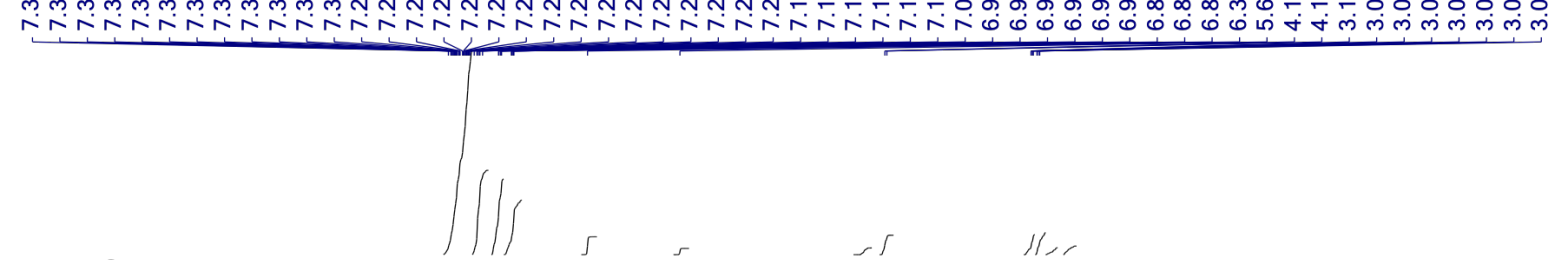<smiles>C(=C1CC(c2ccccc2)c2ccccc2O1)c1ccccc1</smiles>

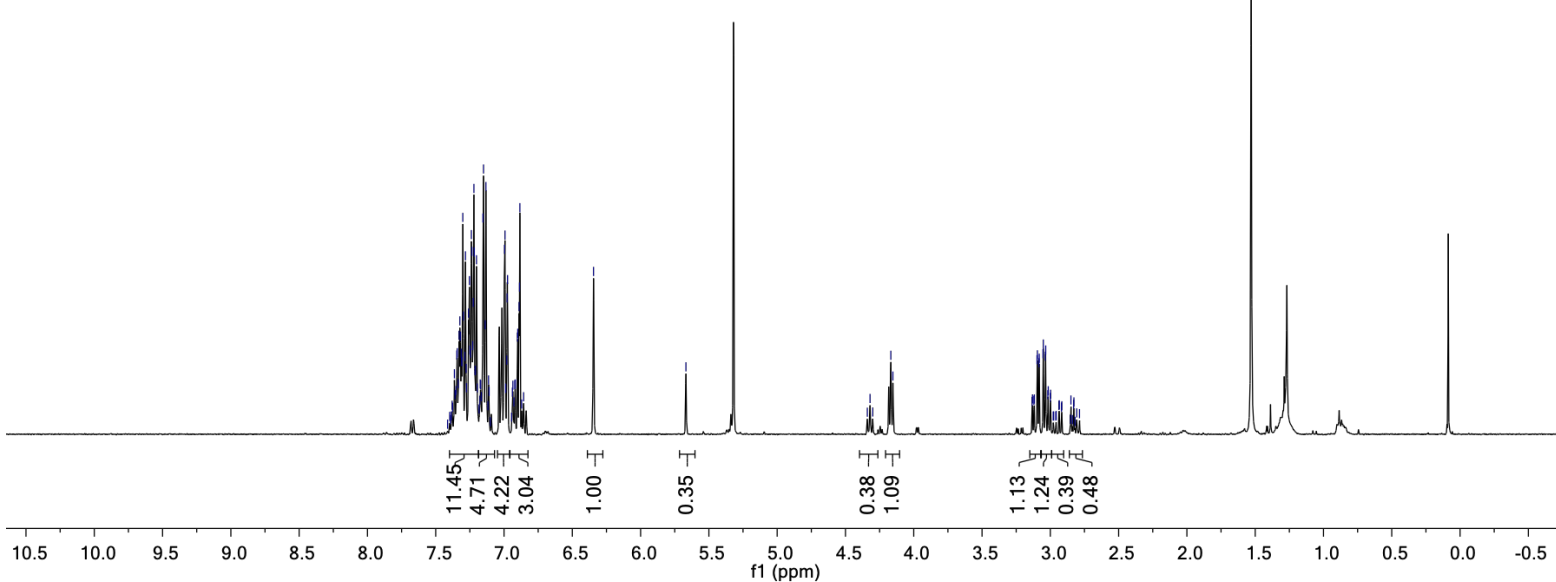



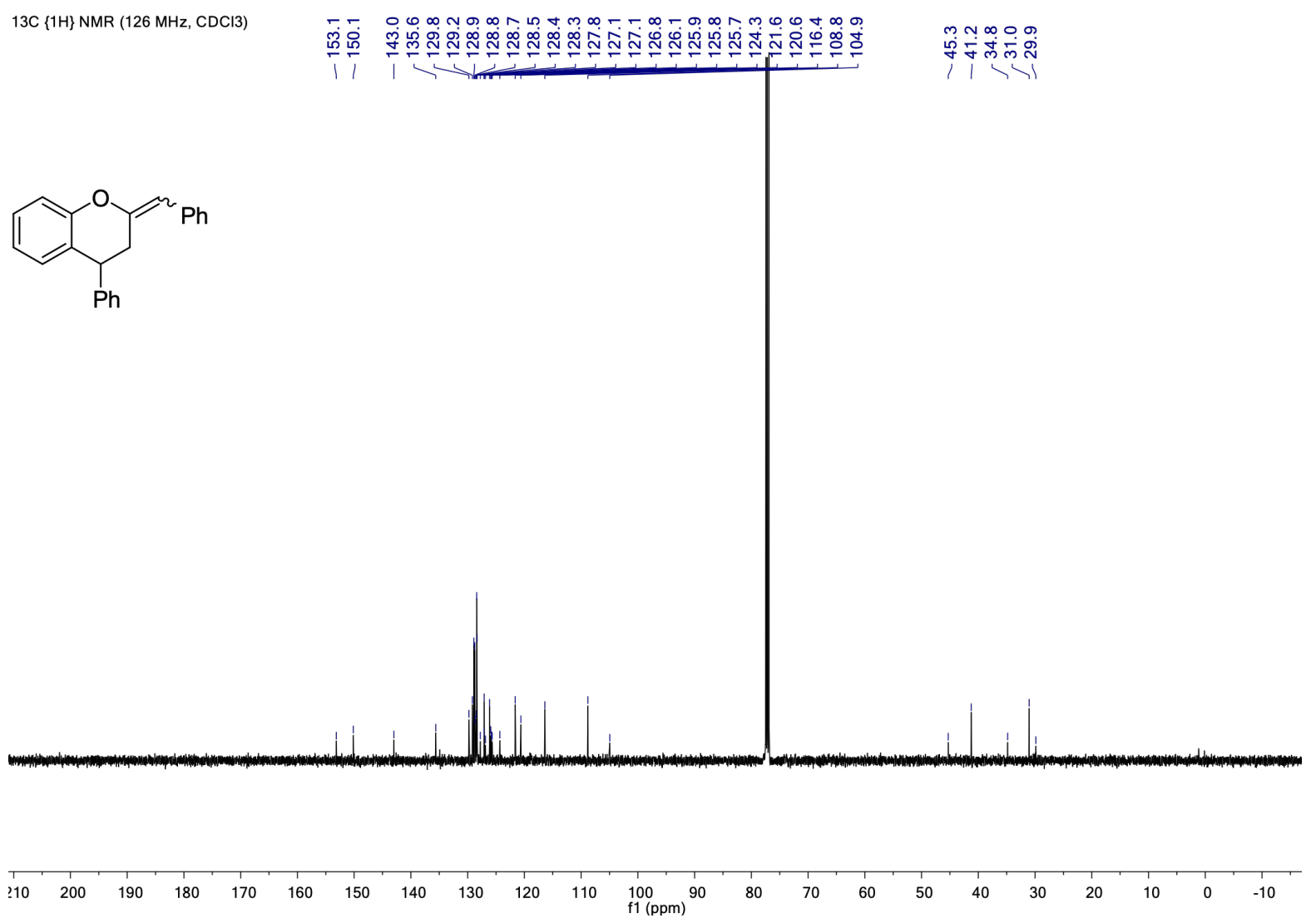

chlorophenyl)chromane (S3)<smiles>Clc1ccc(C2CC(Oc3ccccc3)=C2c2ccccc2)cc1</smiles><smiles>Clc1ccc(C2C/C(=C\c3ccccc3)Oc3ccccc32)cc1</smiles>

s3

The two products were synthesized following general procedure $\mathbf{C}$ starting from (phenoxyethynyl)benzene (39 $\mathrm{mg}, 0.2 \mathrm{mmol})$ and 1-chloro-4-vinylbenzene $(55 \mathrm{mg}, 0.4 \mathrm{mmol})$. The two products were separated by flash chromatography affording $\mathbf{S 3}$ as a yellow oil, $E: Z=4: 1$ (19 $\mathrm{mg}, 28 \%$ ) and 3pp as yellow oil, 2.6:1 mixture of regioisomers $(25.6 \mathrm{mg}, 39 \%)$.

\section{(3-Phenoxycyclobut-2-ene-1,2-diyl)dibenzene (3pp)}

${ }^{1} \mathbf{H}$ NMR $\left(500 \mathrm{MHz}, \mathrm{CD}_{2} \mathrm{Cl}_{2}\right) \delta 7.50-7.42(\mathrm{~m}, 1 \mathrm{H}), 7.43-7.35(\mathrm{~m}, 3 \mathrm{H}, \mathrm{Mr}+\mathrm{mr}), 7.34-7.26(\mathrm{~m}, 7 \mathrm{H}, \mathrm{Mr}+\mathrm{mr})$, $7.26-7.20$ (m, 3H, Mr+mr), $7.21-7.10$ (m, 6H, Mr+mr), 4.29 (dd, $J=4.9,1.6 \mathrm{~Hz}, 0.36 \mathrm{H}, \mathrm{mr}), 3.95$ (dd, $J=4.9$, $1.6 \mathrm{~Hz}, 1 \mathrm{H}, \mathrm{Mr}$ ), 3.22 (dd, $J=13.7,4.8 \mathrm{~Hz}, 1 \mathrm{H}, \mathrm{Mr}$ ), 3.00 (dd, $J=10.6,4.8 \mathrm{~Hz}, 0.36 \mathrm{H}, \mathrm{mr}), 2.47$ (dd, $J=13.7$, $1.6 \mathrm{~Hz}, 1 \mathrm{H}, \mathrm{Mr}), 2.35-2.16(\mathrm{~m}, 0.36 \mathrm{H}, \mathrm{mr})$.

${ }^{13}$ C NMR $\left(126 \mathrm{MHz}, \mathrm{CD}_{2} \mathrm{Cl}_{2}\right) \delta 155.2,154.3$ 144.4, 142.0, 133.3, 130.6, 130.3, 130.0, 129.2, 129.2, 129.0, 128.9, $128.9,128.9,127.41,126.9,126.8,126.7,126.6,124.6,124.2,121.0,119.5,119.2,117.3,46.9,41.2,38.3,32.0$. The signals of ${ }^{13} \mathrm{C}$ NMR were not assigned to each regioisomer.

HRMS (APCI) $m / z$ calculated for $\mathrm{C}_{22} \mathrm{H}_{28} \mathrm{ClO}^{+}[\mathrm{M}+\mathrm{H}]^{+}: 333.1041$, found: 333.1043 . 
${ }^{1} \mathrm{H}$ NMR $\left(500 \mathrm{MHz}, \mathrm{CD}_{2} \mathrm{Cl}_{2}\right.$ ) of $\mathbf{3 p p}$

$1 \mathrm{H} \mathrm{NMR}(500 \mathrm{MHz}, \mathrm{CD} 2 \mathrm{Cl} 2)$

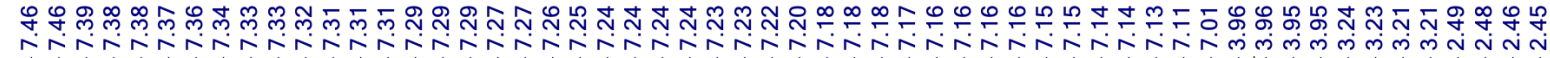<smiles>Clc1ccc(C2CC(c3ccccc3)=C2c2ccccc2)cc1</smiles>
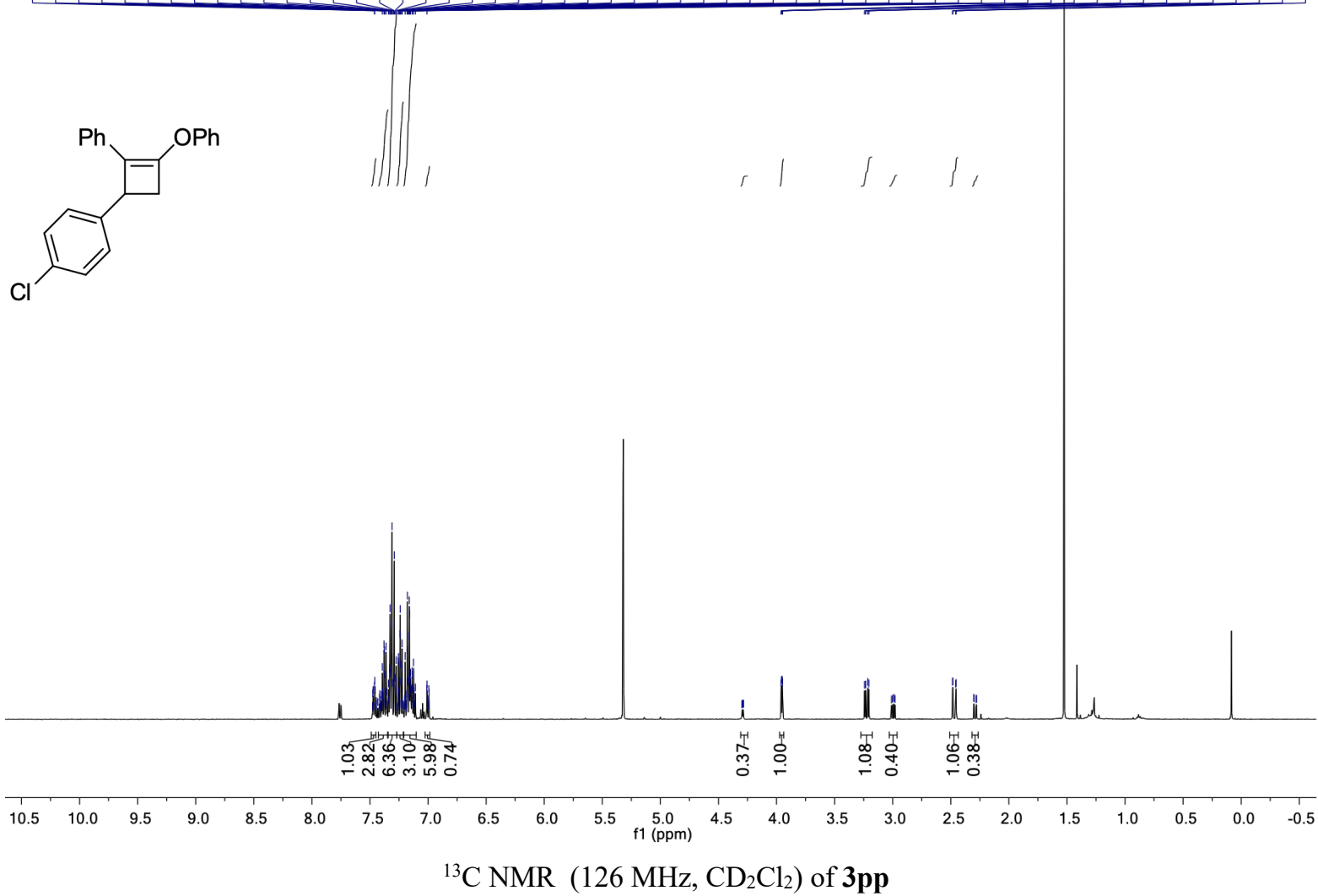

$13 \mathrm{C}\{1 \mathrm{H}\} \mathrm{NMR}(126 \mathrm{MHz}, \mathrm{CD} 2 \mathrm{Cl} 2)$

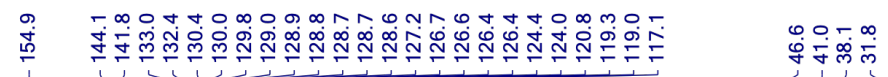<smiles>Clc1ccc(C2CC(Oc3ccccc3)=C2c2ccccc2)cc1</smiles>

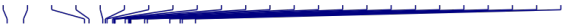

$$
\mathrm{Cl}^{\prime}
$$




\section{2-Benzylidene-4-phenylchromane (S3)}

${ }^{1} \mathbf{H}$ NMR $\left(500 \mathrm{MHz}, \mathrm{CD}_{2} \mathrm{Cl}_{2}\right) \delta 7.42-7.21(\mathrm{~m}, 8 \mathrm{H}, \mathrm{E}+\mathrm{Z}), 7.21-7.10(\mathrm{~m}, 3 \mathrm{H}, \mathrm{E}+\mathrm{Z}), 7.10-7.06(\mathrm{~m}, 2 \mathrm{H}, \mathrm{E})$, $7.02-6.98(\mathrm{~m}, 3 \mathrm{H}, \mathrm{E}+\mathrm{Z}), 6.96-6.82(\mathrm{~m}, 3 \mathrm{H}, \mathrm{E}+\mathrm{Z}), 6.35(\mathrm{~s}, 1 \mathrm{H}, \mathrm{E}), 5.65(\mathrm{~s}, 0.25 \mathrm{H}, \mathrm{Z}), 4.30(\mathrm{t}, J=7.7 \mathrm{~Hz}, 0.25 \mathrm{H}$, Z), 4.16 (t, $J=6.0 \mathrm{~Hz}, 1 \mathrm{H}, \mathrm{E}), 3.08$ (ddd, $J=14.4,5.4,1.2 \mathrm{~Hz}, 1 \mathrm{H}, \mathrm{E}), 3.01$ (ddd, $J=14.5,6.7,0.9 \mathrm{~Hz}, 1 \mathrm{H}, \mathrm{E})$, $3.00-2.91(\mathrm{~m}, 0.25 \mathrm{H}, \mathrm{Z}), 2.82-2.74(\mathrm{~m}, 0.25 \mathrm{H}, \mathrm{Z})$.

${ }^{13}$ C NMR $\left(126 \mathrm{MHz}, \mathrm{CD}_{2} \mathrm{Cl}_{2}\right) \delta 153.5,150.2,142.3,135.9,133.1,130.3,130.2,130.1,130.1,129.5,129.3$, 129.2, 129.1, 129.0, 128.8, 126.6, 125.8, 124.9, 122.2, 121.1, 116.8, 109.5, 40.9, 31.1. for the ${ }^{13} \mathrm{C}$ NMR just the signals of the major isomer are reported.

HRMS (APCI) $m / z$ calculated for $\mathrm{C}_{22} \mathrm{H}_{28} \mathrm{ClO}^{+}[\mathrm{M}+\mathrm{H}]^{+}: 333.1041$, found: 333.1045 .

${ }^{1} \mathrm{H}$ NMR $\left(500 \mathrm{MHz}, \mathrm{CD}_{2} \mathrm{Cl}_{2}\right)$ of $\mathbf{S 3}$

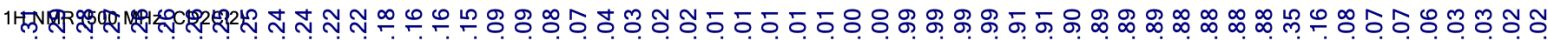

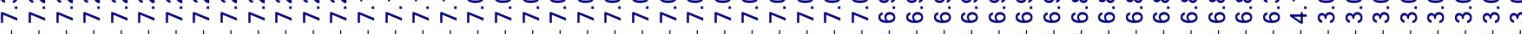<smiles>Clc1ccc(C2CC(=Cc3ccccc3)Oc3ccccc32)cc1</smiles>
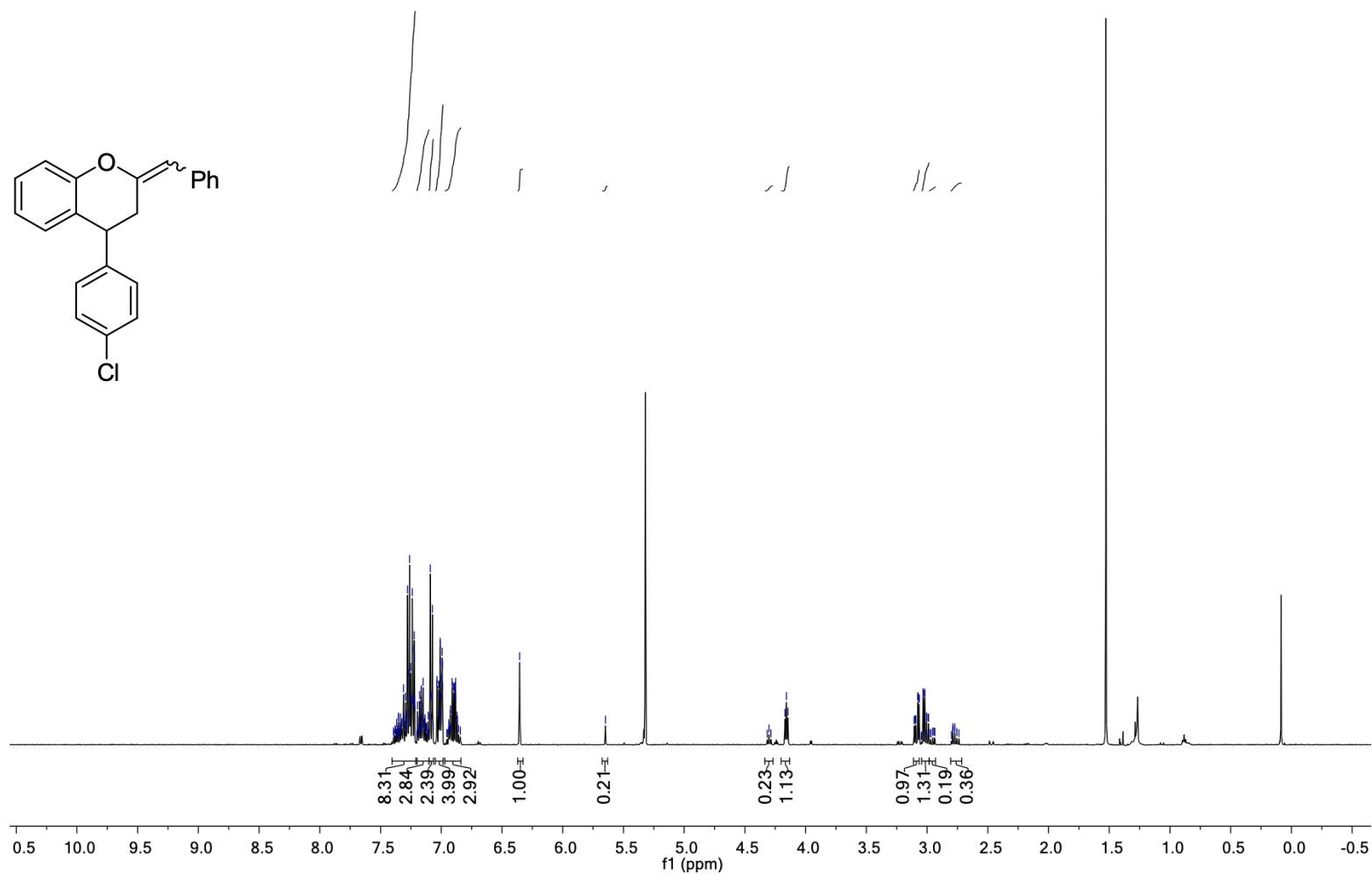
${ }^{13} \mathrm{C}$ NMR $\left(126 \mathrm{MHz}, \mathrm{CD}_{2} \mathrm{Cl}_{2}\right)$ of $\mathbf{S 3}$

$13 \mathrm{C}\{1 \mathrm{H}\}$ NMR (126 MHz, CD2Cl2)

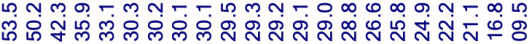

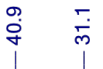<smiles>Clc1ccc(C2C/C(=C\c3ccccc3)Oc3ccccc32)cc1</smiles>

$\begin{array}{lllllllllllllllllllllll}210 & 200 & 190 & 180 & 170 & 160 & 150 & 140 & 130 & 120 & 110 & 100 & 90 & 80 & 70 & 60 & 50 & 40 & 30 & 20 & 10 & 0\end{array}$ 


\section{1-Methoxy-4-(3-methyl-2-phenoxy-3-phenylcyclobut-1-en-1-yl)benzene (3qq)}

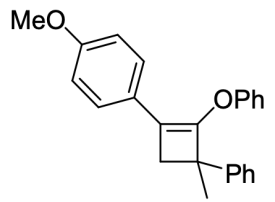

Cyclobutene $\mathbf{3 q q}$ was synthesized following general procedure $\mathbf{C}$ starting from 1-methoxy-4(phenoxyethynyl)benzene $(44.8 \mathrm{mg}, 0.2 \mathrm{mmol})$ and $\alpha$-methylstyrene $(52 \mu \mathrm{L}, 0.4 \mathrm{mmol})$. The crude product was purified by flash chromatography affording 3qq as colorless oil, (29.4 mg, 43\%).

${ }^{1}$ H NMR $\left(400 \mathrm{MHz}, \mathrm{CDCl}_{3}\right) \delta 7.50-7.43(\mathrm{~m}, 2 \mathrm{H}), 7.34-7.28(\mathrm{~m}, 2 \mathrm{H}), 7.26-7.17(\mathrm{~m}, 3 \mathrm{H}), 7.16-7.10(\mathrm{~m}$, 2H), $7.10-7.00(\mathrm{~m}, 3 \mathrm{H}), 6.81-6.74(\mathrm{~m}, 2 \mathrm{H}), 3.78(\mathrm{~s}, 3 \mathrm{H}), 2.70(\mathrm{~d}, J=10.4 \mathrm{~Hz}, 1 \mathrm{H}), 2.61(\mathrm{~d}, J=10.4 \mathrm{~Hz}$, $1 \mathrm{H}), 1.63(\mathrm{~s}, 3 \mathrm{H})$.

${ }^{13}$ C NMR $\left(101 \mathrm{MHz}, \mathrm{CDCl}_{3}\right) \delta$ 158.7, 155.5, 145.5, 145.2, 129.5, 128.4, 128.1, 126.3, 126.2, 123.1, 119.4, 118.1, $113.8,55.4,51.4,39.2,25.2$.

HRMS (APCI) $m / z$ calculated for $\mathrm{C}_{24} \mathrm{H}_{22} \mathrm{O}_{2}{ }^{+}[\mathrm{M}+\mathrm{H}]^{+}: 343.1693$, found: 343.1695 .

\section{${ }^{1} \mathrm{H} \mathrm{NMR}\left(400 \mathrm{MHz}, \mathrm{CDCl}_{3}\right)$ of $\mathbf{3 q q}$}

\section{$13 \mathrm{C}\{1 \mathrm{H}\} \mathrm{NMR}(126 \mathrm{MHz}, \mathrm{CDCl} 3)$}

\begin{tabular}{|c|c|c|c|c|c|}
\hline 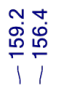 & 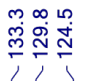 & 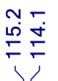 & $\frac{\square}{5}$ & 药苟 & $\dot{j}$ \\
\hline
\end{tabular}
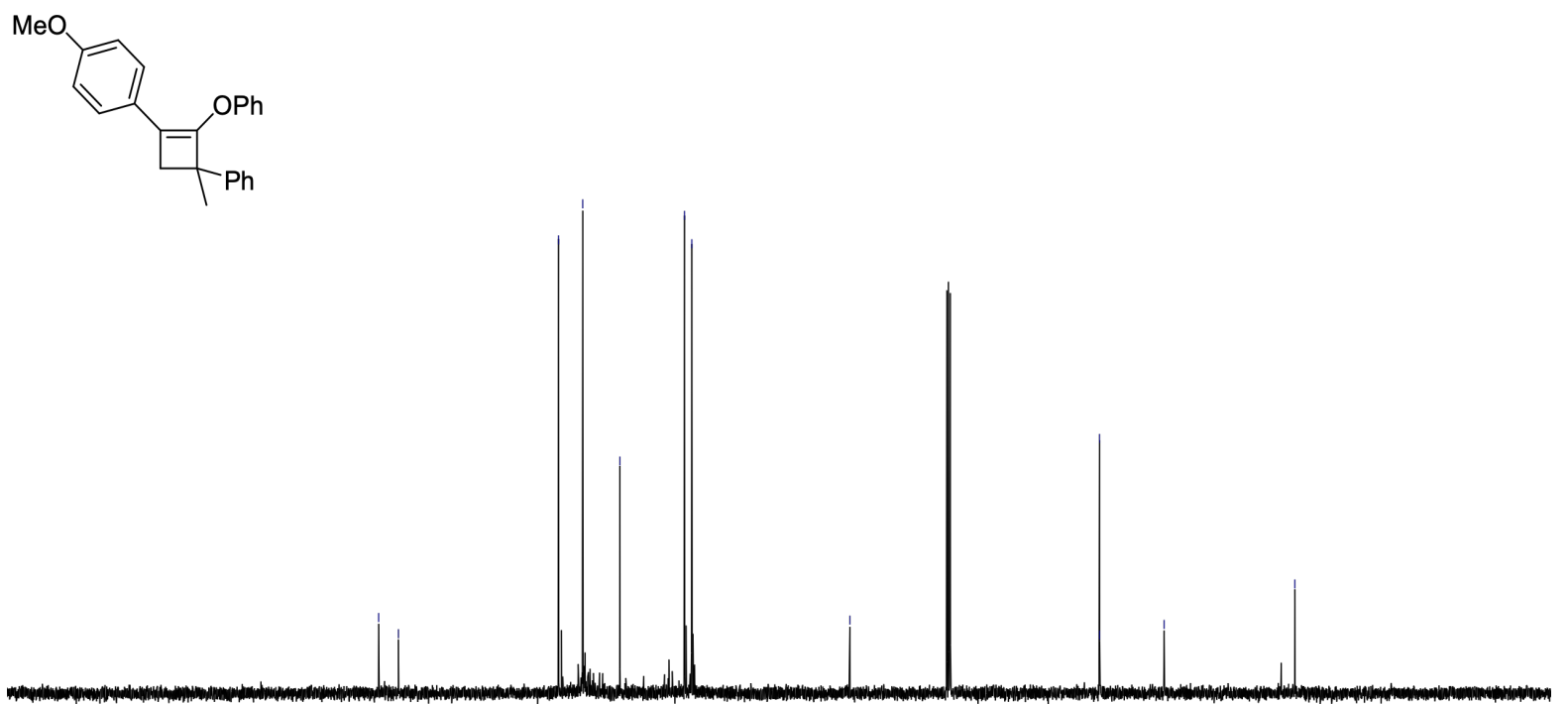

$\begin{array}{llllllllllll}210 & 200 & 190 & 180 & 170 & 160 & 150 & 140 & 130 & 120 & 110 & 100\end{array}$

We performed ${ }^{1} \mathrm{H}$-GOESY experiment on $\mathbf{3 q q}$ irradiating one of the cyclobutene protons to assign the structure of the compound. 

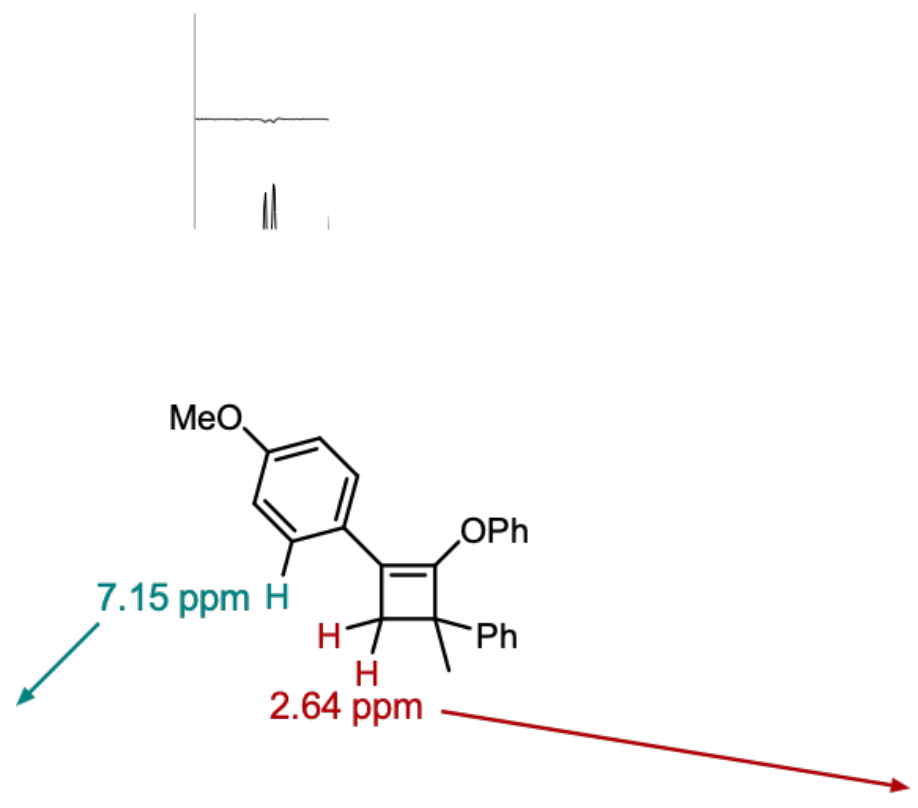

${ }^{13} \mathrm{C}$ NMR $\left(101 \mathrm{MHz}, \mathrm{CDCl}_{3}\right)$ of $\mathbf{3 q q}$

$13 \mathrm{C}\{1 \mathrm{H}\} \mathrm{NMR}(126 \mathrm{MHz}, \mathrm{CDCl} 3)$

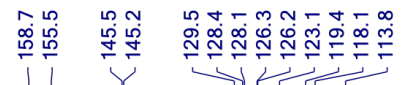

|
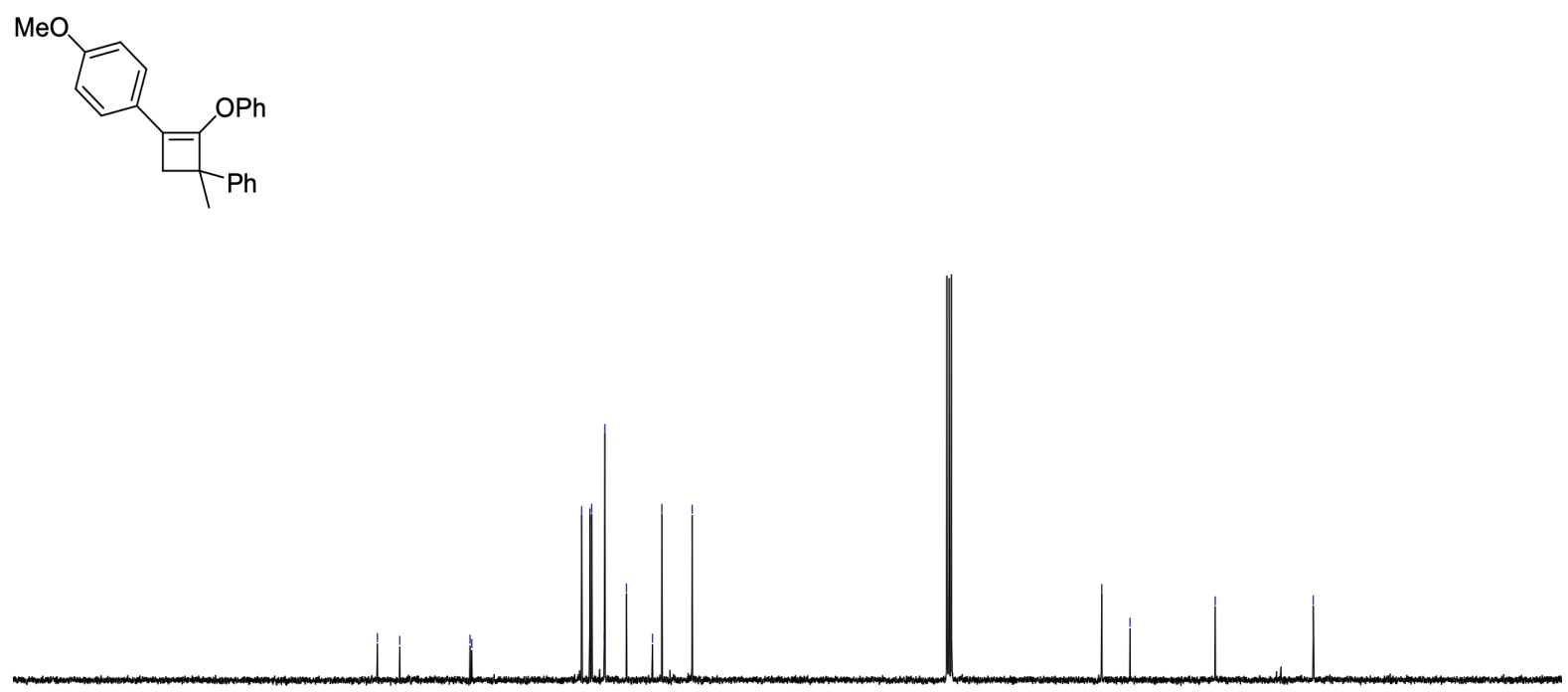

$10 \quad 200$
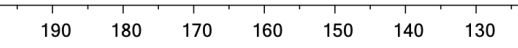

120

$110 \begin{gathered}100 \\ f 1(\mathrm{ppm})\end{gathered}$

S-115 


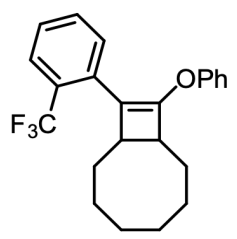

Cyclobutene 3rr was synthesized following general procedure C starting from 1-(phenoxyethynyl)-2(trifluoromethyl)benzene $(52 \mathrm{mg}, 0.2 \mathrm{mmol})$ and $(\mathrm{Z})$-cyclooctene $(52 \mu \mathrm{L}, 0.4 \mathrm{mmol})$. The crude product was purified by flash chromatography affording $3 \mathrm{rr}$ as a yellow oil (52 $\mathrm{mg}, 70 \%)$.

${ }^{1}$ H NMR $\left(400 \mathrm{MHz}, \mathrm{CDCl}_{3}\right) \delta 7.63-7.56(\mathrm{~m}, 1 \mathrm{H}), 7.44-7.33(\mathrm{~m}, 2 \mathrm{H}), 7.29-7.17(\mathrm{~m}, 3 \mathrm{H}), 7.06-6.96(\mathrm{~m}$, $3 \mathrm{H}), 3.15(\mathrm{ddd}, J=11.7,4.4,2.0 \mathrm{~Hz}, 1 \mathrm{H}), 3.07-2.88(\mathrm{~m}, 1 \mathrm{H}), 1.77-1.63(\mathrm{~m}, 2 \mathrm{H}), 1.59$ (ddd, $J=12.3,8.7,3.3$ $\mathrm{Hz}, 3 \mathrm{H}), 1.46$ (dddd, $J=28.3,16.9,13.5,5.1 \mathrm{~Hz}, 6 \mathrm{H}), 1.34-1.13(\mathrm{~m}, 2 \mathrm{H})$.

${ }^{19}$ F NMR $\left(376 \mathrm{MHz}, \mathrm{CDCl}_{3}\right) \delta-60.4$.

${ }^{13}$ C NMR $\left(101 \mathrm{MHz}, \mathrm{CDCl}_{3}\right) \delta$ 155.1, 147.2, 132.3, 131.8, 131.3, 129.4, 126.7, $125.9(\mathrm{q}, \mathrm{J}=5.6 \mathrm{~Hz}), 124.4$ (q, $\mathrm{J}=273.3), 123.3,120.3,118.4,47.4,43.0,42.9,30.2,30.1,26.3,26.3,25.9,24.3$.

HRMS (APCI) $m / z$ calculated for $\mathrm{C}_{23} \mathrm{H}_{24} \mathrm{~F}_{3} \mathrm{O}^{+}[\mathrm{M}+\mathrm{H}]^{+}: 373.1774$, found: 373.1780 .

${ }^{1} \mathrm{H}$ NMR $\left(400 \mathrm{MHz}, \mathrm{CDCl}_{3}\right)$ of $\mathbf{3 r r}$

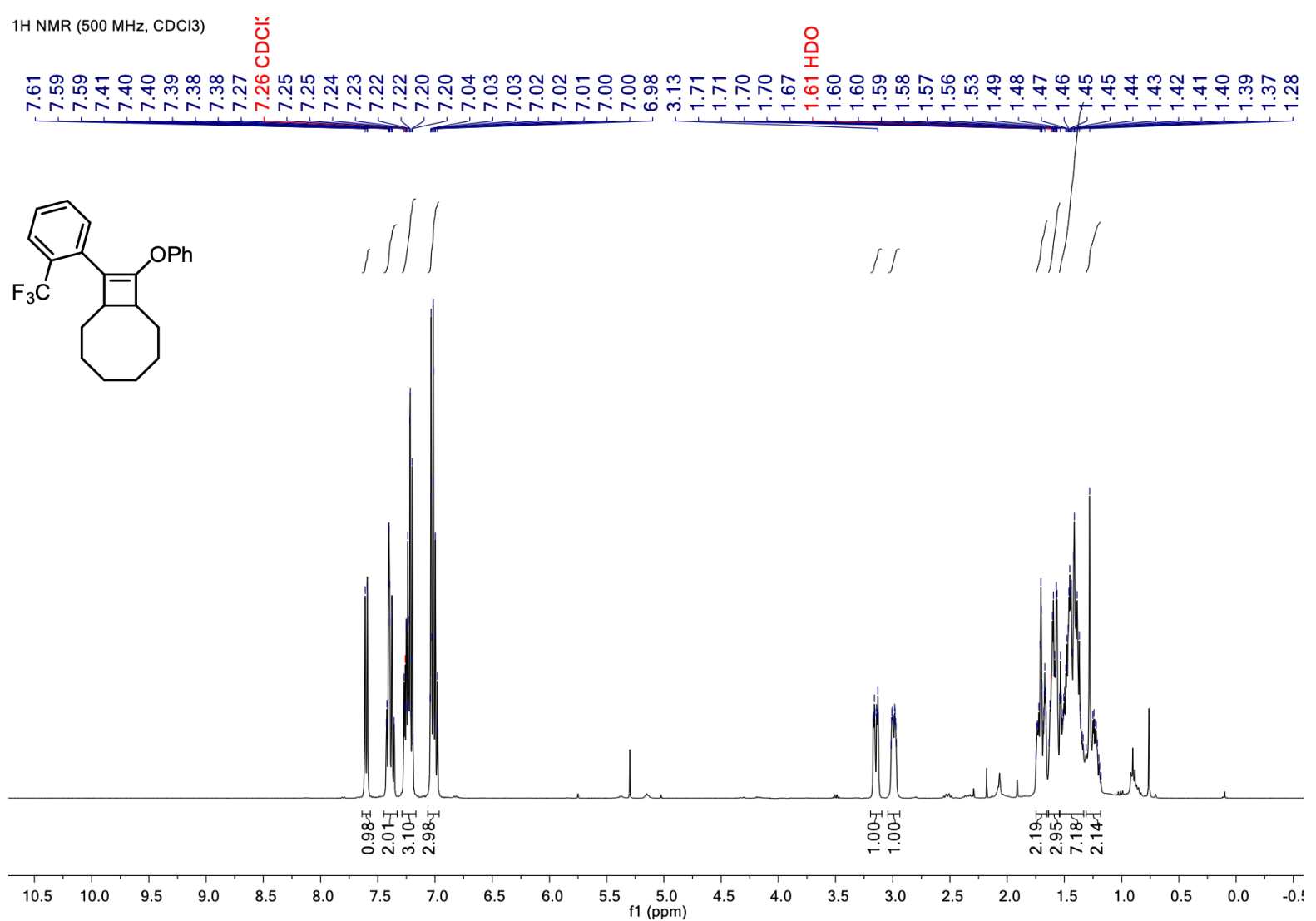

${ }^{19} \mathrm{~F}$ NMR $\left(376 \mathrm{MHz}, \mathrm{CDCl}_{3}\right)$ of $\mathbf{3 r r}$ 


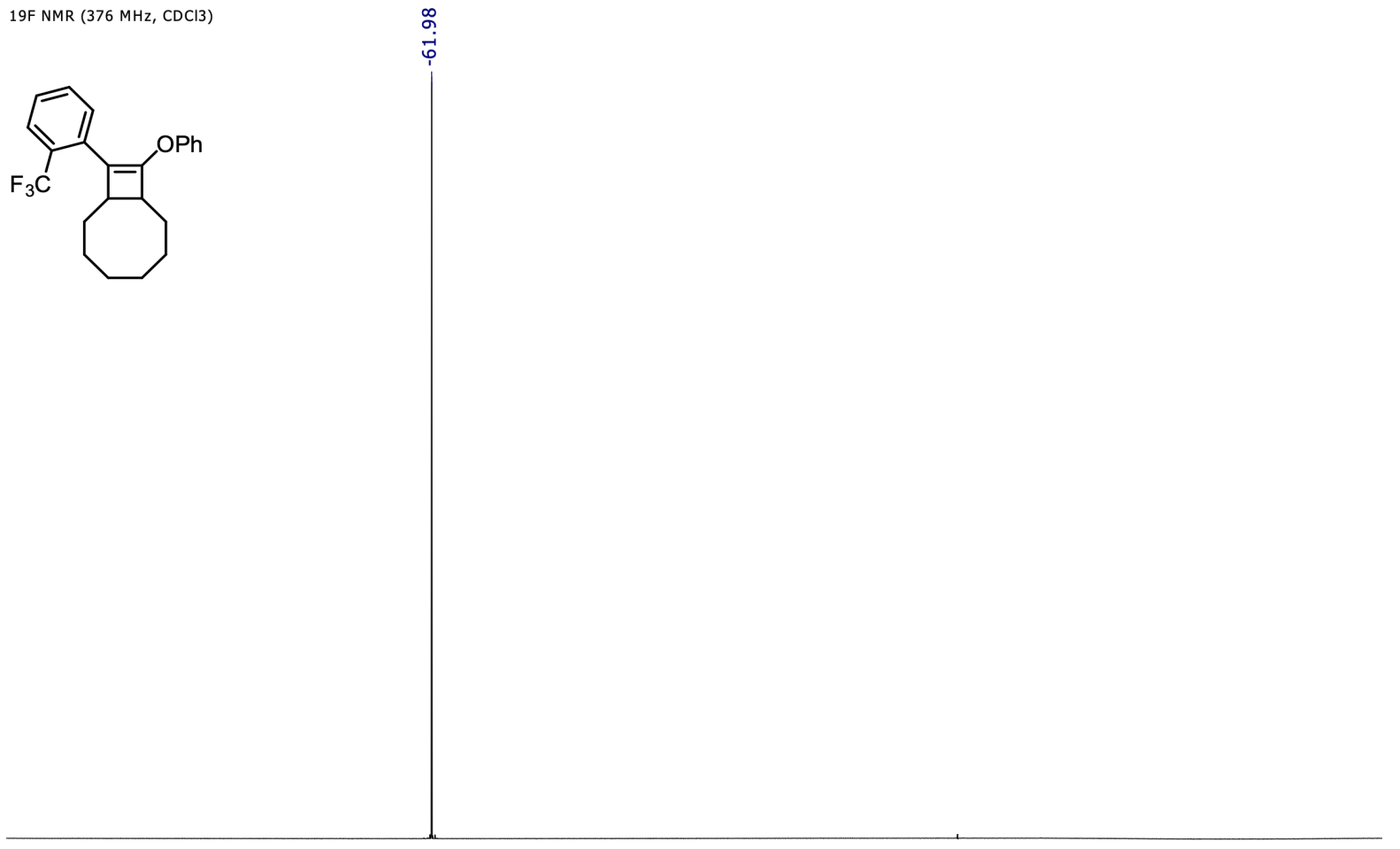

\begin{tabular}{|c|c|c|c|c|c|c|c|c|c|c|c|c|c|c|c|c|c|c|}
\hline-10 & -20 & -30 & -40 & -50 & -60 & -70 & -80 & -90 & 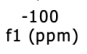 & -110 & -120 & -130 & -140 & -150 & -160 & -170 & -180 & -190 \\
\hline
\end{tabular}

${ }^{13} \mathrm{C}$ NMR $\left(101 \mathrm{MHz}, \mathrm{CDCl}_{3}\right)$ of $\mathbf{3 r r}$

13C \{1H\} NMR (126 MHz, CDCl3)<smiles>[O]</smiles> 


\section{9-Phenoxy-10-(p-tolyl)bicyclo[6.2.0]dec-9-ene (3ss)}

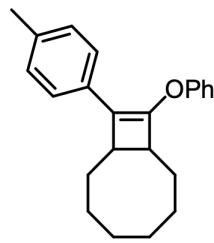

Cyclobutene 3ss was synthesized following general procedure $\mathbf{C}$ starting from 1-methyl-4(phenoxyethynyl)benzene $(41.7 \mathrm{mg}, 0.2 \mathrm{mmol})$ and $(\mathrm{Z})$-cyclooctene $(52 \mu \mathrm{L}, 0.4 \mathrm{mmol})$. The crude product was purified by flash chromatography affording 3 ss as a white oil (46 mg, 72\%).

${ }^{1}$ H NMR (500 MHz, $\left.\mathrm{CDCl}_{3}\right) \delta 7.35$ - $7.29(\mathrm{~m}, 4 \mathrm{H}), 7.15-7.10(\mathrm{~m}, 4 \mathrm{H}), 7.08(\mathrm{dt}, J=7.3,1.2 \mathrm{~Hz}, 1 \mathrm{H}), 3.17$ (ddd, $J=11.7,4.5,2.0 \mathrm{~Hz}, 1 \mathrm{H}), 2.97-2.76(\mathrm{~m}, 1 \mathrm{H}), 2.34(\mathrm{~s}, 3 \mathrm{H}), 2.22-2.04(\mathrm{~m}, 1 \mathrm{H}), 1.71(\mathrm{dtt}, J=8.2,4.5,2.1 \mathrm{~Hz}$, $1 \mathrm{H}), 1.64-1.44(\mathrm{~m}, 8 \mathrm{H}), 1.39(\mathrm{dtd}, J=10.0,7.2,3.4 \mathrm{~Hz}, 1 \mathrm{H}), 1.27-1.15(\mathrm{~m}, 1 \mathrm{H})$.

${ }^{13} \mathbf{C}$ NMR $\left(126 \mathrm{MHz} \mathrm{CDCl}_{3}\right) \delta 155.5,144.1,136.0,130.1,129.6,129.1,126.6,123.3,123.0,117.9,47.9,39.3$, $30.5,30.1,26.4,26.1,25.5,24.4,21.4$.

HRMS (ESI) $m / z$ calculated for $\mathrm{C}_{23} \mathrm{H}_{26} \mathrm{NaO}^{+}[\mathrm{M}+\mathrm{Na}]^{+}: 341.1876$, found: 341.1866 .

${ }^{1} \mathrm{H} \mathrm{NMR}\left(500 \mathrm{MHz}, \mathrm{CDCl}_{3}\right)$ of $\mathbf{3 s s}$

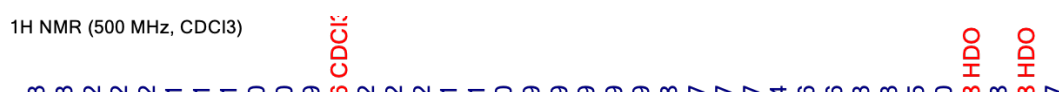

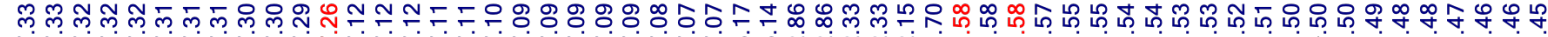

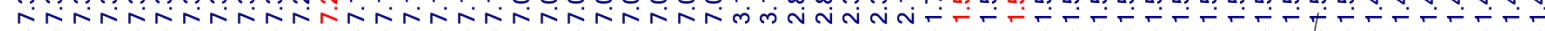<smiles>Cc1ccc(C2=C(Oc3ccccc3)C3CCCCCCC23)cc1</smiles>
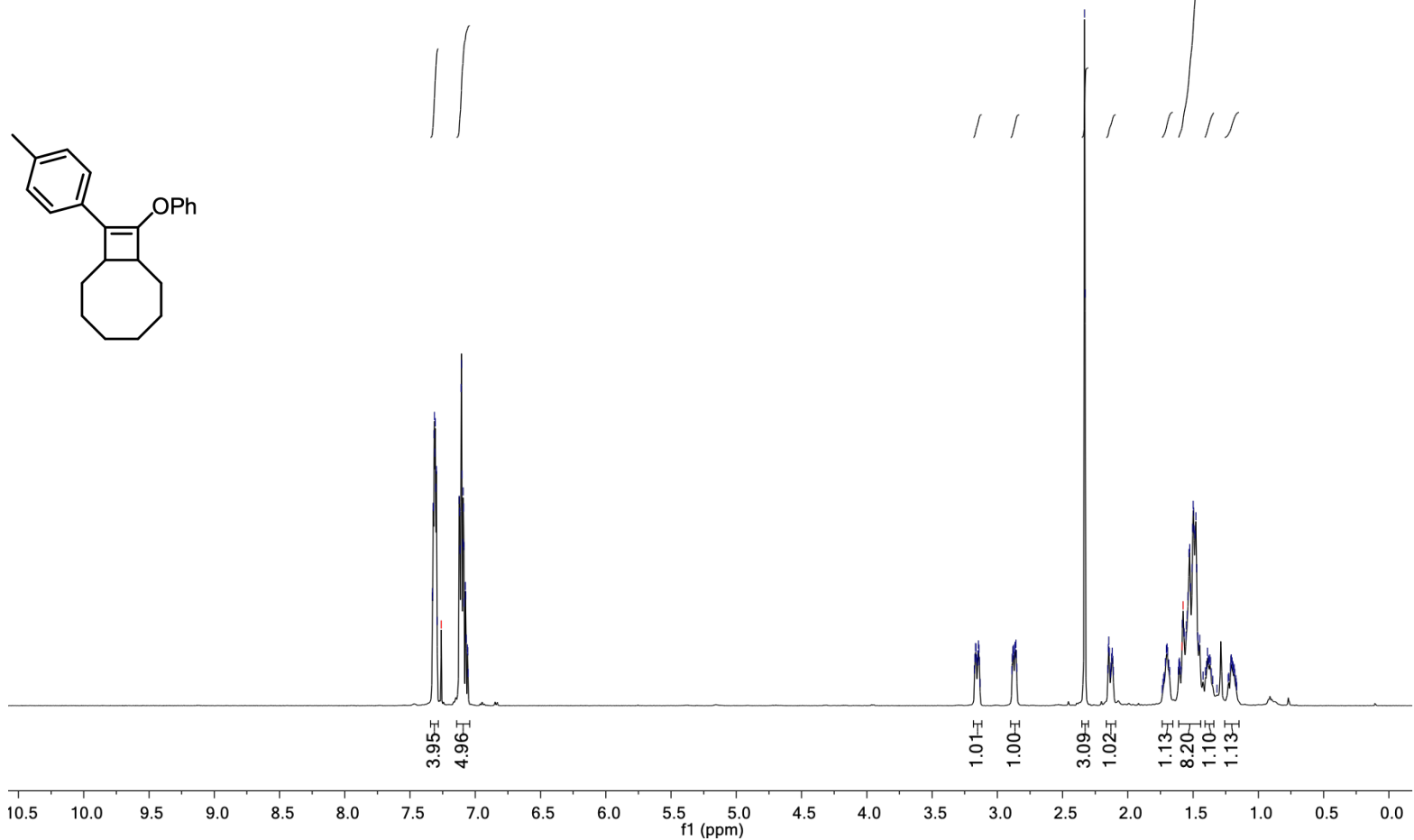

${ }^{13} \mathrm{C} \mathrm{NMR}\left(126 \mathrm{MHz}, \mathrm{CDCl}_{3}\right)$ of 3ss 


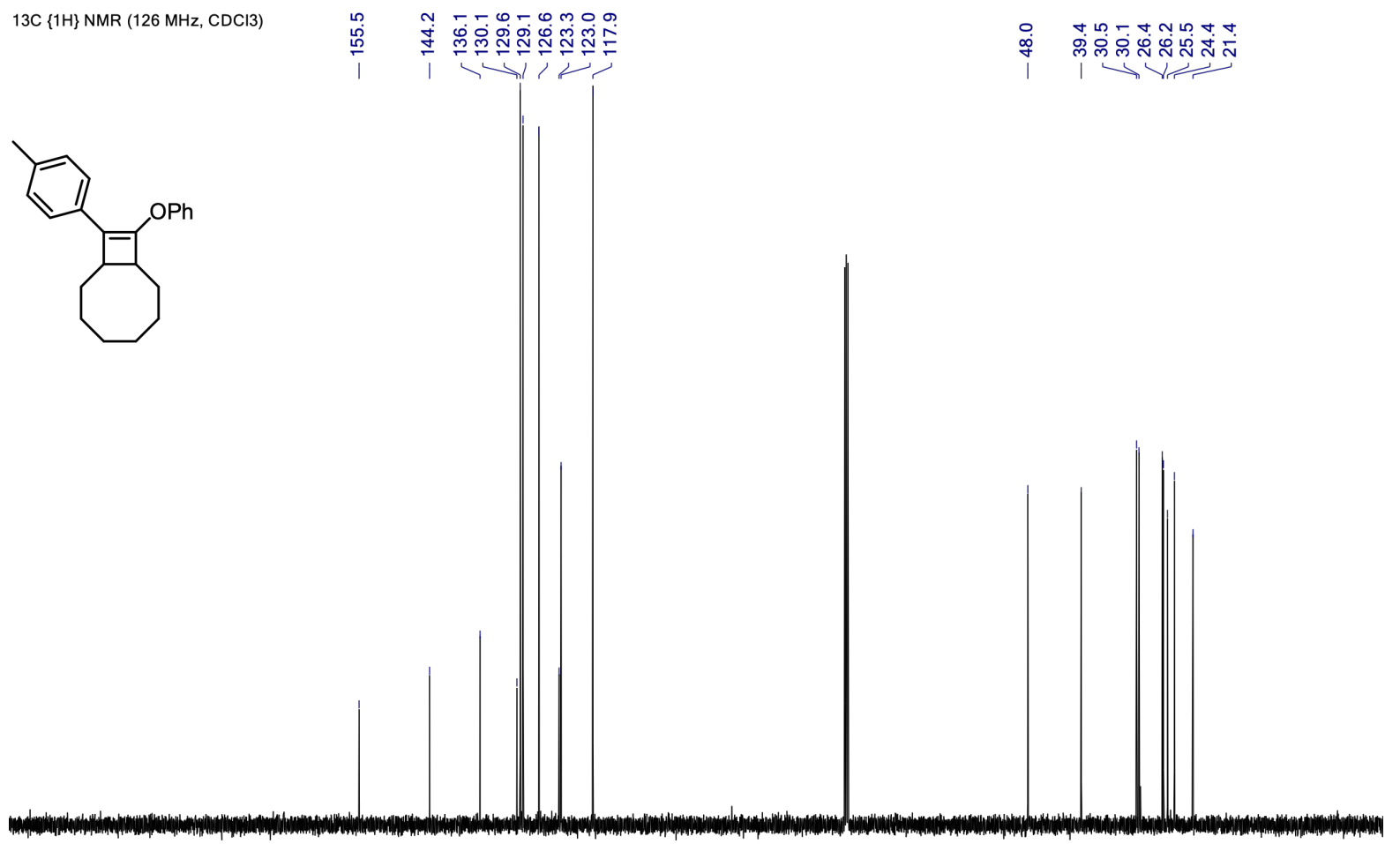

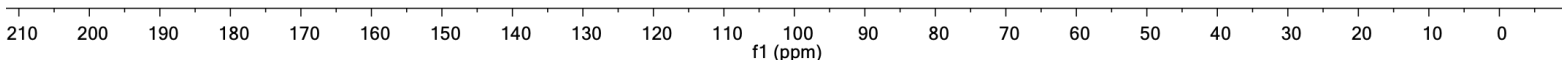




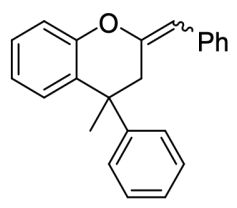

Chromane 4a was synthesized following general procedure $\mathbf{C}$ starting from (phenoxyethynyl)benzene (38 mg, 0.2 mmol) and $\alpha$-methylstyrene $(52 \mu \mathrm{L}, 0.4 \mathrm{mmol})$. The crude product was purified by flash chromatography affording $4 \mathbf{a}$ as a greenish oil $(E: Z=1: 1,47 \mathrm{mg}, 76 \%)$.

${ }^{1} \mathbf{H}$ NMR (400 MHz, $\left.\mathrm{CDCl}_{3}\right) \delta 7.34-7.17(\mathrm{~m}, 13 \mathrm{H}, \mathrm{E}+\mathrm{Z}), 7.17-7.09$ (m, 4H, E+Z), $7.09-7.00$ (m, 6H, E+Z), $6.98-6.88(\mathrm{~m}, 4 \mathrm{H}, \mathrm{E}+\mathrm{Z}), 6.30(\mathrm{~s}, 1 \mathrm{H}, \mathrm{E}), 5.56(\mathrm{~d}, J=1.1 \mathrm{~Hz}, 1 \mathrm{H}, \mathrm{Z}), 3.21(\mathrm{dd}, J=14.5,0.9 \mathrm{~Hz}, 1 \mathrm{H}, \mathrm{E}), 3.06$ (d, $J=16.7 \mathrm{~Hz}, 1 \mathrm{H}, \mathrm{Z}), 2.83(\mathrm{dd}, J=14.5,1.2 \mathrm{~Hz}, 1 \mathrm{H}, \mathrm{E}), 2.71$ (dd, $J=16.7,1.2 \mathrm{~Hz}, 1 \mathrm{H}, \mathrm{Z}), 1.75$ (s, $3 \mathrm{H}), 1.69$ (s, $3 \mathrm{H})$.

${ }^{13}$ C NMR $\left(101 \mathrm{MHz}, \mathrm{CDCl}_{3}\right) \delta 156.9,154.9,152.5,150.1,147.9,146.6,139.9,135.8,134.7,131.1,129.7,129.1$, $128.3,128.3$, 128.2, 128.1, 127.6, 127.2, 127.0, 127.0, 126.6, 126.4, 126.3, 126.1, 125.9, 124.3, 121.7, 120.8, 116.6, 108.7, 104.4, 44.4, 42.1, 40.3, 38.1, 27.8, 27.8 .

HRMS (ESI) $m / z$ calculated for $\mathrm{C}_{2} 3 \mathrm{H}_{20} \mathrm{NaO}^{+}[\mathrm{M}+\mathrm{Na}]^{+}: 335.1406$, found: 335.1420 .

${ }^{1} \mathrm{H} \mathrm{NMR}\left(400 \mathrm{MHz}, \mathrm{CDCl}_{3}\right)$ of $\mathbf{4 a}$

$1 \mathrm{H}$ NMR $(500 \mathrm{MHz}, \mathrm{CDCl} 3)$

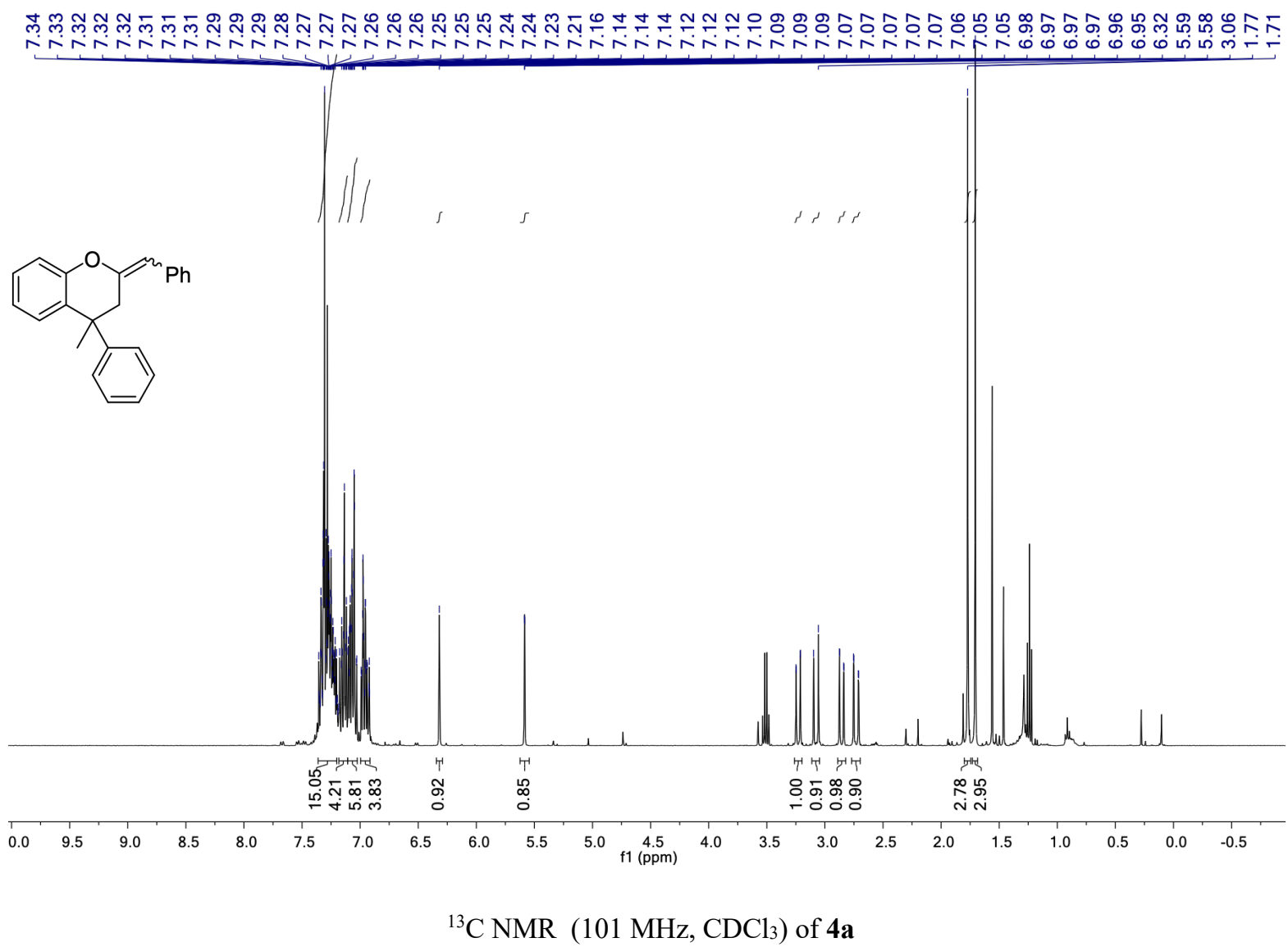




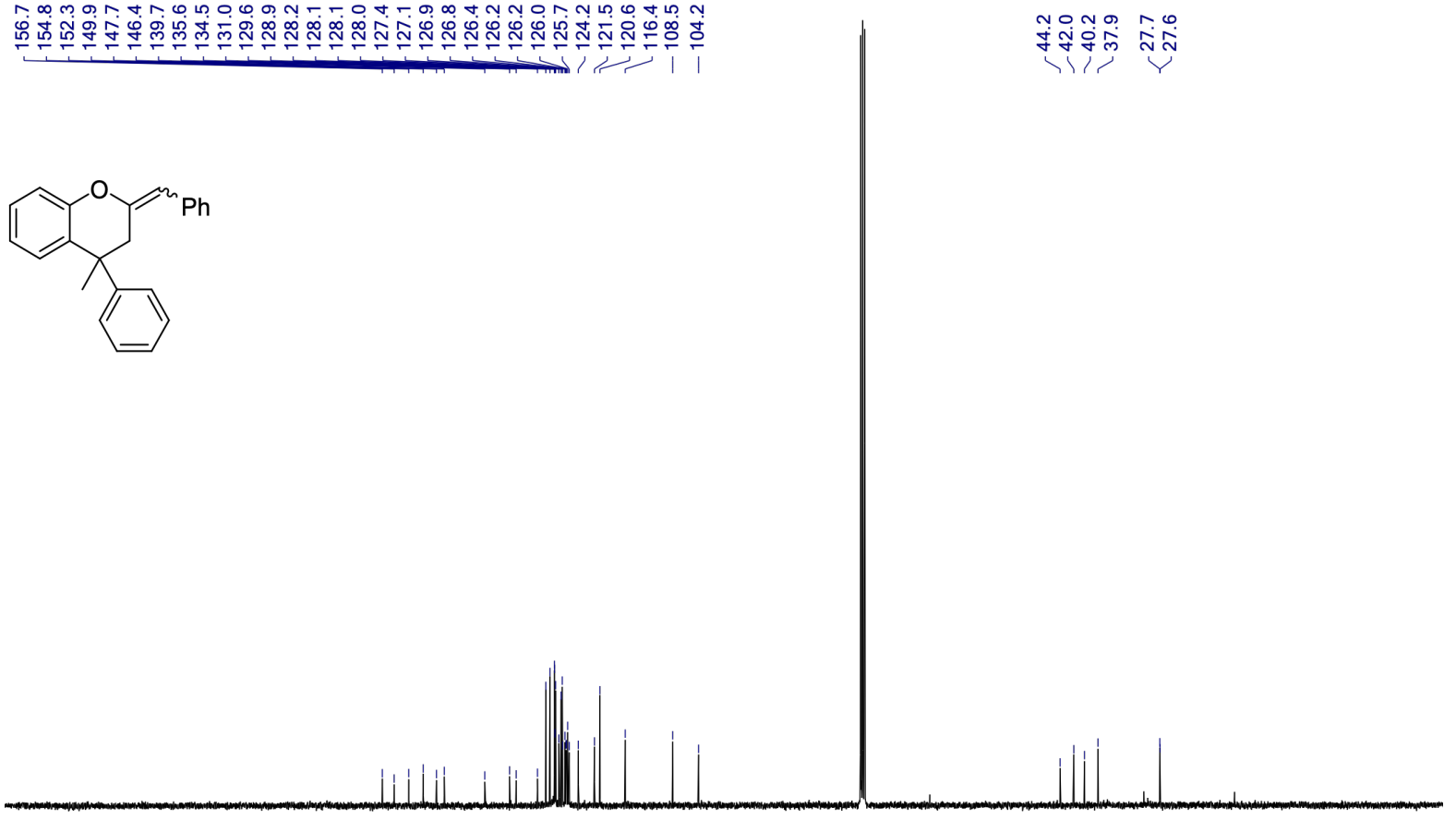

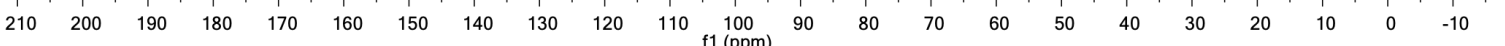


Chromane $\mathbf{4 b}$ was synthesized following general procedure $\mathbf{C}$ starting from (phenoxyethynyl)benzene (38 $\mathrm{mg}$, $0.2 \mathrm{mmol}$ ) and 1-fluoro-4-(prop-1-en-2-yl)benzene $(54 \mu \mathrm{L}, 0.4 \mathrm{mmol}$ ). The crude product was purified by flash chromatography affording $\mathbf{4 b}$ as a colorless oil ( $E: Z=1: 1,46 \mathrm{mg}, 70 \%)$.

${ }^{1} \mathbf{H}$ NMR $\left(500 \mathrm{MHz}, \mathrm{CD}_{2} \mathrm{Cl}_{2}\right) \delta 7.38-7.32(\mathrm{~m}, 2 \mathrm{H}, \mathrm{E}+\mathrm{Z}), 7.31-7.26(\mathrm{~m}, 2 \mathrm{H}, \mathrm{E}+\mathrm{Z}), 7.26-7.21$ (m, 3H, E+Z), $7.21-7.15$ (m, 1H, E+Z), 7.15 - 7.11 (m, 2H, E+Z), $7.10-7.08$ (m, 2H, E+Z), $7.08-7.05$ (m, 2H, E+Z), $7.05-$ $6.94(\mathrm{~m}, 8 \mathrm{H}, \mathrm{E}+\mathrm{Z}), 6.93-6.88(\mathrm{~m}, 3 \mathrm{H}, \mathrm{E}+\mathrm{Z}), 6.25(\mathrm{~s}, 1 \mathrm{H}, \mathrm{E}), 5.52(\mathrm{~d}, J=1.3 \mathrm{~Hz}, 1 \mathrm{H}, \mathrm{Z}), 3.25(\mathrm{dd}, J=14.6,0.7$ Hz, 1H, E), 2.98 (d, $J=16.6 \mathrm{~Hz}, 1 \mathrm{H}, \mathrm{Z}$ ), 2.80 (dd, $J=14.6,1.4 \mathrm{~Hz}, 1 \mathrm{H}, \mathrm{E}), 2.74$ (dd, $J=16.6,1.4 \mathrm{~Hz}, 1 \mathrm{H}, \mathrm{Z}$ ), $1.73(\mathrm{~s}, 3 \mathrm{H}), 1.67(\mathrm{~s}, 3 \mathrm{H})$.

${ }^{19} \mathbf{F}$ NMR $\left(471 \mathrm{MHz}, \mathrm{CD}_{2} \mathrm{Cl}_{2}\right) \delta-117.8,-118.2 .\left({ }^{19} \mathrm{~F}\right.$ NMR shows the presence of an impurity probably arising from the counterion of the cationic gold(I) complex used in the reaction)

${ }^{13}$ C NMR $\left(126 \mathrm{MHz}, \mathrm{CD}_{2} \mathrm{Cl}_{2}\right) \delta 162.9,161.0,157.5,155.2,152.8,150.5,144.1,144.1,143.0,140.0,136.1$, 135.1, 131.4, 130.6, 130.2, 129.4, 129.2, 129.2, 129.1, 129.1, 129.0, 128.8, 128.8, 127.7, 127.5, 126.6, 126.6, $126.5,126.3,125.0,122.2,121.2,117.0,115.4,115.2,115.1,115.0,109.0,104.4,44.2,42.4,40.3,38.3,28.4$, 28.3 .

HRMS (APCI) $m / z$ calculated for $\mathrm{C}_{23} \mathrm{H}_{20} \mathrm{FO}^{+}[\mathrm{M}+\mathrm{H}]^{+}: 331.1493$, found: 331.1495 . 


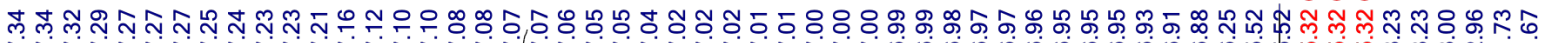

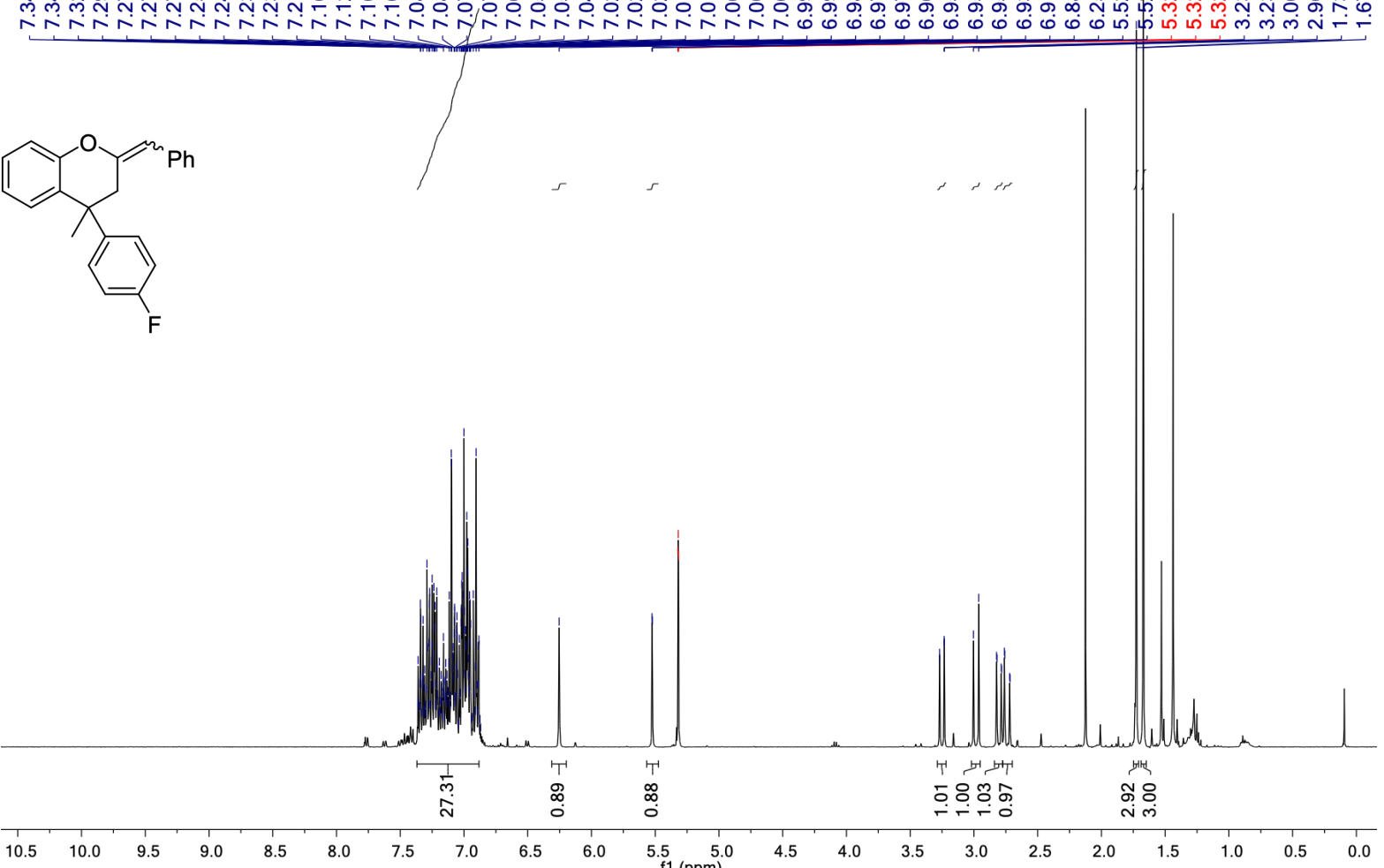

${ }^{19} \mathrm{~F}$ NMR $\left(471 \mathrm{MHz}, \mathrm{CD}_{2} \mathrm{Cl}_{2}\right)$ of $\mathbf{4 b}$

$19 F$ NMR (471 MHz, CD2Cl2)

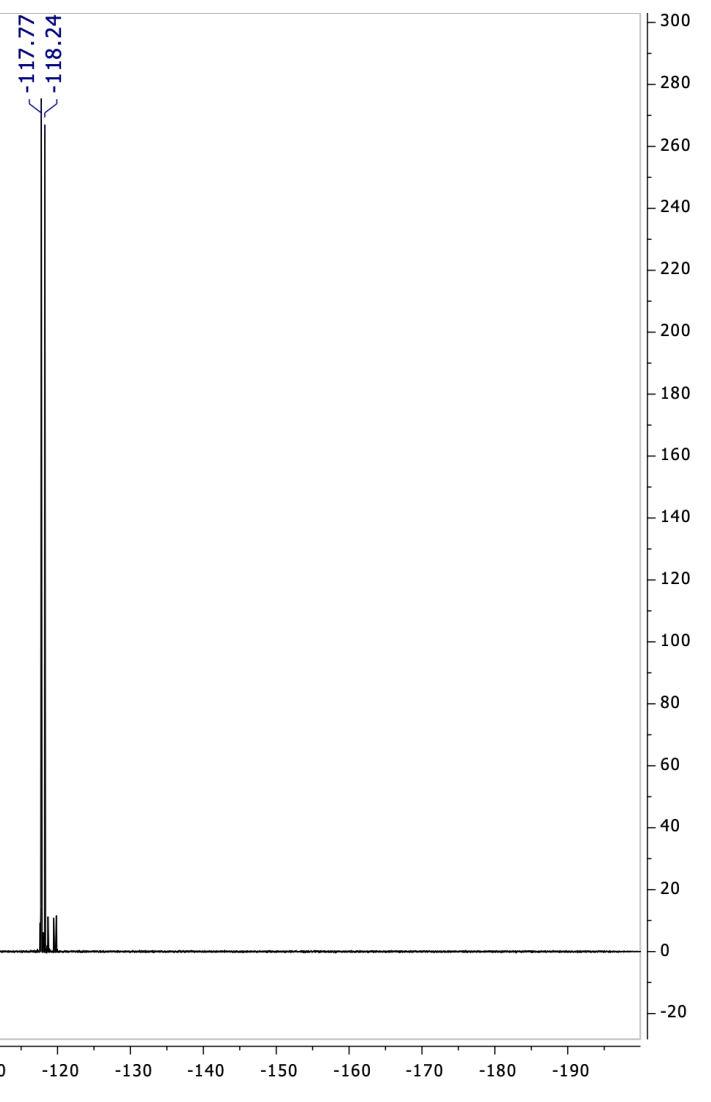


${ }^{13} \mathrm{C}$ NMR $\left(126 \mathrm{MHz}, \mathrm{CD}_{2} \mathrm{Cl}_{2}\right)$ of $\mathbf{4 b}$

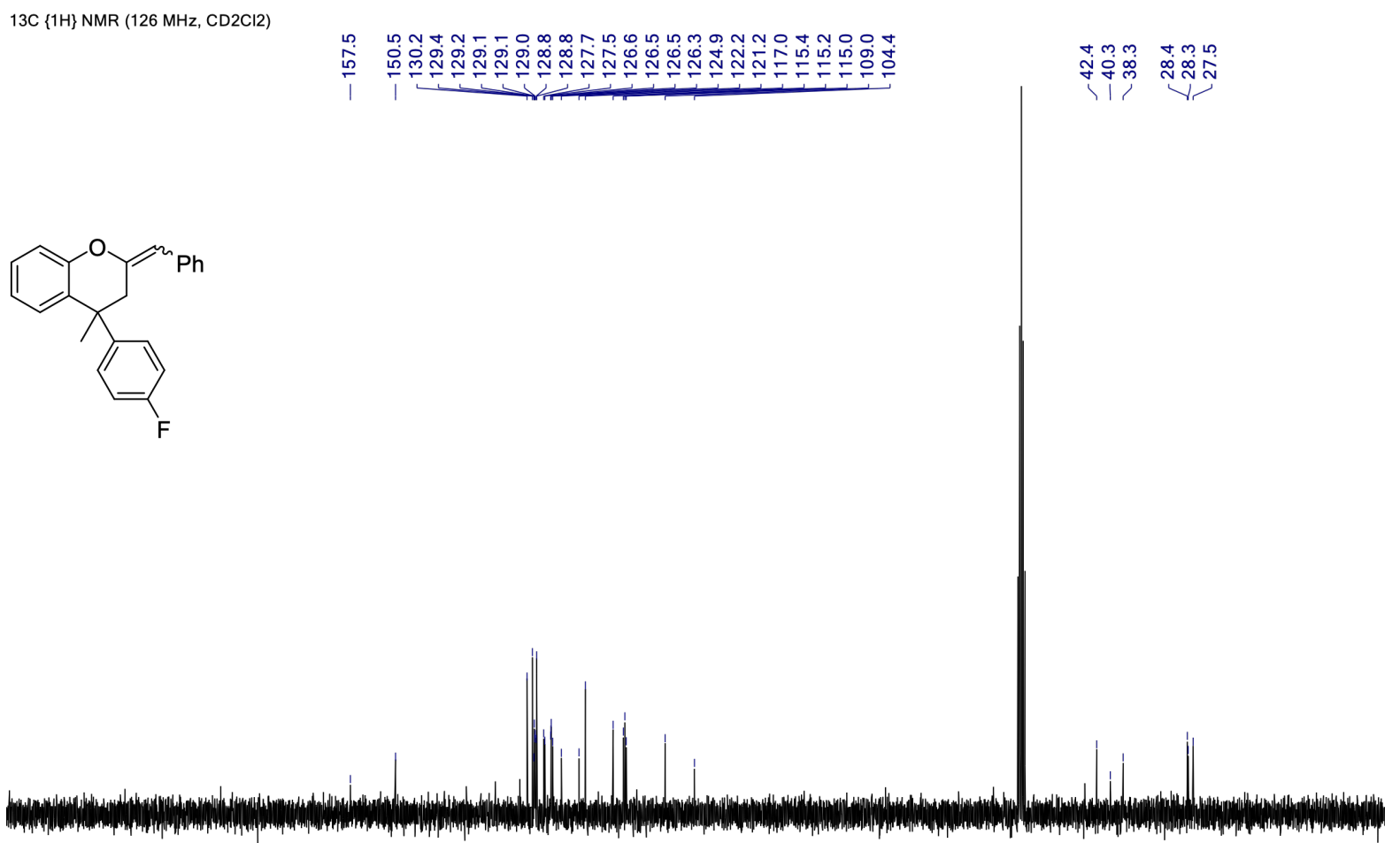

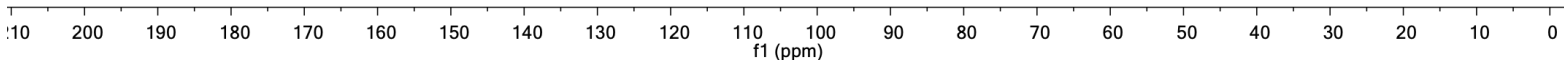


<smiles>CC(C)(C)c1ccc(C2(C)CC(=Cc3ccccc3)Oc3ccccc32)cc1</smiles>

Chromane 4c was synthesized following general procedure $\mathbf{C}$ starting from (phenoxyethynyl)benzene (39 $\mathrm{mg}, 0.2$ $\mathrm{mmol}$ ) and 1-(tert-butyl)-4-(prop-1-en-2-yl)benzene $(70 \mathrm{mg}, 0.4 \mathrm{mmol})$. The crude product was purified by flash chromatography affording $\mathbf{4 c}$ as a yellow oil $(E: Z=1: 2,61 \mathrm{mg}, 83 \%)$.

${ }^{1} \mathbf{H}$ NMR $\left(300 \mathrm{MHz}, \mathrm{CDCl}_{3}\right) \delta 7.38-6.99(\mathrm{~m}, 16 \mathrm{H}, \mathrm{E}+\mathrm{Z}), 6.98-6.89$ (m, 4H, E+Z), $6.34(\mathrm{~s}, 0.4 \mathrm{H}, \mathrm{E}), 5.71-$ $5.55(\mathrm{~m}, 1 \mathrm{H}, \mathrm{Z}), 3.18$ (dd, $J=14.4,1.0 \mathrm{~Hz}, 0.4 \mathrm{H}, \mathrm{E}), 3.06$ (d, $J=16.6 \mathrm{~Hz}, 1 \mathrm{H}, \mathrm{Z}), 2.85(\mathrm{dd}, J=14.4,1.1 \mathrm{~Hz}$, 0,4H, E), 2.72 (dd, $J=16.6,1.3 \mathrm{~Hz}, 1 \mathrm{H}, \mathrm{Z}), 1.78$ (s, 3H, Z), 1.71 (s, 1.2H, E ), 1.36 (s, 9H, Z), 1.33 (d, $J=1.1$ $\mathrm{Hz}, 4 \mathrm{H}, \mathrm{E})$.

${ }^{13}$ C NMR $\left(126 \mathrm{MHz}, \mathrm{CDCl}_{3}\right) \delta 156.7,155.1,150.5,149.0,148.7,147.5,147.3,144.7,140.1,137.9,134.6,129.7$, 129.0, 128.9, 128.5, 127.1, 127.0, 126.9, 126.8, 126.6, 126.4, 126.1, 125.9, 125.1, 125.0, 124.2, 123.2, 120.6, $116.4,108.4,105.0,44.0,42.2,39.9,34.5,31.5,31.5,31.5,30.9,27.8$.

HRMS (APCI) $m / z$ calculated for $\mathrm{C}_{27} \mathrm{H}_{29} \mathrm{O}^{+}[\mathrm{M}+\mathrm{H}]^{+}: 369.2213$, found: 369.2215 .

\section{${ }^{1} \mathrm{H}$ NMR $\left(300 \mathrm{MHz}, \mathrm{CDCl}_{3}\right)$ of $\mathbf{4 c}$}

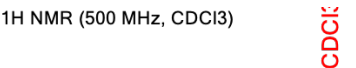

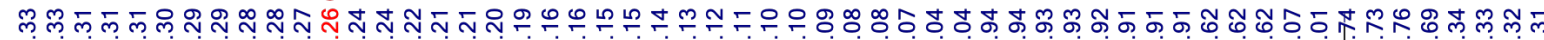

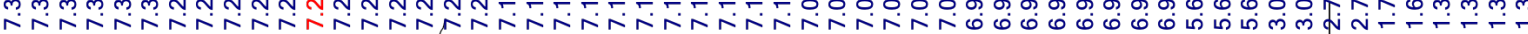

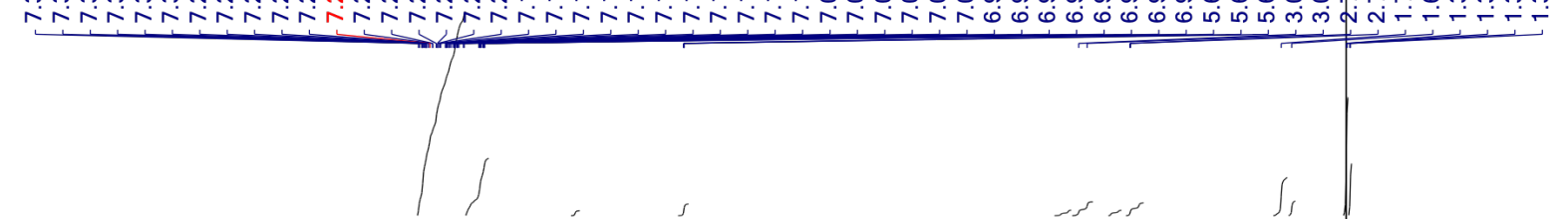<smiles>CC(C)(C)c1ccc(C2(C)C/C(=C\c3ccccc3)Oc3ccccc32)cc1</smiles>

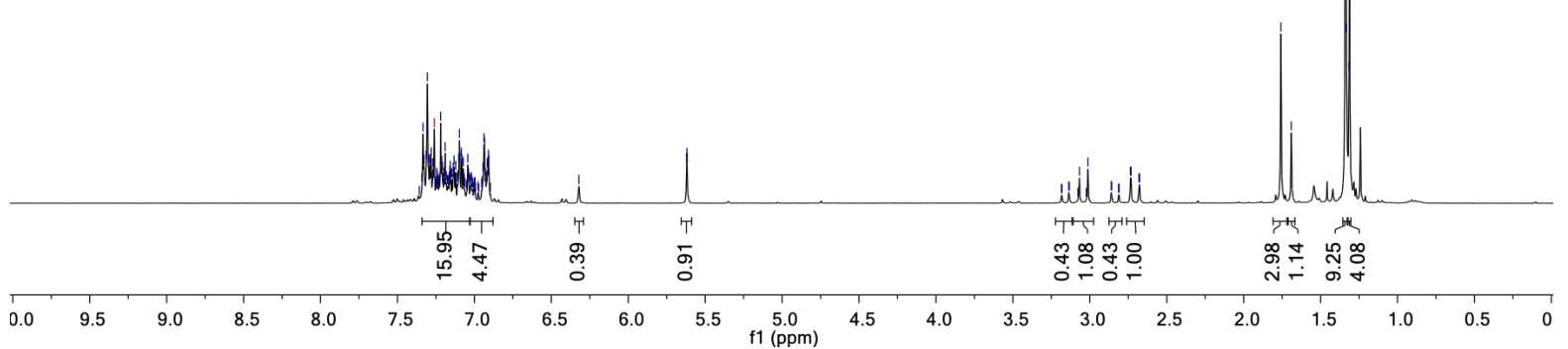


${ }^{13} \mathrm{C}$ NMR $\left(126 \mathrm{MHz}, \mathrm{CDCl}_{3}\right)$ of $\mathbf{4 c}$

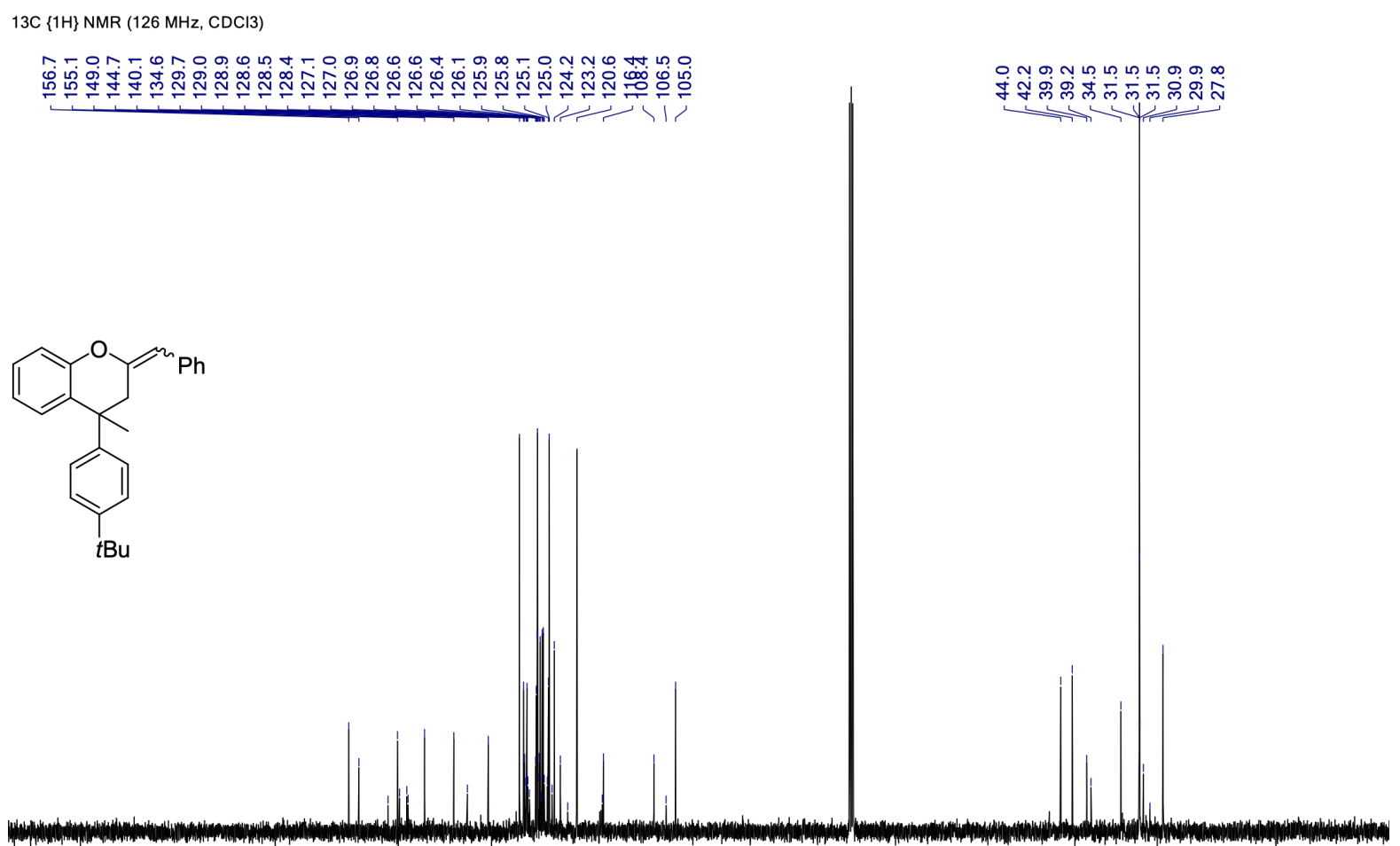

$\begin{array}{llllllllllllllllllllllllll}10 & 200 & 190 & 180 & 170 & 160 & 150 & 140 & 130 & 120 & 110 & \begin{array}{l}100 \\ f 1(\mathrm{pm})\end{array} & 90 & 80 & 70 & 60 & 50 & 40 & 30 & 20 & 10 & 0\end{array}$ 


\section{2-Benzylidene-4-(4-methoxyphenyl)chromane (4d)}<smiles>COc1ccc(C2C/C(=C\c3ccccc3)Oc3ccccc32)cc1</smiles>

Chromane 4d was synthesized following general procedure $\mathbf{C}$ starting from (phenoxyethynyl)benzene (38 $\mathrm{mg}$, $0.2 \mathrm{mmol}$ ) and 1-methoxy-4-vinylbenzene $(54 \mu \mathrm{L}, 0.4 \mathrm{mmol})$. The crude product was purified by flash chromatography affording $\mathbf{4 d}$ as a yellow oil $(E: Z=1: 10,48 \mathrm{mg}, 74 \%)$.

${ }^{1}$ H NMR $\left(500 \mathrm{MHz}, \mathrm{CDCl}_{3}\right) \delta 7.38-7.32(\mathrm{~m}, 2 \mathrm{H}, \mathrm{E}+\mathrm{Z}), 7.25-7.22(\mathrm{~m}, 0.2 \mathrm{H}, \mathrm{E}), 7.22-7.15(\mathrm{~m}, 3 \mathrm{H}, \mathrm{E}+\mathrm{Z})$, $7.15-7.10$ (m, 1H,Z), $7.10-7.06$ (m, 0.2H, E), 6.94 (dd, J=7.5, $1.3 \mathrm{~Hz}, 1 \mathrm{H}, \mathrm{Z}), 6.92-6.87$ (m, 2H, Z), 6.85 $(\mathrm{dq}, J=7.7,0.9 \mathrm{~Hz}, 1 \mathrm{H}, \mathrm{E}+\mathrm{Z}), 6.38(\mathrm{~s}, 0.1 \mathrm{H}, \mathrm{E}), 5.69$ (d, J=1.0 Hz, 1H, Z), $4.36-4.18(\mathrm{~m}, 1 \mathrm{H}, \mathrm{Z}), 4.10$ (dd, $J$ $=7.9,5.2 \mathrm{~Hz}, 0.1 \mathrm{H}, \mathrm{E}), 3.82(\mathrm{~s}, 3 \mathrm{H}, \mathrm{Z}), 3.80(\mathrm{~s}, 0.3 \mathrm{H}, \mathrm{E}), 3.12(\mathrm{ddd}, J=14.3,5.1,0.9 \mathrm{~Hz}, 0.1 \mathrm{H}, \mathrm{E}), 2.97(\mathrm{ddd}, J$ $=14.3,7.9,1.1 \mathrm{~Hz}, 0.1 \mathrm{H}, \mathrm{E}), 2.90(\mathrm{ddd}, J=16.6,7.4,0.9 \mathrm{~Hz}, 1 \mathrm{H}, \mathrm{Z}), 2.84(\mathrm{ddd}, J=16.6,9.3,1.1 \mathrm{~Hz}, 1 \mathrm{H}, \mathrm{Z})$.

${ }^{13}$ C NMR $\left(126 \mathrm{MHz} \mathrm{CDCl}_{3}\right) \delta 158.5,157.0,155.0,135.8,135.3,134.9,129.8,129.4,127.7,127.0,125.8,125.6$, $124.3,120.6,114.1,104.9,55.4,44.5,35.0$.Here just the signal of the major isomer are reported.

HRMS (APCI) $m / z$ calculated for $\mathrm{C}_{23} \mathrm{H}_{21} \mathrm{O}_{2}{ }^{+}[\mathrm{M}+\mathrm{H}]^{+}: 329.1536$, found: 329.1540 .

\section{${ }^{1} \mathrm{H}$ NMR $\left(500 \mathrm{MHz}, \mathrm{CDCl}_{3}\right)$ of $\mathbf{4 d}$}

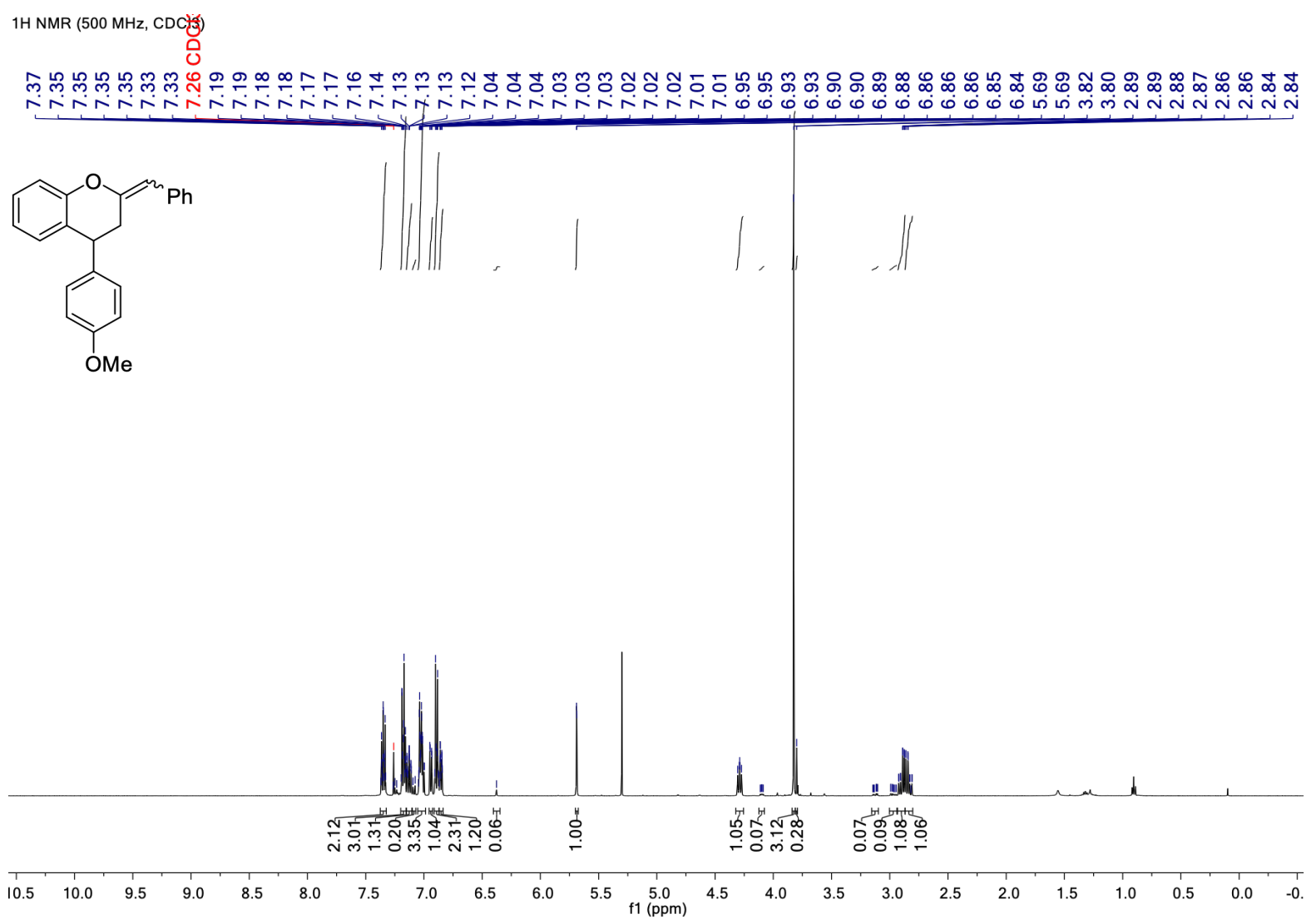


${ }^{13} \mathrm{C}$ NMR $\left(126 \mathrm{MHz}, \mathrm{CDCl}_{3}\right)$ of $\mathbf{4 d}$

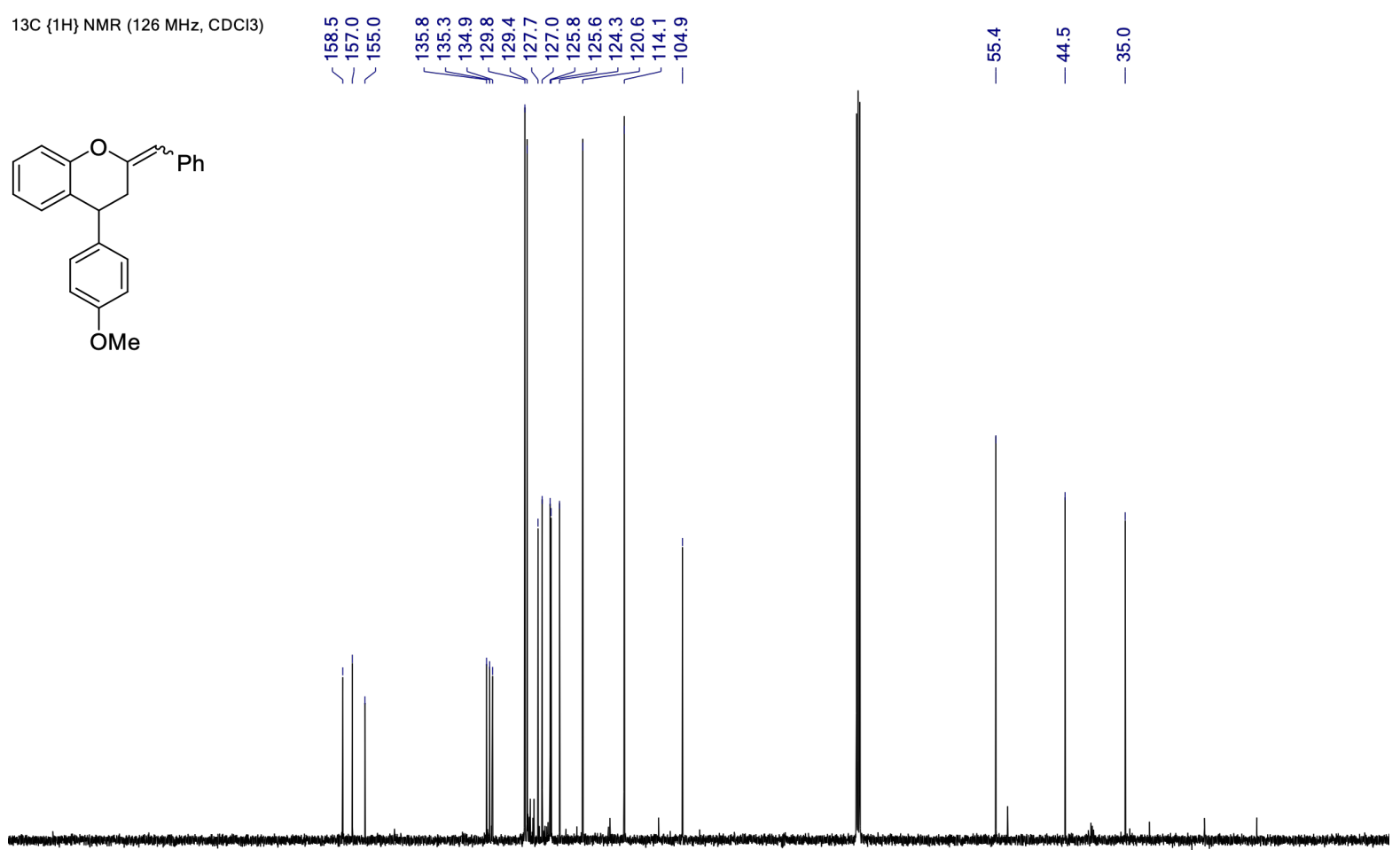

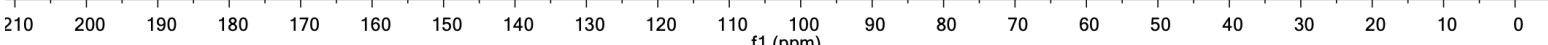




\section{2-Benzylidene-8-methoxy-4-methyl-4-phenylchromane (4e)}

Chromane 4e was synthesized following general procedure $\mathbf{C}$ starting from 1-methoxy-2((phenylethynyl)oxy)benzene ( $45 \mathrm{mg}, 0.2 \mathrm{mmol}$ ) and $\alpha$-methylstyrene $(52 \mu \mathrm{L}, 0.4 \mathrm{mmol})$. The crude product was purified by flash chromatography affording and the two isomers were separated affording (E)-4e as a yellow oil (33 mg, 48\%) and (Z)-4e as a yellow oil (20 mg, 30\%).

\section{(E)-2-benzylidene-8-methoxy-4-methyl-4-phenylchromane}

${ }^{1} \mathbf{H}$ NMR $\left(400 \mathrm{MHz}, \mathrm{CDCl}_{3}\right) \delta 7.37-7.19(\mathrm{~m}, 5 \mathrm{H}), 7.18-7.08(\mathrm{~m}, 3 \mathrm{H}), 7.08-6.99(\mathrm{~m}, 2 \mathrm{H}), 6.99-6.93(\mathrm{~m}$, 2H), $6.92(\mathrm{dd}, J=7.1,1.4 \mathrm{~Hz}, 1 \mathrm{H}), 6.90-6.85(\mathrm{~m}, 1 \mathrm{H}), 5.40(\mathrm{~s}, 1 \mathrm{H}), 3.76(\mathrm{~s}, 3 \mathrm{H}), 3.14(\mathrm{~d}, J=16.6 \mathrm{~Hz}, 1 \mathrm{H}), 2.75$ (dd, $J=16.6,1.1 \mathrm{~Hz}, 1 \mathrm{H}), 1.76(\mathrm{~s}, 3 \mathrm{H})$.

${ }^{13}$ C NMR $\left(101 \mathrm{MHz}, \mathrm{CDCl}_{3}\right) \delta 157.2,151.7,148.1,143.3,139.9,134.9,128.7,128.1,127.3,126.8,126.4,126.2$, $125.9,125.7,125.5,122.8,121.1,113.0,102.0,56.0,44.4,42.0,27.7$.

HRMS (APCI) $m / z$ calculated for $\mathrm{C}_{24} \mathrm{H}_{22} \mathrm{O}_{2}{ }^{+}[\mathrm{M}+\mathrm{H}]^{+}: 343.1693$, found: 343.1692 .

$$
{ }^{1} \mathrm{H} \text { NMR }\left(400 \mathrm{MHz}, \mathrm{CDCl}_{3}\right) \text { of (E)-4e }
$$

\section{$1 \mathrm{H} \mathrm{NMR} \mathrm{(500} \mathrm{MHz,} \mathrm{CDCl3)}$}

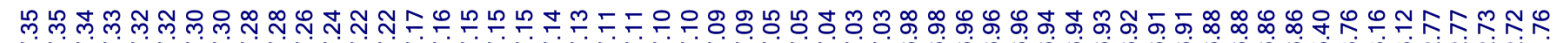

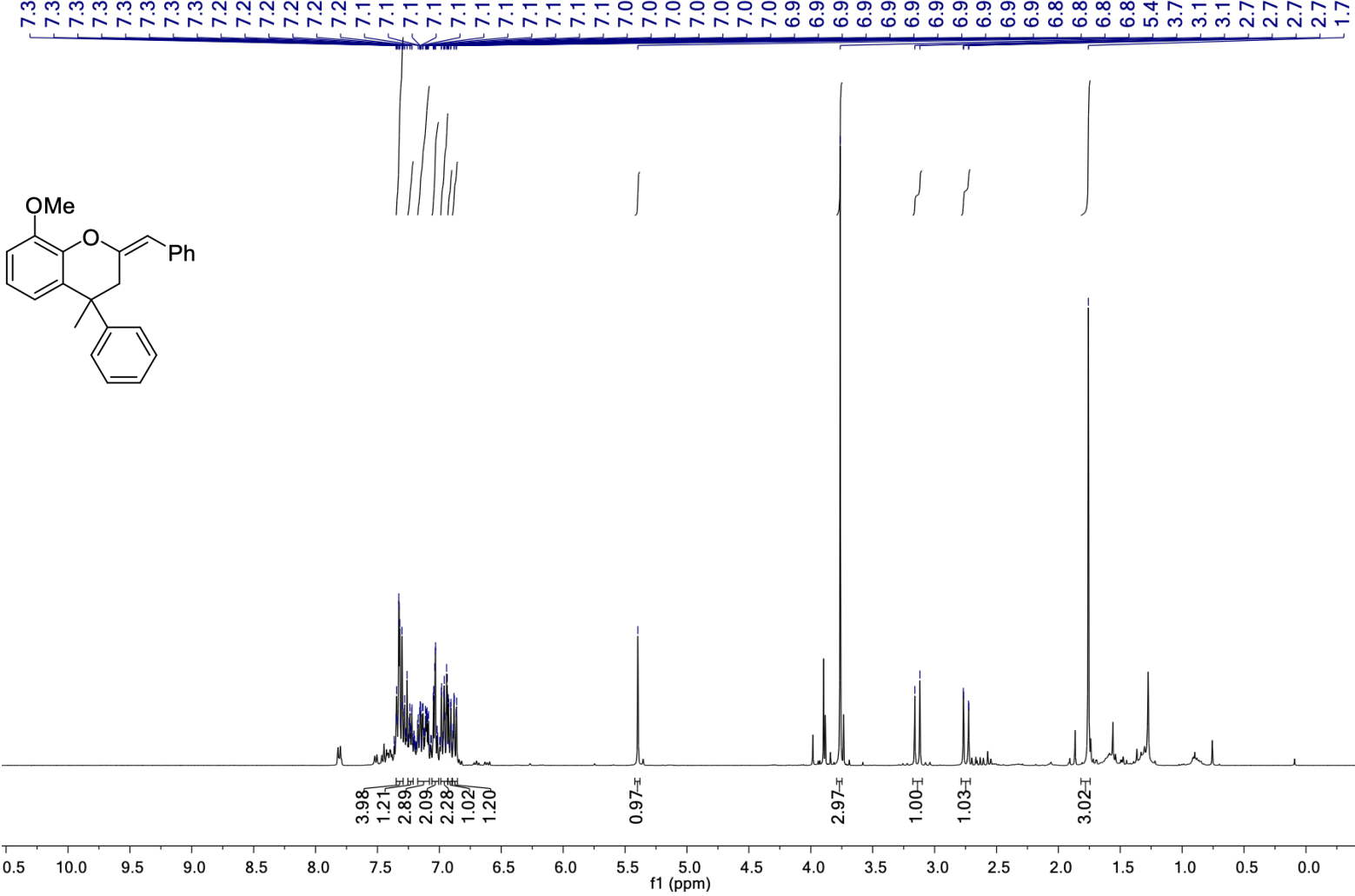

${ }^{13} \mathrm{C}$ NMR $\left(101 \mathrm{MHz}, \mathrm{CDCl}_{3}\right)$ of (E)-4e 
<smiles>COc1cccc2c1O/C(=C/c1ccccc1)C2(C)c1ccccc1</smiles>

(Z)-2-benzylidene-8-methoxy-4-methyl-4-phenylchromane

${ }^{1} \mathbf{H}$ NMR $\left(400 \mathrm{MHz}, \mathrm{CDCl}_{3}\right) \delta 7.30-7.21(\mathrm{~m}, 4 \mathrm{H}), 7.22-7.15(\mathrm{~m}, 2 \mathrm{H}), 7.14-7.09$ (m, 2H), $7.05-6.98(\mathrm{~m}$, 2H), $6.90(\mathrm{dd}, J=8.1,7.4 \mathrm{~Hz}, 1 \mathrm{H}), 6.86(\mathrm{dd}, J=8.1,2.0 \mathrm{~Hz}, 1 \mathrm{H}), 6.64(\mathrm{dd}, J=7.4,1.9 \mathrm{~Hz}, 1 \mathrm{H}), 6.45$ (s, $1 \mathrm{H})$, 3.95 (s, 3H), $3.18(\mathrm{dd}, J=14.4,0.9 \mathrm{~Hz}, 1 \mathrm{H}), 2.84(\mathrm{dd}, J=14.4,1.1 \mathrm{~Hz}, 1 \mathrm{H}), 1.69$ (s, 3H).

${ }^{13}$ C NMR (101 MHz, CDCl3) $\delta 149.6,147.9,146.6,142.0,135.7,131.9,129.0,128.3,127.0,126.5,126.1,121.1$, $119.4,110.3,109.4,56.2,40.5,38.0,27.8$.

HRMS (APCI) $m / z$ calculated for $\mathrm{C}_{24} \mathrm{H}_{22} \mathrm{O}_{2}{ }^{+}[\mathrm{M}+\mathrm{H}]^{+}: 343.1693$, found: 343.1697 . 
${ }^{1} \mathrm{H}$ NMR $\left(400 \mathrm{MHz}, \mathrm{CDCl}_{3}\right)$ of (Z)-4e

$1 \mathrm{H}$ NMR $(500 \mathrm{MHz}, \mathrm{CDCl} 3)$

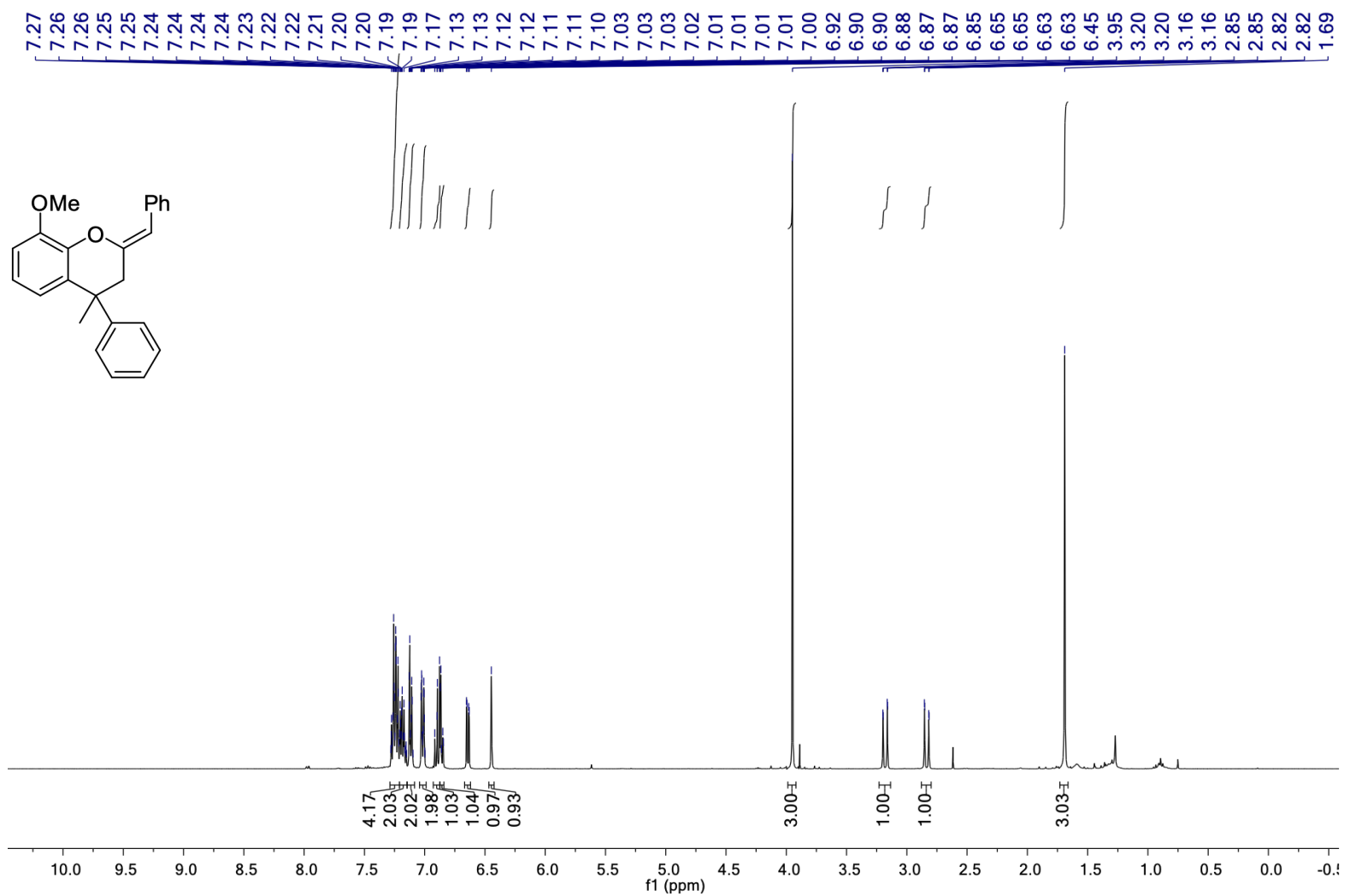

${ }^{13} \mathrm{C}$ NMR (101 MHz, $\left.\mathrm{CDCl}_{3}\right)$ of $(\mathbf{Z})-4 \mathbf{e}$

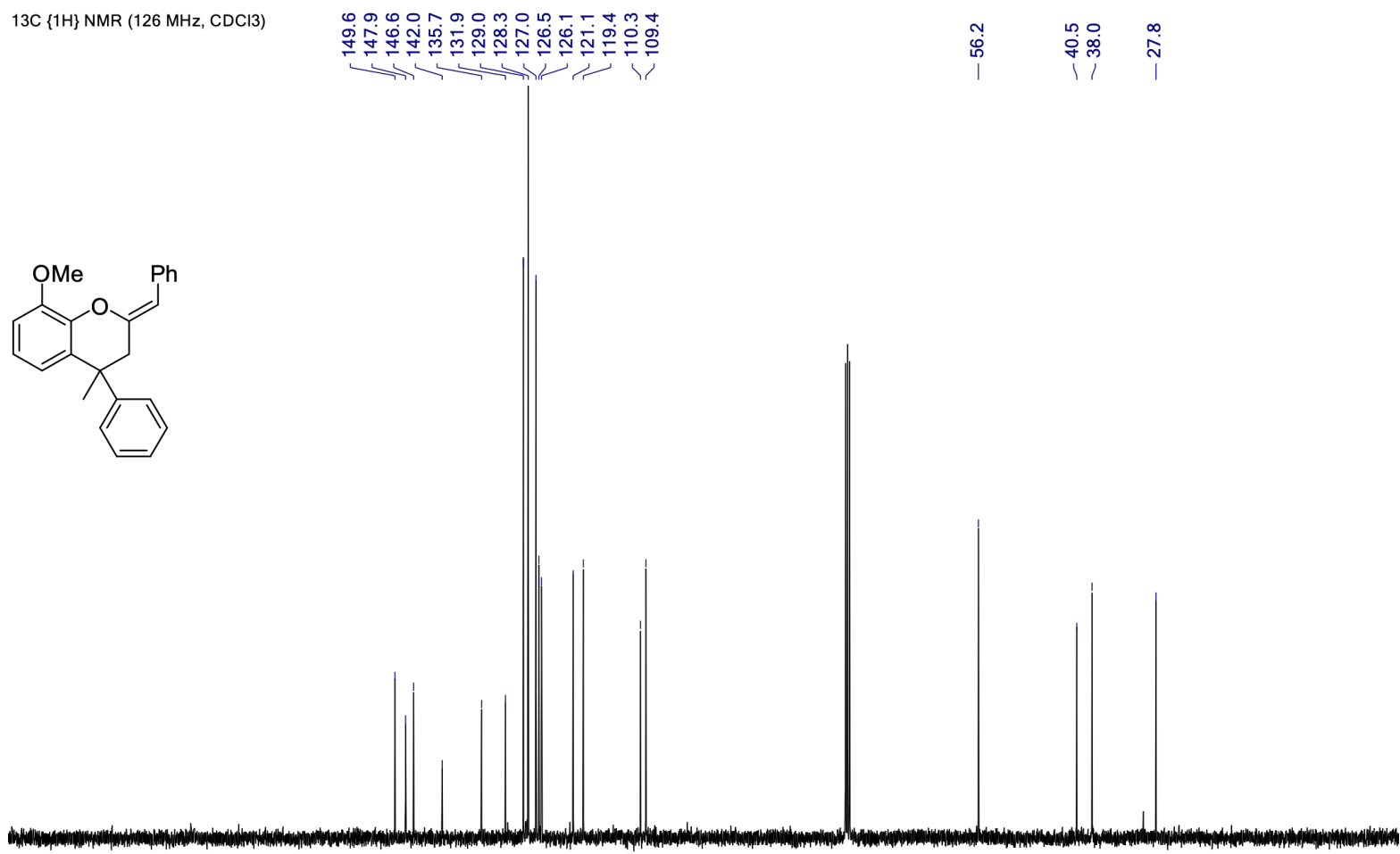

\begin{tabular}{lllllllllllllllllllllllllllll}
\hline & 210 & 200 & 190 & 180 & 170 & 160 & 150 & 140 & 130 & 120 & 110 & 100 & 90 & 80 & 70 & 60 & 50 & 40 & 30 & 20 & 10 & 0 & -1
\end{tabular} 
<smiles>CC1(c2ccccc2)C/C(=C\c2ccccc2C(F)(F)F)Oc2ccccc21</smiles>

Chromane 4f was synthesized following general procedure $\mathbf{E}$ starting from 1-(phenoxyethynyl)-2(trifluoromethyl)benzene $(54.2 \mathrm{mg}, 0.2 \mathrm{mmol})$ and $\alpha$-methylstyrene $(52 \mu \mathrm{L}, 0.4 \mathrm{mmol})$. The crude product was purified by flash chromatography affording $\mathbf{4 f}$ as a colorless oil (70 $\mathrm{mg}, 92 \%)$.

${ }^{1} \mathrm{H}$ NMR $\left(500 \mathrm{MHz}, \mathrm{CD}_{2} \mathrm{Cl}_{2}\right) \delta 7.66-7.61(\mathrm{~m}, 1 \mathrm{H}), 7.43(\mathrm{tdt}, J=7.6,1.4,0.7 \mathrm{~Hz}, 1 \mathrm{H}), 7.37-7.30(\mathrm{~m}, 1 \mathrm{H})$, $7.28-7.22(\mathrm{~m}, 3 \mathrm{H}), 7.22-7.17(\mathrm{~m}, 1 \mathrm{H}), 7.10-7.06(\mathrm{~m}, 3 \mathrm{H}), 7.06-7.02(\mathrm{~m}, 1 \mathrm{H}), 6.98(\mathrm{td}, J=7.5,1.3 \mathrm{~Hz}, 1 \mathrm{H})$, $6.95-6.89(\mathrm{~m}, 1 \mathrm{H}), 6.39(\mathrm{~d}, J=2.8 \mathrm{~Hz}, 1 \mathrm{H}), 3.05(\mathrm{dd}, J=14.6,0.9 \mathrm{~Hz}, 1 \mathrm{H}), 2.70(\mathrm{dd}, J=14.5,1.3 \mathrm{~Hz}, 1 \mathrm{H})$, $1.67(\mathrm{~s}, 3 \mathrm{H})$.

${ }^{19}$ F NMR $\left(471 \mathrm{MHz}, \mathrm{CD}_{2} \mathrm{Cl}_{2}\right) \delta-61.3$.

${ }^{13}$ C NMR $\left(126 \mathrm{MHz}, \mathrm{CD}_{2} \mathrm{Cl}_{2}\right) \delta 152.8,151.9,147.0,134.7,132.3,132.1,131.5,128.8,128.8,128.0,127.4$, 127.1(d, J = 3.9 Hz), $126.3(q, J=5.4 \mathrm{~Hz}), 124.9(q, \mathrm{~J}=273.8 \mathrm{~Hz}), 122.4,116.9$, 105.5, 40.6, 38.8, 30.3, 28.0.

HRMS (APCI) $m / z$ calculated for $\mathrm{C}_{24} \mathrm{H}_{20} \mathrm{~F}_{3} \mathrm{O}^{+}[\mathrm{M}+\mathrm{H}]^{+}: 381.1461$, found: 381.1464 .

${ }^{1} \mathrm{H}$ NMR $\left(500 \mathrm{MHz}, \mathrm{CD}_{2} \mathrm{Cl}_{2}\right)$ of $\mathbf{4 f}$

$1 \mathrm{H}$ NMR $(500 \mathrm{MHz}, \mathrm{CDCl} 3)$

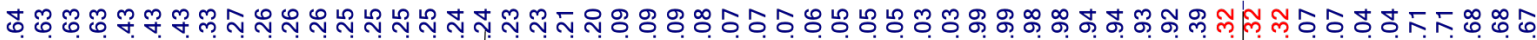

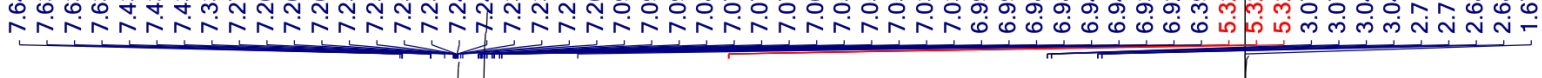<smiles>CC1(c2ccccc2)C/C(=C\c2ccccc2C(F)(F)F)Oc2ccccc21</smiles>
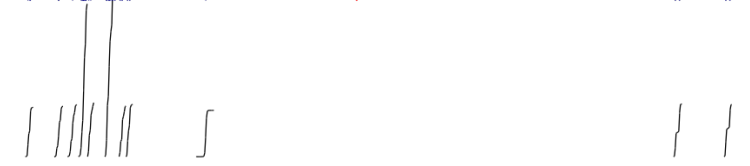

\section{3}


${ }^{19} \mathrm{~F}$ NMR (471 MHz, $\mathrm{CD}_{2} \mathrm{Cl}_{2}$ ) of $\mathbf{4 f}$

19F NMR (471 MHz, CD2Cl2)

$$
\stackrel{m}{i}
$$

(1)

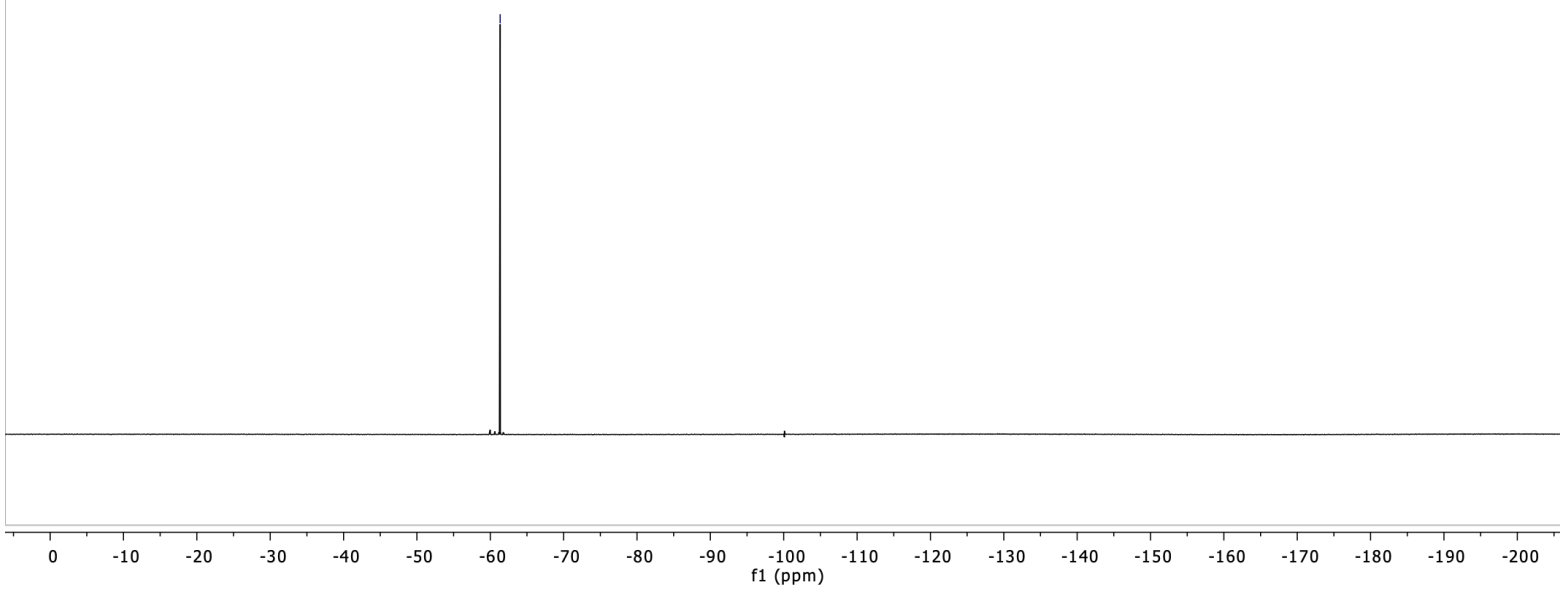

${ }^{13} \mathrm{C}$ NMR $\left(126 \mathrm{MHz}, \mathrm{CD}_{2} \mathrm{Cl}_{2}\right)$ of $\mathbf{4 f}$

13C $\{1 \mathrm{H}\}$ NMR $(126 \mathrm{MHz}, \mathrm{CDCl} 3)$

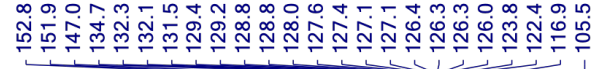

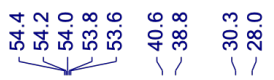

(1)

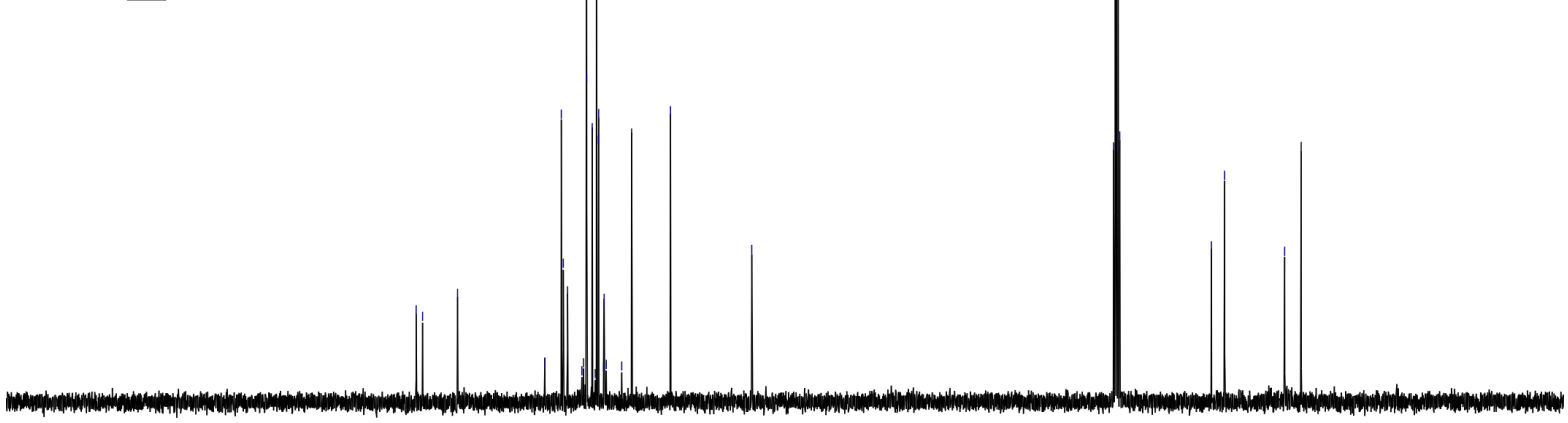

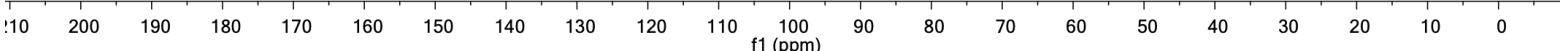




\section{One-pot Synthesis of Cyclobutanones Starting from Ynol ethers}

Table S.5. Optimization of the one pot-two steps synthesis of cyclobutanone $\mathbf{5 a}{ }^{\text {a }}$

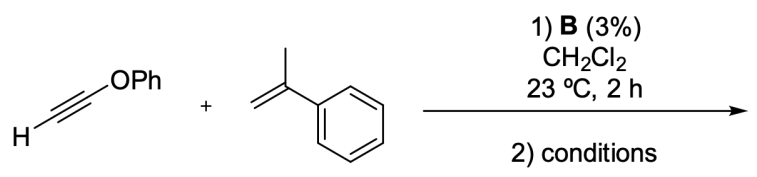

$1 \mathrm{a}$

$\mathbf{2 a}$

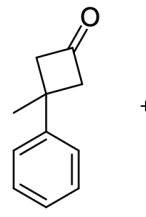

5 a

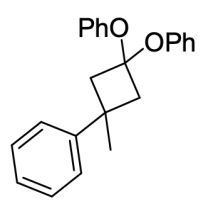

sx

\begin{tabular}{|c|c|c|c|}
\hline Entry & Conditions $^{b}$ & $2.45 \mathrm{~b}(\%)^{c}$ & $2.50(\%)^{c}$ \\
\hline 1 & $\mathrm{H}_{2} \mathrm{O}$ (100 equiv) & $-d$ & $-d$ \\
\hline 2 & $p$-TSA $\cdot \mathrm{H}_{2} \mathrm{O}(0.15$ equiv $)$, water & 37 & 31 \\
\hline 3 & $p$-TSA $\cdot \mathrm{H}_{2} \mathrm{O}$ ( 1 equiv), water & $73(69)$ & - \\
\hline 4 & $p$-TSA $\cdot \mathrm{H}_{2} \mathrm{O}$ ( 2 equiv), water & 68 & 11 \\
\hline 5 & $10 \% \mathrm{HCl}$ in water ( $\mathrm{HCl} 1$ equiv) & 43 & 43 \\
\hline 6 & 1M TFA in water (TFA 1 equiv) & 44 & 35 \\
\hline 7 & $1 \mathrm{M} \mathrm{H}_{2} \mathrm{SO}_{4}$ in water $\left(\mathrm{H}_{2} \mathrm{SO}_{4} 1\right.$ equiv $)$ & $-d$ & $-d$ \\
\hline 8 & $1 \mathrm{M} \mathrm{AcOH}$ in water ( $\mathrm{AcOH} 1$ equiv) & $-d$ & $-d$ \\
\hline
\end{tabular}

${ }^{a} \mathbf{1 a : 2 a}=1: 3 .{ }^{b}$ reaction time $14 \mathrm{~h}$, temperature $=23{ }^{\circ} \mathrm{C} .{ }^{c}$ yield determined by ${ }^{1} \mathrm{H}$ NMR using trichloroethylene as internal standard. Isolated yield in parenthesis. ${ }^{d} \mathbf{3 a}$ fully recovered.

Table S.6. Optimization of the one pot-two steps synthesis of cyclobutanone 5e. ${ }^{\text {a }}$

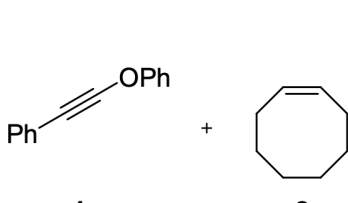

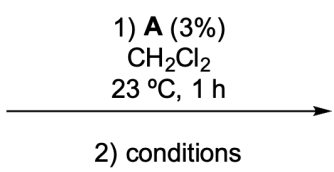

2

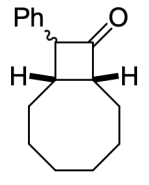

$5 e$ 


\begin{tabular}{|c|c|c|c|c|}
\hline Entry & Conditions ${ }^{b}$ & $\mathrm{~T}\left({ }^{\circ} \mathrm{C}\right)$ & $5 \mathbf{e}(\%)^{c}$ & d.r..$^{c}$ \\
\hline 1 & $p$-TSA $\cdot \mathrm{H}_{2} \mathrm{O}$ ( 1 equiv), water & 23 & $49^{d}$ & $2.5: 1$ \\
\hline 2 & $p$-TSA $\cdot \mathrm{H}_{2} \mathrm{O}$ ( 1 equiv), water & 40 & 75 & $2: 1$ \\
\hline 3 & $p$-TSA $\cdot \mathrm{H}_{2} \mathrm{O}$ ( 1 equiv), water ${ }^{e}$ & 65 & 50 & $4: 1$ \\
\hline 4 & $2 \mathrm{M} \mathrm{HCl}$ in water ( $\mathrm{HCl} 2$ equiv) & 40 & $(75 \%)$ & $4.5: 1$ \\
\hline 5 & $2 \mathrm{M} \mathrm{HCl}$ in water $(\mathrm{HCl} 2 \text { equiv })^{e}$ & 65 & 55 & $3: 1$ \\
\hline
\end{tabular}

${ }^{a} \mathbf{1 : 2}=1: 2 .{ }^{b}$ reaction time $14 \mathrm{~h} .{ }^{c}$ yield determined by ${ }^{1} \mathrm{H}$ NMR using trichloroethylene as internal standard. Isolated yield in parenthesis. ${ }^{d} \mathbf{3 m m}$ was also obtained in $38 \%$ yield. ${ }^{e}$ Reaction performed in toluene.

General procedure D: one-pot synthesis of cyclobutanones 5 starting from ynol ethers 1

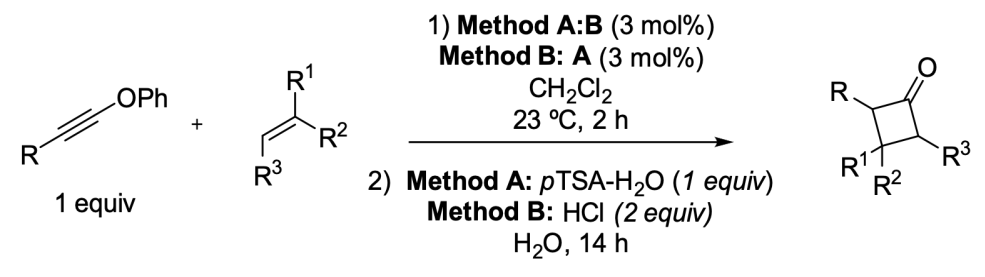

A GC-MS vial equipped with a magnetic stirring bar was charged with the ynol ether $(0.2 \mathrm{mmol}, 1$ equiv) and $\mathrm{CH}_{2} \mathrm{Cl}_{2}$ (HPLC grade, $0.2 \mathrm{~mL}, 1 \mathrm{M}$ ). The alkene (Method A: 0.6 mmol, 3 equiv, Method B: $0.4 \mathrm{mmol}, 2$ equiv) was added followed by the catalyst (Method A: [(JohnPhos)AuNCMe]SbF 6 (2.B, 3 mol\%, 4.6 mg); Method B: by $[(t \mathrm{BuXPhos}) \mathrm{AuNCMe}] \mathrm{BAr}_{4}{ }^{\mathrm{F}}(\mathbf{2 . A}, 3 \mathrm{~mol} \%, 9.2 \mathrm{mg})$. The resulting mixture was stirred at $23{ }^{\circ} \mathrm{C}$ for $3 \mathrm{~h}$. The reaction was monitored by GC-MS or UHPLC-MSD. Once completed the acid was added (Method A: pTSA $\cdot \mathrm{H}_{2} \mathrm{O}$ (38 mg, $0.2 \mathrm{mmol}, 1$ equiv); Method B: $2 \mathrm{M} \mathrm{HCl}$ in $\mathrm{H}_{2} \mathrm{O}$ (200 ml, $0.4 \mathrm{mmol}, 2$ equiv), followed by 0.1 $\mathrm{mL}$ of $\mathrm{H}_{2} \mathrm{O}$. The reaction was stirred overnight and then diluted with $\mathrm{CH}_{2} \mathrm{Cl}_{2}$ and the organic phases was washed three times with $\mathrm{H}_{2} \mathrm{O}$. The collected organic phase was dried over $\mathrm{Na}_{2} \mathrm{SO}_{4}$ and the solvent removed under reduced pressure. The crude product was purified by flash chromatography on silica gel using a pentante:Et $2 \mathrm{O} 20: 1$ as eluent and affording cyclobutanone $\mathbf{5}$

\section{3-Methyl-3-phenylcyclobutan-1-one (5a)}

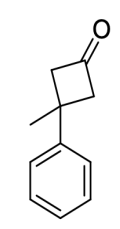


Cyclobutanone 5a was obtained synthesized following general procedure D - Method A starting from (ethynyloxy)benzene (23.6 $\mathrm{mg}, 0.2 \mathrm{mmol}$ ) and $\alpha$-methylstyrene $(78 \mu \mathrm{L}, 0.6 \mathrm{mmol})$. The crude product was purified by flash chromatography affording $\mathbf{5 a}$ as a colorless oil ( $22 \mathrm{mg}, 69 \%)$. The same reaction was performed also on $1.0 \mathrm{mmol}$ scale starting from (ethynyloxy)benzene $(118 \mathrm{mg}, 1 \mathrm{mmol})$ and $\alpha$-methylstyrene $(390 \mu \mathrm{L}, 3$ mmol) and using $p$-TSA $\cdot \mathrm{H}_{2} \mathrm{O}$ (323 mg, $1.7 \mathrm{mmol}, 1.7$ equiv). and $106 \mathrm{mg}$ of $\mathbf{5 a}$ were obtained (66\% yield).

${ }^{1} \mathbf{H}$ NMR $\left(300 \mathrm{MHz}, \mathrm{CDCl}_{3}\right) \delta 7.42-7.29(\mathrm{~m}, 3 \mathrm{H}), 7.29-7.21(\mathrm{~m}, 2 \mathrm{H}), 3.58-3.39(\mathrm{~m}, 2 \mathrm{H}), 3.25-3.00(\mathrm{~m}$, $2 \mathrm{H}), 1.61(\mathrm{~s}, 3 \mathrm{H})$.

${ }^{13}$ C NMR $\left(101 \mathrm{MHz}, \mathrm{CDCl}_{3}\right) \delta 206.9,148.4,128.7,126.4,125.8,59.4,34.1,31.2$.

IR (selected frequency): $1783 \mathrm{~cm}^{-1}$.

\section{IR of $5 \mathbf{a}$}

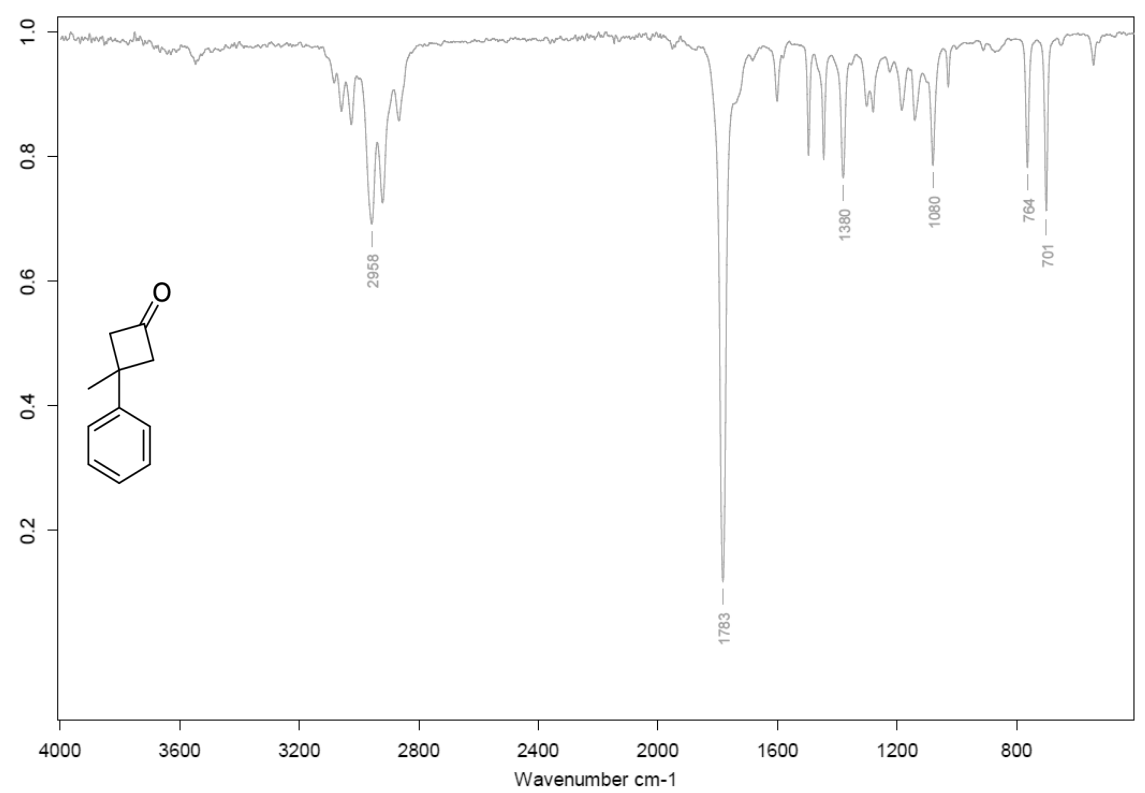

Data in agreement with the one reported in literature ${ }^{\mathrm{x}}$

1 mmol scale one-pot synthesis of cyclobutanones 5 a starting from ynol ethers $1 \mathbf{a}$

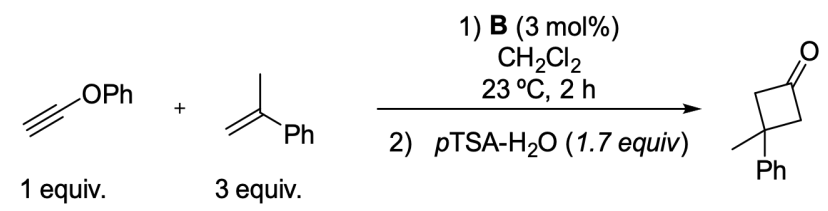

A screw-cap vial equipped with a magnetic stirring bar was charged with the (ethynyloxy)benzene (118 mg, 1 mmol, 1 equiv) and $\mathrm{CH}_{2} \mathrm{Cl}_{2}$ (HPLC grade, $1 \mathrm{~mL}, 1 \mathrm{M}$ ). Then $\alpha$-methylstyrene ( $390 \mu \mathrm{L}, 3 \mathrm{mmol}, 3$ equiv, was added followed by catalyst 2.B, $3 \mathrm{~mol} \%, 23 \mathrm{mg}$ ); The resulting mixture was stirred at $23{ }^{\circ} \mathrm{C}$ for $3 \mathrm{~h}$. The reaction was monitored by GC-MS or UHPLC-MSD. Once completed $p-\mathrm{TSA} \cdot \mathrm{H}_{2} \mathrm{O}(323 \mathrm{mg}, 1.7 \mathrm{mmol}, 1.7$ equiv.) was added followed by $0.3 \mathrm{~mL}$ of $\mathrm{H}_{2} \mathrm{O}$. The reaction was stirred overnight and then diluted with $\mathrm{CH}_{2} \mathrm{Cl}_{2}$ and the organic phases was washed three times with $\mathrm{H}_{2} \mathrm{O}$. The collected organic phase was dried over $\mathrm{Na}_{2} \mathrm{SO}_{4}$ and the solvent 
removed under reduced pressure. The crude product was purified by flash chromatography on silica gel using a pentante: $\mathrm{Et}_{2} \mathrm{O} 20: 1$ as eluent and affording cyclobutanone $\mathbf{5 a}$ (106 $\mathrm{mg}, 66 \%$ yield).

Hydrolysis of $\mathbf{3 a}$ o form cyclobutanone $\mathbf{5 a}$

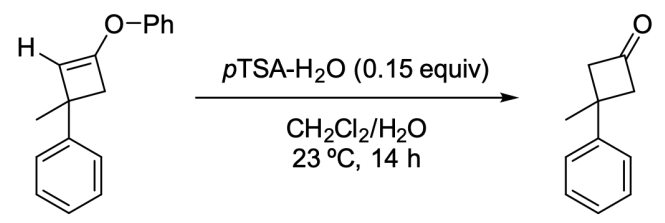

Cyclobutene 3a (47 mg, $0.2 \mathrm{mmol})$ was dissolved in $\mathrm{CH}_{2} \mathrm{Cl}_{2}(0.2 \mathrm{~mL})$ and water $(0.1 \mathrm{~mL})$. $p$-toluenesulfonic acid monohydrate $(5.7 \mathrm{mg}, 0.03 \mathrm{mmol})$ was then added and the biphasic mixture was vigorously stirred at $23^{\circ} \mathrm{C}$ until complete conversion was observed by monitoring the reaction by TLC. The reaction was then diluted with $\mathrm{CH}_{2} \mathrm{Cl}_{2}$ and the organic phases was washed three times with $\mathrm{H}_{2} \mathrm{O}$. The collected organic phase was dried over $\mathrm{Na}_{2} \mathrm{SO}_{4}$ and the solvent removed under reduced pressure. The crude product was purified by flash chromatography on silica gel using a pentane: $\mathrm{Et}_{2} \mathrm{O} 20: 1$ as eluent and affording $\mathbf{5 a}$ as a colorless oil (30 $\left.\mathrm{mg}, 94 \%\right)$. 
<smiles>CC1(c2ccc(-c3ccccc3)cc2)CC(=O)C1</smiles>

Cyclobutanone 5b was obtained synthesized following general procedure C - Method A starting from (ethynyloxy)benzene (23.6 mg, $0.2 \mathrm{mmol}$ ) and 4-(prop-1-en-2-yl)-1,1'-biphenyl (117 mg, $0.6 \mathrm{mmol})$. The crude product was purified by flash chromatography affording $\mathbf{5 b}$ as a white solid (26 $\mathrm{mg}, 56 \%)$.

${ }^{1} \mathbf{H}$ NMR $\left(400 \mathrm{MHz}, \mathrm{CDCl}_{3}\right) \delta 7.65-7.56(\mathrm{~m}, 4 \mathrm{H}), 7.50-7.43(\mathrm{~m}, 2 \mathrm{H}), 7.43-7.39(\mathrm{~m}, 2 \mathrm{H}), 7.39-7.32(\mathrm{~m}$, $1 \mathrm{H}), 3.62-3.39(\mathrm{~m}, 2 \mathrm{H}), 3.26-3.04(\mathrm{~m}, 2 \mathrm{H}), 1.67(\mathrm{~s}, 3 \mathrm{H})$.

${ }^{13}$ C NMR (101 MHz, $\left.\mathrm{CDCl}_{3}\right) \delta 206.7,147.4,140.8,139.4,128.9,127.4,127.2,126.3,59.5,33.9,31.1$.

HRMS (ESI) m/z calculated for $\mathrm{C} 17 \mathrm{H} 16 \mathrm{NaO}^{+}[\mathrm{M}+\mathrm{Na}]^{+}: 259.1093$, found: 259.1091 .

IR (selected frequency): $1771 \mathrm{~cm}^{-1}$.

${ }^{1} \mathrm{H} \mathrm{NMR}\left(400 \mathrm{MHz}, \mathrm{CDCl}_{3}\right)$ of $\mathbf{5 b}$

$1 \mathrm{H}$ NMR $(500 \mathrm{MHz}, \mathrm{CDCl} 3)$

兑

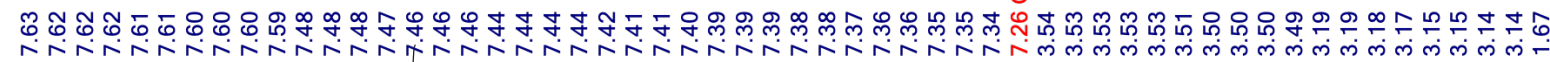

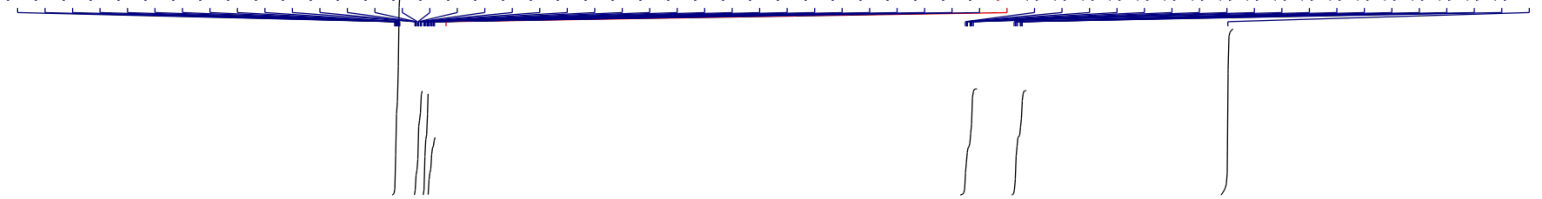

$\underbrace{1}_{P h}$

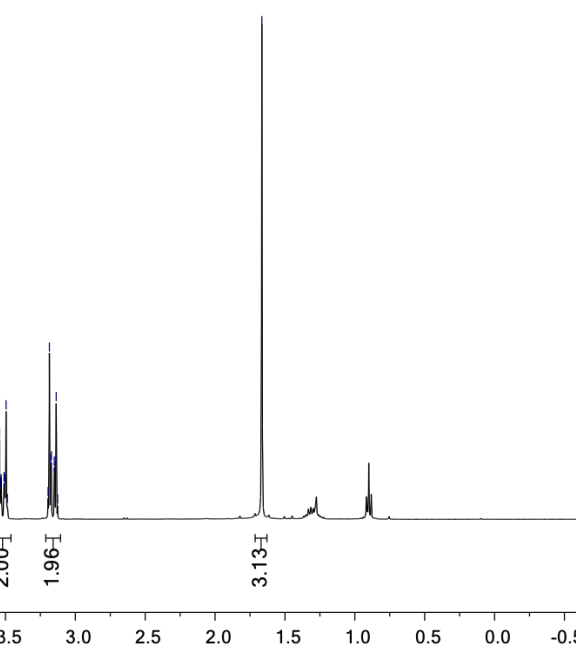


${ }^{13} \mathrm{C}$ NMR $\left(101 \mathrm{MHz}, \mathrm{CDCl}_{3}\right)$ of $\mathbf{5 b}$
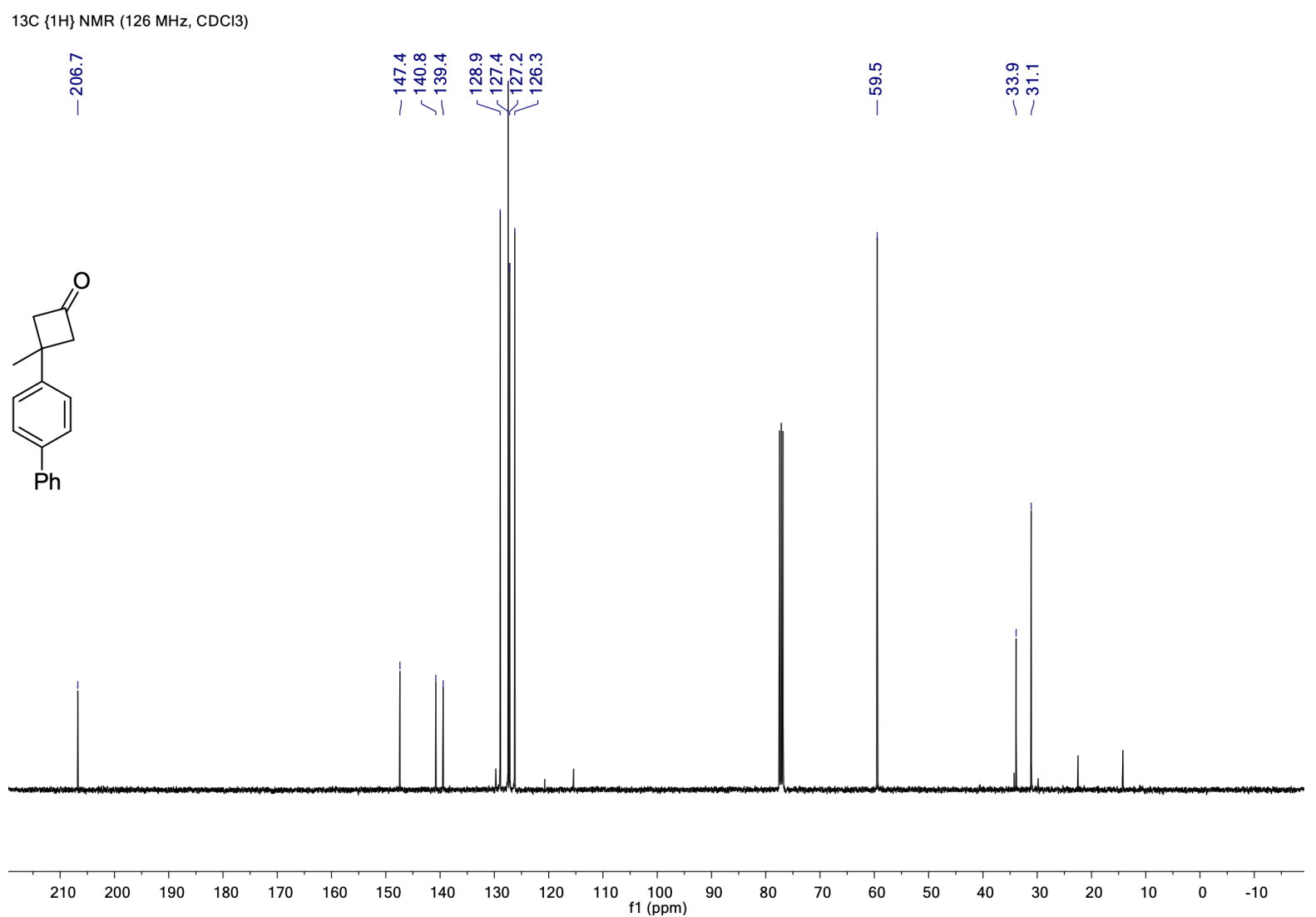

IR of $\mathbf{5 b}$

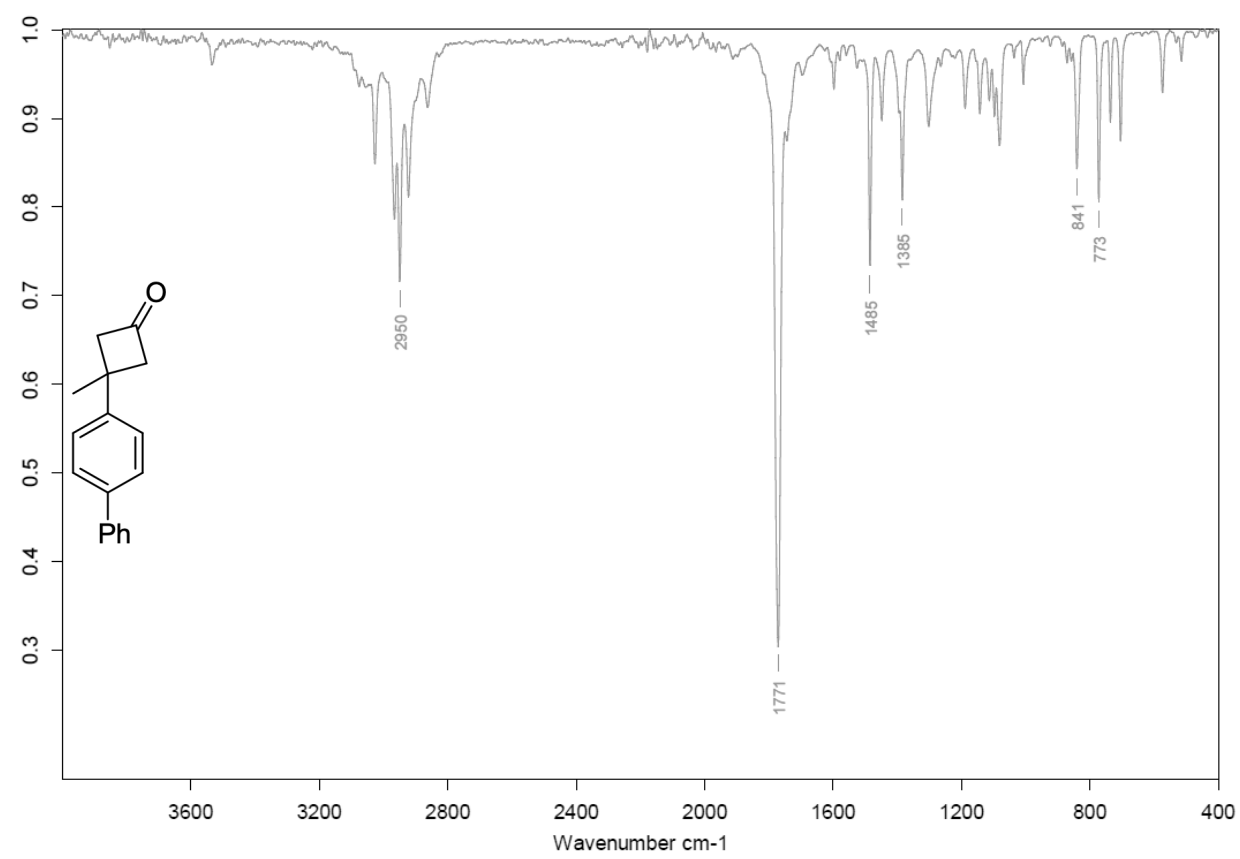


$(1 R, 11 S)-4,13,13-T r i m e t h y l-10-m e t h y l e n e t r i c y c l o[9.2 .0 .04,7]$ tridecan-6-one (5c)

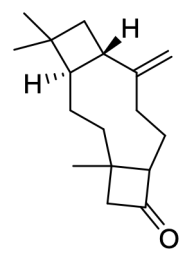

Cyclobutanone 5c was obtained synthesized following general procedure D - Method A starting from(ethynyloxy)benzene $(23.6 \mathrm{mg}, 0.2 \mathrm{mmol})$ and b-Caryophyllene $(170 \mathrm{ml}, 0.6 \mathrm{mmol})$ The crude product was purified by flash chromatography affording $\mathbf{5 c}$ as a yellow oil 3:1 mixture of regioisomers (31 mg, 68\%). See above for the characterization.

\section{3-((tert-Butyldimethylsilyl)oxy)spiro[3.5]nonan-1-one (5d)}

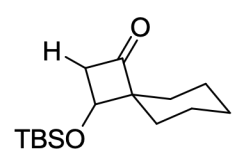

Cyclobutanone 5d was obtained synthesized following general procedure D - Method A starting from (ethynyloxy)benzene (12 mg, $0.1 \mathrm{mmol})$ and tert-butyl(cyclohexylidenemethoxy)dimethylsilane (68 mg, 0.3 $\mathrm{mmol}$ ). The crude product was purified by flash chromatography affording $\mathbf{5 d}$ as a brown oil (11 $\mathrm{mg}, 41 \%)$.

${ }^{1} \mathbf{H}$ NMR $\left(400 \mathrm{MHz} \mathrm{CDCl}_{3}\right) \delta 4.14(\mathrm{dd}, J=6.9,4.7 \mathrm{~Hz}, 1 \mathrm{H}), 3.22(\mathrm{dd}, J=17.8,6.9 \mathrm{~Hz}, 1 \mathrm{H}), 2.86(\mathrm{dd}, J=17.8$, $4.7 \mathrm{~Hz}, 1 \mathrm{H}), 1.76-1.58(\mathrm{~m}, 5 \mathrm{H}), 1.58-1.48(\mathrm{~m}, 1 \mathrm{H}), 1.44(\mathrm{dt}, J=9.1,2.0 \mathrm{~Hz}, 2 \mathrm{H}), 1.34-1.21(\mathrm{~m}, 2 \mathrm{H}), 0.90$ $(\mathrm{s}, 9 \mathrm{H}), 0.09$ (s, 3H), 0.07 (s, 3H).

${ }^{13}$ C NMR $\left(101 \mathrm{MHz}, \mathrm{CDCl}_{3}\right) \delta 214.4,68.2,67.5,52.8,31.7,26.5,25.9,25.8,22.8,22.6,18.2,-4.6,-4.9$.

HRMS (ESI) $m / z$ calculated for $\mathrm{C}_{15} \mathrm{H}_{28} \mathrm{NaO}_{2} \mathrm{Si}^{+}[\mathrm{M}+\mathrm{Na}]^{+}: 291.1751$, found: 291.1744 . 
${ }^{1} \mathrm{H}$ NMR $\left(400 \mathrm{MHz}, \mathrm{CDCl}_{3}\right)$ of $\mathbf{5 d}$

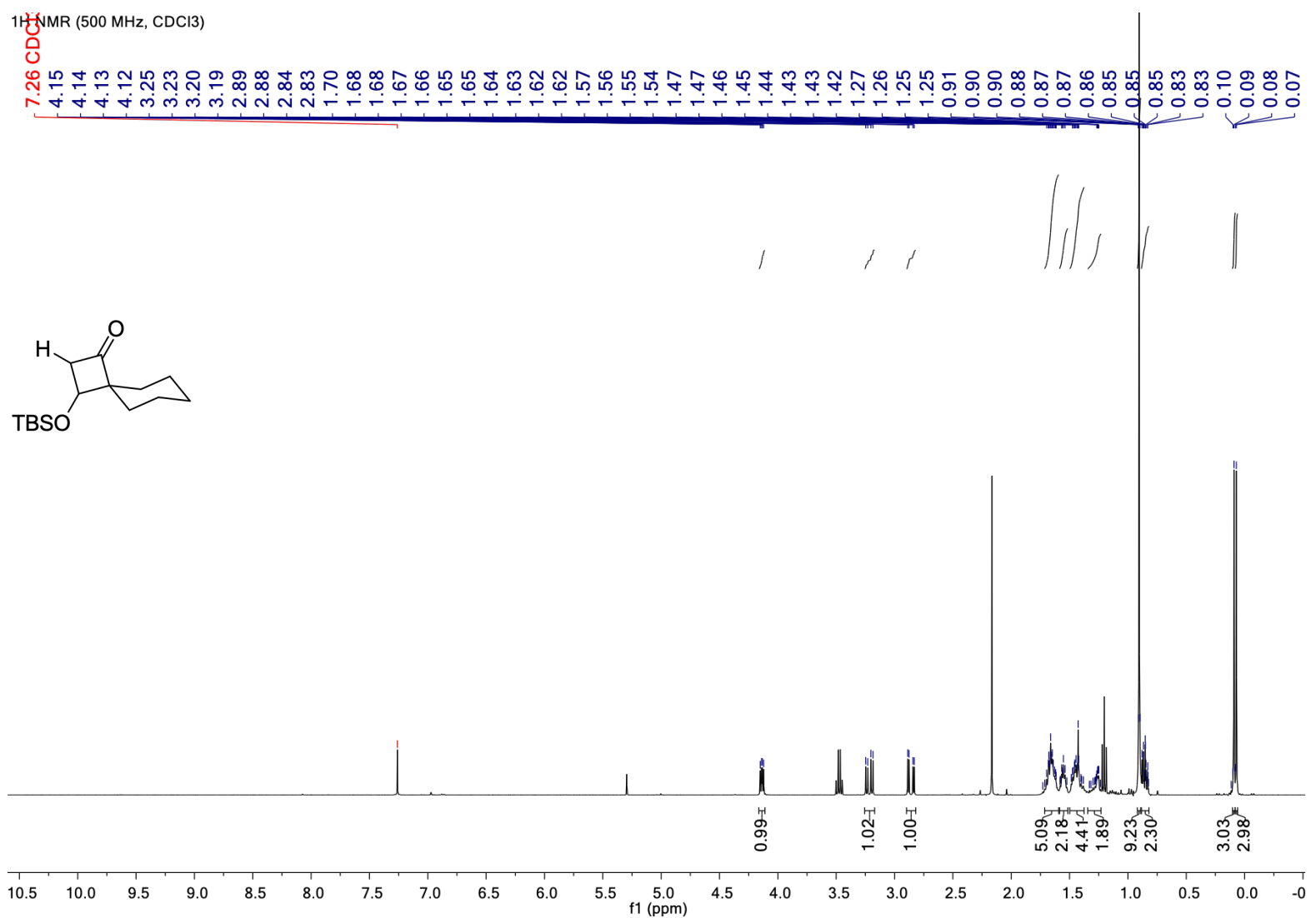

${ }^{13} \mathrm{C}$ NMR $\left(101 \mathrm{MHz}, \mathrm{CDCl}_{3}\right)$ of $\mathbf{5 d}$

13E $\{1 \mathrm{H}\} \mathrm{NMR}(126 \mathrm{MHz}, \mathrm{CDCl} 3)$ $\stackrel{4}{\stackrel{2}{1}}$

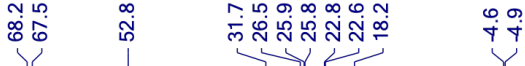

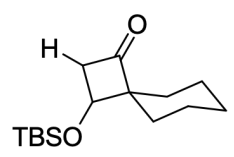

$\begin{array}{lllllllllllllllllllllll}210 & 200 & 190 & 180 & 170 & 160 & 150 & 140 & 130 & 120 & 110 & \begin{array}{l}100 \\ 100\end{array} & 80 & 70 & 60 & 50 & 40 & 30 & 20 & 10 & 0 & -10 & \end{array}$ 


\section{0-Phenylbicyclo[6.2.0]decan-9-one (5e)}

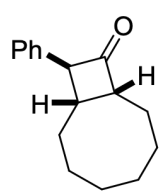

Cyclobutanone 5e was obtained synthesized following general procedure D - Method B starting from (phenoxyethynyl)benzene (39 mg, $0.2 \mathrm{mmol}$ ) and (Z)-cyclooctene $(52 \mathrm{ml}, 0.4 \mathrm{mmol})$. The crude product was purified by flash chromatography affording $\mathbf{5 e}$ as a yellow oil 4:1 mixture of diastereoisomer (34 $\mathrm{mg}, 75 \%$ ). The major diastereoisomer have been assigned by NOESY correlation. $\mathrm{M}=$ major diastereoisomer; $\mathrm{m}=$ minor diastereoisomer.

${ }^{1} \mathbf{H}$ NMR $\left(400 \mathrm{MHz}, \mathrm{CDCl}_{3}\right) \delta 7.37-7.27(\mathrm{~m}, 2 \mathrm{H}, \mathrm{M}+\mathrm{m}), 7.27-7.19(\mathrm{~m}, 3 \mathrm{H}, \mathrm{M}+\mathrm{m}), 7.18-7.11(\mathrm{~m}, 0.4 \mathrm{H}, \mathrm{m})$, $4.74(\mathrm{dd}, J=10.9,2.6 \mathrm{~Hz}, 0.25 \mathrm{H}, \mathrm{m}), 3.98$ (dd, $J=7.9,2.7 \mathrm{~Hz}, 1 \mathrm{H}, \mathrm{M}), 3.30$ (ddt, $J=12.4,9.8,2.6 \mathrm{~Hz}, 0.25 \mathrm{H}$, m), 3.19 (ddt, $J=11.9,9.5,2.4 \mathrm{~Hz}, 1 \mathrm{H}, \mathrm{M}$ ), $2.89-2.75$ (m, 0.25H, m), 2.57 (dddd, $J=11.9,10.0,7.9,2.4 \mathrm{~Hz}$, $1 \mathrm{H}, \mathrm{M}), 2.11-1.91(\mathrm{~m}, 2 \mathrm{H}, \mathrm{M}+\mathrm{m}), 1.90-1.80(\mathrm{~m}, 1 \mathrm{H}, \mathrm{M}), 1.80-1.54(\mathrm{~m}, 3 \mathrm{H}, \mathrm{M}+\mathrm{m}), 1.52-1.44(\mathrm{~m}, 0.4 \mathrm{H}$, $\mathrm{m}), 1.44-1.33(\mathrm{~m}, 2 \mathrm{H}, \mathrm{M}+\mathrm{m}), 1.33-1.09(\mathrm{~m}, 2 \mathrm{H}, \mathrm{M}+\mathrm{m})$.

${ }^{13}$ C NMR (101 MHz, CDCl $) \delta 212.4(\mathrm{M}), 211.2(\mathrm{~m}) 136.7(\mathrm{M}), 134.1(\mathrm{~m}), 129.2(\mathrm{M}), 128.8(\mathrm{M}), 128.5(\mathrm{M})$, $127.2(\mathrm{~m}), 127.1(\mathrm{M}), 69.3(\mathrm{M}), 66.3(\mathrm{~m}), 61.6(\mathrm{M}), 60.4(\mathrm{~m}), 38.0(\mathrm{M}), 36.7(\mathrm{~m}), 30.7(\mathrm{~m}), 29.9(\mathrm{M}), 29.8(\mathrm{~m})$, $29.6(\mathrm{M}), 28.4(\mathrm{M}), 27.9(\mathrm{~m}), 26.2(\mathrm{~m}), 26.1(\mathrm{M}), 25.9(\mathrm{~m}), 25.8(\mathrm{~m}), 25.3(\mathrm{M}), 24.1(\mathrm{M}), 20.7(\mathrm{~m})$.

HRMS (ESI) $m / z$ calculated for $\mathrm{C}_{16} \mathrm{H}_{20} \mathrm{NaO}^{+}[\mathrm{M}+\mathrm{Na}]^{+}: 251.1406$, found: 251.1407. 
${ }^{1} \mathrm{H}$ NMR $\left(400 \mathrm{MHz}, \mathrm{CDCl}_{3}\right)$ of $\mathbf{5 e}$

$1 \mathrm{H}$ NMR $(500 \mathrm{MHz}, \mathrm{CDCl} 3)$

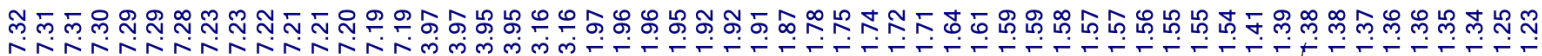
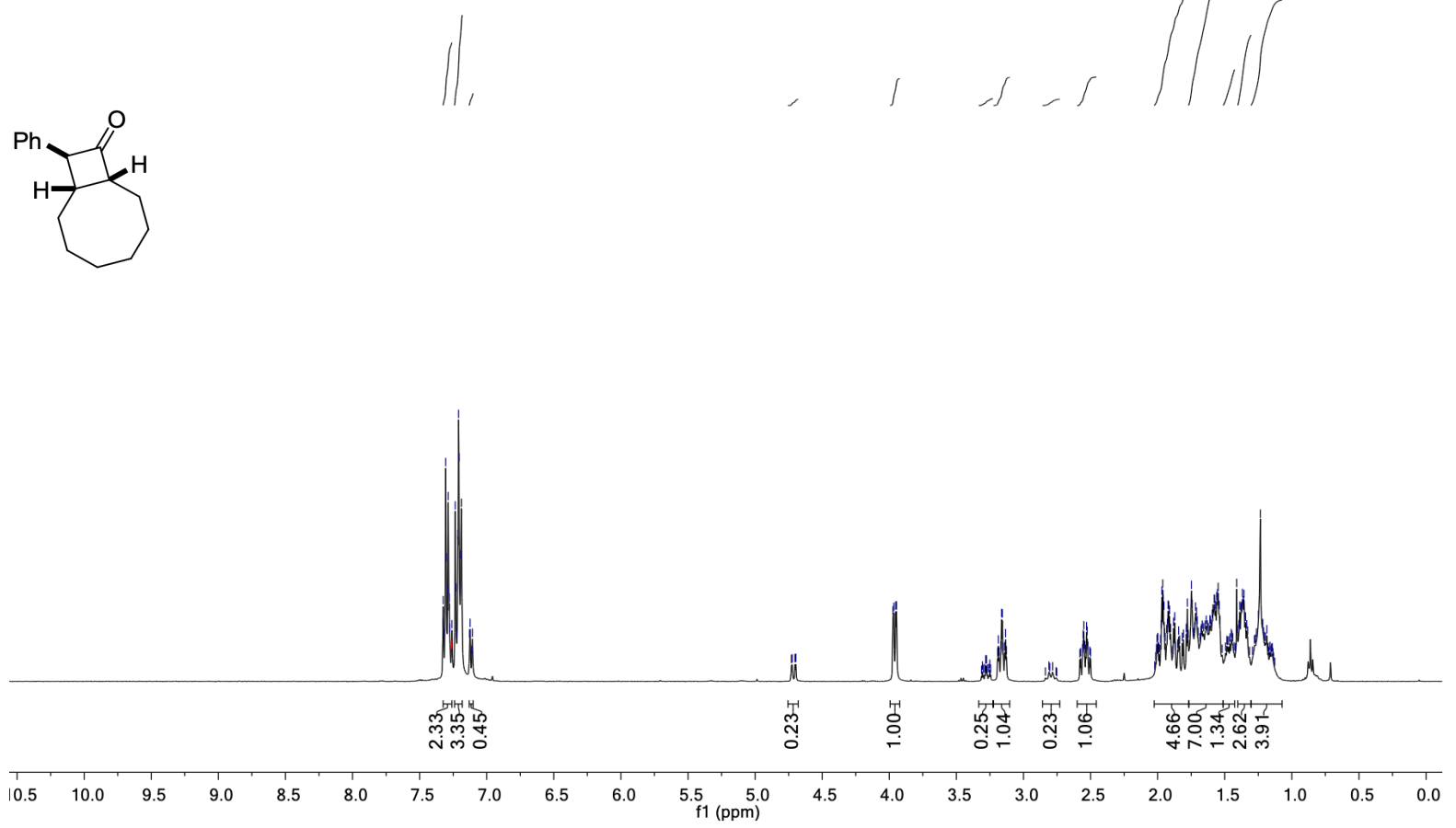

${ }^{13} \mathrm{C}$ NMR $\left(101 \mathrm{MHz}, \mathrm{CDCl}_{3}\right)$ of $\mathbf{5 e}$

$13 \mathrm{C}\{1 \mathrm{H}\} \mathrm{NMR}(126 \mathrm{MHz}, \mathrm{CDCl} 3)$
춘

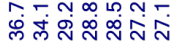
$\underbrace{-12}$

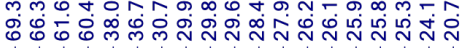

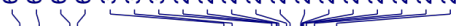

$\longrightarrow$

$\begin{array}{lllllllllll}210 & 200 & 190 & 180 & 170 & 160 & 150 & 140 & 130 & 120 & 110 \\ 100 \\ f 1(\mathrm{ppm})\end{array}$

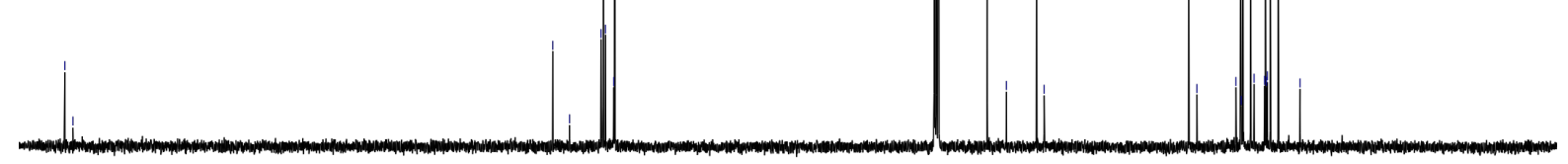




\section{Synthesis of $\alpha$-halo cyclobutanones 6a-c}

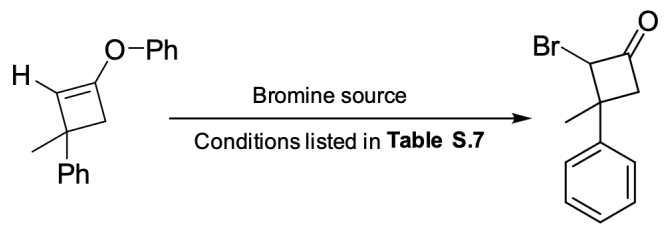

$3 a$

$6 a$

Table S.7. Optimization of the one pot-two steps synthesis of cyclobutanone $\mathbf{6 a}$

\begin{tabular}{|c|c|c|c|c|c|c|}
\hline Entry & Bromine source & Solvent ${ }^{a}$ & $\mathrm{~T}\left({ }^{\circ} \mathrm{C}\right)$ & $\mathrm{t}$ & $6 \mathbf{a}(\%)$ & d.r. \\
\hline 1 & $\mathrm{Br}_{2}$ (1.3 equiv) & $\mathrm{CCl}_{4}$ & 0 & $3 \mathrm{~h}$ & 58 & $3.5: 1$ \\
\hline 2 & $\mathrm{Br}_{2}$ (1.3 equiv) & $\mathrm{CH}_{2} \mathrm{Cl}_{2}$ & 0 & $3 \mathrm{~h}$ & 56 & $5.5: 1$ \\
\hline 3 & $\mathrm{Br}_{2}$ (1.3 equiv) & Toluene & 0 & $3 \mathrm{~h}$ & $80 \mathrm{~b}$ & $6: 1$ \\
\hline 4 & NBS ( 2 equiv) & $\mathrm{CCl}_{4}$ & 23 & $24 \mathrm{~h}$ & / & / \\
\hline 5 & NBS (2 equiv) & $\mathrm{CH}_{2} \mathrm{Cl}_{2}$ & 23 & $24 \mathrm{~h}$ & 20 & n.d. \\
\hline 6 & NBS (2 equiv) & Acetone & 23 & $24 \mathrm{~h}$ & $27^{c}$ & n.d. \\
\hline 7 & NBS ( 2 equiv) & $\mathrm{CH}_{3} \mathrm{CN}$ & 23 & $24 \mathrm{~h}$ & 41 & $4: 1$ \\
\hline
\end{tabular}

${ }^{a}$ Concentration: $0.1 \mathrm{M} .{ }^{\mathrm{b}}$ When performing the gold(I)-catalyzed [2+2] cycloaddition under the optimized conditions followed by bromination under these conditions (entry 3 ) in a one-pot-two-step process, the isolated yield for $6 \mathbf{6}$ went down to $18 \%$. ${ }^{\mathrm{c}}$ Yield determined by ${ }^{1} \mathrm{H}$ NMR using trichloroethylene as internal standard.

2-bromo-3-methyl-3-phenylcyclobutan-1-one (6a)

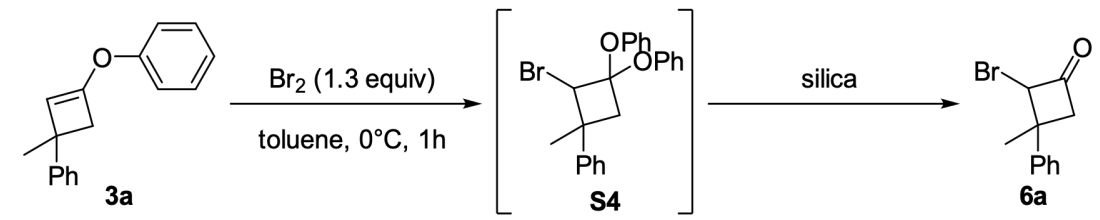

$\mathrm{Br}_{2}$ (62 mg, $0.39 \mathrm{mmol}, 1.3$ equiv) was added dropwise to a solution of (1-methyl-3-phenoxycyclobut-2-en-1yl)benzene (70.9 mg, $0.30 \mathrm{mmol}, 1$ equiv.) in dry toluene $(3.00 \mathrm{~mL}, 0.1 \mathrm{M})$ at $0{ }^{\circ} \mathrm{C}$ and the reaction mixture was left to stir for $1 \mathrm{~h}$ at the same temperature. The solvent was then evaporated and the crude redissolved in $\mathrm{CH}_{2} \mathrm{Cl}_{2}$, silica gel was added and the solvent evaporated again. The crude product absorbed on silica was purified by flash 
chromatography (2:1 DCM:cyclohexane) on silica gel delivering cyclobutanone 6a. in a 6:1 diastereomeric ratio (60 mg, 84\%) . A second column (30:1 pentane: $\left.\mathrm{Et}_{2} \mathrm{O}\right)$ afforded the major diastereomer as a transparent oil (49 $\mathrm{mg}, 68 \%)$.

Notes: After the evaporation of toluene, $\mathbf{S 4}$ was detected as the only product of the reaction. This intermediate completely hydrolyzes over purification on silica gel delivering the final product $\mathbf{6 a}$.

6a: Diastereomeric mixture + impurity: ${ }^{1} \mathrm{H} \mathrm{NMR}\left(500 \mathrm{MHz}, \mathrm{CDCl}_{3}\right) \delta 7.52$ (dddd, $J=6.7,4.1,2.8,1.6 \mathrm{~Hz}, 0.2 \mathrm{H}$, md), $7.44-7.33$ (m, 4.4H Md + md), $7.35-7.27$ (m, 1H, Md), $7.29-7.23$ (m, 0.4H, md), 5.35 (t, $J=2.2 \mathrm{~Hz}$, $1 \mathrm{H}, \mathrm{Md}$ ), 4.95 (dd, $J=4.9,2.2 \mathrm{~Hz}, 0.2 \mathrm{H}, \mathrm{md}$ ), 3.80 (dd, $J=17.1,2.1 \mathrm{~Hz}, 0.2 \mathrm{H}, \mathrm{md}$ ), 3.55 (ddq, $J=16.4,2.5,0.8$ $\mathrm{Hz}, 1 \mathrm{H}, \mathrm{Md}$ ), 3.20 (dd, $J=17.1,4.9 \mathrm{~Hz}, 0.2 \mathrm{H}, \mathrm{md}$ ), 3.13 (dd, $J=16.4,1.9 \mathrm{~Hz}, 1 \mathrm{H}, \mathrm{Md}$ ), 1.84 (s, $0.6 \mathrm{H}, \mathrm{md}$ ), 1.55 (d, $J=0.8 \mathrm{~Hz}, 3 \mathrm{H}, \mathrm{Md}$ ).

\section{${ }^{1} \mathrm{H}$ NMR (500 MHz, $\mathrm{CDCl}_{3}$ ) of $\mathbf{6 a}$ as mixture of diasteroisomers}

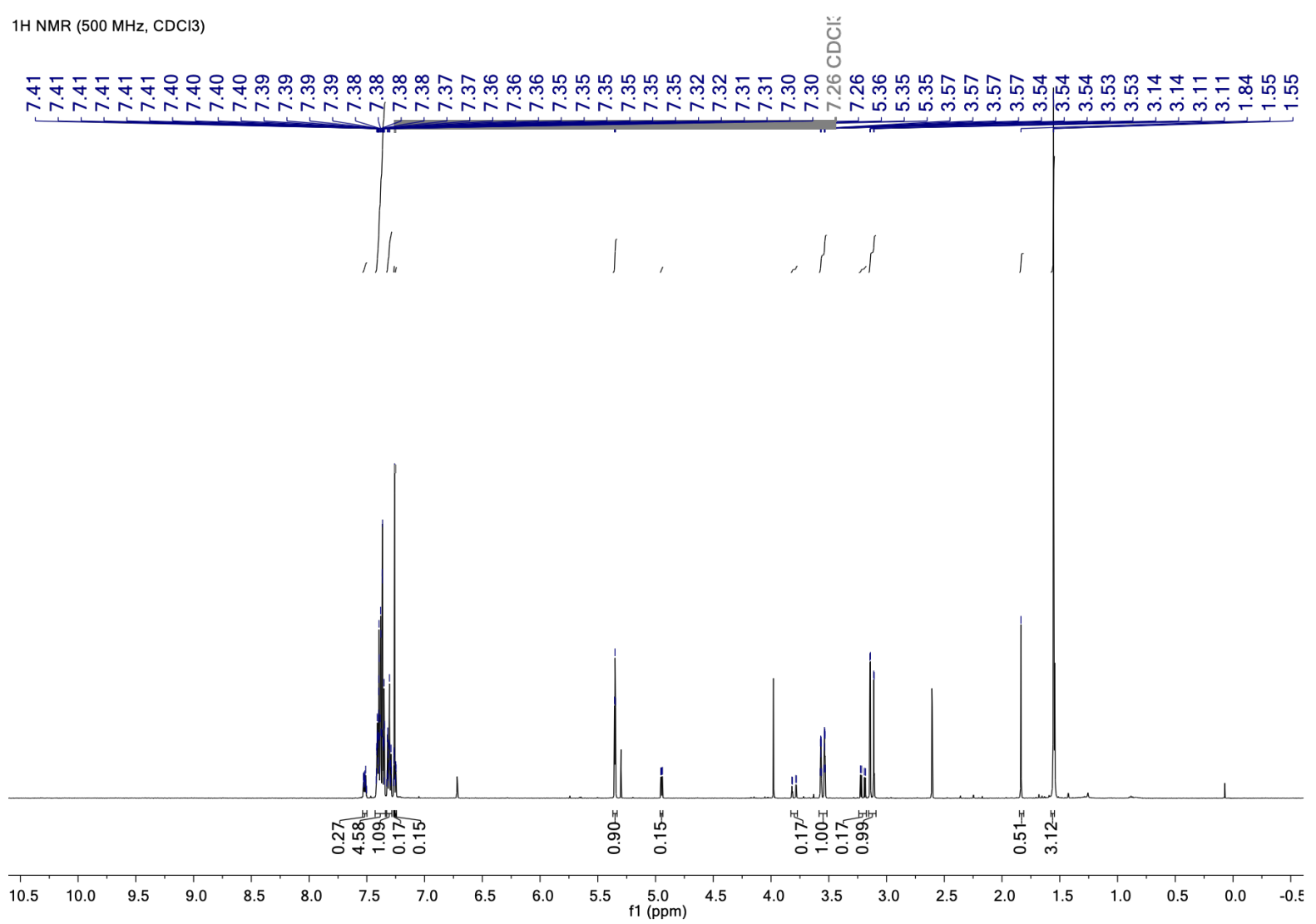

Major diasteromer: ${ }^{1} \mathbf{H}$ NMR $\left(400 \mathrm{MHz}, \mathrm{CDCl}_{3}\right) \delta 7.44-7.32(\mathrm{~m}, 4 \mathrm{H}), 7.36-7.26(\mathrm{~m}, 1 \mathrm{H}), 5.35(\mathrm{t}, J=2.2 \mathrm{~Hz}$, 1H), 3.55 (ddd, $J=16.4,2.6,0.9 \mathrm{~Hz}, 1 \mathrm{H}), 3.12$ (dd, $J=16.4,1.9 \mathrm{~Hz}, 1 \mathrm{H}), 1.55$ (s, $J=0.8 \mathrm{~Hz}, 3 \mathrm{H}$ ).

${ }^{13} \mathrm{C}$ NMR $\left(126 \mathrm{MHz}, \mathrm{CDCl}_{3}\right){ }^{13} \mathrm{C} \mathrm{NMR}\left(126 \mathrm{MHz}, \mathrm{CDCl}_{3}\right) \delta 197.76,146.55,128.93,127.24,125.19,60.54$, $54.59,39.67,28.17$.

HRMS (ESI) $\mathrm{m} / \mathrm{z}$ calculated for $\mathrm{C}_{11} \mathrm{H}_{11} \mathrm{BrNaO}^{+}[\mathrm{M}+\mathrm{Na}]^{+}: 260.9885$, found: 260.9881 . 
IR (selected frequency): $1787 \mathrm{~cm}^{-1}$.

${ }^{1} \mathrm{H}$ NMR $\left(400 \mathrm{MHz}, \mathrm{CDCl}_{3}\right)$ of $\mathbf{6 a}$

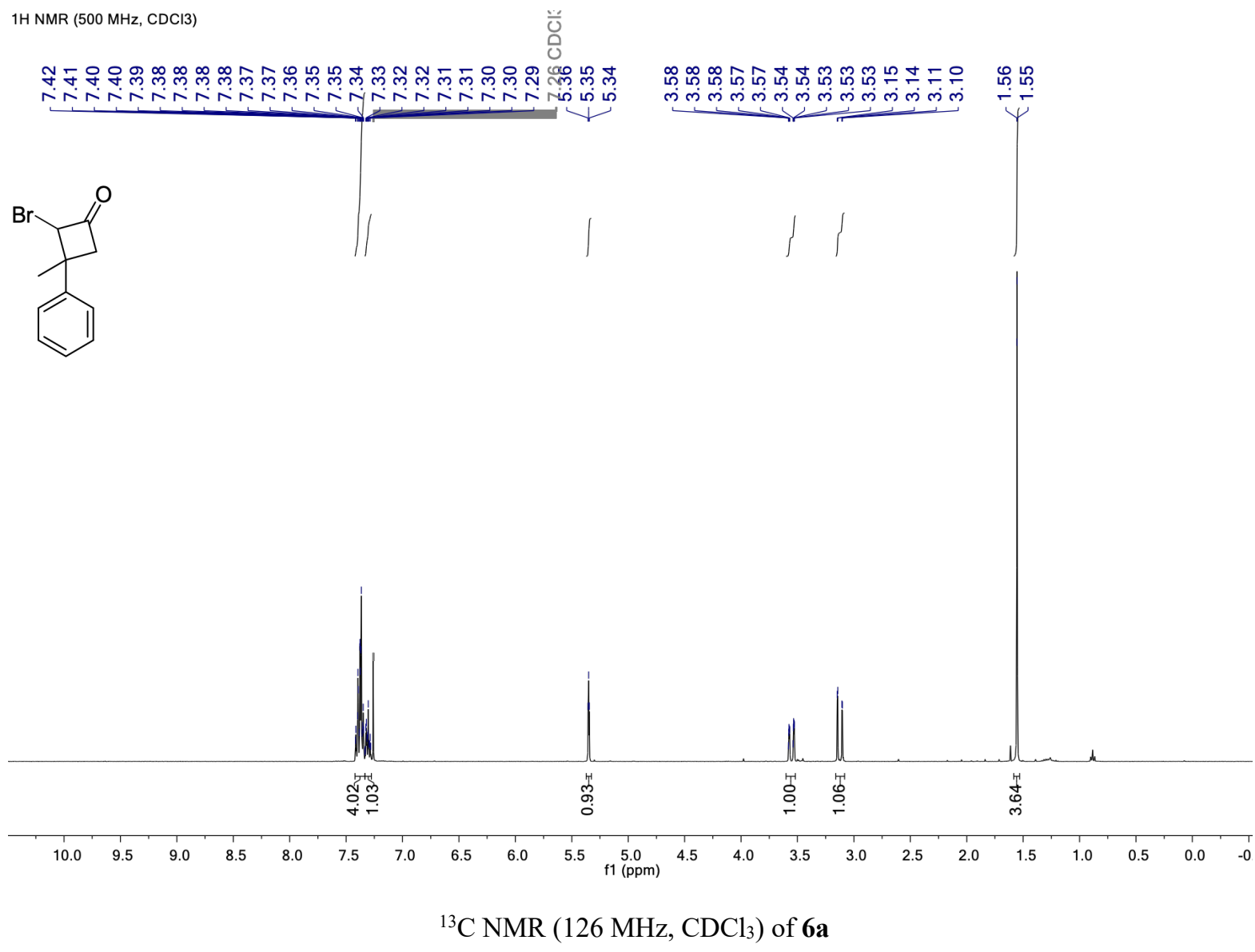


$13 \mathrm{C}\{1 \mathrm{H}\} \mathrm{NMR}(126 \mathrm{MHz}, \mathrm{CDCl} 3)$

$\stackrel{\infty}{\stackrel{\infty}{\sigma}}$

l

$\frac{1}{0}$
0
0

L̊ㅜㅇ<smiles>CC1(c2ccccc2)CC(=O)C1Br</smiles>

IR of $6 a$

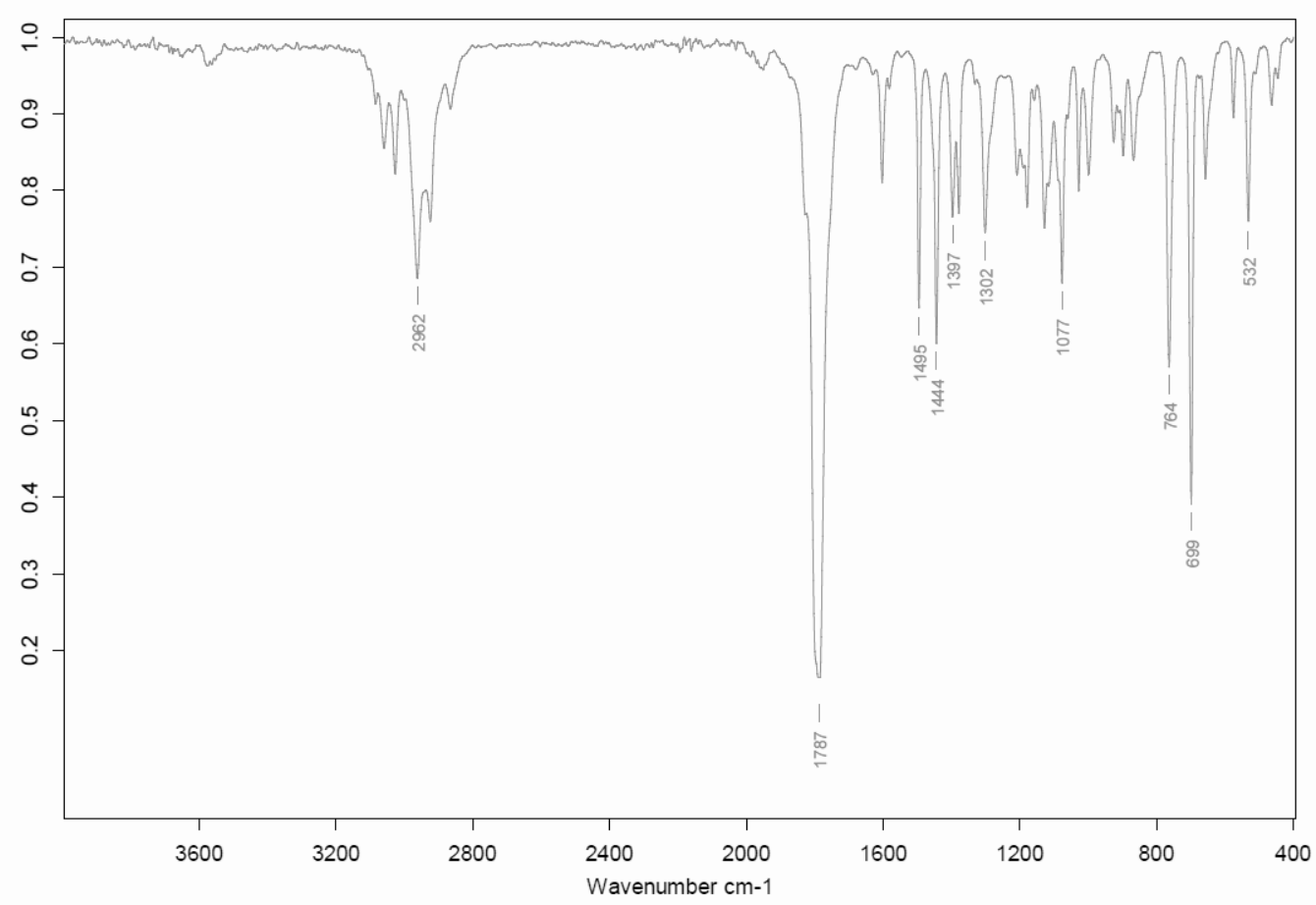


<smiles>CC1(c2ccc(F)cc2)CC(=O)C1Br</smiles>

Cyclobutanone 6b was prepared following the same procedure reported for 6a, starting from 2-bromo-3-(4fluorophenyl)-3-methylcyclobutan-1-one $(42.8 \mathrm{mg}, 0.17 \mathrm{mmol})$ and $\mathrm{Br}_{2}(32 \mathrm{mg}, 0.20 \mathrm{mmol})$ The crude product absorbed on silica was purified by flash chromatography (2:1 DCM:cyclohexane) on silica gel delivering cyclobutanone $6 \mathbf{b}$. in a 3:1 diastereomeric ratio (28 mg, 65\%). A second column (30:1 pentane:Et $2 \mathrm{O})$ afforded the major diastereomer as a yellow solid (20 mg, 46\%).

Diastereomeric mixture: ${ }^{1} \mathbf{H}$ NMR (500 MHz, $\left.\mathrm{CDCl}_{3}\right) \delta 7.36-7.28(\mathrm{~m}, 2 \mathrm{H}, \mathrm{Md}), 7.25-7.18(\mathrm{~m}, 0.8 \mathrm{H}, \mathrm{md}), 7.11$ $-7.03(\mathrm{~m}, 2.8 \mathrm{H}, \mathrm{Md}+\mathrm{md}), 5.30(\mathrm{t}, J=2.2 \mathrm{~Hz}, 1 \mathrm{H}, \mathrm{Md}), 4.94(\mathrm{dd}, J=4.8,2.2 \mathrm{~Hz}, 0.4 \mathrm{H}, \mathrm{md}), 3.74$ (dd, $J=17.1$, $2.1 \mathrm{~Hz}, 0.4 \mathrm{H}, \mathrm{md}), 3.52$ (ddq, $J=16.4,2.6,0.9 \mathrm{~Hz}, 1 \mathrm{H}, \mathrm{Md}), 3.22(\mathrm{dd}, J=17.1,4.8 \mathrm{~Hz}, 0.4 \mathrm{H}, \mathrm{md}), 3.12$ (dd, $J=$ 16.3, 1.9 Hz, 1H, Md), 1.82 (s, 1.2H, md), 1.53 (d, J=0.9 Hz, 3H, Md).

${ }^{1} \mathrm{H}$ NMR $\left(500 \mathrm{MHz}, \mathrm{CDCl}_{3}\right)$ of $\mathbf{6} \mathbf{b}$ as mixture of diasteroisomers

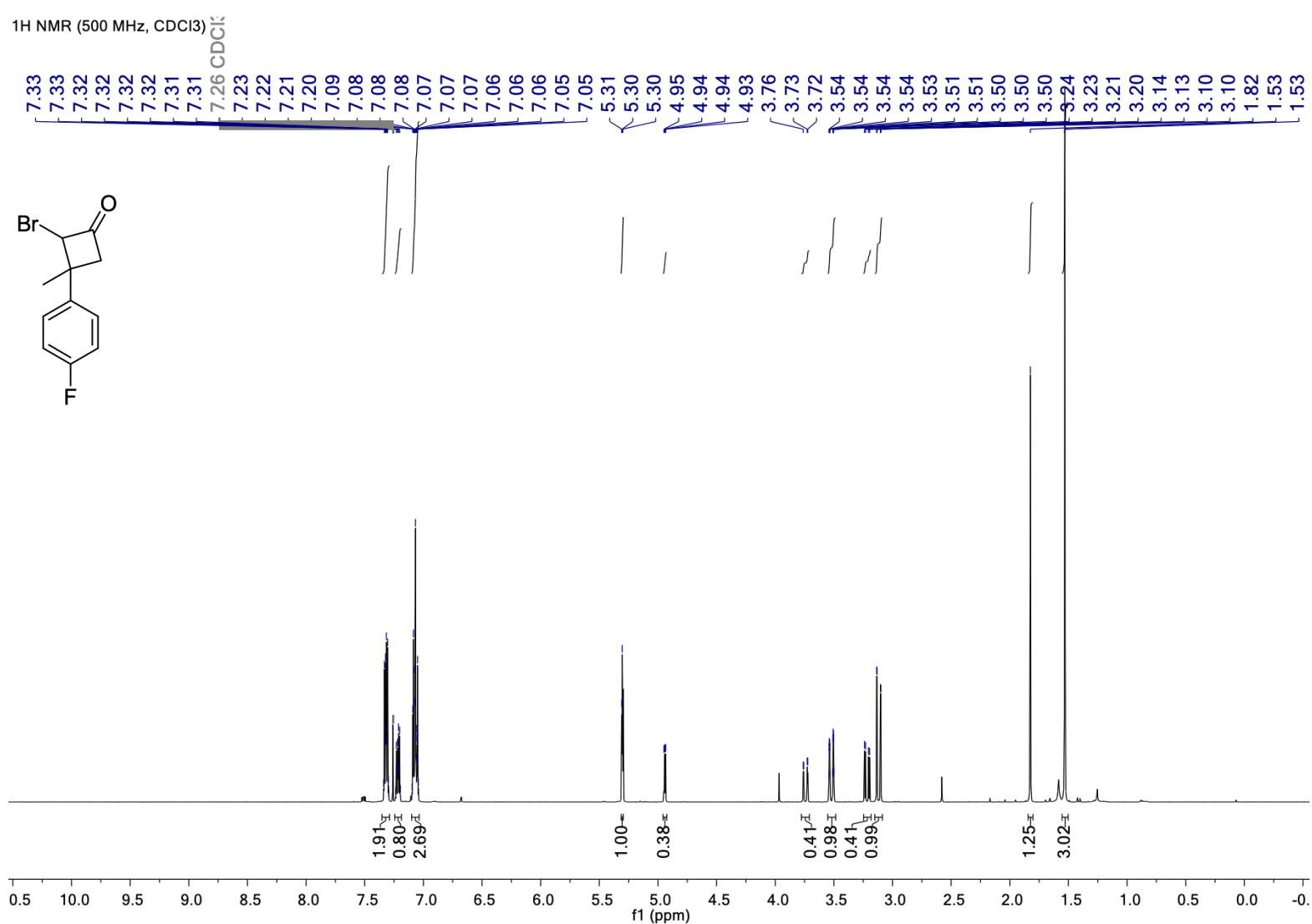

Major diastereomer:

M.p. $=58-62^{\circ} \mathrm{C}$. 
${ }^{1} \mathbf{H}$ NMR $\left(500 \mathrm{MHz}, \mathrm{CDCl}_{3}\right) \delta 7.36-7.28(\mathrm{~m}, 2 \mathrm{H}), 7.11-7.03(\mathrm{~m}, 2 \mathrm{H}), 5.30(\mathrm{t}, J=2.2 \mathrm{~Hz}, 1 \mathrm{H}), 3.52(\mathrm{ddt}, J=$ 16.4, 2.6, $0.9 \mathrm{~Hz}, 1 \mathrm{H}), 3.12$ (dd, $J=16.3,1.9 \mathrm{~Hz}, 1 \mathrm{H}), 1.53$ (d, $J=0.8 \mathrm{~Hz})$.

${ }^{19}$ F NMR $\left(376 \mathrm{MHz}, \mathrm{CDCl}_{3}\right) \delta-114.88$.

${ }^{13} \mathbf{C}$ NMR $\left(126 \mathrm{MHz}, \mathrm{CDCl}_{3}\right) \delta 197.30,161.89(\mathrm{~d}, J=246.2 \mathrm{~Hz}), 142.35(\mathrm{~d}, J=3.3 \mathrm{~Hz}), 126.95(\mathrm{~d}, J=8.0 \mathrm{~Hz})$, $115.83(\mathrm{~d}, J=21.6 \mathrm{~Hz}), 60.54,54.72,39.29,28.29$.

HRMS (ESI) $\mathrm{m} / \mathrm{z}$ calculated for $\mathrm{C}_{11} \mathrm{H}_{11} \mathrm{BrFO}^{+}[\mathrm{M}+\mathrm{H}]^{+}: 256.9972$, found: 256.9968 .

${ }^{1} \mathrm{H}$ NMR $\left(500 \mathrm{MHz}, \mathrm{CDCl}_{3}\right)$ of $\mathbf{6 b}$

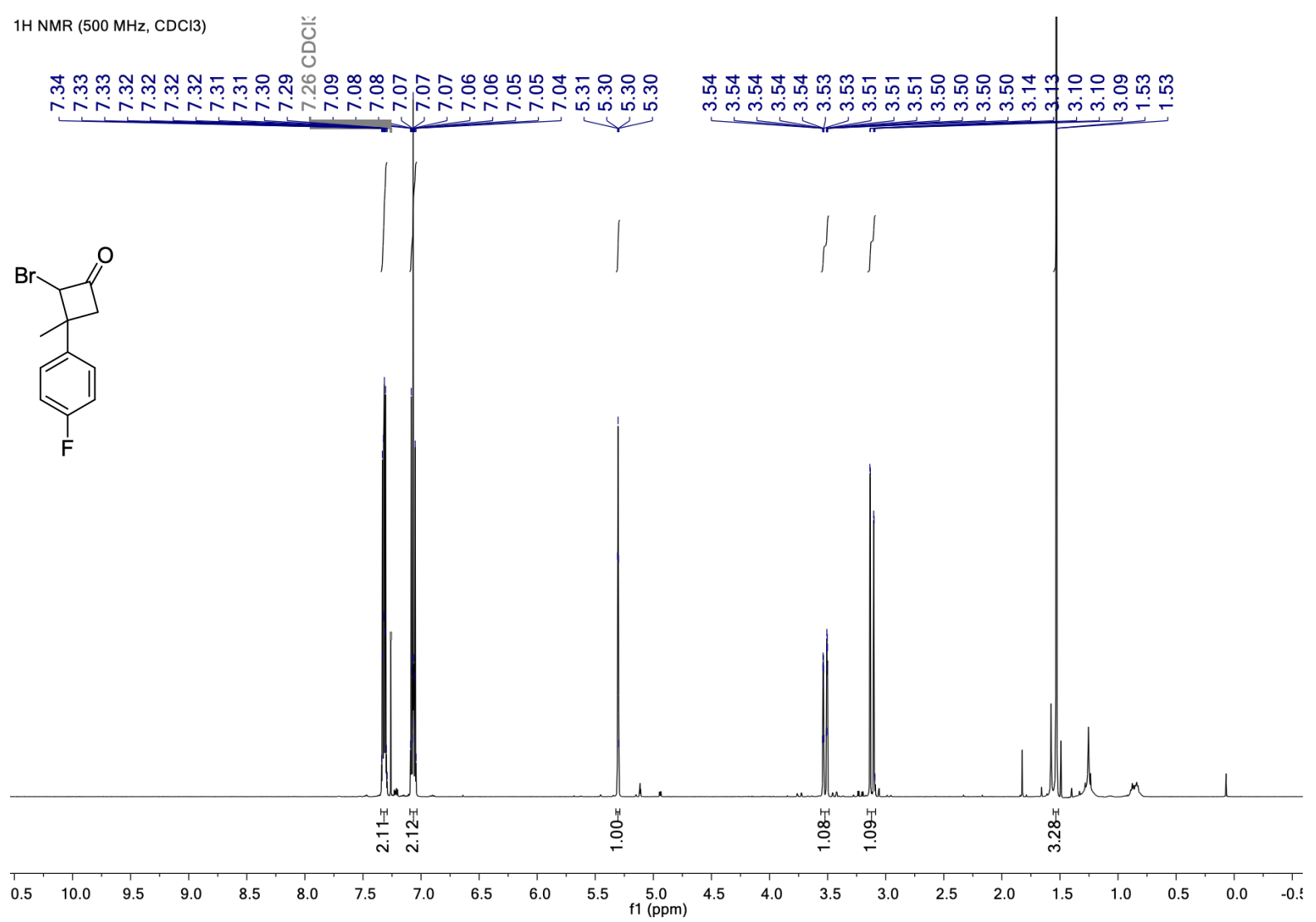


${ }^{19} \mathrm{~F} \mathrm{NMR}\left(376 \mathrm{MHz}, \mathrm{CDCl}_{3}\right)$ of $\mathbf{6 b}$

19F NMR (376 MHz, CDCl3)
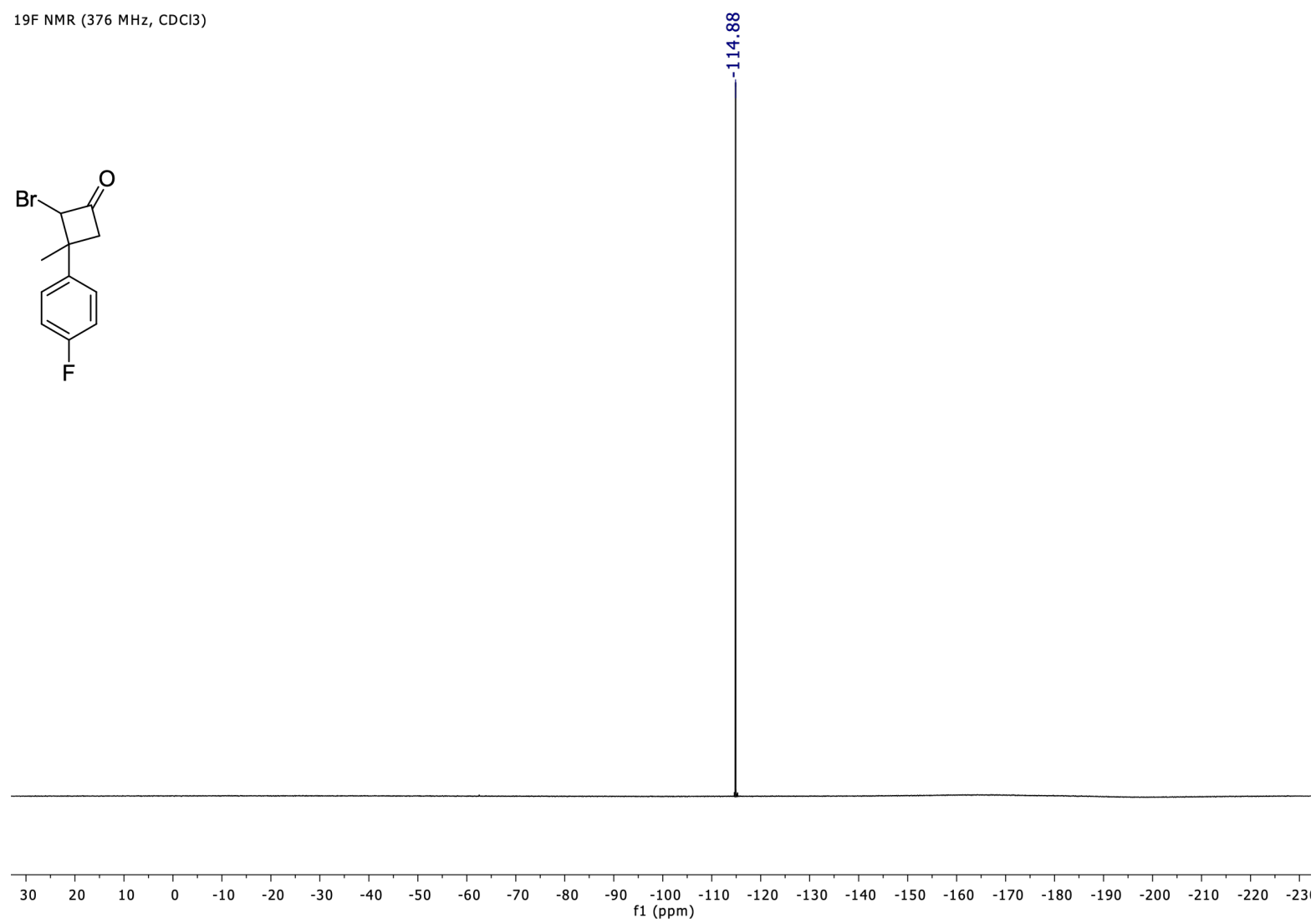

${ }^{13} \mathrm{C} \mathrm{NMR}\left(126 \mathrm{MHz}, \mathrm{CDCl}_{3}\right)$ of $\mathbf{6 b}$

$13 \mathrm{C}\{1 \mathrm{H}\} \mathrm{NMR}(126 \mathrm{MHz}, \mathrm{CDCl} 3)$

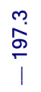

ํํㅇำ

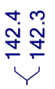

产通

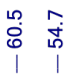

$\stackrel{m}{\infty} \stackrel{\infty}{\infty}$
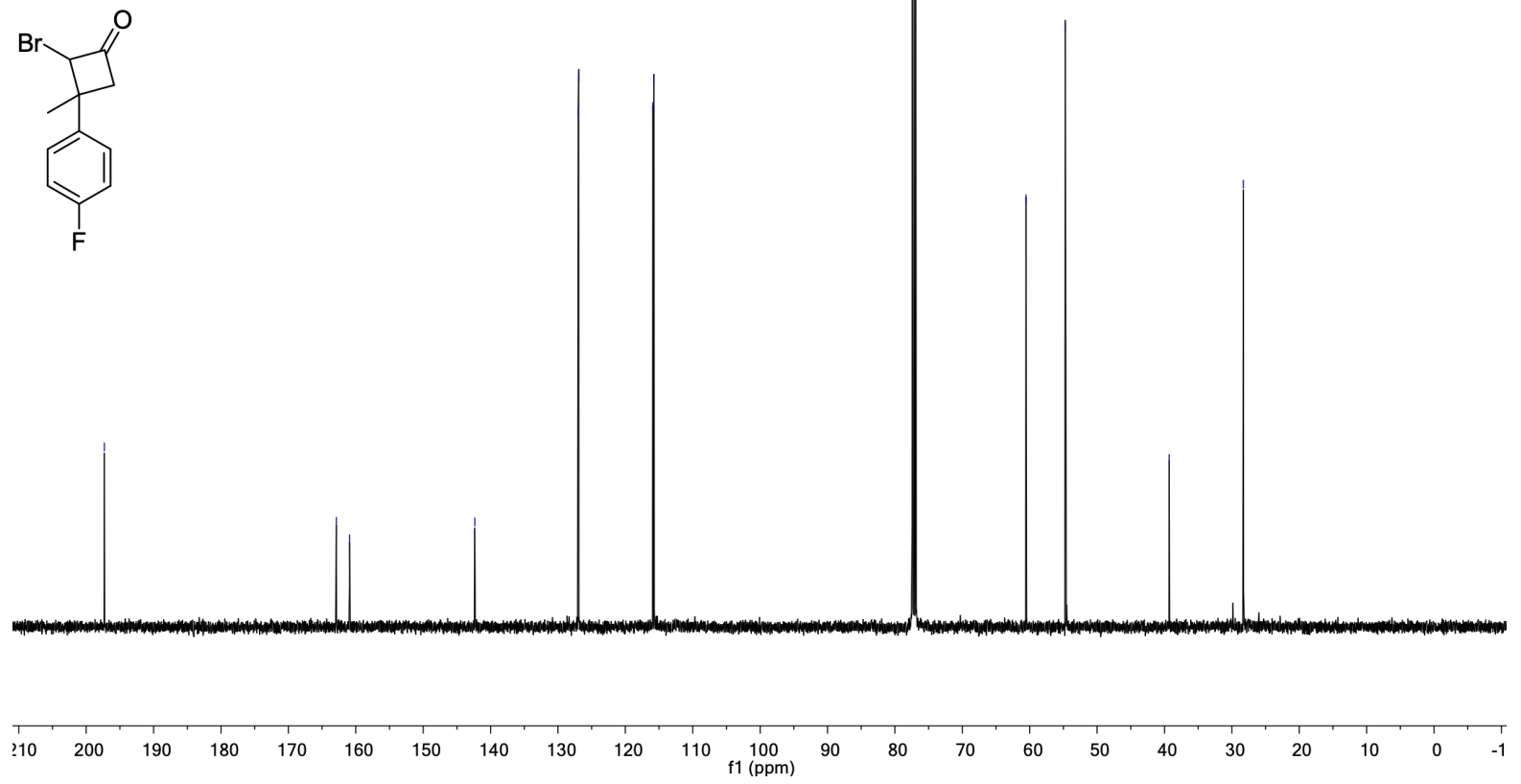

S-150 


\section{2-chloro-3-methyl-3-phenylcyclobutan-1-one (6c)}<smiles>CC1(c2ccccc2)CC(=O)C1Cl</smiles>

NCS (53.4 mg, $0.4 \mathrm{mmol}$ ) was added to a solution of (1-methyl-3-phenoxycyclobut-2-en-1-yl)benzene (47.3 mg, $0.2 \mathrm{mmol})$ in acetonitrile $(2.00 \mathrm{~mL})$ and the mixture was left to stir at $60^{\circ} \mathrm{C}$ for $72 \mathrm{~h}$. The solvent was evaporated and the crude mixture was purified by flash chromatography on silica gel (2:1 DCM:cyclohexane) affording product $6 \mathrm{c}$ as a $4.6: 1$ diastereomeric mixture $\left(20 \mathrm{mg}, 51 \%, d r\right.$ determined by ${ }^{1} \mathrm{H}$ NMR of the crude mixture $=$ 1.3:1). A second column (30:1 pentane:Et $2 \mathrm{O})$ afforded the major diastereomer as a transparent oil (15 $\mathrm{mg}, 39 \%)$.

Diastereomeric mixture: ${ }^{1} \mathbf{H}$ NMR $\left(500 \mathrm{MHz} \mathrm{CDCl}_{3}\right) \delta 7.43-7.36(\mathrm{~m}, 2.4 \mathrm{H} \mathrm{Md}+\mathrm{md}), 7.37-7.32(\mathrm{~m}, 2 \mathrm{H}$, $\mathrm{Md}), 7.34-7.26(\mathrm{~m}, 1.6 \mathrm{H}, \mathrm{Md}+\mathrm{md}), 5.16(\mathrm{t}, J=2.2 \mathrm{~Hz}, 1 \mathrm{H}, \mathrm{Md}), 4.83(\mathrm{dd}, J=4.1,2.2 \mathrm{~Hz}, 0.2 \mathrm{H}, \mathrm{md}), 3.70$ (dd, $J=17.3,2.2 \mathrm{~Hz}, 0.2 \mathrm{H}$, md), 3.47 (ddq, $J=16.4,2.5,0.9 \mathrm{~Hz}, 1 \mathrm{H}, \mathrm{Md}$ ), 3.20 (dd, $J=17.3,4.1 \mathrm{~Hz}, 0.2 \mathrm{H}, \mathrm{md}$ ), $3.08(\mathrm{dd}, J=16.4,2.1 \mathrm{~Hz}, 1 \mathrm{H}, \mathrm{Md}), 1.86$ (s, 0.6H, md), 1.51 (d, $J=0.9 \mathrm{~Hz}, 3 \mathrm{H}, \mathrm{Md})$.

${ }^{1} \mathrm{H}$ NMR $\left(500 \mathrm{MHz}, \mathrm{CDCl}_{3}\right)$ of $\mathbf{6 c}$ as mixture of diasteroisomers

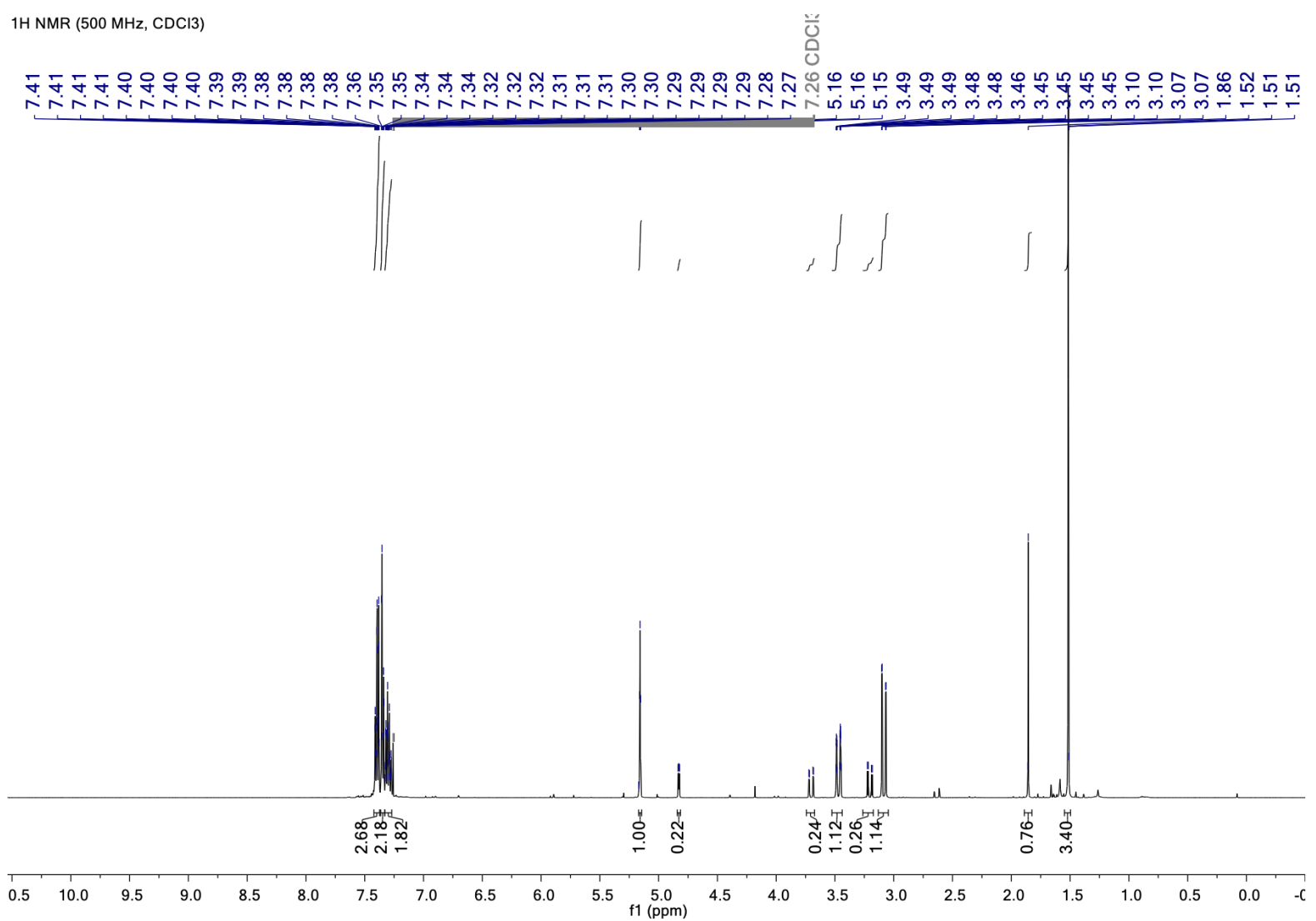

Major diastereomer: ${ }^{1} \mathbf{H}$ NMR $\left(500 \mathrm{MHz}, \mathrm{CDCl}_{3}\right) \delta 7.43-7.36(\mathrm{~m}, 2 \mathrm{H}), 7.38-7.31(\mathrm{~m}, 2 \mathrm{H}), 7.34-7.27$ $(\mathrm{m}, 1 \mathrm{H}), 5.16(\mathrm{t}, J=2.2 \mathrm{~Hz}, 1 \mathrm{H}), 3.51-3.43(\mathrm{~m}, 1 \mathrm{H}), 3.08(\mathrm{dd}, J=16.4,2.0 \mathrm{~Hz}, 1 \mathrm{H}), 1.51(\mathrm{~d}, J=0.9$ $\mathrm{Hz}, 3 \mathrm{H})$. 
${ }^{13}$ C NMR (126 MHz, $\left.\mathrm{CDCl}_{3}\right) \delta 198.4,146.6,129.0,127.3,125.3,70.3,54.5,40.7,25.9$.

HRMS (ESI) $\mathrm{m} / \mathrm{z}$ calculated for $\mathrm{C}_{11} \mathrm{H}_{11} \mathrm{ClNaO}^{+}[\mathrm{M}+\mathrm{Na}]^{+}: 217.0391$, found: 217.0386 .

${ }^{1} \mathrm{H}$ NMR $\left(500 \mathrm{MHz}, \mathrm{CDCl}_{3}\right)$ of $\mathbf{6 c}$

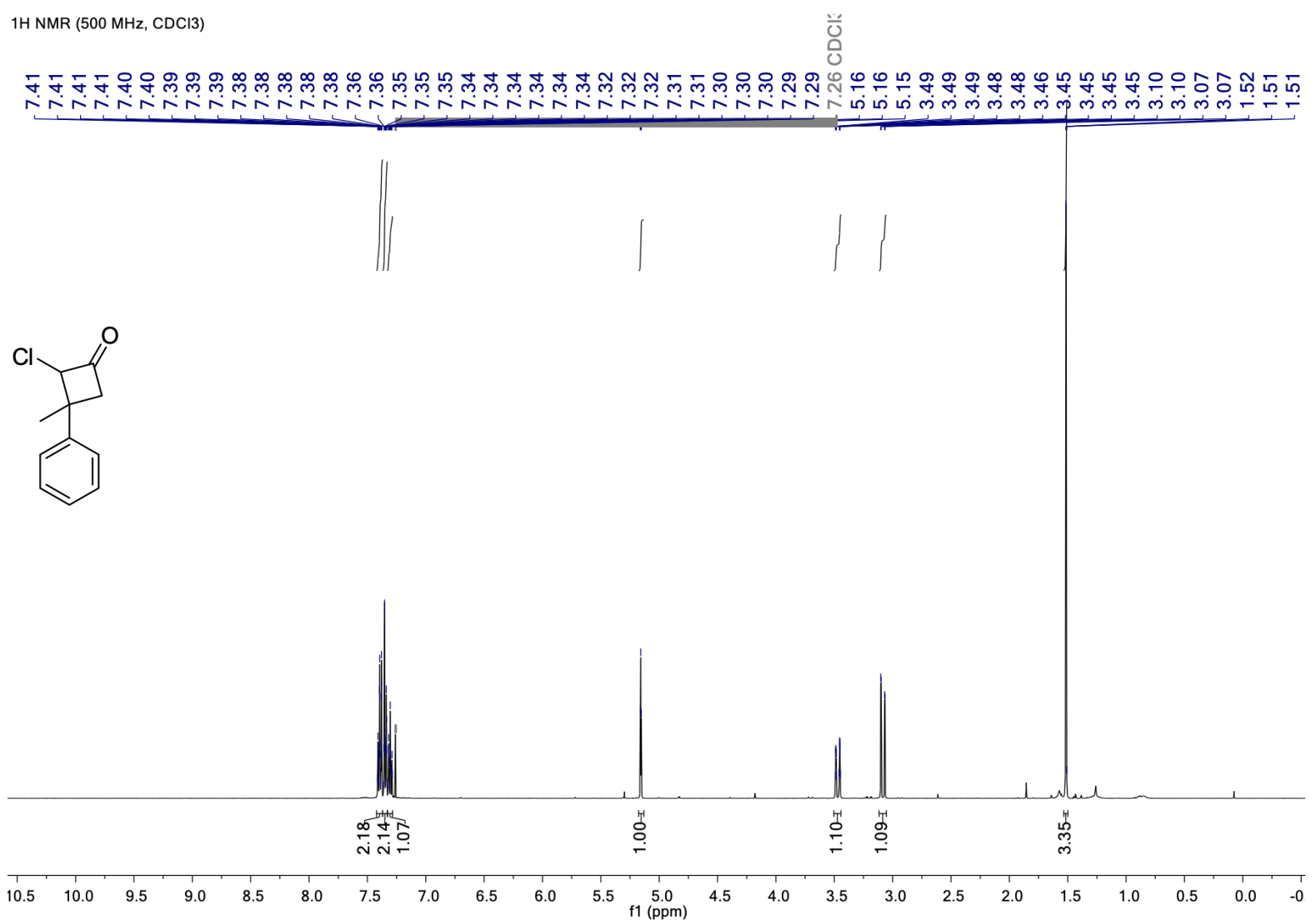


${ }^{13} \mathrm{C}$ NMR (126 MHz, $\left.\mathrm{CDCl}_{3}\right)$ of $\mathbf{6 c}$

$13 \mathrm{C}\{1 \mathrm{H}\} \mathrm{NMR}(126 \mathrm{MHz}, \mathrm{CDCl} 3)$

ণ্

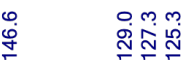

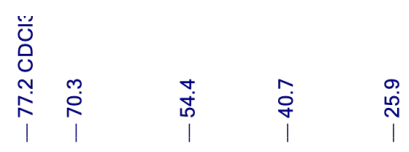<smiles>CC1(c2ccccc2)CC(=O)C1Cl</smiles>

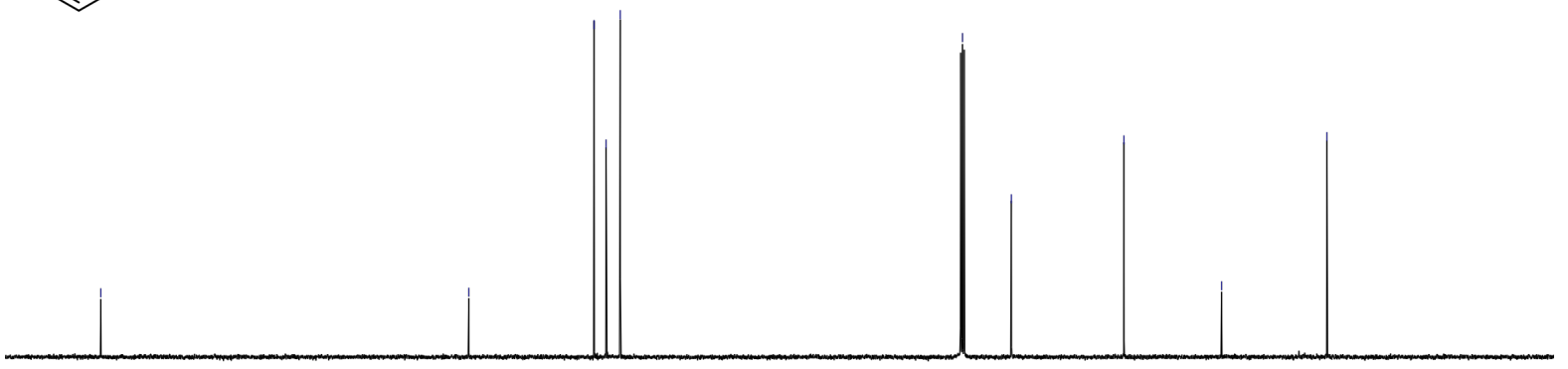

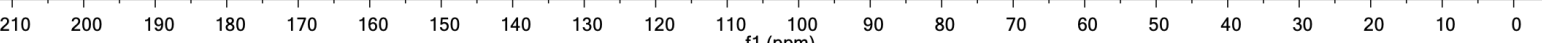




\section{Theoretical DFT Calculations}

\section{Computational Methods}

Calculations were performed by means of the Gaussian 09 suite of programs. ${ }^{\mathrm{xi}}$ DFT was applied using B3LYP ${ }^{\mathrm{xii}}$ The LANL2DZ basis set ${ }^{\mathrm{xii}, \mathrm{xiv}}$ was utilized to describe Au with ECP and additional polarization function $\left(\zeta_{\mathrm{f}}=\right.$ 1.050 for $\left.\mathrm{Au},{ }^{\mathrm{xv}}\right)$. The $6-31 \mathrm{G}(\mathrm{d}, \mathrm{p})$ basis set ${ }^{\mathrm{xvi}}$ was employed for all remaining atoms $(\mathrm{C}, \mathrm{H}, \mathrm{O}$ and $\mathrm{P})$. Full geometry optimizations were carried out in $\mathrm{CH}_{2} \mathrm{Cl}_{2}$, through an implicit solvent SMD. ${ }^{\text {xvii }}$ The stationary points were characterized by vibrational analysis.

Transition states were identified by the presence of one imaginary frequency while minima by a full set of real frequencies. The connectivity of the transition states was confirmed by relaxing each transition state towards both the reactant and the product. Reported energies are potential energies (E) and free energies (G) in solution, computed at $298 \mathrm{~K}$ and $1 \mathrm{~atm}$.

\section{Mechanistic Studies on the Gold(I) Catalyzed Reactions of Ynol Ethers}

\section{Reaction of terminal ynol ethers}

Activation of the alkyne

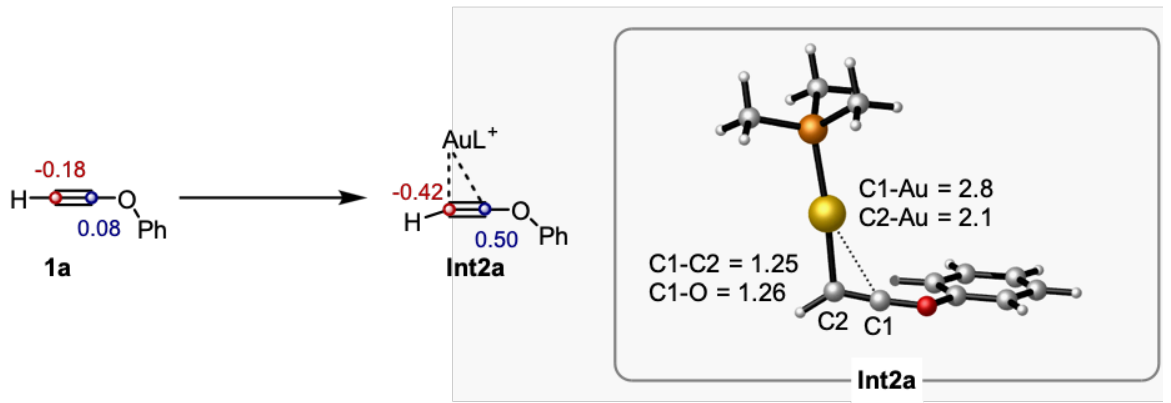

Figure S.1. Computed structures of 2a and Int2a with charge distribution expressed. Positive charges in blue and negative charges in red. $\left(\mathrm{L}=\mathrm{PMe}_{3}\right)$.

The computed structure of 1a displays a short $\mathrm{C}(\mathrm{sp})-\mathrm{O}$ distance $(1.30 \AA)$ and, as expected, a partial charge separation on the alkyne (Figure S.1). The $\eta^{2}$ complex with gold(I) (Int2a) is highly distorted and gold is located at $2.1 \AA$ from $\mathrm{C} 2$ and $2.8 \AA$ from $\mathrm{C} 1$. The coordination also causes an elongation of the $\mathrm{C}$ - $\mathrm{C}$ triple bond and the shortening of the $\mathrm{C}-\mathrm{O}$ bond and the two bonds now have almost the same length (1.25 $\AA$ and $1.26 \AA$, respectively). Hence the resulting structure resemble a metalated ketene. ${ }^{\text {xviii }}$ Also, by coordination of gold(I) the separation of the partial charges on the triple bond increases and $\mathrm{C} 1$ results even more electrophilic. 

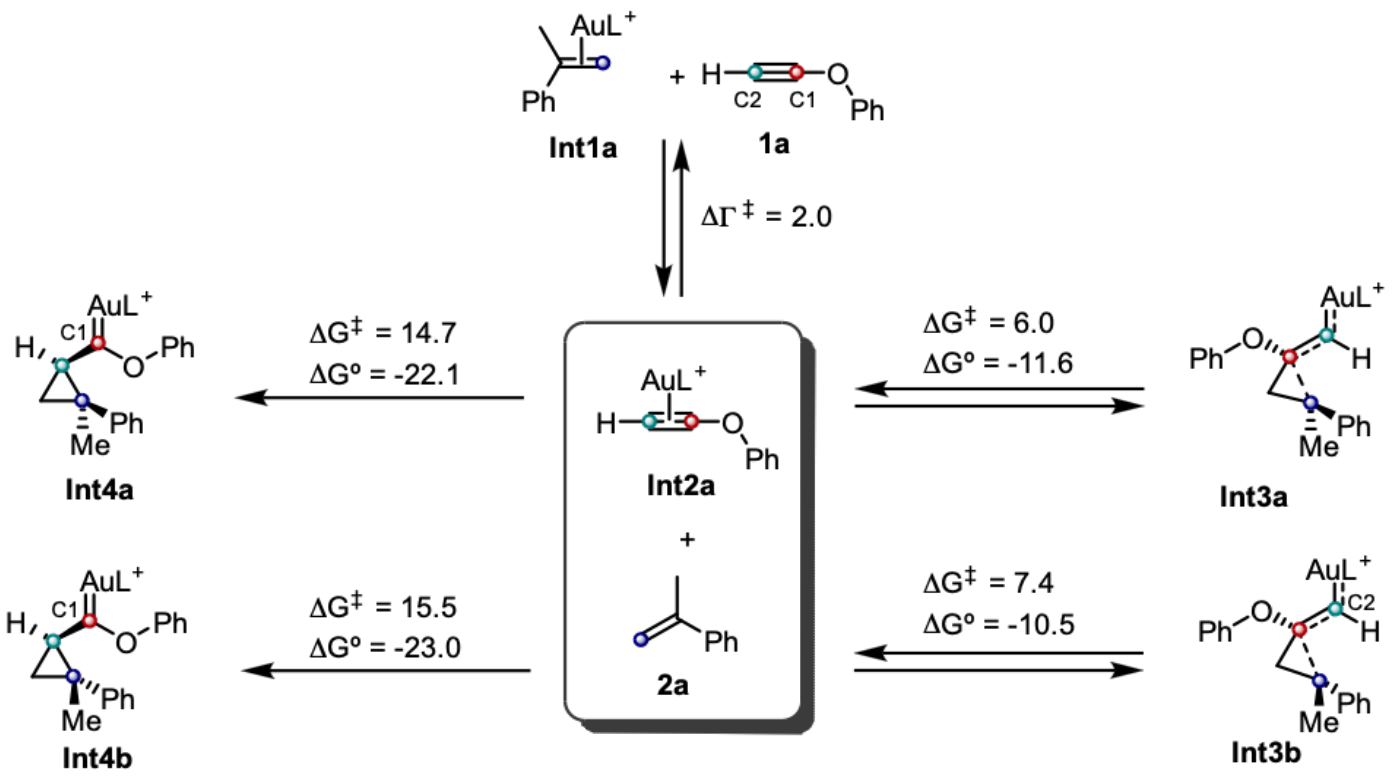

Scheme S.2. Formation of cyclopropyl gold(I) carbene Int3a-b and Int4a-b ( $\mathrm{L}=\mathrm{PMe}_{3}$. Free energies in $\left.\mathrm{kcal} / \mathrm{mol}\right)$.

The associative ligand exchange between Int1a and the ynol ether gives ( $\eta^{2}$-alkyne)gold(I) complex Int2a. Interestingly, in this case the ( $\eta^{2}$-alkene)gold(I) complex Int1a is less stable than Int2a. Comparing the energy barriers for the formation of the four possible cyclopropyl gold(I) carbenes Int3a-b and Int4a-b, the attack of the styrene on carbon $\mathrm{C} 2$ to form cyclopropyl gold(I) carbenes Int4a-b $\left(\Delta \mathrm{G}^{\ddagger}=14.7-15.5 \mathrm{kcal} / \mathrm{mol}\right)$ is highly exothermic due to the stabilization of the oxygen $\left(\Delta G^{\circ}=-22.8--23.0 \mathrm{kcal} / \mathrm{mol}\right)$. However, the formation of Int3ab $\left(\Delta \mathrm{G}^{\ddagger}=6.0-7.4 \mathrm{kcal} / \mathrm{mol}\right)$ is favored kinetically by ca $7-9 \mathrm{kcal} / \mathrm{mol}$. Interestingly, Int4a-b present a highly carbenic character due to the stabilization of the oxygen on C1, while in Int3a-b the cyclopropyl ring is more open and the intermediate is closer to a homoallylic gold(I)-stabilized cation with the benzylic carbon positively charged.

(A)
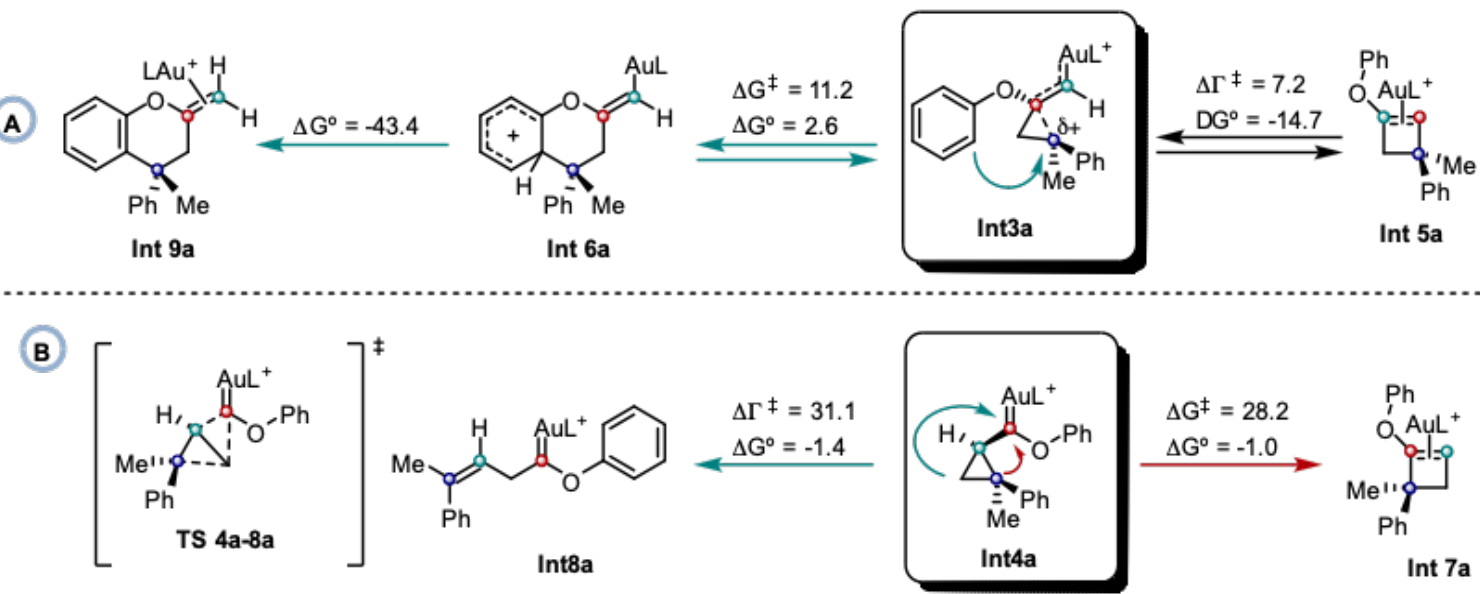

Scheme S.3. Evolution of Int3a and Int4a. $\left(\mathrm{L}=\mathrm{PMe}_{3}\right.$. Free energies in $\left.\mathrm{kcal} / \mathrm{mol}\right)$.

Starting from Int3a, Int5a can be formed upon exothermic ring expansion $\left(\Delta \mathrm{G}^{\ddagger}=7.2 \mathrm{kcal} / \mathrm{mol}\right)$, which will lead to the observed product 3a upon ligand exchange with another molecule of substrate. Alternatively, the aromatic ring can attack the benzylic carbon on the cyclopropyl ring in a Friedel-Craft-type reaction forming Wheland 
intermediate Int6a $\left(\Delta \mathrm{G}^{\ddagger}=11.2 \mathrm{kcal} / \mathrm{mol}\right)$. Thus, for terminal alkynes this second pathway is disfavored both kinetically and thermodynamically.

In the case of Int4a, the attack of the aromatic ring is disfavored because of the increased carbenic nature of the intermediate. Instead Int7a can be formed via ring expansion $\left(\Delta \mathrm{G}^{\ddagger}=28.2 \mathrm{kcal} / \mathrm{mol}\right)$ or Int8a can be generated upon migration of carbon $\mathrm{C} 3$ on the $\operatorname{gold}(\mathrm{I}) \operatorname{carbene}\left(\Delta \mathrm{G}^{*}=31.1 \mathrm{kcal} / \mathrm{mol}\right)$. The energy barriers for these two transformations are too high to compete at room temperature with the formation of Int5a from Int3a, in full agreement with the experimentally observed regioselective formation of the cyclobutene derived from Int5a.

\section{Reaction of internal ynol ethers}
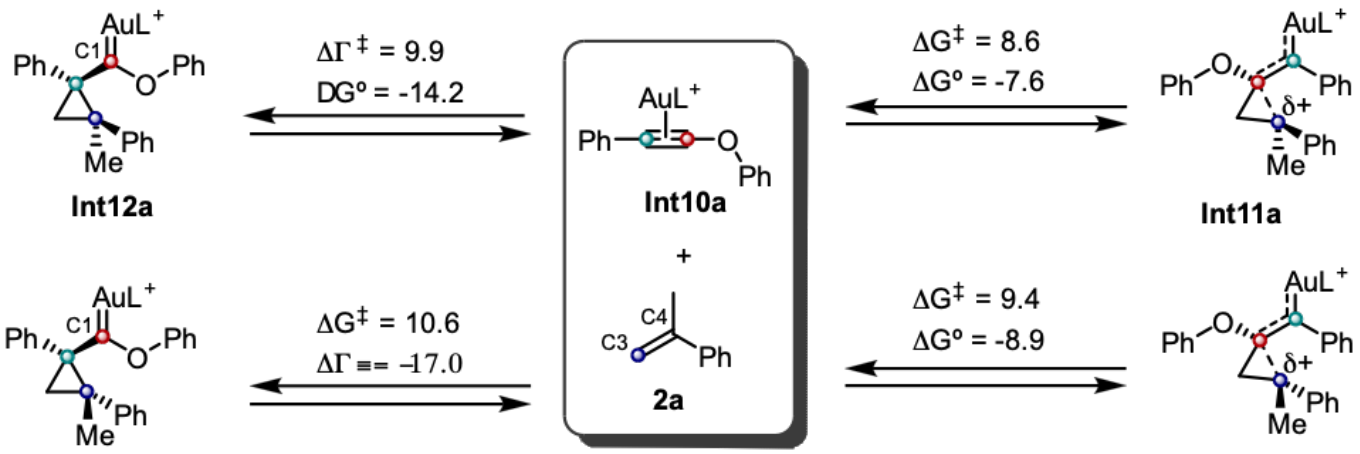

Int12b

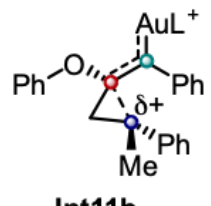

Int11b

Scheme S.4. Formation of cyclopropyl gold(I) carbene Int11a-b and Int12a-b ( $\mathrm{L}=\mathrm{PMe}_{3}$. Free energies in $\left.\mathrm{kcal} / \mathrm{mol}\right)$.

The reaction starts as before with the formation of four possible cyclopropyl gold(I) carbenes Int11a-b and Int12a-b, two diastereoisomers for each of the regioisomers. Compared to the previous case, the difference in activation energy for the formation of the two regioisomers is smaller: for Int11a we found $\Delta \mathrm{G}^{\star}=8.6 \mathrm{kcal} / \mathrm{mol}$ and for Int12a $\Delta \mathrm{G}^{\star}=9.9 \mathrm{kcal} / \mathrm{mol}$, but still the attack of the alkene on carbon $\mathrm{C} 1$ remains favored.

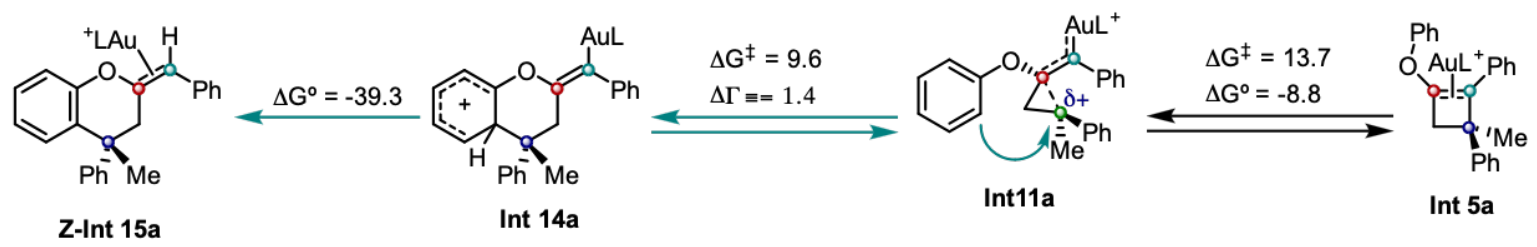

Scheme S.5. Evolution of Int11a. ( $\mathrm{L}=\mathrm{PMe}_{3}$. Free energies in $\left.\mathrm{kcal} / \mathrm{mol}\right)$. and computed structure of structure of Int11a.

The possible evolutions of Int11a are analogous of the one calculated for Int3a. However, in this case the attack of the aromatic ring to form Int14a is favored over the ring expansion towards Int13a $\left(\Delta \mathrm{G}^{\ddagger}=9.6 \mathrm{kcal} / \mathrm{mol} \mathrm{vs}\right.$ $\left.\Delta \mathrm{G}^{*}=13.7 \mathrm{kcal} / \mathrm{mol}\right)$. Wheland intermediate Int14a then undergoes deprotonation and protodeauration in highly an exergonic sequence $\left(\Delta \mathrm{G}^{\circ}=-39.3 \mathrm{kcal} / \mathrm{mol}\right)$ delivering the observed product. This model agreed with the observed selectivity towards the $(4+2)$ cycloaddition for the internal ynol ethers and suggests that the product initially formed has the $E$-configuration. 


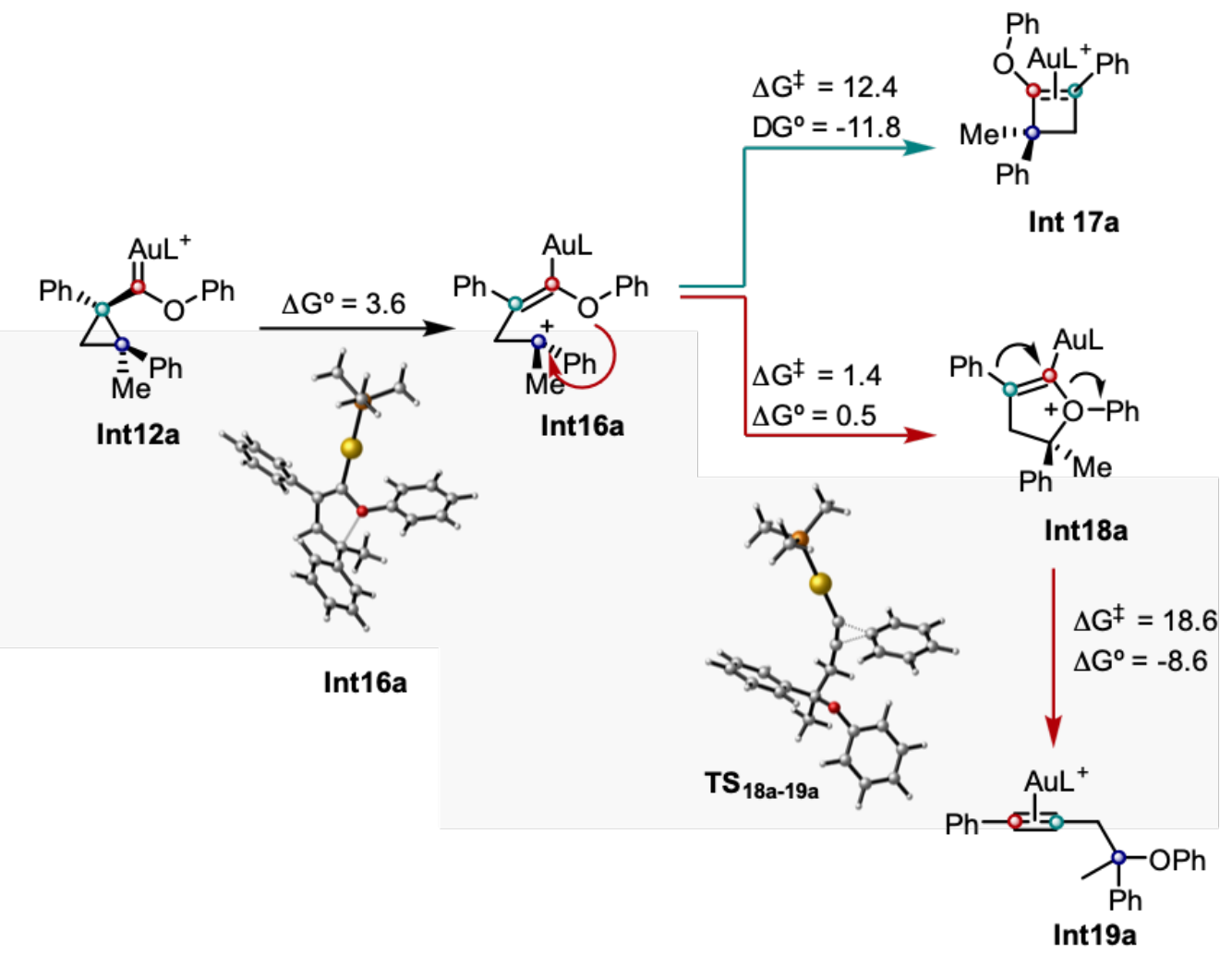

Scheme S.6. Evolution of Int12a. ( $\mathrm{L}=\mathrm{PMe}_{3}$. Free energies in $\left.\mathrm{kcal} / \mathrm{mol}\right)$.

On the other hand, Int12a evolves to the open carbocation Int16a $\left(\Delta \mathrm{G}^{\circ}=3.6 \mathrm{kcal} / \mathrm{mol}\right)$ stabilized by the oxygen. Starting from this intermediate two paths are possible. First, it can undergo ring expansion to form Int17a, regioisomer of Int13a, with an energy barrier of $12.4 \mathrm{kcal} / \mathrm{mol}$. In alternative, the attack of the oxygen on the open carbocation to form the 5-membered oxonium cation Int18a is almost barrierless $\left(\Delta \mathrm{G}^{*}=1.4 \mathrm{kcal} / \mathrm{mol}\right)$. An analogous bromonium cation was already reported by our group as key intermediate for the gold(I) catalyzed formation of skipped enyne from bromoalkynes. ${ }^{\text {xix }} 16$ Int18a can undergo aryl-assisted ring-opening via a vinylidenephenonium gold(I) cationic transition state to from Int19a ${ }^{\mathrm{xx}}$

Studies on the regioselective formation of $3 q q$

In the case of 1e, the formation of Int11a is favored over Int12a accounting for the formation of 4a. However, experimentally we observed that the formation of the cyclobutene $\mathbf{3 q q}$ is possible in presence of electron rich substituents. So, we decided to investigate computationally the step of formation of the cyclopropyl gold(I) carbene between $1 \mathbf{f}$ and 2a. Interestingly, we found that two geometries that differs only by $0.4 \mathrm{kcal} / \mathrm{mol} \mathrm{are}$ possible for the ( $\eta^{2}$-alkyne)gold(I) complex. The first one is analogous of Int2a with the gold atom closer to C2 $(\mathrm{C} 2-\mathrm{Au}=2.20 \AA \mathrm{Vs} \mathrm{C} 1-\mathrm{Au}=2.58 \AA)$ and activating $\mathrm{C} 1$ as electrophile. In the second one, the gold atom stays closer to $\mathrm{C} 1(\mathrm{C} 1-\mathrm{Au}=2.15 \AA \mathrm{Vs} \mathrm{C} 2-\mathrm{Au}=2.63 \AA)$ due to the electron density provided by the electron rich aromatic ring to the alkyne. In this second coordination mode, the distribution of the partial charges is inverted and $\mathrm{C} 2$ is more electrophilic of C1. Starting from Int20a, the formation of Int22a $\left(\Delta \mathrm{G}^{*}=8.0 \mathrm{kcal} / \mathrm{mol}^{-} \Delta \mathrm{G}^{\circ}=\right.$ $13.0 \mathrm{kcal} / \mathrm{mol})$ was found to be favored over Int21a $\left(\Delta \mathrm{G}^{*}=10.5 \mathrm{kcal} / \mathrm{mol}, \Delta \mathrm{G}^{\circ}=-6.4 \mathrm{kcal} / \mathrm{mol}\right)$ in line with the experimental result. 

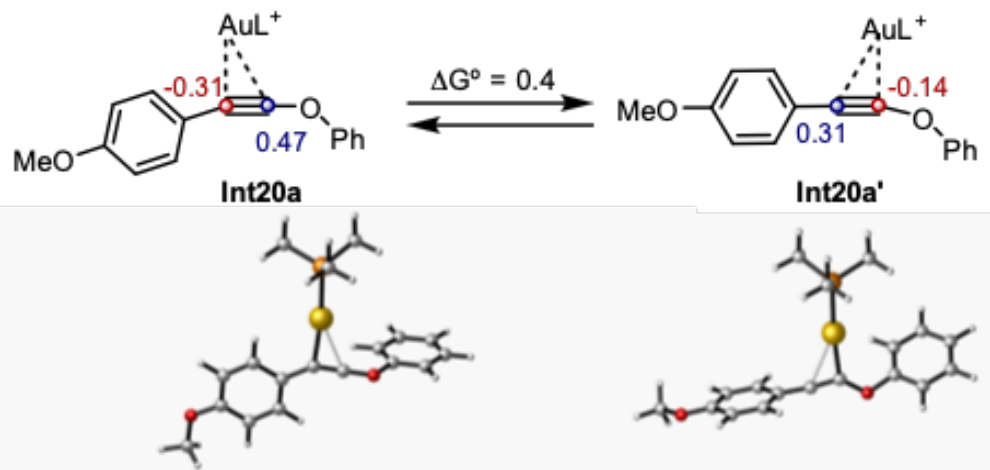

Figure S.2. Computed structures of the two possible ( $\eta^{2}$-alkyne)gold(I) complex with $\mathbf{1 f}$, with charge distribution on the alkyne expressed. Positive charges in blue and negative charges in red. $\left(\mathrm{L}=\mathrm{PMe}_{3}\right.$. Free energies in $\left.\mathrm{kcal} / \mathrm{mol}\right)$.

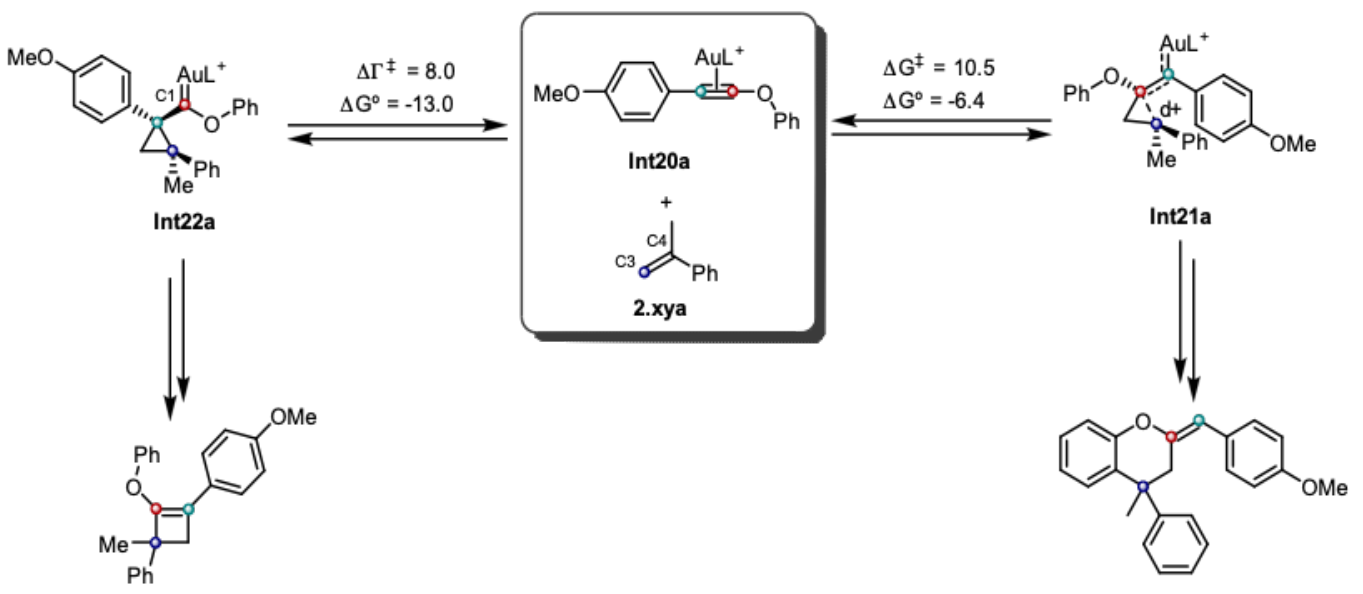

Scheme S.7: Formation of cyclopropyl gold(I) carbene Int21a and Int22a. ( $\mathrm{L}=\mathrm{PMe}_{3}$. Free energies in kcal/mol). 


\section{Computed structures and energies}

E-4a

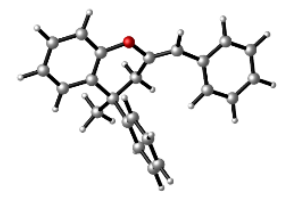

$E=-963.742061$ Hartrees

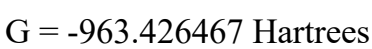

$\begin{array}{lllll}\text { C } & -4.48949200 & 1.98208900 & -0.99569900\end{array}$

$\begin{array}{lllll}\text { C } & -4.62464500 & 0.64701500 & -1.38914000\end{array}$

C $\quad \begin{array}{llll}\text { C } & -3.50321700 & -0.18165600 & -1.41487000\end{array}$

$\begin{array}{lllll}\text { C } & -2.23019900 & 0.28388400 & -1.05374200\end{array}$

$\begin{array}{llll}\mathrm{C} & -2.12786300 & 1.62375000 & -0.64430600\end{array}$

$\begin{array}{llll}\text { C } & -3.24167800 & 2.47133800 & -0.62207900\end{array}$

C $\quad-0.97930900 \quad-0.59895900-1.10810400$

$\begin{array}{lllll}\text { C } & 0.23847200 & 0.36527100 & -1.25529900\end{array}$

$\begin{array}{llll}\text { C } & 0.23225000 & 1.47758800 & -0.25552300\end{array}$

$\begin{array}{llll}\mathrm{O} & -0.95408600 & 2.19209200 & -0.20302300\end{array}$

$\begin{array}{llll}\text { C } & -0.82230800 & -1.446860000 & 0.17407900\end{array}$

$\begin{array}{llll}\text { C } & -1.00861600 & -1.48483100 & -2.37446900\end{array}$

$\begin{array}{llll}\mathrm{C} & 1.22530500 & 1.96234600 & 0.51415200\end{array}$

$\begin{array}{llll}\text { C } & -0.10313700 & -2.65492200 & 0.15903100\end{array}$

$\begin{array}{llll}\text { C } & 0.08530700 & -3.40075200 & 1.32526500\end{array}$

$\begin{array}{llll}\text { C } & -0.43819000 & -2.95551300 & 2.54048900\end{array}$

$\begin{array}{llll}\text { C } & -1.15164700 & -1.75676000 & 2.57387600\end{array}$

$\begin{array}{llll}\text { C } & -1.34181000 & -1.01534800 & 1.40539300\end{array}$

$\begin{array}{llll}\text { C } & 2.61567200 & 1.49803000 & 0.65469100\end{array}$

$\begin{array}{llll}\text { C } & 3.62269900 & 2.46742700 & 0.84123800\end{array}$

$\begin{array}{llll}\text { C } & 4.95965500 & 2.10323300 & 0.99322000\end{array}$

$\begin{array}{llll}\text { C } & 5.32775300 & 0.75505700 & 0.97346800\end{array}$

$\begin{array}{llll}\text { C } & 4.34005600 & -0.22032900 & 0.81542600\end{array}$

$\begin{array}{llll}\text { C } & 3.00074200 & 0.14300200 & 0.66674000\end{array}$

$\mathrm{H} \quad-5.35298000 \quad 2.64077900 \quad-0.97630300$

$\mathrm{H} \quad-5.59536000 \quad 0.25476800 \quad-1.67727600$

$\mathrm{H} \quad \quad-3.61737400 \quad-1.21640700 \quad-1.72240100$

$\mathrm{H} \quad-3.10724000 \quad 3.50039100 \quad-0.30312300$

$\mathrm{H} \quad 0.17284000 \quad 0.82192400 \quad-2.25377400$

$\mathrm{H} \quad 1.17399100 \quad-0.19033000 \quad-1.21381800$ 


$\begin{array}{lrrr}\mathrm{H} & -1.24806800 & -0.87602900 & -3.25118200 \\ \mathrm{H} & -0.03349700 & -1.94681500 & -2.55268600 \\ \mathrm{H} & -1.74852800 & -2.28593600 & -2.30012700 \\ \mathrm{H} & 0.98113700 & 2.87135300 & 1.06205600 \\ \mathrm{H} & 0.31836800 & -3.02870500 & -0.76793600 \\ \mathrm{H} & 0.64098100 & -4.33363600 & 1.27918400 \\ \mathrm{H} & -0.29288500 & -3.53526600 & 3.44772500 \\ \mathrm{H} & -1.56783100 & -1.39456200 & 3.51008600 \\ \mathrm{H} & -1.91200500 & -0.09404700 & 1.45969600 \\ \mathrm{H} & 3.34567000 & 3.51859700 & 0.85799400 \\ \mathrm{H} & 5.71466700 & 2.87326500 & 1.12793700 \\ \mathrm{H} & 6.36862800 & 0.46809700 & 1.09364800 \\ \mathrm{H} & 4.60921200 & -1.27329200 & 0.82372800 \\ \mathrm{H} & 2.24720000 & -0.63379900 & 0.59413000\end{array}$

\section{Z-4a}

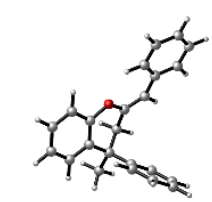

$\begin{array}{lrrr}E=-963.747967 \text { Hartrees } & & \\ \mathrm{G}=-963.431486 \text { Hartrees } & & \\ \mathrm{C} & -4.94446200 & 1.41788100 & -1.27278500 \\ \mathrm{C} & -4.91298400 & 0.03768100 & -1.48770500 \\ \mathrm{C} & -3.69092900 & -0.63683800 & -1.48148800 \\ \mathrm{C} & -2.47915900 & 0.03210200 & -1.25849000 \\ \mathrm{C} & -2.54431000 & 1.41899500 & -1.04172100 \\ \mathrm{C} & -3.75770800 & 2.11191500 & -1.05088000 \\ \mathrm{C} & -1.11405200 & -0.65863200 & -1.26088500 \\ \mathrm{C} & -0.11436300 & 0.41018600 & -1.79077200 \\ \mathrm{C} & -0.16815200 & 1.67325700 & -0.98599700 \\ \mathrm{O} & -1.42769000 & 2.18708000 & -0.77450400 \\ \mathrm{C} & -0.69372600 & -1.13627600 & 0.14837600 \\ \mathrm{C} & -1.09409700 & -1.86184400 & -2.23112400 \\ \mathrm{C} & 0.91825100 & 2.31675500 & -0.51017100 \\ \mathrm{C} & 0.58339700 & -1.69386400 & 0.33965500 \\ \mathrm{C} & 0.99050100 & -2.15815300 & 1.59023300 \\ \mathrm{C} & 0.12741700 & -2.07795900 & 2.68667600 \\ \mathrm{C} & -1.14183500 & -1.52749800 & 2.51351500 \\ \mathrm{C} & -1.54682800 & -1.06239700 & 1.25801200\end{array}$




$\begin{array}{lrrr}\mathrm{H} & -5.88834700 & 1.95524200 & -1.27938900 \\ \mathrm{H} & -5.83304300 & -0.51196700 & -1.66264200 \\ \mathrm{H} & -3.67981100 & -1.70848300 & -1.65124200 \\ \mathrm{H} & -3.75165300 & 3.18460700 & -0.88487400 \\ \mathrm{H} & -0.38852600 & 0.63185900 & -2.83213100 \\ \mathrm{H} & 0.90726500 & 0.02753200 & -1.79861400 \\ \mathrm{H} & -1.44050600 & -1.56478100 & -3.22639000 \\ \mathrm{H} & -0.07758100 & -2.25339800 & -2.32972000 \\ \mathrm{H} & -1.72571400 & -2.68003800 & -1.87473800 \\ \mathrm{H} & 1.27391000 & -1.76921600 & -0.49572400 \\ \mathrm{H} & 1.98378100 & -2.58338800 & 1.70727500 \\ \mathrm{H} & 0.44318500 & -2.43851900 & 3.66167300 \\ \mathrm{H} & -1.82570400 & -1.45606300 & 3.35515300 \\ \mathrm{H} & -2.53972200 & -0.63968900 & 1.14928100 \\ \mathrm{H} & 1.86105000 & 1.83119100 & -0.74681000 \\ \mathrm{C} & 1.04565300 & 3.55580500 & 0.26211800 \\ \mathrm{C} & 2.34699400 & 3.96109100 & 0.63196600 \\ \mathrm{C} & -0.03167700 & 4.37471000 & 0.66636600 \\ \mathrm{C} & 2.56753900 & 5.12303300 & 1.36795900 \\ \mathrm{H} & 3.19322200 & 3.34705200 & 0.33324800 \\ \mathrm{C} & 0.19314100 & 5.53752100 & 1.40297700 \\ \mathrm{H} & -1.04371700 & 4.09870100 & 0.40075300 \\ \mathrm{C} & 1.48899000 & 5.92099200 & 1.75953000 \\ \mathrm{H} & 3.58217000 & 5.40530400 & 1.63648600 \\ \mathrm{H} & -0.65422700 & 6.14980700 & 1.70097000 \\ \mathrm{H} & 1.65582800 & 6.82818600 & 2.33352500\end{array}$

\section{Endo-4a}

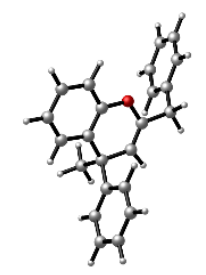

$E=-963.746057$ Hartrees

$\mathrm{G}=-963.431189$ Hartrees

$\begin{array}{llll}\mathrm{C} & -4.74134900 & 1.47015100 & 0.30239200 \\ \mathrm{C} & -4.68187000 & 0.22091300 & -0.32550600 \\ \mathrm{C} & -3.44262600 & -0.34930600 & -0.60914400 \\ \mathrm{C} & -2.23930400 & 0.29710100 & -0.28639500 \\ \mathrm{C} & -2.32892800 & 1.54300500 & 0.34833400\end{array}$




\begin{tabular}{|c|c|c|c|}
\hline $\mathrm{C}$ & -3.56510800 & 2.13062900 & 0.64127900 \\
\hline $\mathrm{C}$ & -0.88193500 & -0.32570000 & -0.64264900 \\
\hline $\mathrm{C}$ & 0.22136300 & 0.55547000 & -0.08540300 \\
\hline $\mathrm{C}$ & 0.02546100 & 1.73042500 & 0.52119800 \\
\hline $\mathrm{O}$ & -1.22526800 & 2.26990500 & 0.73492600 \\
\hline $\mathrm{C}$ & -0.75743200 & -1.72864400 & -0.00250200 \\
\hline $\mathrm{C}$ & -0.73405500 & -0.36686900 & -2.18383300 \\
\hline $\mathrm{C}$ & -0.49037900 & -2.88768400 & -0.74307800 \\
\hline $\mathrm{C}$ & -0.36698900 & -4.13197900 & -0.11322400 \\
\hline $\mathrm{C}$ & -0.50900800 & -4.24067100 & 1.26932900 \\
\hline $\mathrm{C}$ & -0.77577500 & -3.09174900 & 2.02119400 \\
\hline $\mathrm{C}$ & -0.89495200 & -1.85454700 & 1.39104100 \\
\hline $\mathrm{H}$ & -5.70004600 & 1.92721600 & 0.53018300 \\
\hline $\mathrm{H}$ & -5.59459900 & -0.30542500 & -0.58860000 \\
\hline $\mathrm{H}$ & -3.39861300 & -1.32324700 & -1.08891300 \\
\hline $\mathrm{H}$ & -3.57816400 & 3.09790800 & 1.13414700 \\
\hline $\mathrm{H}$ & 1.24215800 & 0.20671300 & -0.20725600 \\
\hline $\mathrm{H}$ & -0.84880200 & 0.64348600 & -2.58590900 \\
\hline $\mathrm{H}$ & 0.25570200 & -0.73385500 & -2.47609600 \\
\hline $\mathrm{H}$ & -1.49010100 & -1.00570400 & -2.65134900 \\
\hline $\mathrm{H}$ & -0.37481900 & -2.83678400 & -1.82005900 \\
\hline $\mathrm{H}$ & -0.15953300 & -5.01534900 & -0.71144100 \\
\hline $\mathrm{H}$ & -0.41412100 & -5.20652400 & 1.75776500 \\
\hline $\mathrm{H}$ & -0.88970500 & -3.15976500 & 3.09991900 \\
\hline $\mathrm{H}$ & -1.10076800 & -0.97003200 & 1.98759000 \\
\hline $\mathrm{C}$ & 1.15311300 & 3.99557800 & 0.38634000 \\
\hline $\mathrm{C}$ & 0.83171500 & 5.16145800 & 1.09186900 \\
\hline $\mathrm{C}$ & 1.53107200 & 4.10732700 & -0.96009400 \\
\hline $\mathrm{C}$ & 0.88749500 & 6.41241600 & 0.47054300 \\
\hline $\mathrm{H}$ & 0.53718400 & 5.08953400 & 2.13608800 \\
\hline $\mathrm{C}$ & 1.58680700 & 5.35418400 & -1.58411100 \\
\hline $\mathrm{H}$ & 1.77998900 & 3.21021100 & -1.52198500 \\
\hline $\mathrm{C}$ & 1.26532900 & 6.51249200 & -0.86971100 \\
\hline $\mathrm{H}$ & 0.63601400 & 7.30651400 & 1.03482800 \\
\hline $\mathrm{H}$ & 1.88333200 & 5.42277600 & -2.62743100 \\
\hline $\mathrm{H}$ & 1.30986900 & 7.48368700 & -1.35480900 \\
\hline $\mathrm{C}$ & 1.10082800 & 2.63327100 & 1.06342700 \\
\hline $\mathrm{H}$ & 2.06034400 & 2.11892400 & 0.94900400 \\
\hline $\mathrm{H}$ & 0.93375200 & 2.77361600 & 2.13827600 \\
\hline
\end{tabular}

AuPMe3 


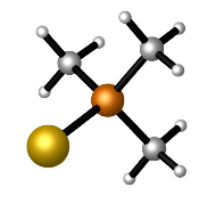

$\begin{array}{lrrr}\text { E }=-596.467300 \text { Hartrees } & & \\ \mathrm{G}=-596.386432 \text { Hartrees } & & \\ \mathrm{Au} & 0.25900600 & -0.14048500 & -0.15605200 \\ \mathrm{P} & 2.42907400 & 0.49273100 & -0.20654700 \\ \mathrm{C} & 2.74203800 & 1.66536100 & -1.56683100 \\ \mathrm{H} & 2.12828700 & 2.55843400 & -1.42654700 \\ \mathrm{H} & 3.80099400 & 1.94356800 & -1.56932800 \\ \mathrm{H} & 2.48186400 & 1.19703300 & -2.51912100 \\ \mathrm{C} & 3.53073900 & -0.93880400 & -0.45478200 \\ \mathrm{H} & 4.56960200 & -0.59501000 & -0.49143300 \\ \mathrm{H} & 3.40648600 & -1.64271200 & 0.37165000 \\ \mathrm{H} & 3.27575300 & -1.43806500 & -1.39260400 \\ \mathrm{C} & 2.93515400 & 1.30934700 & 1.34247400 \\ \mathrm{H} & 3.98752800 & 1.60239400 & 1.26776800 \\ \mathrm{H} & 2.31811400 & 2.19600800 & 1.50628400 \\ \mathrm{H} & 2.80407200 & 0.62059900 & 2.18046900\end{array}$

1a

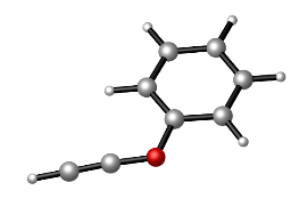

$$
\begin{array}{lrrr}
E=-383.595111 \text { Hartrees } & & \\
\mathrm{G}=-383.514415 \text { Hartrees } & & \\
\mathrm{C} & -0.69067900 & -0.44163000 & 0.34328400 \\
\mathrm{C} & 0.55897000 & -0.25872200 & -0.25170800 \\
\mathrm{C} & 0.95853700 & 1.00453200 & -0.69826900 \\
\mathrm{C} & 0.07910500 & 2.06954100 & -0.53466000 \\
\mathrm{C} & -1.17346100 & 1.91633400 & 0.05496200 \\
\mathrm{C} & -1.55397600 & 0.64841400 & 0.49391000 \\
\mathrm{H} & -0.99226800 & -1.42660800 & 0.68629100 \\
\mathrm{H} & 1.23498900 & -1.10006000 & -0.37311500 \\
\mathrm{H} & 1.92747800 & 1.15581400 & -1.16188200 \\
\mathrm{H} & -1.82591400 & 2.77684000 & 0.16192800 \\
\mathrm{H} & -2.52771200 & 0.51512300 & 0.95590900 \\
\mathrm{O} & 0.38528100 & 3.37940600 & -0.94956500
\end{array}
$$




\begin{tabular}{|c|c|c|c|}
\hline $\mathrm{C}$ & 1.53677500 & 3.63091600 & -1.51189500 \\
\hline & 2.57608800 & 3.94829000 & -2.03851600 \\
\hline & 3.49549100 & 4.22447300 & -2.50372600 \\
\hline
\end{tabular}

2a

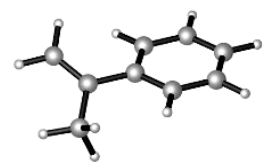

$\begin{array}{lrrr}\mathrm{E}=-348.983441 \text { Hartrees } & & \\ \mathrm{G}=-348.854198 \text { Hartrees } & & \\ \mathrm{C} & 1.12497200 & 0.36006100 & 0.71820200 \\ \mathrm{C} & 0.01988800 & 0.58625000 & 1.44785200 \\ \mathrm{H} & -0.95640000 & 0.73732000 & 0.99703100 \\ \mathrm{H} & 0.06699900 & 0.64354700 & 2.53193100 \\ \mathrm{C} & 2.47321200 & 0.22449100 & 1.39004600 \\ \mathrm{H} & 2.99154600 & -0.68734400 & 1.07111100 \\ \mathrm{H} & 3.13300900 & 1.06606300 & 1.14556100 \\ \mathrm{H} & 2.36421100 & 0.19861600 & 2.47754800 \\ \mathrm{C} & 1.07602300 & 0.25009200 & -0.76778200 \\ \mathrm{C} & 2.19604600 & 0.57345800 & -1.55622100 \\ \mathrm{C} & -0.08599500 & -0.19147600 & -1.43069000 \\ \mathrm{C} & 2.14807500 & 0.48634000 & -2.94891900 \\ \mathrm{H} & 3.11266800 & 0.90912200 & -1.08218300 \\ \mathrm{C} & -0.13391900 & -0.28098800 & -2.82069700 \\ \mathrm{H} & -0.95265900 & -0.49014100 & -0.84893700 \\ \mathrm{C} & 0.98283600 & 0.06115700 & -3.58913100 \\ \mathrm{H} & 3.02555300 & 0.75065900 & -3.53303800 \\ \mathrm{H} & -1.04180200 & -0.63103700 & -3.30466600 \\ \mathrm{H} & 0.94697800 & -0.01251300 & -4.67245600\end{array}$

Int1a

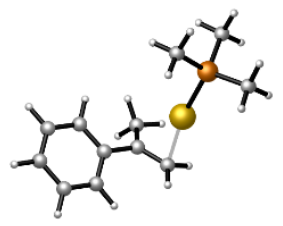

$E=-945.485454$ Hartrees

$G=-945.257300$ Hartrees

$\begin{array}{cccc}\mathrm{Au} & 0.59687300 & 2.89434400 & 1.29936500 \\ \mathrm{P} & 0.85149700 & 5.20930900 & 1.37360600\end{array}$ 


$\begin{array}{llll}\mathrm{C} & 2.26085700 & 5.74573700 & 2.41066200 \\ \mathrm{H} & 2.32420800 & 6.83845800 & 2.42052200 \\ \mathrm{H} & 2.12698200 & 5.37879500 & 3.43167900 \\ \mathrm{H} & 3.18903800 & 5.33244900 & 2.00721500 \\ \mathrm{C} & 1.13333500 & 5.95072500 & -0.27577800 \\ \mathrm{H} & 0.28778300 & 5.71897100 & -0.92888600 \\ \mathrm{H} & 1.23774900 & 7.03671600 & -0.18665900 \\ \mathrm{H} & 2.04245600 & 5.53294200 & -0.71608700 \\ \mathrm{C} & -0.62011700 & 6.05545100 & 2.05660800 \\ \mathrm{H} & -0.46168100 & 7.13835700 & 2.05948900 \\ \mathrm{H} & -1.49657500 & 5.81787000 & 1.44783000 \\ \mathrm{H} & -0.79686100 & 5.71100400 & 3.07910300 \\ \mathrm{C} & 1.14486800 & 0.44992000 & 0.70252300 \\ \mathrm{C} & 0.01066900 & 0.69563900 & 1.45537000 \\ \mathrm{H} & -0.97356200 & 0.72602200 & 0.99536300 \\ \mathrm{H} & 0.04154500 & 0.54624500 & 2.53211400 \\ \mathrm{C} & 2.46032600 & 0.17882100 & 1.38842900 \\ \mathrm{H} & 2.77096100 & -0.85077500 & 1.17391100 \\ \mathrm{H} & 3.25731300 & 0.83300700 & 1.02382200 \\ \mathrm{H} & 2.37627000 & 0.30520100 & 2.46969000 \\ \mathrm{C} & 1.09059200 & 0.32450800 & -0.77805800 \\ \mathrm{C} & 2.19811600 & 0.67111300 & -1.57538600 \\ \mathrm{C} & -0.06131500 & -0.18176400 & -1.41078800 \\ \mathrm{C} & 2.14349800 & 0.54155700 & -2.96221900 \\ \mathrm{H} & 3.10038700 & 1.06117700 & -1.11650800 \\ \mathrm{C} & -0.10732100 & -0.32270800 & -2.79624800 \\ \mathrm{H} & -0.91195900 & -0.49778200 & -0.81570700 \\ \mathrm{C} & 0.99198200 & 0.04370800 & -3.57715400 \\ \mathrm{H} & 3.00314600 & 0.82481300 & -3.56241300 \\ -0.99883200 & -0.72858000 & -3.26519300 \\ \mathrm{H} & 0.95455700 & -0.06644600 & -4.65698700 \\ & & & \\ \mathrm{H} & & & \\ \mathrm{H} & & & \end{array}$

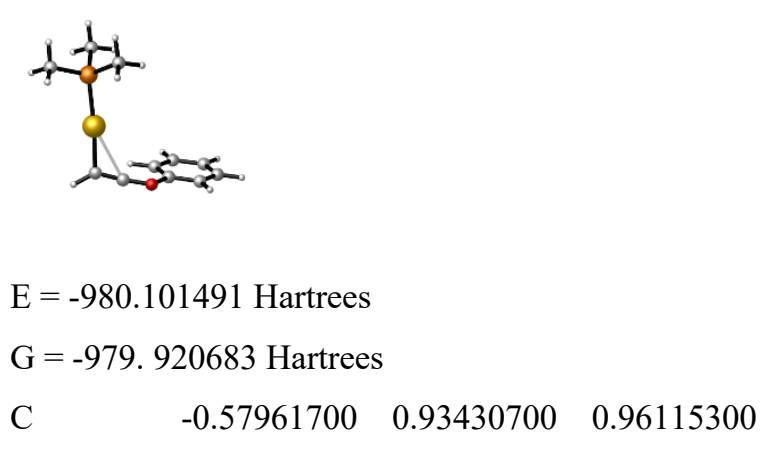




$\begin{array}{lccc}\mathrm{C} & 0.54881500 & 0.60558900 & 0.52963900 \\ \mathrm{O} & 1.74427700 & 0.37906100 & 0.20455100 \\ \mathrm{C} & 2.21897200 & 0.56735000 & -1.14562800 \\ \mathrm{C} & 1.35928200 & 0.90667700 & -2.17885700 \\ \mathrm{C} & 3.58365800 & 0.37796000 & -1.29894000 \\ \mathrm{C} & 1.91881300 & 1.07085700 & -3.44885400 \\ \mathrm{H} & 0.29511800 & 1.03412200 & -2.00999800 \\ \mathrm{C} & 4.11888700 & 0.54744900 & -2.57748400 \\ \mathrm{H} & 4.20048200 & 0.11022800 & -0.44774500 \\ \mathrm{C} & 3.29045800 & 0.89373700 & -3.64837300 \\ \mathrm{H} & 1.27423400 & 1.33497800 & -4.28122800 \\ \mathrm{H} & 5.18412000 & 0.40790500 & -2.73133600 \\ \mathrm{H} & 3.71321300 & 1.02260400 & -4.63971000 \\ \mathrm{Au} & -0.48850900 & 3.01654300 & 1.46405400 \\ \mathrm{P} & -0.55990100 & 5.27290100 & 2.05153300 \\ \mathrm{C} & 0.96687700 & 5.84809600 & 2.88199800 \\ \mathrm{H} & 0.88513900 & 6.91110300 & 3.12988500 \\ \mathrm{H} & 1.12189600 & 5.27192200 & 3.79806200 \\ \mathrm{H} & 1.82293300 & 5.69320800 & 2.22000700 \\ \mathrm{C} & -0.78607500 & 6.37727700 & 0.60862100 \\ \mathrm{H} & -1.72004200 & 6.12584600 & 0.09903900 \\ \mathrm{H} & -0.81814600 & 7.42206000 & 0.93297900 \\ \mathrm{H} & 0.04351900 & 6.23830900 & -0.08959800 \\ \mathrm{C} & -1.93051500 & 5.66841400 & 3.19829400 \\ \mathrm{H} & -1.92990200 & 6.73738600 & 3.43329000 \\ \mathrm{H} & -2.88309400 & 5.39771900 & 2.73522300 \\ \mathrm{H} & -1.81216100 & 5.09390100 & 4.12086400 \\ \mathrm{H} & -1.52344400 & 0.42465500 & 1.09594400 \\ \mathrm{H} & & & \end{array}$

TS2a-3a

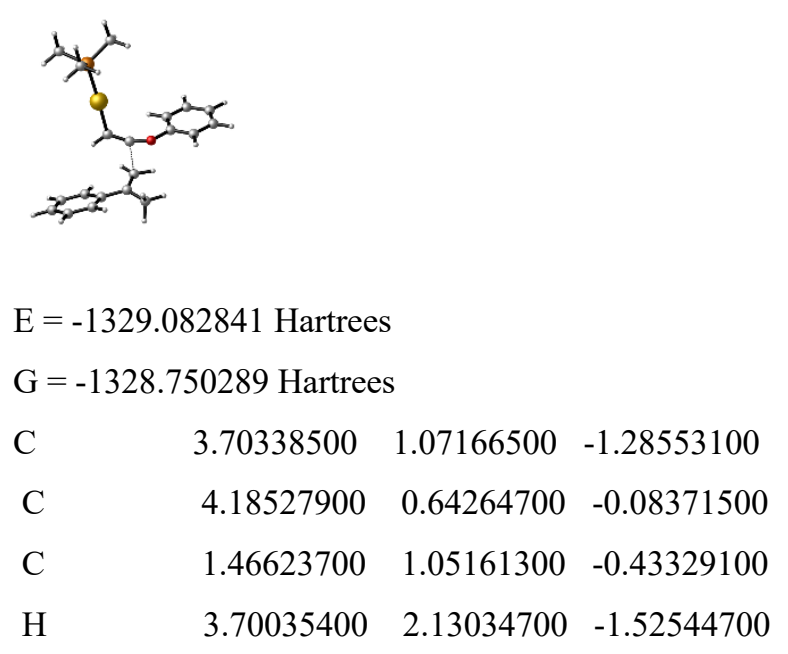




\begin{tabular}{|c|c|c|c|}
\hline $\mathrm{H}$ & 3.48667500 & 0.39422300 & -2.10313000 \\
\hline $\mathrm{C}$ & 0.99498100 & -0.13077800 & -0.59153900 \\
\hline $\mathrm{H}$ & 1.59525400 & -0.98293400 & -0.89030400 \\
\hline $\mathrm{Au}$ & -1.05958800 & -0.40576600 & -0.25645600 \\
\hline$P$ & -3.34293000 & -0.80088900 & 0.10647900 \\
\hline $\mathrm{C}$ & -4.40807200 & 0.56909900 & -0.48360200 \\
\hline $\mathrm{H}$ & -5.46231400 & 0.34459800 & -0.29267200 \\
\hline $\mathrm{H}$ & -4.13381300 & 1.49093500 & 0.03675500 \\
\hline $\mathrm{H}$ & -4.25449000 & 0.71510600 & -1.55621500 \\
\hline $\mathrm{C}$ & -3.96729400 & -2.30375700 & -0.73666900 \\
\hline $\mathrm{H}$ & -3.42602300 & -3.17947300 & -0.36828200 \\
\hline $\mathrm{H}$ & -5.03739800 & -2.43172700 & -0.54495200 \\
\hline $\mathrm{H}$ & -3.79889100 & -2.21557400 & -1.81334600 \\
\hline $\mathrm{C}$ & -3.77563000 & -1.02135200 & 1.87355600 \\
\hline $\mathrm{H}$ & -3.49699800 & -0.12357400 & 2.43213000 \\
\hline $\mathrm{H}$ & -4.84983800 & -1.20067600 & 1.98382000 \\
\hline $\mathrm{H}$ & -3.22212100 & -1.87219700 & 2.28014300 \\
\hline $\mathrm{C}$ & 4.53438400 & 1.65172200 & 0.98091200 \\
\hline $\mathrm{H}$ & 4.45594000 & 2.67042600 & 0.59582600 \\
\hline $\mathrm{H}$ & 3.87204200 & 1.56613400 & 1.84991800 \\
\hline $\mathrm{H}$ & 5.55622100 & 1.49426300 & 1.34373800 \\
\hline $\mathrm{C}$ & 4.35962500 & -0.79673300 & 0.22122200 \\
\hline $\mathrm{C}$ & 4.41722100 & -1.24900200 & 1.55479100 \\
\hline $\mathrm{C}$ & 4.47876300 & -1.75508400 & -0.80729600 \\
\hline $\mathrm{C}$ & 4.56025400 & -2.60438300 & 1.84742000 \\
\hline $\mathrm{H}$ & 4.33506400 & -0.54136600 & 2.37222800 \\
\hline $\mathrm{C}$ & 4.62742000 & -3.10903200 & -0.51281700 \\
\hline $\mathrm{H}$ & 4.48858100 & -1.43773800 & -1.84460800 \\
\hline $\mathrm{C}$ & 4.66440400 & -3.54068700 & 0.81577400 \\
\hline $\mathrm{H}$ & 4.59279300 & -2.92892600 & 2.88347500 \\
\hline $\mathrm{H}$ & 4.72620000 & -3.82698700 & -1.32194000 \\
\hline $\mathrm{H}$ & 4.78245900 & -4.59585500 & 1.04485600 \\
\hline $\mathrm{O}$ & 1.37495600 & 2.25476000 & -0.00446100 \\
\hline $\mathrm{C}$ & 1.15967900 & 3.35560600 & -0.88400900 \\
\hline $\mathrm{C}$ & 1.30641500 & 4.60444500 & -0.29272900 \\
\hline $\mathrm{C}$ & 0.80095400 & 3.17861900 & -2.21465000 \\
\hline $\mathrm{C}$ & 1.07409600 & 5.73388600 & -1.07876000 \\
\hline $\mathrm{H}$ & 1.58825200 & 4.68129400 & 0.75211000 \\
\hline $\mathrm{C}$ & 0.57552100 & 4.32345300 & -2.98371500 \\
\hline $\mathrm{H}$ & 0.69727200 & 2.18600800 & -2.63931600 \\
\hline
\end{tabular}




\begin{tabular}{|c|c|c|c|}
\hline $\mathrm{C}$ & 0.71006200 & 5.59524800 & -2.42126000 \\
\hline $\mathrm{H}$ & 1.17970200 & 6.72058100 & -0.63888500 \\
\hline & 0.29371600 & 4.21409100 & -4.02639800 \\
\hline & 0.53140200 & 6.47740900 & -3.02805600 \\
\hline
\end{tabular}

Int3a

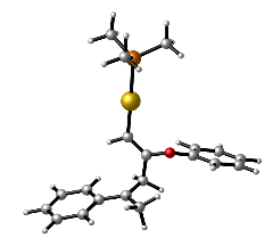

$E=-1329,116422$ Hartrees
$G=-1328.778309$ Hartrees

$\begin{array}{llll}\text { C } & 3.09943500 & 0.99021300 & -0.99728100\end{array}$

$\begin{array}{llll}\text { C } & 3.70698900 & 0.45750100 & 0.24309900\end{array}$

$\begin{array}{lllll}\text { C } & 1.58979500 & 0.93262700 & -0.65755000\end{array}$

$\mathrm{H} \quad 3.39964400 \quad 2.03049400 \quad-1.15079300$

$\mathrm{H} \quad 3.32658400 \quad 0.42497700 \quad-1.89738600$

$\begin{array}{llll}\text { C } & 0.88039200 & -0.20843700 & -0.59493100\end{array}$

$\mathrm{H} \quad 1.46445800 \quad-1.10527300 \quad-0.80417400$

$\mathrm{Au} \quad-1.11993000 \quad-0.40289800 \quad-0.22461200$

$\mathrm{P} \quad-3.44502500 \quad-0.67689300 \quad 0.18783300$

C $\quad \begin{array}{llll}-4.47562900 & 0.72543400 & -0.39946900\end{array}$

$\begin{array}{llll}\mathrm{H} & -5.53526500 & 0.54295400 & -0.19406200\end{array}$

$\mathrm{H} \quad-4.16189200 \quad 1.64325500 \quad 0.10571600$

$\mathrm{H} \quad \begin{array}{llll}\mathrm{H} & -4.33202100 & 0.85715000 & -1.47553200\end{array}$

$\begin{array}{llll}\text { C } & -4.17385400 & -2.15643000 & -0.62062500\end{array}$

$\mathrm{H} \quad-3.67447500 \quad-3.05627700 \quad-0.25046400$

$\mathrm{H} \quad-5.24589500 \quad-2.22559700 \quad-0.41019200$

$\mathrm{H} \quad-4.02001600 \quad-2.09450100 \quad-1.70159200$

$\begin{array}{llll}\text { C } & -3.88344500 & -0.86111700 & 1.96161800\end{array}$

$\mathrm{H} \quad-3.56595400 \quad 0.03077100 \quad 2.50922300$

$\mathrm{H} \quad-4.96286200 \quad-0.99660300 \quad 2.08300800$

$\mathrm{H} \quad-3.36107100 \quad-1.72765000 \quad 2.37675400$

$\begin{array}{llll}\text { C } & 3.73784700 & 1.39822500 & 1.39918600\end{array}$

$\begin{array}{llll}\mathrm{H} & 2.99546300 & 2.18885400 & 1.28263100\end{array}$

$\mathrm{H} \quad 3.60432300 \quad 0.91446800 \quad 2.36741800$

$\mathrm{H} \quad 4.72680000 \quad 1.88211000 \quad 1.40197900$

$\begin{array}{llll}\text { C } & 4.26379900 & -0.86141900 & 0.33135300\end{array}$

$\begin{array}{lllll}\text { C } & 4.84174900 & -1.32288800 & 1.54842300\end{array}$ 


$\begin{array}{llll}\mathrm{C} & 4.29499000 & -1.73413300 & -0.79424800 \\ \mathrm{C} & 5.41956400 & -2.57928000 & 1.62929400 \\ \mathrm{H} & 4.84610500 & -0.68754000 & 2.42502200 \\ \mathrm{C} & 4.86743900 & -2.99278500 & -0.70097600 \\ \mathrm{H} & 3.86544100 & -1.42639500 & -1.73873900 \\ \mathrm{C} & 5.42999900 & -3.41912200 & 0.50832300 \\ \mathrm{H} & 5.85994100 & -2.91268900 & 2.56315300 \\ \mathrm{H} & 4.87976500 & -3.64607900 & -1.56715400 \\ \mathrm{H} & 5.87833300 & -4.40581500 & 0.57688400 \\ \mathrm{O} & 1.04022100 & 2.13489100 & -0.24908300 \\ \mathrm{C} & 1.11389500 & 3.24108700 & -1.07975500 \\ \mathrm{C} & 1.06088100 & 4.49120200 & -0.45542300 \\ \mathrm{C} & 1.18099200 & 3.13693100 & -2.47238300 \\ \mathrm{C} & 1.07741600 & 5.64634600 & -1.23571500 \\ \mathrm{H} & 1.00283900 & 4.54196000 & 0.62735200 \\ \mathrm{C} & 1.20655500 & 4.30455000 & -3.24019900 \\ \mathrm{H} & 1.19839300 & 2.16168300 & -2.94821800 \\ \mathrm{C} & 1.15549700 & 5.55914500 & -2.62963700 \\ \mathrm{H} & 1.03428100 & 6.61769700 & -0.75155200 \\ \mathrm{H} & 1.25918500 & 4.22634600 & -4.32238800 \\ \mathrm{H} & 1.17248800 & 6.46163700 & -3.23303600\end{array}$

\section{TS2a-3b}

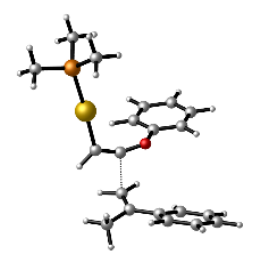

$E=-1329.081996$ Hartrees

$\mathrm{G}=-1328.748126$ Hartrees

C $\quad 3.62472300 \quad 1.25404800 \quad-1.33973400$

$\begin{array}{llll}\text { C } & 4.15197200 & 0.76369300 & -0.18050900\end{array}$

$\begin{array}{llll}\text { C } & 1.42039000 & 1.10191800 & -0.46793200\end{array}$

$\begin{array}{lllll}\mathrm{H} & 3.46898500 & 0.59902900 & -2.19018900\end{array}$

$\mathrm{H} \quad 3.52565900 \quad 2.31815900 \quad-1.52679600$

$\begin{array}{llll}\text { C } & 0.95154500 & -0.07234600 & -0.69183400\end{array}$

$\mathrm{H} \quad 1.54539200 \quad-0.89398000 \quad-1.07280500$

$\mathrm{Au} \quad-1.09222800 \quad-0.38539100 \quad-0.31864000$

$\begin{array}{llll}\mathrm{P} & -3.35534300 & -0.85107800 & 0.08541100\end{array}$

$\begin{array}{lllll}\text { C } & -4.50487300 & 0.14718500 & -0.93482700\end{array}$

$\mathrm{H} \quad \begin{array}{rrrr}\mathrm{C} & -5.54359800 & -0.10596600 & -0.69985700\end{array}$ 


\begin{tabular}{|c|c|c|c|}
\hline $\mathrm{H}$ & -4.33624600 & 1.20926300 & -0.73728300 \\
\hline $\mathrm{H}$ & -4.31363100 & -0.04730000 & -1.99368700 \\
\hline C & -3.79883000 & -2.59716900 & -0.24822500 \\
\hline $\mathrm{H}$ & -3.19501900 & -3.25286000 & 0.38511300 \\
\hline $\mathrm{H}$ & -4.86005300 & -2.77024500 & -0.04346000 \\
\hline $\mathrm{H}$ & -3.58830500 & -2.83177300 & -1.29524300 \\
\hline $\mathrm{C}$ & -3.85290900 & -0.54277400 & 1.82180900 \\
\hline $\mathrm{H}$ & -3.66983300 & 0.50628400 & 2.07008100 \\
\hline $\mathrm{H}$ & -4.91381900 & -0.76997600 & 1.96615200 \\
\hline $\mathrm{H}$ & -3.25260800 & -1.16888100 & 2.48760800 \\
\hline $\mathrm{O}$ & 1.31165600 & 2.27801300 & 0.02716200 \\
\hline $\mathrm{C}$ & 1.02832500 & 3.41169900 & -0.78979900 \\
\hline $\mathrm{C}$ & 1.16709400 & 4.63634800 & -0.14801500 \\
\hline $\mathrm{C}$ & 0.61887200 & 3.28526200 & -2.11164800 \\
\hline $\mathrm{C}$ & 0.87318100 & 5.79303900 & -0.87114000 \\
\hline $\mathrm{H}$ & 1.49709400 & 4.67331700 & 0.88465600 \\
\hline $\mathrm{C}$ & 0.33311900 & 4.45699100 & -2.81780300 \\
\hline $\mathrm{H}$ & 0.52461400 & 2.31062400 & -2.57805300 \\
\hline $\mathrm{C}$ & 0.45797600 & 5.70526500 & -2.20313300 \\
\hline $\mathrm{H}$ & 0.97242600 & 6.76142300 & -0.39084700 \\
\hline $\mathrm{H}$ & 0.01249700 & 4.38673900 & -3.85260900 \\
\hline $\mathrm{H}$ & 0.23362200 & 6.60863200 & -2.76174200 \\
\hline $\mathrm{C}$ & 4.36338500 & -0.72043000 & -0.02449300 \\
\hline $\mathrm{H}$ & 4.13989700 & -1.25097000 & -0.95257100 \\
\hline $\mathrm{H}$ & 5.40204400 & -0.93217900 & 0.25375400 \\
\hline $\mathrm{H}$ & 3.73241500 & -1.13789300 & 0.76796100 \\
\hline $\mathrm{C}$ & 4.52528000 & 1.64880700 & 0.94799600 \\
\hline $\mathrm{C}$ & 4.54374700 & 1.16124300 & 2.26960700 \\
\hline $\mathrm{C}$ & 4.88682100 & 2.99398200 & 0.73147100 \\
\hline $\mathrm{C}$ & 4.88346400 & 1.99349100 & 3.33519300 \\
\hline $\mathrm{H}$ & 4.27610000 & 0.12966700 & 2.47107400 \\
\hline $\mathrm{C}$ & 5.23456500 & 3.82186600 & 1.79677300 \\
\hline $\mathrm{H}$ & 4.92718500 & 3.38585400 & -0.27957400 \\
\hline $\mathrm{C}$ & 5.22947900 & 3.32691800 & 3.10395800 \\
\hline $\mathrm{H}$ & 4.87986900 & 1.59885400 & 4.34708700 \\
\hline $\mathrm{H}$ & 5.52036100 & 4.85212500 & 1.60510300 \\
\hline $\mathrm{H}$ & 5.50193400 & 3.97249100 & 3.93389400 \\
\hline
\end{tabular}

Int3b 


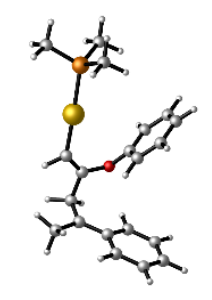

$\mathrm{E}=-1329.114499$ Hartrees

$\mathrm{G}=-1328.776556$ Hartrees

C $\quad 3.52829700 \quad 0.03722700 \quad-0.86171100$

$\begin{array}{llll}\text { C } & 4.00314900 & 0.45340800 & 0.47879600\end{array}$

$\begin{array}{llll}\text { C } & 2.02412700 & 0.37768900 & -0.74879200\end{array}$

$\mathrm{H} \quad 3.96434000 \quad 0.58371000 \quad-1.69734300$

$\mathrm{H} \quad 3.65993100 \quad-1.03244200-1.01559600$

C $\quad 1.10217300 \quad-0.47140200 \quad-0.25523300$

$\mathrm{H} \quad 1.54037600 \quad-1.39337400 \quad 0.12661400$

$\mathrm{Au} \quad-0.94203000 \quad-0.36474600 \quad-0.26988700$

$\mathrm{P} \quad-3.31579200 \quad-0.31822900 \quad-0.28262400$

C $\quad-4.02106400 \quad 0.47421300 \quad-1.78064500$

$\mathrm{H} \quad \quad-5.11510200 \quad 0.47778600 \quad-1.74239600$

$\mathrm{H} \quad \quad-3.65622200 \quad 1.50243900 \quad-1.85183100$

$\mathrm{H} \quad-3.69213700 \quad-0.07168700 \quad-2.66943000$

C $\quad-4.11807400 \quad-1.96782600 \quad-0.21350400$

$\mathrm{H} \quad-3.80865500 \quad-2.48643400 \quad 0.69826500$

$\mathrm{H} \quad-5.20817900 \quad-1.86905900 \quad-0.22215000$

$\mathrm{H} \quad-3.80306800 \quad-2.56285600 \quad-1.07540600$

C $\quad-4.04583000 \quad 0.61706200 \quad 1.11943100$

$\mathrm{H} \quad-3.67454000 \quad 1.64548900 \quad 1.09701500$

$\mathrm{H} \quad-5.13873800 \quad 0.62418900 \quad 1.05766300$

$\mathrm{H} \quad-3.73940200 \quad 0.15820400 \quad 2.06353400$

C $\quad 4.09648800 \quad-0.61941100 \quad 1.51128400$

$\mathrm{H} \quad 3.55815800 \quad-1.51644700 \quad 1.20532400$

$\mathrm{H} \quad 5.16015900 \quad-0.89107900 \quad 1.60033200$

$\mathrm{H} \quad 3.75954000 \quad-0.31380400 \quad 2.50309000$

$\begin{array}{llll}\text { C } & 4.35896200 & 1.81034800 & 0.77606200\end{array}$

$\begin{array}{llll}\text { C } & 4.68948800 & 2.20216300 & 2.10574000\end{array}$

C $\quad 4.43376900 \quad 2.79655900 \quad-0.24889000$

$\begin{array}{llll}\text { C } & 5.06556600 & 3.50434700 & 2.38869800\end{array}$

$\mathrm{H} \quad 4.65013600 \quad 1.48185600 \quad 2.91340100$

$\begin{array}{llll}\mathrm{C} & 4.80402300 & 4.09977100 & 0.04688400\end{array}$

$\mathrm{H} \quad 4.20468100 \quad 2.53773900 \quad-1.27327900$

C $\quad 5.12044600 \quad 4.45709300 \quad 1.36211900$ 


$\begin{array}{lrrr}\mathrm{H} & 5.31484000 & 3.78655300 & 3.40636900 \\ \mathrm{H} & 4.85541300 & 4.83930300 & -0.74551900 \\ \mathrm{H} & 5.41375300 & 5.47791900 & 1.58877700 \\ \mathrm{O} & 1.80486400 & 1.62778600 & -1.28455400 \\ \mathrm{C} & 0.77898100 & 2.45690100 & -0.84666000 \\ \mathrm{C} & 0.56629500 & 2.72741700 & 0.50783700 \\ \mathrm{C} & 0.03077800 & 3.09851200 & -1.83427900 \\ \mathrm{C} & -0.42098600 & 3.64554700 & 0.86898800 \\ \mathrm{H} & 1.16219600 & 2.22771600 & 1.26339900 \\ \mathrm{C} & -0.95166400 & 4.01885300 & -1.45897100 \\ \mathrm{H} & 0.22861300 & 2.87832600 & -2.87864100 \\ \mathrm{C} & -1.18392700 & 4.29239600 & -0.10886900 \\ \mathrm{H} & -0.58669100 & 3.86180300 & 1.92065300 \\ \mathrm{H} & -1.53268600 & 4.52180600 & -2.22667400 \\ \mathrm{H} & -1.94645500 & 5.00935800 & 0.18034400\end{array}$

\section{TS2a-4a}

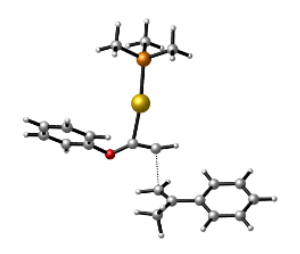

$$
\begin{array}{llll}
\mathrm{E}=-1329.066469 & \text { Hartrees } & & \\
\mathrm{G}=-1328.73645 \text { Hartrees } & & \\
\mathrm{C} & -6.06288600 & -0.84430500 & -0.10043000 \\
\mathrm{C} & -5.39598500 & -1.80912500 & 0.58315400 \\
\mathrm{H} & -4.74583000 & -1.59139300 & 1.42346400 \\
\mathrm{H} & -5.60564100 & -2.85733800 & 0.39585200 \\
\mathrm{C} & -3.45533100 & -2.93535800 & -1.60882100 \\
\mathrm{Au} & -1.43914900 & -2.56671200 & -2.37801100 \\
\mathrm{P} & 0.72795900 & -2.45462800 & -3.25947500 \\
\mathrm{C} & 0.98787300 & -3.62170800 & -4.64435600 \\
\mathrm{H} & 0.29205200 & -3.38750300 & -5.45433100 \\
\mathrm{H} & 2.01540100 & -3.54747600 & -5.01387400 \\
\mathrm{H} & 0.79755900 & -4.64203900 & -4.30116900 \\
\mathrm{C} & 2.01883900 & -2.84813500 & -2.02254300 \\
\mathrm{H} & 1.86145800 & -3.86026500 & -1.64043700 \\
\mathrm{H} & 3.01222400 & -2.78006600 & -2.47695400 \\
\mathrm{H} & 1.95061900 & -2.14204400 & -1.19055000 \\
\mathrm{C} & 1.15004300 & -0.79752100 & -3.91398400
\end{array}
$$




$\begin{array}{llll}\mathrm{H} & 2.16974900 & -0.79923700 & -4.31189500 \\ \mathrm{H} & 0.44894100 & -0.53015300 & -4.70883300 \\ \mathrm{H} & 1.07436400 & -0.05756500 & -3.11265300 \\ \mathrm{O} & -4.12089800 & -4.07931800 & -1.65692900 \\ \mathrm{C} & -3.88902400 & -4.99447000 & -2.69897900 \\ \mathrm{C} & -3.76385900 & -4.57492400 & -4.02138100 \\ \mathrm{C} & -3.87070300 & -6.33876100 & -2.34161300 \\ \mathrm{C} & -3.59170800 & -5.54487500 & -5.01300400 \\ \mathrm{H} & -3.81498900 & -3.52027300 & -4.27242100 \\ \mathrm{C} & -3.70501700 & -7.29472700 & -3.34608000 \\ \mathrm{H} & -3.98340300 & -6.62178200 & -1.30012500 \\ \mathrm{C} & -3.56089500 & -6.90126500 & -4.67940900 \\ \mathrm{H} & -3.49469800 & -5.23462300 & -6.04916900 \\ \mathrm{H} & -3.68578900 & -8.34768800 & -3.08177800 \\ \mathrm{H} & -3.43210500 & -7.64898500 & -5.45595100 \\ \mathrm{C} & -5.85888000 & 0.60301700 & 0.16135900 \\ \mathrm{C} & -6.74178900 & 1.56012500 & -0.37653600 \\ \mathrm{C} & -4.78043800 & 1.07228400 & 0.94165400 \\ \mathrm{C} & -6.56599200 & 2.92266500 & -0.13250000 \\ \mathrm{H} & -7.58257200 & 1.24271400 & -0.98326900 \\ \mathrm{C} & -4.60283500 & 2.43263200 & 1.18035400 \\ \mathrm{H} & -4.06636600 & 0.37109400 & 1.36095200 \\ \mathrm{H} & -5.49672900 & 3.36634200 & 0.64673500 \\ \mathrm{H} & -7.26746600 & 3.63711300 & -0.55394000 \\ \mathrm{H} & -3.76269200 & 2.76597200 & 1.78313200 \\ \mathrm{H} & -5.35751500 & 4.42702500 & 0.83491200 \\ & -3.505338000 & -1.80409400 & -1.05231500 \\ \mathrm{H} & -7.03557600 & -2.32455800 & -1.32807100 \\ \mathrm{H} & -8.07413300 & -0.96490900 & -0.87944600 \\ \mathrm{H} & & -0.79762600 & -0.80982500\end{array}$

\section{Int4a}

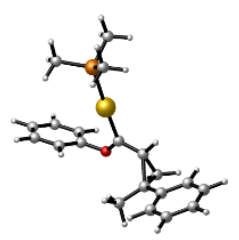

$E=-1329.134230$ Hartrees 


\begin{tabular}{|c|c|c|c|}
\hline \multicolumn{4}{|c|}{$G=-1328.795054$ Hartrees } \\
\hline $\mathrm{C}$ & -5.44218000 & -1.14921000 & -0.43047500 \\
\hline $\mathrm{C}$ & -5.15434500 & -2.11499700 & 0.65355900 \\
\hline $\mathrm{H}$ & -4.99937600 & -1.76346500 & 1.66827800 \\
\hline $\mathrm{H}$ & -5.64083200 & -3.08276000 & 0.57978100 \\
\hline $\mathrm{C}$ & -3.53050400 & -2.83698300 & -1.27836200 \\
\hline $\mathrm{Au}$ & -1.71523600 & -2.59542400 & -2.21066900 \\
\hline $\mathrm{P}$ & 0.41616600 & -2.25169300 & -3.20350500 \\
\hline $\mathrm{C}$ & 0.58579300 & -2.99526700 & -4.87086700 \\
\hline $\mathrm{H}$ & -0.15638200 & -2.55665000 & -5.54359100 \\
\hline $\mathrm{H}$ & 1.58843600 & -2.81580900 & -5.27124000 \\
\hline $\mathrm{H}$ & 0.40734100 & -4.07241700 & -4.80891700 \\
\hline $\mathrm{C}$ & 1.79198600 & -2.96198700 & -2.22071500 \\
\hline $\mathrm{H}$ & 1.63885900 & -4.03846700 & -2.10475700 \\
\hline $\mathrm{H}$ & 2.75075700 & -2.78310000 & -2.71757400 \\
\hline $\mathrm{H}$ & 1.80715900 & -2.50357200 & -1.22814000 \\
\hline $\mathrm{C}$ & 0.84382900 & -0.48191300 & -3.41975200 \\
\hline $\mathrm{H}$ & 1.84566900 & -0.37653200 & -3.84753100 \\
\hline $\mathrm{H}$ & 0.11495000 & -0.01006500 & -4.08444600 \\
\hline $\mathrm{H}$ & 0.80936300 & 0.02298400 & -2.45031500 \\
\hline $\mathrm{O}$ & -4.35140200 & -3.84565900 & -1.48102600 \\
\hline $\mathrm{C}$ & -4.10277800 & -4.80430500 & -2.48671900 \\
\hline $\mathrm{C}$ & -4.12094300 & -4.42794700 & -3.82627500 \\
\hline $\mathrm{C}$ & -3.93518800 & -6.12225000 & -2.07780700 \\
\hline $\mathrm{C}$ & -3.93697900 & -5.42008700 & -4.79311600 \\
\hline $\mathrm{H}$ & -4.29104300 & -3.39267400 & -4.10209100 \\
\hline $\mathrm{C}$ & -3.75140200 & -7.10013800 & -3.05801000 \\
\hline $\mathrm{H}$ & -3.94717000 & -6.37042400 & -1.02161300 \\
\hline $\mathrm{C}$ & -3.74998100 & -6.75117700 & -4.41164000 \\
\hline $\mathrm{H}$ & -3.94992800 & -5.14911100 & -5.84438700 \\
\hline $\mathrm{H}$ & -3.61179500 & -8.13475000 & -2.76005100 \\
\hline $\mathrm{H}$ & -3.61223700 & -7.51733600 & -5.16842100 \\
\hline $\mathrm{C}$ & -5.35175700 & 0.33340600 & -0.17926000 \\
\hline $\mathrm{C}$ & -5.88909300 & 1.24495200 & -1.10505900 \\
\hline $\mathrm{C}$ & -4.74161400 & 0.85521000 & 0.97736500 \\
\hline $\mathrm{C}$ & -5.82455700 & 2.62162100 & -0.88125000 \\
\hline $\mathrm{H}$ & -6.36914800 & 0.88808500 & -2.00850100 \\
\hline $\mathrm{C}$ & -4.67586300 & 2.22942500 & 1.19940800 \\
\hline $\mathrm{H}$ & -4.30845200 & 0.19200700 & 1.71922000 \\
\hline $\mathrm{C}$ & -5.21887300 & 3.12186000 & 0.27131100 \\
\hline
\end{tabular}




$\begin{array}{llll}\mathrm{H} & -6.25190100 & 3.30135800 & -1.61291600 \\ \mathrm{H} & -4.20268900 & 2.60281500 & 2.10309900 \\ \mathrm{H} & -5.17044900 & 4.19260900 & 0.44674200 \\ \mathrm{C} & -6.37615300 & -1.60023300 & -1.53832900 \\ \mathrm{H} & -6.41682800 & -2.68663400 & -1.60870100 \\ \mathrm{H} & -7.38737000 & -1.24831900 & -1.30667000 \\ \mathrm{H} & -6.09585000 & -1.20215800 & -2.51713100 \\ \mathrm{C} & -3.98456000 & -1.89454800 & -0.29935200 \\ \mathrm{H} & -3.22268500 & -1.19319700 & 0.01556400\end{array}$

\section{TS2a-4b}

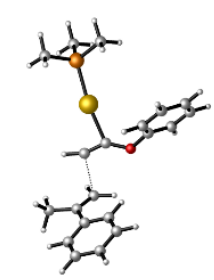

$E=-1329.066219$ Hartrees
$G=-1328.735176$ Hartrees

$\begin{array}{llll}\mathrm{Au} & 1.33144900 & 2.60070400 & -2.86495100 \\ \mathrm{P} & 2.91568800 & 1.49319000 & -4.18501700 \\ \mathrm{C} & 3.57646700 & -0.02659900 & -3.40558600 \\ \mathrm{H} & 4.07361300 & 0.23145700 & -2.46657500 \\ \mathrm{H} & 4.29414800 & -0.51047900 & -4.07560700 \\ \mathrm{H} & 2.75494300 & -0.71505900 & -3.19118300 \\ \mathrm{C} & 2.22227400 & 0.96871400 & -5.79550100 \\ \mathrm{H} & 2.99060000 & 0.46432500 & -6.38979300 \\ \mathrm{H} & 1.85897900 & 1.84529600 & -6.33824800 \\ \mathrm{H} & 1.38424600 & 0.28714400 & -5.62754000 \\ \mathrm{C} & 4.37357200 & 2.52442800 & -4.58693700 \\ \mathrm{H} & 5.06987700 & 1.96234800 & -5.21729600 \\ \mathrm{H} & 4.87805400 & 2.82411300 & -3.66456100 \\ \mathrm{H} & 4.04775900 & 3.42317100 & -5.11745200 \\ \mathrm{C} & -0.29158100 & 3.71415000 & -1.90973700 \\ \mathrm{C} & 0.22842200 & 3.64160200 & -0.76044200 \\ \mathrm{C} & -1.18784700 & 4.35383200 & 1.68896000 \\ \mathrm{C} & -0.91805400 & 5.26185700 & 0.71504100 \\ \mathrm{H} & -1.65706100 & 5.57466100 & -0.01424000 \\ \mathrm{H} & 0.00827800 & 5.82724600 & 0.73521100 \\ \mathrm{C} & -0.14410400 & 4.07005700 & 2.74124900 \\ \mathrm{H} & 0.07628700 & 2.99845600 & 2.81139800\end{array}$




$\begin{array}{llll}\mathrm{H} & -0.48662500 & 4.38753100 & 3.73334100 \\ \mathrm{H} & 0.78344700 & 4.60633300 & 2.52706000 \\ \mathrm{C} & -2.48134100 & 3.62937500 & 1.76996500 \\ \mathrm{C} & -2.85599300 & 2.96601900 & 2.95474300 \\ \mathrm{C} & -3.37345100 & 3.57642700 & 0.67813500 \\ \mathrm{C} & -4.07769800 & 2.29868100 & 3.05315700 \\ \mathrm{H} & -2.19783600 & 2.98012500 & 3.81635900 \\ \mathrm{C} & -4.59032600 & 2.90748200 & 0.77610400 \\ \mathrm{H} & -3.10672400 & 4.04336500 & -0.26397700 \\ \mathrm{C} & -4.95125600 & 2.26623600 & 1.96577100 \\ \mathrm{H} & -4.34364300 & 1.80236900 & 3.98215500 \\ \mathrm{H} & -5.25673800 & 2.87923800 & -0.08144700 \\ \mathrm{H} & -5.90053200 & 1.74346600 & 2.03971200 \\ \mathrm{H} & 0.93235100 & 3.32901000 & -0.01623700 \\ \mathrm{O} & -1.29115600 & 4.32112500 & -2.53429600 \\ \mathrm{C} & -1.72478200 & 3.85637900 & -3.79018800 \\ \mathrm{C} & -1.97051900 & 2.50356300 & -4.01383500 \\ \mathrm{C} & -1.96264200 & 4.82538200 & -4.75916000 \\ \mathrm{C} & -2.45159100 & 2.11405900 & -5.26681700 \\ \mathrm{H} & -1.80594400 & 1.77618200 & -3.22512900 \\ \mathrm{C} & -2.44967300 & 4.41925100 & -6.00375900 \\ \mathrm{H} & -1.76786800 & 5.86959400 & -4.53741400 \\ \mathrm{C} & -2.68931700 & 3.06677700 & -6.26116300 \\ \mathrm{H} & -2.65091500 & 1.06377500 & -5.45762000 \\ \mathrm{H} & -2.63639800 & 5.16346200 & -6.77195900 \\ \mathrm{H} & -3.06602500 & 2.75652800 & -7.23098600\end{array}$

\section{Int4b}

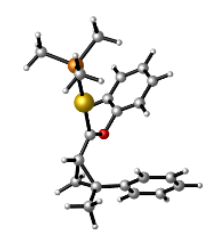

$E=-1329.136242$ Hartrees

$G=-1328.796505$ Hartrees

$\begin{array}{cccc}\mathrm{Au} & 1.12944700 & 2.78618200 & -2.64202400 \\ \mathrm{P} & 2.73024200 & 1.45548500 & -3.78665500 \\ \mathrm{C} & 3.54014200 & 0.21162600 & -2.70894700 \\ \mathrm{H} & 4.04678900 & 0.71827900 & -1.88285400 \\ \mathrm{H} & 4.27091500 & -0.36975100 & -3.27984700\end{array}$




\begin{tabular}{|c|c|c|c|}
\hline $\mathrm{H}$ & 2.78560100 & -0.46305800 & -2.29518600 \\
\hline $\mathrm{C}$ & 2.03814900 & 0.49507300 & -5.18659200 \\
\hline $\mathrm{H}$ & 2.82404800 & -0.08903800 & -5.67554000 \\
\hline $\mathrm{H}$ & 1.59012500 & 1.17901000 & -5.91260700 \\
\hline $\mathrm{H}$ & 1.26139200 & -0.18015000 & -4.81741900 \\
\hline $\mathrm{C}$ & 4.10916000 & 2.42562200 & -4.50741000 \\
\hline $\mathrm{H}$ & 4.82731900 & 1.76107300 & -4.99778400 \\
\hline $\mathrm{H}$ & 4.61609800 & 2.98317500 & -3.71499000 \\
\hline $\mathrm{H}$ & 3.71447400 & 3.13724500 & -5.23770300 \\
\hline $\mathrm{C}$ & -0.19489800 & 3.94201800 & -1.57952400 \\
\hline $\mathrm{C}$ & 0.15548400 & 4.48902800 & -0.30152600 \\
\hline $\mathrm{C}$ & -0.81192800 & 4.34098000 & 0.98153900 \\
\hline $\mathrm{C}$ & -0.61133500 & 5.65411600 & 0.34628300 \\
\hline $\mathrm{H}$ & -1.41024900 & 6.06883400 & -0.25954900 \\
\hline $\mathrm{H}$ & 0.01640200 & 6.38218100 & 0.85182200 \\
\hline $\mathrm{C}$ & -0.03436200 & 4.03040300 & 2.25243500 \\
\hline $\mathrm{H}$ & 0.18410000 & 2.96178300 & 2.33729600 \\
\hline $\mathrm{H}$ & -0.63442600 & 4.31925800 & 3.12265700 \\
\hline $\mathrm{H}$ & 0.90871700 & 4.58305800 & 2.28885300 \\
\hline $\mathrm{C}$ & -2.11947200 & 3.60778500 & 0.85004500 \\
\hline $\mathrm{C}$ & -2.14678500 & 2.20347500 & 0.83306200 \\
\hline $\mathrm{C}$ & -3.33462300 & 4.30557000 & 0.83066500 \\
\hline $\mathrm{C}$ & -3.35945500 & 1.51608200 & 0.78830200 \\
\hline $\mathrm{H}$ & -1.21300700 & 1.64745800 & 0.84974200 \\
\hline $\mathrm{C}$ & -4.55020500 & 3.61749400 & 0.79107900 \\
\hline $\mathrm{H}$ & -3.33250200 & 5.39118800 & 0.85742100 \\
\hline $\mathrm{C}$ & -4.56657200 & 2.22242200 & 0.76784700 \\
\hline $\mathrm{H}$ & -3.36336200 & 0.42972300 & 0.77149300 \\
\hline $\mathrm{H}$ & -5.48277500 & 4.17452300 & 0.77941500 \\
\hline $\mathrm{H}$ & -5.51130800 & 1.68734900 & 0.73521800 \\
\hline $\mathrm{H}$ & 1.20046000 & 4.37524200 & -0.04141100 \\
\hline $\mathrm{O}$ & -1.40205300 & 4.26405500 & -1.98500900 \\
\hline $\mathrm{C}$ & -1.92288700 & 3.76236400 & -3.19907800 \\
\hline $\mathrm{C}$ & -2.28441900 & 2.42267200 & -3.29423700 \\
\hline $\mathrm{C}$ & -2.11744400 & 4.67048300 & -4.23315800 \\
\hline $\mathrm{C}$ & -2.84653500 & 1.97478200 & -4.49279500 \\
\hline $\mathrm{H}$ & -2.14519400 & 1.75739700 & -2.44912400 \\
\hline $\mathrm{C}$ & -2.67814500 & 4.20429100 & -5.42465700 \\
\hline $\mathrm{H}$ & -1.83424200 & 5.71027100 & -4.10658800 \\
\hline $\mathrm{C}$ & -3.04044400 & 2.86067400 & -5.55584100 \\
\hline
\end{tabular}




$\begin{array}{llll}\mathrm{H} & -3.13822200 & 0.93334400 & -4.58831800 \\ \mathrm{H} & -2.83216700 & 4.89525700 & -6.24770300 \\ \mathrm{H} & -3.47923600 & 2.50601500 & -6.48339300\end{array}$

\section{TS3a-5a}

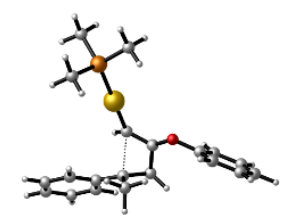

$\begin{array}{llll}\mathrm{E}=-1329.105228 \text { Hartrees } & & \\ \mathrm{G}=-1328.766771 \text { Hartrees } & & \\ \mathrm{C} & 3.05819800 & 1.17575000 & -1.35161100 \\ \mathrm{C} & 2.86829700 & 0.41145100 & -0.05597200 \\ \mathrm{C} & 1.55931400 & 1.12629700 & -1.41113400 \\ \mathrm{H} & 3.48163200 & 2.17516800 & -1.25057500 \\ \mathrm{H} & 3.54874800 & 0.62501000 & -2.15091100 \\ \mathrm{C} & 1.05489900 & -0.10317700 & -0.99314400 \\ \mathrm{H} & 1.60749200 & -0.94723600 & -1.39536200 \\ \mathrm{Au} & -0.80493500 & -0.46813700 & -0.20739500 \\ \mathrm{P} & -2.96469900 & -0.93846300 & 0.66132800 \\ \mathrm{C} & -4.18025000 & 0.40952400 & 0.38689300 \\ \mathrm{H} & -5.16061900 & 0.13049700 & 0.78585100 \\ \mathrm{H} & -3.83315700 & 1.32029800 & 0.88285700 \\ \mathrm{H} & -4.26917600 & 0.60906800 & -0.68468900 \\ \mathrm{C} & -3.74187500 & -2.42362700 & -0.08557500 \\ \mathrm{H} & -3.12153600 & -3.30159300 & 0.11524800 \\ \mathrm{H} & -4.74256100 & -2.58532700 & 0.32738200 \\ \mathrm{H} & -3.81462400 & -2.29055700 & -1.16857500 \\ \mathrm{C} & -3.01343600 & -1.24523100 & 2.47088900 \\ \mathrm{H} & -2.64912900 & -0.36028300 & 3.00022200 \\ \mathrm{H} & -4.03522500 & -1.46539800 & 2.79568600 \\ \mathrm{H} & -2.36466700 & -2.09030600 & 2.71728300 \\ \mathrm{C} & 2.61537100 & 1.25067500 & 1.17348100 \\ \mathrm{H} & 2.04366200 & 2.15403200 & 0.95082700 \\ \mathrm{H} & 2.09464600 & 0.69371700 & 1.95379100 \\ \mathrm{H} & 3.58785000 & 1.56058600 & 1.57728500 \\ \mathrm{C} & 3.51656400 & -0.91467600 & 0.15572900 \\ \mathrm{C} & 3.11055900 & -1.74316400 & 1.22053000 \\ \mathrm{C} & 4.58169300 & -1.33991500 & -0.65716000\end{array}$




$\begin{array}{llll}\mathrm{C} & 3.75232100 & -2.95486200 & 1.46244800 \\ \mathrm{H} & 2.27989600 & -1.44616000 & 1.85237300 \\ \mathrm{C} & 5.23101500 & -2.54883000 & -0.40283400 \\ \mathrm{H} & 4.92975300 & -0.72322700 & -1.47893900 \\ \mathrm{C} & 4.81813300 & -3.36170900 & 0.65356400 \\ \mathrm{H} & 3.42074700 & -3.58327300 & 2.28387800 \\ \mathrm{H} & 6.06159400 & -2.85209100 & -1.03337500 \\ \mathrm{H} & 5.32105100 & -4.30494400 & 0.84571000 \\ \mathrm{O} & 0.79752400 & 2.21654600 & -1.30957400 \\ \mathrm{C} & 1.23463300 & 3.46630100 & -1.78023000 \\ \mathrm{C} & 0.95150300 & 4.56731000 & -0.97560700 \\ \mathrm{C} & 1.83692400 & 3.59778900 & -3.03044400 \\ \mathrm{C} & 1.30453500 & 5.83816300 & -1.43197900 \\ \mathrm{H} & 0.46279500 & 4.42462800 & -0.01744400 \\ \mathrm{C} & 2.19137100 & 4.87624700 & -3.46775000 \\ \mathrm{H} & 2.01336800 & 2.72655700 & -3.65296500 \\ \mathrm{C} & 1.92765600 & 5.99446400 & -2.67338000 \\ \mathrm{H} & 1.09080000 & 6.70548800 & -0.81486300 \\ \mathrm{H} & 2.66458100 & 4.99399500 & -4.43783100 \\ \mathrm{H} & 2.20093100 & 6.98531400 & -3.02284100\end{array}$

\section{Int5a}

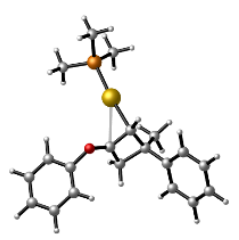

$E=-1329.140088$ Hartrees

$G=-1328.801754$ Hartrees

$\begin{array}{llll}\mathrm{C} & 2.31580000 & 1.18691300 & -0.07025700 \\ \mathrm{C} & 2.31485400 & -0.33020100 & 0.38380200 \\ \mathrm{C} & 1.36313500 & 0.74729700 & -1.14600900 \\ \mathrm{H} & 1.91200700 & 1.90075900 & 0.65266800 \\ \mathrm{H} & 3.26326600 & 1.56598200 & -0.46031900 \\ \mathrm{C} & 1.18842500 & -0.55471000 & -0.68173400 \\ \mathrm{H} & 0.99030400 & -1.43960300 & -1.28428400 \\ \mathrm{Au} & -0.86719700 & -0.25002100 & 0.14556700 \\ \mathrm{P} & -3.03237000 & -0.15095700 & 1.01172900 \\ \mathrm{C} & -3.74885500 & 1.53449800 & 0.98703400 \\ \mathrm{H} & -4.76450500 & 1.51895500 & 1.39502800\end{array}$




\begin{tabular}{|c|c|c|c|}
\hline $\mathrm{H}$ & -3.12690100 & 2.20478600 & 1.58648000 \\
\hline $\mathrm{H}$ & -3.77811200 & 1.90438400 & -0.04123500 \\
\hline C & -4.22471200 & -1.20438300 & 0.10559500 \\
\hline $\mathrm{H}$ & -3.89569900 & -2.24630000 & 0.14402600 \\
\hline $\mathrm{H}$ & -5.21983100 & -1.12028800 & 0.55351000 \\
\hline $\mathrm{H}$ & -4.26927500 & -0.88869000 & -0.94017400 \\
\hline $\mathrm{C}$ & -3.11895100 & -0.69618600 & 2.75772300 \\
\hline $\mathrm{H}$ & -2.47771800 & -0.05837200 & 3.37216800 \\
\hline $\mathrm{H}$ & -4.14880900 & -0.63574100 & 3.12315700 \\
\hline $\mathrm{H}$ & -2.76517700 & -1.72768400 & 2.83624700 \\
\hline $\mathrm{C}$ & 1.95553300 & -0.56763100 & 1.85582100 \\
\hline $\mathrm{H}$ & 1.06446500 & 0.00234500 & 2.14217800 \\
\hline $\mathrm{H}$ & 1.75699900 & -1.62943700 & 2.03695300 \\
\hline $\mathrm{H}$ & 2.78230300 & -0.26501500 & 2.50766400 \\
\hline $\mathrm{C}$ & 3.61661600 & -1.05751900 & 0.04654600 \\
\hline $\mathrm{C}$ & 3.60547500 & -2.34770900 & -0.50321000 \\
\hline $\mathrm{C}$ & 4.85788200 & -0.47846000 & 0.35619000 \\
\hline $\mathrm{C}$ & 4.79795200 & -3.03184200 & -0.75349200 \\
\hline $\mathrm{H}$ & 2.66113500 & -2.83010500 & -0.73880400 \\
\hline $\mathrm{C}$ & 6.05077800 & -1.15926300 & 0.10637000 \\
\hline $\mathrm{H}$ & 4.89645900 & 0.51267500 & 0.80065700 \\
\hline $\mathrm{C}$ & 6.02563100 & -2.43848100 & -0.45447100 \\
\hline $\mathrm{H}$ & 4.76470600 & -4.02880500 & -1.18431100 \\
\hline $\mathrm{H}$ & 6.99933700 & -0.68803000 & 0.34916300 \\
\hline $\mathrm{H}$ & 6.95299800 & -2.96832400 & -0.65289200 \\
\hline $\mathrm{O}$ & 0.94729100 & 1.32090500 & -2.25633500 \\
\hline $\mathrm{C}$ & 1.24026200 & 2.68958000 & -2.47820500 \\
\hline $\mathrm{C}$ & 0.23512700 & 3.62083200 & -2.24160300 \\
\hline $\mathrm{C}$ & 2.48302400 & 3.04093300 & -2.99471700 \\
\hline $\mathrm{C}$ & 0.49690100 & 4.96404100 & -2.52227500 \\
\hline $\mathrm{H}$ & -0.72623300 & 3.29815400 & -1.85503500 \\
\hline $\mathrm{C}$ & 2.72909300 & 4.38912900 & -3.26623000 \\
\hline $\mathrm{H}$ & 3.23200300 & 2.27934500 & -3.18576000 \\
\hline $\mathrm{C}$ & 1.74055500 & 5.34819200 & -3.03085200 \\
\hline $\mathrm{H}$ & -0.27461400 & 5.70716300 & -2.34549700 \\
\hline $\mathrm{H}$ & 3.69341900 & 4.68516100 & -3.66762600 \\
\hline $\mathrm{H}$ & 1.93738800 & 6.39341800 & -3.24885400 \\
\hline
\end{tabular}

TS3a-6a 


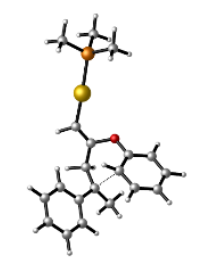

$E=-1329.101443$ Hartrees
$G=-1328.774201$ Hartrees

C $\quad 2.16820100 \quad 1.60894300 \quad-0.34955000$

C $\quad 2.78729300 \quad 1.76110700 \quad 1.03974700$

$\begin{array}{llll}\text { C } & 0.69368800 & 1.24163900 & -0.45858100\end{array}$

$\mathrm{H} \quad 2.30014200 \quad 2.56613600 \quad-0.86641200$

$\mathrm{H} \quad 2.73278700 \quad 0.85574400 \quad-0.90234600$

C $\quad 0.14738800 \quad 0.19635300 \quad-1.08137600$

$\mathrm{H} \quad 0.88875800 \quad-0.47413300 \quad-1.51956600$

$\mathrm{Au} \quad \quad-1.83255200 \quad-0.26691800 \quad-1.31255400$

P $\quad-4.10277900 \quad-0.87520600 \quad-1.66353200$

C $\quad-5.23105900 \quad-0.52800400 \quad-0.25658600$

$\mathrm{H} \quad-6.25439500 \quad-0.83582200 \quad-0.49348300$

$\mathrm{H} \quad-4.88634500 \quad-1.06792500 \quad 0.62978100$

$\mathrm{H} \quad-5.21930100 \quad 0.54324400 \quad-0.03611000$

C $\quad-4.88533900 \quad-0.02988700 \quad-3.09462200$

$\mathrm{H} \quad-4.31866000 \quad-0.25134900-4.00343200$

$\mathrm{H} \quad-5.92045000 \quad-0.36200100 \quad-3.22314300$

$\mathrm{H} \quad-4.86980600 \quad 1.05145700 \quad-2.93216400$

C $\quad-4.33483200 \quad-2.66509800 \quad-2.00571800$

$\mathrm{H} \quad-3.99126400 \quad-3.24678700 \quad-1.14559400$

$\mathrm{H} \quad-5.38850300 \quad-2.89139200 \quad-2.19829300$

$\mathrm{H} \quad-3.73934500 \quad-2.95076400 \quad-2.87738200$

$\begin{array}{llll}\text { C } & 3.40989900 & 3.11045600 & 1.28389500\end{array}$

$\mathrm{H} \quad 2.78061200 \quad 3.91082700 \quad 0.89149900$

$\mathrm{H} \quad 3.63072700 \quad 3.31379600 \quad 2.33108400$

$\mathrm{H} \quad 4.35849200 \quad 3.13606900 \quad 0.72888300$

C $\quad 3.41692100 \quad 0.57657700 \quad 1.65963400$

$\begin{array}{llll}\text { C } & 4.37077500 & 0.71458000 & 2.69175000\end{array}$

C $\quad 3.09059300 \quad-0.72895000 \quad 1.22535400$

C $\quad 4.98229000 \quad-0.40341800 \quad 3.25251400$

$\mathrm{H} \quad 4.64738300 \quad 1.69664800 \quad 3.05582800$

C $\quad 3.69633600 \quad-1.84302600 \quad 1.79530900$

$\mathrm{H} \quad 2.35167900 \quad-0.87276600 \quad 0.44569600$

C $\quad 4.64723100 \quad-1.68516900 \quad 2.80889600$ 


$\begin{array}{lrrr}\mathrm{H} & 5.72117100 & -0.27379800 & 4.03736200 \\ \mathrm{H} & 3.43124300 & -2.83628700 & 1.44618900 \\ \mathrm{H} & 5.12330000 & -2.55603400 & 3.24970300 \\ \mathrm{O} & -0.12050400 & 2.26917200 & 0.07211300 \\ \mathrm{C} & 0.18907500 & 2.69443200 & 1.30489700 \\ \mathrm{C} & 0.93273400 & 1.85591300 & 2.18522800 \\ \mathrm{C} & -0.19514900 & 3.98912500 & 1.68690900 \\ \mathrm{C} & 1.22090300 & 2.33848200 & 3.49662300 \\ \mathrm{H} & 0.86056200 & 0.78691200 & 2.02201600 \\ \mathrm{C} & 0.14091500 & 4.43204700 & 2.95497300 \\ \mathrm{H} & -0.73866800 & 4.61416700 & 0.98651200 \\ \mathrm{C} & 0.85736700 & 3.61238900 & 3.86530400 \\ \mathrm{H} & 1.74327700 & 1.68531300 & 4.18849200 \\ \mathrm{H} & -0.14555500 & 5.43501800 & 3.25772300 \\ \mathrm{H} & 1.09363900 & 3.99131500 & 4.85413100\end{array}$

\section{Int6a}

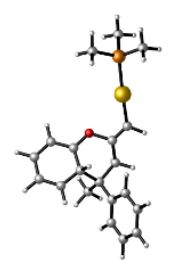

$\begin{array}{lrrr}\mathrm{E}=-1329.115970 \text { Hartrees } & & \\ \mathrm{G}=-1328.774201 \text { Hartrees } & & \\ \mathrm{C} & 2.17997500 & 1.40302600 & -0.26362800 \\ \mathrm{C} & 2.61874100 & 1.74150100 & 1.20744700 \\ \mathrm{C} & 0.72300100 & 1.07995700 & -0.43733700 \\ \mathrm{H} & 2.40021900 & 2.27810500 & -0.88783300 \\ \mathrm{H} & 2.76740800 & 0.57293100 & -0.65676200 \\ \mathrm{C} & 0.12151800 & 0.07760300 & -1.07936800 \\ \mathrm{H} & 0.83537600 & -0.64160500 & -1.48603300 \\ \mathrm{Au} & -1.87221900 & -0.27187600 & -1.38238700 \\ \mathrm{P} & -4.16074400 & -0.76431700 & -1.78856900 \\ \mathrm{C} & -5.29406700 & -0.36455500 & -0.40014400 \\ \mathrm{H} & -6.32594100 & -0.62903500 & -0.65193600 \\ \mathrm{H} & -4.98638800 & -0.91680500 & 0.49229500 \\ \mathrm{H} & -5.23998500 & 0.70568900 & -0.18129300 \\ \mathrm{C} & -4.87724400 & 0.11556100 & -3.23267900 \\ \mathrm{H} & -4.30037200 & -0.12562700 & -4.12989100 \\ \mathrm{H} & -5.92161900 & -0.17528700 & -3.38448200\end{array}$




\begin{tabular}{|c|c|c|c|}
\hline $\mathrm{H}$ & -4.82350100 & 1.19509600 & -3.06625700 \\
\hline $\mathrm{C}$ & -4.46716800 & -2.54136000 & -2.13657300 \\
\hline $\mathrm{H}$ & -4.15732900 & -3.13998200 & -1.27526600 \\
\hline $\mathrm{H}$ & -5.52786400 & -2.72068700 & -2.33959300 \\
\hline $\mathrm{H}$ & -3.87681100 & -2.84979700 & -3.00396500 \\
\hline $\mathrm{C}$ & 3.36222400 & 3.08898100 & 1.19453100 \\
\hline $\mathrm{H}$ & 2.71294400 & 3.86913800 & 0.78558600 \\
\hline $\mathrm{H}$ & 3.67983700 & 3.40522000 & 2.19084600 \\
\hline $\mathrm{H}$ & 4.24834100 & 3.02599900 & 0.55621500 \\
\hline $\mathrm{C}$ & 3.45144100 & 0.59819700 & 1.81309000 \\
\hline $\mathrm{C}$ & 4.71305300 & 0.81518000 & 2.38466000 \\
\hline $\mathrm{C}$ & 2.95574200 & -0.71902800 & 1.79229000 \\
\hline $\mathrm{C}$ & 5.45496400 & -0.24563700 & 2.91582800 \\
\hline $\mathrm{H}$ & 5.13860500 & 1.81132500 & 2.41846200 \\
\hline $\mathrm{C}$ & 3.69264200 & -1.77657900 & 2.32263900 \\
\hline $\mathrm{H}$ & 1.98520200 & -0.92717900 & 1.34896400 \\
\hline $\mathrm{C}$ & 4.94928800 & -1.54400300 & 2.88940000 \\
\hline $\mathrm{H}$ & 6.43097600 & -0.04833700 & 3.35041200 \\
\hline $\mathrm{H}$ & 3.28590800 & -2.78342300 & 2.29038900 \\
\hline $\mathrm{H}$ & 5.52539300 & -2.36686200 & 3.30267300 \\
\hline $\mathrm{O}$ & -0.11090400 & 2.11517100 & 0.11589100 \\
\hline $\mathrm{C}$ & 0.20041300 & 2.57843400 & 1.29882000 \\
\hline $\mathrm{C}$ & 1.27711400 & 1.90397600 & 2.07096000 \\
\hline $\mathrm{C}$ & -0.53259100 & 3.65694500 & 1.81567600 \\
\hline $\mathrm{C}$ & 1.49893000 & 2.46022800 & 3.42898600 \\
\hline $\mathrm{H}$ & 0.90580000 & 0.87043500 & 2.20241500 \\
\hline $\mathrm{C}$ & -0.24743200 & 4.09266700 & 3.09255200 \\
\hline $\mathrm{H}$ & -1.30794700 & 4.11600600 & 1.21279900 \\
\hline $\mathrm{C}$ & 0.76495300 & 3.49348800 & 3.91035500 \\
\hline $\mathrm{H}$ & 2.27791400 & 1.99704500 & 4.02653700 \\
\hline $\mathrm{H}$ & -0.81697800 & 4.92395400 & 3.49862100 \\
\hline & 0.93205500 & 3.88124900 & 4.90926000 \\
\hline
\end{tabular}

Int9a

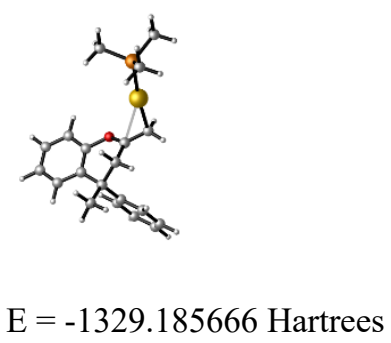




\begin{tabular}{|c|c|c|c|}
\hline \multicolumn{4}{|c|}{$G=-1328.843406$ Hartrees } \\
\hline $\mathrm{C}$ & 1.23198200 & 3.23638900 & -0.23796800 \\
\hline $\mathrm{C}$ & 2.70089400 & 3.69419200 & 0.00574600 \\
\hline $\mathrm{C}$ & 1.05361200 & 1.78314600 & 0.08100700 \\
\hline $\mathrm{H}$ & 0.58209500 & 3.81671300 & 0.43021000 \\
\hline $\mathrm{H}$ & 0.90422900 & 3.42274300 & -1.26114700 \\
\hline $\mathrm{Au}$ & -1.68298800 & 1.26412000 & 0.17351800 \\
\hline $\mathrm{P}$ & -3.88157600 & 1.47520600 & 0.92225900 \\
\hline $\mathrm{C}$ & -4.01260000 & 1.93938700 & 2.68890600 \\
\hline $\mathrm{H}$ & -5.06427100 & 2.00941500 & 2.98424700 \\
\hline $\mathrm{H}$ & -3.51283900 & 1.18463400 & 3.30177500 \\
\hline $\mathrm{H}$ & -3.52459000 & 2.90428400 & 2.85044500 \\
\hline $\mathrm{C}$ & -4.83822400 & 2.73901300 & 0.00599700 \\
\hline $\mathrm{H}$ & -4.85561000 & 2.48496700 & -1.05721200 \\
\hline $\mathrm{H}$ & -5.86360800 & 2.78704500 & 0.38579300 \\
\hline $\mathrm{H}$ & -4.36020300 & 3.71498300 & 0.12613800 \\
\hline $\mathrm{C}$ & -4.82594700 & -0.08370600 & 0.75099100 \\
\hline $\mathrm{H}$ & -4.33601100 & -0.87153500 & 1.32958100 \\
\hline $\mathrm{H}$ & -5.85051800 & 0.04908000 & 1.11210300 \\
\hline $\mathrm{H}$ & -4.84756000 & -0.38317800 & -0.30028800 \\
\hline $\mathrm{C}$ & 2.77062200 & 5.21925500 & -0.22320700 \\
\hline $\mathrm{H}$ & 2.14338900 & 5.75245900 & 0.49818600 \\
\hline $\mathrm{H}$ & 3.79602100 & 5.58660400 & -0.13317600 \\
\hline $\mathrm{H}$ & 2.42035300 & 5.46745900 & -1.22842900 \\
\hline $\mathrm{C}$ & 3.65576500 & 2.97847500 & -0.97961800 \\
\hline $\mathrm{C}$ & 3.44415600 & 3.10581000 & -2.36441000 \\
\hline $\mathrm{C}$ & 4.74997900 & 2.21620000 & -0.54867700 \\
\hline $\mathrm{C}$ & 4.29418300 & 2.48958100 & -3.28255700 \\
\hline $\mathrm{H}$ & 2.61124400 & 3.69591400 & -2.73704200 \\
\hline $\mathrm{C}$ & 5.60342400 & 1.59778700 & -1.46809400 \\
\hline $\mathrm{H}$ & 4.94860900 & 2.10166200 & 0.51140800 \\
\hline $\mathrm{C}$ & 5.37954800 & 1.72968100 & -2.83789600 \\
\hline $\mathrm{H}$ & 4.10778100 & 2.60450300 & -4.34687400 \\
\hline $\mathrm{H}$ & 6.44424100 & 1.01259300 & -1.10594700 \\
\hline $\mathrm{H}$ & 6.04193500 & 1.24907000 & -3.55220500 \\
\hline $\mathrm{O}$ & 1.63770800 & 1.32438400 & 1.18655600 \\
\hline $\mathrm{C}$ & 2.40751600 & 2.17549500 & 1.99176000 \\
\hline $\mathrm{C}$ & 2.59294200 & 1.75673300 & 3.30476500 \\
\hline $\mathrm{C}$ & 3.79748900 & 4.08163900 & 2.32740800 \\
\hline $\mathrm{C}$ & 3.39964200 & 2.52645100 & 4.14279900 \\
\hline
\end{tabular}




$\begin{array}{llll}\mathrm{H} & 2.11514100 & 0.84575400 & 3.65020700 \\ \mathrm{C} & 4.00136400 & 3.68709600 & 3.65210200 \\ \mathrm{H} & 4.27431800 & 4.98546800 & 1.96449000 \\ \mathrm{H} & 3.55564500 & 2.21833900 & 5.17193200 \\ \mathrm{H} & 4.63121500 & 4.28952600 & 4.29944600 \\ \mathrm{C} & 2.99160600 & 3.33192300 & 1.46229200 \\ \mathrm{C} & 0.33100700 & 0.87788900 & -0.68699400 \\ \mathrm{H} & 0.47821900 & -0.18058700 & -0.47915700 \\ \mathrm{H} & 0.15335600 & 1.15649100 & -1.72285500\end{array}$

\section{TS4a-7a}

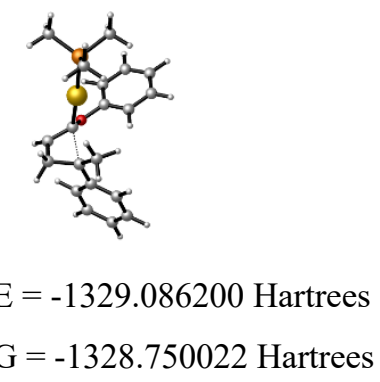

$\begin{array}{llll}\mathrm{Au} & 1.26903000 & 2.16084600 & -1.49057200 \\ \mathrm{P} & 2.35760000 & 0.12816800 & -1.90624800 \\ \mathrm{C} & 3.80735900 & 0.30043200 & -3.01303800 \\ \mathrm{H} & 3.48542200 & 0.70617100 & -3.97604400 \\ \mathrm{H} & 4.28359600 & -0.67260600 & -3.17039600 \\ \mathrm{H} & 4.52939200 & 0.98938900 & -2.56634100 \\ \mathrm{C} & 2.99979200 & -0.69590700 & -0.39943400 \\ \mathrm{H} & 3.49524800 & -1.63526000 & -0.66450100 \\ \mathrm{H} & 2.17481400 & -0.90216900 & 0.28770300 \\ \mathrm{H} & 3.71686000 & -0.03705600 & 0.09806200 \\ \mathrm{C} & 1.27417000 & -1.10924200 & -2.71401700 \\ \mathrm{H} & 0.42061100 & -1.32442400 & -2.06533800 \\ \mathrm{H} & 1.82780900 & -2.03372500 & -2.90557700 \\ \mathrm{H} & 0.90128800 & -0.70652600 & -3.65956900 \\ \mathrm{C} & 0.29657700 & 4.07716300 & -1.34697200 \\ \mathrm{C} & 1.14220000 & 5.12391700 & -1.02492500 \\ \mathrm{C} & 0.12991900 & 4.36567200 & 0.71680700 \\ \mathrm{C} & 1.46645500 & 5.02867900 & 0.43542200 \\ \mathrm{H} & 1.57323600 & 6.01898900 & 0.88422400 \\ \mathrm{H} & 2.33218200 & 4.42153100 & 0.70793200 \\ \mathrm{C} & 0.16651400 & 3.03311400 & 1.42909400 \\ \mathrm{H} & 1.08285100 & 2.48971900 & 1.19826200\end{array}$




$\begin{array}{llll}\mathrm{H} & -0.68941900 & 2.39681800 & 1.19560800 \\ \mathrm{H} & 0.15753300 & 3.23348500 & 2.51067800 \\ \mathrm{C} & -1.07379000 & 5.21991200 & 0.92742600 \\ \mathrm{C} & -2.12045100 & 4.76497500 & 1.75476100 \\ \mathrm{C} & -1.20291300 & 6.50398800 & 0.35899300 \\ \mathrm{C} & -3.22778400 & 5.57050300 & 2.02498200 \\ \mathrm{H} & -2.07051700 & 3.78629300 & 2.21595800 \\ \mathrm{C} & -2.30322800 & 7.30846400 & 0.63420200 \\ \mathrm{H} & -0.44797800 & 6.88015900 & -0.32391700 \\ \mathrm{C} & -3.32482400 & 6.84574200 & 1.47021500 \\ \mathrm{H} & -4.01369300 & 5.19474700 & 2.67355000 \\ \mathrm{H} & -2.37180000 & 8.29332100 & 0.18178900 \\ \mathrm{H} & -4.18987600 & 7.46948500 & 1.67508400 \\ \mathrm{O} & -0.61778400 & 4.36027600 & -2.32401800 \\ \mathrm{C} & -1.45559300 & 3.37044500 & -2.82528300 \\ \mathrm{C} & -2.12676300 & 2.47428900 & -1.98973300 \\ \mathrm{C} & -1.65994100 & 3.36398200 & -4.20524600 \\ \mathrm{C} & -2.99836000 & 1.54229100 & -2.55932600 \\ \mathrm{H} & -1.98312900 & 2.51723000 & -0.91592500 \\ \mathrm{C} & -2.53921200 & 2.43151600 & -4.75927800 \\ \mathrm{H} & -1.13495500 & 4.08355000 & -4.82566800 \\ \mathrm{C} & -3.20632800 & 1.51572000 & -3.94072600 \\ \mathrm{H} & -3.52514700 & 0.84425200 & -1.91495000 \\ \mathrm{H} & -2.70080900 & 2.42248100 & -5.83325300 \\ \mathrm{H} & -3.89009300 & 0.79269600 & -4.37508100 \\ \mathrm{H} & 1.18492200 & 6.05987700 & -1.58631800\end{array}$

Int7a

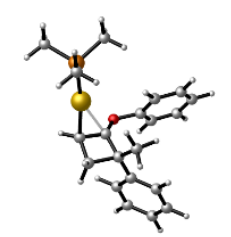

$E=-1329.137342$ Hartrees

$G=-1328.796644$ Hartrees

$\begin{array}{cccc}\mathrm{Au} & 2.23531700 & 2.34655900 & -1.54092200 \\ \mathrm{P} & 3.29969100 & 0.37520800 & -2.18743500 \\ \mathrm{C} & 4.73066700 & 0.66889200 & -3.29020200 \\ \mathrm{H} & 4.39286600 & 1.17987800 & -4.19583600 \\ \mathrm{H} & 5.20202100 & -0.28026900 & -3.56335500\end{array}$




\begin{tabular}{|c|c|c|c|}
\hline $\mathrm{H}$ & 5.46118000 & 1.30294000 & -2.78046300 \\
\hline C & 3.94781700 & -0.58243800 & -0.76796800 \\
\hline $\mathrm{H}$ & 4.42845800 & -1.50135500 & -1.11817600 \\
\hline $\mathrm{H}$ & 3.12517500 & -0.83677500 & -0.09436600 \\
\hline $\mathrm{H}$ & 4.67564500 & 0.02275300 & -0.22082000 \\
\hline $\mathrm{C}$ & 2.19840700 & -0.77207800 & -3.09575700 \\
\hline $\mathrm{H}$ & 1.34754600 & -1.03900400 & -2.46315400 \\
\hline $\mathrm{H}$ & 2.74457300 & -1.67932900 & -3.37284500 \\
\hline $\mathrm{H}$ & 1.82644100 & -0.28202200 & -3.99958000 \\
\hline $\mathrm{C}$ & 0.13286200 & 4.04323600 & -1.17535500 \\
\hline $\mathrm{C}$ & 1.46163000 & 4.36475300 & -0.92726000 \\
\hline $\mathrm{C}$ & -0.27138600 & 3.86554200 & 0.30480800 \\
\hline $\mathrm{C}$ & 1.20339000 & 4.37700800 & 0.59169700 \\
\hline $\mathrm{H}$ & 1.21624800 & 5.38323300 & 1.01601900 \\
\hline $\mathrm{H}$ & 1.81044900 & 3.71193600 & 1.20914500 \\
\hline $\mathrm{C}$ & -0.49531900 & 2.43285900 & 0.79490400 \\
\hline $\mathrm{H}$ & 0.32099200 & 1.79251700 & 0.44622300 \\
\hline $\mathrm{H}$ & -1.43917400 & 2.01198000 & 0.44168600 \\
\hline $\mathrm{H}$ & -0.49540600 & 2.41370600 & 1.88935000 \\
\hline $\mathrm{C}$ & -1.37778400 & 4.84935200 & 0.70406800 \\
\hline $\mathrm{C}$ & -2.48167100 & 4.44692300 & 1.46880900 \\
\hline $\mathrm{C}$ & -1.30357000 & 6.19747200 & 0.30764800 \\
\hline $\mathrm{C}$ & -3.47348900 & 5.36163600 & 1.83488200 \\
\hline $\mathrm{H}$ & -2.57950700 & 3.41687700 & 1.79120600 \\
\hline $\mathrm{C}$ & -2.29492900 & 7.10895300 & 0.66770700 \\
\hline $\mathrm{H}$ & -0.46585100 & 6.54610600 & -0.29071000 \\
\hline $\mathrm{C}$ & -3.38621500 & 6.69494200 & 1.43647700 \\
\hline $\mathrm{H}$ & -4.31548800 & 5.02411200 & 2.43283900 \\
\hline $\mathrm{H}$ & -2.21337800 & 8.14410900 & 0.34817800 \\
\hline $\mathrm{H}$ & -4.15824900 & 7.40446800 & 1.71983500 \\
\hline $\mathrm{O}$ & -0.55208000 & 4.15417800 & -2.30019100 \\
\hline $\mathrm{C}$ & -1.80584300 & 3.52815600 & -2.46308900 \\
\hline $\mathrm{C}$ & -2.88075500 & 4.33664400 & -2.81765100 \\
\hline $\mathrm{C}$ & -1.91155400 & 2.14257900 & -2.37713900 \\
\hline $\mathrm{C}$ & -4.11276500 & 3.73106000 & -3.07194500 \\
\hline $\mathrm{H}$ & -2.75003600 & 5.41128800 & -2.88554100 \\
\hline $\mathrm{C}$ & -3.15270600 & 1.55315400 & -2.63032700 \\
\hline $\mathrm{H}$ & -1.04212100 & 1.54299100 & -2.12785000 \\
\hline $\mathrm{C}$ & -4.25158400 & 2.34353500 & -2.97567800 \\
\hline $\mathrm{H}$ & -4.96406500 & 4.34749200 & -3.34410700 \\
\hline
\end{tabular}




$\begin{array}{llll}\mathrm{H} & -3.25440900 & 0.47404600 & -2.56527900 \\ \mathrm{H} & -5.21227200 & 1.87868500 & -3.17496100 \\ \mathrm{H} & 2.12322500 & 4.99588200 & -1.51846000\end{array}$

\section{TS4a-8a}

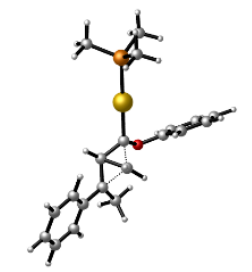

$\mathrm{E}=-1329.084923$ Hartrees

$G=-1328.745549$ Hartrees

$\begin{array}{llll}\text { C } & -5.39816000 & -0.65467500 & -1.25991300\end{array}$

C $\quad-4.19041700 \quad-1.93900500 \quad-0.80015300$

$\mathrm{H} \quad-3.55065700 \quad-1.76604900 \quad 0.05571400$

$\mathrm{H} \quad-4.91731900 \quad-2.73637100 \quad-0.73677200$

C $\quad-3.61412200 \quad-2.09565600 \quad-2.37547900$

$\mathrm{Au} \quad-1.55192200 \quad-2.29151800 \quad-2.68199600$

$\mathrm{P} \quad 0.77169400 \quad-2.48906400 \quad-3.05676600$

$\begin{array}{llll}\text { C } & 1.19365100 & -3.66141500 & -4.40266300\end{array}$

$\mathrm{H} \quad 0.71678900 \quad-3.33715000 \quad-5.33178900$

$\mathrm{H} \quad 2.27752900 \quad-3.70581600 \quad-4.54815200$

$\mathrm{H} \quad 0.82036000 \quad-4.65742400 \quad-4.14895800$

C $\quad \begin{array}{llll}1.70848500 & -3.08909100 & -1.59756800\end{array}$

$\mathrm{H} \quad 1.33114500 \quad-4.07129600 \quad-1.29933600$

$\mathrm{H} \quad 2.77578400 \quad-3.16575700 \quad-1.82766600$

$\mathrm{H} \quad 1.56800500 \quad-2.39521400 \quad-0.76398300$

$\begin{array}{llll}\text { C } & 1.59332300 & -0.91770800 & -3.52854000\end{array}$

$\mathrm{H} \quad 2.66047700 \quad-1.08265100 \quad-3.70767900$

$\mathrm{H} \quad 1.13126900 \quad-0.52365800 \quad-4.43799800$

$\mathrm{H} \quad 1.47063500 \quad-0.18337400 \quad-2.72750900$

$\begin{array}{llll}\mathrm{O} & -4.49973600 & -2.87124000 & -3.12294700\end{array}$

C $\quad-4.27664400 \quad-4.22647100 \quad-3.28100800$

$\begin{array}{llll}\text { C } & -4.83136900 & -4.79658900 & -4.43195600\end{array}$

C $\quad-3.59702100 \quad-5.01707700 \quad-2.34893000$

C $\quad-4.69594400 \quad-6.16546200 \quad-4.65460000$

$\mathrm{H} \quad-5.35893100 \quad-4.15999300 \quad-5.13550600$

C $\quad-3.46465800 \quad-6.38947300 \quad-2.59084900$

$\mathrm{H} \quad-3.18821700 \quad-4.58161200 \quad-1.44514200$

$\begin{array}{llll}\text { C } & -4.00913100 & -6.96844400 & -3.73717000\end{array}$ 


$\begin{array}{llll}\mathrm{H} & -5.12590200 & -6.60517100 & -5.54997200 \\ \mathrm{H} & -2.93780000 & -7.00457600 & -1.86669700 \\ \mathrm{H} & -3.90486900 & -8.03453900 & -3.91453600 \\ \mathrm{C} & -5.52165200 & 0.49125700 & -0.29673500 \\ \mathrm{C} & -6.76768600 & 1.11495700 & -0.11020000 \\ \mathrm{C} & -4.42015200 & 0.97686400 & 0.43406500 \\ \mathrm{C} & -6.90319200 & 2.19616500 & 0.76199300 \\ \mathrm{H} & -7.64054600 & 0.77352100 & -0.65332700 \\ \mathrm{C} & -4.55990900 & 2.05092400 & 1.30911900 \\ \mathrm{H} & -3.44287500 & 0.51375900 & 0.34289300 \\ \mathrm{C} & -5.80249300 & 2.66827800 & 1.47608700 \\ \mathrm{H} & -7.87498200 & 2.66583500 & 0.88152300 \\ \mathrm{H} & -3.69641600 & 2.40159900 & 1.86643600 \\ \mathrm{H} & -5.90981500 & 3.50532400 & 2.15951800 \\ \mathrm{C} & -6.68529400 & -1.28043600 & -1.75394900 \\ \mathrm{H} & -6.50062700 & -2.15115300 & -2.37696500 \\ \mathrm{H} & -7.33553100 & -1.55504600 & -0.92038700 \\ \mathrm{H} & -7.21051900 & -0.53893000 & -2.36557500 \\ \mathrm{C} & -4.14261500 & -0.81354000 & -1.93945600 \\ \mathrm{H} & -3.37925600 & -0.08076700 & -1.70337000\end{array}$

\section{Int8a}

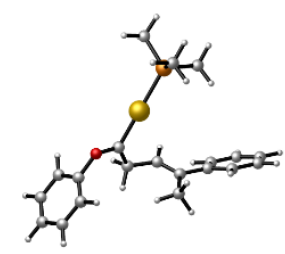

$E=-1329.084923$ Hartrees

$\mathrm{G}=-1328.797298$ Hartrees

C $\quad-4.72500200 \quad-1.00037200 \quad-1.31650200$

C $\quad-4.14462800 \quad-3.47792400 \quad-1.22931800$

$\mathrm{H} \quad-3.91435100 \quad-4.16252600 \quad-0.39553600$

$\mathrm{H} \quad-\quad-5.13320500 \quad-3.78756800 \quad-1.58981800$

C $\quad-3.10436600 \quad-3.78146100 \quad-2.26098900$

$\mathrm{Au} \quad-1.43844600 \quad-2.64113900-2.55646800$

$\mathrm{P} \quad 0.50711600 \quad-1.30374600 \quad-2.85962700$

C $\quad 0.81327800 \quad-0.84388000 \quad-4.60727700$

$\mathrm{H} \quad-0.04426900 \quad-0.28847300 \quad-4.99661900$

$\mathrm{H} \quad 1.71235700 \quad-0.22460800 \quad-4.68548700$

$\mathrm{H} \quad 0.94463700 \quad-1.74982500 \quad-5.20510100$ 


\begin{tabular}{|c|c|c|c|}
\hline $\mathrm{C}$ & 2.05219000 & -2.11928900 & -2.30566500 \\
\hline $\mathrm{H}$ & 2.19335700 & -3.04833700 & -2.86476600 \\
\hline $\mathrm{H}$ & 2.91081200 & -1.46142000 & -2.47231300 \\
\hline $\mathrm{H}$ & 1.98028600 & -2.35827700 & -1.24113000 \\
\hline $\mathrm{C}$ & 0.44231900 & 0.28353700 & -1.94682300 \\
\hline $\mathrm{H}$ & 1.34316900 & 0.87442700 & -2.14000000 \\
\hline $\mathrm{H}$ & -0.43841500 & 0.85001200 & -2.26116400 \\
\hline $\mathrm{H}$ & 0.36291600 & 0.08359600 & -0.87470800 \\
\hline $\mathrm{O}$ & -3.24475100 & -4.83726800 & -3.00494700 \\
\hline $\mathrm{C}$ & -4.32178100 & -5.77507800 & -2.87401600 \\
\hline $\mathrm{C}$ & -5.35973500 & -5.70794100 & -3.79446100 \\
\hline $\mathrm{C}$ & -4.22088300 & -6.76841800 & -1.90779100 \\
\hline $\mathrm{C}$ & -6.36554200 & -6.67465400 & -3.71596000 \\
\hline $\mathrm{H}$ & -5.38008200 & -4.92652600 & -4.54676900 \\
\hline $\mathrm{C}$ & -5.23445600 & -7.72791300 & -1.84966900 \\
\hline $\mathrm{H}$ & -3.37617200 & -6.79437300 & -1.22704500 \\
\hline $\mathrm{C}$ & -6.30395900 & -7.68021400 & -2.74774600 \\
\hline $\mathrm{H}$ & -7.19177100 & -6.64200400 & -4.41936300 \\
\hline $\mathrm{H}$ & -5.18111700 & -8.51405100 & -1.10288400 \\
\hline $\mathrm{H}$ & -7.08654700 & -8.43093700 & -2.69704100 \\
\hline $\mathrm{C}$ & -4.62939000 & 0.35609900 & -0.70343800 \\
\hline $\mathrm{C}$ & -4.66573600 & 1.51232400 & -1.50560200 \\
\hline $\mathrm{C}$ & -4.50887900 & 0.52681200 & 0.68975300 \\
\hline $\mathrm{C}$ & -4.55566200 & 2.78450600 & -0.94144200 \\
\hline $\mathrm{H}$ & -4.76858900 & 1.42110300 & -2.58213700 \\
\hline $\mathrm{C}$ & -4.40179100 & 1.79737100 & 1.25320000 \\
\hline $\mathrm{H}$ & -4.52475000 & -0.34246500 & 1.34002800 \\
\hline $\mathrm{C}$ & -4.42050300 & 2.93402100 & 0.44004000 \\
\hline $\mathrm{H}$ & -4.57766100 & 3.65974100 & -1.58494400 \\
\hline $\mathrm{H}$ & -4.31730900 & 1.90024200 & 2.33158400 \\
\hline $\mathrm{H}$ & -4.34197800 & 3.92408700 & 0.87991500 \\
\hline $\mathrm{C}$ & -5.49936300 & -1.11464400 & -2.60931000 \\
\hline $\mathrm{H}$ & -5.70742100 & -2.15214100 & -2.87783600 \\
\hline $\mathrm{H}$ & -6.45359700 & -0.58129100 & -2.54615600 \\
\hline $\mathrm{H}$ & -4.93975800 & -0.67113000 & -3.44236500 \\
\hline $\mathrm{C}$ & -4.11253800 & -2.05268100 & -0.73442000 \\
\hline $\mathrm{H}$ & -3.52426500 & -1.88723200 & 0.16328400 \\
\hline
\end{tabular}

Int10a 


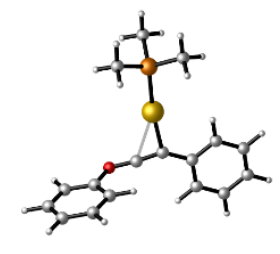

$$
\begin{aligned}
& E=-1211.1696121 \text { Hartrees } \\
& G=-1210.912826 \text { Hartrees }
\end{aligned}
$$

\begin{tabular}{|c|c|c|c|}
\hline $\mathrm{Au}$ & 2.41717900 & 1.61835800 & -2.92066500 \\
\hline$P$ & 3.42538900 & 0.25049900 & -4.51492700 \\
\hline $\mathrm{C}$ & 3.64780200 & -1.47581500 & -3.94954700 \\
\hline $\mathrm{H}$ & 4.25956800 & -1.48799500 & -3.04355300 \\
\hline $\mathrm{H}$ & 4.13773600 & -2.06785000 & -4.72886600 \\
\hline $\mathrm{H}$ & 2.67241800 & -1.91298300 & -3.71985300 \\
\hline C & 2.47506500 & 0.14973100 & -6.07603600 \\
\hline $\mathrm{H}$ & 2.99949400 & -0.48720000 & -6.79539600 \\
\hline $\mathrm{H}$ & 2.35602000 & 1.15200500 & -6.49607300 \\
\hline $\mathrm{H}$ & 1.48531100 & -0.26835800 & -5.87438600 \\
\hline $\mathrm{C}$ & 5.09374100 & 0.84217500 & -4.98069300 \\
\hline $\mathrm{H}$ & 5.02102200 & 1.85148700 & -5.39442100 \\
\hline $\mathrm{H}$ & 5.53665000 & 0.17314600 & -5.72516000 \\
\hline $\mathrm{H}$ & 5.73213200 & 0.87129100 & -4.09352700 \\
\hline $\mathrm{C}$ & 0.51069200 & 2.87109900 & -1.64596300 \\
\hline $\mathrm{C}$ & 1.71683000 & 2.96602100 & -1.32332100 \\
\hline $\mathrm{O}$ & -0.70270100 & 2.63864700 & -1.94470800 \\
\hline $\mathrm{C}$ & -1.38614300 & 3.40143100 & -2.95348100 \\
\hline $\mathrm{C}$ & -2.69931300 & 3.01209900 & -3.17402200 \\
\hline $\mathrm{C}$ & -0.76437100 & 4.43784400 & -3.63425200 \\
\hline $\mathrm{C}$ & -3.42967600 & 3.70712900 & -4.13981800 \\
\hline $\mathrm{H}$ & -3.13070900 & 2.19406100 & -2.60694700 \\
\hline $\mathrm{C}$ & -1.51496600 & 5.11851300 & -4.59713500 \\
\hline $\mathrm{H}$ & 0.26376800 & 4.71297600 & -3.42375000 \\
\hline $\mathrm{C}$ & -2.84036100 & 4.75680500 & -4.84999300 \\
\hline $\mathrm{H}$ & -4.45976400 & 3.42488400 & -4.33334800 \\
\hline $\mathrm{H}$ & -1.05593300 & 5.93550700 & -5.14504600 \\
\hline $\mathrm{H}$ & -3.41405800 & 5.29344100 & -5.59909200 \\
\hline $\mathrm{C}$ & 2.62811700 & 3.61671900 & -0.38782400 \\
\hline $\mathrm{C}$ & 2.11386700 & 4.51851800 & 0.56439200 \\
\hline $\mathrm{C}$ & 4.00853500 & 3.35640000 & -0.41214100 \\
\hline $\mathrm{C}$ & 2.96836700 & 5.14165300 & 1.46984800 \\
\hline & 1.04754700 & 4.72282400 & 0.58531800 \\
\hline
\end{tabular}




$\begin{array}{llll}\mathrm{C} & 4.85793900 & 3.98487600 & 0.49776000 \\ \mathrm{H} & 4.40593800 & 2.65946500 & -1.14393000 \\ \mathrm{C} & 4.34148900 & 4.87723500 & 1.43978700 \\ \mathrm{H} & 2.56220200 & 5.83504800 & 2.20035300 \\ \mathrm{H} & 5.92316400 & 3.77553000 & 0.47090100 \\ \mathrm{H} & 5.00468400 & 5.36515100 & 2.14800200\end{array}$

\section{TS10a-11a}

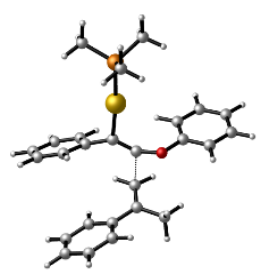

$E=-1560.143145$ Hartrees

$G=-1559.738133$ Hartrees

$\begin{array}{llll}3.79532300 & 0.92448800 & -1.26257000\end{array}$

C $\quad 4.43262300 \quad 0.77021000 \quad-0.06162400$

$\begin{array}{lllll}\mathrm{C} & 1.62878300 & 0.70097100 & -0.48847900\end{array}$

$\mathrm{H} \quad 3.74049400 \quad 0.11694800 \quad-1.98285400$

$\mathrm{H} \quad 3.56574500 \quad 1.92114900 \quad-1.62859800$

C $\quad 1.11039900 \quad-0.41569300 \quad-0.85894500$

$\mathrm{Au} \quad-0.97783500 \quad-0.47977200 \quad-0.36690900$

$\mathrm{P} \quad-3.25903400 \quad-0.71144800 \quad 0.08750300$

$\begin{array}{lllll}\mathrm{C} & -4.25827200 & 0.70971100 & -0.49559600\end{array}$

$\mathrm{H} \quad-5.31844400 \quad 0.54406100 \quad-0.27915500$

$\mathrm{H} \quad-3.92330000 \quad 1.62127700 \quad 0.00665500$

$\mathrm{H} \quad-4.12166800 \quad 0.83351000 \quad-1.57321400$

C $\quad-3.99485200 \quad-2.18711100 \quad-0.71072600$

$\mathrm{H} \quad-3.49150800 \quad-3.08697000 \quad-0.34683600$

$\mathrm{H} \quad-5.06338900 \quad-2.25062900 \quad-0.48190400$

$\mathrm{H} \quad-3.85749500 \quad-2.12284900 \quad-1.79347000$

C $\quad-3.64094300 \quad-0.88010300 \quad 1.87135000$

$\mathrm{H} \quad \begin{array}{llll}\mathrm{H} & -3.28652900 & 0.00566100 & 2.40546300\end{array}$

$\mathrm{H} \quad-4.71992800 \quad-0.98758400 \quad 2.02095700$

$\mathrm{H} \quad-3.12867200 \quad-1.75949900 \quad 2.27102700$

$\begin{array}{llll}\mathrm{O} & 1.48927900 & 1.80548100 & 0.17050800\end{array}$

$\begin{array}{llll}\text { C } & 1.11698300 & 3.01297200 & -0.47260000\end{array}$

$\begin{array}{lllll}\text { C } & 1.13429100 & 4.13645100 & 0.34827300\end{array}$

$\begin{array}{llll}\text { C } & 0.74137900 & 3.06239400 & -1.81074500\end{array}$

$\begin{array}{lllll}\text { C } & 0.74976200 & 5.36095700 & -0.19830700\end{array}$ 


$\begin{array}{llll}\mathrm{H} & 1.43764100 & 4.04383200 & 1.38582300 \\ \mathrm{C} & 0.36259600 & 4.30001800 & -2.33914500 \\ \mathrm{H} & 0.74434700 & 2.16899100 & -2.42449800 \\ \mathrm{C} & 0.36465100 & 5.44523500 & -1.53982400 \\ \mathrm{H} & 0.75400200 & 6.24857100 & 0.42670900 \\ \mathrm{H} & 0.06703900 & 4.36197200 & -3.38202200 \\ \mathrm{H} & 0.06927500 & 6.40129300 & -1.96064000 \\ \mathrm{C} & 4.57531700 & 1.94417800 & 0.87020900 \\ \mathrm{H} & 4.26139300 & 2.87261000 & 0.38957600 \\ \mathrm{H} & 3.97179100 & 1.80929200 & 1.77563700 \\ \mathrm{H} & 5.61541600 & 2.05113300 & 1.19755300 \\ \mathrm{C} & 4.99335500 & -0.53056700 & 0.36840900 \\ \mathrm{C} & 5.12367700 & -0.84293000 & 1.73648200 \\ \mathrm{C} & 5.44074200 & -1.47772100 & -0.57521400 \\ \mathrm{C} & 5.65014400 & -2.06772500 & 2.14367800 \\ \mathrm{H} & 4.79303700 & -0.13416800 & 2.48817900 \\ \mathrm{C} & 5.97690400 & -2.69618800 & -0.16597800 \\ \mathrm{H} & 5.39329500 & -1.24861200 & -1.63472200 \\ \mathrm{C} & 6.07930700 & -2.99884100 & 1.19486200 \\ \mathrm{H} & 5.72792800 & -2.29368000 & 3.20329400 \\ \mathrm{H} & 6.32446900 & -3.40711200 & -0.90991900 \\ \mathrm{H} & 6.49722500 & -3.94963900 & 1.51300500 \\ \mathrm{H} & 1.59830300 & -1.60452200 & -1.57825500 \\ \mathrm{C} & 1.74182600 & -2.82870300 & -0.90151200 \\ \mathrm{C} & 1.87122600 & -1.54957700 & -2.95649500 \\ \mathrm{C} & 2.78682000 & -4.78829000 & -3.48467800 \\ \mathrm{C} & & & \end{array}$

\section{Int11a}

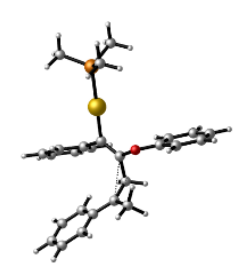




\begin{tabular}{|c|c|c|c|}
\hline \multicolumn{4}{|c|}{$E=-1560.175479$ Hartrees } \\
\hline \multicolumn{4}{|c|}{$G=-1559.7764342$ Hartrees } \\
\hline $\mathrm{C}$ & 3.31776900 & 0.92347400 & -0.87720200 \\
\hline $\mathrm{C}$ & 4.00895800 & 0.74077100 & 0.43598600 \\
\hline $\mathrm{C}$ & 1.80852600 & 0.68065100 & -0.65199400 \\
\hline $\mathrm{H}$ & 3.68892900 & 0.26268200 & -1.65354100 \\
\hline $\mathrm{H}$ & 3.45641000 & 1.95689900 & -1.21353000 \\
\hline $\mathrm{C}$ & 1.12671800 & -0.46621000 & -0.83552700 \\
\hline $\mathrm{Au}$ & -0.91864300 & -0.56471200 & -0.50597300 \\
\hline $\mathrm{P}$ & -3.26330900 & -0.72522200 & -0.19306900 \\
\hline $\mathrm{C}$ & -3.91306700 & 0.27292800 & 1.20405900 \\
\hline $\mathrm{H}$ & -4.99752600 & 0.15466200 & 1.29366300 \\
\hline $\mathrm{H}$ & -3.43620900 & -0.04716600 & 2.13475600 \\
\hline $\mathrm{H}$ & -3.67739900 & 1.32805300 & 1.03885900 \\
\hline $\mathrm{C}$ & -4.23566000 & -0.16572600 & -1.64691100 \\
\hline $\mathrm{H}$ & -3.98442700 & -0.78347500 & -2.51375500 \\
\hline $\mathrm{H}$ & -5.30979200 & -0.23925500 & -1.44859200 \\
\hline $\mathrm{H}$ & -3.98143500 & 0.87291600 & -1.87648200 \\
\hline $\mathrm{C}$ & -3.86447100 & -2.42838800 & 0.13909500 \\
\hline $\mathrm{H}$ & -3.38396800 & -2.81315500 & 1.04319200 \\
\hline $\mathrm{H}$ & -4.95074100 & -2.43904700 & 0.27379000 \\
\hline $\mathrm{H}$ & -3.59786400 & -3.07885100 & -0.69874300 \\
\hline $\mathrm{O}$ & 1.15560700 & 1.77998700 & -0.08976100 \\
\hline $\mathrm{C}$ & 0.97889100 & 2.92997800 & -0.83852500 \\
\hline $\mathrm{C}$ & 0.66905900 & 4.08992300 & -0.11862200 \\
\hline $\mathrm{C}$ & 1.05267700 & 2.95613700 & -2.23471300 \\
\hline $\mathrm{C}$ & 0.43459100 & 5.28078100 & -0.80336500 \\
\hline $\mathrm{H}$ & 0.60986100 & 4.04232100 & 0.96447800 \\
\hline $\mathrm{C}$ & 0.82340300 & 4.16104900 & -2.90609300 \\
\hline $\mathrm{H}$ & 1.26986900 & 2.04909700 & -2.78805600 \\
\hline $\mathrm{C}$ & 0.51526900 & 5.32451400 & -2.19956700 \\
\hline $\mathrm{H}$ & 0.19242500 & 6.17899700 & -0.24234200 \\
\hline $\mathrm{H}$ & 0.88129600 & 4.18094000 & -3.99078200 \\
\hline $\mathrm{H}$ & 0.33599500 & 6.25526800 & -2.72913700 \\
\hline $\mathrm{C}$ & 3.80584200 & 1.85269200 & 1.39653300 \\
\hline $\mathrm{H}$ & 2.74587800 & 2.13059600 & 1.40975600 \\
\hline $\mathrm{H}$ & 4.14996700 & 1.67039300 & 2.41048000 \\
\hline $\mathrm{H}$ & 4.32862800 & 2.73426500 & 0.99541800 \\
\hline $\mathrm{C}$ & 4.81389100 & -0.39908600 & 0.74260100 \\
\hline $\mathrm{C}$ & 5.41914500 & -0.54379300 & 2.02880200 \\
\hline
\end{tabular}




$\begin{array}{llll}\mathrm{C} & 5.07416700 & -1.41297000 & -0.22846000 \\ \mathrm{C} & 6.22571200 & -1.62973000 & 2.31925700 \\ \mathrm{H} & 5.25666600 & 0.19856900 & 2.79858300 \\ \mathrm{C} & 5.88370300 & -2.49512100 & 0.07316000 \\ \mathrm{H} & 4.64094200 & -1.35423600 & -1.21720300 \\ \mathrm{C} & 6.45960900 & -2.60863000 & 1.34427200 \\ \mathrm{H} & 6.67585400 & -1.72159400 & 3.30199200 \\ \mathrm{H} & 6.06780000 & -3.25518300 & -0.67833400 \\ \mathrm{H} & 7.09220400 & -3.46047400 & 1.57594300 \\ \mathrm{C} & 1.76005000 & -1.69963700 & -1.36783200 \\ \mathrm{C} & 1.81066900 & -2.87323700 & -0.59041100 \\ \mathrm{C} & 2.25288400 & -1.75861900 & -2.68612100 \\ \mathrm{C} & 2.35535200 & -4.05113400 & -1.10254100 \\ \mathrm{H} & 1.41647900 & -2.85509200 & 0.42211000 \\ \mathrm{C} & 2.78888500 & -2.94184900 & -3.20071500 \\ \mathrm{H} & 2.19739700 & -0.87531600 & -3.31647500 \\ \mathrm{C} & 2.84665700 & -4.09286700 & -2.41104400 \\ \mathrm{H} & 2.38793000 & -4.94173300 & -0.48058300 \\ \mathrm{H} & 3.15487500 & -2.96381600 & -4.22381700 \\ \mathrm{H} & 3.25881500 & -5.01385500 & -2.81333900\end{array}$

\section{TS10a-11b}

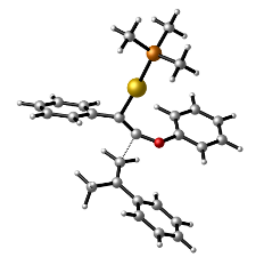

$E=-1560.143667$ Hartrees

$\mathrm{G}=-1559.736920$ Hartrees

C $\quad 3.82958300 \quad 1.07901500 \quad-1.31209000$

$\begin{array}{lllll}\text { C } & 4.44153600 & 0.80574900 & -0.12037100\end{array}$

$\begin{array}{lllll}\mathrm{C} & 1.64646400 & 0.77442100 & -0.57280100\end{array}$

$\mathrm{H} \quad 3.80393900 \quad 0.32477300 \quad-2.09022600$

$\mathrm{H} \quad 3.58293400 \quad 2.09341300 \quad-1.60842700$

C $\quad 1.16510400 \quad-0.35368400 \quad-0.95816500$

$\mathrm{Au} \quad-0.92385600 \quad-0.46797900 \quad-0.47809200$

$\mathrm{P} \quad-3.20095300 \quad-0.71770000 \quad-0.01174200$

$\begin{array}{lllll}\text { C } & -4.28700000 & 0.06174000 & -1.26480200\end{array}$

$\mathrm{H} \quad-5.33867300 \quad-0.07043200 \quad-0.99140300$

$\mathrm{H} \quad-4.06068200 \quad 1.12947900 \quad-1.32937500$ 


\begin{tabular}{|c|c|c|c|}
\hline $\mathrm{H}$ & -4.10560400 & -0.39554800 & -2.24127500 \\
\hline $\mathrm{C}$ & -3.73981800 & -2.46576000 & 0.08509000 \\
\hline $\mathrm{H}$ & -3.18021800 & -2.97670500 & 0.87329700 \\
\hline $\mathrm{H}$ & -4.81044000 & -2.51875000 & 0.30673200 \\
\hline $\mathrm{H}$ & -3.54111900 & -2.96488400 & -0.86713800 \\
\hline $\mathrm{C}$ & -3.69024000 & 0.02834400 & 1.58750600 \\
\hline $\mathrm{H}$ & -3.47430500 & 1.10015300 & 1.57209100 \\
\hline $\mathrm{H}$ & -4.75910300 & -0.12463200 & 1.76722800 \\
\hline $\mathrm{H}$ & -3.11717700 & -0.43323100 & 2.39608700 \\
\hline $\mathrm{O}$ & 1.48313100 & 1.87265300 & 0.08713400 \\
\hline $\mathrm{C}$ & 1.03446400 & 3.06232800 & -0.54342000 \\
\hline $\mathrm{C}$ & 0.98469600 & 4.17545700 & 0.28982500 \\
\hline $\mathrm{C}$ & 0.65117300 & 3.10184700 & -1.87933000 \\
\hline $\mathrm{C}$ & 0.52011200 & 5.37831800 & -0.24193200 \\
\hline $\mathrm{H}$ & 1.30119500 & 4.09176600 & 1.32403100 \\
\hline $\mathrm{C}$ & 0.19180600 & 4.31856200 & -2.39299400 \\
\hline $\mathrm{H}$ & 0.70731700 & 2.21710500 & -2.50298900 \\
\hline $\mathrm{C}$ & 0.12446300 & 5.45261800 & -1.58118700 \\
\hline $\mathrm{H}$ & 0.47086400 & 6.25757500 & 0.39292300 \\
\hline $\mathrm{H}$ & -0.11131000 & 4.37264400 & -3.43413600 \\
\hline $\mathrm{H}$ & -0.23360800 & 6.39233900 & -1.98994800 \\
\hline $\mathrm{C}$ & 4.89132800 & -0.59835300 & 0.19042600 \\
\hline $\mathrm{H}$ & 4.76943900 & -1.25271900 & -0.67413500 \\
\hline $\mathrm{H}$ & 5.94440400 & -0.60415500 & 0.49321800 \\
\hline $\mathrm{H}$ & 4.32159200 & -1.02787500 & 1.02228300 \\
\hline $\mathrm{C}$ & 4.67433900 & 1.85559300 & 0.89528900 \\
\hline $\mathrm{C}$ & 4.79385500 & 1.52655400 & 2.26085200 \\
\hline $\mathrm{C}$ & 4.80013800 & 3.21105700 & 0.52655800 \\
\hline $\mathrm{C}$ & 4.99667300 & 2.51634200 & 3.22101800 \\
\hline $\mathrm{H}$ & 4.70857400 & 0.49368200 & 2.58087100 \\
\hline $\mathrm{C}$ & 5.01377800 & 4.19752400 & 1.48640700 \\
\hline $\mathrm{H}$ & 4.76053700 & 3.49121900 & -0.52099800 \\
\hline $\mathrm{C}$ & 5.10668900 & 3.85549400 & 2.83877100 \\
\hline $\mathrm{H}$ & 5.07174500 & 2.24048600 & 4.26893100 \\
\hline $\mathrm{H}$ & 5.11789100 & 5.23381600 & 1.17802700 \\
\hline $\mathrm{H}$ & 5.27248400 & 4.62545000 & 3.58675800 \\
\hline $\mathrm{C}$ & 1.70478300 & -1.51983000 & -1.67689500 \\
\hline $\mathrm{C}$ & 1.86511200 & -2.74526200 & -1.00581800 \\
\hline $\mathrm{C}$ & 2.02474300 & -1.44317700 & -3.04394500 \\
\hline${ }_{0}$ & 2.36511700 & -3.85830700 & -1.68153200 \\
\hline
\end{tabular}




\begin{tabular}{|c|c|c|c|}
\hline $\mathrm{H}$ & 1.60517300 & -2.81665000 & 0.04623000 \\
\hline & 2.51725400 & -2.56362100 & -3.71542300 \\
\hline & 1.88415200 & -0.50778800 & -3.57671800 \\
\hline & 2.69262300 & -3.77246100 & -3.03779500 \\
\hline & 2.49537200 & -4.79532700 & -1.14761600 \\
\hline & 2.76353800 & -2.48927100 & -4.77088500 \\
\hline & 3.07644800 & -4.64211700 & -3.56302900 \\
\hline
\end{tabular}

\section{Int11b}

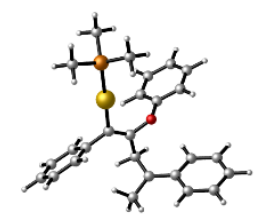

$$
\begin{aligned}
& E=-1560.175714 \text { Hartrees } \\
& G=-1559.765577 \text { Hartrees }
\end{aligned}
$$

$\begin{array}{llll}\mathrm{C} & 3.48354700 & 1.07724900 & -1.23705100 \\ \mathrm{C} & 4.23412000 & 0.75555700 & 0.01122300 \\ \mathrm{C} & 2.00182500 & 0.83750000 & -0.87005900 \\ \mathrm{H} & 3.78387700 & 0.40171900 & -2.03609000 \\ \mathrm{H} & 3.61516500 & 2.10544600 & -1.57637700 \\ \mathrm{C} & 1.28135000 & -0.28709300 & -1.05579500 \\ \mathrm{Au} & -0.70438300 & -0.38908300 & -0.46584300 \\ \mathrm{P} & -2.97848100 & -0.54182600 & 0.19448500 \\ \mathrm{C} & -4.12757600 & 0.40023100 & -0.88447400 \\ \mathrm{H} & -5.15846700 & 0.30427100 & -0.52888900 \\ \mathrm{H} & -3.84244400 & 1.45605200 & -0.88571800 \\ \mathrm{H} & -4.06066600 & 0.02102800 & -1.90805900 \\ \mathrm{C} & -3.65002900 & -2.25058500 & 0.20398700 \\ \mathrm{H} & -3.07477300 & -2.86405100 & 0.90319500 \\ \mathrm{H} & -4.70335400 & -2.25346900 & 0.50168700 \\ \mathrm{H} & -3.55522600 & -2.68449600 & -0.79535400 \\ \mathrm{C} & -3.30925400 & 0.09508400 & 1.88463100 \\ \mathrm{H} & -2.99419100 & 1.14049900 & 1.94652800 \\ \mathrm{H} & -4.37451800 & 0.02460000 & 2.12665900 \\ \mathrm{H} & -2.73356200 & -0.48387200 & 2.61231200 \\ \mathrm{O} & 1.45355100 & 1.90960700 & -0.16551700 \\ \mathrm{C} & 1.02965200 & 3.03166600 & -0.85237300 \\ \mathrm{C} & 0.66815500 & 4.12850500 & -0.05953600 \\ \mathrm{C} & 0.92569000 & 3.09827700 & -2.24527300\end{array}$




$\begin{array}{llll}\mathrm{C} & 0.20222700 & 5.29314600 & -0.66634200 \\ \mathrm{H} & 0.75524700 & 4.05021100 & 1.01967200 \\ \mathrm{C} & 0.46296000 & 4.27748000 & -2.83880500 \\ \mathrm{H} & 1.18666000 & 2.24245500 & -2.85742400 \\ \mathrm{C} & 0.09950400 & 5.37628900 & -2.05957600 \\ \mathrm{H} & -0.08015000 & 6.13992900 & -0.04698200 \\ \mathrm{H} & 0.38370800 & 4.32674300 & -3.92129700 \\ \mathrm{H} & -0.26260800 & 6.28585000 & -2.52902800 \\ \mathrm{C} & 4.80696200 & -0.61137300 & 0.06829800 \\ \mathrm{H} & 4.11448900 & -1.34243600 & -0.35822700 \\ \mathrm{H} & 5.68042400 & -0.60281300 & -0.60450000 \\ \mathrm{H} & 5.14135100 & -0.94156800 & 1.04870900 \\ \mathrm{C} & 4.39503500 & 1.69929400 & 1.07059000 \\ \mathrm{C} & 4.85511800 & 1.29274600 & 2.35872700 \\ \mathrm{C} & 4.11797500 & 3.08314600 & 0.86700400 \\ \mathrm{C} & 5.01950900 & 2.21635000 & 3.37620100 \\ \mathrm{H} & 5.05537300 & 0.24918200 & 2.56477500 \\ \mathrm{C} & 4.30130600 & 4.00142300 & 1.88773500 \\ \mathrm{H} & 3.79373000 & 3.44026200 & -0.10096900 \\ \mathrm{C} & 4.74715300 & 3.57135200 & 3.14329100 \\ \mathrm{H} & 5.35708100 & 1.88984000 & 4.35417800 \\ \mathrm{H} & 4.10114300 & 5.05295200 & 1.71116600 \\ \mathrm{H} & 4.88441400 & 4.29343300 & 3.94282500 \\ \mathrm{H} & 1.83039700 & -1.48053900 & -1.74923000 \\ \mathrm{C} & 1.88403100 & -2.72685400 & -1.09276000 \\ \mathrm{C} & 2.78879100 & -3.78754000 & -3.07511500 \\ \mathrm{C} & 3.151187100 & -4.80828100 & -1.21129800 \\ \mathrm{H} & 2.37047900 & -3.86154600 & -1.74320900 \\ \mathrm{H} & 1.54325600 & -2.79759400 & -0.06315600 \\ \mathrm{H} & 2.71899900 & -2.56534800 & -3.74750500 \\ \mathrm{H} & 2.18826800 & -0.48329100 & -3.63275200 \\ \mathrm{H} & & -2.49730000 & -4.78736400 \\ \mathrm{H} & & -4.67377900 & -3.58485700\end{array}$

TS10a-12a 


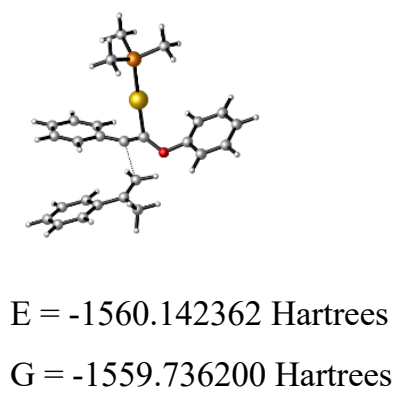

$\begin{array}{llll}\mathrm{Au} & 1.45981400 & 2.38609500 & -2.69052600 \\ \mathrm{P} & 2.97786100 & 1.24552200 & -4.06964700 \\ \mathrm{C} & 3.39079300 & -0.42995400 & -3.45200500 \\ \mathrm{H} & 3.83223900 & -0.35229600 & -2.45476900 \\ \mathrm{H} & 4.09953400 & -0.91946700 & -4.12741100 \\ \mathrm{H} & 2.47836200 & -1.02859800 & -3.38530700 \\ \mathrm{C} & 2.34942100 & 0.99589100 & -5.77198900 \\ \mathrm{H} & 3.08101300 & 0.44385400 & -6.37031700 \\ \mathrm{H} & 2.15975300 & 1.96730400 & -6.23679000 \\ \mathrm{H} & 1.41128600 & 0.43562500 & -5.73698800 \\ \mathrm{C} & 4.58632700 & 2.10274600 & -4.26417200 \\ \mathrm{H} & 4.42356000 & 3.09049800 & -4.70383600 \\ \mathrm{H} & 5.24939200 & 1.52084100 & -4.91234000 \\ \mathrm{H} & 5.05446900 & 2.22802800 & -3.28406200 \\ \mathrm{C} & -0.01018600 & 3.33593900 & -1.46771500 \\ \mathrm{C} & 0.60064700 & 3.79107000 & -0.42341000 \\ \mathrm{C} & -1.55046900 & 4.21576000 & 1.62185200 \\ \mathrm{C} & -1.13435900 & 5.14588500 & 0.71672000 \\ \mathrm{H} & -1.67756900 & 5.28242400 & -0.21156000 \\ \mathrm{H} & -0.37612100 & 5.88287700 & 0.95050500 \\ \mathrm{C} & -2.67709100 & 3.27662300 & 1.26461100 \\ \mathrm{H} & -3.12230400 & 3.54997100 & 0.30720700 \\ \mathrm{H} & -3.45598700 & 3.29423500 & 2.03522600 \\ \mathrm{H} & -2.32997500 & 2.23981800 & 1.18781000 \\ \mathrm{H} & -0.93080300 & 4.08600300 & 2.96027800 \\ \mathrm{C} & -0.66941300 & 1.85676800 & 5.54241800 \\ \mathrm{C} & -1.10941700 & 2.91322400 & 3.72333700 \\ \mathrm{C} & -0.15412400 & 5.12217200 & 3.52225300 \\ \mathrm{C} & -0.52029300 & 2.77366100 & 4.97931800 \\ \mathrm{H} & -1.70415100 & 2.09598600 & 3.33084000 \\ \mathrm{H} & 0.43142500 & 4.98259200 & 4.77751000 \\ \mathrm{H} & -0.02555600 & 6.05605600 & 2.98609700 \\ \mathrm{H} & & & \\ \mathrm{H} & & & \end{array}$




$\begin{array}{llll}\mathrm{H} & 1.01855500 & 5.79841700 & 5.18940100 \\ \mathrm{H} & 0.70932400 & 3.69976700 & 6.49248700 \\ \mathrm{O} & -1.33156600 & 3.32900500 & -1.74833900 \\ \mathrm{C} & -1.76133700 & 3.28184600 & -3.07506300 \\ \mathrm{C} & -2.83458200 & 2.43799800 & -3.35427300 \\ \mathrm{C} & -1.20207000 & 4.10300600 & -4.05465700 \\ \mathrm{C} & -3.35097300 & 2.40862100 & -4.65140100 \\ \mathrm{H} & -3.25128600 & 1.82129100 & -2.56434300 \\ \mathrm{C} & -1.72496900 & 4.05677700 & -5.34983000 \\ \mathrm{H} & -0.38640400 & 4.77341700 & -3.80369000 \\ \mathrm{C} & -2.79656000 & 3.21234100 & -5.65164200 \\ \mathrm{H} & -4.18626800 & 1.75279600 & -4.87863200 \\ \mathrm{H} & -1.29852900 & 4.69398900 & -6.11909600 \\ \mathrm{H} & -3.20103100 & 3.18501600 & -6.65886900 \\ \mathrm{C} & 1.71575100 & 3.93927600 & 0.44531800 \\ \mathrm{C} & 2.46344800 & 5.14149500 & 0.44998800 \\ \mathrm{C} & 2.08912600 & 2.88787300 & 1.31635400 \\ \mathrm{C} & 3.56540100 & 5.27327700 & 1.28609600 \\ \mathrm{H} & 2.16940700 & 5.95117600 & -0.21018300 \\ \mathrm{C} & 3.19527300 & 3.03025500 & 2.14715600 \\ \mathrm{H} & 1.51016400 & 1.97040800 & 1.31655700 \\ \mathrm{C} & 3.93293500 & 4.21922900 & 2.13200400 \\ \mathrm{H} & 4.14152400 & 6.19328400 & 1.28174200 \\ \mathrm{H} & 3.48358500 & 2.21849800 & 2.80754900 \\ \mathrm{H} & 4.79539200 & 4.32675600 & 2.78323900\end{array}$

\section{Int12a}

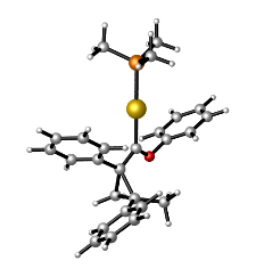

$E=-1560.187043$ Hartrees

$G=-1559.774520$ Hartrees

$\begin{array}{llll}\mathrm{Au} & 1.18462000 & 2.62272000 & -2.28375700 \\ \mathrm{P} & 2.73217200 & 1.27828300 & -3.48031200 \\ \mathrm{C} & 3.03679400 & -0.35265300 & -2.69924800 \\ \mathrm{H} & 3.44812400 & -0.20752900 & -1.69645800 \\ \mathrm{H} & 3.74110800 & -0.93845700 & -3.29815700 \\ \mathrm{H} & 2.09351300 & -0.89906500 & -2.61356800\end{array}$




\begin{tabular}{|c|c|c|c|}
\hline $\mathrm{C}$ & 2.18842000 & 0.89004100 & -5.18814400 \\
\hline $\mathrm{H}$ & 2.92876200 & 0.26119500 & -5.69243100 \\
\hline $\mathrm{H}$ & 2.05892800 & 1.81928000 & -5.74981700 \\
\hline $\mathrm{H}$ & 1.22963500 & 0.36530400 & -5.15599700 \\
\hline $\mathrm{C}$ & 4.39268100 & 2.03208200 & -3.67027800 \\
\hline $\mathrm{H}$ & 4.30093500 & 2.99259400 & -4.18457600 \\
\hline $\mathrm{H}$ & 5.04815200 & 1.37289500 & -4.24820000 \\
\hline $\mathrm{H}$ & 4.83038000 & 2.20503400 & -2.68329100 \\
\hline $\mathrm{C}$ & -0.16373500 & 3.80897500 & -1.26083000 \\
\hline $\mathrm{C}$ & 0.06744200 & 4.35742800 & 0.05549400 \\
\hline $\mathrm{C}$ & -1.14798600 & 4.21178900 & 1.20364500 \\
\hline $\mathrm{C}$ & -0.81513000 & 5.50257300 & 0.57737500 \\
\hline $\mathrm{H}$ & -1.52840000 & 5.90846600 & -0.13304000 \\
\hline $\mathrm{H}$ & -0.27621700 & 6.25031500 & 1.14822100 \\
\hline $\mathrm{C}$ & -2.44566400 & 3.51877300 & 0.81920700 \\
\hline $\mathrm{H}$ & -2.82813700 & 3.85051900 & -0.14434700 \\
\hline $\mathrm{H}$ & -3.19470200 & 3.77494100 & 1.57656300 \\
\hline $\mathrm{H}$ & -2.35383100 & 2.42973000 & 0.80488800 \\
\hline C & -0.65828200 & 3.87907300 & 2.58631700 \\
\hline $\mathrm{C}$ & -0.61849600 & 2.54068000 & 3.01812700 \\
\hline $\mathrm{C}$ & -0.28951300 & 4.88195200 & 3.49703900 \\
\hline $\mathrm{C}$ & -0.21188800 & 2.21706300 & 4.31151300 \\
\hline $\mathrm{H}$ & -0.89984000 & 1.74265700 & 2.33835200 \\
\hline $\mathrm{C}$ & 0.11291500 & 4.55824900 & 4.79417500 \\
\hline $\mathrm{H}$ & -0.33333800 & 5.92626900 & 3.20678000 \\
\hline $\mathrm{C}$ & 0.15725700 & 3.22558200 & 5.20574200 \\
\hline $\mathrm{H}$ & -0.18392500 & 1.17623000 & 4.62117200 \\
\hline $\mathrm{H}$ & 0.38622400 & 5.35223100 & 5.48328900 \\
\hline $\mathrm{H}$ & 0.47109700 & 2.97397800 & 6.21469800 \\
\hline $\mathrm{O}$ & -1.29975100 & 4.19558400 & -1.80773000 \\
\hline $\mathrm{C}$ & -1.71642500 & 3.70639500 & -3.06540700 \\
\hline $\mathrm{C}$ & -2.13750500 & 2.38608000 & -3.18989800 \\
\hline $\mathrm{C}$ & -1.76422600 & 4.61352200 & -4.11753400 \\
\hline $\mathrm{C}$ & -2.60765600 & 1.95771000 & -4.43430600 \\
\hline $\mathrm{H}$ & -2.10776000 & 1.71818600 & -2.33549600 \\
\hline $\mathrm{C}$ & -2.23426600 & 4.16692700 & -5.35481400 \\
\hline $\mathrm{H}$ & -1.44009200 & 5.63818800 & -3.96786800 \\
\hline $\mathrm{C}$ & -2.65345900 & 2.84297900 & -5.51451500 \\
\hline $\mathrm{H}$ & -2.94316500 & 0.93194300 & -4.55276200 \\
\hline $\mathrm{H}$ & -2.27382000 & 4.85797000 & -6.19123300 \\
\hline
\end{tabular}




$\begin{array}{llll}\mathrm{H} & -3.02232200 & 2.50394000 & -6.47763400 \\ \mathrm{C} & 1.49348400 & 4.26954700 & 0.53881900 \\ \mathrm{C} & 2.29545800 & 5.41900200 & 0.56490800 \\ \mathrm{C} & 2.06233200 & 3.04242600 & 0.91514400 \\ \mathrm{C} & 3.63357500 & 5.34682400 & 0.96097000 \\ \mathrm{H} & 1.87479300 & 6.37648500 & 0.27042700 \\ \mathrm{C} & 3.39872200 & 2.96885600 & 1.31203100 \\ \mathrm{H} & 1.45248500 & 2.14451300 & 0.90699400 \\ \mathrm{C} & 4.18863900 & 4.12183900 & 1.33640300 \\ \mathrm{H} & 4.23970000 & 6.24824200 & 0.97437400 \\ \mathrm{H} & 3.81922100 & 2.01296500 & 1.61166500 \\ \mathrm{H} & 5.22715300 & 4.06552000 & 1.64995100\end{array}$

\section{TS10a-12b}

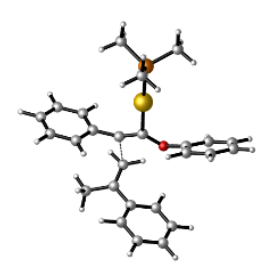

$E=-1560.141184$ Hartrees
$G=-1559.735026$ Hartrees

$\begin{array}{llll}\mathrm{Au} & 1.53834500 & 2.66013800 & -2.80746200 \\ \mathrm{P} & 3.09839800 & 1.61652400 & -4.21700600 \\ \mathrm{C} & 3.08343600 & -0.21091100 & -4.08303500 \\ \mathrm{H} & 3.30925900 & -0.50391400 & -3.05420500 \\ \mathrm{H} & 3.82680900 & -0.64751500 & -4.75747900 \\ \mathrm{H} & 2.09076500 & -0.58690900 & -4.34487300 \\ \mathrm{C} & 2.81696100 & 1.97580300 & -5.99047000 \\ \mathrm{H} & 3.57127200 & 1.47377400 & -6.60449800 \\ \mathrm{H} & 2.87223300 & 3.05499300 & -6.15694000 \\ \mathrm{H} & 1.82172800 & 1.62813100 & -6.28111800 \\ \mathrm{C} & 4.83204700 & 2.11284000 & -3.88967100 \\ \mathrm{H} & 4.93293800 & 3.19359500 & -4.02008700 \\ \mathrm{H} & 5.51018200 & 1.59889100 & -4.57842800 \\ \mathrm{H} & 5.09742000 & 1.85723800 & -2.86022700 \\ \mathrm{C} & 0.05880500 & 3.61038600 & -1.59918100 \\ \mathrm{C} & 0.62257900 & 3.88914800 & -0.46828900 \\ \mathrm{C} & -1.47399600 & 4.53526100 & 1.50463000 \\ \mathrm{C} & -0.97014200 & 5.41110500 & 0.58756500 \\ \mathrm{H} & -1.46614000 & 5.60347000 & -0.35601400\end{array}$




\begin{tabular}{|c|c|c|c|}
\hline $\mathrm{H}$ & -0.15799400 & 6.07597800 & 0.86049700 \\
\hline $\mathrm{C}$ & -0.78598800 & 4.36470700 & 2.83244600 \\
\hline $\mathrm{H}$ & -1.49521900 & 4.49720500 & 3.65742500 \\
\hline $\mathrm{H}$ & 0.02734800 & 5.08274600 & 2.95257600 \\
\hline $\mathrm{H}$ & -0.36316200 & 3.35793100 & 2.93853000 \\
\hline $\mathrm{C}$ & -2.69589600 & 3.73660000 & 1.24560000 \\
\hline $\mathrm{C}$ & -2.92410300 & 2.52310200 & 1.92397200 \\
\hline $\mathrm{C}$ & -3.67870400 & 4.18203900 & 0.33814800 \\
\hline $\mathrm{C}$ & -4.07376800 & 1.77121600 & 1.68237100 \\
\hline $\mathrm{H}$ & -2.19400600 & 2.15312700 & 2.63591700 \\
\hline $\mathrm{C}$ & -4.82956500 & 3.43447600 & 0.10359200 \\
\hline $\mathrm{H}$ & -3.55265900 & 5.13291700 & -0.16871900 \\
\hline $\mathrm{C}$ & -5.03092800 & 2.22250200 & 0.77131800 \\
\hline $\mathrm{H}$ & -4.22299500 & 0.83359300 & 2.21033900 \\
\hline $\mathrm{H}$ & -5.57607900 & 3.80290000 & -0.59421300 \\
\hline $\mathrm{H}$ & -5.93003600 & 1.64101700 & 0.58854600 \\
\hline $\mathrm{O}$ & -1.17061000 & 3.95316300 & -2.03993300 \\
\hline $\mathrm{C}$ & -1.74980000 & 3.26804800 & -3.10765600 \\
\hline $\mathrm{C}$ & -1.77868500 & 1.87376900 & -3.15397100 \\
\hline $\mathrm{C}$ & -2.37457800 & 4.04368200 & -4.08211500 \\
\hline $\mathrm{C}$ & -2.43293000 & 1.24916600 & -4.21919700 \\
\hline $\mathrm{H}$ & -1.31322300 & 1.29220800 & -2.36482000 \\
\hline $\mathrm{C}$ & -3.03138500 & 3.40510700 & -5.13625000 \\
\hline $\mathrm{H}$ & -2.34184100 & 5.12595900 & -4.00763500 \\
\hline $\mathrm{C}$ & -3.05852400 & 2.00968400 & -5.21058800 \\
\hline $\mathrm{H}$ & -2.46293200 & 0.16428200 & -4.26277200 \\
\hline $\mathrm{H}$ & -3.51866200 & 4.00232900 & -5.90132200 \\
\hline $\mathrm{H}$ & -3.56946300 & 1.51779400 & -6.03269800 \\
\hline $\mathrm{C}$ & 1.71435100 & 3.86081100 & 0.44676900 \\
\hline $\mathrm{C}$ & 2.58957600 & 4.96735700 & 0.55681200 \\
\hline $\mathrm{C}$ & 1.94189300 & 2.71646200 & 1.24744400 \\
\hline $\mathrm{C}$ & 3.66592700 & 4.92083300 & 1.43427700 \\
\hline $\mathrm{H}$ & 2.41128500 & 5.84657300 & -0.05379800 \\
\hline $\mathrm{C}$ & 3.02948600 & 2.67670700 & 2.11481300 \\
\hline $\mathrm{H}$ & 1.26872600 & 1.86941000 & 1.16410400 \\
\hline $\mathrm{C}$ & 3.88889600 & 3.77613600 & 2.21046600 \\
\hline $\mathrm{H}$ & 4.33773600 & 5.77003900 & 1.51225700 \\
\hline $\mathrm{H}$ & 3.20863200 & 1.79186700 & 2.71771300 \\
\hline $\mathrm{H}$ & 4.73374600 & 3.74338900 & 2.89223200 \\
\hline
\end{tabular}




\section{Int12b}

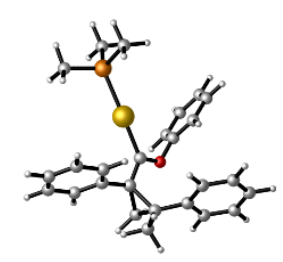

$E=-1560.190329$ Hartrees

$G=-1559.779063$ Hartrees

\begin{tabular}{llll}
$\mathrm{Au}$ & 1.17230400 & 2.59545300 & -2.32560500 \\
$\mathrm{P}$ & 2.78004800 & 1.40621200 & -3.60742300 \\
$\mathrm{C}$ & 2.67627200 & -0.41498900 & -3.41259600 \\
$\mathrm{H}$ & 2.84455900 & -0.68099000 & -2.36523700 \\
$\mathrm{H}$ & 3.42797900 & -0.90847400 & -4.03652400 \\
$\mathrm{H}$ & 1.68022600 & -0.75847100 & -3.70513800 \\
$\mathrm{C}$ & 2.62305800 & 1.70200400 & -5.41013900 \\
$\mathrm{H}$ & 3.38221600 & 1.13659900 & -5.95959600 \\
$\mathrm{H}$ & 2.74740300 & 2.76880900 & -5.61638500 \\
$\mathrm{H}$ & 1.62833900 & 1.39536000 & -5.74530200 \\
$\mathrm{C}$ & 4.51774400 & 1.82790400 & -3.20316100 \\
$\mathrm{H}$ & 4.68537100 & 2.89319300 & -3.38439500 \\
$\mathrm{H}$ & 5.20728900 & 1.24165000 & -3.81884700 \\
$\mathrm{H}$ & 4.70654700 & 1.61963600 & -2.14647200 \\
$\mathrm{C}$ & -0.21601400 & 3.69144600 & -1.26305800 \\
$\mathrm{C}$ & 0.03719100 & 4.30616700 & 0.02377900 \\
$\mathrm{C}$ & -1.14223200 & 4.20660900 & 1.16724100 \\
$\mathrm{C}$ & -0.87343500 & 5.47271300 & 0.46670300 \\
$\mathrm{H}$ & -1.57319200 & 5.82266900 & -0.28469000 \\
$\mathrm{H}$ & -0.36465300 & 6.25566600 & 1.02054500 \\
$\mathrm{C}$ & -0.63036100 & 4.05610200 & 2.59144800 \\
$\mathrm{H}$ & -1.42566200 & 4.37935800 & 3.27273200 \\
$\mathrm{H}$ & 0.25004800 & 4.67180000 & 2.78183300 \\
$\mathrm{H}$ & -0.39128700 & 3.01662000 & 2.83301500 \\
$\mathrm{C}$ & -2.38463500 & 3.40322400 & 0.89022200 \\
$\mathrm{C}$ & -2.36681000 & 2.00345100 & 1.00266200 \\
$\mathrm{C}$ & -3.60241900 & 4.04110400 & 0.61813000 \\
$\mathrm{C}$ & -3.53561900 & 1.26079000 & 0.83388200 \\
$\mathrm{H}$ & -1.43230500 & 1.49267200 & 1.21853300 \\
& -4.77471600 & 3.29897400 & 0.45515700 \\
$\mathrm{H}$ & -3.63823000 & 5.12380700 & 0.54269400 \\
\hline & -4.74502500 & 1.90760800 & 0.56018900
\end{tabular}




$\begin{array}{llll}\mathrm{H} & -3.50370200 & 0.17819600 & 0.91931100 \\ \mathrm{H} & -5.71002200 & 3.81106500 & 0.24736700 \\ \mathrm{H} & -5.65612800 & 1.33038400 & 0.43115500 \\ \mathrm{O} & -1.40821900 & 3.95665600 & -1.74760500 \\ \mathrm{C} & -1.84933400 & 3.39958000 & -2.96863600 \\ \mathrm{C} & -2.16106300 & 2.04574800 & -3.03853300 \\ \mathrm{C} & -2.02983900 & 4.27030700 & -4.03688600 \\ \mathrm{C} & -2.65835700 & 1.54515700 & -4.24483500 \\ \mathrm{H} & -2.03542300 & 1.41073800 & -2.16858200 \\ \mathrm{C} & -2.52500000 & 3.75169100 & -5.23568400 \\ \mathrm{H} & -1.78763800 & 5.32259300 & -3.92987900 \\ \mathrm{C} & -2.83750700 & 2.39325400 & -5.34093800 \\ \mathrm{H} & -2.91269300 & 0.49234600 & -4.32068000 \\ \mathrm{H} & -2.66826000 & 4.41331500 & -6.08439400 \\ \mathrm{H} & -3.22742100 & 1.99798800 & -6.27388200 \\ \mathrm{C} & 1.47131600 & 4.26292900 & 0.48493100 \\ \mathrm{C} & 2.26742700 & 5.41553000 & 0.43469800 \\ \mathrm{C} & 2.04555800 & 3.06398500 & 0.93776600 \\ \mathrm{C} & 3.60698100 & 5.37359600 & 0.83035500 \\ \mathrm{H} & 1.83897400 & 6.35025500 & 0.08388400 \\ \mathrm{C} & 3.38325400 & 3.02154300 & 1.33547200 \\ \mathrm{H} & 1.43950500 & 2.16368500 & 0.98455700 \\ \mathrm{C} & 4.16741300 & 4.17761600 & 1.28431200 \\ \mathrm{H} & 4.20971000 & 6.27623500 & 0.78494200 \\ \mathrm{H} & 3.80955000 & 2.08866700 & 1.69366200 \\ \mathrm{H} & 5.20668300 & 4.14614200 & 1.59861700\end{array}$

\section{TS11a-13a}

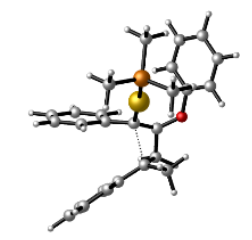

$E=-1560.156474$ Hartrees

$\mathrm{G}=-1559.742500$ Hartrees

$\begin{array}{llll}\text { C } & 4.18605300 & 1.24832200 & -0.75906600\end{array}$

$\begin{array}{llll}\text { C } & 3.55679200 & 0.45622600 & 0.38776500\end{array}$

$\begin{array}{llll}\text { C } & 2.79741400 & 1.34453400 & -1.27048600\end{array}$

$\mathrm{H} \quad 4.84381700 \quad 0.66623600 \quad-1.40076300$

$\begin{array}{lllll}\mathrm{H} & 4.64910900 & 2.20225100 & -0.50100300\end{array}$ 


\begin{tabular}{|c|c|c|c|}
\hline $\mathrm{C}$ & 2.07254900 & 0.14357200 & -0.99819300 \\
\hline $\mathrm{Au}$ & 0.09702300 & 0.16866700 & -0.28366400 \\
\hline $\mathrm{P}$ & -2.09249800 & 0.04147500 & 0.60828600 \\
\hline $\mathrm{C}$ & -2.39770200 & 1.20338100 & 1.99534600 \\
\hline $\mathrm{H}$ & -3.42252200 & 1.10339000 & 2.36669800 \\
\hline $\mathrm{H}$ & -1.69669100 & 0.99442500 & 2.80841600 \\
\hline $\mathrm{H}$ & -2.23620900 & 2.22886400 & 1.65140700 \\
\hline C & -3.42547100 & 0.39552500 & -0.60236400 \\
\hline $\mathrm{H}$ & -3.35939500 & -0.31482000 & -1.43142700 \\
\hline $\mathrm{H}$ & -4.40832500 & 0.30747300 & -0.12875100 \\
\hline $\mathrm{H}$ & -3.30259600 & 1.40706200 & -0.99816300 \\
\hline C & -2.50684300 & -1.61476900 & 1.28292100 \\
\hline $\mathrm{H}$ & -1.80156700 & -1.87244700 & 2.07796700 \\
\hline $\mathrm{H}$ & -3.52474800 & -1.62405000 & 1.68527800 \\
\hline $\mathrm{H}$ & -2.42660500 & -2.36246400 & 0.48867200 \\
\hline $\mathrm{O}$ & 2.31482300 & 2.56461500 & -1.40523000 \\
\hline $\mathrm{C}$ & 1.00870000 & 2.84244900 & -1.86506900 \\
\hline C & 0.27286200 & 3.76785100 & -1.13193700 \\
\hline C & 0.56038900 & 2.31779900 & -3.07511000 \\
\hline $\mathrm{C}$ & -0.97489100 & 4.16169200 & -1.62041300 \\
\hline $\mathrm{H}$ & 0.67419200 & 4.17295200 & -0.20913700 \\
\hline $\mathrm{C}$ & -0.69081200 & 2.72048300 & -3.54648800 \\
\hline $\mathrm{H}$ & 1.17664500 & 1.62426500 & -3.63729800 \\
\hline $\mathrm{C}$ & -1.45815000 & 3.63866300 & -2.82341900 \\
\hline $\mathrm{H}$ & -1.56110600 & 4.88679400 & -1.06426700 \\
\hline $\mathrm{H}$ & -1.05647100 & 2.32540300 & -4.48930400 \\
\hline $\mathrm{H}$ & -2.42378900 & 3.95706600 & -3.20414200 \\
\hline $\mathrm{C}$ & 2.99392200 & 1.31927800 & 1.50104900 \\
\hline $\mathrm{H}$ & 2.59777000 & 2.26541000 & 1.12494200 \\
\hline $\mathrm{H}$ & 2.19566100 & 0.82087600 & 2.05384600 \\
\hline $\mathrm{H}$ & 3.79872900 & 1.55610400 & 2.20782700 \\
\hline $\mathrm{C}$ & 4.10703700 & -0.86728100 & 0.78755200 \\
\hline $\mathrm{C}$ & 3.51749700 & -1.57835900 & 1.85370300 \\
\hline $\mathrm{C}$ & 5.23423400 & -1.43099700 & 0.16273400 \\
\hline 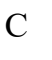 & 4.04400700 & -2.79347000 & 2.28473500 \\
\hline $\mathrm{H}$ & 2.64132800 & -1.18146000 & 2.35361100 \\
\hline $\mathrm{C}$ & 5.76425100 & -2.64495100 & 0.60109500 \\
\hline $\mathrm{H}$ & 5.72162300 & -0.92479800 & -0.66297600 \\
\hline $\mathrm{C}$ & 5.17291800 & -3.33237000 & 1.66209300 \\
\hline & 3.57167300 & -3.31885500 & 3.10984900 \\
\hline
\end{tabular}




$\begin{array}{llll}\mathrm{H} & 6.64271200 & -3.05265800 & 0.10943900 \\ \mathrm{H} & 5.58496700 & -4.27879900 & 1.99989500 \\ \mathrm{C} & 2.44606100 & -1.10460600 & -1.72069100 \\ \mathrm{C} & 2.04853300 & -2.36512300 & -1.22897600 \\ \mathrm{C} & 3.13455400 & -1.06667700 & -2.95017900 \\ \mathrm{C} & 2.34720000 & -3.53449900 & -1.92352200 \\ \mathrm{H} & 1.50086400 & -2.41782100 & -0.29351400 \\ \mathrm{C} & 3.42109300 & -2.23971300 & -3.65143200 \\ \mathrm{H} & 3.43226400 & -0.11178900 & -3.37455800 \\ \mathrm{C} & 3.03296100 & -3.47919100 & -3.14143300 \\ \mathrm{H} & 2.03256700 & -4.49247900 & -1.51899900 \\ \mathrm{H} & 3.94336800 & -2.17919400 & -4.60227700 \\ \mathrm{H} & 3.25197100 & -4.39109600 & -3.68925300\end{array}$

\section{Int13a}

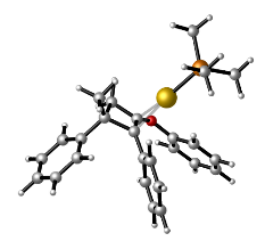

$\begin{array}{lrrc}\mathrm{E}=-1560.192807 \text { Hartrees } & & \\ \mathrm{G}=-1559.778369 \text { Hartrees } & & \\ \mathrm{C} & 2.89226400 & 1.42994700 & 1.07052500 \\ \mathrm{C} & 2.96219600 & -0.13990400 & 1.07021500 \\ \mathrm{C} & 2.10498300 & 1.31237300 & -0.20432300 \\ \mathrm{H} & 3.83528800 & 1.95727700 & 0.92087700 \\ \mathrm{H} & 2.37066100 & 1.87615400 & 1.92056100 \\ \mathrm{C} & 1.99930600 & -0.08532900 & -0.19947500 \\ \mathrm{Au} & -0.16076200 & 0.33427100 & 0.63985400 \\ \mathrm{P} & -2.36655300 & 0.43692000 & 1.37373400 \\ \mathrm{C} & -2.57988000 & 1.43894300 & 2.89030500 \\ \mathrm{H} & -3.63231700 & 1.44369400 & 3.19079000 \\ \mathrm{H} & -1.97477400 & 1.01638300 & 3.69682600 \\ \mathrm{H} & -2.25139600 & 2.46443500 & 2.70210100 \\ \mathrm{C} & -3.50448000 & 1.15649800 & 0.13433900 \\ \mathrm{H} & -3.46222000 & 0.56837700 & -0.78625400 \\ \mathrm{H} & -4.52894400 & 1.15501100 & 0.51973800 \\ \mathrm{H} & -3.20238000 & 2.18337600 & -0.08839500 \\ \mathrm{C} & -3.04782100 & -1.21510100 & 1.77420600 \\ \mathrm{H} & -2.43335700 & -1.68746300 & 2.54537000\end{array}$




\begin{tabular}{|c|c|c|c|}
\hline $\mathrm{H}$ & -4.07592200 & -1.11915800 & 2.13709400 \\
\hline $\mathrm{H}$ & -3.03601100 & -1.84387400 & 0.87980700 \\
\hline $\mathrm{O}$ & 1.91278700 & 2.36022800 & -0.99960400 \\
\hline $\mathrm{C}$ & 1.34695200 & 2.36341200 & -2.28478900 \\
\hline & 1.92441700 & 3.27913100 & -3.16438100 \\
\hline & 0.24266600 & 1.59577800 & -2.64806900 \\
\hline & 1.39059500 & 3.41801400 & -4.44512800 \\
\hline & 2.77651600 & 3.86698700 & -2.83881000 \\
\hline $\mathrm{C}$ & -0.28173600 & 1.75027200 & -3.93516800 \\
\hline & -0.20684700 & 0.89377200 & -1.95472100 \\
\hline $\mathrm{C}$ & 0.28646600 & 2.65405200 & -4.83421600 \\
\hline $\mathrm{H}$ & 1.83851600 & 4.12539100 & -5.13642600 \\
\hline & -1.14100200 & 1.15498700 & -4.22941800 \\
\hline $\mathrm{H}$ & -0.12861700 & 2.76384700 & -5.83136700 \\
\hline $\mathrm{C}$ & 2.42292100 & -0.79654400 & 2.34706400 \\
\hline $\mathrm{H}$ & 1.46980100 & -0.34758100 & 2.64916100 \\
\hline $\mathrm{H}$ & 2.26531500 & -1.87082000 & 2.21428500 \\
\hline $\mathrm{H}$ & 3.13786600 & -0.66309700 & 3.16699200 \\
\hline $\mathrm{C}$ & 4.33287500 & -0.73522600 & 0.72122600 \\
\hline $\mathrm{C}$ & 4.41819600 & -2.10810600 & 0.42297600 \\
\hline $\mathrm{C}$ & 5.52623900 & 0.00257600 & 0.73362900 \\
\hline $\mathrm{C}$ & 5.64043500 & -2.71435500 & 0.13500300 \\
\hline $\mathrm{H}$ & 3.51775300 & -2.71310600 & 0.41446300 \\
\hline $\mathrm{C}$ & 6.75392800 & -0.60345700 & 0.44791700 \\
\hline $\mathrm{H}$ & 5.52262100 & 1.05833200 & 0.98090400 \\
\hline $\mathrm{C}$ & 6.81803000 & -1.96265600 & 0.14373800 \\
\hline $\mathrm{H}$ & 5.67131800 & -3.77577800 & -0.09549400 \\
\hline $\mathrm{H}$ & 7.66094900 & -0.00544100 & 0.46914900 \\
\hline $\mathrm{H}$ & 7.77190000 & -2.43271200 & -0.07835800 \\
\hline $\mathrm{C}$ & 1.83084500 & -1.10769400 & -1.26756700 \\
\hline $\mathrm{C}$ & 1.12146500 & -2.29749000 & -1.01676000 \\
\hline $\mathrm{C}$ & 2.44041700 & -0.94409200 & -2.52381400 \\
\hline $\mathrm{C}$ & 1.00556000 & -3.28147500 & -1.99867800 \\
\hline $\mathrm{H}$ & 0.65834900 & -2.44661800 & -0.04527800 \\
\hline $\mathrm{C}$ & 2.32204100 & -1.92902900 & -3.50592700 \\
\hline & 3.02129100 & -0.05094200 & -2.73025600 \\
\hline$C$ & 1.60307500 & -3.09814500 & -3.24883000 \\
\hline 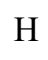 & 0.45019800 & -4.19076400 & -1.78693400 \\
\hline 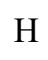 & 2.79989600 & -1.78344600 & -4.47050200 \\
\hline & 1.51386400 & -3.86367000 & -4.01428600 \\
\hline
\end{tabular}




\section{TS11a-14a}

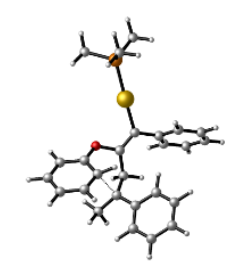

$E=-1560.164042$ Hartrees

$\mathrm{G}=-1559.748998$ Hartrees

C

$\begin{array}{lll}2.78271400 & -0.45695300 & 1.03413500\end{array}$

$\begin{array}{llll}\text { C } & 3.72992500 & 0.70737400 & 0.74497100\end{array}$

$\begin{array}{llll}\text { C } & 1.33248800 & -0.36522300 & 0.57040600\end{array}$

$\begin{array}{llll}\mathrm{H} & 2.76149400 & -0.585666000 & 2.12164600\end{array}$

$\mathrm{H} \quad 3.20780500 \quad-1.36533000 \quad 0.60507200$

C $\quad 0.61792800 \quad-1.21046000 \quad-0.18861900$

$\mathrm{Au} \quad-1.35158600 \quad-0.82317300 \quad-0.71338600$

$\mathrm{P} \quad \quad-3.60812900 \quad-0.44298600 \quad-1.33656100$

C $\quad-3.81200000 \quad 0.50525500 \quad-2.89581400$

$\mathrm{H} \quad-4.87171800 \quad 0.66194400 \quad-3.12098500$

$\mathrm{H} \quad-3.31690900 \quad 1.47548600 \quad-2.79862900$

$\mathrm{H} \quad-3.34399000 \quad-0.04184700 \quad-3.71900300$

C $\quad-4.57627100 \quad-1.98013700 \quad-1.60367700$

$\mathrm{H} \quad-4.57604200 \quad-2.57492700 \quad-0.68602400$

$\mathrm{H} \quad-5.60849200 \quad-1.74522100 \quad-1.88213500$

$\mathrm{H} \quad-4.11108900 \quad-2.56931100 \quad-2.39887100$

$\begin{array}{llll}\text { C } & -4.57921700 & 0.50060500 & -0.09669000\end{array}$

$\mathrm{H} \quad-4.11438300 \quad 1.47689400 \quad 0.06680800$

$\mathrm{H} \quad-5.60939800 \quad 0.64305600 \quad-0.43847800$

$\mathrm{H} \quad-4.58567800 \quad-0.04423500 \quad 0.85155300$

$\begin{array}{lllll}\mathrm{O} & 0.68163800 & 0.73036600 & 1.18116900\end{array}$

$\begin{array}{llll}\mathrm{C} & 1.28702200 & 1.92306700 & 1.09164200\end{array}$

$\begin{array}{llll}\text { C } & 2.25177700 & 2.16047800 & 0.07282200\end{array}$

$\begin{array}{llll}\text { C } & 0.98279300 & 2.90374100 & 2.04899100\end{array}$

$\begin{array}{llll}\text { C } & 2.85786300 & 3.45053800 & 0.00293200\end{array}$

$\mathrm{H} \quad 2.17315400 \quad 1.56226600 \quad-0.82771000$

$\begin{array}{llll}\text { C } & 1.62328600 & 4.12820500 & 1.96785300\end{array}$

$\begin{array}{llll}\mathrm{H} & 0.26183100 & 2.68501200 & 2.82938900\end{array}$

$\begin{array}{llll}\text { C } & 2.57099500 & 4.40645200 & 0.94874100\end{array}$

$\mathrm{H} \quad 3.55878900 \quad 3.65450100 \quad-0.80022000$

$\begin{array}{llll}\mathrm{H} & 1.39915900 & 4.89285000 & 2.70570700\end{array}$ 


$\begin{array}{llll}\mathrm{H} & 3.04564400 & 5.38135100 & 0.91197300 \\ \mathrm{C} & 4.30359300 & 1.34948500 & 1.98231700 \\ \mathrm{H} & 4.74401100 & 2.33009800 & 1.80401100 \\ \mathrm{H} & 3.55056000 & 1.43693000 & 2.76697700 \\ \mathrm{H} & 5.09743800 & 0.68951800 & 2.35897900 \\ \mathrm{C} & 4.58173800 & 0.63733900 & -0.46215000 \\ \mathrm{C} & 5.77267800 & 1.39031600 & -0.55662600 \\ \mathrm{C} & 4.23343500 & -0.19361200 & -1.55105300 \\ \mathrm{C} & 6.58807300 & 1.29960700 & -1.68152500 \\ \mathrm{H} & 6.07489500 & 2.04351300 & 0.25301100 \\ \mathrm{C} & 5.04591200 & -0.27403200 & -2.67674500 \\ \mathrm{H} & 3.32231600 & -0.77961000 & -1.52191900 \\ \mathrm{C} & 6.22799900 & 0.46986500 & -2.74641700 \\ \mathrm{H} & 7.50549700 & 1.87837800 & -1.72655100 \\ \mathrm{H} & 4.75935200 & -0.92207300 & -3.49932700 \\ \mathrm{H} & 6.86378600 & 0.40259100 & -3.62426100 \\ \mathrm{C} & 1.20618900 & -2.48451200 & -0.67287800 \\ \mathrm{C} & 1.71469600 & -3.44773100 & 0.22176500 \\ \mathrm{C} & 1.21978400 & -2.79264500 & -2.04898700 \\ \mathrm{C} & 2.22958300 & -4.66083400 & -0.24190700 \\ \mathrm{H} & 1.68468400 & -3.24941500 & 1.28953100 \\ \mathrm{C} & 1.74885400 & -3.99719600 & -2.51135300 \\ \mathrm{H} & 0.81173000 & -2.07390600 & -2.75475300 \\ \mathrm{C} & 2.25486200 & -4.93936800 & -1.60961200 \\ \mathrm{H} & 2.60845600 & -5.38943600 & 0.46988200 \\ \mathrm{H} & 1.75743100 & -4.20605900 & -3.57782100 \\ \mathrm{H} & 2.65478000 & -5.88282400 & -1.96997300\end{array}$

\section{Int14a}

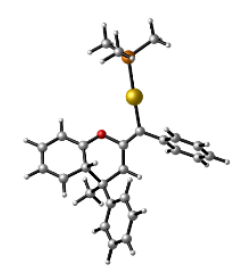

$E=-1560.178410$ Hartrees

$G=-1559.762132$ Hartrees

$\begin{array}{lrrr}\mathrm{C} & 2.80246100 & -0.43475200 & 0.86526300 \\ \mathrm{C} & 3.60313800 & 0.90657600 & 0.66958700 \\ \mathrm{C} & 1.35686200 & -0.39189300 & 0.44839000 \\ \mathrm{H} & 2.83902300 & -0.69017200 & 1.93124600\end{array}$




$\begin{array}{llcc}\mathrm{H} & 3.29063900 & -1.24841800 & 0.32995300 \\ \mathrm{C} & 0.58932600 & -1.24341600 & -0.25216000 \\ \mathrm{Au} & -1.41720700 & -0.88333500 & -0.63673800 \\ \mathrm{P} & -3.71072500 & -0.51103700 & -1.11462500 \\ \mathrm{C} & -3.98168000 & 0.44935500 & -2.65618700 \\ \mathrm{H} & -5.04942400 & 0.61517400 & -2.83175100 \\ \mathrm{H} & -3.47521600 & 1.41525700 & -2.57556000 \\ \mathrm{H} & -3.55648400 & -0.09506900 & -3.50400500 \\ \mathrm{C} & -4.70203300 & -2.03919500 & -1.34207800 \\ \mathrm{H} & -4.66604100 & -2.63676200 & -0.42696300 \\ \mathrm{H} & -5.74326200 & -1.79467500 & -1.57478300 \\ \mathrm{H} & -4.27626100 & -2.62900400 & -2.15852800 \\ \mathrm{C} & -4.60770100 & 0.43447900 & 0.17832500 \\ \mathrm{H} & -4.12529100 & 1.40525800 & 0.32263000 \\ \mathrm{H} & -5.65279900 & 0.58908900 & -0.10839700 \\ \mathrm{H} & -4.57024600 & -0.11544500 & 1.12296400 \\ \mathrm{H} & 3.34304100 & -0.07514200 & -1.87126700 \\ \mathrm{C} & 6.66157900 & 0.41816400 & -2.40268700 \\ \mathrm{O} & & & \\ \mathrm{C} & & & \end{array}$




\begin{tabular}{|c|c|c|c|}
\hline $\mathrm{H}$ & 8.03464400 & 1.21683100 & -0.94585100 \\
\hline$U$ & 5.04902700 & -0.35032700 & -3.61619000 \\
\hline & 7.41698700 & 0.29222100 & -3.17300500 \\
\hline & 1.16720900 & -2.50280500 & -0.78603100 \\
\hline & 1.79872600 & -3.44562200 & 0.04854600 \\
\hline & 1.05208500 & -2.80756800 & -2.15743500 \\
\hline & 2.30608200 & -4.63876900 & -0.47132300 \\
\hline & 1.87291000 & -3.25055500 & 1.11455500 \\
\hline & 1.57672600 & -3.98976200 & -2.67787500 \\
\hline & 0.55016300 & -2.10225000 & -2.81455500 \\
\hline & 2.20456300 & -4.91368500 & -1.83643800 \\
\hline & 2.78121200 & -5.35395400 & 0.19475400 \\
\hline & 1.48718300 & -4.19525500 & -3.74123000 \\
\hline & 2.60191900 & -5.84055300 & -2.24009200 \\
\hline
\end{tabular}

\section{(E)-Int15a}

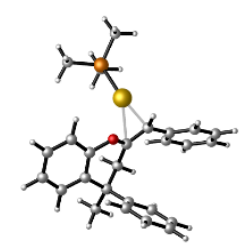

$E=-1560.242023$ Hartrees

$\mathrm{G}=-1559.824770$ Hartrees

$\begin{array}{llll}\text { C } & 1.30196100 & 3.35815900 & -0.49343600\end{array}$

$\begin{array}{llll}\text { C } & 2.73376000 & 3.73386200 & -0.00814500\end{array}$

$\begin{array}{llll}\text { C } & 1.00172100 & 1.90112200 & -0.29375200\end{array}$

$\begin{array}{llll}\mathrm{H} & 0.57910900 & 3.94852200 & 0.08344900\end{array}$

$\mathrm{H} \quad 1.15682200 \quad 3.61594000 \quad-1.54220900$

$\mathrm{Au} \quad \quad-1.61727900 \quad 1.42758000 \quad 0.08275300$

$\begin{array}{llll}\mathrm{P} & -3.66488500 & 1.60244600 & 1.17197200\end{array}$

$\begin{array}{llll}\text { C } & -3.49920000 & 1.98639800 & 2.95353200\end{array}$

$\mathrm{H} \quad \begin{array}{llll}\mathrm{H} & -4.48943900 & 2.07569500 & 3.41151300\end{array}$

$\mathrm{H} \quad-2.94002100 \quad 1.18852600 \quad 3.44946400$

$\mathrm{H} \quad \begin{array}{llll}\mathrm{H} & -2.95571800 & 2.92699200 & 3.07591200\end{array}$

$\begin{array}{llll}\text { C } & -4.73064100 & 2.91900700 & 0.47762600\end{array}$

$\mathrm{H} \quad-4.93450600 \quad 2.70746300 \quad-0.57542700$

$\mathrm{H} \quad \begin{array}{llll}\mathrm{H} & -5.67480800 & 2.96748400 & 1.02934600\end{array}$

$\mathrm{H} \quad \begin{array}{llll}\mathrm{H} & -4.21680700 & 3.88111600 & 0.55177100\end{array}$

$\begin{array}{llll}\text { C } & -4.65001700 & 0.06261400 & 1.08258900\end{array}$

$\mathrm{H} \quad \begin{array}{llll}\mathrm{H} & -4.08940400 & -0.75670300 & 1.54042900\end{array}$ 


\begin{tabular}{|c|c|c|c|}
\hline $\mathrm{H}$ & -5.59951900 & 0.19422600 & 1.61110400 \\
\hline $\mathrm{H}$ & -4.84734600 & -0.18532400 & 0.03620300 \\
\hline $\mathrm{C}$ & 2.89093900 & 5.26415400 & -0.13644900 \\
\hline $\mathrm{H}$ & 2.17168100 & 5.78675100 & 0.50251300 \\
\hline $\mathrm{H}$ & 3.89936100 & 5.58212100 & 0.14051600 \\
\hline $\mathrm{H}$ & 2.72001800 & 5.57518200 & -1.17091500 \\
\hline $\mathrm{C}$ & 3.81001200 & 3.03965600 & -0.87931200 \\
\hline $\mathrm{C}$ & 3.75924000 & 3.15520200 & -2.27977200 \\
\hline $\mathrm{C}$ & 4.88001100 & 2.32898400 & -0.31738400 \\
\hline $\mathrm{C}$ & 4.73826600 & 2.57538800 & -3.08713900 \\
\hline $\mathrm{H}$ & 2.95175200 & 3.70521000 & -2.75432500 \\
\hline $\mathrm{C}$ & 5.86282300 & 1.74844100 & -1.12464600 \\
\hline $\mathrm{H}$ & 4.95644200 & 2.22458100 & 0.75942800 \\
\hline $\mathrm{C}$ & 5.79657900 & 1.86695500 & -2.51250700 \\
\hline $\mathrm{H}$ & 4.67271600 & 2.67872600 & -4.16676900 \\
\hline $\mathrm{H}$ & 6.68042400 & 1.20312700 & -0.66111400 \\
\hline $\mathrm{H}$ & 6.55911700 & 1.41463000 & -3.14020100 \\
\hline $\mathrm{O}$ & 1.53621100 & 1.31057000 & 0.79212600 \\
\hline $\mathrm{C}$ & 2.16298600 & 2.07613200 & 1.77656700 \\
\hline $\mathrm{C}$ & 2.15808100 & 1.54524400 & 3.06228300 \\
\hline $\mathrm{C}$ & 3.44287700 & 3.96130700 & 2.48365600 \\
\hline $\mathrm{C}$ & 2.81199300 & 2.24656000 & 4.07594400 \\
\hline $\mathrm{H}$ & 1.65248900 & 0.60394000 & 3.25246400 \\
\hline $\mathrm{C}$ & 3.45150900 & 3.45387700 & 3.78600600 \\
\hline $\mathrm{H}$ & 3.94930300 & 4.89753900 & 2.27476300 \\
\hline $\mathrm{H}$ & 2.81852100 & 1.84974000 & 5.08639300 \\
\hline $\mathrm{H}$ & 3.95994300 & 4.00294500 & 4.57263600 \\
\hline $\mathrm{C}$ & 2.79938400 & 3.27708700 & 1.44626600 \\
\hline $\mathrm{C}$ & 0.27629000 & 1.05231900 & -1.13129200 \\
\hline $\mathrm{H}$ & 0.41042200 & -0.00219800 & -0.88442700 \\
\hline $\mathrm{C}$ & -0.08605100 & 1.31051700 & -2.55540500 \\
\hline $\mathrm{C}$ & 0.14409700 & 0.27476600 & -3.47971000 \\
\hline $\mathrm{C}$ & -0.65976500 & 2.50669200 & -3.02688400 \\
\hline $\mathrm{C}$ & -0.15737800 & 0.43842900 & -4.83117300 \\
\hline $\mathrm{H}$ & 0.57376500 & -0.66115900 & -3.13248600 \\
\hline $\mathrm{C}$ & -0.95448500 & 2.67045900 & -4.38162500 \\
\hline $\mathrm{H}$ & -0.90128200 & 3.30444000 & -2.33170000 \\
\hline $\mathrm{C}$ & -0.70291600 & 1.64006000 & -5.29018500 \\
\hline $\mathrm{H}$ & 0.03613600 & -0.37398400 & -5.52585300 \\
\hline $\mathrm{H}$ & -1.39494400 & 3.60277400 & -4.72361100 \\
\hline
\end{tabular}




\section{Int16a}

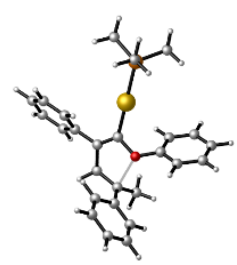

$E=-1560.182048$ Hartrees
$G=-1559.768789$ Hartrees

\begin{tabular}{llll}
$\mathrm{Au}$ & 1.86983200 & 2.45365500 & -2.04002000 \\
$\mathrm{P}$ & 3.22248100 & 1.24034900 & -3.55488300 \\
$\mathrm{C}$ & 3.60334100 & -0.46601400 & -2.99462700 \\
$\mathrm{H}$ & 4.12416300 & -0.42682000 & -2.03387700 \\
$\mathrm{H}$ & 4.23327600 & -0.98129600 & -3.72662800 \\
$\mathrm{H}$ & 2.67156900 & -1.02304100 & -2.86251100 \\
$\mathrm{C}$ & 2.48718100 & 1.02719600 & -5.22284600 \\
$\mathrm{H}$ & 3.14845400 & 0.43663000 & -5.86496400 \\
$\mathrm{H}$ & 2.32327500 & 2.00851200 & -5.67703600 \\
$\mathrm{H}$ & 1.52248300 & 0.52024500 & -5.13307900 \\
$\mathrm{C}$ & 4.86198200 & 2.00398000 & -3.86311900 \\
$\mathrm{H}$ & 4.72593200 & 3.00293400 & -4.28696300 \\
$\mathrm{H}$ & 5.44913500 & 1.39317600 & -4.55613700 \\
$\mathrm{H}$ & 5.40344500 & 2.09986300 & -2.91789800 \\
$\mathrm{C}$ & 0.69679900 & 3.51498300 & -0.70883700 \\
$\mathrm{C}$ & 1.03116500 & 4.51738600 & 0.12734800 \\
$\mathrm{C}$ & -1.38267400 & 4.48806400 & 0.95439100 \\
$\mathrm{C}$ & -0.06762900 & 5.21144500 & 0.95069100 \\
$\mathrm{H}$ & -0.22617500 & 6.22336300 & 0.56205900 \\
$\mathrm{H}$ & 0.28593100 & 5.31517300 & 1.98080500 \\
$\mathrm{C}$ & -2.48194300 & 5.13007500 & 0.18022600 \\
$\mathrm{H}$ & -2.09249100 & 5.62824200 & -0.70980300 \\
$\mathrm{H}$ & -2.91480600 & 5.91188600 & 0.82268000 \\
$\mathrm{H}$ & -3.28341000 & 4.44666100 & -0.10055100 \\
$\mathrm{C}$ & -1.67296600 & 3.49774900 & 1.97903400 \\
$\mathrm{C}$ & -3.00354400 & 3.10424100 & 2.25375600 \\
$\mathrm{C}$ & -0.62676000 & 2.90675700 & 2.72881600 \\
$\mathrm{C}$ & -3.27501100 & 2.16945000 & 3.24709100 \\
$\mathrm{H}$ & -3.82855000 & 3.54413600 & 1.70634300 \\
-0.90305500 & 1.96166600 & 3.70687200 \\
\hline
\end{tabular}




$\begin{array}{llll}\mathrm{H} & 0.40437100 & 3.17605700 & 2.52910000 \\ \mathrm{C} & -2.22792900 & 1.59290800 & 3.97142000 \\ \mathrm{H} & -4.30229600 & 1.88826400 & 3.45608200 \\ \mathrm{H} & -0.09007400 & 1.51131000 & 4.26746900 \\ \mathrm{H} & -2.44183800 & 0.85846500 & 4.74232200 \\ \mathrm{O} & -0.66325800 & 3.13092300 & -0.56617200 \\ \mathrm{C} & -1.43110200 & 2.82691900 & -1.68484400 \\ \mathrm{C} & -2.30064000 & 1.74017100 & -1.57171900 \\ \mathrm{C} & -1.37657800 & 3.59892500 & -2.84884100 \\ \mathrm{C} & -3.12383500 & 1.41593500 & -2.65161100 \\ \mathrm{H} & -2.31570800 & 1.16059700 & -0.65454400 \\ \mathrm{C} & -2.20009200 & 3.25658700 & -3.92299000 \\ \mathrm{H} & -0.70020500 & 4.44460400 & -2.90903300 \\ \mathrm{C} & -3.07373000 & 2.16915100 & -3.82819100 \\ \mathrm{H} & -3.79982900 & 0.56965800 & -2.57369600 \\ \mathrm{H} & -2.16191100 & 3.84718900 & -4.83357400 \\ \mathrm{H} & -3.71239100 & 1.91052100 & -4.66732900 \\ \mathrm{C} & 2.41826100 & 5.00585100 & 0.32359800 \\ \mathrm{C} & 2.69370200 & 6.38496700 & 0.38912200 \\ \mathrm{C} & 3.49247100 & 4.10849500 & 0.48004800 \\ \mathrm{C} & 3.99719600 & 6.84819100 & 0.57119200 \\ \mathrm{H} & 1.88639600 & 7.10394700 & 0.28168000 \\ \mathrm{C} & 4.79655000 & 4.57346100 & 0.65918400 \\ \mathrm{H} & 3.29398800 & 3.04141900 & 0.48239800 \\ \mathrm{C} & 5.05558700 & 5.94520900 & 0.70290000 \\ \mathrm{H} & 4.18644800 & 7.91747000 & 0.60828900 \\ \mathrm{H} & 5.60781800 & 3.86086200 & 0.78156400 \\ & 6.06938800 & 6.30715000 & 0.84847500\end{array}$

\section{TS16a-17a}

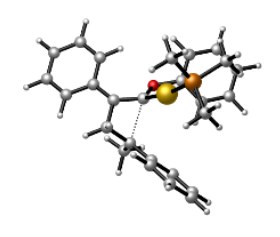

$\mathrm{E}=-1560.162576$ Hartrees

$G=-1559.749074$ Hartrees
$\mathrm{Au}$
$\begin{array}{llll}1.43818700 & 2.17707900 & -1.04288200\end{array}$
$\mathrm{P} \quad 2.56500800 \quad 0.18112400 \quad-1.56666300$
$\begin{array}{llll}\text { C } & 2.19052200 & -1.24206200 & -0.47266900\end{array}$ 


\begin{tabular}{|c|c|c|c|}
\hline $\mathrm{H}$ & 2.47167700 & -0.99743000 & 0.55495300 \\
\hline $\mathrm{H}$ & 2.74536500 & -2.12790700 & -0.79763600 \\
\hline H & 1.11867400 & -1.45517400 & -0.50142000 \\
\hline $\mathrm{C}$ & 2.19447600 & -0.40930200 & -3.26259200 \\
\hline $\mathrm{H}$ & 2.75868200 & -1.32040100 & -3.48520500 \\
\hline $\mathrm{H}$ & 2.46264200 & 0.36833900 & -3.98304500 \\
\hline $\mathrm{H}$ & 1.12421100 & -0.61371700 & -3.35371600 \\
\hline $\mathrm{C}$ & 4.39026300 & 0.35318100 & -1.51314100 \\
\hline $\mathrm{H}$ & 4.70171000 & 1.14410500 & -2.20096400 \\
\hline $\mathrm{H}$ & 4.87229100 & -0.58660800 & -1.80059200 \\
\hline $\mathrm{H}$ & 4.70301100 & 0.62686000 & -0.50162800 \\
\hline $\mathrm{C}$ & 0.59962300 & 4.10629800 & -0.77174500 \\
\hline $\mathrm{C}$ & 1.23892100 & 5.00604600 & 0.10696400 \\
\hline $\mathrm{C}$ & -0.25942100 & 3.93162700 & 1.25705400 \\
\hline $\mathrm{C}$ & 1.15531500 & 4.39342100 & 1.48384400 \\
\hline $\mathrm{H}$ & 1.23699400 & 5.11897300 & 2.29550400 \\
\hline $\mathrm{H}$ & 1.88901600 & 3.60657700 & 1.65663000 \\
\hline $\mathrm{C}$ & -1.32276500 & 5.00441100 & 1.31215500 \\
\hline $\mathrm{H}$ & -0.89626400 & 6.00334800 & 1.21163100 \\
\hline $\mathrm{H}$ & -1.84135100 & 4.94410300 & 2.27760700 \\
\hline $\mathrm{H}$ & -2.08150600 & 4.88738500 & 0.53295900 \\
\hline $\mathrm{C}$ & -0.69498400 & 2.54457700 & 1.51315100 \\
\hline $\mathrm{C}$ & -2.03030000 & 2.15568800 & 1.26914900 \\
\hline $\mathrm{C}$ & 0.17459400 & 1.59430700 & 2.08949800 \\
\hline $\mathrm{C}$ & -2.47002700 & 0.86936300 & 1.57421700 \\
\hline $\mathrm{H}$ & -2.73189900 & 2.86057800 & 0.83768900 \\
\hline $\mathrm{C}$ & -0.27362300 & 0.31592300 & 2.41228100 \\
\hline $\mathrm{H}$ & 1.20470700 & 1.85435500 & 2.30368800 \\
\hline $\mathrm{C}$ & -1.59502700 & -0.05705400 & 2.14692300 \\
\hline $\mathrm{H}$ & -3.50063000 & 0.59318400 & 1.37142100 \\
\hline $\mathrm{H}$ & 0.40952500 & -0.39077600 & 2.87410100 \\
\hline $\mathrm{H}$ & -1.93960000 & -1.05778900 & 2.39049000 \\
\hline $\mathrm{O}$ & -0.02481800 & 4.68939300 & -1.83939300 \\
\hline $\mathrm{C}$ & -0.62610300 & 3.92138600 & -2.83168800 \\
\hline $\mathrm{C}$ & -1.51695900 & 2.88944800 & -2.52744300 \\
\hline $\mathrm{C}$ & -0.37159800 & 4.29003500 & -4.15278000 \\
\hline $\mathrm{C}$ & -2.14310200 & 2.20624900 & -3.57336300 \\
\hline $\mathrm{H}$ & -1.72480500 & 2.63538800 & -1.49427000 \\
\hline $\mathrm{C}$ & -1.00907300 & 3.60223800 & -5.18749200 \\
\hline $\mathrm{H}$ & 0.31129400 & 5.10795400 & -4.35929800 \\
\hline
\end{tabular}




$\begin{array}{llll}\mathrm{C} & -1.89237800 & 2.55740300 & -4.90234000 \\ \mathrm{H} & -2.83905900 & 1.40469200 & -3.34288800 \\ \mathrm{H} & -0.81325900 & 3.88641500 & -6.21731700 \\ \mathrm{H} & -2.38768000 & 2.02631700 & -5.70953200 \\ \mathrm{C} & 1.58848300 & 6.40319600 & -0.14483500 \\ \mathrm{C} & 1.75815400 & 7.32563100 & 0.91287300 \\ \mathrm{C} & 1.86517100 & 6.84884900 & -1.46080600 \\ \mathrm{C} & 2.16831700 & 8.63328700 & 0.66630600 \\ \mathrm{H} & 1.55349700 & 7.02835800 & 1.93490400 \\ \mathrm{C} & 2.29037500 & 8.15065200 & -1.69835500 \\ \mathrm{H} & 1.77257800 & 6.15902900 & -2.28919900 \\ \mathrm{C} & 2.43506000 & 9.05257200 & -0.63894200 \\ \mathrm{H} & 2.27866800 & 9.32607600 & 1.49518800 \\ \mathrm{H} & 2.50919100 & 8.46502200 & -2.71457800 \\ \mathrm{H} & 2.75521900 & 10.07261700 & -0.83012900\end{array}$

\section{Int17a}

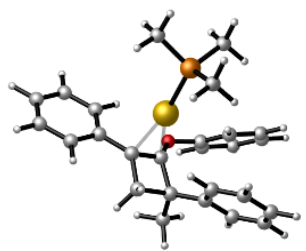

$E=-1560.203229$ Hartrees
$G=-1559.787576$ Hartrees

$\begin{array}{lrrr}\mathrm{Au} & 2.05116300 & 2.82536400 & -0.60291200 \\ \mathrm{P} & 3.44226500 & 1.05088700 & -1.19023500 \\ \mathrm{C} & 3.45160300 & -0.30135700 & 0.04308600 \\ \mathrm{H} & 3.78199200 & 0.08640300 & 1.01042000 \\ \mathrm{H} & 4.12894300 & -1.09807600 & -0.28011600 \\ \mathrm{H} & 2.44155500 & -0.70556500 & 0.15073200 \\ \mathrm{C} & 2.97125600 & 0.27438700 & -2.77943600 \\ \mathrm{H} & 3.65646700 & -0.54678300 & -3.01195700 \\ \mathrm{H} & 3.01274400 & 1.02205700 & -3.57611200 \\ \mathrm{H} & 1.95065100 & -0.11112600 & -2.71066100 \\ \mathrm{C} & 5.18875600 & 1.56310900 & -1.38251600 \\ \mathrm{H} & 5.25996700 & 2.33046800 & -2.15801300 \\ \mathrm{H} & 5.80521200 & 0.70294200 & -1.66228200 \\ \mathrm{H} & 5.55314900 & 1.97987000 & -0.43972300 \\ \mathrm{C} & -0.05617400 & 4.31838700 & -0.63183400 \\ \mathrm{C} & 1.04717100 & 4.81642300 & 0.05823300\end{array}$




\begin{tabular}{|c|c|c|c|}
\hline $\mathrm{C}$ & -0.88966900 & 3.99205000 & 0.63151900 \\
\hline $\mathrm{C}$ & 0.37127600 & 4.53818100 & 1.41108800 \\
\hline $\mathrm{H}$ & 0.13401800 & 5.46023800 & 1.95026400 \\
\hline $\mathrm{H}$ & 0.87486400 & 3.85225800 & 2.09101200 \\
\hline $\mathrm{C}$ & -2.04894700 & 5.00859600 & 0.70180400 \\
\hline $\mathrm{H}$ & -1.67096000 & 6.02836000 & 0.58303500 \\
\hline $\mathrm{H}$ & -2.54993000 & 4.93860700 & 1.67170300 \\
\hline $\mathrm{H}$ & -2.78961600 & 4.83884500 & -0.08318300 \\
\hline $\mathrm{C}$ & -1.33251800 & 2.55737200 & 0.91959000 \\
\hline $\mathrm{C}$ & -2.65527200 & 2.14243700 & 0.69321100 \\
\hline $\mathrm{C}$ & -0.42919300 & 1.60829900 & 1.42616400 \\
\hline $\mathrm{C}$ & -3.05638800 & 0.83022300 & 0.95361400 \\
\hline $\mathrm{H}$ & -3.38935900 & 2.83986000 & 0.30593700 \\
\hline $\mathrm{C}$ & -0.82740200 & 0.29498300 & 1.68812300 \\
\hline $\mathrm{H}$ & 0.60043900 & 1.88634200 & 1.62591200 \\
\hline $\mathrm{C}$ & -2.14464400 & -0.10170500 & 1.45153600 \\
\hline $\mathrm{H}$ & -4.08691600 & 0.54056900 & 0.76758200 \\
\hline $\mathrm{H}$ & -0.10768000 & -0.41331500 & 2.08956200 \\
\hline $\mathrm{H}$ & -2.45752300 & -1.12099400 & 1.65934900 \\
\hline $\mathrm{O}$ & -0.40854800 & 4.55373900 & -1.90100800 \\
\hline $\mathrm{C}$ & -1.35959900 & 3.76699700 & -2.56950400 \\
\hline $\mathrm{C}$ & -1.30644800 & 2.37521700 & -2.54247000 \\
\hline $\mathrm{C}$ & -2.29515500 & 4.45418500 & -3.34021700 \\
\hline $\mathrm{C}$ & -2.23691500 & 1.65674700 & -3.29794000 \\
\hline $\mathrm{H}$ & -0.55492600 & 1.86563000 & -1.94903800 \\
\hline $\mathrm{C}$ & -3.21395000 & 3.72144500 & -4.09305400 \\
\hline $\mathrm{H}$ & -2.29444200 & 5.53938200 & -3.34504800 \\
\hline $\mathrm{C}$ & -3.18926500 & 2.32398000 & -4.07157000 \\
\hline $\mathrm{H}$ & -2.20956300 & 0.57124100 & -3.28228400 \\
\hline $\mathrm{H}$ & -3.94938700 & 4.24617900 & -4.69541100 \\
\hline $\mathrm{H}$ & -3.90670500 & 1.75822200 & -4.65803200 \\
\hline $\mathrm{C}$ & 2.08506700 & 5.81248500 & -0.26628600 \\
\hline $\mathrm{C}$ & 2.91009300 & 6.30028800 & 0.76382400 \\
\hline $\mathrm{C}$ & 2.26945500 & 6.30187200 & -1.57513800 \\
\hline $\mathrm{C}$ & 3.88881500 & 7.25675600 & 0.49560800 \\
\hline $\mathrm{H}$ & 2.77732700 & 5.93202000 & 1.77707200 \\
\hline $\mathrm{C}$ & 3.24997200 & 7.25712600 & -1.83618200 \\
\hline $\mathrm{H}$ & 1.63939800 & 5.93841300 & -2.38013300 \\
\hline $\mathrm{C}$ & 4.06392500 & 7.73699300 & -0.80472900 \\
\hline $\mathrm{H}$ & 4.51417900 & 7.62711100 & 1.30290000 \\
\hline
\end{tabular}


$\begin{array}{llll}\mathrm{H} & 3.37746500 & 7.63109100 & -2.84806200 \\ \mathrm{H} & 4.82610000 & 8.48212500 & -1.01316300\end{array}$

\section{TS16a-18a}

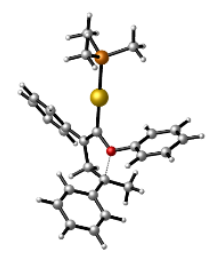

$E=-1560.181746$ Hartrees

$\mathrm{G}=-1559.766609$ Hartrees

$\begin{array}{crrr}\mathrm{Au} & 1.89229800 & 2.47710100 & -2.01685000 \\ \mathrm{P} & 3.14968300 & 1.17670200 & -3.53939400 \\ \mathrm{C} & 3.46206800 & -0.53386500 & -2.95195000 \\ \mathrm{H} & 4.00809900 & -0.50129200 & -2.00509300 \\ \mathrm{H} & 4.04744500 & -1.09392700 & -3.68800700 \\ \mathrm{H} & 2.50796800 & -1.04198500 & -2.78599400 \\ \mathrm{C} & 2.34525600 & 0.96806400 & -5.17553500 \\ \mathrm{H} & 2.95898300 & 0.34081300 & -5.82993700 \\ \mathrm{H} & 2.20557700 & 1.94806800 & -5.64043400 \\ \mathrm{H} & 1.36433400 & 0.50318800 & -5.04291700 \\ \mathrm{C} & 4.81337400 & 1.85125000 & -3.91960400 \\ \mathrm{H} & 4.71342800 & 2.85620400 & -4.33896500 \\ \mathrm{H} & 5.33274500 & 1.20841900 & -4.63727200 \\ \mathrm{H} & 5.40067700 & 1.91577900 & -2.99942900 \\ \mathrm{C} & 0.79438700 & 3.59957000 & -0.67936300 \\ \mathrm{C} & 1.11622400 & 4.59421800 & 0.16648200 \\ \mathrm{C} & -1.30339500 & 4.40206300 & 0.82339300 \\ \mathrm{C} & -0.02415400 & 5.19840400 & 0.98333900 \\ \mathrm{C} & -0.20710100 & 6.23142600 & 0.66747900 \\ \mathrm{H} & 0.26011400 & 5.23203500 & 2.03890200 \\ \mathrm{C} & -2.40197200 & 5.11849100 & 0.10010000 \\ \mathrm{C} & -2.00469100 & 5.68972300 & -0.74095400 \\ \mathrm{C} & -2.83584200 & 5.83729400 & 0.80788900 \\ \mathrm{C} & -3.20033400 & 4.46280200 & -0.24795000 \\ \mathrm{H} & -1.65626400 & 3.40932300 & 1.86328100 \\ \mathrm{H} & -2.99948100 & 3.07364600 & 2.11916700 \\ \mathrm{H} & -3.64694000 & 2.77337400 & 2.61668700 \\ \mathrm{H} & 2.14256900 & 3.10511000 \\ \mathrm{H} & -39600 & 3.54958900 & 1.56485600\end{array}$




$\begin{array}{llll}\mathrm{C} & -0.97311900 & 1.83405400 & 3.58865700 \\ \mathrm{H} & 0.39722100 & 3.00204200 & 2.43201300 \\ \mathrm{C} & -2.31307600 & 1.51782800 & 3.83841800 \\ \mathrm{H} & -4.36499200 & 1.90553400 & 3.29804600 \\ \mathrm{H} & -0.18377100 & 1.34956400 & 4.15509300 \\ \mathrm{H} & -2.56631800 & 0.78937100 & 4.60309700 \\ \mathrm{O} & -0.59857900 & 3.25104000 & -0.51047000 \\ \mathrm{C} & -1.37604800 & 2.88813800 & -1.62428900 \\ \mathrm{C} & -2.18293700 & 1.76093300 & -1.48533200 \\ \mathrm{C} & -1.35970700 & 3.64943900 & -2.79339300 \\ \mathrm{C} & -2.99630700 & 1.38409600 & -2.55683100 \\ \mathrm{H} & -2.16313800 & 1.19305500 & -0.56163700 \\ \mathrm{C} & -2.17153900 & 3.25369900 & -3.85758400 \\ \mathrm{H} & -0.72238500 & 4.52371100 & -2.86730100 \\ \mathrm{C} & -2.99011700 & 2.12588100 & -3.74105300 \\ \mathrm{H} & -3.62865800 & 0.50639100 & -2.46496500 \\ \mathrm{H} & -2.16695900 & 3.83256700 & -4.77616400 \\ \mathrm{H} & -3.62109600 & 1.82647100 & -4.57235600 \\ \mathrm{C} & 2.48280200 & 5.12694400 & 0.36695500 \\ \mathrm{C} & 2.68697900 & 6.50453700 & 0.57242400 \\ \mathrm{C} & 3.60912500 & 4.28155600 & 0.38007000 \\ \mathrm{C} & 3.97075500 & 7.01973000 & 0.75482600 \\ \mathrm{H} & 1.83883600 & 7.18290800 & 0.57591400 \\ \mathrm{C} & 4.89245900 & 4.79896900 & 0.55946800 \\ \mathrm{H} & 3.46852600 & 3.21090500 & 0.27013900 \\ \mathrm{C} & 5.08032700 & 6.17085600 & 0.74496100 \\ \mathrm{H} & 4.10342600 & 8.08768100 & 0.90420400 \\ \mathrm{H} & 5.74503100 & 4.12532200 & 0.57119000 \\ \mathrm{H} & 6.07882300 & 6.57258000 & 0.89149400\end{array}$

\section{Int18a}

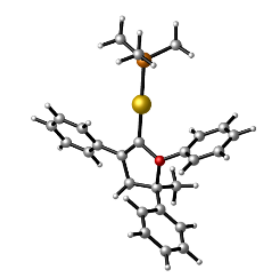

$E=-1560.182487$ Hartrees

$\mathrm{G}=-1559.767991$ Hartrees
$\mathrm{Au}$
$\begin{array}{lll}1.96064600 & 2.56321900 & -2.07559000\end{array}$
$\mathrm{P}$
$\begin{array}{lll}3.12361600 & 1.44536500 & -3.79750500\end{array}$ 


\begin{tabular}{|c|c|c|c|}
\hline $\mathrm{C}$ & 3.49392600 & -0.31344900 & -3.43064800 \\
\hline $\mathrm{H}$ & 4.09949200 & -0.37760400 & -2.52225100 \\
\hline $\mathrm{H}$ & 4.03564100 & -0.77606500 & -4.26168700 \\
\hline & 2.55850600 & -0.85454500 & -3.26289300 \\
\hline $\mathrm{C}$ & 2.21825500 & 1.41118600 & -5.39310300 \\
\hline & 2.80549700 & 0.89648600 & -6.15997700 \\
\hline & 2.01643300 & 2.43543800 & -5.71861600 \\
\hline $\mathrm{H}$ & 1.26405400 & 0.89428000 & -5.25900500 \\
\hline $\mathrm{C}$ & 4.74833800 & 2.19763500 & -4.19654700 \\
\hline $\mathrm{H}$ & 4.60304900 & 3.23786400 & -4.50100400 \\
\hline $\mathrm{H}$ & 5.23916400 & 1.64833300 & -5.00612000 \\
\hline $\mathrm{H}$ & 5.38710900 & 2.18189000 & -3.30914700 \\
\hline $\mathrm{C}$ & 0.97358300 & 3.58451500 & -0.58679200 \\
\hline $\mathrm{C}$ & 1.30677600 & 4.53935600 & 0.29496500 \\
\hline $\mathrm{C}$ & -1.12132700 & 4.33892200 & 0.72063000 \\
\hline $\mathrm{C}$ & 0.17779900 & 4.99467100 & 1.20262900 \\
\hline $\mathrm{H}$ & 0.05416400 & 6.08156000 & 1.17525400 \\
\hline $\mathrm{H}$ & 0.39925200 & 4.72698700 & 2.23963900 \\
\hline $\mathrm{C}$ & -1.95165200 & 5.27140000 & -0.13178400 \\
\hline $\mathrm{H}$ & -1.32014300 & 5.76590600 & -0.87349200 \\
\hline $\mathrm{H}$ & -2.36499500 & 6.04479200 & 0.52352300 \\
\hline $\mathrm{H}$ & -2.77846800 & 4.77040100 & -0.63630700 \\
\hline $\mathrm{C}$ & -1.85467800 & 3.49768100 & 1.73273800 \\
\hline $\mathrm{C}$ & -3.24544800 & 3.58486400 & 1.88716900 \\
\hline $\mathrm{C}$ & -1.13723000 & 2.60214500 & 2.54734700 \\
\hline $\mathrm{C}$ & -3.90317000 & 2.80578600 & 2.84251100 \\
\hline $\mathrm{H}$ & -3.82594800 & 4.26629600 & 1.27621700 \\
\hline $\mathrm{C}$ & -1.79637000 & 1.81539700 & 3.48805900 \\
\hline $\mathrm{H}$ & -0.06063500 & 2.51043800 & 2.43805500 \\
\hline $\mathrm{C}$ & -3.18311800 & 1.91851400 & 3.64166200 \\
\hline $\mathrm{H}$ & -4.97953200 & 2.89382400 & 2.95606500 \\
\hline $\mathrm{H}$ & -1.22885400 & 1.12535800 & 4.10539200 \\
\hline $\mathrm{H}$ & -3.69554500 & 1.31049200 & 4.38151900 \\
\hline $\mathrm{O}$ & -0.44921100 & 3.24216200 & -0.31941900 \\
\hline $\mathrm{C}$ & -1.24168300 & 2.58043300 & -1.29905300 \\
\hline $\mathrm{C}$ & -1.80114000 & 1.36019500 & -0.93785300 \\
\hline $\mathrm{C}$ & -1.43373000 & 3.15611200 & -2.55268700 \\
\hline $\mathrm{C}$ & -2.59298200 & 0.69167700 & -1.87574900 \\
\hline $\mathrm{H}$ & -1.61789300 & 0.94561900 & 0.04662200 \\
\hline $\mathrm{C}$ & -2.22228300 & 2.47084400 & -3.47875800 \\
\hline
\end{tabular}




$\begin{array}{llll}\mathrm{H} & -0.97476700 & 4.10666900 & -2.79896200 \\ \mathrm{C} & -2.80254000 & 1.24400200 & -3.14132500 \\ \mathrm{H} & -3.04010400 & -0.26201700 & -1.61347100 \\ \mathrm{H} & -2.38430500 & 2.90086300 & -4.46228500 \\ \mathrm{H} & -3.41705800 & 0.71933900 & -3.86654300 \\ \mathrm{C} & 2.66802600 & 5.09419800 & 0.46270600 \\ \mathrm{C} & 2.85561500 & 6.45634800 & 0.76165100 \\ \mathrm{C} & 3.80807100 & 4.27466000 & 0.35251800 \\ \mathrm{C} & 4.13801600 & 6.98512400 & 0.91359700 \\ \mathrm{H} & 1.99738700 & 7.11376700 & 0.86247700 \\ \mathrm{C} & 5.08912600 & 4.80589800 & 0.50266200 \\ \mathrm{H} & 3.68049400 & 3.21293100 & 0.16727300 \\ \mathrm{C} & 5.26050600 & 6.16425400 & 0.78154400 \\ \mathrm{H} & 4.25878000 & 8.04146800 & 1.13701200 \\ \mathrm{H} & 5.95390100 & 4.15347000 & 0.41842800 \\ \mathrm{H} & 6.25777400 & 6.57649300 & 0.90533900\end{array}$

TS18a-19a

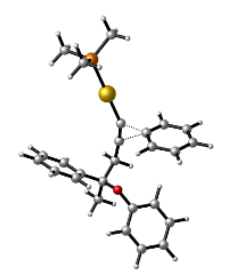

$E=-1560.148883$ Hartrees

$G=-1559.738315$ Hartrees

$\begin{array}{crrr}\mathrm{Au} & 3.21613100 & 1.73191400 & 0.23482600 \\ \mathrm{P} & 4.78779700 & -0.00852400 & 0.32647100 \\ \mathrm{C} & 4.49245600 & -1.19034500 & 1.69530500 \\ \mathrm{H} & 4.52118200 & -0.65698900 & 2.64927000 \\ \mathrm{H} & 5.25671700 & -1.97386000 & 1.69294400 \\ \mathrm{H} & 3.50522400 & -1.64546600 & 1.57762500 \\ \mathrm{C} & 4.82292500 & -1.02559900 & -1.19710500 \\ \mathrm{H} & 5.57486000 & -1.81687200 & -1.11418700 \\ \mathrm{H} & 5.05984700 & -0.38849300 & -2.05354300 \\ \mathrm{H} & 3.83888600 & -1.47474500 & -1.35682900 \\ \mathrm{C} & 6.50830700 & 0.58032400 & 0.55557800 \\ \mathrm{H} & 6.77695600 & 1.24685700 & -0.26842300 \\ \mathrm{H} & 7.20284000 & -0.26538100 & 0.57974600 \\ \mathrm{H} & 6.58200100 & 1.13730600 & 1.49361200 \\ \mathrm{C} & 0.72323600 & 3.76261500 & 0.42643700\end{array}$




\begin{tabular}{|c|c|c|c|}
\hline $\mathrm{C}$ & 1.84172800 & 3.25495400 & 0.14127700 \\
\hline $\mathrm{C}$ & -1.78248600 & 3.68499300 & -0.01805100 \\
\hline $\mathrm{C}$ & -0.59269700 & 4.23762100 & 0.83199000 \\
\hline $\mathrm{H}$ & -0.60954400 & 5.33228800 & 0.82760100 \\
\hline $\mathrm{H}$ & -0.71234200 & 3.94011000 & 1.88181000 \\
\hline $\mathrm{C}$ & -3.09575100 & 4.18550700 & 0.59630500 \\
\hline $\mathrm{H}$ & -3.12141600 & 5.27744600 & 0.64497100 \\
\hline $\mathrm{H}$ & -3.20059400 & 3.79583900 & 1.61176800 \\
\hline $\mathrm{H}$ & -3.94972600 & 3.83827800 & 0.00972500 \\
\hline C & -1.75048700 & 2.15005300 & -0.05593800 \\
\hline $\mathrm{C}$ & -1.70960700 & 1.44674700 & -1.26665400 \\
\hline $\mathrm{C}$ & -1.77502700 & 1.41886000 & 1.14346100 \\
\hline $\mathrm{C}$ & -1.69184500 & 0.04880800 & -1.27820300 \\
\hline $\mathrm{H}$ & -1.69234800 & 1.99499600 & -2.20062200 \\
\hline C & -1.75631200 & 0.02379700 & 1.13142500 \\
\hline $\mathrm{H}$ & -1.81535900 & 1.93570100 & 2.09834800 \\
\hline $\mathrm{C}$ & -1.71397800 & -0.66871600 & -0.08170500 \\
\hline $\mathrm{H}$ & -1.66283100 & -0.47715400 & -2.22862600 \\
\hline $\mathrm{H}$ & -1.78000900 & -0.52098100 & 2.07114200 \\
\hline $\mathrm{H}$ & -1.70267900 & -1.75500100 & -0.09211700 \\
\hline $\mathrm{O}$ & -1.59389700 & 4.13713800 & -1.38045100 \\
\hline $\mathrm{C}$ & -2.22274600 & 5.27636000 & -1.85616100 \\
\hline $\mathrm{C}$ & -3.41139500 & 5.13995200 & -2.58113100 \\
\hline $\mathrm{C}$ & -1.62924500 & 6.53442700 & -1.70771400 \\
\hline $\mathrm{C}$ & -4.01217000 & 6.26670000 & -3.14557500 \\
\hline $\mathrm{H}$ & -3.84942000 & 4.15395800 & -2.70040200 \\
\hline $\mathrm{C}$ & -2.23931400 & 7.65711800 & -2.27336500 \\
\hline $\mathrm{H}$ & -0.69199300 & 6.62774900 & -1.17001800 \\
\hline $\mathrm{C}$ & -3.43109100 & 7.52835800 & -2.99051800 \\
\hline $\mathrm{H}$ & -4.93481200 & 6.15627000 & -3.70840200 \\
\hline $\mathrm{H}$ & -1.77540100 & 8.63269000 & -2.15730400 \\
\hline $\mathrm{H}$ & -3.89982400 & 8.40307900 & -3.43168600 \\
\hline $\mathrm{C}$ & 2.01768600 & 4.60556800 & -0.68737500 \\
\hline $\mathrm{C}$ & 1.69793000 & 4.62965200 & -2.05745600 \\
\hline $\mathrm{C}$ & 2.66783700 & 5.70131900 & -0.08846200 \\
\hline $\mathrm{C}$ & 2.07445200 & 5.72583200 & -2.82968900 \\
\hline $\mathrm{H}$ & 1.16607200 & 3.79442400 & -2.49872500 \\
\hline $\mathrm{C}$ & 3.03493300 & 6.79325100 & -0.87059600 \\
\hline $\mathrm{H}$ & 2.89062700 & 5.67876300 & 0.97350300 \\
\hline $\mathrm{C}$ & 2.74327100 & 6.80400000 & -2.23945000 \\
\hline
\end{tabular}




\begin{tabular}{|c|c|c|c|}
\hline & 1.83775600 & 5.74477100 & -3.88893500 \\
\hline & 3.54472300 & 7.63608300 & -0.41421500 \\
\hline & 3.02951600 & 7.65853000 & -2.84540800 \\
\hline
\end{tabular}

\section{Int19a}

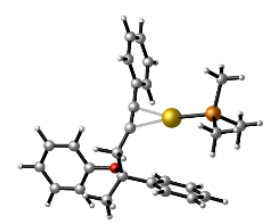

$E=-1560.190640$ Hartrees
$G=-1559.781765$ Hartrees

$G=-1559.781765$ Hartrees

$\begin{array}{llll}\mathrm{Au} & 2.27334300 & 2.39884100 & 0.33571000 \\ \mathrm{P} & 3.63241700 & 0.59144700 & 0.87657900 \\ \mathrm{C} & 3.15919400 & -0.20038900 & 2.45679700 \\ \mathrm{H} & 3.21419900 & 0.53641700 & 3.26272700 \\ \mathrm{H} & 3.83524000 & -1.03280800 & 2.67550000 \\ \mathrm{H} & 2.13379700 & -0.57211200 & 2.38797500 \\ \mathrm{C} & 3.61249800 & -0.73508100 & -0.38266300 \\ \mathrm{H} & 4.26948100 & -1.55506200 & -0.07611400 \\ \mathrm{H} & 3.95382200 & -0.33207500 & -1.33984100 \\ \mathrm{H} & 2.59250200 & -1.10869000 & -0.50325100 \\ \mathrm{C} & 5.38718600 & 1.07488700 & 1.06629700 \\ \mathrm{H} & 5.74981600 & 1.50541000 & 0.12914400 \\ \mathrm{H} & 5.99290400 & 0.19999900 & 1.32318500 \\ \mathrm{H} & 5.47586700 & 1.82466700 & 1.85695300 \\ \mathrm{C} & 0.68207800 & 4.03488200 & 0.10049200 \\ \mathrm{C} & 1.57245100 & 4.52110600 & -0.60682300 \\ \mathrm{C} & -1.71425100 & 3.20889800 & -0.11944400 \\ \mathrm{C} & -0.62440500 & 3.88065600 & 0.77285600 \\ \mathrm{H} & -0.97255600 & 4.88081800 & 1.05591200 \\ \mathrm{H} & -0.51687100 & 3.30733600 & 1.69638900 \\ \mathrm{C} & -3.03861300 & 3.18694200 & 0.65816200 \\ \mathrm{H} & -3.34826100 & 4.19709600 & 0.93890400 \\ \mathrm{H} & -2.93105300 & 2.60157100 & 1.57443700 \\ \mathrm{H} & -3.82671500 & 2.73192900 & 0.05299200 \\ \mathrm{C} & -1.30361800 & 1.78580200 & -0.51738200 \\ \mathrm{C} & -1.15576200 & 1.40950600 & -1.85905100 \\ \mathrm{C} & -1.09361400 & 0.81510300 & 0.47680700 \\ \mathrm{C} & -0.81245600 & 0.09729700 & -2.19859100 \\ & & & \\ \mathrm{H} & & & \\ \mathrm{H} & & & \\ \mathrm{H} & & & \\ \mathrm{H} & & & \end{array}$




$\begin{array}{llrl}\mathrm{H} & -1.32173600 & 2.14340300 & -2.63789800 \\ \mathrm{C} & -0.75936100 & -0.49727200 & 0.13688400 \\ \mathrm{H} & -1.20389700 & 1.07371500 & 1.52634400 \\ \mathrm{C} & -0.61774500 & -0.86307800 & -1.20488300 \\ \mathrm{H} & -0.70936600 & -0.17346000 & -3.24587900 \\ \mathrm{H} & -0.62222900 & -1.23621600 & 0.92174000 \\ \mathrm{H} & -0.36691100 & -1.88610000 & -1.47082900 \\ \mathrm{O} & -1.83427300 & 3.94653400 & -1.35910600 \\ \mathrm{C} & -2.54322000 & 5.13437600 & -1.41704400 \\ \mathrm{C} & -3.87256100 & 5.11176200 & -1.85467400 \\ \mathrm{C} & -1.91124800 & 6.35558100 & -1.15681900 \\ \mathrm{C} & -4.57273400 & 6.30915600 & -2.01093300 \\ \mathrm{H} & -4.34081700 & 4.15814200 & -2.07666000 \\ \mathrm{C} & -2.62052000 & 7.54926500 & -1.31433400 \\ \mathrm{H} & -0.86940200 & 6.37103900 & -0.85552800 \\ \mathrm{C} & -3.95148500 & 7.53100900 & -1.73697000 \\ \mathrm{H} & -5.60416700 & 6.28573300 & -2.35132800 \\ \mathrm{H} & -2.12471800 & 8.49500700 & -1.11366700 \\ \mathrm{H} & -4.49772400 & 8.46139800 & -1.86169000 \\ \mathrm{C} & 2.54274000 & 5.15532600 & -1.44956200 \\ \mathrm{C} & 2.52942600 & 4.91403900 & -2.83822700 \\ \mathrm{C} & 3.49157100 & 6.04040100 & -0.89923200 \\ \mathrm{C} & 3.45343100 & 5.55430800 & -3.65948300 \\ \mathrm{H} & 1.79439500 & 4.23349400 & -3.25570200 \\ \mathrm{C} & 4.41054800 & 6.67297300 & -1.73216700 \\ \mathrm{H} & 3.49552600 & 6.22548400 & 0.17023000 \\ \mathrm{C} & 4.39396000 & 6.43093500 & -3.10949100 \\ \mathrm{H} & 3.44036300 & 5.36947700 & -4.72923800 \\ \mathrm{H} & 5.13957700 & 7.35652600 & -1.30790900 \\ & 5.11350800 & 6.92652700 & -3.75450300\end{array}$

Int20a

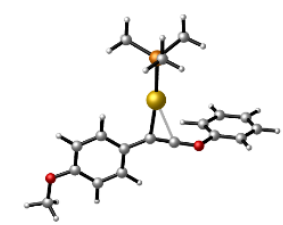

$E=-1674.680629$ Hartrees

$\mathrm{G}=1674.249155$ Hartrees

$\begin{array}{lllll}\text { C } & 4.85950800 & 0.35613500 & -0.77583900\end{array}$

$\begin{array}{lllll}\mathrm{C} & 4.95695000 & 0.63579200 & 0.53558300\end{array}$ 


\begin{tabular}{|c|c|c|c|}
\hline $\mathrm{C}$ & 1.24798200 & 0.68042900 & -1.44351800 \\
\hline $\mathrm{H}$ & 4.83381500 & -0.65969500 & -1.15733500 \\
\hline $\mathrm{H}$ & 4.80435000 & 1.15287200 & -1.51247800 \\
\hline $\mathrm{C}$ & 0.99298200 & -0.50288000 & -1.76386400 \\
\hline $\mathrm{Al}$ & -0.75809700 & -0.59690400 & -0.42558000 \\
\hline $\mathrm{P}$ & -2.64867500 & -0.94930700 & 0.89125700 \\
\hline $\mathrm{C}$ & -3.72641500 & 0.52483900 & 1.00748000 \\
\hline $\mathrm{H}$ & -4.60492500 & 0.30364400 & 1.62194700 \\
\hline $\mathrm{H}$ & -3.16749200 & 1.35028600 & 1.45628800 \\
\hline $\mathrm{H}$ & -4.04793600 & 0.82105200 & 0.00544500 \\
\hline $\mathrm{C}$ & -3.70496300 & -2.29207200 & 0.23440800 \\
\hline $\mathrm{H}$ & -3.12990900 & -3.22116100 & 0.19265600 \\
\hline $\mathrm{H}$ & -4.57979000 & -2.43346700 & 0.87669300 \\
\hline $\mathrm{H}$ & -4.03280300 & -2.03567700 & -0.77654700 \\
\hline C & -2.25965400 & -1.41994700 & 2.61651300 \\
\hline $\mathrm{H}$ & -1.67203600 & -0.62804400 & 3.08822300 \\
\hline $\mathrm{H}$ & -3.18515100 & -1.57192000 & 3.18089000 \\
\hline $\mathrm{H}$ & -1.67450300 & -2.34335100 & 2.62176600 \\
\hline $\mathrm{O}$ & 1.50946600 & 1.84650700 & -1.00185500 \\
\hline $\mathrm{C}$ & 0.89003800 & 3.01061100 & -1.56927600 \\
\hline $\mathrm{C}$ & 1.15568400 & 4.19128300 & -0.88996500 \\
\hline $\mathrm{C}$ & 0.10281300 & 2.94075400 & -2.70963100 \\
\hline $\mathrm{C}$ & 0.59356400 & 5.36903500 & -1.38585700 \\
\hline $\mathrm{H}$ & 1.78168700 & 4.18342600 & -0.00404900 \\
\hline $\mathrm{C}$ & -0.45019700 & 4.13225400 & -3.18768000 \\
\hline $\mathrm{H}$ & -0.06965400 & 1.99651800 & -3.21563100 \\
\hline $\mathrm{C}$ & -0.20848700 & 5.34094300 & -2.53025700 \\
\hline $\mathrm{H}$ & 0.78412800 & 6.30661900 & -0.87303500 \\
\hline $\mathrm{H}$ & -1.06841700 & 4.10856100 & -4.07971000 \\
\hline $\mathrm{H}$ & -0.64205300 & 6.26029300 & -2.91113000 \\
\hline $\mathrm{C}$ & 4.93711900 & 2.07124900 & 1.01029400 \\
\hline $\mathrm{H}$ & 4.95350400 & 2.76067200 & 0.16209000 \\
\hline $\mathrm{H}$ & 4.03657100 & 2.28998500 & 1.59720800 \\
\hline $\mathrm{H}$ & 5.79636000 & 2.29406700 & 1.65375700 \\
\hline 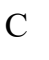 & 5.07992300 & -0.43550200 & 1.56528300 \\
\hline $\mathrm{C}$ & 4.71629000 & -0.19905000 & 2.90427000 \\
\hline $\mathrm{C}$ & 5.57500600 & -1.71394400 & 1.23860800 \\
\hline $\mathrm{C}$ & 4.82121200 & -1.20235200 & 3.87004900 \\
\hline & 4.33983100 & 0.77478300 & 3.19957200 \\
\hline & 5.68020700 & -2.71531700 & 2.20158800 \\
\hline
\end{tabular}




$\begin{array}{llll}\mathrm{H} & 5.90437600 & -1.91859200 & 0.22468100 \\ \mathrm{C} & 5.30056500 & -2.46648600 & 3.52417700 \\ \mathrm{H} & 4.52877000 & -0.99182700 & 4.89524300 \\ \mathrm{H} & 6.07097200 & -3.68971600 & 1.92100200 \\ \mathrm{H} & 5.38799500 & -3.24567700 & 4.27614000 \\ \mathrm{C} & 1.39332700 & -1.61590400 & -2.60931100 \\ \mathrm{C} & 0.70581500 & -2.84506700 & -2.58981600 \\ \mathrm{C} & 2.49589800 & -1.48067900 & -3.47224100 \\ \mathrm{C} & 1.10882000 & -3.89783500 & -3.39650400 \\ \mathrm{H} & -0.14851200 & -2.96828000 & -1.93075300 \\ \mathrm{C} & 2.91093200 & -2.53320800 & -4.28339000 \\ \mathrm{H} & 3.03424900 & -0.53853200 & -3.50766600 \\ \mathrm{C} & 2.21781200 & -3.75419200 & -4.24862000 \\ \mathrm{H} & 0.58145000 & -4.84630200 & -3.38153000 \\ \mathrm{H} & 3.76544100 & -2.39528200 & -4.93501600 \\ \mathrm{O} & 2.53669000 & -4.84083300 & -4.99139000 \\ \mathrm{C} & 3.68719600 & -4.78045700 & -5.83770400 \\ \mathrm{H} & 3.76612000 & -5.76404300 & -6.30315200 \\ \mathrm{H} & 3.57246000 & -4.01973000 & -6.61849100 \\ \mathrm{H} & 4.59686100 & -4.57980900 & -5.26029000\end{array}$

Int20a'

$\begin{array}{lrrr} & \\ \mathrm{E}=-1674.678613 \text { Hartrees } & \\ \mathrm{G}=1674.248496 \text { Hartrees } & \\ \mathrm{Au} & 1.82178300 & 2.24822300 & -2.85891300 \\ \mathrm{P} & 3.21189900 & 1.27372400 & -4.47480800 \\ \mathrm{C} & 3.68670000 & -0.44788900 & -4.06957100 \\ \mathrm{H} & 4.23386000 & -0.46307500 & -3.12315500 \\ \mathrm{H} & 4.31729800 & -0.86396000 & -4.86155100 \\ \mathrm{H} & 2.78555100 & -1.05776200 & -3.96387000 \\ \mathrm{C} & 2.42264700 & 1.19228600 & -6.12488900 \\ \mathrm{H} & 3.10291600 & 0.73195300 & -6.84824700 \\ \mathrm{H} & 2.16748700 & 2.20205300 & -6.45732900 \\ \mathrm{H} & 1.50432100 & 0.60260100 & -6.06141300 \\ \mathrm{C} & 4.78481100 & 2.17922900 & -4.72518800 \\ \mathrm{H} & 4.56816400 & 3.20610400 & -5.03143400\end{array}$




\begin{tabular}{|c|c|c|c|}
\hline $\mathrm{H}$ & 5.38583800 & 1.68727900 & -5.49649900 \\
\hline $\mathrm{H}$ & 5.34596400 & 2.20389300 & -3.78730800 \\
\hline C & 0.38379100 & 3.03789400 & -1.46182700 \\
\hline C & 1.19980400 & 3.32132000 & -0.53250200 \\
\hline $\mathrm{C}$ & -2.02456200 & 4.70033100 & 1.89387400 \\
\hline $\mathrm{C}$ & -1.33679300 & 5.62862500 & 1.20656500 \\
\hline $\mathrm{H}$ & -1.62519600 & 5.90298800 & 0.19565200 \\
\hline $\mathrm{H}$ & -0.46940900 & 6.13571900 & 1.61809200 \\
\hline $\mathrm{C}$ & -3.18823700 & 3.98044800 & 1.25029300 \\
\hline $\mathrm{H}$ & -3.42990000 & 4.42326300 & 0.28078100 \\
\hline $\mathrm{H}$ & -4.08333600 & 4.01874500 & 1.88200600 \\
\hline $\mathrm{H}$ & -2.96264600 & 2.92067500 & 1.08109700 \\
\hline $\mathrm{C}$ & -1.66972400 & 4.33556000 & 3.29518900 \\
\hline $\mathrm{C}$ & -2.00205400 & 3.07416400 & 3.82364100 \\
\hline $\mathrm{C}$ & -1.00313300 & 5.24396200 & 4.14125200 \\
\hline $\mathrm{C}$ & -1.65818200 & 2.72532500 & 5.13131500 \\
\hline $\mathrm{H}$ & -2.52445800 & 2.34899600 & 3.20824900 \\
\hline $\mathrm{C}$ & -0.66122600 & 4.89720300 & 5.44698400 \\
\hline $\mathrm{H}$ & -0.77189100 & 6.24125800 & 3.77967300 \\
\hline $\mathrm{C}$ & -0.98376800 & 3.63300000 & 5.94938100 \\
\hline $\mathrm{H}$ & -1.92157800 & 1.74122000 & 5.50967900 \\
\hline $\mathrm{H}$ & -0.15312900 & 5.62079100 & 6.07888800 \\
\hline $\mathrm{H}$ & -0.72175800 & 3.36432900 & 6.96887800 \\
\hline $\mathrm{O}$ & -0.95042100 & 3.02501800 & -1.59950400 \\
\hline $\mathrm{C}$ & -1.52322300 & 2.96366300 & -2.87469300 \\
\hline $\mathrm{C}$ & -2.60742700 & 2.10247400 & -3.02355100 \\
\hline $\mathrm{C}$ & -1.08649100 & 3.78295900 & -3.91524700 \\
\hline $\mathrm{C}$ & -3.26157100 & 2.05064800 & -4.25629600 \\
\hline $\mathrm{H}$ & -2.92498800 & 1.48958300 & -2.18605200 \\
\hline $\mathrm{C}$ & -1.74693900 & 3.71377300 & -5.14501800 \\
\hline $\mathrm{H}$ & -0.26111300 & 4.47068000 & -3.76114500 \\
\hline $\mathrm{C}$ & -2.83151400 & 2.85010500 & -5.31895300 \\
\hline $\mathrm{H}$ & -4.10597900 & 1.37991400 & -4.38384800 \\
\hline $\mathrm{H}$ & -1.41798800 & 4.34867000 & -5.96244900 \\
\hline $\mathrm{H}$ & -3.34313500 & 2.80491500 & -6.27549000 \\
\hline $\mathrm{C}$ & 2.20554000 & 3.54973300 & 0.40705200 \\
\hline $\mathrm{C}$ & 2.80495000 & 4.83139900 & 0.53174600 \\
\hline $\mathrm{C}$ & 2.63204900 & 2.50947700 & 1.28359000 \\
\hline $\mathrm{C}$ & 3.77953100 & 5.07445400 & 1.48226600 \\
\hline $\mathrm{H}$ & 2.48593700 & 5.62886800 & -0.13148600 \\
\hline
\end{tabular}




$\begin{array}{llll}\mathrm{C} & 3.60413100 & 2.74575600 & 2.22798400 \\ \mathrm{H} & 2.17959100 & 1.52718000 & 1.19683600 \\ \mathrm{C} & 4.18908100 & 4.02921300 & 2.33807000 \\ \mathrm{H} & 4.21962300 & 6.06076300 & 1.56205500 \\ \mathrm{H} & 3.93872200 & 1.96471900 & 2.90234200 \\ \mathrm{O} & 5.12627400 & 4.15304900 & 3.28563700 \\ \mathrm{C} & 5.76952100 & 5.42213300 & 3.48470900 \\ \mathrm{H} & 6.47568800 & 5.26544200 & 4.30017900 \\ \mathrm{H} & 6.30947200 & 5.73345100 & 2.58508000 \\ \mathrm{H} & 5.04263100 & 6.18844900 & 3.77062200\end{array}$

TS20a-21a

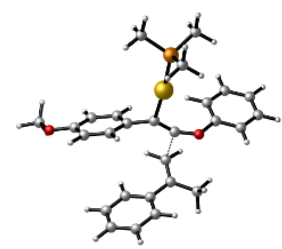

$E=-1674.668192$ Hartrees

$G=1674.232423$ Hartrees

$\begin{array}{llll}\text { C } & 3.86614700 & 0.93222900 & -1.11027000\end{array}$

$\begin{array}{llll}\text { C } & 4.47128600 & 0.84293600 & 0.11463400\end{array}$

$\begin{array}{llll}\text { C } & 1.68767600 & 0.69776600 & -0.42802200\end{array}$

$\mathrm{H} \quad 3.85055600 \quad 0.09569800 \quad-1.79837500$

$\mathrm{H} \quad 3.63413200 \quad 1.90859900 \quad-1.52624400$

C $\quad 1.19933800 \quad-0.43419600 \quad-0.79760200$

$\mathrm{Au} \quad-0.90236500 \quad-0.51285200 \quad-0.36759500$

$\begin{array}{llll}\mathrm{P} & -3.19428700 & -0.76952400 & 0.01641600\end{array}$

$\begin{array}{llll}\text { C } & -4.19272000 & 0.64545600 & -0.58372800\end{array}$

$\mathrm{H} \quad \begin{array}{llll}\mathrm{H} & -5.25646100 & 0.46834000 & -0.39576000\end{array}$

$\mathrm{H} \quad \begin{array}{llll}\mathrm{H} & -3.87967100 & 1.55690700 & -0.06722300\end{array}$

$\mathrm{H} \quad \begin{array}{llll}\mathrm{H} & -4.02930000 & 0.77770700 & -1.65664700\end{array}$

C $\quad-3.89364000 \quad-2.24675000 \quad-0.81226200$

$\mathrm{H} \quad-3.39064800 \quad-3.14439600 \quad-0.44243900$

$\mathrm{H} \quad-4.96688000 \quad-2.32283100 \quad-0.61064800$

$\mathrm{H} \quad-3.72991900 \quad-2.17313500 \quad-1.89075100$

$\begin{array}{llll}\text { C } & -3.62564200 & -0.95720900 & 1.78703800\end{array}$

$\mathrm{H} \quad-3.30673400 \quad-0.06751800 \quad 2.33684300$

$\mathrm{H} \quad-4.70598200 \quad-1.08990200 \quad 1.90295900$

$\mathrm{H} \quad-3.10659300 \quad-1.82801000 \quad 2.19672000$

$\begin{array}{llll}\text { O } & 1.49590900 & 1.81649900 & 0.19765400\end{array}$ 


\begin{tabular}{|c|c|c|c|}
\hline $\mathrm{C}$ & 1.12973400 & 2.99884600 & -0.49105000 \\
\hline $\mathrm{C}$ & 1.09554100 & 4.14490200 & 0.29799100 \\
\hline $\mathrm{C}$ & 0.80865700 & 3.00713300 & -1.84431600 \\
\hline $\mathrm{C}$ & 0.71754400 & 5.34813900 & -0.29812000 \\
\hline $\mathrm{H}$ & 1.35597100 & 4.08542700 & 1.34956600 \\
\hline $\mathrm{C}$ & 0.43485500 & 4.22379600 & -2.42250200 \\
\hline $\mathrm{H}$ & 0.84948500 & 2.09716600 & -2.43179500 \\
\hline C & 0.38798500 & 5.39043800 & -1.65640400 \\
\hline $\mathrm{H}$ & 0.68338900 & 6.25248100 & 0.30155100 \\
\hline $\mathrm{H}$ & 0.18200500 & 4.25258400 & -3.47800900 \\
\hline $\mathrm{H}$ & 0.09819100 & 6.33030000 & -2.11587500 \\
\hline C & 4.55440100 & 2.05993200 & 0.99728700 \\
\hline $\mathrm{H}$ & 4.24981700 & 2.96044000 & 0.46093600 \\
\hline $\mathrm{H}$ & 3.90849500 & 1.95462200 & 1.87720400 \\
\hline $\mathrm{H}$ & 5.57529300 & 2.20009800 & 1.36904000 \\
\hline $\mathrm{C}$ & 5.04238500 & -0.42420400 & 0.62268100 \\
\hline $\mathrm{C}$ & 5.17924000 & -0.64662400 & 2.00797300 \\
\hline $\mathrm{C}$ & 5.49151500 & -1.42969300 & -0.25823700 \\
\hline C & 5.71321000 & -1.83960400 & 2.49250800 \\
\hline $\mathrm{H}$ & 4.84920100 & 0.10817600 & 2.71353500 \\
\hline $\mathrm{C}$ & 6.03304500 & -2.61683200 & 0.22779800 \\
\hline $\mathrm{H}$ & 5.44069600 & -1.27169900 & -1.33028800 \\
\hline $\mathrm{C}$ & 6.14194300 & -2.82954900 & 1.60524500 \\
\hline $\mathrm{H}$ & 5.79655000 & -1.99483200 & 3.56430600 \\
\hline $\mathrm{H}$ & 6.37955300 & -3.37400400 & -0.46947600 \\
\hline $\mathrm{H}$ & 6.56484700 & -3.75594200 & 1.98308400 \\
\hline $\mathrm{C}$ & 1.73063200 & -1.63060300 & -1.46833000 \\
\hline $\mathrm{C}$ & 1.84666300 & -2.84739300 & -0.76724900 \\
\hline $\mathrm{C}$ & 2.08211100 & -1.61002300 & -2.82621800 \\
\hline $\mathrm{C}$ & 2.32688700 & -3.98727300 & -1.39552500 \\
\hline $\mathrm{H}$ & 1.56827300 & -2.89023300 & 0.28144100 \\
\hline $\mathrm{C}$ & 2.56047400 & -2.75433100 & -3.46962800 \\
\hline $\mathrm{H}$ & 1.98092500 & -0.68973900 & -3.39349700 \\
\hline $\mathrm{C}$ & 2.68760200 & -3.95259100 & -2.75396700 \\
\hline $\mathrm{H}$ & 2.42807700 & -4.92161700 & -0.85231200 \\
\hline $\mathrm{H}$ & 2.82430300 & -2.69945000 & -4.51922700 \\
\hline $\mathrm{O}$ & 3.13804100 & -5.12323700 & -3.27677300 \\
\hline $\mathrm{C}$ & 3.49630100 & -5.15858400 & -4.65809500 \\
\hline $\mathrm{H}$ & 3.81716900 & -6.18231700 & -4.85744300 \\
\hline $\mathrm{H}$ & 2.64184800 & -4.91705400 & -5.30152300 \\
\hline
\end{tabular}




\section{Int21a}

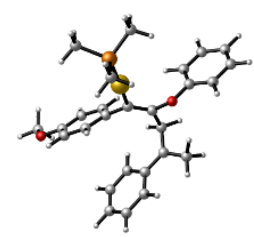

$E=-1674.700136$ Hartrees
$G=1674.259394$ Hartrees

$\begin{array}{llll}\text { C } & 3.41202900 & 1.07892500 & -0.94891700\end{array}$

$\begin{array}{llll}\text { C } & 3.98418400 & 0.72104400 & 0.38255900\end{array}$

$\begin{array}{llll}\text { C } & 1.91225500 & 0.80371400 & -0.72412500\end{array}$

$\mathrm{H} \quad 3.80022400 \quad 0.48428600 \quad-1.76822500$

$\mathrm{H} \quad 3.56861100 \quad 2.13699200 \quad-1.17421500$

$\begin{array}{llll}\text { C } & 1.24679800 & -0.37043000 & -0.86336200\end{array}$

$\mathrm{Au} \quad-0.75043300 \quad-0.50926600 \quad-0.32225900$

$\mathrm{P} \quad-3.03375100 \quad-0.74950100 \quad 0.28461900$

$\begin{array}{llll}\text { C } & -4.08390800 & 0.70571400 & -0.10471400\end{array}$

$\mathrm{H} \quad \begin{array}{llll}\mathrm{H} & -5.12072600 & 0.52721900 & 0.19740900\end{array}$

$\mathrm{H} \quad \begin{array}{llll}\mathrm{H} & -3.69998800 & 1.58478000 & 0.42043800\end{array}$

$\mathrm{H} \quad-4.05009500 \quad 0.90239400 \quad-1.18002000$

C $\quad-3.87176400 \quad-2.15641300 \quad-0.54634800$

$\mathrm{H} \quad-3.34649800 \quad-3.08583800 \quad-0.30847500$

$\mathrm{H} \quad-4.91394300 \quad-2.23572000 \quad-0.22108000$

$\mathrm{H} \quad-3.84123100 \quad-2.00936100 \quad-1.62957600$

C $\quad-3.30245900 \quad-1.04668500 \quad 2.07727500$

$\mathrm{H} \quad-2.91302500 \quad-0.19957200 \quad 2.64892300$

$\mathrm{H} \quad-4.36793200 \quad-1.17179300 \quad 2.29548800$

$\mathrm{H} \quad-2.76329000 \quad-1.94789500 \quad 2.38272800$

$\begin{array}{llll}\mathrm{O} & 1.24658500 & 1.87459700 & -0.12780800\end{array}$

$\begin{array}{llll}\text { C } & 1.08458000 & 3.05559600 & -0.83229700\end{array}$

$\begin{array}{lllll}\text { C } & 0.79748100 & 4.19381300 & -0.07066300\end{array}$

$\begin{array}{llll}\text { C } & 1.14590800 & 3.12733800 & -2.22740900\end{array}$

$\begin{array}{llll}\text { C } & 0.57175300 & 5.40997600 & -0.71340700\end{array}$

$\begin{array}{llll}\mathrm{H} & 0.74885500 & 4.11096600 & 1.01081000\end{array}$

$\begin{array}{llll}\text { C } & 0.92684900 & 4.35649600 & -2.85606900\end{array}$

$\mathrm{H} \quad 1.34619300 \quad 2.23643800 \quad-2.81273000$

$\begin{array}{llll}\text { C } & 0.63971500 & 5.49930600 & -2.10783700\end{array}$

$\begin{array}{llll}\mathrm{H} & 0.34612900 & 6.29199000 & -0.12076700\end{array}$ 
TS20a-22a

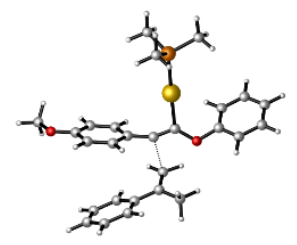

$E=-1674.672595$ Hartrees

$\mathrm{G}=1674.236355$ Hartrees 


$\begin{array}{llll}\mathrm{Au} & 1.44116100 & 2.41411300 & -2.60405300 \\ \mathrm{P} & 3.01670800 & 1.31612900 & -3.95958600 \\ \mathrm{C} & 3.44955900 & -0.35933500 & -3.35284900 \\ \mathrm{H} & 3.87011200 & -0.28505000 & -2.34624900 \\ \mathrm{H} & 4.17942000 & -0.83023800 & -4.01899700 \\ \mathrm{H} & 2.54699300 & -0.97498100 & -3.30921600 \\ \mathrm{C} & 2.43758700 & 1.07018100 & -5.68093500 \\ \mathrm{H} & 3.19584800 & 0.54196000 & -6.26746200 \\ \mathrm{H} & 2.23638100 & 2.04200800 & -6.14012100 \\ \mathrm{H} & 1.51155200 & 0.48893200 & -5.67555600 \\ \mathrm{C} & 4.61545500 & 2.20190900 & -4.10980800 \\ \mathrm{H} & 4.44578800 & 3.19105400 & -4.54381000 \\ \mathrm{H} & 5.30410600 & 1.63885800 & -4.74783900 \\ \mathrm{H} & 5.05861100 & 2.32601400 & -3.11790500 \\ \mathrm{C} & -0.03801900 & 3.32763300 & -1.39370500 \\ \mathrm{C} & 0.48824100 & 3.78915100 & -0.29298100 \\ \mathrm{C} & -1.71228800 & 4.14317000 & 1.59200000 \\ \mathrm{C} & -2.80271000 & 2.45483000 & -3.38088100 \\ \mathrm{C} & -1.17281400 & 4.15274400 & -3.99929100 \\ \mathrm{C} & -3.28111500 & 2.44553000 & -4.69271500 \\ \mathrm{H} & -1.25934900 & 5.08453400 & 0.71200400 \\ \mathrm{H} & -1.77258200 & 5.23880800 & -0.23015900 \\ \mathrm{H} & -0.52759100 & 5.83041600 & 0.99689800 \\ \mathrm{C} & -2.81833500 & 3.20038800 & 1.18822200 \\ \mathrm{H} & -3.23855200 & 3.48216000 & 0.22234100 \\ \mathrm{H} & -3.61703600 & 3.20251600 & 1.93842000 \\ \mathrm{H} & -2.46073600 & 2.16737700 & 1.10832500 \\ \mathrm{C} & -1.13959400 & 4.00776400 & 2.94836100 \\ \mathrm{C} & -1.26263600 & 2.79846000 & 3.66431200 \\ \mathrm{C} & -1.35837100 & 3.33141100 & -1.71458700 \\ \mathrm{C} & -0.46025100 & 5.07610700 & 3.57174500 \\ \mathrm{H} & -0.70733300 & 2.65577800 & 4.93483100 \\ \mathrm{H} & -1.78159900 & 1.95610900 & 3.22014900 \\ \mathrm{H} & 0.08789800 & 4.93411300 & 4.84359300 \\ \mathrm{H} & -0.38711100 & 6.03501800 & 3.07015600 \\ \mathrm{H} & -1.74841400 & 3.30552300 & -3.05069000 \\ \mathrm{H} & & & \end{array}$




$\begin{array}{llll}\mathrm{H} & -3.23399700 & 1.81598200 & -2.61669500 \\ \mathrm{C} & -1.65722100 & 4.12699100 & -5.30983900 \\ \mathrm{H} & -0.37302900 & 4.82756300 & -3.71206300 \\ \mathrm{C} & -2.70864500 & 3.27604100 & -5.66043600 \\ \mathrm{H} & -4.10095500 & 1.78406700 & -4.95708600 \\ \mathrm{H} & -1.21666700 & 4.78519700 & -6.05315900 \\ \mathrm{H} & -3.08317400 & 3.26427900 & -6.67949300 \\ \mathrm{C} & 1.56360400 & 3.88626400 & 0.61712700 \\ \mathrm{C} & 2.34223600 & 5.06728700 & 0.69933900 \\ \mathrm{C} & 1.89236500 & 2.80277000 & 1.47786400 \\ \mathrm{C} & 3.41705300 & 5.16236800 & 1.56801200 \\ \mathrm{H} & 2.09456200 & 5.90762800 & 0.05843000 \\ \mathrm{C} & 2.96073500 & 2.88979700 & 2.34625100 \\ \mathrm{H} & 1.29938000 & 1.89539100 & 1.43384700 \\ \mathrm{C} & 3.73512400 & 4.06921500 & 2.39961300 \\ \mathrm{H} & 4.00288000 & 6.07251000 & 1.60122000 \\ \mathrm{H} & 3.22599000 & 2.06450000 & 2.99865600 \\ \mathrm{O} & 4.75378600 & 4.05631000 & 3.27501200 \\ \mathrm{C} & 5.60716700 & 5.20538200 & 3.37449500 \\ \mathrm{H} & 6.34697000 & 4.95413300 & 4.13499800 \\ \mathrm{H} & 6.11281200 & 5.40381400 & 2.42386200 \\ \mathrm{H} & 5.04461100 & 6.08948200 & 3.69123200\end{array}$

Int22a

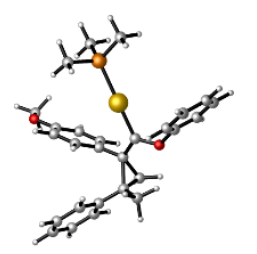

$E=-1674.712284$ Hartrees

$G=1674.269795$ Hartrees

$\begin{array}{llll}\mathrm{Au} & 1.15496900 & 2.65853200 & -2.14076100 \\ \mathrm{P} & 2.87259800 & 1.46546700 & -3.26170100 \\ \mathrm{C} & 3.24203400 & -0.16457100 & -2.50611900 \\ \mathrm{H} & 3.54411100 & -0.02308700 & -1.46471900 \\ \mathrm{H} & 4.04690200 & -0.66747100 & -3.05137000 \\ \mathrm{H} & 2.34510100 & -0.78976800 & -2.52653700 \\ \mathrm{C} & 2.51960300 & 1.10514900 & -5.02419600 \\ \mathrm{H} & 3.35281900 & 0.56159400 & -5.48054000 \\ \mathrm{H} & 2.36105500 & 2.04320500 & -5.56337800\end{array}$




\begin{tabular}{|c|c|c|c|}
\hline $\mathrm{H}$ & 1.60988400 & 0.50285500 & -5.09863400 \\
\hline $\mathrm{C}$ & 4.47275100 & 2.36141800 & -3.26656000 \\
\hline $\mathrm{H}$ & 4.35562000 & 3.31251900 & -3.79330700 \\
\hline $\mathrm{H}$ & 5.24696800 & 1.76597400 & -3.76030200 \\
\hline $\mathrm{H}$ & 4.77477800 & 2.56878700 & -2.23626900 \\
\hline $\mathrm{C}$ & -0.27856100 & 3.77875100 & -1.16132500 \\
\hline $\mathrm{C}$ & -0.11091800 & 4.30836400 & 0.17082700 \\
\hline $\mathrm{C}$ & -1.39661800 & 4.18341900 & 1.25015600 \\
\hline $\mathrm{C}$ & -1.00364600 & 5.46779000 & 0.64962200 \\
\hline $\mathrm{H}$ & -1.66714700 & 5.88759300 & -0.10009000 \\
\hline $\mathrm{H}$ & -0.48559300 & 6.20624500 & 1.25119800 \\
\hline $\mathrm{C}$ & -2.68538900 & 3.51764800 & 0.79469500 \\
\hline $\mathrm{H}$ & -3.01290300 & 3.86337700 & -0.18382700 \\
\hline $\mathrm{H}$ & -3.46683100 & 3.78101900 & 1.51639200 \\
\hline $\mathrm{H}$ & -2.61249000 & 2.42715800 & 0.77589700 \\
\hline $\mathrm{C}$ & -0.98754700 & 3.83382400 & 2.65372000 \\
\hline $\mathrm{C}$ & -1.01609600 & 2.49393700 & 3.08109600 \\
\hline $\mathrm{C}$ & -0.62960500 & 4.82027000 & 3.58636100 \\
\hline $\mathrm{C}$ & -0.68817500 & 2.15197800 & 4.39197100 \\
\hline $\mathrm{H}$ & -1.29058700 & 1.70910600 & 2.38339000 \\
\hline $\mathrm{C}$ & -0.30594200 & 4.47875700 & 4.90088700 \\
\hline $\mathrm{H}$ & -0.61976600 & 5.86628200 & 3.29952400 \\
\hline $\mathrm{C}$ & -0.33011800 & 3.14429200 & 5.30848900 \\
\hline $\mathrm{H}$ & -0.71255400 & 1.10983300 & 4.69754300 \\
\hline $\mathrm{H}$ & -0.03969300 & 5.26052800 & 5.60656900 \\
\hline $\mathrm{H}$ & -0.07678900 & 2.87924700 & 6.33094100 \\
\hline $\mathrm{O}$ & -1.39106400 & 4.16231100 & -1.75838000 \\
\hline $\mathrm{C}$ & -1.74973800 & 3.67245700 & -3.03168700 \\
\hline $\mathrm{C}$ & -2.10518900 & 2.33579000 & -3.18647500 \\
\hline $\mathrm{C}$ & -1.81667300 & 4.59295400 & -4.07123200 \\
\hline $\mathrm{C}$ & -2.52573500 & 1.90612800 & -4.44805500 \\
\hline $\mathrm{H}$ & -2.06639200 & 1.65687400 & -2.34100300 \\
\hline $\mathrm{C}$ & -2.23660600 & 4.14517500 & -5.32583600 \\
\hline $\mathrm{H}$ & -1.54491300 & 5.62914100 & -3.89842700 \\
\hline $\mathrm{C}$ & -2.58926500 & 2.80575500 & -5.51540500 \\
\hline $\mathrm{H}$ & -2.81055700 & 0.86803100 & -4.58980700 \\
\hline $\mathrm{H}$ & -2.28986900 & 4.84713400 & -6.15236600 \\
\hline $\mathrm{H}$ & -2.92059000 & 2.46577000 & -6.49177600 \\
\hline $\mathrm{C}$ & 1.27815300 & 4.18248100 & 0.74084700 \\
\hline $\mathrm{C}$ & 2.11193200 & 5.30087000 & 0.84242500 \\
\hline
\end{tabular}




$\begin{array}{llll}\mathrm{C} & 1.79809100 & 2.93724900 & 1.14074700 \\ \mathrm{C} & 3.41999200 & 5.20234500 & 1.32733700 \\ \mathrm{H} & 1.74420100 & 6.27702500 & 0.53834100 \\ \mathrm{C} & 3.09506300 & 2.81990100 & 1.62343000 \\ \mathrm{H} & 1.17453900 & 2.05062200 & 1.08249100 \\ \mathrm{C} & 3.91888000 & 3.95461800 & 1.72258300 \\ \mathrm{H} & 4.03137300 & 6.09448800 & 1.39105600 \\ \mathrm{H} & 3.48860600 & 1.85931500 & 1.94161800 \\ \mathrm{O} & 5.16874800 & 3.73828000 & 2.21180300 \\ \mathrm{C} & 6.05344500 & 4.85175900 & 2.33420500 \\ \mathrm{H} & 6.98429600 & 4.45027200 & 2.73840100 \\ \mathrm{H} & 6.25355300 & 5.31587000 & 1.36102300 \\ \mathrm{H} & 5.65729700 & 5.60632700 & 3.02391400\end{array}$




\section{References}

i. de Orbe, M. E. Intermolecular [2+2] Cycloaddition of Alkynes with Alkenes Catalyzed by Gold(I). Org. Synth. 2016, 93, 115-126.

ii. Johnson, J. S.; Chong, E.; Tu, K. N.; Blum, S. A. Kinetic Study of Carbophilic Lewis Acid Catalyzed Oxyboration and the Noninnocent Role of Sodium Chloride. Organometallics 2016, 35 (5), 655-662.

iii Graf, K.; Rühl, C. L.; Rudolph, M.; Rominger, F.; Hashmi, A. S. K. Metal-Free Oxidative Cyclization of Alkynyl Aryl Ethers to Benzofuranones. Angew. Chem. Int. Ed. 2013, 52, 12727-12731.

iv . García-Morales, C.; Ranieri, B.; Escofet, I.; López-Suarez, L.; Obradors, C.; Konovalov, A. I.; Echavarren, A. M. Enantioselective Synthesis of Cyclobutenes by Intermolecular [2+2] Cycloaddition with Non-C2 Symmetric Digold Catalysts. J. Am. Chem. Soc. 2017, 139, 13628-13631.

v. Lux, M.; Klussmann, M. Additions of Aldehyde-Derived Radicals and Nucleophilic N-Alkylindoles to Styrenes by Photoredox Catalysis. Org. Lett. 2020, 22, 3697-3701.

vi. Iwai, R.; Suzuki, S.; Sasaki, S.; Sairi, A. S.; Igawa, K.; Suenobu, T.; Morokuma, K.; Konishi, G. Bridged Stilbenes: AIEgens Designed via a Simple Strategy to Control the Non-Radiative Decay Pathway. Angew. Chem. Int. Ed. 2020, 59, 10566-10573.

vii. Song, J. J.; Tan, Z.; Reeves, J. T.; Fandrick, D. R.; Yee, N. K.; Senanayake H. N-Heterocyclic CarbeneCatalyzed Silyl Enol Ether Formation†. Org. Lett. 2008, 10, 877-880.

viii. Mato, M.; García-Morales, C.; Echavarren, A. M. Synthesis of Trienes by Rhodium-Catalyzed Assembly and Disassembly of Non-Acceptor Cyclopropanes. ACS Catal. 2020, 10, 3564-3570.

ix. Sosa, J. R.; Tudjarian, A. A.; Minehan, T. G. Synthesis of Alkynyl Ethers and Low-Temperature Sigmatropic Rearrangement of Allyl and Benzyl Alkynyl Ethers. Org. Lett. 2008, 10, 5091-5094.

x. Petersen, K. S.; Stoltz, B. M. Palladium-Catalyzed, Asymmetric Baeyer-Villiger Oxidation of Prochiral Cyclobutanones with PHOX Ligands. Tetrahedron 2011, 67, 4352-4357.

xi. Gaussian 09, Revision B.1, Frisch, M. J., Trucks, G. W., Schlegel, H. B., Scuseria, G. E., Robb, M. A., Cheeseman, J. R., Scalmani, G., Barone, V., Mennucci, B., Petersson, G. A., Nakatsuji, H., Caricato, M., Li, X., Hratchian, H. P., Izmaylov, A. F., Bloino, J., Zheng, G., Sonnenberg, J. L., Hada, M., Ehara, M., Toyota, K., Fukuda, R., Hasegawa, J., Ishida, M., Nakajima, T., Honda, Y., Kitao, O., Nakai, H., Vreven, T., Montgomery, J. A., Peralta, Jr. J. E., Ogliaro, F., Bearpark, M., Heyd, J. J., Brothers, E., Kudin, K. N., Staroverov, V. N., Kobayashi, R., Normand, J., Raghavachari, K., Rendell, A., Burant, J. C., Iyengar, S. S., Tomasi, J., Cossi, M., Rega, N., Millam, J. M., Klene, M., Knox, J. E., Cross, J. B., Bakken, V., Adamo, C., Jaramillo, J., Gomperts, R., Stratmann, R. E., Yazyev, O., Austin, A. J., Cammi, R., Pomelli, C., Ochterski, J. W., Martin, R.L., Morokuma, K.,, Zakrzewski, V. G., Voth, G. A., Salvador, P., Dannenberg, J. J., Dapprich, S., Daniels, A. D., Farkas, Ö., Foresman, J. B., Ortiz, J. V., Cioslowski, J., Fox, D. J. Gaussian, Inc., Wallingford CT 2009.

xii. Becke, A. D. Density-functional Thermochemistry. III. The Role of Exact Exchange. J. Chem. Phys. 1993, 98, $5648-5652$.

xiii. Hay, P. J.; Wadt, W. R. Ab Initio Effective Core Potentials for Molecular Calculations. Potentials for the Transition Metal Atoms Sc to Hg. J. Chem. Phys. 1985, 82, 270-283. 
xiv. Hay, P. J.; Wadt, W. R. Ab Initio Effective Core Potentials for Molecular Calculations. Potentials for K to Au Including the Outermost Core Orbitals. J. Chem. Phys. 1985, 82, 299-310.

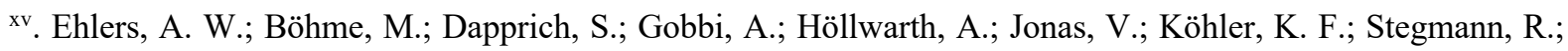
Veldkamp, A.; Frenking, G. A Set of F-Polarization Functions for Pseudo-Potential Basis Sets of the Transition Metals Sc-Cu, Y-Ag and La-Au. Chemical Physics Letters 1993, 208, 111-114.

xvi . Hehre, W. J.; Ditchfield, R.; Pople, J. A. Self —Consistent Molecular Orbital Methods. XII. Further Extensions of Gaussian-Type Basis Sets for Use in Molecular Orbital Studies of Organic Molecules. J. Chem. Phys. 1972, $56,2257-2261$.

xvii . Marenich, A. V.; Cramer, C. J.; Truhlar, D. G. Universal Solvation Model Based on Solute Electron Density and on a Continuum Model of the Solvent Defined by the Bulk Dielectric Constant and Atomic Surface Tensions. J. Phys. Chem. B 2009, 113, 6378-6396.

xviii. Sweis, R. F.; Schramm, M. P.; Kozmin, S. A. Silver-Catalyzed [2 + 2] Cycloadditions of Siloxy Alkynes. J. Am. Chem. Soc. 2004, 126, 7442-7443.

${ }^{\text {xix }}$. de Orbe, M. E.; Zanini, M.; Quinonero, O.; Echavarren, A. M. Gold- or Indium-Catalyzed Cross-Coupling of Bromoalkynes with Allylsilanes through a Concealed Rearrangement. ACS Catal. 2019, 9, 7817-7822.

xx. (a)Yamataka, H.; Biali, S. E.; Rappoport, Z. Structural Sensitivity of 1,2-Aryl Rearrangements in Triarylvinyl Cations: Bridged Transition States vs Bridged Intermediates and Inversion vs Helicity Retention. J. Org. Chem. 1998, 63, 9105-9108. (b) Rappoport, Z.; Kobayashi, S.; Stanger, A.; Boese, R. Crystal Structure of 1,2-Diphenyl5,7-Di-Tert-Butylspiro[2.5]Octa-1,4,7-Trien-6-One, a Possible Model for Diphenylvinylidenephenonium Ions. $J$. Org. Chem. 1999, 64, 4370-4375. (c) Gronheid, R.; Zuilhof, H.; Hellings, M. G.; Cornelisse, J.; Lodder, G. Photochemical Generation of a Primary Vinyl Cation from (E)-Bromostyrene: Mechanisms of Formation and Reaction. J. Org. Chem. 2003, 68, 3205-3215. 